\title{
Verwaltete Vermarktlichung
}

Zur Sonderstellung der öffentlichen Verwaltung in der Ideenproduktion für die Politik am Beispiel der Wohneigentumsförderung in der Bundesrepublik Deutschland

\author{
DISSERTATION \\ zur Erlangung des Doktorgrades \\ der Sozialwissenschaftlichen Fakultät \\ der Georg-August-Universität Göttingen
}

vorgelegt von

Dipl.-Volksw. Heide Haas

geboren in Bergisch Gladbach

Göttingen, 2019 
Erstbetreuer: $\quad$ Prof. Dr. Sascha Münnich

Weitere Betreuer: Prof. Dr. Timo Weishaupt

Dr. Alexander Engel

Tag der mündlichen Prüfung: 15. März 2019 
Für Chococino. 



\section{Zusammenfassung}

Angesichts weitreichender Folgen der US-amerikanischen Subprime-Krise und steigender Wohnungspreise in zahlreichen Ländern haben die Sozialwissenschaften Wohnungspolitik als Forschungsgegenstand wiederentdeckt. Zahlreiche Untersuchungen weisen Trends der Vermarktlichung und Finanzialisierung nach: ein Rückzug des Staates aus der Wohnungsversorgung scheint weltweit mit einer zunehmenden Förderung von Wohneigentum einher zu gehen. Allerdings führen diese globalen Trends nicht zu einer Vereinheitlichung der Wohnungsmärkte. So ist Deutschland weiterhin das einzige Land in der Europäischen Union, in dem mehr Menschen zur Miete wohnen als in ihren eigenen vier Wänden. Die vorliegende Dissertationsschrift beschreibt detailliert Besonderheiten von Vermarktlichung und Finanzialisierung in der Wohneigentumsförderung in Deutschland als kleinteiligen institutionellen Wandel seit dem Ende des Zweiten Weltkrieges bis 2008.

Basierend auf einer Analyse ausgewählter Bundestagsdebatten zeigt die Arbeit, wie mehrere Reformen dazu beigetragen haben, die Wohneigentumsförderung allmählich von einer familien- und gesellschaftspolitischen Maßnahme im Rahmen des Sozialen Wohnungsbaus zu einem Bestandteil der staatlich geförderten, privaten Altersvorsorge zu machen. Anhand der langfristigen Betrachtung wird deutlich, dass ideeller Wandel ein entscheidender Faktor für den Wandel des Regelgehalts der Eigenheimförderung war: Die Entwicklung von sozialpolitischen Zielsetzungen der Eigenheimförderung zu ökonomischen Begründungsmustern machten eine weitere Vermarktlichung und Finanzialisierung in der Wohnungspolitik erst möglich.

Als zentrale Faktoren für diesen Wandel identifiziert die Autorin Akteure und Mechanismen der politikfeldspezifischen Wissensproduktion: Akteure dieses knowledge regimes konkretisieren abstraktere Weltbilder zu politischen Zielsetzungen und Instrumenten. Sie stellen Problemdefinitionen sowie analytische Tools zu deren Erfassung für politische 
Akteure bereit. Für die Bedeutungszunahme der ökonomischen Begründungsmuster waren zwei Bedingungen entscheidend: Erstens wurde eine Entpolitisierung der sozialpolitischen Debatten anhand ökonomischer Analysen dadurch begünstigt, dass für die politischen Parteien Wohnungspolitik im hier betrachteten Zeitraum an Bedeutung verloren hat. Zweitens ist der gestiegene Einfluss der wirtschaftlichen Deutungen nicht auf eine Initiative von Wirtschaftswissenschaftlern zurückzuführen, sondern auf deren Einbeziehung durch die öffentliche Verwaltung. In der Wissensproduktion nehmen öffentliche Stellen eine doppelte Funktion ein: Zum einen bietet die amtliche Statistik mit zunehmenden Datenbeständen eine notwendige Grundlage zur wirtschaftswissenschaftlichen Analyse der Eigenheimförderung. Zum anderen verschafft die Ministerialbürokratie wirtschaftswissenschaftlicher Expertise über Auftragsgutachten und Beiratstätigkeiten den Zugang zur politikfeldspezifischen Wissensmaschinerie.

Die öffentliche Verwaltung ist Produzent und gatekeeper der Wissensproduktion zugleich. Vermarktlichung und Finanzialisierung können somit in der langen Frist als nicht-intendierte Folgen von Verwaltungshandeln in der Wissensproduktion gesehen werden: die zunehmende ökonomische Betrachtung der Eigenheimförderung in der Bundesrepublik wäre nicht denkbar ohne die durch die Ministerialverwaltung verfolgte Zielvorstellung, wohnungspolitische Arbeit an exaktes statistisches Berichtswesen und externe Expertise zu knüpfen. Vermarktlichung und Finanzialisierung sind weder Ausdruck eines internationalen, neoliberalen Trends oder anonymer Globalisierungsprozesse. Stattdessen sind Ideen als eine Bedingung institutionellen Wandels von den an ihrer Produktion beteiligten Akteuren und den Kontextbedingungen der Wissensproduktion abhängig. 


\section{Abstract}

Due to far-reaching consequences of the US subprime crisis and rising housing prices in many countries, social sciences have rediscovered housing policy as a research topic. Numerous studies show trends in marketisation and financialisation: a withdrawal of the state from housing provision is accompanied by an increasing promotion of home ownership worldwide. However, these global trends do not lead to a uniformity of housing markets. Germany is still the only country in the European Union where more people live for rent than in their own home. This dissertation describes in detail peculiarities of the marketisation and financialisation of home ownership promotion in Germany as small-scale institutional change since the end of the Second World War until 2008.

Based on an analysis of selected parliamentary debates, the study shows how several reforms have helped to gradually change home ownership promotion from a family and socio-political measure within social housing to a part of state-subsidized private retirement provision. Based on a long-term perspective, it becomes clear that changing ideas are decisive for the change in home ownership promotion in Germany: The transformation of socio-political objectives of home ownership promotion into economic justification enabled further marketisation and financialisation in housing politics.

The author identifies actors and mechanisms of knowledge production as key factors for this transformation: knowledge regime actors concretise abstract worldviews into political objectives and instruments. They provide problem definitions as well as analytical tools for political actors. Two conditions were decisive for increasing the significance of the economic models of justification. First, a depoliticisation of sociopolitical debates based on economic analyses was favoured by the fact that housing politics became less important for political parties over a long period. Second, the increased influence of economic interpretations is not due to an initiative of economists, but to the inclusion of eco- 
nomics expertise by public administration. With respect to knowledge production, public authorities take on a dual function: on the one hand, official statistics, with an increasing amount of data, provide a necessary basis for the economic analysis of home equity promotion. On the other hand, ministerial bureaucracy provides economic experts access to the field-specific knowledge machinery with commissioned research.

Public administration is producer and gatekeeper of knowledge production at the same time. Marketisation and financialisation can therefore be seen as unintended consequences of administrative action in knowledge production: the increasing economic consideration of home ownership in the Federal Republic of Germany would be inconceivable without the objective of ministerial administration to rest housing politics on accurate statistical reporting and scientific expertise. Marketisation and financialisation are neither an expression of an international, neo-liberal trend nor anonymous globalization processes. Instead, ideas as a condition of institutional change depend on the actors involved in their production and the contextual conditions of knowledge production. 


\section{Danksagungen}

Um die Jahrtausendwende hatten Fragen der Wohnraumversorgung große Teile ihrer einstigen Bedeutung auf bundespolitischer Ebene verloren. Die auf sozialpolitischen Zielvorstellungen aufbauende Wohnungspolitik der Nachkriegszeit hatte sich zu einer Wohnungsmarktpolitik entwickelt: im Mietrecht wurde die Vertragsfreiheit gestärkt, der Soziale Wohnungsbau auf immer kleinere Personenkreise eingegrenzt. 2005 wurde sogar die Eigenheimzulage abgeschafft - ein deutlicher Bruch mit der lange ausgebauten Wohneigentumsförderung. 2015, zu Beginn meiner Arbeiten an dieser Dissertation, hatte sich das Blatt gewendet. Die erstmals seit Jahrzehnten deutlich ansteigenden Wohnkosten in mehreren Städten brachten wohnungspolitische Debatten zurück auf die bundespolitische Agenda. Beschlüsse wie die Mietpreisbremse und das Baukindergeld sowie Debatten um eine Mietendeckelung, Enteignungen großer, privater Wohnungsunternehmen und die Stärkung des Sozialen Wohnungsbaus deuten auf eine Renaissance der klassischen Wohnungspolitik hin. Für ein besseres Verständnis heutiger wohnungspolitischer Entscheidungen und Debatten lohnt ein Blick in deren Geschichte, die, mit Fokus auf die Eigenheimförderung, in dieser Arbeit mit aufgearbeitet ist.

Das vorliegende Buch ist das Ergebnis meines Dissertationsprojekts an der Georg-August-Universität Göttingen, das mit der mündlichen Prüfung am 15. März 2019 abgeschlossen wurde. Nach Abschluss des Studiums der Volkswirtschaftslehre an der Universität zu Köln hatte ich zunächst nicht vor an der Uni zu bleiben und zu promovieren. Ich wollte mit wirtschaftswissenschaftlicher Expertise konkrete Handlungsempfehlungen erarbeiten, vermitteln und politische Veränderungen bewirken. In meiner Zeit als Referentin am Institut der deutschen Wirtschaft Köln vertiefte ich mich erstmals in den in Deutschland noch jungen Bereich der Immobilienökonomie mit zahlreichen Überschneidungen zu gesellschaftspolitischen Fragestellungen. Mit steigender Bedeutung des 
Themenfeldes im tagespolitischen Geschehen und dem doch sehr überschaubaren Expertennetzwerk wuchs mein Interesse an der Frage, wie sich dieses politische Feld genau zusammensetzt, wie es funktioniert und wie Ideen in den politischen Prozess hineinkommen. Als Wissensproduzentin stellte ich mir zunehmend die Frage, unter welchen Bedingungen meine Arbeit überhaupt Einfluss auf politische Entscheidungen haben kann. Schließlich zog es mich aus der privatwirtschaftlichen Forschung doch wieder zurück an die Uni - genauer gesagt in die politische Soziologie, wo ich mich mit dieser und weiteren Fragen auseinandersetzen konnte.

Auf diesem nicht immer leichten Weg haben mich viele Menschen inspiriert, begleitet und ermutigt. Mein erster Dank gilt meinem Erstbetreuer Sascha Münnich, der mir die Freiheit gegeben hat, mich mit den Fragestellungen zu beschäftigen, die mich am meisten interessieren und mich stets darin bestärkt hat bei meinem Kurs zu bleiben. Dank gebührt außerdem Timo Weishaupt und Alexander Engel, die mir als Zweit- und Drittbetreuer jederzeit für Fragen und Anmerkungen zur Verfügung standen. Im Jahr 2017 durfte ich in der Bibliothek des Bundesinstituts für Bau-, Stadt- und Raumforschung recherchieren. Für die Unterstützung bei den aufwendigen Archivrecherchen möchte ich mich vor ausdrücklich bei Stephanie Simon und Martina Schneider bedanken.

Besonderer Dank gilt auch meinen Kolleginnen und Kollegen an der Georg-August-Universität Göttingen, die mich über den gesamten Prozess mit wertvollem Feedback, detaillierten Kommentierungen und ihrer Freundschaft unterstützt haben. Hierfür danke ich vor allem Katharina Legantke, Julia Rohringer, Luzie Sennewald, Jan Weckwerth, Judith Czepek, Vincent Lindner und Lukas Drögemeier. Die Begeisterung für immobilienwirtschaftliche Forschung im Zusammenhang mit Wohnungspolitik verdanke ich meinen ehemaligen Kollegen am Institut der deutschen Wirtschaft Köln, allen voran Michael Voigtländer, Ralph Henger und Philipp Deschermeier. Der fachliche Austausch mit 
den Experten Andrej Holm, Björn Egner und Tobias Just hat viele Erkenntnisse erst möglich gemacht.

Für beständigen Rückhalt - komme was wolle - danke ich meiner Familie, allen voran meinen Eltern. Begleitet und bestärkt haben mich in all den Jahren zudem sehr gute Freunde, denen ich nicht genug danken kann. Für den wiederkehrenden und beständigen Rat das zu tun, was mich inspiriert, danke ich Mark Benecke. Für das Immer-da-sein, auch wenn uns viele Kilometer trennen, danke ich Felix Holtermann, Karina Copil, Julia Schleper, Johanna Füllmann, Lene Karpp und Dorothee Krause. Für die gute Zeit in Göttingen danke ich Klaus Raeker, Katja Schymitzek, Sarah Müller, Rieke Wolters, Peter Walther und Lukas Nykamp. Die Ruhe und Zuversicht, auch in schwierigen Zeiten mit viel Vorfreude in die Ferne zu sehen, verdanke ich Tobias Doeubler. 



\section{Inhaltsverzeichnis}

I Einleitung 1

1 Vermarktlichung \& Ideenproduktion 3

1.1 Vermarktlichung \& Finanzialisierung in der Wohnungs-

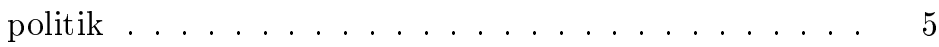

1.2 Ursachen von Vermarktlichung und Finanzialisierung . . 8

1.3 Wohneigentumsförderung in Deutschland . . . . . . . 12

1.4 Methodisches Vorgehen . . . . . . . . . . . . . 16

1.5 Aufbau der Arbeit . . . . . . . . . . . . . . . . 17

II Institutioneller Wandel und Ideenproduktion 21

2 Institutioneller Wandel \& Ideen 23

2.1 Einflussfaktoren institutionellen Wandels . . . . . . . . . 24

2.1.1 Wirtschaftliche \& politische Rahmenbedingungen 28

2.1.2 Interessenbasierte Akteurshandlungen . . . . . . 30

2.2 Ideen und Institutionen $\ldots \ldots \ldots \ldots$

2.2.1 Techniken \& Analytische Tools . . . . . . . . . 40

2.2 .2 Paradigmen . . . . . . . . . . . . . . 42

2.3 Ideenwandel als institutioneller Wandel . . . . . . . . 45 
3.1 Akteure im Regime der Ideenproduktion . . . . . . . . 54

3.1 .1 Parteien . . . . . . . . . . . . . . . 54

3.1.2 Verbände \& Denkfabriken . . . . . . . . . . 58

3.1.3 Experten aus der Wissenschaft . . . . . . . . 64

3.1.4 Öffentliche Verwaltung . . . . . . . . . . . 73

3.2 Mechanismen im Regime der Ideenproduktion . . . . . . 84

3.2.1 Fremdbestimmung der Politik: Technokratie \& political capture . . . . . . . . . . . . 85

3.2.2 Politik bestimmte Ideenproduktion: Dezisionistische Inanspruchnahme von Wissenschaft \& Verbänden . . . . . . . . . . . . 90

3.2.3 Wechselseitige Beziehungen zwischen policy- und Ideenproduzenten: das pragmatistische Modell . 93

3.2.4 Mechanismen der Ideenproduktion für die Politik in der Bundesrepublik Deutschland . . . . . . . . 95

3.3 Zusammenhang zwischen Ideenproduktion und institutionellem Wandel . . . . . . . . . . . . . . 100

4 Methodik 105

4.1 Fallstudiendesign . . . . . . . . . . . . . . 105

4.2 Identifikation wohnungspolitischer Ideen . . . . . . . . 113

4.3 Identifikation von Akteuren \& Mechanismen der Ideenproduktion . . . . . . . . . . . . 117

4.3.1 Institutionalisierte Politikberatung . . . . . . . 118

4.3.2 Nicht institutionalisierte Politikberatung . . . . . 120

4.4 Kritische Würdigung der Methodik . . . . . . . . 127 


\section{Wissensproduktion \& Vermarktlichung in der Eigenheimförderung}

\section{Phase 1: Der Weg zum Fördervorrang des Familien-}

heims

5.1 Wiederaufbau \& Institutionalisierung der Eigenheimförderung auf Bundesebene . . . . . . . . . . . . . . 136

5.2 Familienförderung und Gesellschaftsstabilisierung durch das Familienheim . . . . . . . . . . . . . . . . 143

5.2 .1 Familienförderung . . . . . . . . . . . 149

5.2.2 Gesellschaftsstabilisierung gegen Vergemeinschaftung . . . . . . . . . . . . . 152

5.3 Politische Ideenproduktion . . . . . . . . . . . . . 158

5.3.1 Bündnisse aus Parteien und Verbänden . . . . . 159

5.3.2 Theologie statt Wirtschaftswissenschaften . . . . 171

5.3.3 Aufbau staatlicher Wissensproduktion . . . . . . 182

5.4 Phase 1: Vermarktlichung ohne Marktwissen . . . . . . . 190

6 Phase 2: Vom Sozialen Wohnungsbau zur individuellen steuerlichen Förderung $\quad 197$

6.1 Ende der Wohnungsnot und Ideenwandel . . . . . . . . . 199

6.2 Individuelle Vermögensbildung durch

Wohneigentum ............... . 211

6.2.1 Zielgenauigkeit \& individualisierte Förderung . . 216

6.2.2 Erlebbares und anlegbares Vermögen . . . . . . . 221

6.2.3 Familienförderung als Rahmenerzählung . . . . . 226

6.3 Nachfragegesteuerte Ideenproduktion . . . . . . . . . . 230

6.3.1 Parteien \& Verbände in Wissenschaftseuphorie . 231

6.3.2 Wissenschaft auf Nachfrage . . . . . . . . . . 241

6.3.3 Etablierung von Ministerialbürokratie \& amtlicher Statistik . . . . . . . . . . . . . . 248

6.4 Phase 2: Vermarktlichung mithilfe von Marktexperten . 261 
7 Phase 3: Verringerte Wohneigentumsförderung als Teil der privaten Altersvorsorge

7.1 Bedeutungsverlust der Wohnungspolitik \& Haushaltsdisziplin . . . . . . . . . . . . . . . . . 270

7.2 Schlanker Staat \& Wohneigentum als Finanzanlage . . . 277

7.2 .1 Austerität . . . . . . . . . . . . . . . . 282

7.2 .2 Private Altersvorsorge . . . . . . . . . . . . . 284

7.3 Vielfalt datenbasierter Ideenproduktion . . . . . . 288

7.3.1 Gelockerte Bande in Parteien \& Verbandslandschaft . . . . . . . . . . . . . . . 289

7.3.2 Aufstieg der Wirtschaftswissenschaften . . . . . . 295

7.3.3 Herausforderungen für Ministerialbürokratie \& amtliche Statistik . . . . . . . . . . . . . . . . . 304

7.4 Phase 3: Finanzialisierung anhand wissenschaftlicher Expertise . . . . . . . . . . . . . . . . 318

\section{Zusammenfassung und Ausblick 325}

8 Vermarktlichung und ihre Produzenten $\quad 327$

8.1 Dimensionen der Vermarktlichung \& Wohneigentumsförderung . . . . . . . . . . . . . 329

8.2 Wahlverwandtschaft: Verwaltung \& Vermarktlichung . . 333

8.3 Forschungsperspektiven und Ausblick . . . . . . . . . 344

$\begin{array}{ll}\text { Literaturverzeichnis lvi } & \text { lvis }\end{array}$

Anhang lix

A Rahmenbedingungen lix

A.1 Wechselnde Zuschnitte des Wohnungsbauministeriums . lix 
B Methodik

$\operatorname{lxv}$

B.1 Schriftenreihen des Bundesministeriums für Wohnungsbau lxv

B.2 Wohnimmobilienmarktspezifischen Veröffentlichungen der

Deutschen Bundesbank . . . . . . . . . . . lxvii

B.3 Transkriptionsregeln .............. lxix 



\section{Abbildungsverzeichnis}

2.1 Politisches Modell policy-bezogenen Wandels. . . . . . . 32

2.2 Interessenformierung unter Berücksichtigung von Ideen. 37

2.3 Politisches Modell institutionellen Wandels mit Ideenbezug im policy-Subsystem. . . . . . . . . . . . . . . 46

3.1 Ideenproduktion und institutioneller Wandel innerhalb eines policy-Subsystems . . . . . . . . . . . . . 53

3.2 Ideenproduzenten und institutioneller Wandel innerhalb eines policy-Subsystems . . . . . . . . . . . . 101

5.1 Förderung von Eigenheimen auf Wahlplakaten zur Bundestagswahl von 1949 von CDU und SPD. . . . . . . 170

7.1 Eigentümerquote nach Bundesländern im Zeitvergleich. 273 



\section{Tabellenverzeichnis}

4.1 Bedeutende Gesetzgebungen zur Wohneigentumsförderung in der Bundesrepublik Deutschland und zur Ideenidentifikation herangezogene Bundestagsdrucksachen . 131

8.1 Phasen der Ideenproduktion für die Wohneigentumsförderung . . . . . . . . . . . . . . . . 330 



\section{Teil I}

\section{Einleitung}





\section{Kapitel 1}

\section{Vermarktlichung \& Ideenproduktion}

Angesichts der weitreichenden Folgen der US-amerikanischen SubprimeKrise und steigender Wohnungspreise in zahlreichen Ländern haben die Sozialwissenschaften Wohnungspolitik als Forschungsgegenstand wieder entdeckt. Zahlreiche Untersuchungen auf nationaler wie auf internationaler Ebene konnten dabei Trends der Vermarktlichung und Finanzialisierung nachweisen, die sich durch einen Rückzug des Staates aus der Wohnungsversorgung und einer zunehmenden Förderung von Wohneigentum auszeichnen. Wie in der wohnungspolitischen Forschung stellt sich auch für andere sozialpolitische Bereiche die Frage nach den Ursachen derartiger oft als Liberalisierung beschriebener Prozesse. Einigkeit besteht darin, dass sich die Vermarktlichung wohlfahrtsstaatlicher Institutionen meist in kleinteiligen Schritten vollzieht und auf verschiedene Faktoren gleichzeitig zurückzuführen ist (Streeck u. Thelen 2005b; Mahoney u. Thelen 2010; Campbell u. Pedersen 2001c). Neben sich ändernden Rahmenbedingungen, dem vorhandenen institutionellen Gefüge, den Positionierungen politischer Parteien und der Macht von 
Interessengruppen verweisen immer mehr Untersuchungen auf die Bedeutung von Ideen für politischen Wandel. In Form von Problemdefinitionen, konkreten Lösungsansätzen und Zielvorstellungen ermöglichen und formen Ideen die Interessenformulierung und damit auch die Handlungen politischer Akteure. $\mathrm{Ob}$ und wie sich wandelnde Rahmenbedingungen politische Entscheidungen beeinflussen, hängt also maßgeblich von deren Wahrnehmung durch die beteiligten Akteure ab. Trotz der anerkannten Bedeutung von Ideen für institutionellen Wandel bleibt oftmals unklar, woher diese Ideen kommen (Campbell u. Pedersen 2014; Ban 2016). Die vorliegende Dissertation leistet einen Beitrag zur Beantwortung der Frage, welchen Einfluss Akteure und Mechanismen der Wissensproduktion auf die Vermarktlichung wohlfahrtsstaatlicher Institutionen haben.

Anhand einer Fallstudie zur Förderung von Wohneigentum in der Bundesrepublik Deutschland zeigt die Untersuchung, unter welchen Kontextbedingungen institutioneller Wandel innerhalb des policy-Subsystems der Wohneigentumsförderung als zentralem Merkmal staatlich vorangetriebener Wohnungsmarktprivatisierung stattgefunden hat und welche Bedeutung der Wissensproduktion dabei zukam. Dabei wird deutlich, dass Deutschland einen besonderen Pfad der Vermarktlichung und Finanzialisierung beschritten hat: Schrittweise wurde aus der familien- und gesellschaftspolitisch motivierten Förderung des Sozialgutes ,Familienheim“ der jungen Bundesrepublik ein vermögens- und finanzpolitisch diskutiertes Wirtschaftsgut und schließlich ein Anlageprodukt der privaten Altersvorsorge. Mit diesen Befunden hebe ich nicht nur die Bedeutung ideellen Wandels für den institutionellen Wandel in der Wohnungspolitik hervor, sondern frage in einem weiteren Schritt auch nach den Ursprüngen dieses ideellen Wandels. Mit einem Fokus auf knowledge regimes (Campbell $\mathrm{u}$. Pedersen, 2014) als organisationale und institutionelle Maschinerie der Ideen- und Wissensproduktion für die Politik zeige ich, dass Vermarktlichung nicht nur durch Faktoren wie die wirtschaftlichen Rahmenbedingungen oder dem interessengelei- 
teten Verhalten von Parteien und Interessenverbänden zu erklären ist. Stattdessen argumentiere ich, dass sich die Entwicklungen besser unter Berücksichtigung der Sonderrolle der staatlichen Produktion und Verwaltung von Wissen und Ideen für die politischen Entscheidungen in der Wohneigentumsförderung verstehen lassen: Staatliche Stellen waren und sind nicht nur selber wesentliche Produzenten von Ideen, sondern können auch durch ihre Rolle als gatekeeper die Aufnahme anderweitig produzierter Wissensbestände maßgeblich mit beeinflussen.

Die Befunde liefern Implikationen für die Forschung zur langfristigen Entwicklungen der bundesdeutschen Wohnungspolitik (Egner, 2004b), der Weiterentwicklung von Theorien institutionellen Wandels durch Ideen (Béland u. Cox, 2011), sowie für die jüngeren Untersuchungen zu knowledge regimes (Campbell u. Pedersen, 2014), die bislang die Sonderrolle staatlicher Akteure in der politikbezogenen Wissensproduktion nur unzureichend berücksichtigen.

\subsection{Vermarktlichung \& Finanzialisierung in der Wohnungspolitik}

Vor dem Hintergrund der weitreichenden Relevanz wohnungspolitischer Maßnahmen ${ }^{1}$ wundert es wenig, dass Wohnungspolitik in Deutschland zu einem der ältesten Felder staatlichen Eingreifens gehört (Jenkis, 1996, 69) ${ }^{2}$. Noch bis zur Zwischenkriegszeit des 20. Jahrhunderts wurde

1 Unter Wohnungspolitik werden hier in Anlehnung an Lampert „alle Maßnahmen staatlicher Träger und Organe der Wirtschafts- und Sozialpolitik [verstanden], mit denen das Ziel verfolgt wird, die Wohnungsversorgung der Bevölkerung zu beeinflussen. Wohnungspolitik umfaßt die Wohnungsbaupolitik und die Wohnungs bestandspolitik" (Lampert, 1994, 306).

2 Jenkis (1996) sieht die Anfänge aktiver staatlicher Wohnungspolitik bereits kurz nach dem Dreißigjährigen Krieg, als mitteleuropäische Kleinstaaten erstmals einen aktiven Wohnungs- und Siedlungsbau betrieben (vgl. Jenkis, 1996, $69)$. 
die „Wohnungsfrage“ als Teilaspekt der ,sozialen Frage“ gesehen (Heinelt u. Egner 2006, 204; vgl. Lowe 2011), die vor allem aus der Verstädterung im Zuge der Industrialisierung resultierte.

Trotz dieser vielfältigen Ansatzpunkte staatlichen Eingreifens wurde die Wohnungspolitik über einen langen Zeitraum hinweg vonseiten der Wohlfahrtsstaatenforschung vernachlässigt (Aalbers u. Christophers 2014; Schwartz u. Seabrooke 2008; Kohl 2015). Allerdings ist seit dem Ausbruch der Subprime-Krise eine Wiederbelebung der wohnungspolitischen Forschung in den Sozialwissenschaften zu beobachten (Schwartz, 2012). Befunde zur Entwicklung der Wohnungspolitik westlicher Industrienationen in der zweiten Hälfte des zwanzigsten Jahrhunderts beschreiben zunehmende Tendenzen der Vermarktlichung und Finanzialisierung. Während mit einer Vermarktlichung meist ein „Rückzug des finanziellen Engagements des Staates für die Wohnungsversorgung mit einer Verlagerung des Schwerpunkts zum Eigenheim“ (Jaedicke u. Wollmann, 1991, 432) verbunden wird, die mit einer Privatisierung öffentlichen Wohnraums einhergeht (Schipper 2017; Holm 2010; Glatter 2007; Hesse u. Preckwinkel 2009), steht der Begriff Finanzialisierung für eine „Deregulierung und globale Verflechtung von Immobilien- und Finanzmärkten" (Schipper 2017, 1; siehe auch Aalbers 2016; Belina 2017; Seabrooke u. Schwartz 2009; Wijburg u. Aalbers 2017). Das Gebrauchsgut Wohnen wird in diesem Prozess zunehmend von einem Sozial- zu einem Finanz- und Anlageprodukt (Heeg 2013, 76; siehe auch Rolnik u. Rabinovich 2014; Forrest u. Hirayama 2014). Diese Beschreibungen von Vermarktlichungsprozessen in der Wohnungspolitik decken ein breites Spektrum politischer Maßnahmen ab, allerdings auf Kosten der Genauigkeit in der Beschreibung des eigentlichen policy-Wandels. So beschreiben die meisten Untersuchungen lediglich eine im Ergebnis stärkere Bedeutung marktlicher gegenüber staatlichen Regelungsmechanismen und lassen die ursprünglichen Intentionen des Politikwechsels außen vor.

Auch in anderen sozialpolitischen Bereichen wurden für die zweite 
Hälfte des zwanzigsten Jahrhunderts Tendenzen von Vermarktlichung und Finanzialisierung westlicher Wohlfahrtsstaaten festgestellt (Streeck u. Thelen 2005a; Thelen 2012; Schimank u. Volkmann 2017; Heires u. Nölke 2014). Bezugnehmend auf Polanyi wird mit Vermarktlichung eine institutionalistische Perspektive verbunden, wonach ,Marktmechanismen in zuvor nicht-marktlich koordinierten gesellschaftlichen Feldern" ausgeweitet werden (Ebner, 2014, 49). Dabei bleiben die zeitgenössischen Diagnosen der Vermarktlichung oftmals nicht auf Kommodifizierung der von Polanyi als fiktive Waren ${ }^{3}$ bezeichneten Güter beschränkt. Stattdessen wird sie mit Bezug auf Polanyi breiter definiert ,im Sinne der Ausweitung der Marktbeziehungen und der Angleichung aller anderen gesellschaftlichen Institutionen an marktförmige Regeln" (Brie, 2015, 111). Auch das oftmals zitierte Konzept der Finanzialisierung nach Epstein (2005a) ist überaus breit angelegt als ,the increasing role of financial motives, financial markets, financial actors and financial institutions in the operation of the domestic and international economies" (Epstein, 2005b, 3).

Derart umfassende Definitionen von Vermarktlichung und Finanzialisierung im Sinne einer allgemeinen Liberalisierung sind allerdings zu breit, um als analytisches Konzept von Nutzen zu sein (Thelen, 2012). Daher bietet es sich an, Vermarktlichung und Finanzialisierung nach den unterschiedlichen Dimensionen, in denen sie sich bemerkbar machen können, zu unterscheiden: Zum einen können Vermarktlichung und Finanzialisierung die regulierende Dimension betreffen, ,that consists of economically consequential rules of action" (Campbell u. Pedersen, 2001a, 250). Eine Vermarktlichung in dieser Dimension wird also in einem Wandel der policies erkennbar, die das Handeln der Ak-

$3 \quad$ Als fiktive Waren bezeichnet Polanyi Arbeit, Boden und Kapital, die nicht für den Handel auf Märkten produziert wurden (Polanyi, 1978, 107). Eine Kommodifizierung dieser fiktiven Waren hat eine gesellschaftliche Gegenbewegung zur Folge, mit der die fiktiven Waren vor ihrer Vermarktlichung geschützt werden sollen. 
teure regulieren. Die weiteren Dimensionen beschreiben Campbell u. Pedersen (2001a) als positive und normative Dimension, die im Rahmen dieser Arbeit als ideelle Dimension zusammen betrachtet werden sollen. Die positive, oder auch kognitive Dimension ,makes predictive claims about the effects [...] on economic performance" (Campbell u. Pedersen, 2001a, 250). Sie ist primär mit Erkenntnissen der ökonomischen Theorie und ihren Vorhersagen verknüpft, wonach freie Märkte durch ihre Effizienz die besten Ergebnisse liefern. Unter der normativen Dimension sind demgegenüber Idealvorstellungen betroffen, die mit freien (Finanz-)Märkten in Verbindung gebracht werden. Mit diesen zusätzlichen kognitiven und normativen Dimensionen von Liberalisierung weisen Campbell u. Pedersen (2001a) nicht nur auf die Vielschichtigkeit des beobachteten Phänomens hin. Vielmehr enthält diese Unterteilung auch den Hinweis, dass nicht alle Dimensionen gleichermaßen von Vermarktlichungsprozessen betroffen sein müssen (vgl. Hall u. Thelen, 2009, 22). Beispielsweise kann eine Verschiebung hin zu liberaleren Werten und Theorien stattgefunden haben, die sich allerdings nicht in einer Anpassung von Gesetzestexten äußert. So lässt sich die Einteilung in Dimensionen nutzen, um auf die Spezifika einzelner Maßnahmen einzugehen und zu präzisieren, worin die Vermarktlichung oder Finanzialisierung einer bestimmten wohlfahrtsstaatlichen Maßnahme genau besteht.

\subsection{Ursachen von Vermarktlichung und Fi- nanzialisierung}

Zahlreiche sozialwissenschaftliche Studien befassen sich mit möglichen Ursachen für Vermarktlichung und Finanzialisierung wohlfahrtsstaatlicher Institutionen. Während Vertreter der klassischen Institutionalismen (vgl. Rueschemeyer, 2009, 206) die Bedeutung von Rahmenbedingungen und interessenbasierten Handlungen politischer Akteure für 
politischen Wandel hervorheben, stellen Untersuchungen des diskursiven Institutionalismus (vgl. Schmidt, 2008) die Bedeutung von Ideen in den Vordergrund. So beschreiben einige ideenfokussierte Forscher Prozesse der Vermarktlichung als neoliberale Wende, die sich vor allem auf wirtschaftswissenschaftliche Theorien zurückführen lässt (so Centeno u. Cohen 2012; vgl. Lessenich 2013; vgl. Peck 2010). Die Verbreitung der ökonomischen Theorien von Hayek und Popper bildeten demnach die entscheidende Grundlage für den liberalen Politikwechsel von Margaret Thatcher and Ronald Reagan und leiteten so den Abbau westlicher Wohlfahrtsstaaten ein (vgl. Mirowski u. Plehwe, 2015). Beeinflusst durch die Arbeiten des Geografen David Harvey (Harvey 2007b; Harvey 2007a) beurteilen viele Studien auch die Entwicklung der deutschen Wohnungspolitik als Ausdruck eines globalen ,neoliberalen" Trends (Aalbers, 2012, 120). So beschreibt Holm (2010) die zunehmende Privatisierung öffentlicher Wohnungsbestände als „Teil einer globalen Enteignungsökonomie“ und stellt diese in den Kontext internationaler Kapitalkreisläufe (Holm 2010, 46; Holm 2011, 13). Auch Rolnik u. Rabinovich (2014) beschreiben Entwicklungen auf nationaler Ebene als Trend einer ,hegemonic neoliberal economic doctrine“, derzufolge ,governments should renounce their role as suppliers of affordable housing and become facilitators, supporting market demand rather than directly providing outcomes" (Rolnik u. Rabinovich, 2014, 59).

Eine grenzübergreifende Verbreitung liberaler Ideen hätte theoretisch zu einer zunehmenden Konvergenz westlicher Wohlfahrtsstaaten führen müssen. Allerdings haben zahlreiche Studien gezeigt, dass in der wohnungspolitischen Realität weiterhin starke Unterschiede in der Ausgestaltung nationaler Wohnungspolitiken bestehen (vgl. Kohl 2015; Schwartz u. Seabrooke 2008; Heinelt u. Egner 2006). Gegen die Theorie der neoliberalen Wende spricht zusätzlich, dass ,neoliberales Denken auch in der politischen Elite gar nicht so stark verbreitet ist, wie es eigentlich sein müsste, um die breite Tendenz zur Ausbreitung des Marktes zu erklären“ (Münnich u. Sachweh, 2017, 12). Als empirisch 
haltbarer erweisen sich demgegenüber Studien, welche die Handlungen von Akteuren im Kontext unterschiedlicher institutioneller Arrangements ins Zentrum ihrer Analysen stellen. Vermarktlichung oder auch Finanzialisierung als Ergebnis institutionellen Wandels sind kein semi-automatischer Prozess, ,but the work of situated actors"(Peck, 2010, xii). So haben Ansätze der international vergleichenden Wohlfahrtsstaatenforschung entgegen der Konvergenzthese einer neoliberalen Orthodoxie gezeigt, dass weiterhin nationale Besonderheiten und damit auch Divergenzen in wohlfahrtsstaatlichen Institutionen bestehen (Scharpf u. Schmidt 2004; Campbell u. Pedersen 2001b). Diese Ansätze verweisen auf mehrere potenzielle Ursachen für Vermarktlichung und Finanzialisierung. Statt lediglich einem internationalen Politiktrend zu folgen, können Staaten ,block, adapt to, mediate, and in some cases even reverse neoliberal tendencies" (Campbell u. Pedersen, 2001b, 3). Der am häufigsten genannte Grund für diese Unterschiede sind national unterschiedliche Rahmenbedingungen. So führen Jaedicke u. Wollmann (1991) wohnungspolitische Entscheidungen in Deutschland ,auf auftretende Engpässe auf den Wohnungsmärkten, also auf wachsenden Problemdruck" zurück (Jaedicke u. Wollmann, 1991, 433). Weitere Erklärungsfaktoren beziehen sich auf den Wandel interessenbasierter Handlungen von Akteuren, die institutionellen Wandel bewirken. Vermarktlichung wird demnach ,von politischen Akteuren unter Maßgabe strategischer Interessen durchgesetzt" (Ebner, 2014, 49) und ist damit nicht einfach das Ergebnis anonymer Globalisierungsprozesse (siehe auch Nölke 2009, 128f.).

So wie die auf Ideen fokussierten Studien oftmals Rahmenbedingungen und Akteurskonstellationen vernachlässigen, lassen Untersuchungen mit Fokus auf Rahmenbedingungen und Akteursinteressen Ideen als Erklärungsfaktoren oft außen vor. Für eine Analyse von Vermarktlichungsprozessen, die möglichst viele potenzielle Erklärungsfaktoren mit berücksichtigen soll, sollten jedoch beide Perspektiven aufeinander bezogen werden, um herauszustellen, ,wie der symbolische und mate- 
rielle Kontext die diskursive Produktion und Rezeption von Wissen beeinflusst" (Hamann 2012, 372; siehe auch Fourcade 2009, 15). Um zu klären, wie Kontextfaktoren und Interessenformierung der klassischen Institutionalismen mit der Bedeutung von Ideen die Entscheidungen politischer Akteure beeinflussen, müssen handlungstheoretische Grundannahmen getroffen werden. In Anlehnung an Münnich wird die Interessenformierung der politischen Akteure verstanden ,als ein Prozess der ideengeleiteten Sinnstiftung, der positional eingeschränkt ist" (Münnich, 2011, 380). Mit dieser Modellierung sind Ideen zwar nicht die alleinige Ursache institutionellen Wandels, bilden allerdings als Hintergrund vor dem die beteiligten Akteure ihre Interessen formulieren zentrale Erklärungsfaktoren für deren Handlungen.

Hieraus ergibt sich Erklärungsbedarf, wie diese Ideen den Akteuren zur Verfügung gestellt werden. So weisen Campbell u. Pedersen (2014) in ihrer international vergleichenden Studie nach, dass Ideen der Vermarktlichung maßgeblich innerhalb knowledge regimes durch diverse Akteure und ein national spezifisches Set von Mechanismen debattiert und für die Politik bereitgestellt werden (Campbell u. Pedersen, 2014, 6). Daher sind Regime der Wissensproduktion ein wesentlicher Erklärungsfaktor für die Frage, warum internationale Trends wie Vermarktlichung oder Finanzialisierung auf nationaler Ebene unterschiedliche Ausprägungen annehmen (siehe hierzu auch Fourcade, 2009, 239). Sie bilden aller internationalen Vernetzung zum Trotz nationenspezifische Konstrukte, deren Strukturen und Praktiken nicht vollständig durch das dazugehörige politische Regime und das Produktionsregime determiniert werden. Basierend auf empirischen Studien zur politikorientierten Wissensproduktion in der Bundesrepublik Deutschland können verschiedene Wissensproduzenten identifiziert werden. Neben politischen Parteien, Interessenverbänden und Think Tanks gehören dazu auch Akteure aus dem Bereich der wissenschaftlichen Politikberatung und der öffentlichen Verwaltung. Es zeigt sich, dass vor allem Akteure aus dem Bereich der öffentlichen Verwaltung und insbesondere die 
Ministerialbürokratie wesentliche Schlüsselpositionen in der Wissensproduktion für politische Entscheider darstellen. Als Triebkräfte der Vermarktlichung können somit nicht nur sich ändernde Rahmenbedingungen und Akteursinteressen angesehen werden, sondern auch die policy-spezifische Ausgestaltung der Wissensproduktion.

\subsection{Wohneigentumsförderung in Deutschland}

Obwohl Wohnungspolitik in westlichen Wohlfahrtsstaaten ein zentraler sozialpolitischer Bereich ist, wurde sie über einen langen Zeitraum hinweg vonseiten der Wohlfahrtsstaatenforschung vernachlässigt (Mayer 1998; Aalbers u. Christophers 2014; Schwartz u. Seabrooke 2008; Lowe 2011; Ronald 2008; Kohl 2015). Dabei ist sie nicht nur historisch ein wichtiges Feld staatlichen Eingreifens (Jenkis, 1996), sondern auch eines, in dem der Wandel wohlfahrtsstaatlicher Institutionen im Verlauf der zweiten Hälfte des 20. Jahrhunderts erkennbar wird. In Deutschland hat sich Wohnungspolitik mit zahlreichen Verbindungen zu weiteren Politikbereichen zu einem komplexen Politikfeld weiter entwickelt. $\mathrm{Zu}$ den relevantesten nahestehenden Politikfeldern zählen der Städtebau, die Familienpolitik, die Altersvorsorge (Heinelt u. Egner, 2006, 210) sowie die Finanzpolitik (Mertens, 2015, 303). Neben dem Mietrecht, das auf erste Gesetzgebungen aus der Weimarer Republik zurückgeht (vgl. hierzu Kofner, 2004), zählt Egner (2014) den Sozialen Wohnungsbau, das Wohngeld sowie die Wohneigentumsförderung zu den bedeutendsten Instrumenten der deutschen Wohnungspolitik nach dem Zweiten Weltkrieg. Im Rahmen dieser Ausdifferenzierung der Wohnungspolitik lässt sich eine Aufspaltung in eine Politik, für sozial Schwache und eine Vermögens-und Familienpolitik für den Mittelstand" in der zweiten Hälfte des 20. Jahrhunderts beobachten (Hilpert, 2012, 297). Neue Studien zu Prozessen zur Entwicklung der Wohnungspolitik beschreiben für diesen Zeitraum vor allem Liberalisierungsrozesse (Schipper 2017; Holm 2010; Glatter 2007; Hesse u. Preckwinkel 2009; Schipper 2017; 
Aalbers 2016; Belina 2017; Seabrooke u. Schwartz 2009; Heeg 2013; Rolnik u. Rabinovich 2014). Dabei wird unter Liberalisierung nicht nur ein Rückzug des staatlichen Engagements in der Bereitstellung von Sozialem Wohnungsbau verstanden, sondern auch die zunehmende Tendenz zu staatlicher Eigenheimförderung (Lowe 2011; Häußermann u. Siebel 1981; Bourdieu 1998a; Rolnik u. Rabinovich 2014). Eigenheimförderung kommt somit eine besondere Stellung zu: die mit ihr zusammenhängenden policies werden zwar oftmals in der Wohnungs- oder Sozialpolitik verortet, sind aber meist eng mit wirtschafts- und finanzpolitische Maßsnahmen verknüpft (Heinelt u. Egner 2006; Mertens 2015). Somit bietet die Eigenheimförderung als ursprünglich sozialpolitisch motivierte Maßnahme die Möglichkeit Vermarktlichung und Finanzialisierung im Rahmen einer Tiefenanalyse auf der Ebene konkreter policies zu erfassen.

Nicht zuletzt wegen der US-amerikanischen Subprime-Krise, die eng mit Maßnahmen der staatlichen Eigenheimförderung verknüpft war, werden diese Maßnahmen erneut kontrovers diskutiert (Bentzien 2012, 23; Arentz u.a. 2010, 2; Forrest u. Hirayama 2014; Schwartz 2012, 54). Dabei ist die Eigenheimförderung in Bezug auf die Fragestellungen nach der Ideenproduktion für Vermarktlichung und Finanzialisierung in zweierlei Hinsicht von besonderem Interesse. Erstens ist sie wie kaum eine andere wohnungspolitische Maßnahme Ausdruck einer staatlich geförderten Vermarktlichung (Rolnik u. Rabinovich, 2014). Bourdieu (1998b) weist darauf hin, dass nur wenige Märkte ,so sehr wie der Häusermarkt vom Staat nicht nur kontrolliert, sondern regelrecht konstruiert [werden], und zwar ganz besonders durch die Vergabe von Fördermitteln für Privatpersonen" (Bourdieu 1998b, 62; vgl. Bourdieu 2014). Durch Eigenheimförderung werden Personen vor allem zu einem ,selbstständigen Agieren am Wohnungsmarkt" als Nachfrager und Anbieter von selbstgenutztem Wohnraum befähigt (Heinelt u. Egner, 2006, 218): 
„Indem sie Wohnungsversorgung letztlich zur Frage des privaten Eigentumserwerbs macht, weist sie nur den Weg individueller Anstrengung und befestigt so die Basisideologie einer Marktwirtschaft." (Häußermann u. Siebel, 1981, 328)

Zweitens betrifft Eigenheimförderung nicht nur die policy-Dimension von Vermarktlichung, sondern verfügt über einen starken symbolischen und ideellen Gehalt ,als materielles Gut, das wie die Kleidung, aber auf Dauer für alle wahrnehmbar wird" (Bourdieu, 1998b, 26, HvH.i.O.). Sie war nie eine rein wohnungspolitisch gerechtfertigte Maßnahme, sondern stets mit einem breiten Spektrum sozial- und gesellschaftspolitischer Motive wie auch wirtschafts- und finanzpolitischer Überlegungen verknüpft (Hämmerlein, 1996, 57). Keine andere wohnungspolitische Maßnahme hat einen derart starken ideellen Bezug (DeSalvo, 1976), weswegen Egner u. a. (2004) sie auch als ,fragmentiert-überfrachtetes Instrument" der Wohnungspolitik beschreiben, bei dem Zustandsbeschreibungen, Funktionsdeutungen und Problemwahrnehmungen inhaltlich stark divergieren (Egner u.a., 2004, 254). Politische Debatten um ideelle Konzepte sollten demnach besonders gut in der Wohneigentumsförderung zu erfassen sein.

In der international vergleichenden Wohlfahrtsstaatenforschung spielt die unterschiedliche Bedeutung und Förderung von Wohneigentum eine wichtige Rolle zur Charakterisierung verschiedener Marktmodelle (Arbaci 2007; Schwartz u. Seabrooke 2008). Deutschland gilt dabei stets als Sonderfall: Einerseits weist die staatliche Förderung von Wohneigentum eine vergleichsweise lange Geschichte auf, die im Gegensatz zu vielen anderen Ländern nicht erst in den 70er Jahren ihren Anfang nahm (Rolnik u. Rabinovich 2014; Ronald 2008; Bourdieu 2014), sondern bereits in den ersten Legislaturperioden nach dem Zweiten Weltkrieg. Andererseits erweisen sich die im internationalen Vergleich geringen Wohneigentumsquoten als auffällig: Deutschland ist bis heute das einzige Land in der EU, in dem mehr Menschen zur Miete wohnen 
als in ihren eigenen vier Wänden (Housing Europe 2017, 68; Kleinman 1996). Nach Angaben des Statistischen Bundesamtes lag der Anteil der von Eigentümern selbst genutzten Wohnungen an allen bewohnten Wohnungen 2014 lediglich bei 45,5 Prozent (Statistisches Bundesamt, 2014).

Erklärt werden diese Besonderheiten bislang mit Eigenheiten der Finanzierungsstruktur (Hüther u.a. 2015; Schwartz u. Seabrooke 2008; Jäger u. Voigtländer 2006). Sie werden in der international vergleichenden Forschung auf historische Besonderheiten der institutionellen Rahmenbedingungen und der daraus resultierenden Pfadabhängigkeiten zurückgeführt (Kohl 2015; Kohl 2016; Kohl 2017). Allerdings weist Mertens darauf hin, dass Perspektiven wie die der Varieties of Capitalism häufig dazu tendieren, ,institutionelle Pfadabhängigkeiten und Unterschiede zwischen Ländern zu akzentuieren [und zugleich] die institutionellen Veränderungen über Zeit [zu verwischen]" (Mertens, 2015, 45). Tatsächlich können mit der These der Pfadabhängigkeiten einzelne Besonderheiten der Finanzierungsstruktur erklärt werden, allerdings nicht die Wandlungsprozesse, die sich in wohnungspolitischen Institutionen wie der Eigenheimförderung abzeichnen (Heinelt u. Egner, 2006). Daher sollen hier die langfristigen Entwicklungstendenzen innerhalb eines policy-Subsystems offen gelegt werden, um die Eigenheiten des kleinteiligen Institutionenwandels in der Eigenheimförderung der Bundesrepublik zwischen Pfadabhängigkeiten und Trends zur Vermarktlichung und Finanzialisierung beschreiben im Zusammenhang mit dem sich ändernden Regime der Wissensproduktion beschreiben zu können. Akteure und Mechanismen der politikfeldspezifischen Wissensproduktion, so die These, sind wesentliche Faktoren für institutionellen Wandel - auch in Bezug auf Vermarktlichung und Finanzialisierung. 


\subsection{Methodisches Vorgehen}

Um die Singularität der Wohneigentumsförderung in Deutschland mit ihren spezifischen Kontexteigenschaften herauszustellen, führe ich eine Einzelfallstudie durch. Anhand mehrerer Beobachtungen im Zeitverlauf soll ermöglicht werden, den Ideenwandel im policy-Subsystem der Wohneigentumsförderung rückblickend genauer zu erklären (vgl. Ebbinghaus, 2009, 485). Der Untersuchungszeitraum wurde auf den Beginn der Nachkriegszeit bis Ende 2008 festgelegt, da erst nach dem Zweiten Weltkrieg von einer Wohneigentumsförderung auf gesamtstaatlicher Ebene die Rede sein kann, und 2008 mit der Eingliederung der Wohneigentumsförderung in das System der Riesterrente die zum Zeitpunkt der empirischen Arbeiten wesentlichste Neuerung im System der Eigenheimförderung zustande kam. Für die vorliegenden Untersuchungen wurde ein breites Spektrum historischer Literatur zur Wohnungspolitik in Deutschland ergänzt um Primäranalysen von Bundestagsprotokollen, eine eigene Archivrecherche sowie Experteninterviews, um eine unkritische Replikation bestehender Forschung zu vermeiden (Starke, 2015, 465).

Für die Analyse des ideellen Wandels wurden bewusst ausgewählte ${ }^{4}$ Plenarprotokolle des Deutschen Bundestags ausgewertet. Dabei wurden in einem ersten Schritt in Anlehnung an Halls Paradigmenkonzept (Hall, 1993) ein Codesystem mit den Kategorien „Zielvorstellungen“, „Instrumentarien“ und „Problemstellungen“ gebildet. Aus den zugewiesenen Codes wurden in einem weiteren Schritt Subkategorien gebildet und nach den Maßgaben der qualitativen Inhaltsanalyse zusammengefasst (vgl. Döring u. Bortz, 2016, 542), um die zentralen Ideen der Eigenheimförderung in der Bundesrepublik und deren Entwicklungen bestimmen zu können.

4 Die Auswahl der relevanten Debatten erfolgte nach Lesungen jener Gesetze, die - nach der einschlägigen Literatur zu urteilen - die wichtigsten Meilensteine für die Wohneigentumsförderung in der Bundesrepublik bilden. 
Zur Beschreibung der Maschinerie der Wissensproduktion in Anlehnung an Campbell u. Pedersen (2014) wird zunächst nach den Mitgliedern jener institutionellen Maschinerie gesucht, „die Daten, Forschung, Politikempfehlungen und weitere Ideen generiert, welche die politischen Debatten und die Ausgestaltung der Politik beeinflussen" (Campbell u. Pedersen, 2014, 3). Die Recherchen erfolgten zunächst durch eine Literaturrecherche zu Akteuren im Politikfeld „Wohnen“. Darüber hinaus wurden Recherchen zur Wissensproduktion anhand von Protokollen öffentlicher Ausschusssitzungen sowie im Rahmen der inhaltsanalytischen Betrachtungen der Bundestagsprotokolle durchgeführt. Neben den Ausschussanhörungen lässt sich die für den politischen Prozess relevante Wissensproduktion auch über gutachterliche Tätigkeiten ausfindig machen. Eine vollständige Auflistung der in den Reihen des Ministeriums veröffentlichten Gutachten wurde anhand von Recherchen in der Bibliothek des Bundesinstituts für Bau-Stadt und Raumforschung (BBSR) in Bonn ermöglicht. Ergänzt wurden die Recherchen durch leitfadengestützte Experteninterviews mit Vertretern von halbstaatlichen Forschungsinstituten, einem privaten Beratungsunternehmen und Mitarbeitern des Bundesinstituts für Bau-, Stadt- und Raumforschung, die mit der Vergabe, Durchführung und Betreuung von Forschungsaufträgen vonseiten des Bundesministeriums betraut sind.

\subsection{Aufbau der Arbeit}

Zunächst konzeptioniere ich in Teil II dieser Arbeit den theoretische Rahmen und die methodische Herangehensweise für die durchgeführten Analysen. Um Vermarktlichungs- und Finanzialisierungstendenzen in der bundesdeutschen Wohnungspolitik adäquat beschreiben zu können, werden in Kapitel 2 zunächst verschiedene Erklärungsfaktoren institutionellen Wandels diskutiert. Die Erklärungsansätze beziehen sich dabei oft auf externe Effekte, welche die am politischen Prozess beteiligten wirtschaftlichen und politischen Akteure zu einer Anpassung 
bestehender institutioneller Arrangements veranlassen. Ergänzt werden diese Ansätze durch Studien, welche die Bedeutung von Ideen und Wissen für den institutionellen Wandel betonen. Basierend auf dem Stand der Forschung zu institutionellem Wandel skizziere ich ein politisches Modell institutionellen Wandels, mit dem sich mögliche Zusammenhänge zwischen ideellem und institutionellem Wandel beschreiben und Hypothesen generieren lassen. Entscheidend sind in diesem Konzept nicht nur die bereits genannten Akteure, die ihre individuellen Interessen verfolgen, sondern auch diejenigen, die an der Wissens- und Ideenproduktion für den politischen Prozess beteiligt sind. In Kapitel 3 werden diese Akteure und ihre Rolle in der Wissensproduktion für die Politik beschrieben. Neben der wissenschaftlichen Politikberatung, der klassischerweise eine zentrale Rolle in diesem Prozess zugestanden wird, sollen auch die Funktionen von Parteien und Verbänden innerhalb der Wissensmaschinerie eingehender diskutiert werden. Als letzte und oftmals in der Literatur vernachlässigte Akteursgruppe beschreibe ich die Rolle der öffentlichen Verwaltung in der Wissensmaschinerie.

Bevor in Teil III die Ergebnisse der empirischen Arbeit dargestellt werden, wird in Kapitel 4 das methodische Vorgehen detailliert dargelegt. In den Kapiteln 5, 6 und 7 beschreibe ich drei Phasen der Wissensproduktion für die Eigenheimförderung in der Bundesrepublik Deutschland nach dem Zweiten Weltkrieg. Neben den (wohnungs-)wirtschaftlichen und politischen Rahmenbedingungen steht dabei zunächst der mit der Eigenheimförderung verbundene institutionelle und ideelle Wandel im Vordergrund. Dabei soll spezifiziert werden, in welchen Dimensionen Liberalisierungsprozesse nachweisbar sind. Anschließend beschreibe ich genauer, welche Akteure über welche Mechanismen maßgeblich an der Bereitstellung der politisch relevanten Ideen beteiligt waren. Dabei wird deutlich, dass die klassischen Erklärungsfaktoren institutionellen Wandels wie wirtschaftliche und gesellschaftspolitische Rahmenbedingungen, der Wandel von Partei- und Verbandsinteressen oder auch der zunehmende Einfluss wirtschaftswissenschaftlicher Politikberatung nicht 
ausreichen, um den Wandel in der bundesdeutschen Eigenheimförderung zu erklären. Erstens wird dieser Wandel nachvollziehbarer, wenn er als Teilergebnis eines ideellen Wandels gesehen wird, der einen besonderen Pfad der Vermarktlichung bedingte. Wurde die Eigenheimförderung in den jungen Jahren der Bundesrepublik noch durch familienpolitisch motivierte Ideen mit deutlichen Bezügen zur christlichen Soziallehre dominiert, gewannen nach und nach vermögenspolitische Konzepte und ökonomische Kennzahlen wie die Wohneigentumsquote an Bedeutung. Ohne diesen Ideellen Wandel wäre die frühere Abschaffung der Eigenheimförderung zugunsten einer Wiedereinführung als geförderte Finanzanlage im Rahmen der Riesterrente nicht möglich gewesen. Dieser grundlegende ideelle Wandel lässt sich zweitens zu großen Teilen auf die Rolle der öffentlichen Verwaltung in der politikbereichspezifischen Wissensproduktion zurückführen: Sie produziert nicht nur selber Ideen und Wissen für die Politik, sondern fungiert auch als zentrale vermittelnde Schnittstelle zwischen Regierung, Wissenschaft und Interessengruppen und bestimmt daher die Rahmenbedingungen der Wissensproduktion wesentlich mit. In Teil IV fasse ich die wesentlichen Erkenntnisse der Untersuchung in theoretischer und empirischer Hinsicht zusammen und schließe die Arbeit mit einem wohnungspolitischen Ausblick und Anregungen für künftige Forschungsprojekte (Kapitel 8). 


\section{Teil II}

\section{Institutioneller Wandel und Ideenproduktion}





\section{Kapitel 2}

\section{Institutioneller Wandel \& Ideen}

Der Wandel wohlfahrtsstaatlicher Institutionen ist seit längerem Bestandteil sozialwissenschaftlicher Forschung. Dabei ist insbesondere der kleinteiligere, inkrementelle Wandel, der mit Prozessen der Vermarktlichung einhergeht, in den Fokus empirischer Untersuchungen gerückt (Streeck u. Thelen, 2005a). Um diese Vermarktlichungsprozesse sowie ihre Ursachen beschreiben zu können, skizziert Abschnitt 2.1 ein allgemeines politisches Modell institutionellen Wandels für ein policySubsystem $^{1}$. Dabei soll anhand von bestehenden Theorien institutionellen Wandels auf dessen potenzielle Ursachen eingegangen werden. Neben sich ändernden Rahmenbedingungen und Akteursinteressen, weisen zahlreiche sozialwissenschaftliche Untersuchungen auf die Bedeutung von Ideen als Ursache und Bestandteil institutionellen Wandels

1 Mit der Untersuchungseinheit des policy-Subsystems werden in Anlehnung an den advocacy-Koalitionen-Ansatz grundsätzlich alle Akteure aus Politik, Verwaltung, Wissenschaft und Gesellschaft mit einbezogen, „die ein besonderes Interesse an politischen Regulierungsfragen artikulieren und versuchen, Wirkung im Politikfeld zu erzeugen“ (Schneider u. Janning, 2006, 195). 
hin. So zeigen Studien zu Vermarktlichungsprozessen, dass diese nicht nur auf der policy-Ebene stattfinden, sondern auch kognitive und normative Aspekte betreffen (vgl. Campbell u. Pedersen, 2001a). Daher soll in Abschnitt 2.2 dargelegt werden, wie Ideen und Institutionen zusammenhängen und welche unterschiedlichen Arten von Ideen Einfluss auf institutionellen Wandel ausüben können. Abschließend sollen in Abschnitt 2.3 mögliche Zusammenhänge zwischen ideellem Wandel und institutionellem Wandel beschrieben werden. Gegenüber den klassischen Theorien institutionellen Wandels zeigt sich, dass Ideen nicht nur einen wesentlichen Bestandteil von Institutionen bilden, sondern auch die Interessenformierung der relevanten Akteure institutionellen Wandel beeinflussen können. Basierend auf diesen Erkenntnissen soll im anschließenden Kapitel 3 auf die Produktion dieser Ideen eingegangen werden.

\subsection{Einflussfaktoren institutionellen Wandels}

Zunächst soll darauf hingewiesen werden, dass auch policies als Institutionen verstanden werden

„to the extent that they constitute rules for actors other than for the policymakers themselves - rules that can and need to be implemented and that are legitimate in that they will if necessary be enforced by agents acting on behalf of the society as a whole." (Streeck u. Thelen, 2005b, 12)

Sie sind „nicht nur politische Gebilde, sondern auch Gegenstand politischer Auseinandersetzungen“ und spielen ,ähnlich wie andere etablierte Verfahrensweisen eine große Rolle für die Erwartungsbildung von Akteuren“" (Mertens, 2015, 34f.). Dementsprechend zielen institutionentheoretische Ansätze darauf ab ,to illuminate how political struggles are mediated by the institutional setting in which they take place" (Steinmo u.a., 1992, 2). In den vergangenen Jahrzehnten 
hat die sozialwissenschaftliche Forschung zahlreiche Erklärungsansätze für den Wandel wohlfahrtsstaatlicher Institutionen hervorgebracht. Dabei befasst sich ein Großteil der Untersuchung mit Tendenzen der Vermarktlichung, wobei vor allem von Vertretern der Modernisierungstheorie oftmals suggeriert wird, dass sich die westlichen Wohlfahrtsstaaten immer ähnlicher werden (vgl. Dobbin u. a. 2007; Lee u. Strang 2006). Demgegenüber haben insbesondere Vertreter der international vergleichenden Wohlfahrtsstaatenforschung und des Varieties of Capitalism-Ansatzes dazu beigetragen die weiterhin bestehenden Divergenzen westlicher Wohlfahrtsstaaten zu erklären. Entgegen der Vorstellung einer allgemeinen Konvergenz zur Liberalisierung betonen diese institutionenbasierten Ansätze nationale Eigenheiten und deren Pfadabhängigkeiten und sind dadurch imstande trotz gleichlaufender internationaler Trends nationale Besonderheiten von Wohlfahrtsstaaten zu erklären.

Klassische Perspektiven auf Institutionen in der sozialwissenschaftlichen Forschung lassen sich in Anlehnung an Hall u. Taylor (1996) in drei Idealtypen einteilen (vgl. Rueschemeyer, 2009, 206): den RationalChoice-Institutionalismus, den Historischen Institutionalismus sowie den Soziologischen Institutionalismus. Dabei konzentriert sich jeder dieser Ansätze auf unterschiedliche Ausgangsfragen, benennt eigene Bedingungen und Mechanismen institutionellen Wandels und ist mit einer eigenen Epistemologie und methodischen Herangehensweisen verbunden (Campbell u. Pedersen, 2001b). Theoriegeschichtlich suchen Vertreter des Rational Choice Institutionalismus (RCI) wie Douglas North (1981) oder Oliver Williamson (1985) für jene Verhandlungsprobleme Lösungen zu entwickeln, die mit den Modellen der neoklassischen Ökonomie nicht oder nur unzureichend erklärt werden konnten (Campbell 2004, 12; Rueschemeyer 2009, 207). Mithilfe von Institutionen - verstanden als formelle wie auch informelle Normen, Regeln und Verfahrensweisen - werden Verhandlungsprozesse effizienter lösbar als dies unter perfekten Marktbedingungen der Modellökonomie der Fall ist. So 
liefert der RCI, den Rueschemeyer (2009) wegen seiner theoretischen Nähe zur neoklassischen Ökonomie auch als ,new economic institutionalism" bezeichnet, beispielsweise Antworten auf die Frage, inwiefern Institutionen dabei helfen können, bestehende Koordinationsprobleme in der Bereitstellung öffentlicher Güter zu lösen (Campbell, 2004, 11). Zugleich zeigen Arbeiten der RCI-Perspektive, dass bestehende Institutionen auch zu Ineffizienzen auf Märkten beitragen können (Campbell, 2004, 12f.). Entgegen der auf nutzenmaximierende Akteure konzentrierten Perspektive des RCI stellen Vertreter des Historischen Institutionalismus (HI) die historisch einzigartigen Umstände, die zur Entstehung von Institutionen führen können, ins Zentrum ihrer Analysen. Institutionen werden verstanden als formelle und informelle Regeln und Verfahrensweisen (Campbell, 2004, 12), die entgegen der RCI-Perspektive nicht nur als Restriktionen für die Strategien der Akteure funktionieren, sondern auch die Präferenzen und Zielsetzungen der Akteure selber mit beeinflussen (Campbell, 2004, 25). Studien in der Tradition des HI befassen sich - oftmals inspiriert durch die Arbeiten von Weber und Marx - mit Transformationen von Staaten, dem Verhältnis zwischen Produktionsweisen und Klassendynamiken oder der Ausbreitung von Märkten (Rueschemeyer, 2009, 206). Dabei geht es insbesondere um die Frage, wie bereits bestehende Institutionen zu einem bestimmten Zeitpunkt den Rahmen für die Entstehung neuer Institutionen beeinflussen konnten (Campbell, 2004, 11). Campbell weist jedoch darauf hin, dass bestehende Institutionen dabei nicht direkt neue institutionelle Arrangements determinieren, sondern vielmehr den Rahmen einschränken, in dem sie sich wandeln können (Campbell, 2004, 24). Institutionen reflektieren daher immer selber auch ihre eigenen historischen Wurzeln:

„once institutions have been established through complex struggles and bargaining among organized groups, they have a continuing effect on subsequent decision-making and institution-building episodes." (Campbell, 2004, 25) 
Aufgrund dieser selbstverstärkenden Wirkung bestehender Institutionen, betonen HI-Studien die Relevanz von Pfadabhängigkeiten (Pierson, 2000) und legen somit den Fokus auf die Konstanz von Institutionen. Große Vorteile bietet dieser Ansatz vor allem in der international vergleichenden Perspektive von Institutionen, da diese auf historische Besonderheiten im Entstehungsprozess des jeweiligen Institutionengefüges verweisen (Mahoney u. Rueschemeyer, 2003). Insbesondere die vergleichende Wohlfahrtsstaatenforschung hat von derartigen Ansätzen profitieren können (Campbell, 2004, 23f.). In Bezug auf die unterschiedlichen Wohneigentumsquoten in den USA und Deutschland zeigt Kohl, dass die bis heute bestehenden Unterschiede in den historischen Wurzeln der Wohnungssysteme begründet sind: Während in den USA vor allem die private Wohnungskreditaufnahme unterstützt wurde, erfolgte ein Großteil der Unterstützung in Deutschland für gemeinnützige Wohnungsgesellschaften bereits vor dem Ersten Weltkrieg. Die Gemeinnützigen stellten Mietwohnungen bereit und boten damit schon früh eine Alternative zum Eigenheim anboten (Kohl 2015; Kohl 2016; Kohl 2017).

Gegenüber dem RC-Institutionalismus und dem Historischen Institutionalismus nimmt der Soziologische - oder auch Organisationale - Institutionalismus (SI) deutlich stärkeren Bezug auf die Bedeutung von Normen, kulturellen Faktoren und kognitiven Rahmungen (Campbell u. Pedersen, 2001b, 7), was gemeinhin auch als cognitive turn bezeichnet wird (Rueschemeyer, 2009, 206). Institutionen werden in dieser Perspektive nicht nur als formale Regeln definiert, sondern auch als etablierte kulturelle Rahmungen und kognitive Schemata verstanden (Campbell, 2004, 11). Dabei umfasst der Institutionenbegriff auch Routinen, Symbole und Schemata, die selber dazu beitragen, dass sich Institutionen reproduzieren und verstetigen (Campbell, 2004, 19). Insgesamt tendieren alle klassischen Institutionalismus-Perspektiven dazu die Konstanz von Institutionen gegenüber ihrem Wandel zu betonen.

Gegenüber den konstanzbetonenden klassischen Institutionalismen haben sich zunehmend Forschungsansätze etabliert, die weder den radi- 
kalen Wandel von Institutionen noch deren Konstanz fokussieren (Mahoney u. Thelen, 2010, 2). Daher betonen Streeck u. Thelen (2005b), dass Prozesse der Vermarktlichung oft in kleinteiligen Schritten erfolgten (Streeck u. Thelen, 2005b, 33). Dieser Wandel lässt sich dabei vor allem auf zwei Ursachen zurückführen, die bereits in den klassischen Institutionalismen angelegt sind: Rahmenbedingungen und das Handeln von Akteuren. Im Folgenden sollen diese Ursachen institutionellen Wandels genauer betrachtet werden, um alternative Erklärungsmuster gegenüber der Bedeutung der Wissensproduktion aufzuzeigen. Dabei erweist sich vor allem die Modellierung der Handlungsentscheidung von Akteuren als bedeutsam für den Fortgang der Untersuchung.

\subsubsection{Wirtschaftliche \& politische Rahmenbeding- ungen}

$\mathrm{Zu}$ einem institutionellen Wandel kommt es primär dann, wenn sich die exogenen Rahmenbedingungen ändern. Im RCI-Ansatz passen bei die Akteure bei sich ändernden Rahmenbedingungen ihr individuelles Nutzenkalkül an. Dementsprechend ist diese Sichtweise besonders anschlussfähig an Konzepte institutionellen Wandels, die Machtressourcen der Akteure als Ursache institutionellen Wandels in den Vordergrund stellen (Korpi u. Palme 1998). Im Rahmen des HI-Ansatzes wird der auf Rahmenbedingungen beruhende Wandel häufig als punctuated equlilibrium, also eine Aneinanderreihung gleichgewichtiger Zustände, beschrieben, die durch Krisen erschüttert werden (Hall 1993; Campbell 2004, 26; Campbell u. Pedersen 2001b, 10; Campbell u. Pedersen 2001a, 257). Zu diesen Umbrüchen gehören Kriege, ökonomische Krisen, technischer Fortschritt sowie weitreichendere Politikwechsel (Campbell, 2004, 174). Für die empirische Arbeit im Rahmen dieses Forschungsprojektes legen die Erkenntnisse des HI somit nahe, dass institutioneller Wandel stets vor dem Hintergrund der zum jeweiligen Zeitpunkt gegebenen historischen Ereignisse und der entsprechenden Vorbedingun- 
gen erfolgen muss. Damit sind sie besonders anschlussfähig an Erklärungsansätze institutionellen Wandels, die vor allem sozioökonomische Entwicklungen in den Vordergrund stellen (Wilensky 1975). Ergänzend kann die SI-Perspektive dazu beitragen, dass Wandel oft als Reaktion auf Druck der sie umgebenden Umwelt zustande kommt - vor allem dann, wenn sie von ihrer Umwelt als nicht mehr akzeptabel angesehen wird (Rueschemeyer 2009, 224; Campbell 2004, 17). Dabei spielt die Legitimität oder auch Angemessenheit einer Institutionen eine entscheidende Rolle:

„Organizations adopt whatever practices they believe their institutional envrironment deems appropriate or legitimate regardless of whether these practices increase organizational efficiency or otherwise reduce costs relative to benefits." (Campbell, 2004, 18)

Für die vorliegende Untersuchung ist es also wichtig auch die Entwicklung ökonomischer, politischer und gesellschaftlicher Rahmenbedingungen mit zu berücksichtigen, um die Relevanz der Wissensproduktion für die beobachteten Wandlungsprozesse nicht überzubewerten. Im Hinblick auf Wandlungsprozesse im Bereich der Wohnungspolitik sind dabei insbesondere Informationen zur Versorgungslage mit Wohnungen zu berücksichtigen (vgl. Jaedicke u. Wollmann, 1991, 433). Durch die Betrachtung eines policy-Subsystems stellt sich zudem die Herausforderung auch Entwicklungen in anderen Politikbereichen mit einzubeziehen. Für die Eigenheimförderung im Besonderen sind die Mietenpolitik sowie die Förderung des Sozialen Mietwohnungsbaus relevant, da Konflikte in diesen Bereichen auch in den Debatten zur Eigenheimförderung ausgetragen wurden und die politischen Koalitionen mit bestimmten. Darüber hinaus sind politikfeldübergreifende Wechselwirkungen in der allgemeinen Wohnungspolitik besonders ausgeprägt, ,weil dieses Politikfeld keine festen Grenzen aufweist, sondern sich durch fließende Grenzen auszeichnet - etwa zur Sozialpolitik allgemein, aber auch zur 
Städtebaupolitik und zur Familienpolitik sowie bei der Eigenheimzulage zur Vermögens- und darüber zur Renten-/Alterssicherung" (Heinelt, 2004, 46). Veränderte Kontextbedingungen können in allen hier diskutierten Modellen dazu führen, dass Akteure ihre Handlungen anpassen. Auf welcher Grundlage dies geschehen kann, soll im folgenden Abschnitt beschrieben werden.

\subsubsection{Interessenbasierte Akteurshandlungen}

Eine wesentliche Gemeinsamkeit der klassischen Institutionalismen bildet die Forschungsheuristik des akteurszentrierten Institutionalismus, in der das Akteurshandeln vor allem von zwei Faktoren bestimmt wird: dem Eigennutz der Akteure und von Institutionen (Scharpf, 2000, 49ff.). Die wesentliche Annahme im Modell des RCI besteht in der Gewinnbzw. Nutzenmaximierung der beteiligten Akteure. Basierend auf ihren eigenen Präferenzen und den ihnen zur Verfügung stehenden Ressourcen treffen sie Entscheidungen in Verhandlungssituationen. Eine auf diesen Annahmen basierende Analyse institutionellen Wandels muss einerseits die kalkulatorisch relevanten Rahmenbedingungen identifizieren und andererseits die den Kalkulationen zugrundeliegenden Interessen der Akteure kennen. Zu berücksichtigen ist insbesondere bei den Rahmenbedingungen, dass bereits bestehende Institutionen im RCI Strukturen erzeugen, ,die es für die Akteure profitabel macht, auf einem einmal eingeschlagenen Weg zu bleiben“ (Münnich, 2010, 30). Die Akteure tendieren also häufig dazu, nur kleinteilige Änderungen an bestehenden Institutionen vorzunehmen, sodass aus dieser Perspektive gleichbleibende Entwicklungen wahrscheinlicher sind als institutioneller Wandel (Campbell, 2004, 13). Auch aus der HI-Perspektive kann institutioneller Wandel einerseits über die Beeinflussung der Interessenstrukturen der Akteure zustande kommen (Campbell u. Pedersen, 2001b, 11). Andererseits können Institutionen angepasst werden, wenn sich die Logiken bestehender Institutionen widersprechen (Campbell u. 
Pedersen, 2001b, 10).

Somit ist klar, dass Akteurshandlungen von entscheidender Bedeutung für institutionellen Wandel sind. Allerdings wurde noch nicht spezifiziert, welche Akteure für den Wandel von policies von Bedeutung sind. Da Prozesse, aus denen policies als Institutionen hervorgehen, stets politisch sind, bedarf es zu dessen Verständnis ein ,Modell der Politik institutionellen Wandels" (Hall, 2006, 192). Dabei sind laut Hall zwei Akteursgruppen von entscheidender Bedeutung:

1. Die Regierung ändert die politischen Rahmenbedingungen, ,,[a]usgestattet mit den Mitteln, neue Verhaltensformen per Gesetz durchzusetzen" (Hall, 2006, 193)

2. Ökonomische Akteure ändern ihr Verhalten „und zwar oft dadurch, dass sie bestehenden kooperativen Arrangements die Gefolgschaft kündigen oder sich untereinander darauf einigen, die Bedingungen ihrer Interaktion zu ändern“ (Hall, 2006, 192)

Damit erweisen sich ökonomische Akteure auf der einen Seite und die politischen Machthaber auf der anderen Seite als die wesentlichen Triebfedern institutionellen Wandels - unabhängig von ihnen ist dieser nicht denkbar (Sachweh, 2011, 374). Streeck u. Thelen (2005b) benennen die relevanten Akteursgruppen in ihrem Modell weitreichender als rule makers und rule takers (Streeck u. Thelen, 2005b, 13) und berücksichtigen damit alle Akteure, die von den Regeln und ihren Änderungen betroffen sind. Die Miteinbeziehung der von den Regeländerungen betroffenen Akteure in das Modell institutionellen Wandels ist deswegen von Bedeutung, weil die Wirksamkeit von policies maßgeblich von deren Umsetzung abhängt. Zudem ist von einem direkten Feedback der rule takers gegenüber der rule makers auszugehen, worin die demokratischen Strukturen Ausdruck finden sollen (Streeck u. Thelen, 2005b, 14). Institutioneller Wandel ist somit laut Streeck u. Thelen (2005b) auch dann möglich, wenn sich zwar die formelle Institution nicht än- 
dert, aber die Umsetzung durch die Akteure nicht stattfindet (vgl. Abbildung 2.1).

Gemein ist den hier diskutier-

Abbildung 2.1: Politisches Modell policy-bezogenen Wandels.

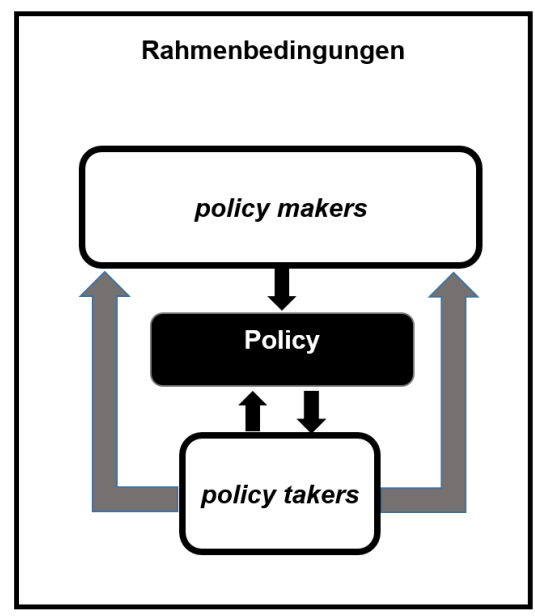

Quelle: eigene Darstellung in Anlehnung an Streeck u. Thelen (2005b). ten Modellierungen institutionellen Wandels nicht nur, dass sie die Entscheidungen von Akteuren vor dem Hintergrund diverser Rahmenbedingungen als wesentlichen Faktor institutionellen Wandels ansehen. Eine weitere Gemeinsamkeit findet sich in der Annahme, dass das Handeln der Akteure vor allem durch eine Orientierung an ihren eigenen Interessen beeinflusst wird. Rueschemeyer verweist in diesem Zusammenhang auf das sich ändernde Effizienzkalkül beim RCInstitutionalismus, sich wandelnde Machtverhältnisse für den historischen Institutionalismus oder aber der Wandel kultureller Kontextfaktoren für den soziologischen Institutionalismus (Rueschemeyer, 2009, 224). Institutionen, die auf Effizienzkalkülen, Machtstrukturen oder kulturellen Rahmenbedingungen beruhen ,should change as a result of shifts in those underlying conditions" (Thelen 2004, 211f.).

Allerdings bilden die bislang diskutierten Ansätze mit ihrem Fokus auf den Wandel von policies nur die regelbezogene Ebene von Vermarktlichungsprozessen ab. Dabei müssen zur Untersuchung von Vermarktlichungsprozessen auch kognitive und normative Gehalt von Institutionen berücksichtigt werden (vgl. Campbell u. Pedersen, 2001a). Zudem wird mit dem bloßen Verweis auf Interessen der Akteure allenfalls unzureichend erklärt, wie die Interessen und Handlungsstrategien 
der Akteure überhaupt zustande kommen (Blyth, 2001, 3). Um auch diese Lücke zu schließen, soll im im folgenden Abschnitt gezeigt werden, dass Ideen (1) selber ein Bestandteil von Institutionen sind und (2) eine wichtige Rolle in der Sinnstiftung der Akteurshandlungen spielen.

\subsection{Ideen und Institutionen}

Gegenüber den drei klassischen Institutionalismen werden im diskursiven Institutionalismus (DI) auch diskursive Elemente ${ }^{2}$ zu einer Bedingung institutionellen Wandels (Campbell u. Pedersen 2001b; Schmidt 2008; Béland u. Cox 2011). Das skizzierte Modell institutionellen Wandels lässt sich durch die DI-Perspektive in zweierlei Hinsicht ergänzen. So wird erstens von einem breiteren Institutionenbegriff ausgegangen als dies beispielsweise im RCI der Fall ist. Während die engere Definition von Institutionen als „norms with teeth“ ausgeht (Rueschemeyer, 2009, 210) und sich damit auf deren Regelgehalt konzentriert, der mit starken, aber durchaus variablen Mechanismen durchgesetzt wird, finden sich in der DI-Perspektive zugeordneten Studien Definitionen, in denen Ideen selber ein Teil der Institutionen sind. Nach dieser Vorstellung, die auch bei Vertretern des Soziologischen Institutionalismus vorherrscht, gewinnen Institutionen Stabilität dadurch, „dass sie eine Leitidee institutionalisiert und so einen symbolischen Wertbezug ausdrückt" (Münnich, 2010, 34). Institutionen haben somit nicht nur einen festen Regelbezug, sondern als Träger von Ideen auch einen ideellen Bezug (vgl. Schmidt 2010b, 9; Fourcade 2009, 25). Dieser ideeller Bezug von Institution wird von Lessenich (2003) auch als „Leitidee“ bezeich-

2 Allerdings erscheint in den meisten Ansätzen unklar, was alles unter diskursiven Elementen zu verstehen ist. So wird in der sozialwissenschaftlichen Forschung einer „Gesamtheit der sozial verfügbaren kognitiven, evaluativen und normativen Orientierungsstandards wie soziale Deutungsmuster, Wertvorstellungen, Normen und Handlungsorientierungen" sozialpolitische Relevanz zugeschrieben (Ullrich 2003, 9f., zitiert nach Sachweh 2011, 373). 
net. Institutionen im Sinne von policies regulieren nicht nur einen bestimmten Politikbereich, sondern ,,vermitteln - über ihre Leitideen zunächst einmal Ordnungssinn und Stabilitätsgewissheit" (Lessenich, 2003, 43). Mit diesem erweiterten Institutionenkonzept lassen sich sowohl institutionelle Stabilität als auch deren Wandel beschreiben. Hinsichtlich der stabilisierenden Funktion von Ideen als Teil von Institutionen verweist Blyth (2001) auf ihre Funktion als kognitive Schlösser. Mit Verweis auf Studien zu Pfadabhängigkeiten im historischen Institutionalismus zeigt er, dass institutionalisierte Ideen dazu beitragen, dass weitere policy-Änderungen nur in Bezug auf diese Ideen stattfinden können:

„Whereas historical institutionalists have hitherto understood policy continuity in the face of environmental changes as a function of the path dependence of institutions, it may also be a function of cognitive locking and is thus an ideational rather than an institutional phenomenon." (Blyth, 2001, 4, HvH.i.O.)

Allerdings lässt sich auch institutioneller Wandel durch das erweiterte Institutionenverständnis begreifen. Lessenich führt hierzu an, dass eine ,allmähliche Verselbständigung und faktische Fraglosigkeit der Ordnungsvorstellungen einer Institution [...] den Möglichkeitsraum für flexible Interpretationen und innovative Implementationen derselben" öffnen (Lessenich, 2003, 48). Institutionen können sich demnach auch ändern ,as actors' ideas and discourse about them change in tandem with changes in their performance" (Schmidt, 2010b, 9). Mit Institutionen verknüpfte Ideen können so Bestandteil inkrementellen Wandels werden: entweder über den Prozess der Aushöhlung oder auch durch eine wechselnde Zuschreibung von Ideen zu bestehenden Institutionen. Dementsprechend zeigen Campbell u. Pedersen (2001a) anhand einer Zusammenstellung von Studien zu institutionellem Wandel, dass sich verschiedene Wirkungsdimensionen von Vermarktlichung unterscheiden 
lassen (Campbell u. Pedersen, 2001a, 250): Zum einen kann die Liberalisierung die regulierende Dimension, also den Regelgehalt einer Institution betreffen, ,that consists of economically consequential rules of action" (Campbell u. Pedersen, 2001a, 250). Eine Vermarktlichung in dieser Dimension wird also in einem Wandel von policies erkennbar, die das Handeln der Akteure regulieren. Die weiteren Dimensionen, die von einer Vermarktlichung betroffen sein können, beschreiben Campbell u. Pedersen (2001a) als positive und normative Dimension. Die positive, oder auch kognitive Dimension ,makes predictive claims about the effects [...] on economic performance" (Campbell u. Pedersen, 2001a, 250). Sie ist primär mit Erkenntnissen der ökonomischen Theorie und ihren Vorhersagen verknüpft, wonach freie Märkte durch ihre Effizienz die besten Ergebnisse liefern. In der normativen Dimension sind demgegenüber mit den policies verknüpfte Idealvorstellungen betroffen, die mit Vermarktlichungsprozessen einhergehen.

Die zweite Ergänzung des engen strukturalistischen Modells institutionellen Wandels liegt hingegen im Handlungsmodell der Akteure selbst begründet. Während in einer engen Definition Interessen als, ,verhaltensorientierende Ziele und Bedürfnisse von Einzelnen und Gruppen in einem sozialen Umfeld" (Weber, 1977, 31) dargestellt werden, erscheinen Ideen oftmals als den materiellen Interessen entgegengesetzte konstruktivistische Einflussfaktoren menschlichen Handelns. So rückt der wissenspolitologische Ansatz - anstelle von politischen Rahmenbedingungen, Entwicklungen, konkreten Problemen, Anforderungen, Institutionen und weiteren Machtkonstellationen - das ,in seiner Geltung strittige und veränderbare Wissen politischer Akteure über Rahmenbedingungen, Probleme und Konstellationen" in das Zentrum der Analysen (Nullmeier u. Rüb, 1993, 24). Dies ist insofern eine bemerkenswerte Abweichung von anderen politikwissenschaftlichen Konzepten, da viele in der Literatur als bedeutend geltende Kausalfaktoren wie ökonomische, soziale oder demographische Rahmenbedingungen, politische Institutionen, Klassenkonstellationen oder -bedürfnisse, Parteienkon- 
stellationen und Machtverhältnisse keine unmittelbare Beziehung zu Politikergebnissen mehr zugestanden wird. Stattdessen ist es aus Sicht der konstruktivistischen policy-Analyse den politischen Akteuren selbst überlassen ihren Handlungsraum durch Deutung von Situationen und möglichen Handlungszielen selbst zu konstituieren, um in Deutungskämpfen bestehen zu können (Nullmeier u. Rüb, 1993, 19f.).

Dabei sind Ideen und Interessen faktisch enger miteinander verknüpft als es ihre dualistische Gegenüberstellung vermuten ließe. So weist Münnich (2011) darauf hin, dass eine mangelnde Trennschärfe in der Definitionen von ideengeleitetem Handeln einerseits und interessengeleitetem Handeln andererseits besteht. Münnich plädiert daher dafür die Konzepte von Ideen und Interessen handlungstheoretisch miteinander zu verknüpfen (Münnich, 2011, 373). Da sich die Formierung von Interessen nicht eindeutig von ideellen Einflüssen auf deren Handeln trennen lässt, wird diese hier in Anlehnung an Münnich verstanden ,als Ausdruck der situativen Vermittlung von materiellen Positionen und ideellen Wahrnehmungsmustern“ (Münnich, 2011, 371). Ideen haben somit keinen unabhängigen Stellenwert von Interessen oder sind diesen entgegenzusetzen. Stattdessen wird die „Interessenformierung [selbst] als ein Prozess der ideengeleiteten Sinnstiftung [verstanden], der positional eingeschränkt ist" (Münnich, 2011, 380). Dies bedeutet, dass Ideen wie in den oben dargestellten Konzepten durchaus handlungsanleitend sein können, aber die Handlungen der Akteure niemals vollends bestimmen (vgl. Abbildung 2.2). Ein Wandel von Ideen erzwingt in dieser Modellierung somit keinen institutionellen Wandel, kann diesen aber über die Sinnstrukturierung der Akteure ermöglichen (vgl. Seeleib-Kaiser, 2003b). Das Set der zur Verfügung stehenden Ideen wirkt gemeinsam mit der materiellen Position des Akteurs im jeweiligen situativen Kontext als sinnstiftendes Element der Handlung. Selbst wenn Interessen für institutionellen Wandel besonders betont werden, sind Ideen also wesentlicher Bestandteil der Interessenformulierung und damit auch handlungsentscheidend. 
Abbildung 2.2: Interessenformierung unter Berücksichtigung von Ideen.

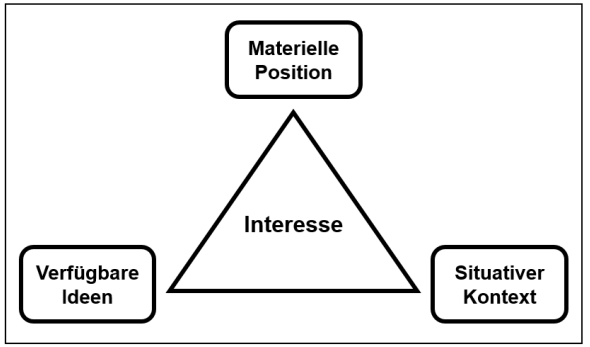

Quelle: eigene Darstellung in Anlehnung an Münnich (2011, 384)

Besonders deutlich wird die Bedeutung von Ideen zur Formierung von Interessen in Krisensituationen, die von Unsicherheit geprägt sind. So zeigt Blyth (2002) in seiner Arbeit zur Transformation der schwedischen Wirtschaftspolitik, dass Kämpfe um wirtschaftswissenschaftliche Theorien in Krisenmomenten einen paradigmatischen Wandel hervorrufen können, wodurch sich schließlich auch Institutionen wandeln können. Antizipieren Akteure nicht einmal mit gewissen Wahrscheinlichkeiten die Auswirkungen ihrer Handlungen antizipieren, geben ihnen Ideen als blueprints Handlungsorientierungen:

„By defining what the economy is, how it operates, and the place of the individual or collectivity within the economy, economic ideas both reduce uncertainty by defining a given moment of crisis and project the institutional forms that arguably will resolve it" (Blyth, 2001, 4).

Bezogen auf institutionellen Wandel bedeutet dies, dass nicht nur die jeweiligen Kontextbedingungen des Handelns, Macht- und Ressourcenstrukturen eine Rolle für die Positionierungen der Akteure spielen, sondern ebenso die ihnen zur Verfügung stehenden Ideen. Sie beeinflussen auf unterschiedliche Weise die Handlungsorientierungen der Akteure und können somit Einfluss auf institutionellen Wandel nehmen: Sie formen ,political and economic perceptions, the definition of actors' interests and, ultimatively, behavior" (Campbell u. Pedersen, 2001b, 6). Im hier verfolgten akteurszentrierten Ansatz bilden Wissen und Ideen 
handlungsrelevante Informationen, die zwischen den Akteuren transferierbar sind: Wissen und Ideen können ,won einem Anbieter hin zu einem Empfänger und Nachfrager von Beratung übermittelt werden“ (Blätte, 2018, 8).

$\mathrm{Da}$ in der Literatur zur Bedeutung von Ideen für Vermarktlichungsprozesse vor allem die Bedeutung ökonomischer Ideen hervorgehoben wird, soll im Rahmen der Darstellungen bereits Beispiele zu ökonomischen Ideen angeführt werden. Als handlungsanleitende und damit im Rahmen dieser Arbeit relevante Ideen werden hier all jene Elemente bezeichnet, ,die Wertigkeiten von Informationen, Problemen und Maßnahmen festlegen und realitätsstrukturierende Effekte für die Akteure zeitigen, die mit ihnen operieren" (Schneider u. Janning, 2006, 211). Dabei sei an dieser Stelle explizit darauf hingewiesen, dass sowohl kognitive als auch normative Ideen zu verorten sind (Sachweh 2011, 372; Münnich 2011, 378; Rueschemeyer u. Skocpol 1996, 300). Während die kognitiven Wahrnehmungen anzeigen sollen, was ist und was zu tun ist, deuten die Inhalte normativer Leitbilder darauf hin, was getan werden sollte und was als gut oder schlecht anzusehen ist (Schmidt, 2008, 306f.). Allerdings lassen sich selbst positivistische Beschreibungen ${ }^{3}$ und theoretische Analysen nie vollends von normativen Ideenkonzepten trennen (Rueschemeyer u. Skocpol, 1996, 300). Blyth (2002) bringt diesen Umstand in Bezug auf ökonomische Ideen wie folgt auf den Punkt:

„Economic ideas provide agents with both a ,scientific“ and a ,normative“ account of the existing economy and polity, and a vision that specifies how these elements should be constructed." (Blyth, 2002, 11)

3 Die Vorstellung einer positiven Ökonomie als Gegenstück zu einer normativen Ökonomie geht auf Friedman (1953) zurück. Er vertrat die Auffassung die positive Ökonomie könne ,in principle independent of any particular ethical position or normative judgements" bestehen und sich damit von der normativen Ökonomie abgrenzen (Friedman, 1953, 4). 
Bislang existieren keine allgemeingültigen Definitionen von Ideen, die für die vorliegenden empirischen Untersuchungen tragfähig wären (Béland u. Cox, 2011). Dies ist jedoch nicht einer mangelnden Forschung geschuldet, sondern vielmehr dem Umstand, ,that there are so many ideas about ideas" (Schmidt, 2008, 306). Metha (2011) und Schmidt (2008) unterscheiden Ideenkonzepte nach ihrer Abstraktionsebene. Die abstrakteste und zugleich am schwersten zu erfassende Ebene politischer Ideen ist jene der umfassenden Weltanschauungen, die Metha (2011) auch als Zeitgeist bezeichnet. Zu dieser abstrakten IdeenEbene zählt Schmidt (2008) neben den public philosophies von Campbell (1998) auch dessen Konzept der public sentiments (Campbell, 2004). Unter dem public sentiment versteht Campbell (1998) jene normativen Hintergrundannahmen, die zwar nur selten offen zutage treten, jedoch den Handlungsspielraum von politischen Akteuren insofern begrenzen als dass sie gewisse Handlungsalternativen als politisch inakzeptabel ausschließen (Campbell, 1998, 8). Hinweise für diese pfadweisende Funktion abstrakter Ideen finden sich bereits in den Arbeiten von Weber: Hier bestimmen Weltbilder die Bahnen, ,in denen die Dynamik der Interessen das Handeln fortbewegt" (Weber, 1988, 252) und bilden in diesem Sinne für die Akteure den Rahmen der denkbaren Handlungsoptionen. Allerdings weisen derart abstrakte Weltbilder keinen direkten policy-Bezug auf, der die Handlungen von Akteuren im Hinblick auf konkrete politische Entscheidungen leiten könnte. Deutlich konkreter fallen die handlungsanweisenden Aspekte demgegenüber bei den weniger abstrakten Paradigmen und Politiklösungen aus, die als relevante Analyseebenen für den weiteren Gang der empirischen Arbeiten bestimmt werden und in den folgenden Abschnitten erläutert werden. 


\subsubsection{Techniken \& Analytische Tools}

Den Gegensatz zur abstrakten Ebene der Weltbilder bilden policy-solutions auf Ebene einzelner Politikinstrumente. Diese Ideen liefern den Akteuren die wohl konkretesten Handlungsoptionen angesichts spezifischer politischer Problemlagen. Somit bezeichnet Metha (2011) diese Ideen auch als policy-solutions. Im Rahmen des advocacy-KoalitionenAnsatzes (Advocacy-Coalition-Framework, ACF) von Sabatier u. JenkinsSmith (1999b) werden derart konkrete Politiklösungen aufgrund ihrer Ableitbarkeit aus abstrakteren Ideen auch als secondary beliefs bezeichnet. Sie beinhalten vor allem technische Aspekte, ,wie etwa Detailwissen über legislative Einzelheiten" (Sachweh, 2011, 373) und sind aufgrund ihrer Situationsbezogenheit wohl die Ideen, die am häufigsten neuen Umständen angepasst oder aufgehoben werden (Weible u. a., 2009, 123). Sie sind empirisch besonders gut zu erfassen, da sie explizit im politischen Prozess diskutiert werden.

Derartiges Detailwissen zu politischen Instrumenten wird im Rahmen ökonomisch geprägter Debatten meist in Form ökonomischer Objekte wie Kennzahlen bereitgestellt. Arbeiten, die sich mit den Wirkungen von Kennzahlen auf wirtschaftliche Akteure auseinandersetzen, finden sich im Bereich der Wissen(schaft)s- und Techniksoziologie (Science and Technology Studies, STS), die sich in der Tradition von Callon vor allem mit der Frage beschäftigen, wie "[e]conomics, in the broad sense of the term, performs, shapes and formats the economy" (Callon, 1998, 2). Laut Callon existiert der in ökonomischen Modellen beschriebene homo oeconomicus durchaus als, the result of a process of configuration" (Callon, 1998, 22). Basierend auf der Annahme der Performativität ökonomischer Theorien bringen wirtschaftswissenschaftliche Ideen selber diejenigen Objekte hervor, die sie vorgeben zu beobachten. Wirtschaftswissenschaftliche Ideen sind somit als ,an engine, not a camera" zu verstehen (MacKenzie, 2008). Bei Kennzahlen oder anderen ökonomischen Objekten handelt es sich demnach nicht bloß um Abbil- 
dungen bereits existierender Objekte, die durch Kalkulation und ihre Darstellung sichtbar gemacht werden, sondern um solche die ,erst durch die Verfahren der Kalkulation hervorgebracht" werden (Kalthoff, 2007, 153). Durch ihre Modelle und deren Anwendung tragen ökonomische Ideen in dieser überaus konstruktivistischen Perspektive nicht bloß dazu bei, bestimmte Realitäten qua Informationen darzustellen, sondern auch diese maßgeblich mit zu konstruieren. Besonders sichtbar werden diese Zusammenhänge zwischen wirtschaftswissenschaftlicher Theorie und wirtschaftlicher Praxis in der Konstruktion von Finanzmärkten:

„Inscribed in the theory, as in financial economics more generally, was a vision of a market: not of how markets actually were, but of an 'ideal' market, where the ideal was not just a matter of eleminating mathematical complexities but became hitched to how markets were, historically, being transformed“ (MacKenzie, 2010, 99, e.HvH.).

Ökonomische Objekte als Bestandteil von policies können demnach dazu beitragen, dass Akteure den Gegenstand der jeweiligen Politiken anders betrachten. Sind die ökonomischen Objekte als neutrale und sachliche Beschreibung der gegebenen Rahmenbedingungen von den entscheidenden Akteuren akzeptiert, können sie einen massiven politischen, aber auch moralischen Einfluss auf die Entscheidungen der Akteure haben:

„Sheer factual description can become a tool for political agenda setting. [...] Such description establishes the authors as experts and lends authority to their views. This authority is rarely confined just to the factual information and analysis; it typically spills over to the broader ideas, conceptions, and policy poposals presented along with the „facts“." (Rueschemeyer u. Skocpol, 1996, 300f.)

Eine Analyse der Verwendung von Kennzahlen als Bestandteil von 
policies und deren Wirkung auf die Institution stellt Erkenntnisgewinne hinsichtlich der Wirksamkeit ökonomischen Wissens innerhalb politischer Institutionen in Aussicht. Allerdings bieten bloße Kalkulationen und deren Darstellungen den Akteuren noch kein ausreichendes, tatsächlich handlungsanleitendes Wissen. Dieses handlungsanleitende Wissen kommt erst durch eine über das policy-Instrument hinausgehende Interpretation der kalkulierten, dargestellten und verbreiteten Information zustande. Zusätzlich verbleibt der resultierende policy-Wandel überaus kleinteilig und nur innerhalb einer bereits bestehenden policy. Damit erscheinen konkrete policy-solutions einerseits überaus passend als Untersuchungsgegenstand für kleinteiligen institutionellen Wandel. Allerdings würden Analysen dieser Art in technischen Details einer policy verbleiben und einen darüber hinausgehenden Wandel nicht mit berücksichtigen.

\subsubsection{Paradigmen}

Als sinnvolle Ergänzung der policy-solutions in analytischer Hinsicht erweisen sich Paradigmen, die sich auf der mittleren Abstraktionsebene verorten lassen und gegenüber der weniger abstrakten Ebene ein breiteres politisches Rahmenprogramm umfassen (Schmidt, 2008, 306). Zu dieser Ebene zählt Schmidt (2008) die programmatic beliefs von Berman (1998) die policy cores von Sabatier u. Jenkins-Smith (1999a), sowie die policy paradigms von Hall (1993). Eine kurze Beschreibung dieser Konzepte soll die Verortung entsprechender Ideen sowie deren Bedeutung im politischen Kontext verdeutlichen.

Berman (1998) beschreibt mit dem Konzept der programmatic beliefs Ideen, die ,provide a relatively clear and distinctive connection between theory and praxis“ (Berman, 1998, 21). Dies liegt vor allem daran, dass in den programmatischen Ideen gegenüber den breiter gefassten Weltbildern die Zusammenhänge klar und in sich stimmig definiert sein müssen. Unter den policy cores, oder auch policy core beliefs verste- 
hen Weible u.a. (2009) Ideen, die gegenüber der konkreteren policyEbene mit einem gesamten policy-Subsystem ${ }^{4}$ einen weiteren Bereich abdecken (Weible u.a., 2009, 122) und grundlegende Wertvorstellungen, Kausalannahmen und Problemperzeptionen zu diesem politischen Subsystem beinhalten (Sabatier u. Jenkins-Smith 1999a, 127; so auch Béland u. Cox 2011). Da der Bezugspunkt dieser policy core beliefs eindeutig ist, bieten auch sie eine bessere Grundlage für politische Handlungsanleitungen als dies bei breiter gefassten Weltbildern der Fall sein kann. Diese Definition entspricht auch dem Konzept, das in der Analyse von Campbell u. Pedersen (2014) als policy paradigm bezeichnet wird. Paradigmen bilden in ihrem Sinne den kognitiven Rahmen, welcher die Kernannahmen und kausale Argumente über die Wirkungen einzelner policies in verschiedenen Situationen umfasst und somit den Rahmen der von den politischen Akteuren als Handlungsalternativen angenommenen policies vorgibt (Campbell u. Pedersen, 2014, 10). ${ }^{5}$ Eine insbesondere für die empirische Arbeit dieses Forschungsvorhabens geeignete Definition bietet Hall mit seinem Konzept der policy paradigms. Unter diesen Paradigmen versteht er einen interpretativen Rahmen, der nicht nur (1.) die Ziele verschiedener policies und (2.) die zu deren Erreichung benötigten Instrumente vorgibt, sondern (3.) auch die Probleme definiert, die durch diese Maßnahmen zu adressieren sind (Hall, 1993, 279).

Aus einer stärker konstruktivistischen Perspektive heraus bieten diese Paradigmen zunächst einen klaren Rahmen, in dem die Akteure ihre

4 „Die Untersuchungseinheit des policy-Subsystems geht nicht von einer typischen oder dominanten Akteurskonfiguration [...] aus, sondern bezieht grundsätzlich alle Akteure aus Politik, Verwaltung, Wissenschaft und Gesellschaft mit ein, die ein besonderes Interesse an politischen Regulierungsfragen artikulieren und versuchen, Wirkung im Politikfeld zu erzeugen“ (Schneider u. Janning, 2006, 195).

$5 \quad$ Als Ergänzung von Campbell u. Pedersen (2014) sei noch genannt, dass Ideen auf dieser Ebene im ökonomischen Kontext meist mit Daten und Analysen verschiedener Art unterlegt sind (Campbell u. Pedersen, 2014, 28). 
Welt interpretieren können und somit Handlungen überhaupt erst möglich machen. Münnich (2011) verweist in diesem Zusammenhang auf die Bedeutung von frames, die bestimmen, ,welche Faktoren einer gegebenen Situation [von den Akteuren] überhaupt als interessenrelevant wahrgenommen werden“ (Münnich, 2011, 379). Bezogen auf die Wohnungspolitik bedeutet dies, dass die politischen Akteure erst anhand von Kennzahlen und deren Interpretationen die Lage auf dem Wohnungsmarkt erfassen können (Lowe, 2011, 17). Erst im Rahmen eines solchen umfassenderen Paradigmas können die in Abschnitt 2.2.1 beschriebenen ökonomischen Objekte im politischen Prozess Handlungen der beteiligten Akteure anleiten. Ohne Interpretationen der Objekte und eine damit einhergehende Beschreibung der Wirksamkeit politischer Instrumente, sind diese für die Akteure in einer Entscheidungssituation ohne größeren Nutzen. So zeigen Kalthoff u. Köllner (2015), dass Entscheidungen über die Ermöglichung von wirtschaftlichen Handlungen nicht bloß auf den technischen Möglichkeiten und kalkulativen Praktiken beruhen, ,,sondern in und durch eine soziale Praxis der ganz uneinheitlichen und sich widersprechenden Auslegung und Verständigung über den ökonomischen Sinn dieser Zahlenwelten fabriziert werden“ (Kalthoff u. Köllner, 2015, 406, e.HvH.). Da die auf Techniken und Kalkulation beruhenden „Zahlenwelten“ alleine durchaus ambivalente Deutungen zulassen - so das Argument von Kalthoff u. Köllner (2015) - bedürfen diese stets einer Interpretation (so auch Langenohl 2009 und Wansleben 2011). Anhand von Interpretationen der Zahlenwelten kann ökonomischen Ideen auch eine legitimierende Funktion zugeschrieben werden (Spruijt u. a., 2014, 20). Einen Hinweis auf eine derartige legitimierende Funktion liefern MacKenzie u. Millo (2003) für die Regulierung des Marktes für Finanzderivate in Chicago. Demnach bot das Modell von Black, Scholes und Merton nicht nur ein Idealbild für das Funktionieren der Märkte, an dem sich die wirtschaftlichen Akteure orientieren konnten, sondern lieferte auch ,the capstone, decisively undermining the regulatory view that derivatives were morally dubious 
instruments of gambling" (MacKenzie u. Millo, 2003, 136f.). So konnte ein Marktmodell als umfassendes politisches Paradigma mit einer eigenen Problemdefinition, einer Zielsetzung sowie konkreten Instrumentarien dazu beitragen, dass neue Politiken und damit auch neue Märkte geschaffen wurden.

Insgesamt bieten Paradigmen den Akteuren ein breites Set an Annahmen und Vorstellungen für ein bestimmtes Politikfeld, die deutlich über die bereits beschriebene Dimension bloßer policy solutions hinausgehen. Daher bieten Paradigmen gemeinsam mit policy solutions eine geeignete Analyseebene für den Ideenwandel im policy-Subsystem der Wohneigentumsförderung in der Bundesrepublik Deutschland. Im folgenden Abschnitt soll das in in Abschnitt 2.1 skizzierte Modell institutionellen Wandels um die hier erläuterten Ideenkonzepte ergänzt werden.

\subsection{Ideenwandel als institutioneller Wandel}

Wie in Abschnitt 2.1 gezeigt wurde, lässt sich institutioneller Wandel nicht unabhängig von den Handlungen von policy makers und policy takers erklären. Anpassungen im Akteurshandeln wird in Bezug auf institutionellen Wandel meist mit sich wandelnden Rahmenbedingungen begründet: Erst wenn sich diese ändern, haben die Akteure einen Anlass basierend auf ihren eigenen Interessen ihre Handlungsmuster zu ändern und institutionellen Wandel herbeizuführen. Eine Einschränkung institutionellen Wandels als bloßes Ergebnis von Krisen wird dabei von Streeck u. Thelen (2005b) kritisiert, die anmerken, dass es insbesondere der langsame, endogene Wandel von Institutionen ist, der in den Vermarktlichungsprozessen der vergangenen Jahrzehnte eine bedeutende Rolle gespielt hat. Basierend auf dieser Erkenntnis wird hier ein Modell institutionellen Wandels vorgeschlagen, dass nicht nur Bedingungen des externen institutionellen Wandels, sondern auch des inkrementellen Wandels einbezieht. Beide Akteursgruppen können 
durch Anpassungen ihrer interessenbasierten Handlungsstrategien institutionellen Wandel herbeiführen (Streeck u. Thelen, 2005b).

Zusätzlich wird im Rahmen dieser Arbeit die Bedeutung von Ideen für institutionellen Wandel betont. Zwar reflektieren Ideen selber ihr institutionelles Umfeld - vor allem in stabilen Zuständen (Blyth, 2002, 10). Allerdings sind sie in zweierlei Hinsicht für institutionellen Wandel von Belang. Erstens haben Institutionen nicht nur einen Regelbezug, sondern stets auch einen ideellen, diskursiven Bezug, der zu ihrem eigenen Wandel beitragen kann. Institutioneller Wandel dürfte demnach nicht nur den Wandel exogener Faktoren und interessengeleitetem Handeln der Akteure berücksichtigen, sondern muss auch den Wandel der mit den Institutionen verknüpften Ideen einbeziehen.

\section{Abbildung 2.3: Politisches Modell institutionellen Wandels mit Ideen- bezug im policy-Subsystem.}

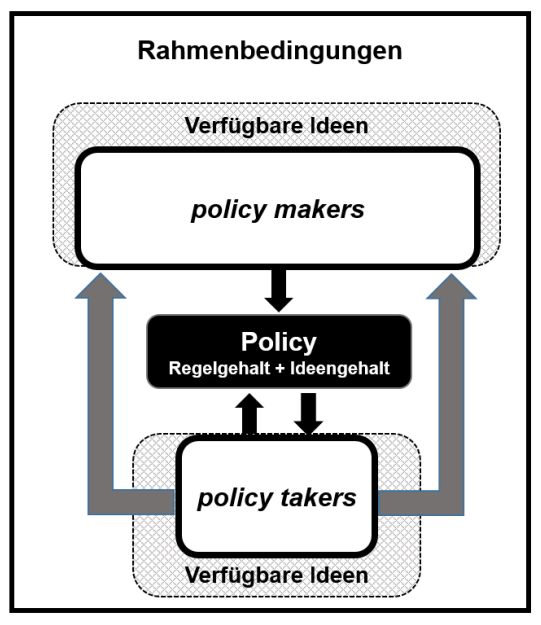

Quelle: eigene Darstellung
In diesem erweiterten Modell wird vonseiten der Akteure demnach nicht mehr nur der Regelgehalt einer Institution verhandelt, sondern auch ihr ideeller Gehalt. Zweitens wurde herausgearbeitet, dass Ideen dann einen bedeutsamen Einfluss auf institutionellen Wandel haben können, wenn sie sich als handlungsanleitend in der jeweils situativen Interessenformulierung der am institutionellen Wandel beteiligten Akteure erweisen. Mit dieser akteurszentrierten Perspektive soll darauf hingewiesen werden, dass jene Ideen, die den Akteuren zur Verfügung stehen, zwar das Handeln politischer Akteure anders strukturieren und die Weichen zu manchen Entscheidungen anders stellen können, jedoch niemals das 
Handeln der Akteure vollständig determinieren. Ausgehend von unterschiedlichen Abstraktionsgraden können policy-Paradigmen sowie Techniken und analytische Tools als wesentliche Ideen für das Akteurshandeln identifiziert werden. Aus den Überlegungen ergibt sich nicht nur ein erweitertes Institutionenkonzept, sondern auch ein um ideelle Aspekte erweitertes politisches Modell institutionellen Wandels (Abbildung 2.3). Insbesondere in Bezug auf Vermarktlichungstendenzen erweist es sich als wesentlich normative und kognitive Elemente für die Handlungsorientierungen von Akteuren in den Blick zu nehmen (Münnich u. Sachweh, 2017). Mit der anerkannten Bedeutung von Ideen für institutionellen Wandel ist jedoch nicht nicht geklärt, wie die für das Akteurshandeln entscheidenden Ideen den Akteuren überhaupt verfügbar gemacht werden. Dieser Zusammenhang soll im folgenden Kapitel genauer dargestellt werden. 


\section{Kapitel 3}

\section{Regime der}

\section{Ideenproduktion}

Wie im vorhergegangenen Kapitel herausgearbeitet wurde, werden Akteure als der zentrale Ausgangspunkt institutionellen Wandels angesehen. Dabei wird ihr Handeln weder vollkommen durch objektivierbare Interessen noch durch abstrakte Ideen determiniert: Handlungsleitende Interessen werden durch Ideen sowie den situativen Kontext beeinflusst. Damit ist allerdings noch nicht geklärt, wie und durch wen den Akteuren handlungsanleitende Ideen für die Politikgestaltung zur Verfügung gestellt werden. Basierend auf den Erkenntnissen der international vergleichenden Wohlfahrtsstaatenforschung haben Campbell u. Pedersen (2014) das Konzept der knowledge regimes entwickelt. Diese Regime umfassen die organisationale und institutionelle Maschinerie, die Daten, Forschungsergebnisse, Politikempfehlungen und andere Ideen für die politische Entscheidungsfindung produzieren (Campbell u. Pedersen, 2014, 3). Unter diesem analytischen Rahmen erhalten Ideen im politischen Prozess eine verortbare Herkunft, wobei es weniger darum geht, auf welchen Urheber abstraktere Ideenkonzepte wie Weltbilder 
zurückgehen. Vielmehr geht es darum, welche Akteure Ideen wie für den Politikprozess übersetzen und aufbereiten und damit gezielt policyInstrumente und umfassendere Paradigmen für die Politik bereitstellen (siehe auch Hustedt u. Houlberg Salomonsen 2014, 204; vgl. Mayntz 2009, 5). Besonders deutlich wird dieser Zusammenhang in der Rolle der knowledge regime als Übersetzer internationaler Ideentrends für die nationale Politik:

„We argue, however, that the structure and practices of knowledge regimes - not just political and economic institutions - also had important mediating effects. This is because knowledge regimes are where neoliberal ideas were often formulated and debated and because the nationally specific organization of knowledge regimes affected how these and other ideas were craftes in the first place." (Campbell u. Pedersen, 2014, 4)

Die Ausgestaltung des knowledge regimes ist demnach entscheidend dafür wie Ideen der Vermarktlichung und Finanzialisierung auf nationaler Ebene formuliert und debattiert und schließlich für die Akteure bereitgestellt werden (Campbell u. Pedersen, 2014, 6). Hier entscheidet sich also, welche Ideen den rule makers und den rule takers als Handlungsorientierung zur Verfügung stehen. Indem sie einen Politikbereich rahmen, können die Wissensproduzenten nicht nur zu policy-Wandel beitragen, sondern auch zum Ausbleiben von Änderungen. Die Wissensproduzenten ,liefern Zuarbeit zum Set der Annahmen, die sich um einen Glaubenskern gruppieren, sie reproduzieren und rekonstruieren diese" (Blätte, 2018, 8). So werden die Regime der Wissensproduktion im Modell von Campbell und Pedersen zu einem wesentlichen Erklärungsfaktor für die Frage, warum Ideentrends auf nationaler Ebene unterschiedliche Ausprägungen annehmen (siehe hierzu auch Fourcade, 2009, 239). Sie bilden aller internationalen Vernetzung zum Trotz nationenspezifische Konstrukte, deren Strukturen und Praktiken nicht 
vollständig durch das dazugehörige politische Regime und das Produktionsregime determiniert werden.

Sofern ein internationaler Vergleich unterschiedlicher knowledge regime Aufschluss über unterschiedliche Ergebnisse von Vermarktlichungstendenzen liefern kann, ist zu erwarten, dass auch eine Untersuchung der Entwicklung dieser knowledge regime über einen längeren Zeitraum hinweg Erkenntnisse über die generellen Möglichkeiten von Vermarktlichungsideen im jeweiligen politischen Kontext liefert. Von dieser Annahme ausgehend verwende ich das Modell von Campbell und Pedersen als Ausgangspunkt für meine Analyse ideellen Wandels in der bundesdeutschen Eigenheimförderung. Wenn sich die Ideen im politischen Prozess über einen längeren Zeitverlauf ändern, so die These, müssen diese Änderungen nicht nur mit den sich ändernden wirtschaftlichen und politischen Rahmenbedingungen verknüpft sein, sondern können auch auf einen Wandel in der Maschinerie der Ideenproduktion zurückgeführt werden.

In ihrer international vergleichenden Untersuchung ${ }^{1}$ stellen Campbell u. Pedersen (2014) fest, dass Ideen und Wissen in Deutschland gezielt vonseiten der Politik nachgefragt werden, und die Wissensproduzenten entsprechend auf die Nachfrage reagieren (Campbell u. Pedersen, 2014, 156). Dabei werden vor allem die halböffentlichen Forschungsinstitute als relevante Akteursgruppe sowie deren Beirats- und Gutachtertätigkeiten als entscheidende Mechanismen genannt (Campbell u. Pedersen, 2014, 129ff.). Zudem sei durch das Ende wirtschaftlichen Aufschwungs der Nachkriegsjahre auch der Korporatismus als Koordinationsform gesellschaftlicher Gruppen in eine Krise geraten (vgl. Czada, 1994), wodurch zunehmend mehr Wissensproduzenten aufgetreten sind, die Campbell und Pedersen als advocacy policy research organizations beschreiben (Campbell u. Pedersen, 2014, 143ff.). Aller-

1 Campbell u. Pedersen (2014) untersuchen in ihrem Ländervergleich knowledge regimes und deren Einflüsse in den USA, Frankreich, Dänemark und Deutschland. 
dings haben diese privaten Forschungseinrichtungen, die oft auch als Think Tanks bezeichnet werden, nicht so einen bedeutenden Stellenwert für die Wissensproduktion wie in den USA. Für eine Analyse der Ideenproduktion im policy-Subsystem der Eigenheimförderung können allerdings die Erkenntnisse von Campbell u. Pedersen (2014) zur Ausgestaltung des deutschen knowledge regimes nicht eins zu eins auf die Wohnungspolitik übertragen werden. Dies wird bedingt durch unterschiedliche Akteurskonstellationen, institutionelle Strukturen sowie inhaltliche Eigenheiten wie spezifische Konfliktlinien einzelner Politikfelder (vgl. Beyme u. Schmidt 2013; Lowe 2011, 10). So finden sich in unterschiedlichen Politikfeldern jeweils andere Konstellationen von beteiligten Akteuren, die auch Unterschiede in der Zusammensetzung der Wissensproduzenten erwarten lassen. Wie für einzelne Teilbereiche der wissenschaftlichen Politikberatung unterschiedliche Akteure beteiligt sind, so ist auch eine unterschiedliche Beteiligung von Verbänden und Parteien an der politikfeldspezifischen Wissensproduktion zu erwarten. Eine tiefergehende Betrachtung des knowledge regimes eines einzelnen Politikfeldes und dessen Entwicklung verspricht daher einen besseren Einblick in die Einflussmöglichkeiten der Wissensproduzenten innerhalb eines bestimmten Politikfeldes und auch in die Dynamiken, denen diese in einem längeren Zeitraum unterliegen. Der idealtypische Zusammenhang zwischen Ideenproduktion und institutionellem Wandel ist in Abbildung 3.1 dargestellt.

Mit dem Verweis auf Regime sei an dieser Stelle angemerkt, dass es sich dabei nicht nur um ein Set beteiligter Akteure handelt, sondern auch die entsprechenden Institutionen wie die dazugehörigen Regeln, deren Kontroll- und Durchsetzungsmechanismen umfasst, die das Verhalten der Akteure strukturieren (vgl. Campbell u. Pedersen, 2014, 29f.). In diesem Sinne gilt es für die Beschreibung der Wissensproduktion im Rahmen dieser Arbeit nicht nur die Akteure (Abschnitt 3.1), sondern auch die Mechanismen (Abschnitt 3.2) der Ideenbereitstellung für die Politik zu identifizieren (vgl. Evans u. a., 1985b, 358). In empirischen 
Abbildung 3.1: Ideenproduktion und institutioneller Wandel innerhalb eines policy-Subsystems

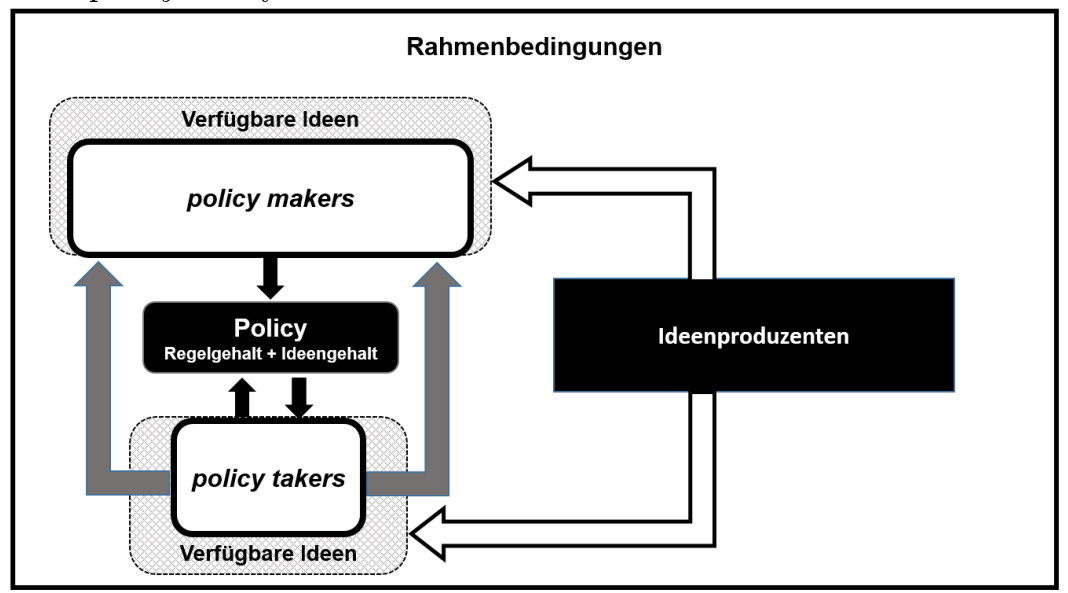

Quelle: eigene Darstellung

Arbeiten zur Wissensproduktion in Deutschland wird die gesonderte Stellung der öffentlichen Verwaltung nicht nur in dessen Produktion, sondern auch in ihrer Rolle als zentrale und vermittelnde Schnittstelle zwischen Regierung, Wissenschaft und Interessengruppen erkennbar. Das Kapitel endet mit einer Darstellung des Analyserahmens für die Ideenproduktion anhand eines erweiterten politischen Modells institutionellen Wandels (vgl. Abschnitt 2.3) und einer Zusammenstellung der zu überprüfenden Thesen zum Zusammenhang zwischen dem Regime der Wissensproduktion und dem ideellen Wandel in der Eigenheimförderung der Bundesrepublik Deutschland (Abschnitt 3.3). 


\subsection{Akteure im Regime der Ideenprodukti- on}

Erkenntnisse der jüngeren wissenspolitologischen Forschung lassen darauf schließen, dass entgegen dem idealtypischen Modell von Campbell $\mathrm{u}$. Pedersen (2014) in Deutschland keine vollkommene Trennung zwischen Ideenproduzenten und anderen Akteursgruppen besteht. Stattdessen sind ebenso Parteien und Interessenverbände an der Ideenproduktion beteiligt. Daher soll in den Abschnitten 3.1.1 und 3.1.2 auf die Ideenproduktion dieser Akteure eingegangen werden. Da im Hinblick auf Prozesse der Vermarktlichung und Finanzialisierung oftmals den Wirtschaftswissenschaften eine große Bedeutung zugeschrieben wird, sollen diese in Abschnitt 3.1.3 eingehender betrachtet werden. Als letzte und oftmals in der Literatur vernachlässigte Akteursgruppe stelle ich die Rolle der öffentlichen Verwaltung in der Wissensmaschinerie dar (Abschnitt 3.1.4). Im anschließenden Abschnitt 3.2 skizziere ich Mechanismen der Ideenproduktion im Sinne einer Bereitstellung für den politischen Prozess zwischen den unterschiedlichen Akteursgruppen.

\subsubsection{Parteien}

In ihrer Studie zur Entwicklung der Sozialpolitik in Deutschland weisen Nullmeier u. Rüb (1993) darauf hin, dass der Anteil jenes Wissens von zentraler Bedeutung ist, das von politischen Akteuren selbst produziert wird (Nullmeier u. Rüb 1993, 30; siehe auch Blätte 2018, 10). Sofern sie in Regierungsverantwortung kommen und mächtige Koalitionen bilden können, sind sie die handlungsfähigen rule maker und verfügen hinsichtlich des institutionellen Wandels über die wohl wichtigste Schlüsselposition für den Aufbau wohlfahrtsstaatlicher Institutionen (Esping-Andersen 1998, 49; vgl. Hall 1989). In der parteienstaatlichen Demokratie Deutschlands sind Parteien die wesentlichen Akteure im politischen System und gelten ,als Scharniere der politischen Willens- 
bildung zwischen Wahlbürgern und Staat" (von Alemann u.a., 2018, 115). Aus systemtheoretischer Perspektive haben Parteien vor allem die Funktion bestehende Debatten in politische Themen und Probleme zu transformieren (Luhmann, 2000, 245f.), ,indem sie politisches Personal selektieren, was wiederum zur politischen Integration und zur Sozialisation beiträgt und zur Selbstregulation führen kann, um damit die gesamte Legitimation des politischen Systems zu befördern" (von Alemann u.a., 2018, 4). In praktischer Hinsicht liefern parteieigene Experten für ein spezifisches Politikfeld neben policy-Ideen auch politisch taktisches Wissen für ihre jeweiligen Regierungsvertreter (Hustedt u. Houlberg Salomonsen, 2014, 207).

In der Literatur zu institutionellem Wandel finden sich zahlreiche Hinweise darauf, dass Parteien stets eine wesentliche Schlüsselposition zur Herbeiführung institutionellen Wandels einnehmen. So zeigt Hall (1989) in seiner Analyse zur Verbreitung des Keynesianismus, dass die politische Ideologie der dominanten politischen Partei eine Rolle spielt: Für sie müssen die produzierten Ideen anschlussfähig sein. Zudem produzieren die Parteien auch selber meist überaus konkrete Ideen für institutionellen Wandel: ,parties produce policy alternatives“ (Heclo, 1975, 293). Demnach sollte es in Anlehnung an die Parteidifferenztheorie (vgl. Schmidt, 2001) sowohl in Bezug auf die angewendeten Ideen als auch auf die Wissensproduktion einen Unterschied machen, welche Partei an der Macht ist. Auch in Untersuchungen zur bundesdeutschen Wohnungspolitik wird die Bedeutung von Parteien und deren inhaltlicher Positionierungen hervorgehoben. Demnach sind es vor allem die konkurrierenden Positionen der großen Parteien in Regierungsverantwortung, die für die Entwicklung der Wohnungspolitik in der Nachkriegszeit verantwortlich gewesen sind (so Mayer, 1998).

Um eine Vorstellung für die Ideenproduktion durch Parteien zu bekommen, muss an dieser Stelle die inhaltliche Positionierung von Parteien genauer konzeptioniert werden. Dabei lassen sich intra-organisatorische Programmselektionen einerseits von der Programmsteue- 
rung durch den Parteienwettbewerb andererseits unterscheiden (Lehmbruch, 1979, 594). Im Prozess der intra-organisationalen Programmselektion sollte die Parteianhängerschaft über das Programm bestimmen. Theoretisch lassen sich hierfür sozialstrukturelle Theorien von Milieuund Interessentheorien unterscheiden (von Alemann u. a., 2018, 140ff.). Aus sozialstruktureller Perspektive (vgl. Lipset u. Rokkan, 1967) dienen Parteien als parlamentarische Träger der mit ihnen verbundenen Klasseninteressen, was sie im politischen System zum „Ausdruck sozialstruktureller Konfliktlinien" (von Alemann u. a., 2018, 141) wie Arbeit und Kapital werden lässt. Ein ähnlicher Wirkungsmechanismus besteht in mikro- und mesosozilogischen Milieutheorien, die jedoch gegenüber dem auf international vergleichender Forschung beruhenden sozialstrukturellen Ansatz stärker auf die deutsche Parteienentwicklung bezogen sind (von Alemann u.a., 2018, 147ff.). Nach Lepsius (1966) spiegelten Parteien im Kaiserreich und in der Weimarer Republik die folgenden ,sozialmoralische Milieus“ wider: Das katholische Sozialmilieu (Zentrumspartei), das konservativ-protestantische Sozialmilieu (Konservative), das protestantisch-bürgerliche Sozialmilieu (Liberale) sowie das sozialdemokratische Sozialmilieu (Sozialisten und Kommunisten) (von Alemann u.a., 2018, 148). Beiden Ansätzen zufolge lassen sich parteipolitische Positionierungen und damit die von ihnen vertretenen Ideen als eine Spiegelung der in ihrer Sozialstruktur oder aber in ihren Milieus vertretenen Ideen und Interessen formulieren. Allerdings folgt aus einer ,zunehmenden Abnahme der Bedeutung von Klassen- und Religionszugehörigkeit in der Gesellschaft" auch eine Auflösung traditioneller Milieus und der traditionellen Wählerschaft der jeweiligen Parteien (Seeleib-Kaiser 2003a, 15; vgl. Kirchheimer 1965).

Eine andere Perspektive auf die programmatische Ausgestaltung bietet der Parteienwettbewerb, wobei interessenbasierte Ansätze für die inhaltliche Positionierung von Parteien anzuführen sind: Parteien sind demnach nicht einfach Repräsentanten ihrer eigenen Umwelt im parlamentarischen System, sondern Vertreter ihrer eigenen Inter- 
essen. Im Extremfall bedeutet dies, dass Parteien in ihrem Handeln vor allem danach streben, möglichst viel politische Macht zu erhalten. In dieser Perspektive sind Parteien vor allem „Organisationen zur Stimmenmaximierung auf dem Wählermarkt, [...] die ideologisch völlig uninteressiert sind" (von Alemann u.a., 2018, 154). Dementsprechend müssten sich Parteien nach dem mehrheitlichen Interesse ausrichten, um die Stimmen möglichst vieler Wähler auf sich zu vereinen. In Bezug auf die Wohnungspolitik könnte somit argumentiert werden, dass in der Bundesrepublik insbesondere die Bedeutung der Mieter als großer Wählergruppe hervorzuheben ist, die das Zustandekommen eines regulierten Mietwohnungsmarktes erst ermöglichte (so Mayer, 1998, 426). Eine vollkommene Dominanz der Umfragenorientierung von Parteien muss allerdings empirisch verneint werden. Laut von Alemann u. a. (2018) ,kann man heute nicht ernsthaft behaupten, dass die bundesdeutschen Parteien reine entideologisierte politische Maschinen zur Mehrheitsgewinnung wären" (von Alemann u. a., 2018, 157f.). Stattdessen sei ,ihre soziodemographische Struktur [...] nach wie vor geprägt von gesellschaftlichen Konfliktlinien, die sich in neuen soziopolitischen Milieus verdichten“ (von Alemann u. a., 2018, 157ff.).

Diese Feststellung lässt sich mit dem in Kapitel 2 formulierten Handlungsmodell in Bezug auf Ideen und Interessen in Einklang bringen: Zwar verfolgen Parteien ihr Interesse, möglichst viele Stimmen auf sich zu vereinen. Um diesem Interesse allerdings gerecht zu werden, verfügen sie über ein Set von Ideen auf verschiedenen Abstraktionsgraden, das ihre Zielvorstellungen vorgibt und die Interessenverfolgung mit prägt. Parteien treten somit nicht nur als Träger, sondern auch als Produzent und Übersetzer von Ideen im politischen Prozess auf (Nullmeier u. Rüb, 1993, 30), womit eine distinkte Trennung zwischen Wissensproduktion und Politik aufgehoben werden muss (Friedrich, 1970, 7).

Für sozialpolitische Entscheidungen in der jungen Bundesrepublik wird meist das Augenmerk auf die CDU/CSU-geführten Bundesregierungen gelegt, die ,in ihrer Politik der ,sozialen Marktwirtschaft“ neben 
einer marktfreundlichen Politik in erheblichem Umfang einen sozialpolitischen Staatsinterventionismus" praktizierten (Ostheim u. Schmidt, 2007a, 163) und damit den deutschen Sozialstaat prägten (Ostheim u. Schmidt 2007a, 162; vgl. Kaufmann 1988). Dabei standen für die Sozialpolitik der christlichen Parteien jene Instrumente im Vordergrund, die den Einzelnen dazu befähigen sollten, Verantwortung zu übernehmen (van Kersbergen, 1995). Aufgrund ihrer regierungsleitenden Verantwortung in den ersten Jahren der Bundesrepublik werden vor allem die ersten christlich-konservativen Parteien als pfadprägend hinsichtlich der Wohneigentumsförderung gesehen (Schulz 1994; vgl. Abschnitt 5). Von ebensolcher Bedeutung für die Ausgestaltung der Sozialpolitik in Deutschland muss die SPD angesehen werden (Seeleib-Kaiser, 2003a). Dabei ist nicht nur das Wirken der Sozialdemokratie innerhalb der großen Koalitionen mit den christlich-konservativen Parteien, sondern vor allem die Regierungsjahre der sozialliberalen Koalition (Streeck, 2015) und die rot-grüne Bundesregierung unter Gerhard Schröder (Frenzel, 2002) zu nennen. Dabei ist für die SPD als klassischer Arbeiterpartei gegenüber CDU und CSU ein stärkerer programmatischer Wandel im Verlauf der hier betrachteten Zeitspanne zu verzeichnen gewesen. So sind Mitglieder und Wähler der SPD inzwischen deutlich heterogeneren Milieus zuzuordnen (Seeleib-Kaiser, 2003a, 16).

\subsubsection{Verbände \& Denkfabriken}

Eine weitere Akteursgruppe, der innerhalb des korporatistischen Systems der Bundesrepublik Deutschland eine bedeutende Rolle zugesprochen wird, ist die der Interessenverbände (Streeck, 1994). Sie unterscheiden sich von Parteien vor allem dadurch, dass sie zwar die Gesetzgebung beeinflussen möchten, allerdings nicht durch die Übernahme von Wahlämtern (Bernhagen 2018, 2; von Alemann u.a. 2018, 5). Mit Schmidt lassen sich Interessenverbände definieren als ,ein auf formaler Mitgliedschaft und ausdifferenzierter Binnenstruktur beruhender Verband, der 
vor allem die Berufs- und Standesinteressen einer Gesellschaftsgruppe artikuliert, bündelt und gegenüber den Mitgliedern, anderen Organisationen, der Öffentlichkeit und vor allem gegenüber der Regierung und der Verwaltung durch direkte oder indirekte Einflussnahme oder Druckausübung vertritt" (Schmidt, 2010a, 367f.). Der Kern der Verbandstätigkeit im Zusammenspiel mit der Politik ,besteht in der Übermittlung von Informationen [...] an politische Entscheider" (Bernhagen 2018, 4; vgl. Austen-Smith 1993; vgl. Beyme 1986, 96). Dementsprechend erweist sich eine genauere Betrachtung der Verbandslandschaft und deren Rolle in der Wissensproduktion als unerlässlich.

Gegenüber den Parteien, die je nach politischer Agenda mal mehr und mal weniger Ressourcen auf einzelne politische Themen verwenden können, zeigen sich Verbände, die abseits von der politischen Agenda ein dauerhaft bestehendes Interesse an wohnungspolitischen Fragen aufweisen, überaus starke Aktivitäten im Bereich der Wissensproduktion:

„Soweit sie mächtige, konzentrierte und finanzstarke Spitzenverbände sind [...] haben sie weit bessere Möglichkeiten Informationen $\mathrm{zu}$ sammeln und diese geschickt an die öffentliche Meinung zu verkaufen." (Beyme, 1986, 96)

Ähnlich wie Parteien können sie zunächst als ,intermediäre Institutionen" (Wagschal u. Grasl, 2015, 204) dazu beitragen, die Interessen anderer in das politische System zu tragen. Basierend auf der Definition der Interessen ,als Ausdruck der situativen Vermittlung von materiellen Positionen und ideellen Wahrnehmungsmustern" (Münnich, 2011, 371) stellt sich an dieser Stelle allerdings ähnlich wie bei der Positionsfindung von Parteien die Frage, woher die Verbände ihre ideellen Wahrnehmungsmuster beziehen. Einerseits wird über ihre Funktion in der Interessenaggregation, -selektion und -artikulation (vgl. Wagschal u. Grasl, 2015) deutlich, dass sie eine Vermittlerposition von bestehenden Ideen ihrer Mitglieder haben und somit handlungsleitendes Wissen 
an andere Akteure vermitteln können (siehe auch Bernhagen, 2018). Neben dieser Vermittlerfunktion übernehmen die Verbände allerdings auch ordnende Funktionen hinsichtlich der Ideen und Interessen ihrer Mitglieder, wodurch parlamentarische Anhörungen effektiver ausgestaltet werden können (Wagschal u. Grasl, 2015, 205). So lässt sich der Einfluss der Verbände auf die Wissensproduktion als eine Weitergabe von Informationen ihrer Mitglieder beschreiben. In dieser Funktion können sich die Verbände allerdings nicht auf die Interessen ihrer Mitglieder berufen, sondern ihre fachliche Expertise außerhalb des politischen Spektrums in den Vordergrund stellen. Aus systemtheoretischer Perspektive können sie dabei behilflich sein, ihre Wissensbestände über ein bestimmtes gesellschaftliches Funktionssystem dem politischen System zugänglich zu machen (vgl. Kusche, 2008, 135).

In der Bundesrepublik existiert eine Vielzahl an Interessenverbänden, von denen im August 20182.346 in der sogenannten „Lobbyliste“ des Deutschen Bundestages aufgeführt werden (Deutscher Bundestag 2018; siehe hierzu Wagschal u. Grasl 2015). Eine erstmalige Zusammenstellung der wesentlichen Interessenverbände der bundesdeutschen Wohnungspolitik liefert Egner (2004b) auf Grundlage einer experteninterviewbasierten Netzwerkanalyse von 2004. Er zeigt, dass sich die Wohnungspolitik durch eine ,komplexe, aus einem breiten Spektrum verschiedenster Verbände und Interessengruppen bestehenden Akteurslandschaft" zusammensetzt, von denen einige, ,über erhebliche Verhandlungsmacht [verfügen], weil von ihrer Mitwirkungsbereitschaft [...] die wohnungspolitisch intendierten Effekte abhängen. Zum anderen haben sie politischen Einfluss, weil sie personell über Funktions- und Mandatsträger mit Parteien und parlamentarischen Entscheidungsgremien verbunden sind - was besonders beim Deutschen Mieterbund markant ist" (Heinelt u. Egner, 2006, 211).

$\mathrm{Zu}$ den relevantesten Akteuren aus der Verbandslandschaft zählen laut Egner u. Georgakis (2004) der Deutsche Mieterbund, der 1951 als Nachfolgeverband des bereits 1900 gegründeten ,Verband deutscher 
Mietervereine" hervorging, der Zentralverband der Deutschen Haus-, Wohnungs- und Grundeigentümer „Haus und Grund“, der aus dem 1879 gegründeten Dachverband der örtlichen Haus- und Grundbesitzer hervorging, der Bundesverband deutscher Wohnungsunternehmen GdW, der 1946 noch als Verband gemeinnütziger Wohnungsunternehmen gegründet wurde, das Deutsche Volksheimstättenwerk (vhw), das mit seiner Neugründung von 1947 auf den „Bund deutscher Bodenreformer“ und den „Deutschen Verein für Wohnungsreform“ zurückgeht, der katholische Siedlungsdienst, der als Spitzenverband kirchlich gebundener Wohnungs- und Städtebauunternehmen fungiert, der Verband der deutschen Pfandbriefbanken (vdp), ehemals Verband der Deutschen Hypothekenbanken (VDH), einer der größten Interessenverbände der deutschen Kreditwirtschaft (Verband deutscher Pfandbriefbanken, 2016) sowie die Verbände der Bauwirtschaft. Seit 2006 ist mit dem Zentralen Immobilienausschuss (ZIA) ein weiterer Verband hinzugekommen, der vor allem die Interessen für kapitalmarktorientierte Immobilienunternehmen vertritt (Zentraler Immobilien Ausschuss e.V., 2018). Eine besondere Stellung hat der Deutsche Verband für Wohnungswesen, Städtebau und Raumordnung - kurz Deutscher Verband -, der als eingetragener Verein ,seine Unabhängigkeit von Standes- und Berufsinteressen" hervorhebt und seine Tätigkeit als ,selbstlos" darstellt (Egner u. Georgakis, 2004, 176). Hinsichtlich der Wissensproduktion ist der eingetragene Verein von besonderem Interesse, da er seit seiner Gründung 1946 insbesondere Symposien, Fachgespräche und Arbeitsgruppen ,in Zusammenarbeit mit anderen Verbänden und Instituten im In- und Ausland" organisiert und somit eine wichtige Vernetzungsplattform für die anderen Wissensproduzenten bietet (Egner u. Georgakis 2004, 176; vgl. Wiese-von Ofen 2016).

Besonders anschaulich zeigt sich die Rolle von Interessenvertretern als Wissensproduzenten für die Politik in der Ausgestaltung sogenannter Think Tanks wie auch in der zunehmenden Tätigkeit von Stiftungen (Baur 2018; Heisterkamp 2017). Think Tanks sind ,privat oder öffent- 
lich finanzierte praxisorientierte Forschungsinstitute, zu deren Hauptaufgabe die wissenschaftlich fundierte, häufig interdisziplinär angelegte Untersuchung und Kommentierung eines breiten Spektrums politisch relevanter Themen und Vorhaben gehört" (Thunert, 1999, 10). $\mathrm{Zu}$ diesen advocacy-Forschungseinrichtungen, die zunehmend an Bedeutung in der Wissensproduktion für die bundesdeutsche Politik gewinnen zählen Campbell u. Pedersen 2014 das Institut der deutschen Wirtschaft Köln (IW), das Wirtschafts- und Sozialwissenschaftliche Institut (WSI), das Institut für Makroökonomie und Konjunkturforschung (IMK), die DB Research als Forschungsabteilung der Deutschen Bank (Campbell u. Pedersen 2014, 135ff.; siehe auch Thunert 1999, 11). Hinzu kommt die 1983 gegründete Stiftung Marktwirtschaft (ehemals Frankfurter Institut für wirtschaftspolitische Forschung) als einem prominenten Verfechter marktwirtschaftlicher Ideen in allen Politikbereichen (vgl. Pätsch 2018; Campbell u. Pedersen 2014, 145). Die Think Tanks veröffentlichen Forschungsarbeiten und Positionspapiere zu aktuellen und vorrangig wirtschaftspolitischen Themen (Thunert, 2001, 235) und verstehen sich dabei nicht als Vertreter bestimmter Unternehmen, sondern als Verfechter globaler Ideen (Campbell u. Pedersen, 2014, 146). Dabei konkurrieren sie seit den 80er Jahren mit den klassischen Forschungsinstituten (vgl. Abschnitt 3.1.3 um die Erstellung von Auftragsgutachten, was insbesondere deren finanzielle Position deutlich geschwächt hat (Thunert, 2001, 236). Während Think Tanks beispielsweise in den USA maßgeblich auf Öffentlichkeitsarbeit konzentriert sind, geschieht dies in Deutschland vor allem durch die direkte Kommunikation mit der Politik (Pätsch, 2018, 15). Trotz ihrer beachtlichen Größe, existieren in Deutschland keine, ,themen- und politikfeldübergreifenden Denkfabriken sowie an interdisziplinär zusammengesetzten Forschungsgruppen“ (Thunert, 1999, 13). Dies ist ein Hinweis darauf, dass sich insbesondere die Think-Tank-Landschaft in den einzelnen $p o-$ licy-Subsystemen stark voneinander unterscheiden kann.

Stiftungen sind im allgemeinen ,auf Dauer zweckgebundene Ver- 
mögensmasse[n], die aus den Erträgen der Vermögensbewirtschaftung sowie durch weitere Zuwendungen ihre zumeist gemeinnützigen Zwecke selbstlos verwirklich[en]" (Pätsch, 2018, 3). Als besonders relevant für die politische Wissensproduktion in Deutschland sind die parteinahen Stiftungen einzustufen (Jun 2018; Campbell u. Pedersen 2014, 141ff.). Die parteinahen Stiftungen verfügen über einen „Vertrauensvorschuss, recht kurze Wege und ein privilegierter Zugang zur Mutterpartei“ (Jun, $2018,8)$ und damit auch über eine privilegierte Position in deren Wissensproduktion. Die für Deutschland relevanten parteinahen Stiftungen sind die Konrad Adenauer Stiftung mit Nähe zur CDU, die Friedrich Ebert Stiftung mit Nähe zur SPD, die Heinrich Böll Stiftung mit Nähe zu Bündnis 90/Grüne, die Rosa Luxemburg Stiftung mit Nähe zur Linken, die Hanns Seidel Stiftung mit Nähe zur CSU und die Friedrich Naumann Stiftung mit Nähe zur FDP. Teilweise gehen sie auf Gründungen zu Zeiten der Weimarer Republik zurück. Zu ihren Aufgaben zählen der Unterhalt von dezentralen und zentralen Bildungsstätten, wobei „die zentralen Institute dienen besonders der zeithistorischen, sozialwissenschaftlichen und ökonomischen Grundlagenforschung" dienen (von Alemann u.a., 2018, 203). Hinzu kommt die Vergabe von Stipendien aus dem Bundeshaushalt sowie die internationale Arbeit zum Aufbau demokratischer Strukturen (Pätsch 2018, 10f.; von Alemann u. a. 2018, 203). Entscheidend für die Aufnahme in die staatliche Globalförderung und die Festlegung der Zuschüsse entscheidet der deutsche Bundestag, während sich die Mittelverteilung an Durchschnittsergebnissen der Bundestagswahlen orientiert (von Alemann u. a., 2018, 202).

Als „Think Tanks der Parteien“ (Heisterkamp, 2017) verfügen sie mit einer großzügigen Ausstattung mit staatlichen Fördergeldern² über die Ressourcen die Parteien auch inhaltlich zu beraten. Dazu verfügen die Konrad-Adenauer-Stiftung sowie die Friedrich-Ebert-Stiftung über

2 Die staatliche Förderung der parteinahen Stiftungen lag 2015 bei über 513 Mio. $€-$,mehr als das Dreifache der absoluten Obergrenze jenes Betrages, den die Parteien selbst direkt vom Staat erhalten" (von Alemann u.a., 2018, 131). 
eigene Abteilungen (die Hauptabteilung „Politik und Beratung“ bei der KAS und die Arbeitseinheit „Politische Bildung und Beratung“" bei der FES) (Jun, 2018, 8). Gegenüber den Parteien sind die Stiftungen im Vorteil, da sie über die Zeit wie auch die Ressourcen verfügen ,aktuelle Ereignisse zu reflektieren, langfristige Entwicklungen zu beobachten und abseits des politischen Alltagsgeschäfts Interpretationen und Perspektiven anzubieten“ (Jun, 2018, 8). Neben der Beratung der Parteien treten die Stiftungen mit ihren Positionen auch durch eigene Veröffentlichungen an die Öffentlichkeit. Darüber hinaus leisten sie auf diversen Themengebieten Vernetzungsarbeit zu anderen Wissensproduzenten beispielsweise durch die Organisation von Veranstaltungen mit Experten aus der Wissenschaft und anderen Think Tanks(Jun, 2018, 8). Somit können die Stiftungen neben den Parteien an sich als der zentrale Ort politischer Ideenproduktion angesehen werden.

\subsubsection{Experten aus der Wissenschaft}

„The ideas of economists and political philosophers, both when they are right and when they are wrong, are more powerful than is commonly understood.

Indeed the world is ruled by little else. Practical men, who believe themselves to be quite exempt from any intellectual influence, are usually the slaves of some defunct economist." (Keynes, 2008)

Seit Keynes in seiner General Theory die Bedeutung wirtschaftswissenschaftlicher Ideen und Konzepte als entscheidenden Baustein für politische Entscheidungen betonte, wird dieser Zusammenhang diskutiert. Hervorzuheben sind zunächst die bereits genannten Arbeiten von Peter Hall zur Verbreitung des Keynesianismus und der späteren monetaristischen Trendwende (Hall 1989; Hall 1993). Zahlreiche sozialwissenschaftliche Arbeiten befassen sich mit Vermarktlichungsprozessen und suchen die Ursache in der wirtschaftswissenschaftlichen Wissens- 
produktion (Crouch 2013, 35; Centeno u. Cohen 2012, 328). Erwähnung finden dabei meist Gruppen von Wissenschaftlern wie die Mont-PèlerinGruppe oder die sogenannten Chicago Boys (Plehwe 2015; van Horn u. Mirowski 2015; Harvey 2007a).

Wissenschaftliche Wissensproduktion für die Politik wird in der Literatur meist als wissenschaftliche Politikberatung verstanden, die das ,institutionalisierte Liefern wissenschaftlicher Informationen an politisch Handelnde" (Falk u.a., 2006, 13) umfasst. Vor dem Hintergrund des in Kapitel 2 verfassten Ideenkonzeptes für diese Arbeit konzentriere ich mich in den folgenden Ausführungen auf wissenschaftliche Informationen, die sich auf die Konzeptionierung von policies und die damit verbundene policy-Beratung ${ }^{3}$ richten. Die Fülle an Literatur zur wissenschaftlichen policy-Beratung in der Bundesrepublik ist nicht zuletzt darauf zurückzuführen, dass sich bereits früh zahlreiche institutionalisierte Beratungsmechanismen und Forschungsorganisationen etablieren konnten (vgl. Abschnitt 3.2). Einen Überblick über die historisch gewachsenen Zusammenhänge der institutionalisierten wirtschaftswissenschaftlichen Politikberatung in Deutschland liefert Nützenadel (2005) mit seiner Studie „Die Stunde der Ökonomen“. Diese schlug in den ersten Jahrzehnten nach dem Zweiten Weltkrieg. In dieser ersten Phase wissenschaftlicher Politikberatung trugen vor allem die angestrebte Rationalisierung und Verwissenschaftlichung der wirtschaftspolitischen Entscheidungen zu einer tiefgreifenden Veränderung der politischen wie auch der wirtschaftlichen Verfassung Deutschlands bei, da fortwährend ,Experten und Sachverständige in die politische Entscheidungsfindung und deren administrative Umsetzung eingebunden werden sollten“ (Nützenadel 2005, 134f.; siehe auch Weingart 2006, 42). Die Strukturen der Forschungslandschaft lassen sich allerdings bereits

$3 \quad$ Zwar spielt wissenschaftliche Politikberatung auch als organisatorische und institutionelle Beratung (polity-Beratung) und als strategische und taktische Beratung (politics-Beratung) eine wichtige Rolle, soll jedoch im Rahmen dieser Arbeit nicht behandelt werden (siehe hierzu Bogumil, 2016, 432). 
auf die Zeit vor dem Zweiten Weltkrieg zurückführen, da die alliierten Besatzungsmächte auf eine Grundsatzreform des Hochschulwesens verzichtet hatten. So bestanden auch nach dem Zweiten Weltkrieg organisatorische und personelle Kontinuitäten (Lengwiler, 2016, 6), sodass das Kriegsende weder für die Wirtschaftsstatistik noch für die angewandte Konjunkturforschung eine „Stunde Null“ darstellte (Nützenadel, 2005, 93).

Ein besonderes Kennzeichen der politikbezogenen Wissensproduktion in Deutschland sind die halbstaatlichen und zum Teil auch staatlichen Forschungseinrichtungen als Teil des knowledge regimes (Campbell u. Pedersen, 2014, 135). Einige dieser Einrichtungen gehen auf eine Transformation der Forschungslandschaft ,,von einem wirtschaftsnahen zu einem stärker staatsorientierten, öffentlich finanzierten Forschungssystem“, zu Zeiten des Ersten Weltkrieges zurück (Lengwiler, 2016, 5). Gegründet wurden die ersten staatlich bezuschussten wirtschaftswissenschaftlichen Forschungsinstitute bereits in der Weimarer Republik wie das IfW in Kiel 1924 und das DIW in Berlin 1925. Das deutsche Institut für Wirtschaftsforschung ging als Institut für Konjunkturforschung (IfK) ,als eine Art Ausgliederung“ als selbstständiges Institut aus dem Statistischen Reichsamt 1925 hervor (Egner 2004b, 194; zur Geschichte des IfK siehe Tooze 2001). Für die Finanzierung der Institute bildete sich ,ein ausgesprochen merkwürdiges System“ heraus (Hesse, 2010, 138): Mit einem festen Pauschalbetrag wurden das IfW und das DIW aus der Wirtschaftsverwaltung finanziert und sollten dafür Gutachten für sämtliche Bundesministerien verfassen. Dies führte zu einer festen ,Alimentierung der beiden Wirtschaftsforschungsinstitute aus dem Bundeshaushalt, denn das System wurde 1949 fortgeführt"; ,allerdings [wurde diese Tätigkeit] etatmäßig als Auftragsarbeiten abgerechnet" (Hesse, 2010, 138). Mit einer regelrechten Neugründungswelle nach dem Zweiten Weltkrieg waren vor allem die Verselbständigungen der Außenstellen des DIW verbunden wie 1949 das ifo-Institut und das RWI in Essen (Hesse, 2010, 135). 
Die Forschungsinstitute wurden zum ,eigentlichen Ort der empirischen Wirtschaftswissenschaft in Deutschland" (Hesse 2010, 250). Bis heute kann die wirtschaftswissenschaftliche Wissensproduktion ,an der Schnittstelle von Wissenschaft, Ökonomie, Staat und Öffentlichkeit" verortet werden (Maeße 2013a, 242; vgl. Campbell u. Pedersen 2014, 135ff.). Die Förderung der außeruniversitären Forschung bedingt die bis heute in der Bundesrepublik bestehende Zweiteilung zwischen einem universitären und einem außeruniversitären Forschungssystem (Lengwiler, 2016, 4). Insgesamt kann es als Kennzeichen der wirtschaftswissenschaftlichen Forschung in Deutschland angesehen werden, dass sich ihr Aufstieg nach dem Zweiten Weltkrieg fast vollständig außerhalb der universitären Forschungseinrichtungen abspielte (Nützenadel, 2005, 98). Daher bietet es sich an, im Kontext der wissenschaftlichen Politikberatung in Deutschland von Expertise im Sinne einer Übersetzung von Forschungsergebnissen in Anwendungsgebiete anstatt von Forschung im universitären Sinne zu sprechen (Grundmann u. Stehr, 2011, 37). Das Aufblühen und die zahlreichen (Neu-)Gründungen außeruniversitärer wirtschaftswissenschaftlicher Forschungseinrichtungen nach dem Zweiten Weltkrieg sind vor allem auf zwei Ursachen zurückzuführen: Erstens waren die Universitäten nur mit wenigen Ressourcen ausgestattet und zudem mit einer steigenden Anzahl von Studienanfängern überlastet (Nützenadel, 2005, 98). Zweitens stieg die Nachfrage nach wirtschaftswissenschaftlicher Expertise vonseiten der Politik im Rahmen des genannten Trends zur Verwissenschaftlichung rapide an und konnte von den überlasteten Hochschulen nicht befriedigt werden:

„So sehr nämlich die Überlastung der Universitäten durch die hochschulpolitische Zurückhaltung der fünfziger Jahre und die Indienstnahme des Universitätssystems für die Restitutionspolitik verursacht war, erhielt die Blüte der außeruniversitären Forschung die zentralen Impulse von einer bewussten Förderungsentscheidung des Bundes und der 
Länder“ (Hesse, 2010, 141).

Der tatsächliche Einfluss, den Wirtschaftswissenschaftler auf die Ideen in der Politik haben können, hängt von drei Faktoren ab. Erstens kann ihnen der Einfluss gegeben werden, indem ihnen professionelle Autorität zugesprochen wird. Zweitens können sie selber vor allem durch Vernetzung und Zertifizierung untereinander diese Autorität positiv beeinflussen. Drittens kann ihr Einfluss auch indirekt über eine diskursive Überformung der Gesellschaft mit ökonomischen Kategorien erfolgen: Sobald sich wirtschaftswissenschaftliche Ideen innerhalb politischer Debatten als legitim herausgestellt haben, werden Wirtschaftswissenschaftler als Experten unabdingbar. In den folgenden Abschnitten soll auf die einzelnen Faktoren genauer eingegangen werden.

$\mathrm{Zu}$ besonderen Faktoren, welche die Einflussmöglichkeiten der Wirtschaftswissenschaftler ausmachen, gehört die den Beratern zugeschriebene professionelle Autorität. Ein bereits erreichter Expertenstatus bedingt selber bereits eine größere Autorität und damit auch eine wahrscheinlichere Deutungshoheit in den entsprechenden Diskursen (vgl. Abbott, 1988). Dieser Status ist verknüpft mit den Wirtschaftswissenschaften als Disziplin und hat einen direkten Einfluss darauf, wie stark der Einfluss der Ökonomen zu einem bestimmten Zeitpunkt ausfallen kann (Hirschman u. Berman, 2014, 781). Bereits in den jungen Jahren der Bundesrepublik kann von einem Mangel zugeschriebener Autorität in den Wirtschaftswissenschaften nicht die Rede sein. Volkswirten wie dem Vater des Wirtschaftswunders Ludwig Erhard und seinem Nachfolger Karl Schiller wurde eine entscheidende Rolle für das Gelingen des Wirtschaftswunders zugeschrieben. So waren nach Hesse (2010) die Zeitgenossen gerne dazu bereit ,den wirtschaftlichen Wiederaufstieg auf die Anwendung der ,richtigen“ Wirtschaftspolitik zurückzuführen und Krisen als Resultate von Fehlern [zu sehen], die durch Inkompetenz entstünden" (Hesse, 2010, 105). Vor diesem Hintergrund ist es wenig verwunderlich, dass Wirtschaftswissenschaftler in zahlreichen beraten- 
den Gremien vertreten waren und auch aus dieser Tätigkeit wiederum Reputationsgewinne erzielen konnten (Hesse, 2010, 130f.). Zwar hatten Wissenschaftler durch ihre Einbindung in politische Gremien schon früh ,in ihrer Entwicklung einen Stand erreicht, der sie in die Lage versetzte, durch Informationen, Erkenntnisse und Handlungstechnologien direkt in die politische Praxis einzugreifen und deren Zielsetzungen mitzubestimmen“ (Friedrich, 1970, 18). Insbesondere „ökonomische Expertise [hatte sich] in der politischen Kultur der Bundesrepublik fest eingebürgert" (Nützenadel, 2005, 362). Allerdings zeigte sich die Überlastung des universitären Systems im Bereich der Wirtschaftswissenschaften in dem Umstand, dass es kaum ausreichend Professoren gab, um die zahlreichen ins Leben gerufenen Beiräte und Kommissionen zu besetzen (Hesse 2010, 123f.; siehe auch Abschnitt 5.3.2).

Darüber, wie Wirtschaftswissenschaftler ihren eigenen Expertenstatus in Diskursen im Allgemeinen konstruieren, finden sich mehrere Hinweise im Bereich der Professionssoziologie, wobei jedoch nur selten Bezug zu politischen Entscheidungen hergestellt wird (vgl. Hirschman u. Berman, 2014, 7f.). Einen Ansatzpunkt bieten die ländervergleichenden Studien von Fourcade „Economists and Societies" von Fourcade (2006; 2009). Reputationsgewinne von Ökonomen als Experten hängen laut Fourcade maßgeblich mit der Etablierung der gesamten Ökonomie als wissenschaftliche Disziplin an Hochschulen zusammen. Demnach ist auch in öffentlichen Diskursen die ökonomische Methode der Informations- und Ideengenerierung von Belang, sondern auch der Expertenstatus, den Ökonomen im Rahmen des akademischen Systems erlangen. Damit wird zwar der akademische Diskurs auf der inhaltlichen Ebene noch nicht relevant für den öffentlichen oder gar den politischen Diskurs, bedingt aber mit seinem Ergebnis der Deutungshoheit bestimmter Ökonomen, zum Beispiel durch bestimmte Veröffentlichungen, Auszeichnungen oder Berufungen im akademischen Bereich, deren stärkere Wahrnehmung als Experte in anderen diskursiven Bereichen (vgl. McCloskey 2009, 119; Maeße 2013b, 205). Mit der zunehmenden 
Spezialisierung der Volkswirtschaftslehre in einzelne Subdisziplinen im Rahmen eines universitären Strukturwandels seit den 60er Jahren (Hesse, 2010, 250), erscheint es sinnvoll, einen Blick auf mögliche Spezialisierungen in der VWL hinsichtlich wohnungswirtschaftlichen Themenbereichen zu werfen. Gegenüber Fachgebieten, wie der Arbeitsmarktökonomik oder der Geldpolitik, erweist sich dieser Bereich jedoch als wenig entwickelt. Während in den USA der Themenbereich real estate bereits seit längerem einen festen Bestandteil der universitären Lehre und Forschung bildete, konnte „Immobilienbildung auf universitärem Niveau“ (Rottke, 2009, 39) erst seit den 1990er Jahren Bedeutung an deutschen Hochschulen gewinnen. Nach Angaben der Gesellschaft für immobilienökonomische Forschung (gif) zeugten bereits 2012 mehr als 1.300 Mitglieder der gif und mehr als 120 Studiengänge mit immobilienwirtschaftlichen Programmen von der Etablierung der Immobilienökonomik in Deutschland (Gesellschaft für Immobilienwirtschaftliche Forschung e.V., 2012). Auch in den halböffentlichen Forschungsinstituten spielte wohnungspolitische Wissensproduktion meist eine untergeordnete Rolle. So wurden lediglich innerhalb des DIW wohnungspolitische Fragen standardmäßig bearbeitet, allerdings stets in Verbindung mit bauwirtschaftlichen Fragestellungen (Egner u. Georgakis, 2004, 196). Erst deutlich später richteten andere Forschungsinstitute und Think Tanks eigene Teams für immobilienökonomische Forschung ein. Eine Ausnahme bildet das 1971 vom Land Hessen und der Stadt Darmstadt gegründete Institut für Wohnen und Umwelt (IWU), das vor allem Grundlagenforschung zu Formen des Wohnens untersuchen und ,insbesondere auf die Verbesserung der Wohnverhältnisse der sozial schwächeren Schichten hinwirken" sollte (Egner u. Georgakis 2004, 199; vgl. Wullkopf u. Zinn 1981).

In der Konstruktion des Expertenstatus von Ökonomen haben internationale Eliteuniversitäten eine doppelte Funktion: Einerseits bieten sie den dort aktiven Wissenschaftlern jene Reputationsgewinne, die ihnen auch in politischen Diskursen bessere Einflussmöglichkeiten 
verschaffen. Andererseits sind sie auch der Ort, an dem sich Wissenschaftler vernetzen und Ideen austauschen können. So werden die reputationsstarken Universitäten in der Erforschung von Vermarktlichungsprozessen auch zu den Orten, über die sich liberale Denkweisen am besten verbreiten können (vgl. Harvey 2007a, 71f.; siehe auch Gormley 2007). Angesichts zunehmender globaler Interdependenzen auch im Bereich der Wohnungspolitik (Schwartz u. Seabrooke, 2008, 237) erscheint es notwendig auf internationale Zusammenhänge in der Wissensproduktion näher einzugehen (Morgan, 2001). In der sozialwissenschaftlichen Forschung rücken dabei zunehmend transnationale Netzwerke von Wissenschaftlern in den Fokus, die sich in Anlehnung an Haas (1992) als epistemische Gemeinschaften beschreiben lassen. Sie bestehen aus Netzwerken wissensbasierter Experten, die eine wichtige Rolle in der Vermittlung von Ursache- Wirkungszusammenhängen, komplexer Probleme sowie in der Formulierung bestimmter Sachverhalte in politischen und öffentlichen Debatten spielen können (Haas, 1992, 2). Dabei ist zu beachten, dass es sich bei epistemischen Gemeinschaften nicht bloß um eine Gruppe von Wissenschaftlern und Experten auf ihrem Gebiet, sondern um eine Gemeinschaft von Gleichgesinnten handelt, „die den Glauben an die Beziehung von Ursache und Wirkung, an Gültigkeitstests und grundlegende Werteprinzipien teilen und gemeinsame politische Ziele verfolgen. Ihre Einstellung lässt sich vielleicht am besten mit den Worten eines der ihren ausdrücken, der sich bereiterklärte, die „Plausibilität kausaler Zusammenhänge auch ohne Gewissheit zu akzeptieren“ (Haas, 1993, 187f.). Weitere Autoritätsgewinne können Wissenschaftler demnach erlangen, wenn der Konsens in ihrer Disziplin zu einem bestimmten Beratungsthema vorhanden ist (Weingart 2006, 39; siehe auch Gormley 2007). Allerdings war die VWL in der jungen Bundesrepublik insbesondere von einer Heterodoxie in den Theorien geprägt, die eine einstimmige Positionierung zu Politikinhalten nahezu ausschloss. Die VWL umfasste 
„eine komplizierte Vermischung von keynesianischen, (neo)liberalen, historischen und sogar sozialistischen Theoriebestandteilen [und war] nicht ein vom Ordoliberalismus und der Euckenschule dominiertes Feld, wie aufgrund der großen Bedeutung dieser Richtung für die Wirtschaftspolitik in der Vergangenheit häufig behauptet wurde." (Hesse, 2010, 17)

Dass epistemische Gemeinschaften durchaus zu politischem und allgemeinem institutionellen Wandel beitragen können, lässt sich für zahlreiche Prozesse politischen Wandels bestätigen. Dies ist vor allem dann der Fall, wenn policy-making zunehmend mit internationalen Standards und Verträgen zusammenhängt (Schneider u. Janning, 2006, 212). So zeigt King (2005), wie akademische Ideen, die durch eine epistemische Gemeinschaft aus wirtschaftswissenschaftlichen Beratern und Geldexperten wesentlich zu einer Zentralbankenreform in Großbritannien beigetragen haben. McNamara (1998) weist nach, dass epistemische Gemeinschaften mit einem ,neoliberalen policy consensus“ auch Einfluss auf die Ausgestaltung der Europäischen Währungsunion nehmen konnten. Allgemein wird den vernetzten Experten auf internationaler Ebene eine entscheidende Funktion in der Verbreitung von Ideen der Vermarktlichung zugeschrieben (Mirowski u. Plehwe 2015; Bonanno 2017, 109f.). Für die Untersuchung des Einflusses wirtschaftswissenschaftlicher Ideen auf die Eigenheimpolitik sind somit nicht nur die organisationalen Zugehörigkeiten der Experten relevant. Stattdessen kann auch deren Vernetzung untereinander sowie im internationalen Kontext eine Rolle für deren Wirksamkeit spielen.

Einhergehend mit ihrer zunehmenden Bedeutung im politischen Betrieb beschreibt auch Fourcade (2006) eine zunehmende diskursiv-performative Überformung der globalen Gesellschaft mit ökonomischen Kategorien (vgl. Maeße, 2013a, 90). Demnach beeinflussen sie die kognitive Infrastruktur der Akteure, worunter (1.) die Art der vorherrschenden und akzeptierten Begründungsmuster (style of reasoning) und 
(2.) die bereits im Politikfeld gebräuchlichen ökonomischen Instrumente und Kennzahlen (economic policy devices) zu verstehen sind (Hirschman u. Berman 2014, 794ff.; vgl Abschnitt 2.2.1). Als weitere länderübergreifend zutreffende Eigenschaft der ökonomischen Disziplin nennt Fourcade die zunehmenden Möglichkeiten der Anwendung formalisierter ökonometrischer Modelle, die gegenüber anderen wissenschaftlichen Herangehensweisen nicht nur in ökonomischen, sondern auch in akademischen und politischen Institutionen besonders honoriert wurden (Fourcade, 2009, 426). Auch die zunehmende Etablierung immobilienökonomischer Forschung und Bildung in Deutschland ging mit Bestrebungen zu einem stärkeren Fokus auf finanzwirtschaftliche und hoch mathematisierte Methoden angelsächsischer Prägung einher (Hoesli u. MacGregor, 1997, 5).

\subsection{4 Öffentliche Verwaltung}

Betrachtet man das System der Wissensproduktion für die Politik in Deutschland genauer, fällt auf, dass Staatsstrukturen - und damit die Rolle der öffentlichen Verwaltung - in der Modellierung des deutschen knowledge regimes von Campbell $\mathrm{u}$. Pedersen (2014) kaum mit berücksichtigt wurden. Zwar finden sich in ihren Darstellungen ministerielle Forschungseinheiten als Wissensproduzenten (Campbell u. Pedersen, 2014, 140f.), deren darüber hinausgehende Bedeutung als gatekeeper für die Mechanismen der Ideenproduktion wurde jedoch nicht ausreichend mit berücksichtigt. Bevor in Abschnitt 3.2 auf diese Sonderrolle der öffentlichen Verwaltung genauer eingegangen werden kann, soll in diesem Abschnitt auch auf die Bedeutung öffentlicher Stellen für die Wissensproduktion eingegangen werden.

Mit ihrem Sammelband „Bringing the State back in“ haben Evans u. a. (1985a) bereits auf die Bedeutung staatlicher Strukturen für die Analyse von Politiken hingewiesen. Dabei ergeben sich Strategien der Zielerreichung politischer Akteure erst durch die bestehenden staatli- 
chen Strukturen:

„The forms of collective action through which groups make political demands or through which political leaders seek to mobilize support are also partially shaped in relation to the structures and activities of the states." (Skocpol, 1985, 22)

Somit bilden Staatsstrukturen an sich bereits relevante Rahmenbedingungen, die von den bereits beschriebenen Akteursgruppen berücksichtigt werden müssen. In den deutschsprachigen Sozialwissenschaften sind staatliche Strukturen und ihre Bürokratien in der Tradition von Max Weber ein festes Themengebiet sozialwissenschaftlicher Forschung (vgl. Skocpol 1985, 7; Habermas 1990, 269). In Webers idealtypischer Beschreibung von Politik zum Betrieb in der Organisationsform der legalen Herrschaft bildet die strikte arbeitsteilige Organisation zwischen den Politikern und dem fachlich qualifizierten Beamtenapparat die Grundlage politischer Entscheidungsprozesse. Dabei sind es die Bürokratien selber, die Wissen produzieren und für den politischen Prozess bereitstellen (Bohne, 2018, 76). Für Weber geschieht dies in erster Linie durch die Herausbildung eines Fachmenschentums im Zusammenhang mit der Bürokratisierung der Herrschaft (Weber, 2013, 578). Durch die ständig zunehmende Bedeutung von Fachwissen und der Rationalisierung wächst die Bedeutung dieses Fachmenschentums an und macht es zum „Berufs“-Ideal der modernen Bürokratie (Weber, 2013, 752): „Erst die moderne Vollbürokratisierung bringt das rationale, fachmäßige Examenswesen zur unaufhaltsamen Entfaltung." (Weber, 2013, 676). Auch Rueschemeyer u. Skocpol (1996) sehen in dem gut ausgebildeten Fachmenschentum einen zentralen Vorteil der staatlichen Verwaltung hinsichtlich der Ideenproduktion:

,[...] the body of higher civil servants tends to be professionally educated and as capable of indeendent interpretation and analysis of information. They are likaly to come to judgements of their own and have an interest in controlling the 
political agenda at least as it pertains to their own agencies." (Rueschemeyer u. Skocpol, 1996, 309)

Der bereits in Abschnitt 3.1.3 thematisierte steigende Bedarf an Wissen vonseiten des Staates betraf allerdings nicht nur die wissenschaftliche Expertise, sondern geht auch mit einem Ausbau eigener staatlicher Expertise im Rahmen von Bürokratien einher (Skocpol u. Rueschemeyer 1996, 6; Friedrich 1970, 17; Rueschemeyer u. Skocpol 1996, 297). So wird die Wissensproduktion von Bürokratien auch von Vertretern politischer Lerntheorien hervorgehoben, denn „to officials has fallen the task of gathering, coding, storing and interpreting policy experience" (Heclo, 1975).

Für eine Beschreibung der Wissensproduktion durch die öffentliche Verwaltung in der Bundesrepublik Deutschland muss zunächst definiert werden, welche Akteure zu dieser Gruppe gezählt werden können. Vereinfachend ließen sich Staatsstrukturen als ein Bestandteil des policy-making-Regimes beschreiben. In dieser Sichtweise, die auch der Tradition in der deutschen Verwaltungswissenschaft entspricht, wird die öffentliche Verwaltung als Subsystem des politisch-administrativen Systems betrachtet (Fricke u. Siewert, 2015, 239). Dem entgegen steht die US-amerikanische Perspektive der Public Administration, die eine Trennung beider Bereiche modelliert (Fricke u. Siewert, 2015, 239). Eine generelle Trennung von Politik und öffentlicher Verwaltung erscheint zwar nicht empirisch haltbar, aber ,auch die ausnahmslose Verbindung von Politik und Verwaltung im Begriff des politisch-administrativen Systems [erscheint] empirisch unbegründet" (Bohne, 2018, 31). Der wesentliche Unterschied zwischen den gewählten politischen Akteuren auf der einen Seite und jenen der öffentlichen Verwaltung besteht in der Dauerhaftigkeit ihrer Position. Das von Weber beschriebene Fachmenschentum ist auf Dauerhaftigkeit hinaus angelegt, während politische Akteure stets nur für eine gewisse Zeitspanne gewählt werden. Dementsprechend lassen sich all jene Positionen als Teil der Verwaltung be- 
schreiben, ,deren Besetzung sich nicht direkt aus politischen Legitimationsmechanismen ergibt, und bei deren Besetzung die Laufbahnlogik des Staatsdienstes weitgehend in Kraft bleibt" (Schnapp, 2004, 23). Auch Skocpol verweist auf die auf Dauer angelegte öffentliche Verwaltung als eine wesentliche Ressource dieser Akteursgruppe (Skocpol, 1985, 7). So sichert neben der eigenen fachlichen Expertise vor allem die kontinuierliche Arbeitsorganisation der Beamten ,einen hervorragenden Platz im politischen Entscheidungsprozeß" (Friedrich, 1970, 20). Tendenziell können ,administrative Denkweisen politische Zielsetzungen überformen, je mehr die delegierte Normsetzung der Verwaltung zunimmt" (Friedrich, 1970, 14). Im Hinblick auf die Wissensproduktion bedeutet dies maßgebliche Vorteile der öffentlichen Verwaltung gegenüber politischen Amtsträgern und Interessenverbänden (vgl. Friedrich, 1970, 446f).:

,While parties and interest groups did occasionally play extremely important parts, it was the civil services that provided the most constant analysis and review underlying most courses of government action." (Heclo, 1975, 301)

Dementsprechend werden hier alle Akteure zum staatlichen Verwaltungsapparat gezählt, die entgegen der politischen Machthaber auf Dauer ausgerichtete Positionen innehaben. Eine derartige Trennung von politischen Akteuren und Verwaltung ,ermöglicht in empirischer Hinsicht das Verständnis der öffentlichen Verwaltung als einer selbstständigen sozialen Organisation sowie eine situative Unterscheidung administrativen und politischen Handelns" (Bohne, 2018, 156). In Bezug auf die Aufgabenbereiche dieser auf Dauer angelegten Staatsapparate schlägt Skocpol zwei Modellierungen vor:

„On the one hand, states may be viewed as organizations through which official collectives may pursue distinctive goals, realizing them more or less effectively given the 
available state resources in relation to social settings. On the other hand, states may be viewed more macroscopically as configurations or organization and action that influence the meanings and methods of politics for all groups and classes in society.“ (Skocpol, 1985, 28)

In der ersten Variante haben staatliche Administrationen eine dienende Funktion gegenüber anderen Akteuren: Sie helfen dabei, die Interessen gesellschaftlicher Gruppen zu verhandeln und zu einem Ausgleich zu bringen. Diese funktionalistische Perspektive ähnelt dem systemtheoretischen Ansatz, wonach die Verwaltung gegenüber der Politik zunächst ein Handlungssystem darstellt, das einerseits Komplexität reduzieren soll und zugleich auf die Herstellung bindender Entscheidungen für Dritte ausgerichtet ist (Luhmann, 1966, 70). Nicht nur die Funktionszuschreibung der Komplexitätsreduktion kann als eine Bereitstellung handlungsanleitenden Wissens interpretiert werden, sondern auch die Herstellung bindender Entscheidungen, die für Luhmann als „Mitteilung des Ergebnisses einer Informationsverarbeitung" gelten (Luhmann, 1966, 69). Aus dieser Perspektive heraus wird insbesondere die ausführende Funktion der staatlichen Verwaltung in der Informationsbeschaffung für die Politik sowie in der „Umsetzung und Kontrolle von Gesetzen und Vorschriften" verstanden (Fricke u. Siewert, 2015, 238). Dabei muss berücksichtigt werden, dass es sich bei den staatlichen Strukturen nie um einen neutralen Rahmen für die Handlungen anderer Akteure handelt. Stattdessen beinhalten Staatsstrukturen selber Akteure, die ihrerseits wiederum eigene Interessen vertreten:

,$[$,... autonomous state actions will regularly take forms that attempt to reinforce the authority, political longevity, and social control of the state organizations whose incumbents generated the relevant policies or policy ideas." (Skocpol, $1985,15)$ 
Die zweite von Skocpol (1985) angeführte Modellierung verweist auf einen entgegengerichteten Mechanismus, bei dem die staatlichen Akteure und ihre Handlungen sinngebend und handlungsanleitend für andere Akteursgruppen sein können. Im Hinblick auf die Wissensproduktion kann dieser Hinweis in zwei Varianten gelesen werden: Einerseits produziert die staatliche Verwaltungsmaschinerie selber Wissen und Ideen, die sich für die übrigen politischen Akteure als handlungsleitend erweisen können. Andererseits hat die staatliche Verwaltung eine Sonderrolle für die Bereitstellung von Wissen und Ideen durch andere Akteure: Die Bürokratie greift damit auch aktiv in den Politikprozess mit ein und erfüllt entsprechend der beiden genannten Definitionsmöglichkeiten nach Skocpol eine „Doppelrolle als Subjekt und Objekt der Politik“ (Bogumil u. a., 2012, 154). Durch agenda setting und strategische Interaktion wie auch der Überwachung der Implementation der policies gewinnt sie besonderes Einflusspotenzial.

Besonders relevant hinsichtlich der Wissensproduktion für die Politik erscheint die Ministerialbürokratie. Ihre Aufgabe besteht zunächst darin, politische Entscheidungen vorzubereiten, wobei ihr Einfluss auf den späteren Entscheidungsprozess als erheblich einzustufen ist (Bogumil u.a. 2012, 154; Hustedt u. Houlberg Salomonsen 2014, 206). $\mathrm{Zu}$ den Aufgaben der Ministerialbürokratie gehören in der Bundesrepublik Deutschland ,die Vorbereitung der Gesetzgebung, das Sammeln von notwendigen Informationen und Daten und die Aufbereitung dieses Materials für den Gebrauch der politischen Instanzen, die Beratung der Politiker, die Vorklärung von geplanten Maßnahmen mit den interessierten Gruppen zusammen mit den entsprechenden Verhandlungen, schließlich die Unterstützung der politischen Leitung bei dem Versuch, politische Kompromisse zwischen divergierenden Interessen herbeizuführen“" (Friedrich, 1970, 100). Aus dieser Aufgabenvielfalt heraus wird deutlich, dass die Verwaltung zunächst administrativ an allen wesentlichen Stufen des policy-cycle im Sinne von Lasswell (1956) beteiligt ist: Sie bereitet Gesetzesentwürfe vor (Agenda-Setting), im- 
plementiert diese und führt auch selber Evaluationen durch (Fricke u. Siewert 2015, 239; Schnapp 2004, 81ff.; Niederhafner u. Speth 2004). Sie unterstützt die Regierungstätigkeit sowohl mit der Vorbereitung als auch mit der Durchführung von Gesetzen und ist in diesem Rahmen auch mit der ,Aufbereitung und Zusammenstellung relevanter Informationen" betraut (Bogumil 2007, 50; Hustedt u. Houlberg Salomonsen 2014, 207). Dazu verfügen die Bundesministerien in ihren Fachabteilungen ,über ein hohes Maß an Sachkompetenz in Form von Erfahrungsund Überblickswissen“ (Tiemann u. Wagner, 2013, 2). Die Mitarbeiter zeichnen sich vor allem durch eine eigene große Expertise aus, die einerseits auf deren Fachausbildung beruht und andererseits in der arbeitsteiligen Organisation des Ministeriums beruhen, in der jeder Beamte über seinen klaren Zuständigkeitsbereich verfügt Friedrich 1970, 111f.; Siefken 2007, 59f.). So erstellt das Bundesministerium für Verkehr, Bauund Stadtentwicklung bereits seit 1966 auf Basis des Wohngeldgesetzes einen Wohngeld- und Mietenbericht, in dem die Entwicklungen im Wohnungsbau und der Mietkosten beschrieben werden (Tiemann u. Wagner, 2013, 9). Zusätzlich erläutert das Ministerium in diesen Berichten seine Politik, wie dies auch in den Politikbereichsberichten anderer Ministerien üblich ist (Tiemann u. Wagner, 2013, 9).

Zur nichtministeriellen Verwaltung gehören die nachgeordneten Behörden. Die prominentesten sind das Bundesamt für Verfassungsschutz, das Umweltbundesamt sowie das Statistische Bundesamt (Bogumil, 2007, 49). Für die politikfeldübergreifende Wissensproduktion kommt dabei vor allem dem Statistischen Bundesamt eine Sonderrolle zu. Evans u. a. (1985b) weisen darauf hin, dass der Aufbau statistischer Kenntnisse ein zentraler Faktor für den Aufbau wohlfahrtsstaatlicher Institutionen darstellte (Evans u.a., 1985b, 357f.). So zeigt Kuhnle (1996) in seiner Analyse skandinavischer Sozialstaaten, dass gerade die Entwicklung von staatlichen Stellen zum Aufbau einer amtlichen Statistik zentral war für die Entstehung der ersten wohlfahrtsstaatlichen Institutionen. Dabei wurde nicht nur der ministerielle Apparat selber von 
den Statistiken beeinflusst, sondern darüber hinaus auch damit assoziierte Interessengruppen. Der Aufbau der amtlichen Statistik folgte einem europäischen Trend, in dessen Rahmen in den meisten europäischen Ländern zwischen 1830 und 1850 statistische Ämter errichtet wurden (Kuhnle 1996, 245; Hölder u. Ehling 1991, 16). Im Rahmen des Deutschen Zollvereins wurden ab 1934 regelmäßige Volkszählungen nach einheitlichem Muster durchgeführt, die als erste zentralstaatliche Aktivitäten im Rahmen der amtlichen Statistik gewertet werden können (Hölder u. Ehling, 1991, 17). Nach der Gründung des Deutschen Reiches wurde, basierend auf den Erfahrungen des Zollvereins, 1979 das Kaiserliche Statistische Amt ins Leben gerufen, das als zentrale Stelle für die statistischen Landesämter fungierte (Hölder u. Ehling, 1991, 18). In den drei Haupteinheiten des Amtes (Bevölkerungsstatistiken, Statistiken der Landwirtschaft und des Gewerbes und Statistiken des Verkehrs, der gemeinschaftlichen Einnahmen und der Steuer- und Zollverwaltung) arbeiteten von Beginn an vor allem Wissenschaftler, welche die gewonnenen Daten auch selber wissenschaftlich bearbeiten und veröffentlichen sollten (Hölder u. Ehling, 1991, 18f.). In diesen Statistiken spielten Erfassungen der Gebäudebestände oder gar der Wohneinheiten jedoch noch keine Rolle. Dieser Umstand lässt sich auch darauf zurückführen, dass die statistischen Ämter von Anfang an nur auf staatlichen Beschluss hin Statistiken erstellten und dem wohnungspolitischen Feld in dieser Epoche nur eine vergleichsweise geringe Bedeutung beigemessen wurde.

In der Weimarer Republik wurde aus dem Kaiserlichen Statistischen Amt das Statistische Reichsamt, an das immer mehr Kompetenzen vonseiten der Länder abgegeben wurden (Hölder u. Ehling, 1991, 20). Zur inhaltlichen Neuausrichtung heben Hölder u. Ehling (1991) insbesondere das neue Interesse ,an der zukünftigen Bevölkerungsentwicklung und [an der] Familien- und Haushaltsstatistik als neues Element" hervor (Hölder u. Ehling, 1991, 21). Im Rahmen dieser Arbeit erscheint von besonderem Belang, dass die Produktionsstatistik erstmals um den Be- 
reich der Bautätigkeitsstatistik ergänzt wurde (Hölder u. Ehling, 1991, 22). Zu Zeiten des Nationalsozialismus wandelte sich die Wirtschaftsstatistik der Weimarer Republik zunehmend zu einer Bewirtschaftsungsstatistik (Hölder u. Ehling, 1991, 23): ,[J]etzt [sollten] die Einzelangaben für die Verwaltung und Bewirtschaftung für die Zuteilung an den einzelnen Betrieb über die Kontrolle seiner Leistungen dienstbar gemacht werden" (Hölder u. Ehling, 1991, 23). Das statistische Instrumentarium wurde allerdings weitestgehend beibehalten und laut Hölder u. Ehling (1991) auch nicht um zahlreiche Sachverhalte ${ }^{4}$ erweitert.

In der ersten Phase nach dem Zweiten Weltkrieg (1945 bis 1949) waren Befugnisse sowie Organisation der sich neu konsolidierenden statistischen Ämter je nach Besatzungszone sehr unterschiedlich geregelt (Hölder u. Ehling, 1991, 24). Über die Zonengrenzen hinweg kam es zwar 1946 zur gemeinsamen Volkszählung, ,die jedoch nur unzureichende Vorbereitung und den Zuzug von Flüchtlingen und Vertriebenen zu wenig brauchbaren und bald überholten Ergebnissen führte" (Hölder u. Ehling, 1991, 24). In dieser Phase wurden jedoch die Grundlagen für die heutige Wohnungs- und Bevölkerungsstatistik gelegt, die für die weitere Ausgestaltung der Wohnungspolitik in der Bundesrepublik entscheidend sein sollte (vgl. Abschnitt 5).

Andere nachgeordnete Behörden sind speziellen Ministerien zugeordnet. Im Bereich Wohnungsbau ist dabei das Bundesinstitut für Bau-, Stadt- und Raumforschung (BBSR) zu nennen, das in unterschiedlichen Konstellationen dem für Wohnungsbau zuständigen Ministerium zugeordnet ist, und sich innerhalb der letzten Jahre zu einem entscheidenden Akteur in der politikfeldspezifischen Wissensproduktion entwickelt hat (vgl. Abschnitt 7.3.3). Zu den Kernaufgaben des BBSR zählen unter an-

4 Die wesentlichen Anpassungen bestanden stattdessen in einem Ausbau der Industrie- und Agrarstatistik im Rahmen der Vorbereitung der wehrwirtschaftlichen Planungen sowie der Autarkiebestrebungen des Dritten Reichs sowie in einer Ausweitung der Volkszählung im Rahmen der nationalsozialistischen Bevölkerungspolitik (Hölder u. Ehling, 1991, 24). 
derem die „Unterstützung des Ministeriums bei der fachlichen und administrativen Umsetzung einschlägiger Ressortforschungsprogramme", die „Mitarbeit bei Fachgremien sowie Vorbereitung und/oder Vertretung des Ministeriums in nationalen und internationalen politischen Gremien“, die Aufbereitung von Expertisen und Fachanalysen, die „Bereitstellung von Berichten zur Raum- und Stadtentwicklung sowie zur Entwicklung des Wohnungs- und Immobilienwesens und Bauwesens als Grundlage der Politikberatung von europäischen Gremien, der Bundesregierung und der Länderregierungen sowie zur Information der Fachöffentlichkeit“, die „Beschaffung, Aufbereitung und Auswertung von raumrelevanten Daten/Informationen als Grundlage zur Beschreibung und Bewertung räumlicher Entwicklungen sowie von Entwicklungen auf dem Wohnungs- und Immobilienmarkt" (vgl. Bundesamt für Bauwesen und Raumordnung, 2018b). Mit dieser Aufgabenzuweisung nimmt das Bundesinstitut einen hohen Stellenwert in der ministeriumseigenen Informationsinfrastruktur und der breit gefächerten Politikberatung ein. Gegründet wurde das Institut jedoch erst 2009 ,durch Zusammenfassung des Wissenschaftlichen Bereichs des Bundesamtes für Bauwesen und Raumordnung unter Integration des Instituts für die Erhaltung und Modernisierung von Bauwerken e.V. (IEMB), Berlin“(Bundesamt für Bauwesen und Raumordnung, 2018a), und stellt somit eine recht junge Einrichtung der Ministerialbürokratie dar. Das BBSR informiert in eigener Verantwortung über die Publikation der Raumordnungsberichte ,über Trends und Entwicklungstendenzen der räumlichen Entwicklungen" (Tiemann u. Wagner, 2013, 12).

Ein gesonderter Akteur der Wissensproduktion für die Politik ist zusätzlich die Forschungszentrale der Deutschen Bundesbank, die vor allem die Regierung in Fragen von geldpolitischem Belang berät (Campbell u. Pedersen, 2014, 140). Zusätzlich erstellt das Forschungszentrum der Bundesbank Publikationen für den Bereich der akademischen Forschung wie auch zur Information eines breiteren Fachpublikums bereit und ist somit hinsichtlich der Wissensproduktion vergleichbar mit den 
halböffentlichen Forschungsinstituten (Deutsche Bundesbank, 2018). Im Zusammenhang mit wohnungspolitischen Themen sind insbesondere die regelmäßig erscheinenden Monatsberichte wie auch die Finanzstabilitätsberichte der Deutschen Bundesbank von Interesse, die vor allem in der jüngeren Phase der Wissensproduktion an Bedeutung gewonnen haben (vgl. Abschnitt 7.3.3).

Die Potenziale der staatlichen Wissensproduktion sind zwar deutlich geworden, dürfen jedoch nicht generalisierend überbewertet werden. So konnte Friedrich (1970) in Umfragen im Rahmen der Ministerialbürokratie feststellen, dass sich die Experten in der Ministerialbürokratie häufig auch als Konkurrenten der wissenschaftlichen Politikberater verstehen. Demzufolge erwarten sie von einer zunehmenden Stärkung der Position der Wissenschaftler eine Verminderung des Gewichts der eigenen Position (Friedrich, 1970, 450). Die staatlichen Wissensproduzenten sehen sich auch in einem Konkurrenzverhältnis zu anderen Wissensproduzenten gegenüber den politischen Entscheidungsträgern. Ebenso können Ideenproduzenten aus der Wissenschaft oder der Verbandslandschaft wichtige Positionen innerhalb der Ministerialbürokratie einnehmen (Niederhafner u. Speth, 2004, 29). So können Akteure aus dem Spektrum der wissenschaftlichen Politikberatung durchaus über die Bekleidung von Positionen innerhalb der Ministerialbürokratie ihren Einfluss ausüben (Hirschman u. Berman, 2014, 781). Ebenso nachgewiesen werden konnten für diverse Länder und Zeiträume starke personelle Verflechtungen zwischen Interessenverbänden und der Ministerialbürokratie (vgl. Benzner, 1989). Wie bei Verbänden, Parteien und Wissenschaftlern hängt auch der Einfluss der Verwaltung von den weiteren Ressourcen ab, die sie brauchen ,to generate and assess pertinent knowledge" (Rueschemeyer u. Skocpol 1996, 309; Schnapp 2004, 333f.). Damit ist der Einfluss der Verwaltung zwischen einzelnen Politikfeldern durchaus unterschiedlich ausgeprägt (Hustedt u. Houlberg Salomonsen, 2014, 206). Darüber hinaus ist es wichtig darauf hinzuweisen, dass insbesondere die Ministerialbürokratie mit ihren politisch besetzten Füh- 
rungspositionen nicht vollkommen unabhängig von der Parteipolitik agieren kann (Bogumil u. a. 2012; Mayntz u. Derlien 1989; Niederhafner u. Speth 2004, 30f.; Niederhafner u. Speth 2004, 30). Schließlich haben Verwaltungen in der Regel nicht die Macht policy-Wandel auf eigene Initiative hin herbeizuführen:

„This corrective - or if one prefers, reactive - role does not minimize the administrative contribution, for much of social policy development has been and remains an elaboration rather than redirection of the original liberal framework." (Heclo, 1975, 304)

Im folgenden Abschnitt soll auf die Mechanismen der Wissensproduktion und damit insbesondere auf die Interaktionen der unterschiedlichen Akteursgruppen zur Bereitstellung von Wissen und Ideen für die Politik eingegangen werden.

\subsection{Mechanismen im Regime der Ideenpro- duktion}

In den vorhergegangenen Abschnitten wurde deutlich, dass mehrere Akteursgruppen am Prozess der Ideenproduktion für politische Entscheidungen beteiligt sein können: Neben den Parteien formulieren auch Verbände und Think Tanks sowie Akteure der wissenschaftlichen Politikberatung und der öffentlichen Verwaltung Ideen und stellen diese den am institutionellen Wandel beteiligten Akteuren zur Verfügung. Dabei existiert eine Vielzahl von Mechanismen, über die diese Ideen an verschiedene Adressaten im politischen Prozess bereitgestellt werden (Siefken, 2007, 40). In der sozialwissenschaftlichen Forschung existieren zahlreiche Arbeiten zur Fragestellung, ob überhaupt von einem Einfluss von policy-Beratung ausgegangen werden kann. Die wohl bedeutendste Typologie zu möglichen Zusammenhängen von Wissensproduktion 
und Politik in Deutschland stammt von Jürgen Habermas (Thunert, 2001, 224). Mit ihr lassen sich drei Idealtypen für das Zusammenspiel von Wissensproduktion und Politik beschreiben: das technokratische Modell, in dem eine Abhängigkeit der policy-maker von der externen Ideenproduktion ausgegangen wird (Abschnitt 3.2.1), das entgegengesetzte dezisionistische Modell, das der Wissensproduktion lediglich eine dienende Funktion zuweist (Abschnitt 3.2.2), sowie das pragmatistische, das von einem wechselseitigen Verhältnis zwischen Wissensproduktion und policy-makers ausgeht (Abschnitt 3.2.3). Ausgehend von einem wechselseitigen Verhältnis zwischen Wissensproduktion und Politik werden potenzielle Mechanismen über die Bereitstellung von Wissen für policy-Wandel dargestellt und für die Bundesrepublik Deutschland spezifiziert werden (Abschnitt 3.2.4). Gemeinsam mit den Erkenntnissen zum Zusammenhang zwischen institutionellem Wandel und Ideen (Kapitel 2) sowie deren Produzenten (Abschnitt 3.1) bilden die folgenden Darstellungen die Grundlage für die Thesen, die im empirischen Teil der vorliegenden Arbeit überprüft werden sollen (Abschnitt 3.3).

\subsubsection{Fremdbestimmung der Politik: Technokratie \& political capture}

Ausgehend von einer zunehmenden Verwissenschaftlichung der Politik in den 50er und 60er Jahren (vgl. Abschnitt 3.1.3) stellt das technokratische Modell die Prämisse, dass policy-maker in eine Abhängigkeit gegenüber wissenschaftlichen Erkenntnissen verfallen. Als wegweisend in dieser Debatte ist Schelskys Rede „Der Mensch in der wissenschaftlichen Zivilisation“ anzusehen (Schelsky, 1961), in der Schelsky das überlegene naturwissenschaftliche Wissen als Möglichkeit zur Überwindung politischer Auseinandersetzungen darstellt. Der Politiker wird in dieser Vorstellung ,zum Vollzugsorgan einer wissenschaftlichen Intelligenz, die unter konkreten Umständen den Sachzwang der verfügbaren Techniken und Hilfsquellen sowie der optimalen Strategien und Steuerungsvor- 
schriften entwickelt" (Habermas, 1968, 122). In diesem Modell wird eine vollständige Umsetzung der wissenschaftlichen Politikberatung in die Politik für möglich gehalten und folgt einem Leitbild, das als ,schlüsselfertige Beratung" bezeichnet werden kann (Bröchler, 2004, 20). Eine technokratische Politik wurde von Schelsky noch überaus positiv bewertet: Der Staat sollte als „technischer Staat“ nur noch eine „fiktive Entscheidungstätigkeit" übernehmen (Schelsky, 1961). Auch Lasswell und Kaplan gehen in ihrem linear-rationalen Modell der Politik davon aus, dass wissenschaftliche Expertise bei der Lösung gesellschaftlicher Probleme helfen wird (Lasswell u. Kaplan, 1968). Voraussetzung für diesen Zusammenhang von Wissensproduktion und Politik ist allerdings, dass Politikberatung genau die Expertise liefert, welche die Politik nicht selber aufbringen kann und diese auch in einer Form bereitstellt, die direkt in den Politikprozess eingespeist werden kann, sodass die Umsetzung der Beratung 1:1 möglich ist (Bröchler, 2004, 20).

Zwar existieren vereinzelte Fälle des „Gesetzgebungsoutsourcings“ ${ }^{\star 5}$ (Kropp u. Kuhlmann, 2014, 8), eine dauerhafte Institutionalisierung direkter Umsetzung von Expertisen in policy-Wandel ist jedoch in der Gesetzgebung der Bundesrepublik überaus unwahrscheinlich. So stellt Bröchler heraus, dass es sich bei Politikberatung um ,einen vielfach vorstrukturierten Prozess [handelt], in dessen Verlauf die Umsetzung der Beratungsvorschläge zunächst offen ist" (Bröchler, 2004, 35). Diese Ergebnisoffenheit begründet er vor allem mit den Eigenheiten des bundesdeutschen Regierungssystems als Verhandlungsdemokratie, in der wissenschaftliche Expertise zu ihrer Umsetzung auf Mehrheiten im Parlament sowie auf die „Durchsetzungsfähigkeit in gesellschaftlich

$5 \quad$ Kropp u. Kuhlmann (2014) verweisen im Zusammenhang mit dem Outsourcing der Gesetzgebung auf die Debatten um die Großkanzlei Linklaters, die 2009 ,anstelle des Wirtschaftsministeriums einen Entwurf zum Gesetz zur Ergänzung des Kreditwesengesetzes anfertigte, der versehentlich noch mit dem Logo der Rechtsanwaltskanzlei in den Bundestag eingebracht wurde" (Kropp u. Kuhlmann, 2014, 8). 
pluralistisch zusammengesetzten Ausschüssen und Kommissionen angewiesen" sind (Bröchler, 2004, 24). Zudem beruht wissenschaftliche Politikberatung in Deutschland in der Regel auf Nachfrage vonseiten der policy-maker, die sich Problemen gegenübersehen, deren Lösung sie sich nicht selber zutrauen (Mayntz, 2006, 212). Hinzu kommt der Umstand, dass mehrere Wissensproduzenten zugleich verschiedene Ideen produzieren und gemeinsam in den beratenden Gremien vertreten sind: Neben Wissenschaftlern dominieren oftmals Vertreter aus ,der Praxis", und damit aus Verbänden und der freien Wirtschaft, politische Beratungsgremien, sodass die einzelnen Akteure in der Regel „nur eine Stimme unter vielen" haben (Cassel u. Baumann, 2018, 15). Ein Mechanismus, wonach vonseiten der Wissensproduzenten direkte Vorschläge an politische Organisationen adressiert werden und auch zum Erfolg führen, ist demgegenüber höchst unwahrscheinlich (Buchholz, 2008, 78).

Darüber hinaus werden technokratische Tendenzen aus einer demokratietheoretischen Sichtweise überaus kritisch bewertet (Fischer 1990; Rickert 1983). Eng verwandt mit der demokratietheoretisch fundierten Kritik an technokratischer Regierungsbeeinflussung ist die Kritik an Lobbyismus. Dabei bezieht sich Lobbying auf ,beratende Tätigkeiten, mit denen Lobbyisten Unternehmen und andere Organisationen in ihrem Umfang mit politischen Problemstellungen unterstützen" (Bernhagen, 2018, 2). Weingart spricht in diesem Zusammenhang von korporatistischer Politikberatung, in der „Repräsentanten der jeweils in Frage kommenden Interessengruppen in die Beratungsgremien eingebunden oder aber die Experten von den jeweiligen Interessengruppen bestimmt" werden (Weingart, 2006, 43). So kann der zunächst angenommene enge Begriff der wissenschaftlichen Politikberatung erweitert und beschrieben werden als ,das Verfügbarmachen von Informationen und Handlungsempfehlungen für politisch Handelnde und Entscheidende durch Wissenschaftler (wissenschaftliche Politikberatung) sowie durch Fachleute aus Wirtschaft und Gesellschaft" (Wollmann 2002, 376; siehe auch 
Grundmann u. Stehr 2011, 12f.). Gegenüber einer Herrschaft des Volkes sahen bereits frühe Kritiker der Verbandspolitik eine „Herrschaft der Verbände, die die Funktionsweise der parlamentarischen Demokratie untergraben und die innere Souveränität des Staates bedrohen würde“ (Wagschal u. Grasl, 2015, 208). Politikberatung durch Lobbyisten kann so auch interpretiert werden als Austausch von Fachwissen gegen politischen Einfluss (Bernhagen, 2018, 2). Hinsichtlich der Wissensproduktion in der immer komplexer werdenden Gesellschaft und dem Mangel an Expertise innerhalb der Parteien merkt Forsthoff (1971) bereits an, dass sich insbesondere das Fachwissen für den jeweiligen Politikbereich bei den Verbandsexperten konzentrierte (Forsthoff, 1971, 19). Somit sieht er die Gefahr, dass sich gewählte Volksvertreter nicht mehr auf ihre eigenen parteipolitischen Maximen verlassen, sondern zunehmend abhängig von Verbandsinteressen gesteuert würden, die nur noch wenig mit den Interessen der Allgemeinheit zu vereinbaren sein müssen. Diese Vereinnahmung lässt sich, wie Wagschal u. Grasl (2015) anmerkt, bestens über den Begriff der „capture“ (Gefangennahme) beschreiben. Dabei erfolgt der Einfluss der Interessenvertreter nicht nur über die klassischen Tätigkeiten in Beratungsgremien oder durch eigene Expertisen, sondern auch über die enge Bindungen zu Parteien. Diese Verbindungen, die Lehmbruch als „Elitenverflechtung“ bezeichnet (Lehmbruch, 1979), ,können zu einer Zielharmonisierung führen und so zentrale Ankerpunkte korporatistischer Politik darstellen" (Hassel u. Trampusch, 2006, 114). Das Netzwerk personeller Verknüpfungen zwischen Interessenvertretern und Parteien kann demnach innerhalb einzelner politischer Subsysteme zur Politikentwicklung der Parteien selber beitragen (Lehmbruch, 1979, 606f.).

Dabei wird Lobbyismus nicht nur kritisch beurteilt. So betonen Vertreter der pluralistischen Verbändeforschung, dass ein möglichst freies Kräftespiel unter den Verbänden entscheidend ist für ein „dem Allgemeinwohl verhältnismäßig gut entsprechendes Politikergebnis“ (Wagschal u. Grasl, 2015, 207). Ebenso kann korporatistische Konsensbe- 
schaffung durch seine höhere Flexibilität der reinen Parteipolitik überlegen sein (Lehmbruch, 1977). Bezogen auf die Weitergabe von Informationen und Ideen würde dieser Zusammenhang auch nahelegen, dass alle wesentlichen Informationen und Ideen aus dem entsprechenden Politikfeld auf einer Art freiem Markt der Ideen geteilt und demokratisch verhandelt werden können. Basierend auf der optimistischen Annahme offener und neutraler Wissensmärkte würden somit Ideen gegeneinander abgewogen mit dem Ergebnis, dass die beste Idee gewinnt (Rueschemeyer, 2009, 61). Diese Neutralitätsvermutung der Ideenproduzenten ist allerdings nur unter der Annahme gegeben, dass alle gesellschaftlichen Kräfte gleich stark organisierbar sind, und ein freier Wettbewerb unter deren Positionen eine demokratietheoretisch einwandfreie Lösung für alle beteiligten hervorbringen kann (Wagschal u. Grasl, 2015, 207), also dann, wenn kein klassisches Marktversagen vorliegt, wie es in der Realität von Märkten jedoch meist der Fall ist (Rueschemeyer, 2009, 61f.). Problematisch ist diese Sichtweise auf die Wissensproduktion vor allem, wenn sich nicht alle Interessen über Verbände artikulieren können. So zeichnen sich korporatistische Systeme wie das der Bundesrepublik Deutschland durch ,institutionalisierte Beteiligung gesellschaftlicher Gruppen an der politischen Willensbildung und der Umsetzung politischer Entscheidungen“ aus (Wagschal u. Grasl, 2015, 208), was eine begrenzte Anzahl funktional differenzierter Verbände mit sich bringt. In Deutschland hat sich weitgehend ein Vertretungsmonopol größerer Dachverbände durchgesetzt (Lehmbruch, 1986, 110). Das Eintreten neuer Verbände und damit die freie Wissensproduktion aller Beteiligten ist in diesem System erschwert. Zudem muss die These berücksichtigt werden, dass Ideen, die von Eliten gefördert werden, ein größeres Potenzial aufweisen auch Gehör zu finden (Goldstein u. Keohane, 1993). Diese Einschätzung deckt sich mit zahlreichen Thesen zur Vermarktlichung, die von einer groß angelegten Finanzierung von den Wirtschaftswissenschaftlern ausgehen, deren Theorien dem politischen Willen der Machthabenden entsprechen (Harvey, 2007a, 32). 
Verbraucherinteressen gelten demgegenüber oft als schwer organisierbar und dementsprechend den gegenüberstehenden Interessen vonseiten der Industrie als unterlegen (Wagschal u. Grasl, 2015, 212). Gerade in Bezug auf Wissens- und Ideenproduktion bezogen zeigen sich deutliche Unterschiede in der Ressourcenausstattung verschiedener Akteursgruppen. So sind in der Wohnungspolitik die Interessen der Mieter oftmals schlechter gestellt als diejenigen der Vermieter, da ihre Organisation durch eine starke Fragmentierung innerhalb der Mieterschaft erschwert wird und auch über geringere Ressourcen verfügt als die der Wohnungsanbieter (Egner, 2004a).

Gegenüber der These der Fremdbestimmung der Politik muss eingewendet werden, dass Grenzen für den Einfluss der Wissensproduktion sowohl vonseiten der Wissenschaften als auch durch Interessenvertreter bestehen. So ist der Erfolg von Politikberatung nur mit einer entsprechenden Ressourcenausstattung und guten Beziehungen zu den politischen Entscheidern, bzw. einer Etablierung als Wissensproduzent möglich (Bernhagen, 2018, 8). Eine reine Abhängigkeitsthese der Regierung von externer Wissensproduktion kann nicht vertreten werden, da „Berater eben nur beraten und nicht legitimiert sind, Entscheidungen zu treffen“ (Cassel u. Baumann, 2018, 15).

\subsubsection{Politik bestimmte Ideenproduktion: Dezisio- nistische Inanspruchnahme von Wissenschaft \& Verbänden}

Bezugnehmend auf die Unterscheidung von Fachwissen und Politik bei Weber beschreibt Habermas das dezisionistische Modell anhand einer klaren Arbeitsteilung, ,zwischen den sachlich informierten und technisch geschulten“ Experten auf der einen und den „machtinstinktiven und willensintensiven Führern" auf der anderen Seite (Habermas, 1968, 121f.). Überspitzt lässt sich dieses Modell wie folgt darstellen: „[D]er Politiker liefert das Wollen, der Wissenschaftler das Wissen und Kön- 
nen, der eine das Ziel, der andere die Mittel“ (Streeck, 2016, 490). In diesem Modell haben Wissenschaftler eine dienende Funktion gegenüber den politischen Entscheidungsträgern, indem sie ,politisch nützliche Beratung“" (Bröchler, 2004, 21) in Form von Problemdefinitionen und policy-solutions liefern, während die Zielvorstellungen aus politischen Wertvorstellungen heraus formuliert werden. Positiv lässt sich dieses Modell wie bei Weber als eine Art sinnvolle Arbeitsteilung beschreiben (Streeck, 2016). Negativ gewendet erscheint wissenschaftliche Expertise in diesem Modell allerdings lediglich als Erfüllungsgehilfe der Machthabenden. In dieser Leseart kann wissenschaftliche Politikberatung nur dazu dienen, bereits vorhandene politische Optionen zu legitimieren (Pielke 2007; Krasner 1993; Blattmann u. a. 2014) oder lediglich zu einem Instrument der Öffentlichkeitsarbeit werden, ,nämlich im Verhältnis zu Teilöffentlichkeiten, bei denen das Imprimatur eines eindrucksvoll benannten Forschungsinstituts noch einen legitimierenden Eindruck macht" (Streeck 2016, 495; siehe auch Weingart 2006, 37f.; Brown u.a. 2006, 55). Expertise und die mit ihr verknüpften Ideen können somit betrachtet werden als Waffe im Sinne von symbolischem Kapital, „das in Politik und Gesellschaft als Machtmittel eingesetzt werden kann“ (Maeße 2013a, 90; siehe auch Blyth 2001).

Bezieht man einen zunehmenden Grad der Verwissenschaftlichung in das dezisionistische Modell der Politikberatung mit ein, so ergibt sich ein erweitertes dezisionistische Modell, das laut Habermas den Spielraum purer Dezisionen einschränkt, ,,in dem der Politiker über ein vervielfachtes und verfeinertes Arsenal technologischer Mittel verfügt und sich der strategischen Entscheidungshilfen bedienen kann" (Habermas, 1968, 124). Der eigentliche Kern der politischen Zielvorstellungen bleibt jedoch weiterhin den gewählten Volksvertretern und deren normativen Wertvorstellungen vorbehalten. Gegenüber dem technokraischen und dem rein-dezisionistischen Verständnis vom Zusammenspiel zwischen Wissensproduktion und Politik zeigen sich in dieser Sichtweise empirisch haltbarere Vorstellungen von Mechanismen zwischen 
Wissensproduktion und Politik. Zwar beinhaltet diese Vorstellung der Machtverteilung zwischen Wissensproduktion und Politik noch keine eingehendere Beschreibung der damit verknüpften Mechanismen. Allerdings wird deutlich, dass die Wissensproduktion vonseiten der Politik nachgefragt wird, da sie deren „Opportunitätsgesichtspunkten“ zu entsprechen hat (Bröchler, 2004, 21). Unter dieser Voraussetzung ist vor allem eine weniger institutionalisierte Politikberatung zu erwarten, da jedwede Form der Institutionalisierung der Beratertätigkeiten mit einem Machtzuwachs der Wissensproduzenten einhergehen würde (Hirschman u. Berman 2014, 781; Cassel u. Baumann 2018, 4ff.).

Je unüberschaubarer die politische Lage vonseiten der policy-Produzenten eingestuft wird, desto größer ist die Wahrscheinlichkeit, dass sie auf externe Expertisen zurückgreifen. Dies sind auch diejenigen Momente, in denen beispielsweise Akteure aus dem Bereich der Wissenschaft verstärkte Einflussmöglichkeiten haben können (Hall 1989; Haas 1992; Blyth 2002). Die Unsicherheit politischer Entscheidungsträger ist gemeinhin die ,wohl [...] beliebteste allgemeine Begründung für den Bedarf an Politikberatung" (Kusche, 2008, 17) - ob vonseiten der Wissenschaft oder durch die korporatistischen Akteure. Hinzu kommen jene Momente, in denen Parteien über keine eigenen starken Positionen verfügen, die eng mit ihren Grundwerten verknüpft sind (Hirschman u. Berman, 2014, 6). Politischer Einfluss ist demgegenüber in solchen Fällen zu erwarten, in denen ein Problemfeld stark wertebasiert ist. Dabei wächst die Wahrscheinlichkeit, dass die Regierung starke Positionen vertreten wird, die ,prinzipiell wissenschaftlich nicht abschließend zu klären“ sind (Siefken, 2007, 30). 


\subsubsection{Wechselseitige Beziehungen zwischen policy- und Ideenproduzenten: das pragmatistische Mo- dell}

Problematisch für die technokratische wie auch die dezisionistische Perspektive erweist sich in empirischer wie auch in analytischer Hinsicht die strikte Trennung zwischen Wissensproduktion und Politik. So konnte bereits in Abschnitt 3.1 gezeigt werden, dass neben der wissenschaftlichen und korporatistischen Politikberatung auch Wissens- und Ideenproduktion innerhalb politischer Parteien und der öffentlichen Verwaltung erfolgt. Die analytischen Grenzen einer solchen Trennung werden demgegenüber besonders deutlich in der funktionalistisch-systemtheoretischen Perspektive von Luhmann. Dem politischen Teilsystem kommt die Funktion zu, Kapazitäten für kollektiv bindendes Entscheiden bereit zu halten (Luhmann, 2000, 84). Diese Entscheidungen werden von politischen Organisationen wie Parlamenten und Regierungen oder politischen Personen getroffen (Luhmann, 2000, 228ff.). Demgegenüber hat die Wissenschaft die Funktion neues Wissen zu schaffen, das als wahr gelten kann (Luhmann, 1992, 216f.). Politik und Wissenschaft werden somit als funktional voneinander getrennte Bereiche konzeptioniert und sind maßgeblich über Beratungstätigkeiten sowie Beratungsorganisationen strukturell gekoppelt (Luhmann, 2000, 393ff.). Wissens- und Ideentransfer zwischen den beiden Systemen werden in diesem Zusammenhang problematisiert, da jedes Teilsystem über seinen eigenen Sinnhorizont verfügt (Blätte, 2018, 6). Aufgrund ihrer Makroorientierung können reine systemtheoretische Ansätze nicht definieren, wie diese Kopplungen genau beschaffen sind: Sie können für die Reibungen zwischen Politik und Wissenschaft zwar sensibilisieren, doch sind sie ,nicht darauf ausgelegt, herleiten zu können, wie wissenschaftliche Politikberatung zu Verschränkungen der Sphären führen könnte“ (Blätte, 2018, 7).

Eine deutlich offenere Modellierung des Zusammenspiels von Wis- 
sensproduktion und Politik entwickelt Habermas gleichsam ergänzend und als Kompromiss zu den genannten Modellen mit seinem pragmatistischen Modell:

\begin{abstract}
,Anstelle einer strikten Trennung zwischen den Funktionen des Sachverständigen und des Politikers tritt im pragmatistischen Modell gerade ein kritisches Wechselverhältnis, das eine ideologisch gestützte Ausübung von Herrschaft nicht etwa nur einer unzuverlässigen Legitimationsbasis entkleidet, sondern im ganzen der wissenschaftlich angeleiteten Diskussion zugänglich macht und dadurch substanziell verändert." (Habermas, 1968, 126)
\end{abstract}

Somit ergibt sich ein Modell, dem gegenüber einer reinen Herrschaft der policy-maker oder einer bloß durch wissenschaftliche Erkenntnisse getriebenen Politik der größte empirische Realitätsgehalt zugesprochen werden kann (Bogumil, 2016, 433). Ausgehend vom pragmatistischen Modell lässt sich somit die Frage stellen, über welche Mechanismen die produzierten Ideen in die Politik getragen werden. Denn alleine aus dem Angebot vonseiten der Wissensproduzenten folgt noch kein Handlungswissen. Dieses muss erst durch Interaktionen geschaffen werden (Blätte, 2018, 11). Die Herausforderung an die sozialwissenschaftliche Forschung zur Analyse der Zusammenhänge zwischen Wissensproduktion und Politik besteht also darin, „den realen Stand der Kontakte aufzuzeigen und die Formen der Wechselbeziehungen zu analysieren" (Friedrich, 1970, 26). Mit den Wechselbeziehungen zwischen den Akteuren liege ich nahe an der Vorstellung des kooperativen Staates, wie er dem GovernanceKonzept von Renate Mayntz zugrunde liegt (siehe hierzu ausführlicher Bohne, 2018, 137). Diese Vorstellung steht dem Ansatz von Steuerung entgegen, wonach sich konkrete Steuerungssubjekte von Steuerungsobjekte unterscheiden lassen würden: Welche Akteursgruppe wann eine bedeutendere Rolle spielt, muss jeweils empirisch festgestellt werden. Daher soll vor den Analysen zur spezifischen Wissensproduktion zur 
Eigenheimförderung im Folgenden auf die empirisch nachgewiesenen Mechanismen der Wissensproduktion in der Bundesrepublik Deutschland eingegangen werden.

\subsubsection{Mechanismen der Ideenproduktion für die Po- litik in der Bundesrepublik Deutschland}

In Anlehnung an Siefken (2007) institutionalisierte von weniger institutionalisierten Formen der Wissensproduktion unterschieden. Ist von Politikberatung in Deutschland die Rede, so sind deren Ansprechpartner in erster Linie die policy-maker, die sich aus der für einen begrenzten Zeitraum gewählten Regierung und der auf Dauer angelegten Ministerialverwaltung zusammen setzt. Dabei ist es die Ministerialbürokratie, die Probleme identifiziert, Informationen sammelt und konkrete Vorschläge für die politischen Entscheidungsträger entwickelt (Tiemann u. Wagner, 2013, 2). Die Ministerialbeamten sind es, die Gesetzesentwürfe und Vorlagen der Bundesministerien für neue Gesetze erstellen (Wehlau, 2009, 45f.) und daher auch die wichtigsten Auftraggeber, Adressaten und Partner für die Wissensproduzenten darstellen (Beyme 1986, 95; Friedrich 1970, 27; Sebaldt 2001). Sobald der Entwurf einmal im Plenum des Bundestages diskutiert wird, ,ist es oftmals schon zu spät, den Gesetzgebungsprozess erfolgreich zu beeinflussen“" (Bernhagen 2018, 6; vgl. Niederhafner u. Speth 2004, 29). Für Parteien und ihre Vertreter nimmt die policy-Beratung gegenüber der externen Politikberatung für Kommunikationsstrategien in Wahlkämpfen für Parteien hingegen einen überaus geringeren Stellenwert ein (Jun, 2018), da sie selber politische Ideen vermitteln und auch für den Politikprozess konkretisieren. Vonseiten der Wissensproduzenten der Interessenvertreter bietet sich zudem eine engere Zusammenarbeit mit den Ministerialbeamten an, da es für politische Akteure ,durchaus mit negativen Konsequenzen verbunden sein kann, in der Öffentlichkeit mit einem Wirtschaftsverband oder einem einzelnen Unternehmen in Verbindung gebracht zu werden“ 
(Niederhafner u. Speth, 2004, 29).

Als nicht-institutionalisierte Kanäle der Wissensproduktion sind zunächst gutachterliche Tätigkeiten zu nennen (Siefken, 2007, 42). Während einige Gutachten Sachstandsberichte und oftmals keine wirklichen Empfehlungen beinhalten, werden einzelne Studien gezielt von policy makers und policy takers in Auftrag gegeben um politische Problemlösungen zu entwickeln (Buchholz, 2008, 78). Gutachten haben dabei die Eigenheit, ,dass sie vom Auftraggeber vergleichsweise gut auf spezifische Fragestellungen steuerbar sind, beispielsweise über die Auswahl der Gutachter, die detaillierte Formulierung des Arbeitsauftrags und über die häufig begleitende Diskussion von Zwischenergebnissen“ (Siefken, 2007, 55). Anhand des Mechanismus der Expertisen wäre es für die am politischen Prozess beteiligten Akteure somit möglich im Sinne eines dezisionistischen Modells ihre eigenen Positionen zu legitimieren, oder auch in der eingeschränkten dezisionistischen Sichtweise einzelne Expertisen gezielt zur Schließung ihrer Wissenslücken nachzufragen. Auftragsnehmer dieser Gutachten sind oftmals außeruniversitären Forschungseinrichtungen, aber auch diverse Think Tanks. In einzelnen Fällen können Gutachten auch von Professoren oder wissenschaftlich arbeitende Abteilungen von Beratungsunternehmen erstellt werden (Buchholz, 2008, 86). Da gutachterliche Tätigkeiten für die policy-maker als Aufgabenbereich der Verwaltung zugeordnet sind, verfügen die zuständigen Ministeriumsmitarbeiter als eigener Expertenstab über die letztendliche „Verwertungs- und Interpretationsmacht über die Ergebnisse der Beratung [und kann] alle Versuche, die über von ihr vorgenommene Funktionszuweisung hinausgehen, abwehren" (Friedrich, 1970, 446).

Institutionalisierte Formen des Ideentransfers bestehen in Anhörungen, wie sie beispielsweise von Bundestagsausschüssen oder Ministerien durchgeführt werden (Buchholz 2008, 86f.; Siefken 2007, 42). Einfluss durch die Politik kann dabei bereits durch die Auswahl der anzuhörenden Experten ausgeübt werden (Krevert, 1993, 136). Je dauerhafter die Politikberatung und damit der Kommunikationsprozess verläuft, desto 
institutionalisierter und potenziell einflussreicher kann dieser vonseiten der Wissensproduzenten angesehen werden (vgl. Siefken 2007, 42; Schulte 2013, 126). Zu den zwar immer noch zeitlich begrenzten Mechanismen der Wissensproduktion, die jedoch über einmalige Anhörungen hinausgehen sind neben Enquete-Kommissionen und Untersuchungsausschüssen auch eingesetzte Expertenkommissionen zu nennen. Bei seiner Untersuchung verschiedener Beratungsgremien auf Bundesebene stellt Siefken (2007) heraus, dass auch die Resultate von Expertenkommissionen von politischen Akteuren überaus pragmatisch genutzt werden (Siefken, 2007, 317f.). Allerdings weist er auch darauf hin, dass Expertenkommissionen nicht vorrangig dazu verwendet werden „PolicyPositionen politischer Akteure argumentativ zu stärken“ (Siefken, 2007, 318), da die Steuerungsmöglichkeiten der Politik in diesem Mechanismus deutlich eingeschränkter sind als dies beispielsweise in der Auftragsforschung der Fall ist.

Ein deutlich höherer Grad der Organisation ist bei Beiräten anzunehmen, die auf eine längere Dauer hin angelegt sind und nicht selten „mit einer eigenständigen Geschäftsstelle und eigenen wissenschaftlichen Mitarbeitern ausgestattet sind" (Buchholz, 2008, 88). Während systemtheoretisch inspirierte Ansätze wie auch die Wissenssoziologie ihren Schwerpunkt auf kommunikative Handlungen im Rahmen der Beratung legen, fokussieren politikwissenschaftliche wie auch institutionelle Ansätze das Machtgefüge der Wissensproduzenten bzw. deren institutionelle Position im politischen Prozess. So gewinnen aus politikwissenschaftlicher Perspektive „Probleme der organisatorischen Eingliederung wissenschaftlicher Berater in das politische System in den Blick“" (Weingart, 2006, 35). Neben der eigenen Geschäftsstelle und damit einer verbesserten Ressourcenausstattung nennt Mayntz (2006) als Erfolgskriterien für den tatsächlichen Einfluss der Wissensproduktion die direkte Anbindung an den Adressaten der Beratung, den Anteil der im Gremium vertretener Akteure sowie die vorgegebenen Arbeitsverfahren (Mayntz, 2006, 116). Je besser die Ressourcenausstattung in 
Kombination mit der direkten Anbindung an die Politik und der Freiheit der Themenwahl, desto stärker kann der Einfluss eines Beirates auf die Ideenproduktion und damit auch für die Ausgestaltung von policies sein (siehe auch Cassel u. Baumann, 2018). Beiräte, ob korporatistisch oder wissenschaftlich dominiert, sind meist direkt den Ministerien zugeordnet, was ihre oftmals postulierte Unabhängigkeit infrage stellt. So weisen Cassel u. Baumann (2018) darauf hin, dass Vertreter der Ministerien in der Regel an den Beiratssitzungen teilnehmen und diese auch organisatorisch unterstützen (Cassel u. Baumann, 2018, 9). Zusätzlich würden auch Themenwünsche der Ministerien mit berücksichtigt.

Ein besonders frühzeitig und nahe der Politik installiertes Gremium stellt der wissenschaftliche Beirat beim Bundeswirtschaftsministerium dar, dessen Vertreter 1948 auf Einladung der Verwaltung für Wirtschaft des Vereinigten Wirtschaftsgebietes in einer ersten konstituierenden Sitzung zusammen traten (BMWi, 2017). Es war das erste unabhängige Gremium der wissenschaftlichen Politikberatung der Bundesrepublik (Cassel u. Baumann, 2018, 9). Diesem Gremium wird in der zeithistorischen Forschung oftmals als fast mythisch beschrieben ,hinsichtlich der politischen Wirkungsmächtigkeit der bundesdeutschen Volkswirtschaftslehre" (Hesse, 2010, 123). Vor dem Hintergrund der bereits in Abschnitt 3.1.3 beschriebenen geringen Ressourcenausstattung der universitären Volkswirtschaftslehre muss diesem mythischen Charakter jedoch entgegengehalten werden, dass in den mehr oder minder wissenschaftlich besetzten Beiräten auf Bundesebene zunächst nicht viele Professoren der Volkswirtschaftslehre vertreten waren. Da es nur wenige Professoren gab, Beiratsmitglieder aber zugleich den Professorentitel tragen mussten, herrschte zumindest statistisch gesehen eine Vollversorgung der Professoren mit Beiratsmandaten (Hesse, 2010, 123f.).

Gegenüber Wissensproduzenten anderer Akteursgruppen verfügt die Ministerialbürokratie über eine wesentliche Schaltstellen- und Türsteherfunktion im System der Wissensproduktion. Wissensproduzenten, 
die einen Einfluss auf die Ideenproduktion haben wollen, müssen vor allem Zugang zu diesem Feld erlangen. Neben engen Verbindungen zu politischen Parteien und Funktionsträgern, ist für Wissensproduzenten, die Einfluss ausüben wollen, bleiben ihnen als dauerhafte Ansprechpartner die Vertreter der Ministerialbürokratie innerhalb des policy-Subsystems. So steuert die öffentliche Verwaltung maßgeblich die Prozesse „by which the state itself influences the development and application of social knowledge“" (Evans u.a., 1985b, 357f.). Hall (1989) betont dabei vor allem den Einfluss der fachlich geschulten Bürokratie auf die Anwendung politischer Ideen. Sie hängt maßgeblich mit der administrative viability der Ideen selber zusammen. So wurde seiner Einschätzung zufolge das Paradigma des Keynesianismus wahrscheinlicher akzeptiert, "if it accorded with the long-standing administrative biases of the officials responsible for approving it and seemed feasible in light of the existing implementational capacities of the state" (Hall, 1989, 373). Das von anderen Akteuren außerhalb des politischen Systems produzierte Wissen muss also gegenüber jenen Akteuren aufgenommen werden (können), die sich mit der Umsetzung der Ideen in konkrete policies befassen. Je mehr Experten mit einem vergleichbaren Bildungshintergrund innerhalb der Administration tätig sind, und je mehr die Lösungen des neuen Paradigmas zu den von ihnen wahrgenommenen Problemlagen haben, desto wahrscheinlicher wird der fit des Expertenwissens mit den Umsetzungstätigkeiten der Ministerialbürokratie (Hall, 1989, 291, 374). Dabei ist jedoch zu berücksichtigen, dass sich staatliches Handeln, die Zuständigkeitsbereiche staatlicher Akteure sowie die Zusammenarbeit mit anderen politischen Akteuren Wandlungsprozessen unterliegen und je nach Politikbereich unterscheiden können (vgl. Skocpol, 1985, 14). Bevor diese Wandlungsprozesse im empirischen Teil dieser Arbeit genauer untersucht werden, sollen im folgenden Abschnitt die zentralen Ergebnisse der theoretischen Vorüberlegungen sowie die Thesen für den weiteren Gang der Arbeit zusammengefasst werden. 


\subsection{Zusammenhang zwischen Ideenproduk- tion und institutionellem Wandel}

Aus den theoretischen Überlegungen der Kapitel 2 und 3 ergeben sich zunächst Anforderungen für den empirischen Teil dieser Arbeit, da für die Beantwortung der Frage, warum sich Ideen und die mit ihnen verbundenen policies ändern, ein komplexes Geflecht verschiedener Erklärungsfaktoren herangezogen werden kann. Neben sich ändernden politischen, wirtschaftlichen und gesellschaftlichen Rahmenbedingungen können in den Modellierungen der klassischen Institutionalismen sich ändernde Interessen der beteiligten Akteure zu einem Wandel herbei führen (Abschnitt 2.1). Für die Analyse der Ursachen ideellen Wandels in der Eigenheimförderung müssen somit die Rahmenbedingungen, aber auch die Formierung der Akteursinteressen vor dem Hintergrund ihrer materiellen Position und dem jeweiligen situativen Kontext mit berücksichtigt werden. Daher werden die Rahmenbedingungen in den Darstellungen der empirischen Ergebnisse bei jeder Phase der Ideenproduktion für die Eigenheimförderung in Deutschland vorangestellt (Abschnitte 5.1, 6.1, refrahmen3). Neben materiellen und situativen Kontextfaktoren wurden in Abschnitt 2.2 Ideen als wesentlicher Faktor für diese Interessenformierung identifiziert. Sie sind nicht nur handlungsanleitend und sinnstiftend für die Handlungsentscheidungen der Akteure, sondern auch selber Bestandteil der Institutionen. Institutioneller Wandel kann sich somit auf deren Regelgehalt als auch auf deren normative und kognitive Elemente beziehen. Angesichts der Vielschichtigkeit institutionellen Wandels und den für die Wohnungspolitik postulierten Vermarktlichungstendenzen stehen die folgenden Analysen daher vor der Herausforderung zu präzisieren, welche policy-Bestandteile der Eigenheimförderung von welchen Wandlungsprozessen betroffen waren. Dies geschieht für jede im empirischen Teil beschriebene Phase im Anschluss an die Beschreibung der Rahmenbedingungen (Abschnitte 5.2, 
6.2, 7.2). Eine Darstellung des um Wissensproduzenten erweiterten Modells institutionellen Wandels findet sich in Abbildung 3.2.

Abbildung 3.2: Ideenproduzenten und institutioneller Wandel innerhalb eines policy-Subsystems

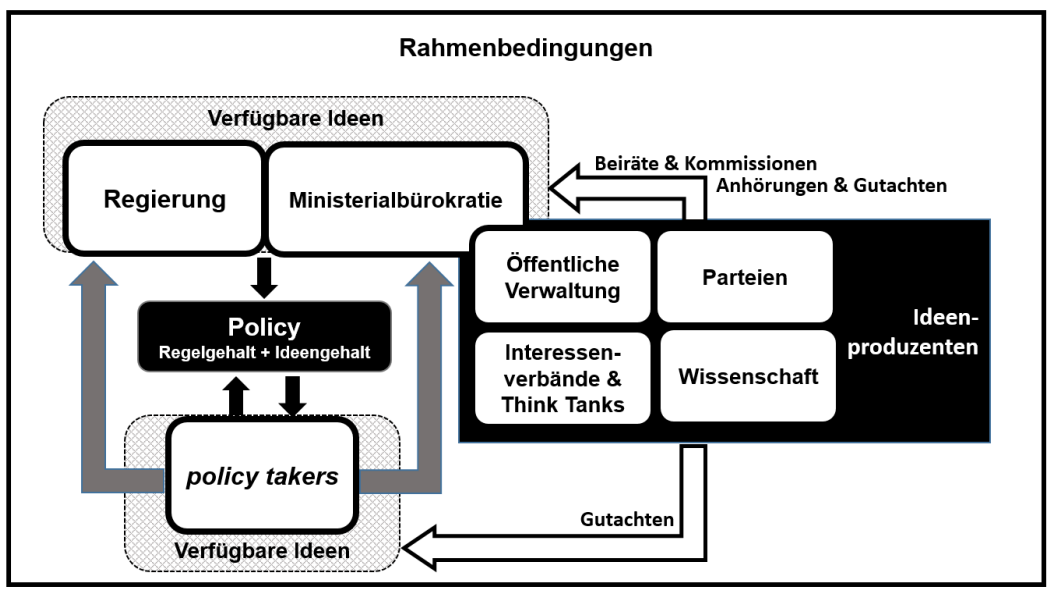

Quelle: eigene Darstellung

Dieses Modell lässt mehrere Möglichkeiten institutionellen Wandels zu und berücksichtigt damit die bestehenden empirischen Erkenntnisse zu institutionellem Wandel. Es kann demnach zu institutionellem Wandel kommen, wenn sich die wirtschaftlichen, politischen wie auch gesellschaftlichen Rahmenbedingungen für die Akteure ändern. Darüber hinaus sind Änderungen erwartbar, wenn sich die Akteurskonstellationen ändern. Dies kann der Fall sein, wenn beispielsweise ein Regierungswechsel stattfindet oder aber neue policy-takers im policySubsystem auftreten, während andere es verlassen. Ebenso können sich die Interessen der Akteure ändern, über deren Formierung neue Ideen die Möglichkeit haben, in dem politischen Subsystem Eingang zu finden. Weitere Wandlungsmöglichkeiten ergeben sich durch die Einbeziehung des Regimes der Ideenproduktion. So können sich neben der Zusammensetzung der Akteure des knowledge regimes (vgl. Abschnitt 
3.1) auch die Mechanismen der Wissensproduktion ändern (vgl. Abschnitt 3.2).

Die zentrale theoretische Fragestellung für die vorliegende Arbeit lautet, welchen Einfluss Akteure und Mechanismen der Wissensproduktion auf die Vermarktlichung wohlfahrtsstaatlicher Institutionen haben. Hierzu bieten die Erkenntnisse aus den Abschnitten 3.1 und 3.2 die Grundlagen für die im Empirieteil zu überprüfenden Hypothesen. Die erste zu prüfende Hypothese lautet dabei:

Hypothese 1. Ein Wandel in der Akteursstruktur und den Mechanismen der politikfeldspezifischen Wissensproduktion hat einen Einfluss auf ideellen Wandel wohlfahrtsstaatlicher Institutionen.

Dieser Hypothese folgend sollte sich der Wandel des ideellen Gehalts einer Institution nicht nur durch allgemeine Rahmenbedingungen oder die situativen und materiellen Bedingungen des Interessenwandels der beteiligten Akteure erklären, sondern auch auf Änderungen innerhalb des knowledge regimes zurückführen lassen. Dementsprechend werden im dritten Teil der Ergebnisdarstellungen die Akteure, die von ihnen zum ideellen Wandel beigesteuerten Ideen und die dazugehörigen Mechanismen genauer beschrieben (Abschnitte 5.3, 6.3, 7.3). Um die institutionelle Reproduktion von knowledge regimes nicht zu überbewerten (Kelstrup, 2016, 23), sollen dabei nicht nur Kontinuitäten in der Wissensproduktion, sondern vor allem deren Entwicklung beschrieben werden (Ban, 2016, 20).

Die zweite zu prüfende Hypothese bezieht sich auf die unterschiedliche Bedeutung, die den einzelnen Akteursgruppen für die Ideenproduktion zukommt. Während für policy-Wandel im allgemeinen die Positionierungen von politischen Parteien angesehen werden, die an der Regierung beteiligt sind (Abschnitt 3.1.1), wird im Hinblick auf Vermarktlichung und Finanzialisierung meist die Bedeutung von Wirtschaftswissenschaftlern in der Wissensproduktion und Interessenvertretern als Lobbyisten hervorgehoben (vgl. Abschnitte 3.1.2, 3.1.3 sowie 3.2.1). Al- 
lerdings liefert die Literatur zur Bereitstellung von Wissen und Ideen für Politikgestaltung Hinweise darauf, dass öffentliche Stellen dabei eine entscheidende Schlüsselrolle einnehmen. So produzieren Ämter und Behörden einerseits selber Ideen und bringen diese in den Politikprozess mit ein (vgl. Abschnitt 3.1.4). Allerdings ist vor allem die Ministerialbürokratie durch ihre privilegierte Position in der Vorformulierung von policies einerseits, aber auch durch ihre Stellung in der Wissensverwaltung durch das Ausschreiben von Gutachten und die Organisation von Beiratstätigkeiten der zentrale Ansprechpartner für alle übrigen Wissensproduzenten (vgl. Abschnitt 3.2.4). Über die Eigenschaft als Wissensproduzent hinaus sind Akteure der öffentlichen Verwaltung da$\mathrm{zu}$ in der Lage als wesentlicher gatekeeper für die Bereitstellung von Ideen im politischen Prozess zu fungieren. Aus diesen Feststellungen ergibt sich die zweite zu überprüfende Hypothese:

Hypothese 2. Die öffentliche Verwaltung hat gegenüber den anderen Ideenproduzenten den größten Einfluss auf den ideellen Wandel wohlfahrtsstaatlicher Institutionen.

Im folgenden Kapitel soll darauf eingegangen werden, mit welchen Methoden die genannten Hypothesen im Rahmen einer Fallstudie zur Eigenheimförderung in der Bundesrepublik Deutschland überprüft werden sollen. 


\section{Kapitel 4}

\section{Methodik}

Vor der Darstellung der empirischen Ergebnisse in Teil III sollen in diesem Kapitel die dazu verwendeten Methoden beschrieben werden. Dabei gehe ich zunächst auf das Untersuchungsdesign der Fallstudie und die getroffene Fallauswahl ein (Abschnitt 4.1), bevor ich detaillierter auf die Identifikation wohnungspolitischer Paradigmen (Abschnitt 4.2) sowie der Akteure und Mechanismen in der Wissensproduktion eingehe (Abschnitt 4.3).

\subsection{Fallstudiendesign}

Zur Untersuchung der Hypothesen über den Zusammenhang zwischen der Ideenproduktion und dem ideellen Wandel wohlfahrtsstaatlicher Institutionen bietet sich angesichts des breiten Spektrums wohlfahrtsstaatlicher Maßnahmen eine Vielzahl von Untersuchungsmöglichkeiten. Da diese im Rahmen der vorliegenden Untersuchung nicht umfassend betrachtet werden können, bedarf es einer gezielten Auswahl des konkreten Untersuchungsgegenstandes. Wie in Abschnitt 3.3 herausgestellt wurde, bedarf es zur Überprüfung der entworfenen Hypothesen neben einer Erfassung von Akteuren und Mechanismen der policy-bezogenen 
Ideenproduktion auch einer Erfassung der daraus resultierenden Ideen sowie den in der Literatur als relevant beschriebenen Kontextfaktoren. Eine derart intensive Analyse in ihrem ganzheitlichen Kontext wird bei Fallstudien durch eine möglichst geringe Fallzahl ermöglicht (Ebbinghaus, 2009, 489). Dabei sollte die Fallauswahl ,aufgrund bestimmter vorab bekannter, theoretisch relevanter Eigenschaften getroffen werden" (Ebbinghaus, 2009, 488). Evans u. a. (1985b) empfiehlt die Konzentration auf einzelne Bereiche des Wohlfahrtsstaates, um offenzulegen wie verschiedene Wissensformen, the overall shape and content of governmental interventions for economic and social welfare purposes" beeinflusst haben (Evans u.a., 1985b, 359). Einen außergewöhnlichen Fall im Hinblick auf Vermarktlichung bietet Wohneigentumsförderung in zweierlei Hinsicht. So ist sie erstens wie kaum eine andere wohnungspolitische Maßnahme Ausdruck einer staatlich geförderten Vermarktlichung (Rolnik u. Rabinovich, 2014). Bourdieu (1998b) weist darauf hin, dass nur wenige Märkte ,so sehr wie der Häusermarkt vom Staat nicht nur kontrolliert, sondern regelrecht konstruiert [werden], und zwar ganz besonders durch die Vergabe von Fördermitteln für Privatpersonen“ (Bourdieu 1998b, 62; vgl. Bourdieu 2014). Durch Eigenheimförderung werden Personen vor allem zu einem ,selbstständigen Agieren am Wohnungsmarkt" als Nachfrager und Anbieter von selbstgenutztem Wohnraum befähigt (Heinelt u. Egner, 2006, 218):

„Indem sie Wohnungsversorgung letztlich zur Frage des privaten Eigentumserwerbs macht, weist sie nur den Weg individueller Anstrengung und befestigt so die Basisideologie einer Marktwirtschaft." (Häußermann u. Siebel, 1981, 328)

Zweitens betrifft Eigenheimförderung nicht nur die policy-Dimension von Vermarktlichung, sondern verfügt über einen starken symbolischen und ideellen Gehalt ,als materielles Gut, das wie die Kleidung, aber auf Dauer für alle wahrnehmbar wird" (Bourdieu, 1998b, 26, HvH.i.O.). Sie war nie eine rein wohnungspolitisch gerechtfertigte Maßnahme, sondern 
stets mit einem breiten Spektrum sozial- und gesellschaftspolitischer Motive wie auch wirtschafts- und finanzpolitischer Überlegungen verknüpft (Hämmerlein, 1996, 57). Keine andere wohnungspolitische Maßnahme hat einen derart starken ideellen Bezug (DeSalvo, 1976), weswegen Egner u.a. (2004) sie auch als ,fragmentiert-überfrachtetes Instrument" der Wohnungspolitik beschreiben, bei dem Zustandsbeschreibungen, Funktionsdeutungen und Problemwahrnehmungen inhaltlich stark divergieren (Egner u. a., 2004, 254). Politische Debatten um ideelle Konzepte sollten demnach besonders gut in der Wohneigentumsförderung zu erfassen sein.

Die Analysen der vorliegenden Arbeit konzentrieren sich auf den ideellen Wandel im Rahmen der Wohneigentumsförderung in der Bundesrepublik Deutschland, da dieser Fall in der Literatur stets als Sonderfall betrachtet wird. So weist die staatliche Förderung von Wohneigentum eine vergleichsweise lange Geschichte auf, die im Gegensatz zu vielen anderen Ländern nicht erst in den 70er Jahren ihren Anfang nahm (Rolnik u. Rabinovich 2014; Ronald 2008; Bourdieu 2014), sondern bereits in den ersten Legislaturperioden nach dem Zweiten Weltkrieg. Demgegenüber erweisen sich die im internationalen Vergleich geringen Wohneigentumsquoten als auffällig. Deutschland ist bis heute das einzige Land in der EU, in dem mehr Menschen zur Miete wohnen als in ihren eigenen vier Wänden (Housing Europe 2017, 68; Kleinman 1996). Nach Angaben des Statistischen Bundesamtes lag der Anteil der von Eigentümern selbst genutzten Wohnungen an allen bewohnten Wohnungen 2014 lediglich bei 45,5 Prozent (Statistisches Bundesamt, 2014). Mit der Einzelfallstudie soll die Einmaligkeit dieses Zusammenhanges herausgearbeitet und der außergewöhnliche Fall der Wohneigentumsförderung in Deutschland herausgehoben werden (Ebbinghaus, 2009, 486).

Anhand mehrerer Beobachtungseinheiten soll ermöglicht werden, den Ideenwandel im policy-Subsystem der Wohneigentumsförderung im Zeitverlauf rückblickend genauer zu erklären (vgl. Ebbinghaus, 2009, 
485ff.) und damit möglichst viele alternative Erklärungsfaktoren für den ideellen Wandel mit einbeziehen zu können. Die hierzu verwendete Untersuchungsmethode lässt sich als Prozessanalyse verstehen „bei der vielfältige empirische Beobachtungen innerhalb eines oder mehrerer Fälle als potenzielle Implikationen theoretischer Kausalmechanismen verstanden werden" (Starke, 2015, 454). Da die direkte Kausalität oft nicht beobachtbar ist, spielt die zeitliche Abfolge der betrachteten Ereignisse eine entscheidende Rolle:

„Es geht also um möglichst gesicherte Informationen über den Ablauf und die Reihenfolge von Ereignissen, ihre Dauer und ihr Tempo, beteiligte Akteure und deren relative Position und Ressourcen, Erwartungen vor und Einschätzungen nach zentralen Ereignissen, (informelle oder formelle) Entscheidungsprozesse, relevante und verworfene Optionen und ähnliches" (Starke, 2015, 464).

Evidenz wird in diesen Untersuchungen somit ,aufgrund zeitlicher, räumlicher oder disaggregierter Prozessbeschreibungen innerhalb der Fallstudie (within-case analysis) erbracht" (Ebbinghaus, 2009, 491). Dabei ist interne Validität entscheidend, also die Frage ob Alternativerklärungen für den empirischen Sachverhalt ausgeschlossen werden können (Döring u. Bortz, 2016, 766). Dazu ,ist es unerlässlich, dass die empirischen Implikationen alternativer Erklärungen (inklusive ihrer Mechanismen!) systematisch herausgearbeitet und miteinander verglichen werden, um theoretische Einzigartigkeit zu erfassen" (Starke, 2015, 469). In Kapitel 2 konnten bereits die in der Literatur genannte alternative Erklärungen für institutionellen Wandel herausgestellt werden. Diese umfassen neben den wirtschaftlichen und politischen Rahmenbedingungen auch Interessenkonstellationen der beteiligten Akteure. Zudem besteht die Möglichkeit, dass unterschiedliche Akteursgruppen einen Einfluss auf die Wissensproduktion im policy-Subsystem ausüben konnten. Um der Frage nach den Ursachen von Vermarktlichung nach- 
zugehen, stellt sich für die empirische Arbeit die Herausforderung jenen alternativen Erklärungsmustern nachzugehen und die Alternativhypothesen zu überprüfen. Dabei ist nicht davon auszugehen, dass die Effekte als Ganzes beobachtbar sind. Stattdessen müssen sie aus dem Material heraus weitgehend indirekt erschlossen werden: „Bestimmte Beobachtungen von Zuständen und Ereignissen können demnach als empirische Implikationen eines Mechanismus verstanden werden, insbesondere wenn sie in dem Kontext und der Reihenfolge auftreten, die theoretisch erwartbar ist" (Starke, 2015, 456f.).

Neben der Auswahl des Falles ist auch die des betrachteten Zeithorizontes eine wichtige Eingrenzung des Forschungsprojektes (Ebbinghaus, 2009, 389). Entscheidend ist dabei, dass Ursache und Wirkung innerhalb des gewählten Zeitfensters richtig analysiert werden können (Ebbinghaus, 2009, 489). Laut (Schneider u. Janning, 2006) muss sich eine Untersuchung der ideellen Entwicklung innerhalb eines policySubsystems über eine Zeitspanne von mindestens einem Jahrzehnt erstrecken (Schneider u. Janning, 2006, 13f.). Da hier nicht nur die Ideen selbst, sondern auch deren zuvor erfolgte Bereitstellung für die Politik von Interesse sind, wird von einem längeren notwendigen Zeithorizont für die Analysen ausgegangen. Vor allem mit Blick auf staatliche Wissensproduktion empfiehlt sich eine längerfristige Perspektive, da es notwendig ist ,to identify conditions of persistence or nonpersistence to explain many outcomes" (Evans u.a., 1985b, 348). Auch Siefken verweist darauf, dass die Einflussnahme von Beratung auf die Entscheidungsträger oftmals erst mit einer Verzögerung von Jahren bis Jahrzehnten eintritt (Siefken, 2007, 44). So wurde der Untersuchungszeitraum auf den Beginn der Nachkriegszeit bis Ende 2008 möglichst breit angelegt: Erst nach dem Zweiten Weltkrieg kann von einer Wohneigentumsförderung auf gesamtstaatlicher Ebene die Rede sein. 2008 kam mit der Eingliederung der Wohneigentumsförderung in das System der Riesterrente die zum Zeitpunkt der empirischen Arbeiten wesentlichste Neuerung im System der Eigenheimförderung zustande. Die Materialsammlung 
für die prozessanalytische Forschung orientiert sich somit an grundlegenden policy-Reformen der Eigenheimförderung in der Bundesrepublik Deutschland. Für die vorliegenden Untersuchungen wurde ein breites Spektrum historischer Literatur zur Wohnungspolitik in Deutschland um Primäranalysen von Bundestagsprotokollen sowie eine eigene Archivrecherche sowie Experteninterviews ergänzt, um eine unkritische Replikation bestehender Forschung zu vermeiden (Starke, 2015, 465). Die genaueren Analyseschritte sollen in den folgenden Abschnitten dargestellt werden.

Sowohl für einen Vergleich der sich ändernden wohnungspolitischen Zielsetzungen als auch hinsichtlich der Wissensproduktion bietet es sich an, den betrachteten Zeitraum seit dem Ende des Zweiten Weltkrieges in Phasen einzuteilen und die genannten Debatten entsprechend zu gruppieren. Mit Fokus auf die Wissensproduktion wird dabei in Anlehnung an Thunert (2001) und Weingart (2006) eine Gruppierung anhand unterschiedlicher Phasen der Politikberatung in Deutschland nach dem Zweiten Weltkrieg vorgenommen. Beide teilen die Entwicklung der Politikberatung in der Bundesrepublik in drei Phasen ein. Ohne den in Teil III eingehender beschriebenen Phasen der Wissensproduktion vorweg greifen zu wollen, sollen die von Thunert (2001) und Weingart (2006) identifizierten Kriterien zur Unterscheidung der Phasen der Wissensproduktion an dieser Stelle skizziert werden.

Die Beratung für Politikinhalte spielte sich nach der Einteilung von Weingart und Thunert innerhalb der ersten 20 Jahre der Bundesrepublik maßgeblich innerhalb der Organe der Bundesregierung ab. Aufgrund der engen und geschlossenen Kooperation zwischen der Amtsbürokratie und den von ihnen ausgewählten Beratern beschreibt Thunert die Beratungspraxis dieser Phase in Anlehnung an Habermas auch als dezisionistisch. Diese „Dominanz der Regierungsberatung und geschlossener Beratungsformen" öffnete sich nach Thunert schrittweise gegen Ende der 60er Jahre und vollzog sich primär mit dem Wechsel zur sozialliberalen Koalition (Thunert, 2001, 226, Fußnote 6). Durch eine 
zunehmende Einbeziehung externer wissenschaftlicher Expertise sollte dabei vor allem ein Gegengewicht zur starken Stellung der Ministerialbürokratie gewährleistet werden (Thunert, 2001, 227). Dementsprechend spricht Weingart in seiner Beschreibung dieser ersten Phase von einer zunehmenden Verwissenschaftlichung der Politik (Weingart, 2006, 42), die durch eine wachsende Einbindung wissenschaftlicher Politikberater gekennzeichnet wurde.

In der zweiten von Thunert beschriebenen Phase seit dem Ende der 60er Jahre bis ca. 1980 kam es demgegenüber zu einer ,Institutionalisierung regierungsunabhängiger Beratung und Öffnung der Beratungsformen" (Thunert, 2001, 228ff.). Dabei kamen vor allem die Mechanismen der öffentlichen Anhörungen von Sachverständigen und die EnqueteKommissionen zum Einsatz, womit das „Beratungsmonopol der Exekutive" zugunsten der Legislative gebrochen werden sollte (Thunert, 2001, 229). Ebenso kam es zu einer Öffnung der Beratung der Exekutive, in deren Rahmen vor allem die externen Expertengutachten einen besonderen Boom erlebten (Thunert, 2001, 229). Der Trend zu mehr wissenschaftlicher Expertise in der politischen Wissensproduktion zeigte sich ebenso in einem zunehmenden Ausbau der Forschungskapazitäten bei politischen Parteien und Verbänden (Thunert, 2001, 230). Weingart interpretiert diese Ausweitung der wissenschaftlichen Expertise im politischen Bereich als eine zunehmende Politisierung der Wissensproduktion (Weingart, 2006, 42). Mit einer zunehmenden Inanspruchnahme der wissenschaftlichen Expertise durch Bürgerinitiativen und Interessengruppen ,wird die politische Interessenvertretung auf die Expertise selbst ausgedehnt mit der Folge der De-Legitimierung der Expertise" (Weingart 2006, 42; siehe auch Bogner u. Menz 2002). Auch Hesse (2010) zeigt in seiner zeitgeschichtlichen Untersuchung, dass Politik in die Wissenschaft zurückwirkte. Die „Politisierung der Wissenschaft" äußerte sich ihm zufolge vor allem in einer ,Irritation des Systems Wissenschaft durch die Politik [...], weil sich die fachliche Reputation von Ökonomen mit öffentlichen oder politischen Reputations- 
mechanismen immer stärker vermischten“ (Hesse, 2010, 106). Friedrich (1970) stellt dazu fest, dass auch politische Organisationen und Verbände zunehmend ,Methoden und Forschungsergebnisse insbesondere der Statistik, Psychologie, Soziologie und Ökonomie im Kampf um die Erringung von politischen Positionen und in der Geltendmachung ihrer Interessen und Ziele im politischen Entscheidungsprozeß verwenden müssen" (Friedrich, 1970, 18f.). Diese Zunahme der wissenschaftlichen Argumentationsmuster in zahlreichen politischen Bereichen führte da$\mathrm{zu}$, dass ,der wissenschaftliche Nimbus eines überparteilichen und unparteiischen Wahrheitsanspruches schwindet" (Blätte, 2018, 7). Von einer Politisierung kann also insofern die Rede sein, als dass die in diese Phase stattfindende Wissensproduktion weniger unabhängig von den Auftraggebern eingestuft wird. Insbesondere bei Auftragsgutachten ist dieser Zusammenhang gut erkennbar. Dieses geöffnete Verhältnis der planungseuphorischen Sozialdemokratie schloss sich allerdings bereits mit dem Übergang der Regierung Brandt zu Schmidt, die wieder zu den geschlosseneren Beratungsformen tendierte (Thunert, 2001, 229).

In der dritten von Thunert genannten Phase seit den 1980er Jahren, die durch „Öffentlichkeitsorientierung und Pluralität der Beratungsformen" gekennzeichnet ist, stand zunehmend die Beratung des Bundestages durch Kommissionen im Interesse der Öffentlichkeit (Thunert 2001, 232ff.; Siefken 2007). Die in dieser Phase zunehmende „Demokratisierung der Politikberatung“ (Weingart, 2006, 42) zeigt sich vonseiten der wirtschaftswissenschaftlichen Politikberatung in einem verstärkten Trend zu einer Beratung der Öffentlichkeit (Pitlik 2001; Frey u. Kirchgässner 2002). Entgegen der reinen Beratung des politischen Fachpublikums sollten sich die Wirtschaftswissenschaften deutlich stärker an eine breitere Öffentlichkeit wenden, sodass sich „durch die Vermittlung ökonomischen Wissens an die Bevölkerung ein Gegengewicht zu den im politischen Prozess dominierenden speziellen Gruppeninteressen" entwickeln kann (Pitlik, 2001, 64f.). Auch weitere gesellschaftliche Gruppen wie Bürgerinitiativen und sektor-spezifische Interessengruppen tra- 
ten nun zunehmend als Auftraggeber und Adressaten der Wissensproduktion für die Politik auf (Thunert, 2001, 232).

\subsection{Identifikation wohnungspolitischer Ide- en}

Ausgehend von der Einschränkung des Untersuchungszeitraumes ergibt sich eine breite Grundgesamtheit an potentiell interessantem Untersuchungsmaterial. Alleine in den Debatten des deutschen Bundestages finden sich bis einschließlich zur 18. Legislaturperiode über 650 Plenarprotokolle $^{1}$, in denen mindestens einmal von „Eigenheim“ oder von „Wohneigentum“ die Rede ist. Hinzu kommen Debatten in Landtagen und Kommunen, die ebenfalls über wohnungspolitische Kompetenzen ${ }^{2}$ verfügen. Aufgrund der nur schwerlich zu bewältigenden Textmengen erscheint die Bildung einer Stichprobe unerlässlich. Für die Stichprobenbildung wurde hier auf eine bewusste Auswahl aus Plenarprotokollen des Deutschen Bundestags zurückgegriffen. Die Auswahl der relevanten Debatten erfolgte nach Lesungen jener Gesetze, die - nach der einschlägigen Literatur zu urteilen - die wichtigsten Meilensteine für die Wohneigentumsförderung ${ }^{3}$ in der Bundesrepublik bilden. Analy-

1 Die Ermittlung erfolgte über Suchanfragen unter http://pdok.bundestag.de/

2 Der Wohnungsbau ist einer jener Politikbereiche, der unter die konkurrierende Gesetzgebung nach Art 74 des Grundgesetzes fällt. Dabei wurden die „zu erreichenden Ziele für die Wohnungspolitik [...] vom Bund festgelegt [...], mit der Durchführung der wohnungspolitischen Maßnahmen waren jedoch die Länder betraut" (Simian, 2000,74).

3 Zur Wohneigentumsförderung werden dabei sowohl Maßnahmen der Sparförderung, der Einkommenssteuergesetzgebung sowie des Sozialen Wohnungsbaus gezählt, welche die Förderung des Erwerbs von Wohneigentum betreffen. In der getroffenen Auswahl sind sowohl Fälle enthalten, in denen die Eigentumsförderung ausgebaut wurde als auch solche, bei denen die entsprechenden Schranken für den Zugang zu Fördermitteln verschärft oder Förderwege abgeschafft wurden. 
siert wurden jeweils die Inhalte der ersten, zweiten und dritten Lesung der jeweiligen Gesetzesänderungen. Eine Zusammenstellung der Gesetze und Quellen der dazugehörigen Debatten in den Drucksachen des Deutschen Bundestages (BT-Drs.) findet sich in Tabelle 4.1.

So wurden gezielt jene Debatten in das Sample mit aufgenommen, die als besonders aussagekräftig für die Fragestellung gelten können (vgl. Döring u. Bortz, 2016, 302). Neben der Reduktion der Datenbasis auf eine bearbeitbare Textmenge wird durch dieses Auswahlverfahren zusätzlich gewährleistet, dass (1.) in den untersuchten Debatten tatsächlich Wohneigentumsförderung im Zentrum der Diskussion steht und nicht nur am Rande erwähnt wird und dass (2.) vergleichbares Material für die verschiedenen Zeiträume vorliegt. Zwar können die Debatten im Deutschen Bundestag nicht als der tatsächliche Ort für politische Entscheidungen angesehen werden. Allerdings bilden sie einen Rahmen, um „die andernorts getroffenen Entscheidungen öffentlich zu begründen bzw. zu kritisieren“ (Marschallek 2004, 291; Burkhardt 2003, 147ff.). Somit wird in dieser Arbeit angenommen, dass alle relevanten Deutungsangebote der im Bundestag vertretenen Parteien sich in diesen Debatten nicht nur wiederfinden, sondern diese auch in ausreichendem Umfang widerspiegeln.

Wie in Abschnitt 2.2 bereits erläutert, lassen sich Paradigmen in politischen Debatten nach Hall in drei Variablen unterteilen: (1.) Zielvorstellungen, (2.) Instrumentarien zur Zielerreichung sowie (3.) eine Vorstellung von Problemen, die mit der entsprechenden policy in Verbindung gebracht werden (Hall 1993, 279; siehe auch Ban 2016, 10). Für eine Codierung ${ }^{4}$ der genannten Debatten nach diesen Dimensionen bietet sich die folgende Vorgehensweise an: In einem ersten Schritt wird ein Codesystem mit den Kategorien „Zielvorstellungen“, „Instrumentarien“ und „Problemstellungen“ in Anlehnung an das Hall'sche Paradigmenkonzept gebildet (vgl. Abschnitt 2.2.2). Zusätzlich wurden verwende-

4 Die Codierung erfolgten mithilfe der Software MaxQDA 12. 
te Kennzahlen und ökonomische Objekte mit erfasst, die sich als Bestandteil von policy-solutions beschreiben lassen (vgl. Abschnitt 2.2.1). Durch dieses vorgefertigte Kategoriensystem sollte vor allem vermieden werden zu viele Allgemeinplätze politischer Debatten oder Anmerkungen zum politischen Prozess in die Analysen mit einfließen zu lassen. Für die darauf folgenden Codierarbeiten waren die folgenden anleitenden Fragen relevant:

1. Zielvorstellungen: Welche Ziele sollen mit diesem oder einem alternativen Gesetzentwurf verfolgt werden?

2. Instrumentarien: Über welche konkreten Maßnahmen sollen diese Ziele verfolgt werden?

3. Problemstellungen: Was sind die Probleme, die als Begründungen politischer Zielsetzungen oder Maßnahmen diskutiert werden?

4. Techniken \& analytische Tools: Auf welche konkreten Techniken und Kennzahlen beziehen sich die Redner in den Debatten?

Aufgrund der herausragenden Bedeutung von politischen Zielvorstellungen in der Eigenheimförderung wurden im zweiten Schritt aus den Textstellen mit dem Code „Zielvorstellungen“ neue Subcodes entwickelt, um inhaltliche Unterschiede der einzelnen Zielvorstellungen genauer herauszuarbeiten. Die Vergabe der Codings entsprach dabei der Vorgehensweise einer zusammenfassenden qualitativen Inhaltsanalyse, bei der in einem ersten Schritt die Textpassagen mit paraphrasierenden Codes versehen wurden. Nach diesem Schritt wurden die Paraphrasierungen in mehreren Runden generalisiert, um eine Reduktion der Inhalte auf das Wesentliche zu gewährleisten (vgl. Döring u. Bortz, 2016, 542). Anhand der Paraphrasierungen konnten mehrere Zieldimensionen der Wohneigentumsförderung in Deutschland zusammengefasst werden. Anhand der Vielfalt der Zielsetzungen zeigt sich, dass diese in Bezug auf die Wohneigentumsförderung stark fragmentiert sind und ,neben 
der Zuordnung zur Wohnungspolitik von Zuweisungen zur Familien-, Sozial-, Alterssicherungs-, Konjunktur- und Steuerpolitik oder zur Förderung der Vermögensbildung" reicht (Egner u.a., 2004, 254):

1. Zieldimensionen pro Eigenheimförderung:

- Familienpolitik: Ziel der Wohneigentumsförderung ist eine Form der Familienförderung

- Sozialpolitik: Ziel der Wohneigentumsförderung ist den aufgrund von Bedürftigkeit Geförderten sozioökonomische Absicherung zukommen zu lassen

- Wohnungspolitik: Ziel der Wohneigentumsförderung ist eine Verbesserung der allgemeinen Wohnraumversorgung

- Wirtschaftspolitik: Ziel der Wohneigentumsförderung ist die Förderung bestimmter Wirtschaftszweige wie der Bauwirtschaft und die Anregung privater Investitionen

- Vermögenspolitik: Ziel der Wohneigentumsförderung ist die Anregung zum Sparen und die Stärkung der privaten Altersvorsorge

- Umweltpolitik: Ziel der Wohneigentumsförderung ist die gezielte Förderung umweltfreundlichen und energiesparenden Bauens

- Demokratietheorie \& Gesellschaftsstabilisierung: Ziel der Wohneigentumsförderung ist die Förderung der Individualität und Unabhängigkeit und der Teilhabemöglichkeiten der Eigentümer

2. Zieldimensionen contra Eigenheimförderung:

- Austeritätspolitik: Wohneigentumsförderung ist abzulehnen, da der Staat sparen muss 
- Eigentum als Falle: Wohneigentumsförderung ist abzulehnen, da die Dauerhaftigkeit der Wohnverhältnisse angesichts des Flexibilitätsanspruchs auf Arbeitsmärkten nicht (mehr) gegeben ist

- Fehlgeleitete Förderung: Wohneigentumsförderung ist abzulehnen, da nicht die Ärmeren, sondern diejenigen von der Förderung profitieren, die sie nicht benötigen

- Gegen Spekulation: Wohneigentumsförderung ist abzulehnen, da Wohnraum keine Kapitalanlage sein darf, von der vor allem Banken profitieren

Basierend auf diesen Hauptzielen ${ }^{5}$ lassen sich die Zielvorstellungen der Befürworter und Gegner einer bestimmten policy nicht nur genauer gegenüberstellen, sondern auch intertemporal miteinander vergleichen, um einen paradigmatischen Wandel zu identifizieren.

\subsection{Identifikation von Akteuren \& Mecha- nismen der Ideenproduktion}

Im Sinne von Campbell u. Pedersen (2014) wird nach den Mitgliedern jener institutionellen Maschinerie gesucht, ,die Daten, Forschung, Politikempfehlungen und weitere Ideen generiert, welche die politischen Debatten und die Ausgestaltung der Politik beeinflussen" (Campbell u. Pedersen, 2014, 3). Basierend auf den Erkenntnissen über potenzielle Wissensproduzenten in Abschnitt 3.1 sind neben den reinen Forschungsinstituten zahlreiche weitere Akteure für die Ideenproduktion

$5 \quad$ Neben diesen Hauptzielen, die zahlreiche Anwendung finden, gibt es noch weitere Ziele, die sich nicht in die genannten Oberkategorien einfügen ließen. Dazu zählen die Förderung der neuen Bundesländer in den Debatten nach der Wende, das technokratische und daher auch stärker als Instrumentarium verstandene Ziel der Vereinfachung der Beantragung. 
im Politikbereich der Wohneigentumsförderung von Interesse. Eine erstmalige Zusammenstellung der wesentlichen Akteursgruppen der bundesdeutschen Wohnungspolitik liefert Egner (2004b) auf Grundlage einer experteninterviewbasierten Netzwerkanalyse. Er zeigt, dass sich das Politikfeld aus einer Vielzahl von Akteuren zusammensetzt: von Bundesministerien über Parteien, Bundesländer, zahlreiche Interessenverbände aus Wirtschaft und Zivilgesellschaft und Forschungsinstituten. Zwar ist diese Zusammenstellung nicht für den gesamten hier relevanten Untersuchungszeitraum aussagekräftig, bildet dabei aber eine gute Ausgangsbasis für weitere Recherchen. Die Wissensproduktion muss jedoch nicht immer in einer auf Dauer angelegten Form ablaufen. Im Folgenden soll auf die methodischen Möglichkeiten zur Erfassung sowohl der institutionalisierten wie auch der weniger institutionalisierten Mechanismen der Wissensproduktion eingegangen werden.

\subsubsection{Institutionalisierte Politikberatung}

Die ministeriellen Zuständigkeiten im weiteren Bereich der Wohnungspolitik lassen sich nicht ausschließlich dem Ministerium für Wohnungswesen zuordnen. So zeigen Egner u. Georgakis (2004), dass neben dem in diesem Zuschnitt seit 1998 bestehenden Bundesministerium für Verkehr, Bau- und Wohnungswesen 2004 auch das Bundesministerium der Finanzen - ,weil die meisten wohnungspolitischen Gesetze mit direkten Kosten für den Bund im Rahmen von direkten Finanzhilfen oder steuerrechtlichen Fragen verbunden sind“ (Egner u. Georgakis, 2004, 137) - sowie das Bundesjustizministerium - ,[d]a das Mietrecht in den Bereich des BGB fällt" (Egner u. Georgakis, 2004, 137) - eine bedeutende Rolle spielten. Darüber hinaus zeigt die Beteiligung weiterer Ministerien auch, dass das Politikfeld Wohnen unter einem besonders starken Einfluss anderer Politikfelder steht und ,sich durch fließende Grenzen auszeichnet - etwa zur Sozialpolitik allgemein, aber auch zur Städtebaupolitik und zur Familienpolitik sowie bei der Eigenheimzula- 
ge zur Vermögens- und darüber zur Renten-/Alterssicherung" (Heinelt, 2004, 46). Eine über mehrere Ministerien geteilte Zuständigkeit lässt sich auch im Teilbereich der Wohneigentumsförderung feststellen.

Über den längsten hier betrachteten Zeitraum hinweg war vor allem das Bundesministerium für Wohnungswesen federführend und tonangebend in der Eigenheimförderung. Dabei hat sich der Zuschnitt und damit auch der Zuständigkeitsbereich des Ministeriums seit Bestehen der Bundesrepublik mehrmals geändert: „Hieß das Ministerium bis 1961 Ministerium für Wohnungsbau, danach Ministerium für Wohnungswesen, Städtebau und Raumordnung, später nur noch Ministerium für Wohnungswesen und Städtebau, blieb die Bezeichnung des Ministeriums zwischen 1972 und 1998 mit Ministerium für Raumordnung, Bauwesen und Städtebau gleich“ (Egner u. Georgakis, 2004, 134). Für eine bessere Übersicht der zahlreichen Umbenennungen des Ministeriums aufgrund sich wandelnder Zuständigkeiten und Ressortzuschnitte findet sich in Anhang A.1 eine Tabelle des entsprechenden Wandels mit dem verantwortlichen Minister sowie der zugehörigen Regierungskonstellation.

Hinsichtlich der Beiräte und Expertengremien als einer weiteren Form der institutionalisierten Politikberatung erweisen sich die Akteursrecherchen als umständlich, da in Deutschland keine offizielle Statistik oder gar Auflistung von Expertengremien existiert (vgl. Siefken, 2007, 17ff.). Allerdings konnten im Rahmen der Recherchen nach Expertisen (vgl. Abschnitt 4.3.2) sowie in der weiteren Sekundärliteratur um die Eigenheimförderung mehrere Gremien identifiziert werden, die als Beiräte bzw. Expertenkommissionen mit der Wohneigentumsförderung befasst waren. Besonders prominent sind dabei der wohnungswirtschaftliche Beirat aus den frühen Jahren der Bundesrepublik sowie die Expertenkommission Wohnungspolitik, die in den 90er Jahren einberufen wurde. Hinzu kommen der Sachverständigenbeirat des Bundesministeriums für Wohnungswesen, Städtebau und Raumordnung sowie die Expertenkommission Wohnungsgenossenschaften. Angesichts der 
erweiterten ministeriellen Zuständigkeiten verwundert es zudem nicht, dass auch Expertengremien anderer Bundesministerien mit dem Thema Eigenheimförderung befasst waren. $\mathrm{Zu}$ nennen sind dabei vereinzelte Gutachten des wissenschaftlichen Beirats beim Bundesministerium für Wirtschaft sowie des wissenschaftlichen Beirats beim Bundesfamilienministeriums.

\subsubsection{Nicht institutionalisierte Politikberatung}

Der nicht-institutionalisierte Bereich der Wissensproduktion erfolgte in einem ersten Schritt über die Sichtung der öffentlichen Ausschussprotokolle sowie im Rahmen der in Abschnitt 4.2 beschriebenen Dokumentenanalyse. Bei der Nennung von weiteren Akteuren wurden diese mit dem Code „Beteiligte“ versehen und in eine Datenbank aufgenommen. An dieser Stelle zeigt sich die größte Vielfalt der beteiligten Akteure. Dazu zählen Vertreter von Gewerkschaften, Wohlfahrtsverbänden, der Kirche, Mieter- und Hauseigentümerverbänden, Verbänden von Industrie und Wohnungswirtschaft, Verbänden der Kredit- und Finanzwirtschaft wie auch Familienverbänden. Ebenso finden sich in diesen Dokumenten Berufungen auf Beiräte und Expertenkommissionen sowie auf Forschungsinstitute aus dem öffentlichen, privaten wie auch dem halböffentlichen Bereich sowie auf Einzelexperten.

Neben den Ausschussanhörungen lässt sich die für den politischen Prozess relevante Wissensproduktion auch über gutachterliche Tätigkeiten für Ministerien und Regierung ausfindig machen. Als Gutachten definiert Siefken (2007) die ,unabhängige, fachlich geprägte, methodisch gefestigte, selbständige Analyse und nachvollziehbar wertende Beantwortung gezielter Fragen" (Siefken, 2007, 55). Im Gegensatz zu Wissen und Ideen, die in Anhörungen oder Expertenkommissionen (re)produziert werden, sind die Inhalte der Gutachten bereits über die Art der zugrundeliegenden Fragestellung durch den Auftraggeber steuerbar, ebenso „über die Auswahl der Gutachter, die detaillierte Formulierung 
des Arbeitsauftrags und über die häufig begleitende Diskussion von Zwischenergebnissen" (Siefken, 2007, 55).

Gutachten zum Themenbereich Wohneigentumsförderung wurden unter anderem durch das für den Wohnungsbau zuständige Ministerium in Auftrag gegeben und in den entsprechenden Schriftenreihen veröffentlicht. Aufgrund der wandelnden Ministeriumsstruktur (siehe Abschnitt 4.3.1) haben sich auch die Titel der Schriftenreihen im Zeitverlauf geändert. Um einen Überblick über Forschungsaufträge zu erhalten, mussten daher zunächst potenziell relevante Schriftenreihen recherchiert werden, in denen die Forschungsergebnisse veröffentlicht wurden oder die zumindest Hinweise auf Forschungsprojekte geben. Eine vollständige Auflistung der Reihen mit Titelangaben wurde anhand von Recherchen in der Bibliothek des Bundesinstituts für Bau-Stadt und Raumforschung (BBSR) in Bonn ermöglicht (siehe Anhang B.1). Diese Reihen umfassen inhaltlich eine große Bandbreite wohnungspolitischer Themengebiete. Im Rahmen eines mehrtägigen Forschungsaufenthaltes wurden die Titelangaben sowie die Inhaltsverzeichnisse der nicht anderweitig verfügbaren Publikationen gesichtet. So konnten jene Studien ermittelt werden, die für den Bereich der Wohneigentumsförderung eine besondere Relevanz aufweisen. Wurde für den Bereich der Wohneigentumsförderung oder einer damit verwandten Thematik wie dem Eigenheimbau nur ein geringer Anteil der Studie gewidmet, wurde diese nicht mit aufgenommen. Neben den Veröffentlichungen in den Reihen des Bundesministeriums wurden im Rahmen weiterer Literaturrecherchen in der BBSR-Bibliothek weitere Veröffentlichungen aus Reihen recherchiert, die ebenfalls aus Forschungsaufträgen des Bundesbauministeriums resultierten. Neben den bibliographischen Daten wurden auch Auftraggeber und Auftragsnehmer der Studien erfasst sowie der Publikation entnehmbare Informationen zur Fragestellung und Hintergrund des Forschungsauftrags. Insgesamt konnten so 43 Publikationen zum Themenbereich Wohneigentumsförderung von 1951 bis einschließlich 2008 ausfindig gemacht werden. Davon handelt es sich 
bei sieben Publikationen um veröffentlichte Gutachten von Beiräten (vgl. Abschnitt 4.3.1). Aus den Publikationen des Bundesministeriums für Wirtschaft lassen sich ergänzend drei Publikationen anführen, die sich mit dem Thema der Eigenheimförderung befassen. Für diese Recherchen konnte auf die Zusammenstellung der Publikationen des wissenschaftlichen Beirats beim Bundesministerium für Wirtschaft sowie weiterer Beiräte zurückgegriffen und die entsprechenden Exemplare bestellt werden (Bundesministerium für Wirtschaft und Energie, 2018).

Als deutlich anspruchsvoller erwies sich demgegenüber die Recherche nach weiteren informellen Prozessen der Ideenproduktion und vermittlung für die Politik. Buchholz (2008) bezeichnet die persönliche und informelle Beratung von Politikern durch Wissenschaftler als die am wenigsten erforschte, aber zugleich auch wirksamste Form der Politikberatung (Buchholz, 2008, 86). Die Forschung zu policy networks legt nahe, dass ,in modernen Demokratien zunehmend die formalen und von den Verfassungen vorgegebenen Prozesse und Verfahren politischer Entscheidungsfindung an inhaltlicher Bedeutung verlieren" (Schubert, 1991, 36). Eine Möglichkeit zur Erfassung informeller Netzwerke und der Anreicherung des Hintergrundwissens über den Untersuchungsgegenstand besteht in der Befragung von Akteuren aus dem Feld (vgl. Starke, 2015, 466). Dabei handelt es sich im Rahmen dieses Forschungsprojektes um Experteninterviews in zweifacher Hinsicht, da sowohl mit „Experten als institutionelle[n] Figur[en]“ (Walter, 1994, 271) gesprochen wird, die über einen Expertenstatus verfügen, welcher ihnen vonseiten politischer Instanzen zugesprochen wird. Andererseits handelt es sich auch um Experten aus methodologischer Sicht, da ihnen der der Expertenstatus für den Politikbereich vonseiten der Forscherin zugeschrieben wird (vgl. Walter, 1994, Meuser u. Nagel, 1991). Der methodologische Expertenstatus bezieht sich also nicht primär auf die Wissensbestände, welche bereits über die Publikationen der Experten verbreitet werden, sondern auf jene, welche die befragten Experten durch ihre Tätigkeit im ihrem Tätigkeitsfeld selbst entwickeln. Für die ge- 
führten Experteninterviews mit Experten auf dem Gebiet der Eigenheimförderung haben sich die folgenden Fragestellungen zu deren Tätigkeitsbereich ergeben:

1. Wie kam es dazu, dass die ersten Analysen zu Wohnimmobilien erstellt wurden?

2. Wie und nach welchen Maßgaben werden die Immobilienmarktanalysen erstellt?

3. Hat sich das Feld der Expertentätigkeit in Deutschland in den letzten Jahren geändert und wenn ja: wie?

4. Wie bewerten die Analysten ihren eigenen Expertenstatus?

5. Welche Zielgruppe soll mit den veröffentlichten Berichten angesprochen werden?

6. Wie beurteilen die Experten ihre eigene Tätigkeit im Hinblick auf das politische Feld?

Diese Fragestellungen verweisen auf verschiedene Wissensbestände der zu interviewenden Experten. Neben dem technischen Wissen, ,das sich durch die Herstellbarkeit und Verfügung über Operationen und Regelabläufe, fachspezifische Anwendungsroutinen, bürokratische Kompetenzen usw. charakterisiert" (Bogner u. Menz, 2009, 70), umfasst das Forschungsinteresse auch Prozesswissen, „das sich auf Einsichtnahme und Informationen über Handlungsabläufe, Interaktionsroutinen, organisationale Konstellationen sowie vergangene oder aktuelle Ereignisse bezieht" (Bogner u. Menz, 2009, 70) sowie Bestandteile des Deutungswissens, das ,,subjektive Relevanzen, Regeln, Sichtweisen und Interpretationen des Experten umfasst" (Bogner u. Menz, 2009, 71). Da bereits aus dem beruflichen Werdegang der Autorin ${ }^{6}$ feldspezifische Kenntnisse vorhanden sind und theoretische Vorüberlegungen auch anhand der

6 Sowohl in der Vorbereitung als auch bei der Durchführung und der späteren Analyse der Interviews ist der Werdegang der Verfasserin dieser Arbeit mit zu berücksichtigen. Aufgrund der ehemaligen Tätigkeit im Bereich der wis- 
Dokumentenanalyse bereits getroffen werden konnten, bietet sich die Führung von Leitfaden-Interviews an (vgl. Rosenthal, 2008, 128). Dieser Leitfaden soll vor allem dabei helfen, Antworten auf die bereits stärker konkretisierten Fragestellungen zu finden. Allerdings sollte den Experten sowohl durch die Ausgestaltung des Leitfadens als auch durch die Art der Gesprächsführung genügend Raum für eigene Erzählungen ihrer Tätigkeit eingeräumt werden. Hierzu empfiehlt Hopf (2013) die Interviews keiner vollen Standardisierung zu unterziehen, sondern den Interview-Leitfaden lediglich als Orientierung zu nutzen, der ,viele Spielräume in den Frageformulierungen, Nachfragestrategien und in der Abfolge der Fragen eröffnet" (Hopf, 2013, 351). Insbesondere für die Darstellung des praktischen Ablaufs einer Expertise oder aber der grundlegenden Motivation für diese Tätigkeit, ist es zudem angebracht, Fragen zu formulieren, die zu einer Erzählung anregen (vgl. Czarniaw-

senschaftlichen Beratung wohnungspolitischer Akteure kann ihr vonseiten der Interviewten der Status als „Co-Expertin“ (Bogner u. Menz, 2009, 88-89) zugestanden werden. Dieser Status ist zunächst aufgrund des erleichterten Feldzuganges als auch im hohen fachlichen Niveau des Interviews als positiv zu bewerten. Ein potenzieller Nachteil besteht laut Bogner u. Menz (2009) jedoch in die Gefahr eines Verbleibens ,im professionellen Relevanzrahmen des Befragten“(Bogner u. Menz, 2009, 89), was zu einer Konservierung der fachlichen Blackbox anstelle eines Einblickes führen kann. Bei den interviewten Experten ließ sich jedoch kein Bezug zu dem beruflichen Werdegang der Forscherin feststellen. Dies liegt zum einen daran, dass die Experten die Forscherin nicht im Rahmen ihrer vorherigen Tätigkeit persönlich kennen gelernt haben und biographische Informationen vonseiten der Forscherin weder in der Expertenansprache noch im Rahmen der Interviews thematisiert wurden. Dies erscheint sinnvoll, da die Forscherin so auf ihrer Naivität auch in Nachfragesituationen beharren kann und ihr ,tatsächliches oder vermeintliches - Nicht-Wissen so lange wie möglich methodisch nutzen zu können"(Wolff, 2013, 349). 
ska, 2009, 71), sich als Interviewer von den gegebenen Antworten leiten zu lassen und Verständnisfragen zu stellen (vgl. Breidenstein u. a., 2013, $81)$.

Um diesen Anforderungen gerecht zu werden, wurde zur Strukturierung der ersten Interviews eine Einteilung in vier Phasen in Anlehnung an Rosenthal (2015) vorgenommen. Die erste Phase beinhaltet neben einer sogenannten Regieanweisung auch eine Erzählaufforderung in geschlossener Form, welche sich auf den Kontext der Expertentätigkeit in der Analyse von Wohnimmobilienmärkten konzentriert:

Ich bin an den persönlichen Erfahrungen von Wissenschaftlern und Wissenschaftlerinnen interessiert, die sich mit Wohnimmobilienmärkten beschäftigen. Vielleicht fangen Sie einmal an zu erzählen, als Sie begannen sich im Rahmen Ihrer Tätigkeit mit Märkten für Wohnimmobilien zu beschäftigen, was sie in der Anfangszeit erlebten, wie sich dies weiterentwickelt hat und erzählen mir von Ihren Erfahrungen bis heute.

Regieanweisung: Sie können sich dazu so viel Zeit nehmen, wie Sie möchten. Ich werde Sie auch nicht unterbrechen, mir nur einige Notizen machen, und später noch darauf zurückkommen.

Mithilfe dieser zeitlichen Strukturierung soll dem Interviewten dabei geholfen werden sich am zeitlichen Ablauf seiner Karriere zu orientieren, sodass er ,nicht lange überlegen [muss], welche Situationen erwähnenswert sind und welche nicht" (Rosenthal, 2015, 171). Zugleich ermöglicht es die Erzählaufforderung den eigenen Relevanzen der interviewten Experten ausreichenden Raum zu geben und somit das Feld nicht aus 
zu eng gehaltenen Fragen aus der Perspektive der Forscherin vorzugeben. Die auf die Eingangsfrage folgende Haupterzählung der Experten wurde entsprechend der Regieanweisung nicht von der Forscherin unterbrochen, sondern lediglich mit parasprachlichen Bekundungen begleitet. Zudem wurden zu den vom Experten angesprochenen Episoden und Ereignissen Notizen gemacht, um in der späteren Nachfragephase auf diese erneut einzugehen. ${ }^{7}$ dabei wurde ein Großteil dieser Fragen ebenfalls mit einer Erzählaufforderung verbunden, um hierzu möglichst detaillierte und ausführliche Informationen vonseiten der Experten zu erhalten (vgl. Rosenthal, 2015, 175ff.). In der dritten Phase des Interviews wurden schließlich jene Aspekte angesprochen, die noch nicht durch die Erzählungen der Experten geklärt werden konnten, jedoch für das Forschungsvorhaben von gesonderter Bedeutung sind. Es wurden sechs Experteninterviews mit Vertretern von halbstaatlichen Forschungsinstituten, privaten Beratungsunternehmen und Mitarbeitern des Bundesinstituts für Bau-Stadt und Raumforschung geführt, die vor allem mit der Vergabe von Forschungsaufträgen vonseiten des Bundesministeriums betraut sind.

Die Ergebnisse aller Akteursrecherchen wurden mit der entsprechenden Quellenzuordnung in eine Akteursdatenbank aufgenommen. Dank der zeitlichen Verortung der Zuständigkeiten auf ministerieller Ebene, der Beiratstätigkeiten sowie der Expertisen und Angaben von Experten konnten die jeweiligen Akteurskonstellationen der Wissensmaschinerie

7 Zugunsten des Gesprächsflusses und der Konzentration der Interviewerin auf die Inhalte des Interviews sowie auf den Interviewten werden die Interviews mithilfe eines Diktiergerätes aufgezeichnet und im Nachgang teilweise mit Unterstützung des Programmes f4transcript transkribiert. Die verwendeten Transkriptionsregeln finden sich in Anhang B.3. 
zu Wohneigentum in Deutschland für unterschiedliche Zeitabschnitte rekonstruiert werden.

\subsection{Kritische Würdigung der Methodik}

Sowohl die Fallauswahl als auch die Auswahl der einzelnen Methoden ermöglichen zwar Aussagen im Hinblick auf die Forschungsfragen, sind aber zugleich auch mit Einschränkungen hinsichtlich ihrer Generalisierbarkeit verbunden. So sind die Thesen zum Zusammenhang von Wissensproduktion und ideellem Wandel im Bereich der Wohneigentumsförderung nicht auf alle wohlfahrtsstaatlichen Bereiche übertragbar. So sind einzelne Politikbereiche oftmals durch unterschiedliche Akteurskonstellationen, institutionelle Strukturen sowie inhaltliche Eigenheiten gekennzeichnet (vgl. Beyme u. Schmidt 2013; Lowe 2011, 10). Somit trägt die vorliegende Einzelfallbetrachtung weniger zur Aufstellung generalisierbarer Aussagen bei, verspricht jedoch eine erste tiefergehende, verstehende Erklärung zum Zusammenhang von Wissensproduktion und ideellem Wandel im Rahmen der bundesdeutschen Wohnungspolitik (vgl. Ebbinghaus, 2009, 494).

Auch die Analyse ideellen Wandels anhand der Bundestagsprotokolle geht mit Einschränkungen in der Ergebnisinterpretation einher. Zwar bieten sie einen Rahmen um „die andernorts getroffenen Entscheidungen öffentlich zu begründen bzw. zu kritisieren“ (Marschallek 2004, 291; Burkhardt 2003, 147ff.). Allerdings ist dies nicht der einzige Ort, an dem die entscheidenden Debatten geführt werden. Neben nicht-öffentlichen Ausschusssitzungen und informellen Kontakten verweist Siefken auf die Bedeutung indirekter Politikberatung durch den öffentlichen Diskurs (Siefken, 2007, 44), bei dem die Expertisen und 
Veröffentlichungen nicht direkt an die Politik gerichtet sind oder von dieser nachgefragt werden (siehe auch Pitlik, 2001, 64-65). Dabei fungieren vor allem Medienberichte und nicht die mehr oder minder institutionalisierten Beratungsformen ,als Vermittler zwischen Ereignissen der sozialen Realität und deren möglichen Interpretationen, indem sie versuchen diesen Ereignissen Sinn zu geben“ (Mützel 2009, 231; siehe auch Blattmann u. a. 2014, 392). Im Rahmen der Debattenanalyse konnten nur vereinzelte Verweise auf mediale Debatten ausfindig gemacht werden. Deren genereller Einfluss auf den ideellen Wandel kann jedoch mit dem vorliegenden Untersuchungsdesign nicht präzise bestimmt werden. Der Vorgehensweise liegt jedoch die Annahme zugrunde, dass relevante Ideenproduzenten zumindest in einer der untersuchten Quellen auffindbar sind. Das Erkenntnisinteresse erfordert an dieser Stelle keine Vollständigkeit der Erfassung aller tatsächlich über den formalisierten Prozess hinaus beteiligten Akteure, sondern vielmehr eine Untersuchung der Frage, welchen Akteure zu welchem Zeitpunkt eine größere Relevanz vonseiten der Politik zugeschrieben wurde und wie sich dies im Zeitverlauf gewandelt hat.

Die geführten Experteninterviews erweisen sich als überaus reichhaltig bezüglich der Informationen über Funktionsweisen der Wissensproduktion und ihrem Zusammenspiel mit der Politik und deren jüngsten Wandlungsprozessen und geben auch Hinweise über weitere beteiligte Akteure. Allerdings sind sie nicht ohne weiteres für den gesamten hier betrachteten Zeitraum anwendbar, da zahlreiche relevante Akteure insbesondere aus früheren Betrachtungszeiträumen mittlerweile nicht mehr als Ansprechpartner zur Verfügung stehen. Es ist somit durchaus möglich und wahrscheinlich, dass bereits im Vorfeld der Institutiona- 
lisierung des formellen Netzwerkes bereits informelle policy-Netzwerke bestanden haben, die mithilfe der hier angewendeten Methodik nicht erfasst werden können.

Auch die gutachterliche Tätigkeit lässt sich über die hier betrachteten Veröffentlichungen nicht vollständig abbilden. Nach Angaben des BBSR ist davon auszugehen, dass nicht jeder vergebene Forschungsauftrag auch zu einer Veröffentlichung der Ergebnisse führt. Entscheidend seien dabei vor allem qualitative Kriterien der Forschungsarbeiten. In früheren Veröffentlichungen des Bundesministeriums werden zudem politisch-motivierte Gründe für verspätete Veröffentlichungen genannt. So erklärt Staatssekretär Bellinger im Vorwort einer erst 1966 veröffentlichten Referatssammlung:

„Nachdem die Referate [...] druckreif eingereicht waren und inzwischen wieder längere Zeit verstrichen war, hat das Ministerium dann [...] mitgeteilt, daß es die Veröffentlichung vorerst zurückstellen möchte. Die Referate [...] böten umfangreiches Material für die Änderung des bestehenden Rechts. Es können nur einer breiten und gründlichen Diskussion der gesamten Wohnungswirtschaft ausreichend gewürdigt und so ausgewertet werden, daß vielseitige Anregungen für den Entwurf eines Wohnungswirtschaftsgesetzes zu erwarten seien." (Bundesministerium für Wohnungwesen und Städtebau, 1967, XIII)

Somit haben Gutachten nicht nur die Eigenheit, ,dass sie vom Auftraggeber vergleichsweise gut auf spezifische Fragestellungen steuerbar sind, beispielsweise über die Auswahl der Gutachter, die detaillierte Formulierung des Arbeitsauftrags und über die häufig begleitende Dis- 
kussion von Zwischenergebnissen" (Siefken, 2007, 55), sondern auch, dass deren Veröffentlichung eng mit den Vorstellungen des Auftraggebers verknüpft ist.

Trotz der genannten methodischen Einschränkungen bietet das hier verwendete Untersuchungsdesign die Möglichkeit Eigenheiten der Wohneigentumsförderung in der Bundesrepublik Deutschland und den mit ihr verbundenen Ideenkonzepten hermeneutisch aus ihren Kontexteigenschaften heraus zu verstehen (vgl. Ebbinghaus, 2009, 491). 
Tabelle 4.1: Bedeutende Gesetzgebungen zur Wohneigentumsförderung in der Bundesrepublik Deutschland und zur Ideenidentifikation herangezogene Bundestagsdrucksachen

\begin{tabular}{|c|c|}
\hline Jahr & Gesetz \\
\hline 1956 & $\begin{array}{l}\text { Zweites Wohnungsbaugesetz: Erstmaliger Förder- } \\
\text { vorrang für die Eigentumsförderung im Sozialen Woh- } \\
\text { nungsbau (BT-Drs. 02036, 02143) }\end{array}$ \\
\hline 1965 & $\begin{array}{l}\text { Wohnungsbauänderungsgesetz: Verstärkung der } \\
\text { Eigentumsförderung im Sozialen Wohnungsbau (BT- } \\
\text { Drs. 04163, 04194) }\end{array}$ \\
\hline 1976 & $\begin{array}{l}\text { Gesetz zur Förderung von Wohneigentum und } \\
\text { Wohnbesitz im sozialen Wohnungsbau: Stärkung } \\
\text { des Einzeleigentums in der Wohnungsbauförderung } \\
\text { (BT-Drs. 07036, 07162) }\end{array}$ \\
\hline 1986 & $\begin{array}{l}\text { Gesetz zur Neuregelung der steuerrechtlichen } \\
\text { Förderung des selbstgenutzten Wohneigen- } \\
\text { tums: } \S 7 \text { b EStG wird durch den } \S 10 \text { e ersetzt: Aus- } \\
\text { bau der steuerlichen Absetzbarkeit (BT-Drs. 10156, } \\
10208 \text { ) }\end{array}$ \\
\hline 1996 & $\begin{array}{l}\text { Gesetz zur Neuregelung der steuerlichen } \\
\text { Wohneigentumsförderung (Eigenheimzulagege- } \\
\text { setz): Ersatz der steuerlichen Absetzbarkeit durch } \\
\text { einkommensunabhängige Bauzulage (BT-Drs. 13055, } \\
\text { 13065) }\end{array}$ \\
\hline 2005 & $\begin{array}{l}\text { Ersatzlose Streichung der Eigenheimzulage für } \\
\text { Neufälle ab } 2006 \text { (BT-Drs. 16008) }\end{array}$ \\
\hline 2008 & $\begin{array}{l}\text { Eigenheimrentengesetz: selbstgenutztes Wohnei- } \\
\text { gentum wird gleichberechtigte Anlageform in staat- } \\
\text { lich geförderter privater Altersvorsorge (Riesterrente) } \\
\text { (BT-Drs. } 16158,16170 \text { ) }\end{array}$ \\
\hline
\end{tabular}

Quellen: eigene Darstellung in Anlehnung an Jokl u. Zehnder (1996), Eigenheimerverband Deutschland e.V (2016), Deutsche Bundesbank (1996), Gesamtverband der Wohnungswirtschaft (1992), Kornemann (2000), Leutner (1990). 


\section{Teil III}

\section{Wissensproduktion \&}

Vermarktlichung in der

Eigenheimförderung 



\section{Kapitel 5}

\section{Phase 1: Der Weg zum}

\section{Fördervorrang des}

\section{Familienheims}

Vor dem Hintergrund des Wiederaufbaus werden mit den ersten Gesetzen zur Eigenheimförderung auf Bundesebene die wesentlichen Grundlagen für die spätere Entwicklung der Eigenheimförderung in Deutschland in Form von policies institutionalisiert (Abschnitt 5.1). Dies betrifft nicht nur den Regelgehalt der policies, sondern auch die mit ihnen verknüpften Ideen (Abschnitt 5.2). Maßgeblich ist dabei erstens die in der christlichen Soziallehre wurzelnde Vorstellung, dass eine Förderung des freistehenden „Familienheims“ einen wichtigen familienpolitischen Beitrag zu leisten vermag. Zweitens bildet die Förderung des Eigenheims einen Gegenentwurf zu vergemeinschafteten Wohnformen - insbesondere in Form der gemeinnützigen Wohnungswirtschaft - und sollte 
in der Anfangsphase des Kalten Krieges als „Bollwerk gegen den Kommunismus“ dienen. Entscheidend für die Ausgestaltung dieser ideellen Zielvorstellungen ist eine Wissensmaschinerie, die nicht von wirtschaftswissenschaftlicher Expertise, sondern von einem Bündnis aus christlichen Parteien, diversen Verbänden und einzelnen Theologen dominiert wird (Abschnitt 5.3). Ebenso wurden in dieser Phase die Grundlagen für die staatliche Wissensproduktion und -verwaltung gelegt, die einen entscheidenden Einfluss auf die Ausgestaltung der analytischen Tools der Wohnungspolitik für die kommenden Jahrzehnte haben sollte. Die wesentlichen Erkenntnisse im Hinblick auf die Hypothesen über den Zusammenhang zwischen Ideenproduktion und institutionellem Wandel werden in Abschnitt 5.4 zusammengefasst.

\subsection{Wiederaufbau \& Institutionalisierung der Eigenheimförderung auf Bundesebene}

Die erste Phase wohnungspolitischer Zielsetzungen nach dem Zweiten Weltkrieg ist durch eine bereits seit längerem bestehende Wohnungsnot gekennzeichnet, die jedoch in ihrem Ausmaß angesichts zerstörter Städte und umfangreicher Zuwanderungen von Vertriebenen deutlich intensiviert wurde (vgl. Leutner 1990; Leidner 1981; Kofner 2004). ${ }^{1}$ Nicht nur im Bereich der Wohnungspolitik, sondern im gesamten sozialpolitischen Bereich zeigten sich die Folgen des Krieges. So waren

1 Basierend auf der Wohnungsversorgung der Vorkriegszeit schätzt Kofner (2004) den Wohnungsbedarf im Jahr 1949 auf 5 Millionen Wohnungen im westlichen Deutschland (Kofner, 2004, 152). Andere Berechnungen gehen von einem Mangel von bis zu 5,9 Millionen Wohnungen aus (Schulz, 2000,65). 
„die finanziellen Grundlagen des Sozialstaats weitgehend zusammengebrochen" (Ostheim u. Schmidt, 2007a, 153) und wesentliche Bestandteile der nationalsozialistisch ausgerichteten wohlfahrtsstaatlichen Leistungen wurden schon früh vonseiten der Besatzungsmächte beseitigt, was vor allem familienpolitische Leistungen wie das Kindergeld betraf (vgl. Ostheim u. Schmidt, 2007a, 155). Während die Besatzungsmächte im Bereich der Sozialversicherungssysteme eine institutionelle „Restauration der alten Sozialversicherungssysteme" (Ostheim u. Schmidt, 2007a, 156) verfolgten und damit einzelne Pfadabhängigkeiten der bundesdeutschen Sozialpolitik wieder herstellten, musste die Sozialpolitik „ganz neue Wege [...] für die Bewältigung der direkten Kriegsfolgen einschlagen" (Ostheim u. Schmidt, 2007a, 157). Zu diesen neuen Wegen gehörten auch wesentliche Bestandteile der Wohnungspolitik.

Während alle Parteien nach 1918 noch bessere Wohnungen bauen wollten, konzentrierten sie sich nach 1945 ,angesichts der schier unüberwindlich erscheinenden Wohnungsnot [darauf] überhaupt Wohnungen [zu] bauen" (Schulz, 1994, 107). Dementsprechend stand in der Nachkriegszeit zunächst das rein quantitative Ziel der Wohnraumgewährleistung für die Bevölkerung im Vordergrund, welches durch Notstandsmaßnahmen des Alliierten Kontrollrates realisiert werden sollte. $\mathrm{Zu}$ den ersten Notstandsmaßnahmen des Alliierten Kontrollrates gehörten neben einem Einfrieren der Mieten auf dem Stand von 1936 und einer Einschränkung der Kündigungsmöglichkeiten von Mietverhältnissen auch die Einführung der Wohnraumbewirtschaftung durch kommunale Wohnungsämter (Mayer, 1998, 177). Zusammen werden diese Maßnahmen häufig auch als „Wohnungszwangswirtschaft" bezeichnet. Im Rahmen 
des sogenannten „Lücke-Plans"2 sollte diese ,Zwangswirtschaft“ allerdings beendet werden. Das Programm sah einen stufenweisen Abbau staatlicher Eingriffe in den Wohnungsmarkt und somit die Überführung der Wohnungsversorgung in die freie Marktwirtschaft vor und wurde mit dem „Gesetz über den Abbau von Wohnungszwangswirtschaft und über ein soziales Miet- und Wohnrecht" (Abbaugesetz) von 1960 umgesetzt (Leutner, 1990, 84ff.). Nachdem bereits zu Beginn der 50er Jahre einige Deregulierungen des vorab stark regulierten Mietwohnungsmarktes vorgenommen worden waren, wurden durch das Abbaugesetz die strikten Preisvorschriften für Mietwohnungen in Westdeutschland ab 1963 schrittweise $^{3}$ aufgehoben (Kofner, 2004, 164f.). Diese Freigabe der Altbaumieten $^{4}$ führte im Nachgang zu einer massiven Steigerung der Mietbelastung in einer Höhe von rund 60 Prozent (Leidner, 1981, 36). ${ }^{5}$

Als eine Art Vorbereitung auf die wegfallenden Mietpreisbindungen wird mitunter die ,soziale Ausgestaltung des BGB-Kündigungsrechts

2 Benannt wurde der Plan nach dem damaligen Bundesminister für Wohnungsbau Paul Lücke.

3 Als Entscheidungsgrundlage für die Freigabe der Mieten bildete auf lokaler Ebene sogenannte Defizitberechnungen, mit denen ermittelt werden sollte, ob für die vorhandenen Haushalte auch ausreichende Wohnungen verfügbar waren (Leidner, 1981, 37f.)

4 Die Mietpreise für Neubarwohnungen, welche ab Januar 1950 bezugsfertig waren, wurden bereits Anfang der 50er Jahre von der noch geltenden Mietpreisbindung ausgenommen. Galt der lokale Wohnungsmarkt auf Basis dieses Kriteriums als ausgeglichen, konnten die sogenannten weißen Kreise von der Mietpreisbindung freigestellt werden (Kofner, 2004, 165).

$5 \quad$ Von den Mietpreissteigerungen war lediglich privatwirtschaftliche Mietwohnungen betroffen, da für die Wohnungen des sozialen Wohnungsbaus weiterhin die bestehenden Miet- und Belegungsbedingungen fortbestanden (Mayer, 1998, 187). 
angesehen" (Kofner, 2004, 166). Als weitere soziale Abfederung der Mietpreisfreigabe wurde 1965 das Wohngeld eingeführt (Mayer, 1998, 186). Mit jenem „Gesetz über die Gewährung von Miet- und Lastenbeihilfen“ von 1960 wurde laut Jenkis (1996), ,der Übergang von der Kapital- zur Subjektförderung vollzogen“(Jenkis, 1996, 93). Der Grundgedanke der Mieterförderung über das Wohngeld besteht darin, dass der Mieter auf Antrag Mietbeihilfe erhalten kann, sofern ,die Miete über die sogenannte tragbare Miete hinausging"(Jenkis, 1996, 93; Hvh.i.O.). Mit dem Ersten Wohngeldgesetz von 1965 kodifizierte und erweiterte der Bund die Förderung von Mietern über das Wohngeld, da es als ein ,zielgenaueres Instrument als die Objektförderung [angesehen wurde], um die Versorgung der einkommensschwachen Bevölkerungsgruppen mit Wohnraum zu gewährleisten"(Schulz, 2000, 71). Mit dem Zweiten Wohngeldgesetz wurde die ursprüngliche Fassung 1970 ersetzt. Anstelle der Gewährleistung eines „Mindestmaßes an Wohnraum“ trat die ,wirtschaftliche Sicherung angemessenen und familiengerechten Wohnens" (Mayer, 1998, 189). Mit den Neuerungen der Wohnungspolitik waren bereits Ende der 60er-Jahre die entscheidenden wohnungspolitischen Instrumentarien der heutigen Wohnungspolitik vorhanden.

Die massive Bedeutungszunahme wohnungspolitischer Maßnahmen in der unmittelbaren Nachkriegszeit zeigt sich besonders deutlich in der Errichtung eines ersten eigenständigen Wohnungsbauministeriums auf Bundesebene im Rahmen des Ersten Wohnungsbaugesetzes (Erstes WoBauG) von 1950 (vgl. Schulz, 2000, Mayer, 1998). Mit dieser Entscheidung gingen zahlreiche Regelungskompetenzen von der Länderebene erstmals auf die Bundesebene über, was zu der bis heute bestehenden Komplexität in Entscheidungsprozessen rund um die Wohnungspolitik 
in Deutschland beiträgt (vgl. Heinelt u. Egner, 2006, 211). ${ }^{6}$

Für den Großteil der Bevölkerung erfolgte die Wohnraumversorgung - insbesondere in den Städten - primär über den Mietwohnungsmarkt. So besaßen 1950 lediglich 6,2 Prozent der Arbeiter- und 7,1 Prozent der Angestelltenhaushalte Wohneigentum (Hilpert, 2012, 277). Den wohnungspolitischen Herausforderungen begegnete die Politik in der Nachkriegszeit daher mit einer Neukonzeption des Sozialen Wohnungsbaus und einer Mietpreisbindung (Mayer, 1998, 179). Dabei hatte noch bis in die 40er-Jahre hinein außer Frage gestanden, ,dass der Staat ausschließlich Mietwohnungen förderte" (Hilpert, 2012, 277). Eine spezifische Förderung von Einzeleigentum ${ }^{7}$ war somit ein Novum der bundesdeutschen Wohnungspolitik der Nachkriegszeit (Schulz 1994). ${ }^{8}$

Als erste Fördermaßnahmen für den Bau von Eigenheimen müssen die steuerlichen Abschreibungsmöglichkeiten nach §7b EStG von 1949 (Kofner 2004, 182; Simian 2000, 77; Leutner 1990, 46f.) und ihrer Ausweitung durch die Einkommenssteuerreform von 1952 genannt werden,

6 Weite Bereiche der Wohnungspolitik wie der Wohnungsbau, aber auch das Wohngeld und das Wohnbauprämienrecht fallen unter die konkurrierende Gesetzgebung nach des Art. 74 GG. Demnach werden zwar die Zielsetzungen wohnungspolitischer Maßnahmen zwar vom Bund festgelegt, werden jedoch von den Ländern durchgeführt (vgl. Simian, 2000, 74).

7 Im Gegensatz zur Förderung des Einzeleigentums war die Förderung von Genossenschaftswohnungen bereits im 19. Jahrhundert in den großen Städten aufgekommen: ,,Das erste soziale Wohnungsunternehmen war die Berliner Gemeinnützige Baugesellschaft, die es sich 1848 zur Aufgabe machte, Wohneigentum für breite Bevölkerungsschichten zugänglich zu machen (Bentzien, 2012, 18f.)".

$8 \mathrm{Zu}$ angedachten Förderungen des Eigenheims in den Jahren vor 1945 siehe Lütge (1949, 313f.) 
welche eine steuerliche Förderung des Mietwohnungsbaus auch auf das Wohneigentum ausdehnte. Ergänzt wurden diese Maßnahmen durch die Förderung von Bausparverträgen durch das Wohnungsbauprämiengesetz $^{9}$ von 1952 (vgl. Biedenkopf u. Miegel 1979, 49; Schulz 1994, 274; Leutner 1990, 56f.). Zusätzlich wurde mit dm Wohnungseigentumsgesetz von 1951 die Möglichkeit geschaffen Einzeleigentum an einer Wohnung zu erwerben. Damit sollte es Personen ermöglicht werden auch Eigentum zu erwerben, ,die die Mittel zum Bau eines Eigenheims nicht aufbringen oder aus anderen Gründen nicht in die Stadtrandsiedlung wandern" können (Weitnauer, 1951, 161). Diese Fördermaßnahmen bestanden zunächst nur aus mittelbaren und keinen direkten monetären Förderungen für Wohneigentum.

Als die größten Wohnungsnotlagen der Nachkriegszeit jedoch beseitigt schienen, verlor die Eigentumsförderung nach und nach ihre breite Unterstützung aus dem gesamten parteipolitischen Spektrum und wurde immer mehr zu einer „Domäne der CDU“ (Schulz 1994, 98; vgl. auch Mayer 1998, 180). Währenddessen forcierte die SPD zunehmend den sozialen Mietwohnungsbau gegenüber der Eigenheimförderung (Schulz, 1994, 172). Tatsächlich nimmt die Förderung privaten Wohneigentums bereits in den frühen Jahren der Bundesrepublik einen besonders hohen Stellenwert in wohnungspolitischen Diskussionen ein und findet sich bereits in den frühesten wohnungspolitischen Gesetzgebungen wieder. Dies ist zunächst verwunderlich, denn ein umfassender Einsatz staatli-

9 Das Wohnungsbauprämiengesetz garantierte denjenigen staatliche Prämien, die für den Wohnungsbau sparten, eine staatliche Prämie, die nach der Kinderzahl gestaffelt war und bis zu 400 DM pro Jahr betragen konnte. Damit wurde das Ziel verfolgt, die Sparleistung anzuregen um auch über diesen Weg Kapital für den Wohnungsbau zu mobilisieren (Hilpert, 2012, 282). 
cher Mittel zur Förderung privaten Wohneigentums bedarf angesichts der gravierenden Wohnungsnöte im Nachkriegsdeutschland einer stärkeren Rechtfertigung als die Förderung des kostengünstigeren Mietwohnungsbaus für breite Bevölkerungsschichten (Hämmerlein, 1996, 57). Zwar war eindeutig der Bedarf an privatem Kapital zum Wiederaufbau der Bundesrepublik gegeben (Hilpert, 2012, 281), wäre jedoch über den Bau von Mehrfamilienhäusern auch deutlich kostengünstiger gewesen, was allen am Politikprozess beteiligten auch klar war (Kramper, 2008, 48). Aus der Wohnungsnot allein lässt sich die staatliche Wohneigentumsförderung also nicht rechtfertigen. Vielmehr stellt bereits DeSalvo (1976) heraus, dass diese Art der Förderung nur dann logisch erscheint, sofern Wohneigentum als meritorisches Gut $^{10}$ anerkannt wird. Somit finden sich in den Begründungen für die Förderung von Wohneigentum auch keine harten wohnungspolitischen Notwendigkeiten, sondern vorrangig normative Begründungen wie Motive aus dem sozial- und gesellschaftspolitischen Bereich (vgl. Leidner, 1981, 283f.). Daher lässt sich die frühe Etablierung der Eigentumförderung in Deutschland nicht alleine aus den Nachkriegsbedingungen heraus erklären, sondern muss vor dem Hintergrund der dazugehörigen Ideen betrachtet werden. Konkret gehörten dazu Leitbilder der Familienpolitik, der Vermögenspolitik, aber auch der Konjunktur- und Finanzpolitik (vgl. Jokl u. Zehnder,

10 Unter einem meritorischen Gut werden jene Güter verstanden, die nicht über den freien Markt bereitgestellt werden können, sondern einen staatlichen Eingriff erfordern. Dies setzt einen politischen Willensbildungsprozess voraus, in dem definiert wird, welche Verbreitung von Hauseigentum in der Bevölkerung als die gesellschaftlich wünschenswerte Menge angesehen wird (Musgrave, 2005, 127ff.). Bleibt die tatsächlich konsumierte Menge hinter dem gesellschaftlich wünschenswerten Niveau zurück, so ergibt sich der Bedarf für staatliches Handeln. 
1996, 406), die im folgenden Abschnitt eingehendere Betrachtung finden sollen.

\subsection{Familienförderung und Gesellschaftssta- bilisierung durch das Familienheim}

Ein Meilenstein in der Wohneigentumsförderung in Deutschland ist das sogenannte Zweite Wohnungsbaugesetz von 1956. Während das Erste Wohnungsbaugesetz von 1950 noch als ein „Bauprogramm für Standardwohnungen" (Kofner, 2004, 175), als Finanzierungsgesetz (Schulz, 1994, 309) flankierend zur Gesetzgebung des Lastenausgleichs ${ }^{11}$ (Ostheim u. Schmidt, 2007a, 158) und als eine Übergangslösung gedacht war, wird das Zweite Wohnungsbaugesetz (Zweites WohnbauG) häufig als das „Grundgesetz des Sozialen Wohnungsbaus“ bezeichnet (vgl. Kofner 2004, 176; Jokl u. Zehnder 1996, 406). Von zentraler Bedeutung erscheint im Rahmen dieser Arbeit, dass im Zweiten Wohnungsbaugesetz erstmals das „Ziel der breiten Streuung von Wohneigentum“ (Mayer, 1998, 182) im Vordergrund stand. Hierzu sollte das Ideal des Familienheimes ${ }^{12}$ nicht mehr nur indirekt über Sparförderung oder

11 Aufgrund der massiven Ungleichheiten nach dem Zweiten Weltkrieg im Hinblick auf die Vermögensverhältnisse verabschiedete der erste Deutsche Bundestag in den Jahren 1952/53 die Gesetzgebung zum Lastenausgleich mit starkem Umverteilungscharakter - vor allem um die Wiedereingliederung sicherzustellen (Ostheim u. Schmidt, 2007a, 158).

12 Die finale rechtliche Definition des Familienheims lautet „Eigenheime, Kaufeigenheime und Kleinsiedlungen, die nach Größe und Grundriß ganz oder teilweise dazu bestimmt sind, dem Eigentümer und seiner Familie oder einem Angehörigen und dessen Familie als Heim zu dienen“ (Schulz, 1994, 298f.) 
steuerliche Maßnahmen gefördert, sondern zur neuen Norm des Sozialen Wohnungsbaus ${ }^{13}$ werden (Schulz, 1994, 288).

Das Zweite Wohnungsbaugesetz war allerdings nicht unumstritten und somit auch das Ergebnis langer Debatten und umfassender Verhandlungen, welche sich über mehrere Jahre hinweg zogen. Trotz umfassender und langwieriger Beratungen in den Wiederaufbauausschüssen von Bundesrat und Bundestag zur Vermeidung des Vermittlungsausschusses erfolgten die Debatten im Plenarsaal überaus kontrovers, sodass vor der abschließenden dritten Sitzung eine weitere Überarbeitung unausweichlich wurde (vgl. Schulz, 1994, 297). Auch der Vermittlungsausschuss musste tätig werden, um eine Reihe formaler Regelungen anzupassen. ${ }^{14}$ Schließslich stimmte auch die sozialdemokratische Fraktion dem finalen Entwurf vom 8. Juni 1956 zu. Laut Hilpert stellt das Zweite Wohnungsbaugesetz ,einen Höhepunkt der Bemühungen dar, Eigenheim-, Eigentums- und Familienförderung zu verbinden" (Hilpert, 2012, 285). So heißt es bereits in $§ 1$ des Zweiten Wohnungsbaugesetzes: „Die Förderung des Wohnungsbaues soll überwiegend der Bildung von Einzeleigentum (Familienheimen und eigengenutzten Eigentumswohnungen) dienen".

So wurde vor allem ein klarer Vorrang des Familienheims gegenüber dem Mietwohnungsbau im Rahmen des Sozialen Wohnungsbaus

13 Der Finanzierungssystematik des Sozialen Wohnungsbaus folgend füllten die öffentlichen Mittel die Lücke „Zwischen den Baukosten einerseits und der erststelligen Hypothek, der Eigenleistung des Bauherren und den sonstigen Mitteln andererseits" (Schulz, 1994, 308)

14 Das wichtigste Argument der Länder den Vermittlungsausschuss anzurufen bestand in der vorgesehenen Berichtspflicht der Länder gegenüber der Bundesregierung, wodurch dem Bund ein Druckmittel gegeben worden wäre die Fördermittelvergabe der Länder stärker zu kontrollieren (Schulz, 1994, 300). 
festgeschrieben (Kofner, 2004, 177). Hinzu kam der Vorrang weiterer Eigentumswohnungen vor Mietwohnungen, sodass eine doppelte Zielvorstellung verfolgt wurde: Familienheime vor anderen Wohnungen und Eigentumswohnungen vor anderen Wohnungen in Mehrfamilienhäusern (Müller-Heine, 1984, 14). Zuvor war lediglich festgelegt, dass ein „angemessener Teil“" der Mittel zum Bau von Eigenheimen verwendet werden sollte, der jedoch nicht weiter spezifiziert wurde (Müller-Heine, 1984, 12). Eine ganze Reihe von Einzelvorschriften bevorzugte den Bau von Eigenheimen gegenüber dem Mietwohnungsbau. Zwar wurde eine feste Förderquotierung des Eigenheimbaus von den Bundesländern blockiert, allerdings gab es überaus konkrete Prioritätsfolgen in der Vergabe öffentlicher Fördermittel. Wären diese Rangfolgen stets eingehalten worden, ,hätte keine Mietwohnung gefördert werden dürfen, solange auch nur ein einziger Antrag auf ein Baudarlehen für ein Familienheim nicht positiv beschieden war" (Schulz 1994, 302; siehe auch Eichener u. a. 2000, 70). Darüber hinaus erhielten Bauherren, die mehr als zwei Kinder hatten ein zinsloses öffentliches Familienzusatzdarlehen (§45 II WoBauG). Auch bei der Rückzahlung der öffentlichen Darlehen wurden Eigentümer von Familienheimen begünstigt.

Tatsächlich hatte das Zweite Wohnungsbaugesetz zwar zu einer stärkeren Bautätigkeit im Bereich der Einfamilienhäuser beigetragen. Diese vermehrte Bautätigkeit blieb jedoch hinter der grundlegenden Intention der Eigenheimbefürworter zurück. Als Ursache hierfür werden meist Widerstände in den Ländern und den kommunalen Wohnungsämtern angeführt (so Schulz, 1994). Allerdings spielten auch die vom Mietrecht weiterhin künstlich niedrig gehaltenen Altbaumieten und das somit geringe Interesse einkommensschwacher Haushalte an Wohneigentum eine 
Rolle (Kofner, 2004, 185). So war die Eigentumsförderung zwar in der wohnungspolitischen Praxis schwer durchzusetzen, in den parlamentarischen Debatten allerdings auch für Oppositionskreise ein wesentliches Ziel des Sozialen Wohnungsbaus. So stellte sich auch die SPD in mehreren Äußerungen grundsätzlich hinter die Förderung von Wohneigentum (vgl. Ausführungen des MdB und wohnungspolitischen Sprechers der SPD-Fraktion Jacobi in BT-Drs. 04/136, 8052D; 04/194, 9881C; Abg. Meermann 04/194, 9885A-B).

Wie das Zweite Wohnungsbaugesetz scheint auch das Wohnungsbauänderungsgesetz („Gesetz zur verstärkten Eigentumsbildung im Wohnungsbau und zur Sicherung der Zweckbestimmung von Sozialwohnungen“, WoBauÄndG) von 1965 den Befürwortern des Mietwohnungsbaus mit ihren Befürchtungen recht zu geben. Zwar sollten beide Gesetze einerseits dazu dienen „Bevölkerungsgruppen den Zugang zu preisgünstigem Wohnraum [zu erleichtern], die am stärksten benachteiligt waren (Simian, 2000, 81). Allerdings markierten beide Gesetze ebenso den von nun an zunehmenden Rückzug des Staates aus der Förderung des Sozialen Mietwohnungsbaus (Kofner, 2004, 178). Da beide Gesetze zugleich zu einem weiteren Ausbau der Eigentumsförderung beitragen sollten, intensivierte sich das Argumentationsmuster der Mietwohnungsbaubefürworter, die Eigenheimförderung würde nur zulasten des Mietwohnungsbaus geschehen.

Die Gesetzesnovelle zum Wohnungsbauänderungsgesetz ist eine Reaktion der konservativ-liberalen Bundesregierung unter Erhard auf einen Entwurf der SPD für ein Drittes Wohnungsbaugesetz, wonach die Wohnungspolitik neu geordnet und dem Staat ein größeres Gewicht bei der Wohnungsversorgung zugestanden werden sollte (Hilpert, 2012, 288f.). 
Dies widersprach fundamental den Plänen der Regierung, die bereits mit dem sogenannten Abbaugesetz vom 23. Juni 1960 den schrittweisen Abbau der sogenannten „Wohnungszwangswirtschaft“"15 einleiteten. Der Plan für einen Ausweg aus dieser „Zwangswirtschaft“ sah vor, den Mietwohnungsmarkt weitestgehend zu deregulieren und somit auch die Miethöhen wieder dem Markt zu überlassen. Besondere Härten sollten dabei durch staatliches Wohngeld gemildert werden. ${ }^{16}$ Dieser Gesetzgebung im Mietwohnungsbereich steht das Wohnungsbauänderungsgesetz von 1965 als Pendant in der Eigentumsförderung gegenüber. So führt der nun amtierende Bundesbauminister Lücke zur Begründung der Gesetzesnovelle an, das Hauptziel des Regierungsentwurfes sei erstens eine „Verstärkung der Maßnahmen zur Eigentumsbildung im sozialen Wohnungsbau für die breiten Schichten der Bevölkerung, zweitens wirkungs-

15 Als Zwangswirtschaft wird eine Konstellation aus Verordnungen des Alliierten Kontrollrates bezeichnet, die neben einem Einfrieren der Mieten auf dem Stand von 1936 auch eine Einschränkung der Kündigungsmöglichkeiten von Mietverhältnissen sowie die Wohnraumbewirtschaftung durch kommunale Wohnungsämter umfasste (Mayer, 1998, 177) eingeleitet hatte. Erste Maßnahmen zu deren Abschaffung wurden bereits seit 1950 ergriffen (Kofner, 2004, 161). Die Deregulierung sollte in einzelnen Städten und Landkreisen erfolgen, in denen das Wohnungsdefizit unter drei Prozent ausmachte (Müller-Heine, 1984, 20). Das Erste Wohngeldgesetz vom 1. April 1965 zielte in seinem Schwerpunkt darauf $\mathrm{ab}$, ,zur Vermeidung sozialer Härten ein Mindestmaß an Wohnraum zu sichern“ ( $\$ 1$ Wohngeldgesetz in BGBl. I, S. 177. e.HvH.). Dabei ist zu berücksichtigen, dass ein Bedarf sozialer Härten im Mietwohnungsbereich vor allem aufgrund der Aufhebung der Mietpreisbindungen und den damit verbundenen Mietsteigerungen für Altbauwohnungen bestand (Müller-Heine 1984, 25; Leidner 1981, 36). Auch im Wohngeldbereich sollten Familien unterstützt werden, deren Einkommenssituation einen Rückgriff auf die Maßnahmen zur Objektförderung unrealistisch erscheinen ließ (Müller-Heine, 1984, 26). 
volle Maßnahmen zur Privatisierung der mit öffentlichen Mitteln erstellten Sozialwohnungen und drittens die Sicherung der Zweckbestimmung der öffentlich geförderten Sozialwohnungen zugunsten der wohnberechtigten Bevölkerungskreise, vornehmlich der kinderreichen Familien, der alten Menschen und der jungen Familien." (BT-Drs. 04/163, 8044C).

Die weiterhin bestehende und noch weiter ausgebaute Förderung der Familien ${ }^{17}$ stand mit dem neuen Gesetz eine Abschaffung weiterer Förderungebegünstigungen für einkommensschwache Schichten gegenüber, die im Zweiten Wohnungsbaugesetz noch gesichert werden sollten. Auch die Einkommensgrenzen im Sozialen Wohnungsbau wurden nicht angepasst - mit Ausnahme einer Erhöhung der Familienzuschläge - was maßgeblich eine Förderung der mittleren Einkommensgruppen begünstigte (Hilpert, 2012, 289). Die Gegenargumentation zu dieser erneuten Bevorzugung des Wohneigentums hielt sich in Grenzen - abgesehen von der bereits im Zweiten Wohnungsbaugesetz von der SPD angeführten Kritik, der Mietwohnungsbau dürfe darunter nicht zu kurz kommen. Diese besondere Förderung der eigenen vier Wände wurde im Rahmen der untersuchten Bundestagsdebatten vor allem vor dem Hintergrund von drei Zielvorstellungen diskutiert: die Familienförderung, demokratietheoretischen Idealvorstellungen sowie der Anspruch der Privatisierung der Wohnungswirtschaft. In den folgenden Abschnitten sollen diese Zielsetzungen eingehender erläutert werden.

17 Als wesentliche Zusatzförderung kann hier die Anhebung der Beträge für das Familienzusatzdarlehen und dessen Gewährung ab dem zweiten Kind genannt werden (Hilpert, 2012, 289). 


\subsubsection{Familienförderung}

Ein wesentliches Ergebnis des Zweiten Wohnungsbaugesetzes war die Einführung des Familienheimes in die Gesetzgebung. Entsprechend dominant erweisen sich familienpolitische Zielvorstellungen auch als Zieldimension in den parlamentarischen Debatten. So bekennt der CDUAbgeordnete und Vorsitzende des Ausschusses für Wiederaufbau und Wohnungswesen Lücke in der dritten Lesung, dass der „Leitgedanke, der über allen Beratungen dieses Gesetzes stand“ wie folgt zusammen zu fassen sei: „Bauen, wie es die Familie braucht“ (BT-Drs. 02/143, 7574A). Die CDU-Abgeordnete Brauksiepe erklärt, man wolle mit der Gesetzesinitiative,zum Ausdruck zu bringen, daß endlich der Familienstand hiermit noch einmal eine gerechte Bewertung erfährt" (BT-Drs. 02/142, 7410A). Als immer wiederkehrender Leitsatz der eigenheimpolitischen Zielvorstellungen lässt sich ein Zitat des CDU-Abgeordneten Brönner anführen: „Das Familienheim ist und bleibt die Grundlage für eine gesunde und wachsende Familie“ (BT-Drs. 02/142, 7380D). Hinter derartigen Gesamtkonzeptionen stand die Idee, dass die Wohn- und Eigentumsverhältnisse ,,von erheblicher Bedeutung für das gesamte Leben und den inneren Zusammenhalt der Familien" seien (Hilpert, 2012, 297). Dabei wird das Argument, wie Wohneigentum letzten Endes zur Förderung der Familien beiträgt, nicht näher erläutert.

Eng verbunden mit der Eigenheimförderung für Familien war dabei das Gebot der Sparsamkeit. So sollte im Zuge der Eigenheimförderung vor allem der Sparwillen in der Bevölkerung angeregt werden und nicht einfach Zuschüsse gewährt werden. Wie bereits durch das Bausparprämiengesetz von 1952 das Ansparen eigenen Bauvermögens staatlich gefördert wurde, sollte auch das zweite Wohnungsbaugesetz jene Bauher- 
ren bevorzugen, die einen Großteil der Baukosten selber angespart hatten. So erklärt der CDU-Abgeordnete Brönner in der Zweiten Lesung: „wer für ein eigenes Heim gespart hat, soll belohnt werden, und diese Belohnung besteht in der bevorzugten Zuteilung öffentlicher Mittel für das Familienheim“ (BT-Drs. 02/143, 7381B).

Auch die Abgeordneten der SPD sprechen sich in den Debatten wiederholt für eine Förderung des Eigenheims für Familien aus. Allerdings enthielten ihre Forderungen auch eine weitere Förderung des Mietwohnungsbaus für die einkommensschwächeren Schichten. Die Reihe der Bedürftigen, die unter dem neuen Gesetzeswerk eine bevorzugte Förderung erhalten sollten, erstreckten sich jedoch nicht nur auf Einkommensschwache, sondern auch auf Flüchtlinge aus den ehemaligen deutschen Ostgebieten, Evakuierte sowie Kriegsversehrte und Witwen (vgl. BT-Drs. 02/7573-7574). So standen neben familienpolitischen Zielsetzungen, die im Rahmen des Zweiten Wohnungsgesetzes die Debatten dominierten, auch weitere als bedürftig definierte Zielgruppen im Fokus der sozialpolitischen Eigenheimförderung. Diese Zielsetzung sollte sich jedoch im Rahmen des Wohnungsbauänderungsgesetzes von $1965 \mathrm{zu}-$ gunsten einer Förderung breiter Schichten mehr und mehr auflösen. Die grundlegende Intention der Familienförderung erwies sich mindestens ebenso zentral in den Debatten rund um das Wohnungsbauänderungsgesetz. Dies wird besonders in den Ausführungen zur Ersten Lesung des Wohnungsbauförderungsgesetzes (1965) des CDU/CSU-Abgeordneten Czaja deutlich:

„Wer in den Länderparlamenten und den Landesregierungen würde denn ernstlich daran denken oder es öffentlich vertreten wollen, daßs nicht gerade der Wohnungsbau für 
große Familien stärker subventioniert werden soll und die Familienzusatzdarlehen der derzeitigen Baukostenhöhe angepaßt werden sollen? Wer würde eigentlich öffentlich gegen die verstärkte Verwendung der Mittel beim Eigenheimbau zu sprechen wagen, nachdem jährlich 125000 förderungsfähige Familienheimanträge unerledigt bleiben?“ (BT-Drs. 04/163, 8047B-C)

Für weite Kreise in der CDU/CSU stand fest, dass „das Umziehen aus einer Mietwohnung etwa in ein Familienheim - wenn auch mit nur wenigen Quadratmetern Garten - der größte soziale Fortschritt für eine Familie ist" (BT-Drs. 04/163, 8054C). Besonders deutlich wird die breite Unterstützung der Idee des Eigenheimes als förderungswürdiges Familienheim anhand einer Suggestivfrage der SPD-Abgeordneten Meermann:

„Wer sollte denn nicht dafür sein, daß große Familien in den eigenen vier Wänden wohnen können, wenn sie den Wunsch dazu haben und wenn sie bereit sind, die erforderlichen Eigenmittel aufzubringen?“ (BT-Drs. 04/194, 9885D)

Gegenüber den Debatten um das Zweite Wohnungsbaugesetz fällt in diesen Debatten jedoch auf, dass nun Wohneigentum nicht mehr nur gezielt für Kreise von Bedürftigen, sondern für möglichst breite Schichten gefördert werden soll. Zwar wird weiterhin auch auf die Förderung bedürftiger Kreise hingewiesen, doch die Zielrichtung hat sich auch in der Argumentation merklich zu einer breiter angelegten Zielgruppe verschoben. So erklärt der nunmalige Bundesbauminister Lücke:

„Hierbei steht ein Anliegen im Vordergrund: die Eigentums- 
bildung für die breiten Schichten der Bevölkerung zu verstärken. Der Herr Bundeskanzler [Erhard] hat bereits in seiner Regierungserklärung am 15. Oktober des vergangenen Jahres darauf hingewiesen, daßs die Bundesregierung seit vielen Jahren bestrebt ist, die Eigentums- und Vermögensbildung in breitesten Schichten der Bevölkerung zu fördern.“ (BT-Drs. 04/163, 8045A)

Um möglichst breiten Schichten Wohneigentum zu ermöglichen, sollte auch die Möglichkeit der Schaffung von Wohneigentum in Wohnungen geprüft werden. Insbesondere auf Betreiben der FDP hin sollten verstärkt Eigentumswohnungen in Mehrfamilienhäusern gefördert werden - auch wenn diese im Förderungsumfang nicht mit dem Familienheim gleichgestellt wurden (Peters, 1984, 205). Eines der dahinterstehenden Ziele war das Wohneigentum auch innerhalb der Städte zu ermöglichen, wo Bauland entweder nicht verfügbar oder aber zu kostspielig war für ein Einfamilienhaus (vgl. Ausführungen des CDU-Abg. Mick, BT-Drs. 04/163, 8055B). Mit der Förderung des Wohnungseigentums sollten daher ,[d]ie Eigentumswünsche der Menschen [...] auch in Form von Stockwerkseigentum befriedigt werden" (FDP-Abg. KiepAltenloh BT-Drs. 04/194, 9895C).

\subsubsection{Gesellschaftsstabilisierung gegen Vergemein- schaftung}

Als zweite überaus dominante Idee der Befürworter der Eigenheimidee erscheint in den Debatten die demokratiefördernde Wirkung der eigenen vier Wände. Dabei ist vor allem die geopolitische Situation der jungen Bundesrepublik mit zu berücksichtigen: Das Eigenheim sollte 
sich als „Bollwerk gegen Kommunismus und Kollektivismus“ zu Zeiten des Kalten Krieges dienen (Jaedicke u. Wollmann, 1991, 421). Durch Eigentumsbildung sollten möglichst breite Kreise der Bevölkerung „unempfänglich für kommunistische Gesellschaftsentwürfe" gemacht werden (Hanke, 1994, 10). Entsprechend finden sich bei den Vertretern von FDP und CDU/CSU Äußerungen, in denen sich das Ziel die Förderung von Einzeleigentum direkt als Abgrenzung vom Gemeinschaftseigentum verstehen. Besonders prägnant wird dieses Ziel in den Äußerungen des FDP-Abgeordneten Will:

„In der Vergangenheit ist leider ein sehr wesentlicher Teil in den Besitz großer Wohnungsgesellschaften gekommen. Dadurch haben sich großstädtische Latifundienbesitze ansammeln können. Das liegt nicht im Sinne unserer Anschauung; denn uns kommt es ja darauf an, eine breitere Streuung dieser öffentlichen Gelder im Sinne einer Mehrung des Kleinkapitals, des Kleineigentums herbeizuführen." (BT-Drs. 02/7577, C)

Doch auch die Sozialdemokratie positionierte sich positiv zur Förderung von Wohneigentum, allerdings mit der Forderung der Beteiligung der Arbeiter am Eigentum (Hanke, 1994, 11). Das Ziel der Gesellschaftsstabilisierung durch Wohneigentum „den sozialen Frieden zu sichern" (Schulz, 1994, 260) bildet bis in die Bundestagsdebatten der 70er Jahre hinein eine der dominanten Zieldimensionen der Wohneigentumsförderung und wird sogar noch im Bericht einer Expertenkommission aus den 90er Jahren als eines der Argumente für die Wohneigentumsförderung genannt. Dort heißt es, dass ,das Erleben von Eigentum und der Gewinn an Unabhängigkeit im eigenen Heim Lerneffekte in 
Gang setzt, die für den Zusammenhalt des Gemeinwesens nützlich sind, eine Bejahung der Gesellschaftsordnung und eine größere Unabhängigkeit bei Einkommens- und Arbeitsplatzverlust und somit eine geringere Neigung zur Radikalisierung" beinhalten würde (Expertenkommission, 1994, 58, zitiert nach Szypulski 2008, 82).

Die Eigentumsförderung breiter Schichten ist dabei untrennbar mit bereits im Rahmen des Zweiten Wohnungsbaugesetzes diskutierten demokratietheoretischen Begründungsmustern für die Wohneigentumsförderung verbunden. Dabei ist jedoch anzumerken, dass die demokratiefördernden Elemente der Eigenheimförderung hier als ein wichtiges Element der Privatisierung diskutiert werden und sich im Rahmen dessen vor allem gegen die „Entprivatisierung“ durch öffentliche Wohnungsgesellschaften richten. Diese Ängste vor einer möglichen Entprivatisierung sind mit einer grundsätzlich positiven Konnotation der Deregulierung des Wohnungsmarktes verbunden, wie in den Ausführungen des CDU-Abgeordneten Czaja deutlich wird: „Der gewaltige Wohnungsbau nach dem Zweiten Weltkrieg trug in dem Tempo, in dem er gestartet werden mußte, die Gefahr einer gewaltigen Entprivatisierung in sich“" (BT-Drs. 04/194, 9881, C-D). In diesen Passagen finden sich weitere Vorbehalte, die gegen die gemeinnützigen Wohnungswirtschaft gewendet sind. Diese sind zwar vereinzelt bereits in den Debatten um das Zweite Wohnungsbaugesetz zu finden, intensivierten sich jedoch insbesondere im Rahmen der Debatten um die Deregulierung der Mietwohnungsmärkte. Auch Mayer erklärt in ihrer Analyse der deutschen Mietenpolitik die Eigentumsförderung hätte auch dadurch eine zusätzliche Bedeutung erfahren, ,um überhaupt erst eine Deregulierung der Mietpreisregelung und Kündigungsschutzbestimmungen 
politisch durchsetzbar zu machen" (Mayer, 1998, 426).

Interessant ist in diesem Zusammenhang, dass eben das Begründungsmuster der Regierungsparteien angegriffen wird, wonach eine Deregulierung der Wohnungsmärkte erst nach einer möglichen Funktionstüchtigkeit des Marktmechanismus eben diesem überlassen werden könne. Als wesentliche Grundannahme für die wohnungspolitischen Zielvorstellungen der Regierungsparteien muss zudem die erstmalig vorliegende amtliche Statistik zur Wohnungsmarktsituation in Deutschland gezählt werden, die somit deutlich stärker als zuvor ins Blickfeld politischer Akteure geriet (Kramper, 2008, 221). Die Erhebungen wurden erstmals nach dem Zweiten Wohnungsbaugesetz 1956 basierend auf dem Gesetz über eine Statistik der Wohn- und Mietverhältnisse und des Wohnungsbedarfs durchgeführt (BGBl 1956, 427ff.; vgl. Abschnitt 5.3.3). Getragen von der Vorstellung, dass ,daß die Wohnungswirtschaft auf die Dauer keine marktwirtschaftsfremde Oase sein dürfte, weil sie mit den sie bestimmenden Kosten eindeutig in die Marktwirtschaft und ihre Abhängigkeiten hineingestellt ist" (Kramper, 2008, 221), sollten die marktwirtschaftlichen Elemente nach dem sogenannten „Lücke-Plan“ gestärkt werden. Mit dem sogenannten Abbaugesetz vom 23. Juni 1960 sollte die noch bestehende Mietpreisbindung nach der sogenannten ,Wohnungszwangswirtschaft" schrittweise abgeschafft werden, sofern das Wohnungsdefizit in den entsprechenden Bereichen weniger als 3 Prozent des Wohnungsbestandes ausmachte (Mayer 1998, 158; vgl. Statistisches Bundesamt 1959). Dabei wurden die anzuwendenden Berechnungsmethoden durch das Gesetz selber mitgeliefert.

Diese Statistiken bilden die Grundlagen für den Großteil der Problemanalysen der Regierungsparteien, sind jedoch zugleich in den De- 
batten überaus umstritten. Ihnen zufolge wären die Wohnungsnöte der Nachkriegsjahre nahezu vollständig beseitigt gewesen, was vor allem den Widerstand des DGB erregte (Kramper, 2008, 223). Bereits in den vorherigen Jahren hatten sowohl der DGB als auch die Neue Heimat beständig auf ein weiterhin zu bekämpfendes Wohnungsdefizit hingewiesen (vgl. Kramper, 2008, 232). So wurde vonseiten des DGB die Bestimmungsmethode der zugrundeliegenden Haushalte kritisiert. Im Abbaugesetz zählten dazu zwar ,alle Mehrpersonenhaushalte [aber lediglich] die Hälfte der Einpersonenhaushalte“ (Mayer, 1998, Fußnote 45). Sowohl der Mieterbund als auch der DGB sowie die SPD lehnten den Gesetzesentwurf ab, was ihnen jedoch nur wenig half. Wie Kramper herausstellt, war die Bundesregierung vor allem an einer grundsätzlichen ordnungspolitischen Weichenstellung interessiert ,und die Kriterien, die sie für die Freigabe der Mietpreise und der Bewirtschaftungsmaßnahmen angesetzt hatte, waren einzig und allein dazu bestimmt, den Prozeß der Überführung des Wohnungswesens in die Marktwirtschaft in den Gang zu bekommen" (Kramper, 2008, 224). Allerdings konnten die Widerstände bewirken, dass der Abbau deutlich langsamer vollzogen wurde als geplant: Erst 1975 kam es zur Aufhebung der letzten Ausnahmeregelungen (Kramper, 2008, 230)

Als Argument gegen die bestehenden Statistiken der Bundesregierung wiesen in den Debatten rund um das Wohnungsbauänderungsgesetz von 1965 mehrere SPD-Abgeordnete auf deren mangelnde Verlässlichkeit hin. Als Beispiel seien die Aussagen des SPD-Abgeordneten Jacobi angeführt:

„Einer der Indikatoren, von denen die Bundesregierung ihre Schlußfolgerungen für ihre zukünftige Wohnungspolitik 
ableitet, ist nach wie vor die Berufung auf die Statistik. So fingiert sie weitgehend eine Wohnungsmarktlage, die mit den Realitäten nicht im Einklang steht.“ (04/136, 8050C)

Auch in der zweiten und dritten Lesung verwies der SPD-Abgeordnete wiederholt darauf, dass diese Ausgeglichenheit des Wohnungsmarktes nicht gegeben sein könne (vgl. BT-Drs. 04/194, 9882, A). ${ }^{18}$

Bei der Zusammenstellung der Ideen zeigt sich, dass die Befürwortung staatlicher Wohneigentumsförderung in Deutschland maßgeblich von gesellschaftspolitischen Idealvorstellungen getragen wurde. Sie waren wichtig für die politischen Entscheidungen, da alleine die bloße Schaffung von Wohnraum nach dem Zweiten Weltkrieg noch keine ausreichenden Ausschlag für die Förderung geben konnte. Mit ihr sollten familienpolitische Zielsetzungen verwirklicht werden und zeitgleich die Gesellschaft gegenüber dem kommunistischen Modell von Gemeinschaftseigentum immunisiert werden. Somit wurde die Vermarktlichung nicht vom Ideal der freien Märkte alleine getragen. Politiker bedienten sich in ihren Begründungsmustern stattdessen an einem Spektrum anderer Ideen. Wie weit entfernt dieses Ideenspektrum von jenen idealisierten Zahlenwelten der Marktbefürworter entfernt ist, zeigt sich be-

18 Tatsächlich lassen sich die Einschätzung eines ausgeglichenen Wohnungsmarktes für die Mitte der sechziger Jahre keineswegs ohne Einschränkungen halten. So hatten sich bedingt durch neue Wohnbedürfnisse und Haushaltsstrukturen sowie deutliche Preissteigerungen vor allem im Altbausegment neue Verteilungsprobleme und Versorgungsmängel ergeben (Hilpert, 2012, 290). Auch Müller-Heine geht davon aus, dass es sich beim damaligen statistischen Wohnungsdefizit nur um einen sehr groben Maßstab der tatsächlichen Wohnungsversorgung handelt, ,der über marktwirtschaftliche Vorgänge zunächst nichts aussagt, und der im besonderen die Struktur von Wohnungsangebot und Wohnungsnachfrage unberücksichtigt läßt" (Müller-Heine, 1984, 21). 
sonders deutlich anhand der Debatten um die Ausgeglichenheit der Wohnungsmärkte: Zahlen und Rechenwerke stellten keine annehmbare Begründungsmuster in den wohnungspolitischen Debatten dar. Die zunehmende Vermarktlichung des Wohnungsmarktes durch policies folgten keinem ökonomischen Effizienzgedanken, wie man annehmen könnte, sondern einem gesellschaftspolitischen Ideal.

Was durch diese Darstellung der politischen Debatte jedoch noch nicht geklärt werden konnte ist, woher die politischen Akteure in den Debatten die von ihnen vorgetragenen Begründungsmuster nahmen. Auf die Bereitstellung dieser Ideen soll daher im kommenden Abschnitt genauer eingegangen werden.

\subsection{Politische Ideenproduktion}

Wie bereits in Kapitel 3 dargestellt wurde, sind an der Wissensproduktion für die Politik meist eine Vielzahl von Akteuren beteiligt. Basierend auf der in Kapitel 4 dargestellten Methodik zeigt sich ein ebenso breites Akteursspektrum in der Wissensproduktion rund um die Wohneigentumsförderung in Deutschland. Im Hinblick auf die Mechanismen lässt sich zudem meist keine klare Grenze ziehen. So sind an Anhörungen wie auch an Gutachtenerstellungen und Beiratstätigkeiten sowohl Vertreter der öffentlichen Verwaltung als auch Wissenschaftler und Verbandsvertreter beteiligt. Zur Klarifizierung des Einflusses und der Form der Wissensproduktion verschiedener Akteure soll im Folgenden eine getrennte Betrachtung der einzelnen Akteursgruppen vorgenommen werden. In Abschnitt 5.3.1 zeige ich, dass vor allem die Koalition rund um die christdemokratischen Regierungsparteien in dieser ersten Phase eine gesonderte Rolle für die dominanten Ideen hinsichtlich der 
Eigenheimförderung gespielt hat. Wissenschaftliche Unterstützung erhielt diese Koalition maßgeblich aus dem Bereich der Theologie, während wirtschaftswissenschaftlich fundierte Ideen allenfalls nur am Rande eine Rolle spielten (Abschnitt 5.3.2). Eine gesonderte Bedeutung für die Ausgestaltung der weiteren Wissensproduktion für das gesamte Politikfeld Wohnen kommt demgegenüber der Wissensproduktion der öffentlichen Verwaltung zu (Abschnitt 5.3.3). In dieser ersten Phase wurden dort die Grundlagen für die heutigen Wohnungsstatistiken sowie die weitere Mechanismen der Wissensproduktion für die Wohneigentumsförderung geschaffen.

\subsubsection{Bündnisse aus Parteien und Verbänden}

Im Bereich der Wohneigentumsförderung lassen sich insbesondere in der ersten Phase die wohnungspolitischen Großlager entlang der großen parteipolitischen Linien zwischen CDU und CSU auf der einen Seite und der SPD auf der anderen Seite nachzeichnen. Schulz beschreibt die Lage der Koalitionen wie folgt:

Zum Klientel der CDU/CSU gehörten die katholischen Verbände wie die katholischen Siedlungsdienste, der Deutsche Siedlerbund, der sich für die Verbreitung der Kleinsiedlung einsetzte, die kleineren Kommunen und ein Teil der Gemeinnützigen. Der FDP standen die Organisationen des Kreditgewerbes und der Freiberufler nahe. Die Unterstützung der Haus- und Grundeigentümer und der Spitzenorganisationen der Wirtschaft mußten sich CDU/CSU und FDP teilen. Die SPD arbeitete besonders eng mit der Mehrzahl der Gemeinnützigen zusammen. darüber hinaus fand sie typischerweise 
Unterstützung bei den großen Städten, beim Siedlungsverband Ruhrkohlenbezirk, beim Mieterbund und beim Deutschen Gewerkschaftsbund. (Schulz, 1994, 209)

Wie bereits in Abschnitt 5.1 beschrieben, können die Unionsparteien als die stärksten Fürsprecher der Wohneigentumsförderung in der ersten Phase angesehen werden (vgl. Leutner, 1990, 28, 32). Geprägt von einer hohen „Wertschätzung des Eigenheims“(Mayer, 1998, 180), verfolgten sie in der direkten Nachkriegszeit eine Politik, in der das Wohneigentum im Zentrum staatlicher Wohnungspolitik stehen sollte (vgl. Eichener u. a., 2000, 69f.). Bereits in ihren „Kölner Leitsätzen“, dem ersten vorläufigen Programmentwurf der in der Nachkriegszeit neu gegründeten CDU von 1945 (vgl. Lill, 2005, 159f.), formulierte die CDU die Forderung: „Die Großstädte werden durch Anlage gesunder Aussensiedlungen aufgelockert. Das Eigenheim wird gefördert" (Christliche Demokraten Kölns 1945; siehe hierzu auch Hilpert 2012, 277). Dabei sollte Die Familie „die Grundlage der sozialen Lebensordnung“ bilden, denn ,[i]hr Lebensraum ist heilig" (Christliche Demokraten Kölns, 1945). Die Grundsätze dieses ersten und in der weiteren Geschichte der CDU bedeutenden Programmes, welches für ,die Gründung und geistige Orientierung weiterer christlicher Parteien in Westdeutschland wegweisend" sein würde (Uertz, 1981, 9), wurden maßgeblich von den an der Verfassung beteiligten Dominikanern geprägt (Lill, 2005, 160). In die Programmatik der jungen Partei flossen somit nicht nur über die Politiker selber christlich-katholische Wertvorstellungen mit ein, sondern wurden gemeinsam von Politikern und Vertretern der katholischen Kirche verfasst, was eine tiefe Verwurzelung der Partei im katholischen Milieu widerspiegelt (vgl. Lill, 2005). Zwar wurden in dem von Adenauer 
entworfenen späteren CDU-Programm einige Aspekte der katholischen Soziallehre in den Kölner Leitsätzen verworfen (Uertz, 1981, 12); die Eigenheim-Idee blieb dabei jedoch nicht nur bestehen, sondern wurde noch weiter gestärkt, da dem Privateigentum eine zunehmende Bedeutung zugeschrieben wurde (vgl. Uertz, 1981, 77).

Zu den Düsseldorfer Leitsätzen vom 15. Juli 1949, dem ersten Bundestagswahlprogramm von CDU und CSU, gehörte angesichts der immensen bestehenden Wohnungsnot ein eigenes Wohnungsbauprogramm, in dem deutlich wird, dass das Ideal des Einfamilienhauses und der Kleinsiedlung besteht, jedoch angesichts der herrschenden Wohnungsnot vorerst mit möglichst geringem Mitteleinsatz eine möglichst große Anzahl an Wohnungen geschaffen werden sollten:

„Bei der Notwendigkeit, eine möglichst große Zahl von Wohnungen mit dem geringsten Aufwand von Kapital und Baustoffen zu erstellen, fordert die CDU/CSU, daß beim Neubau wie auch beim Wiederaufbau von Dauerwohnungen in erster Linie Kleinwohnungen mit sozial tragbaren Mieten für breite Bevölkerungsschichten gebaut werden. Zur stärkeren Verwurzelung der Bevölkerung, insbesondere der Vertriebenen, mit dem Grund und Boden, ist einer wirtschaftlichen Form des Kleinhauses - Einfamilienhaus und Kleinsiedlung - überall dort der Vorzug zu geben, wo nicht aus städtebaulichen Gründen mehrgeschossige Mietwohnungen gefördert werden müssen. Eine Eingliederung der Vertriebenen in den Wirtschaftsprozeß ist vor allem in mittleren und kleineren Gemeinden durch planmäßigen Ausbau von Arbeits- und Wohnstätten zu fördern.“ (CDU/CSU, 1949) 
Dies bedeutet jedoch nicht, dass das Ideal der Eigenheimförderung aus den Zielsetzungen der Partei verschwunden war. So startete die Partei 1950 eine groß angelegte ,gesetzgeberische, publizistische und personalpolitische Offensive“ in enger Zusammenarbeit mit ,führende[n] Vertreter[n] der Wohnungswirtschaft aus dem gesamten Bundesgebiet", die in der Gründung der Arbeitsgemeinschaft ${ }^{19}$ für christlich-soziale Wohnungspolitik am 18. November 1950 in Essen mündete (Schulz, 1994, 264). Diese Entwicklung ging einher mit der Eigenheiminitiative der katholischen Kirche und verstand sich ,als dritter Weg zwischen der "sozialistischen Gedankenwelt" einerseits, die sie im Genossenschaftshaus, Gemeinschaftseigentum und in Wohnblocks verwirklicht sah, und der wirtschaftspolitischen Orientierung des Wohnungsbauministeriums andererseits, die schließlich auch Großbaustellen und Wohnblocks hervorbringe" (Schulz, 1994, 257).

Entscheidende Akteure waren in diesem Zusammenhang die kirchlichen Siedlungsdienste - allen voran der katholische Siedlungsdienst. Dieser fungierte seit seiner Gründung durch alle 27 deutschen Bistümer $1930^{20}$ als ,,Spitzenverband kirchlich gebundener und kirchlich organisierter Wohnungs- und Städtebauunternehmen“ (Egner u. Georgakis, 2004, 174), die ihr Handeln auch heute noch als ,praktische Ausformung der katholischen Soziallehre" verstehen (Egner u. Georgakis,

19 Die Arbeitsgemeinschaft ,sollte die Plattform für die enge Zusammenarbeit der christlich-sozialen Politiker in Bund und Ländern sein, um die Gesetzgebung zu beeinflußen und um die Tätigkeit christlich-demokratischer Vertreter in den Führungsorganen der Gemeinnützigen Wohnungswirtschaft abzustimmen" (Schulz, 1994, 264).

20 Der Verband wurde 1947 nach seiner Auflösung 1941 wiedergegründet (Egner u. Georgakis, 2004, 174). 
2004, 174). Weitere Mitglieder sind überregionale katholische Einrichtungen wie die Caritas, das Zentralkomitee der Katholiken sowie der Familienbund. Ähnlich wie die katholischen Siedlungsdienste bestanden auch zwischen dem Volksheimstättenwerk und dem späteren Wohnungsbauminister Lücke enge Verbindungen (Schulz, 1994, 203). In diesem Bereich ist auch der Deutsche Siedlerbund zu verorten, „der sich für die Verbreitung der Kleinsiedlung einsetzte“ (Schulz, 1994, 209).

Die Siedlungsdienste waren zwar insgesamt kein herausragender Faktor in der damaligen Bau- und Wohnungswirtschaft ${ }^{21}$, ,hatten [jedoch] großen politischen Einfluß, weil sie engagierte Anhänger in der CDU/CSU fanden" (Schulz, 1994, 257). Die gelungene Einflussnahme der kirchlichen Siedlungsdienste wird auch in den Bundestagsdebatten deutlich, wo sie von Mitgliedern der Unionsparteien positiv hervorgehoben werden, obwohl sie weder zu den bedeutenderen Bereitstellern von Wohnraum noch zu jenen Akteuren gehörten, die sich wie der Gesamtverband der gemeinnützigen Wohnungsunternehmen durch eine Eingabe beim Ausschuß für Wohnungsbau und dem Ausschuß für Bau- und Bodenrecht $^{22}$ positionierten:

„Ich darf hier darauf hinweisen, daß gerade die Siedlungsdienste der beiden Kirchen - der Evangelischen Kirche und der Katholischen Kirche - in den vergangenen Jahren solche verlorenen Zuschüsse in Millionenbeträgen eingesetzt haben und damit der Wohnungsversorgung der einkommensschwa-

21 „Von 1945 bis Anfang 1950 errichteten die 20 Siedlungsdienste weniger als 5.000 Wohnungen, vornehmlich in Selbsthilfe“ (Schulz, 1994, 257).

22 An der Stelle der Siedlungsdienste leisteten die mit ihnen assoziierten Familienverbände Eingaben an die entsprechenden Ausschüsse (vgl. BT-Drs. 02/142, $7450 \mathrm{~B})$. 
chen Familien in großem Umfang gedient haben" (Abg. Hesberg, BT-Drs. 02/142, 7427A)

Die zentralen eigenheimpolitischen Vorstellungen der katholischen Verbände lassen sich am besten innerhalb des sogenannten Altenberger Programms identifizieren, das ,als Ergebnis einer Fachtagung 1951 die Grundzüge der Eigenheimförderung festlegte (Clemens, 1994, 14). Darin heißt es: „Der Staat solle [1.] das private individuelle Eigentum fördern, nicht das "Kollektiveigentum" der großen Wohnungsunternehmen. Und er solle [2.] große, familiengerechte Wohnungen fördern, keine Kleinwohnungen. Beide Forderungen erfülle nur das Eigenheim. Insofern stelle das I. Wohnungsbaugesetz die Weichen falsch" (Schulz, 1994, 265). Die groß angelegte Schaffung von Eigenheimen sollte auch nach der Vorstellung Konrad Adenauers ,als sozial wertvollster und am meisten förderungswürdiger Zweck staatlicher Wohnungsbau- und Familienpolitik angesehen werden“ (Clemens, 1994, 15). Schließlich gewannen die katholischen Vereinigungen mit Paul Lücke ${ }^{23}$ einen Vertreter der CDU für ihre Eigenheimkampagne, der ab 1950 zunächst in der Position des Vorsitzenden des Ausschusses für Wiederaufbau und Wohnungswesen und von 1957 bis 1965 als Wohnungsbauminister die Rolle als „Eigenheimapostel [...], wie ihn der "Spiegel" 1958 in einer Titelgeschichte bezeichnete“" innehatte (Schulz, 1994, 258).

Für den Aspekt der Eigenheimförderung erscheint ebenso das Deutsche Volksheimstättenwerk von Interesse. Der 1946 gegründete Verband

23 „Er war streng katholisch, als eines von 14 Kindern im kargen oberbergischen Dörfchen Schönborn aufgewachsen, wo sein Vater Steinbruchmeister war. Er war Jugendführer beim katholischen Jungmännerverband und in der Deutscher Pfadfinderschaft Sankt Georg“ (Schulz, 1994, 258f.). 
ist der Nachfolger der ,Vorkriegsorganisationen Bund Deutscher Bodenreformer und Deutscher Verein für Wohnungsreform" (Egner u. Georgakis, 2004, 172) und trat maßgeblich für die ,Schaffung von preiswerten Eigenheimen in hoher Zahl und eine Bodenreform" ein (Egner u. Georgakis, 2004, 172). Zudem wurde der Hauptgeschäftsführer des Deutschen Volksheimstättenwerks Simon auch von Minister Lücke als Mitglied des Sachverständigenbeirates für die Gemeinnützigkeitsreform und das Wohnungswirtschaftsgesetz berufen, der ,[z]ur Beratung des Ministeriums bei der Neugestaltung des Gemeinnützigkeitsrechts und bei der Ausarbeitung eines umfassenden Wohnungswirtschaftsgesetzes" dienen sollte (Bundesministerium für Wohnungwesen und Städtebau, $1967, \mathrm{X}) .{ }^{24}$ Sowohl die kirchlichen Verbände wie auch die Volksheimstätten beteiligten sich durch Eingaben im beratenden Ausschuss für Bau- und Bodenrecht (BT-Drs. 02/142, 7450B). Auch der Zentralverband der deutschen Haus- und Grundbesitzer ist in diesem Zusammenhang zu nennen, der wie der Mieterbund bereits seit dem Kaiserreich existierte und nach dem Zweiten Weltkrieg neu gegründet wurde und traditionell für eine starke Eigenheimförderung plädiert (Egner, 2004b, $162)$.

Gegenüber den christlichen Parteien sah die SPD eine straffe staatliche Lenkung der Wohnungspolitik als zentrale staatliche Aufgabe an (Leutner, 1990, 28). Als wichtigster Partner ${ }^{25}$ der SPD können im Be-

24 Der Sachverständigenbeirat tagte mehrmals und erstellte zwischen 1962 und 1963 eine Reihe an Expertisen, die jedoch erst später auch der Öffentlichkeit zugänglich gemacht wurden (Bundesministerium für Wohnungwesen und Städtebau, 1967).

25 Schulz bezeichnet die SPD in diesem Zusammenhang sogar als ,'parlamentarische[n] Arm' der Gemeinnützigen und des DGB“ (Schulz, 1994, 170) 
reich der Wohnungspolitik die gemeinnützigen Wohnungsunternehmen sowie der Deutsche Gewerkschaftsbund (DGB) angesehen werden, die gemeinsam eine Intensivierung des Gemeinnützigen Mietwohnungsbaus sowie die Niedrighaltung des Mietniveaus forderten (Schulz, 1994, 170). Auch der 1947 gegründete Gesamtverband gemeinnütziger Wohnungsunternehmen (heute GdW - Bundesverband deutscher Wohnungsunternehmen), bei dem die öffentlichen Wohnungsunternehmen organisiert sind (Egner u. Georgakis, 2004, 166), prägte maßgeblich die Positionen der SPD und beteiligte sich auch über eigene Eingaben beim beratenden Ausschuss für Bau- und Bodenrecht an den Debatten zum Zweiten Wohnungsbaugesetz (BT-Drs. 02/142, 7450B) und gab selber wissenschaftliche Gutachten in Auftrag (vgl. Ifo-Institut für Wirtschaftsforschung, 1960). Gegenüber den Vertretern von Gewerkschaften erscheint der Mieterbund - damals Zentralverband Deutscher Mieter - nicht explizit in den Debatten um das Zweite Wohnungsbaugesetz. Allerdings zeichnet sich der 1951 in seiner heutigen Form gegründete Verein aufgrund seiner Mitgliederstruktur durch eine zunehmende Nähe zu Gewerkschaften aus und gilt bis heute als eng verknüpft mit der SPD (Egner, 2004b, 157f.). Erst in den 60er Jahren scheint dieser Verband auch zu der Bedeutung der Gewerkschaften als eigenständiger Akteur aufgeschlossen zu haben und wird in den Debatten explizit benannt.

Dabei kam den deutschen Gewerkschaften vor allem in Form des $\mathrm{DGB}^{26}$ eine gesonderte Stellung zu. So war er einerseits an hohen Bauarbeiterlöhnen interessiert, was die Baukosten verteuerte und forderte

26 Neben dem DBG zeigt sich im Rahmen der Akteursrecherchen auch die Industriegewerkschaft Bau, Steine und Erden (IG Bau. IG BSE) innerhalb der ersten Debatten rund um die Eigenheimförderung (vgl. Schulz 1994, 206; Egner u. Georgakis 2004, 185). 
zugleich niedrige Mieten für die von ihnen vertretenen Arbeitnehmer (Schulz, 1994, 209). Andererseits traten sie jedoch auch selber als Vermietergesellschaft im Rahmen des Unternehmens „Neue Heimat“ auf. Ihre Gründung geht auf die Enteignung der gewerkschaftlichen Wohnungsunternehmen während des Nationalsozialismus zurück, wonach die Benennung in „Neue Heimat" 1939 erfolgte. 1952 wurde das Unternehmen von den Alliierten an den DGB übergeben und sollte in den Folgejahren zum größten Wohnungsbaukonzern Europas werden (Kunz, 2003, 31). Der „Zusammenschluß einer Vielzahl kleiner, in der Weimarer Republik gegründeter Wohnungsbaugesellschaften“ (Kramper, 2008, 12) gewann als das größte Wohnungsbauunternehmen Europas und eines der wichtigsten Unternehmen der Nachkriegszeit, das „etwa eine halbe Million Wohnungen gebaut [hatte], vor allem Sozialwohnungen" (Kunz, 2003, 31) nach und nach großen Einfluss auf die Sozialpolitik in Deutschland (Kramper, 2008, 12). Das politische Spannungsfeld, in dem sich die Neue Heimat dabei befand, beschreibt Kramper wie folgt:

„Zum einen war sie der mit Abstand größte Vertreter der gemeinnützigen Wohnungswirtschaft, die durch das [...] Wohngemeinnützigkeitsgesetz (WGG) eine Reihe von Steuerbefreiungen erhielten. [...] Zum anderen war die Neue Heimat aber auch [...] das Flaggschiff der gewerkschaftlichen Gemeinwirtschaft, einer Gruppe von Unternehmen, deren Anteilseigner der Deutsche Gewerkschaftsbund (DGB) und die in ihm zusammengeschlossenen Einzelgewerkschaften waren." (Kramper, 2008, 13f.) 
Somit waren für die Neue Heimat deutlich stärker als für andere Unternehmen ,politische Diskurse und kulturelle Normen ein integraler Bestandteil der Unternehmenspolitik“ (Kramper, 2008, 33). Insbesondere in der wohnungspolitischen Konzeptionierung der SPD in der sozialliberalen Koalition sollten DGB, Mieterbund und Neue Heimat eine wichtige Schlüsselposition einnehmen (siehe hierzu Abschnitt 6.3.2).

Gegner der zunehmenden Deregulierungsbestrebungen der Bundesregierung auf Seiten der SPD waren bereits qua Eigeninteresse die öffentlichen Wohnungsunternehmen. Der Konflikt um die Förderung gemeinnütziger Wohnungsunternehmen war an dieser Stelle keineswegs neu. Bereits 1954 hatte Lücke im Bundestag erklärt, Steuergelder sollten nicht dafür verwendet werden dürfen, „um Rieseneigentum in den Händen einiger weniger Wohnungsunternehmen zu schaffen" (Schulz, 1994, 290). Diese Zielsetzung wurde somit einen auch in den kommenden Jahren immer wiederkehrenden Konflikt vor allem mit der SPD, welche den Mietwohnungsbau - insbesondere durch die Gemeinnützigen - zumindest gleichberechtigt neben der Schaffung von Einfamilienhäusern etablieren wollte. Um die eigenen Positionen zu bekräftigen und die Deregulierungspläne des späteren Wohnungsbauministers Lücke zu bekämpfen, erstellten Mieterbund, DGB und Neue Heimat Gegendarstellungen zu den Defizitzahlen auf dem Mietwohnungsmarkt. Diese sollten belegen, dass der Wohnungsmarkt anders als in den Darstellungen des Statistischen Bundesamtes mitnichten im Gleichgewicht war, sondern noch ein deutlicher Mangel an Wohnraum bestand (vgl. BT-Drs. 04/136, 8051B). Somit können sowohl die Mieterbund als auch DGB als wesentliche Wissensproduzenten in diesem Zeitraum in Konkurrenz zur amtlichen Statistik gesehen werden. Welche Bedeutung diesen Ge- 
gendarstellungen beigemessen werden muss, zeigt sich in der entsprechenden Entrüstungen auf der Gegenseite. So wurde dem Mieterbund vonseiten eines CDU-Abgeordneten gemeinsam mit dem DGB und der IG-Metall vorgeworfen ,Angriffe auf den Familienminister, auf das Ministerium und auf die Koalition zu führen“ (BT-Drs. 04/136, 8027B).

Entgegen der klassischen Darstellung von zwei politischen Lagern, die einander bekämpften, wurde die Förderung des Eigenheims in den politischen Debatten der direkten Nachkriegszeit allerdings von nahezu allen Parteien überaus positiv bewertet. So findet sich auch in einem Wohnungsbauprogrammentwurf der SPD von 1946 die Forderung, dass an Stelle des Miet- und Mehrfamilienhauses künftig das Ein- und Wenigfamilienhaus zu bevorzugen sei (Hilpert 2012, 277; Leutner 1990, 28f.). Schulz stellt trotz des eigenheimpolitischen Übergewichtes der Unionsparteien in seiner Untersuchung fest, dass „die Wohnungspolitik in den frühen fünfziger Jahren nicht nur das Werk der Regierungsparteien war“ und ,die Parteien [vielmehr] auf diesem Feld so eng kooperierten, daß man von einer begrenzten informellen großen Koalition sprechen kann“ (Schulz, 1994, 324). So wurde die Förderung von Eigenheimen von beiden Parteien bereits bei den Wahlkampagnen zum ersten Wahl zum Deutschen Bundestag gefordert (vgl. Abbildung 5.1). Die CDU/CSU-Fraktion hatte bereits 1952 auf Initiative von Paul Lücke einen Entwurf für ein Gesetz ,zur Schaffung von Familienheimen“ vorgelegt, das in der darauf folgenden Legislaturperiode zur Verhandlung kam (Hilpert, 2012, 284) und auch die SPD brachte 1954 einen eigenen Entwurf für das Zweite Wohnungsbaugesetz ein (BT-Drs. 02/722), das vor allem den Wohnungsbau für die Bevölkerung mit niedrigem Einkommen fördern sollte. In den frühen Bundestagswahlkämpfen sah 
auch die SPD die Eigenheimförderung als einen Beitrag zur Förderung der Subsistenzwirtschaft, welche die allgemeine Versorgungslage sicherstellen sollte.

Abbildung 5.1: Förderung von Eigenheimen auf Wahlplakaten zur Bundestagswahl von 1949 von CDU und SPD.

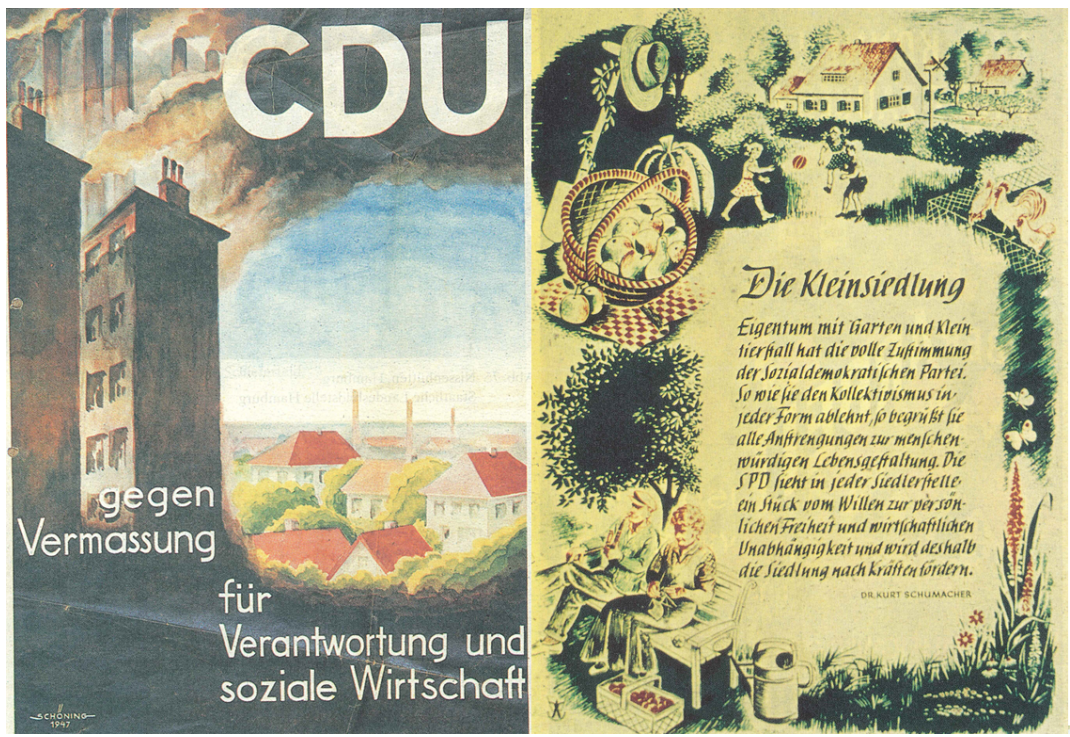

Quelle: Schulz (1994)

Das Zweite Wohnungsbaugesetz gilt zwar als ,legislatorische Umsetzung der Familien- und Eigenheimvorstellungen der Union" (Schulz, 1994, 288), muss jedoch als eine Mischung der wohnungspolitischen Ziele mehrerer Parteien angesehen werden. Während die CDU/CSU den Vorrang des Familienheims durchsetzte, konnte die SPD die Priorität für die unteren Einkommensbezieher im Gesetz verankern und die FDP setzte in Teilen eine weitere Deregulierung des Wohnungsmarktes durch (Schulz, 1994, 310). 


\subsubsection{Theologie statt Wirtschaftswissenschaften}

Wie bereits in Abschnitt 3.1.3 geschildert, wird die erste Phase der Politikberatung in der jungen Bundesrepublik durch eine zunehmenden Verwissenschaftlichung der Politik beschrieben (Weingart, 2006, 42). Gegenüber den in der Literatur vielfach beachteten Beratungsprozessen im Bereich der Wirtschaftspolitik hat die wirtschaftswissenschaftliche Politikberatung im Bereich der Wohnungspolitik in der ersten Phase nur eine geringe Rolle gespielt. Dies wird vor allem deutlich bei einem Vergleich von Zusammensetzung und Arbeitsweise des wissenschaftlichen Beirates beim Bundesministerium für Wirtschaft (vgl. Abschnitt 3.1.3) mit dem sogenannten wohnungswirtschaftlichen Beirat, der ursprünglich die Bezeichnung ,Wohnungspolitischer Sachverständigenbeirat" tragen sollte (Schulz, 1994, 210). Dieser Beirat erstellte Gutachten für das Bundeswohnungsbauministerium, aber auch für den Bundestagsausschuss für Wiederaufbau und Wohnungswesen (Wilhelm, 1968, 48). Mitglieder waren ,führende Vertreter des Wohnungsbaus und der Wohnungswirtschaft [aber nur] in geringer Zahl der Wissenschaft", was einen deutlichen Unterschied zum wissenschaftlichen Beirat beim Bundesministerium für Wirtschaft ausmachte, dem ,ausschließ̧lich Hochschullehrer der Wirtschafts- und Sozialwissenschaften" angehörten (Schulz, 1994, 210). Der durch Vertreter der Ministerialbürokratie berufene Beirat setzte sich aus einer Vielfalt von Vertretern aus „Theorie und Praxis“ zusammen (vgl. Nell-Breuning, 1959, 35f.): Von neunzehn Gründungsmitgliedern waren ,lediglich vier Inhaber von Lehrstühlen der Wirtschafts- und Sozialwissenschaften. Von den zehn Mitgliedern, die bis 1962 den Beirat ergänzten [...] waren [nur] zwei Lehrstuhlinhaber der genannten Wissenschaften“ (Wilhelm, 1968, 47). 
Demgegenüber dominierten die „Praktiker“27 die Beiratstätigkeit, was an dem Vorsitzenden des Beirates Arnold Knoblauch deutlich wird. Dieser war während des gesamten Tätigkeitszeitraums des Beirates der Erste Vorsitzende (Wilhelm, 1968, 44) und war bereits seit mehreren Jahren Generaldirektor der gemeinnützigen Gagfah (Lücke, 1959, 5). In dieser Funktion hatte Knoblauch bereits in den 30er Jahren über seine Vorschläge zur Reform des Bausparwesens politisch Einfluss genommen (Schloder, 1959, 83). Auch die übrigen Mitglieder und damit die Mehrzahl waren meist ,Sachkenner aus den Verbänden, Unternehmen und der öffentlichen Verwaltung“ (Wilhelm, 1968, 47). An dieser Stelle kann somit herausgestellt werden, dass die Praxis der Beiratsberufung kein Spezifikum der Wohnungspolitik in dieser frühen Phase der Bundesrepublik darstellt, sondern vielmehr einem Trend entspricht, dem auch weitere Ministerien folgten.

Inhaltlich verfolgte der Wohnungswirtschaftliche Beirat starke Deregulierungsvorstellungen, ,deren Ziel radikal war - der Wohnungsbau solle von öffentlichen Subventionen völlig unabhängig werden -, doch er empfahl moderate Schritte zu ihrer Realisierung“" (Schulz, 1994, 210). Damit entsprechen seine Empfehlungen in weiten Teilen vollständig den Auffassungen, die auch der Wissenschaftliche Beirat beim Bundeswirtschaftsministerium bereits 1950 in seiner Stellungnahme festhielt: „Auf lange Sicht sollte auch die Wohnungswirtschaft marktwirtschaft-

27 Allerdings setzte sich der Beirat aus solchen Praktikern zusammen, die „mit starkem Übergewicht wissenschaftlich hochgebildet [...] [waren und] deren einige auch Lehraufträge am wissenschaftlichen Hochschulen ausübten oder nochmals zu Honorarprofessuren berufen wurden" (Nell-Breuning, 1959, 35f.). Von einer mangelnden Präsenz wirtschafts- und sozialwissenschaftlicher Expertise kann also auch innerhalb dieses Praktikergremiums keine Rede sein. 
lich geordnet werden. Dieses Ziel darf nicht aus dem Auge gelassen, kann aber nur schrittweise erreicht werden." (Wissenschaftlicher Beirat beim Bundesministerium für Wirtschaft, 1950, 89) Dies erscheint wenig verwunderlich, da innerhalb des Beirates große personelle Überschneidungen mit dem wirtschaftswissenschaftlichen Beirat beim Bundesministerium für Wirtschaft bestanden. Das wohl entscheidendste Gutachten des wohnungswirtschaftlichen Beirates für die Fragestellung der vorliegenden Arbeit ist das 1952 erschienene Gutachten zur Frage der familiengerechten Wohnungen vom 17. Dezember 1952, das zwei Jahre später vom Bundesministerium veröffentlicht wurde (Wohnungswirtschaftlicher Beirat, 1954). In diesem Gutachten orientierte sich der Beirat nicht an Idealvorstellungen, wie Wilhelm feststellt, ,sondern [zeigt] lediglich Mindestanforderungen für eine Familienwohnung auf" (Wilhelm, 1968, 137). Wohnungspolitik sollte demnach so gestaltet werden, „daß die wohnungssuchenden Familien selbst frei unter den verschiedenen Wohn- und Rechtsformen wählen könnten“ (Schulz, 1994, 211). Den Empfehlungen des Beirates in diesem Gutachten folgte die Politik nach der Analyse von Wilhelm weitestgehend (Wilhelm, 1968, 164). Diese betonte Neutralität des Gutachtens lässt sich jedoch auch als ein Ergebnis beiratsinterner Uneinigkeiten interpretieren. So führt der spätere Vorsitzende des Gremiums Nell-Breuning an, dass es bei einigen vorherigen Gutachten bereits schwierig gewesen sei, eine gemeinsame Stellungnahme zu formulieren, allerdings ,fehlte für die Behandlung dieses auch in der öffentlichen Meinung so heiß umstrittenen Themas geradezu die gemeinsame Sprache, deren es bedarf, um sich auch nur untereinander zu verstehen“" (Nell-Breuning, 1959, 39). Er kommt jedoch zu dem Schluss, dass ,ein wohnungswirtschaftlicher Beirat nicht 
Ideale zu propagieren, sondern Realitäten zu analysieren und Lösungen anzugeben hat, die nicht bei besonderer Gunst der Verhältnisse im Einzelfall, sondern aufs Ganze gesehen praktikabel sind und zum Ziele führen" (Nell-Breuning, 1959, 40). Größere Einigkeit bestand demgegenüber anscheinend hinsichtlich der notwendigen Schaffung von Sparanreizen. Insbesondere sparfördernde Maßnahmen wie das Bausparen wurden vonseiten des Wohnungswirtschaftlichen Beirates entsprechend den Empfehlungen des Wissenschaftlichen Beirats beim Bundeswirtschaftsministerium dringend angeraten (vgl. Wissenschaftlicher Beirat beim Bundesministerium für Wirtschaft, 1950, 93). Überschneidungen der inhaltlichen Ausrichtungen beider Beiräte dürften jedoch weniger verwundern, wenn man beachtet, dass einige der Theoretiker des Wohnungswirtschaftlichen Beirates zeitgleich Mitglieder des Wissenschaftlichen Beirates beim Bundesministerium für Wirtschaft waren (vgl. NellBreuning, 1959, 37).

Als Nachfolgebeirat des Wohnungswirtschaftlichen Beirates konstituierte sich entsprechend der geänderten Ministeriumsstruktur 1962 der Beirat für Städtebau und Raumordnung, der abermals ,,gemischtes Gremium aus Praktikern und Wissenschaftlern“ bildete (Wilhelm, 1968, 49). Dieser befasste sich jedoch, wie der Name bereits vermuten lässt, vermehrt mit Fragen der Raumordnung, in denen die Eigenheimförderung - wenn überhaupt - nur am Rande thematisiert wurde. Gleiches gilt für die 1968 tagende Gutachterkommission „Wohnungsbau in der Marktwirtschaft", in der ein Wissenschaftler zehn Verbandsvertretern und vier Sachverständigen aus der Ministerialbürokratie gegenüberstand (BT-Drs. V/4585, 37).

Über den Wohnungswirtschaftlichen Beirat hinaus wurden inner- 
halb der ersten Phase kaum wissenschaftliche Gutachten in Auftrag gegeben. Allerdings wurden einzelne Gutachten vonseiten des Ministeriums aus der eigentlichen Beiratstätigkeit ausgegliedert und von einzelnen Mitgliedern übernommen, ,die einen entsprechenden Mitgliederstab einsetzen konnten“ (Maurach, 1963, 160). Zu diesen Berichten zählen auch die Arbeiten von Seraphim, seit 1948 Professor in Münster, auf dessen Initiative hin an der Universität zwei neue Institute entstanden waren - ,für Genossenschaftswesen und für Wohnungs- und Siedlungsfragen“" (Maurach, 1963, 160). Insbesondere das Institut für Wohnungsund Siedlungsfragen sollte auch nach Seraphims plötzlichem Tod 1962 weiterhin Expertisen zu aktuellen wohnungspolitischen Themen veröffentlichen und Tagungen veranstalteten (so Schneider 1964a; Schneider 1964b). Zwar galt er im Nachkriegsdeutschland als anerkannter Experte für wohnungspolitische Themen, hatte sich jedoch wie die anderen Ökonomen auch bis dahin nur am Rande mit dem Themengebiet auseinandergesetzt. Vor dem Ende des Zweiten Weltkriegs hatte er sich vor allem mit Osteuropastudien auseinandergesetzt und gilt als einer der typischen Vertreter der völkischen Wirtschaftslehre zu Zeiten des Nationalsozialismus (Janssen, 2012, 616). Unter der Herausgeberschaft von Seraphim erschien das Buch „Das Eigenheim. Eine soziologische und volkswirtschaftliche Analyse" (Seraphim 1955; Michels 2004). Dieses Gutachten geht jedoch über die bis dato vorherrschende Neutralitätsanforderung in der Wahlfreiheit der Wohnform vonseiten des Beirates hinaus und kommt zu dem folgenden Schluss:

„Die ökonomische Beweisführung hat die Vorteile der Eigenheimförderung klar zutage treten lassen. Ihr Wert ist im Verein mit der ungleich gewichtigeren soziologischen und 
sozialpolitischen Bedeutung des Eigenheimes umfassender und nachhaltiger als dies mit einer noch so exakten quantitativen Analyse auszudrücken ist." (Seraphim, 1955, 223224)

Zwar wird innerhalb des Gutachtens auch davor gewarnt, ,in dem Eigenheim ein soziales Allheilmittel zu sehen“, allerdings „dürfte doch für breitere Schichten der Arbeitnehmerschaft die Eigenheimbildung das erfolgversprechendste Mittel sein, die Peersönlichkeitsbildung zu fördern, die wirtschaftliche Unabhängigkeit und damit gleichzeitig die soziale Sicherheit zu stärken und darüber hinaus den Familien, insbesondere den aufsteigenden, Daseinsbedingungen zu bieten, die ihren Bestand und ihre Entfaltung begünstigen“ (Seraphim, 1955, 39). Zudem sollte das „Einfamilienhaus mit Garten [...] der Vermassung und Kollektivierung besonders entgegenwirk[en]" (Seraphim, 1955, 39).

Auch eine Fachtagung von Vertretern wohnungs- und kreditwirtschaftlicher Verbände wie auch von Wissenschaftlern am bereits erwähnten Institut für Siedlungs- und Wohnungswesen in Münster kommt im Wesentlichen zu dem Schluss, dass ,es in einer Sozialen Marktwirtschaft ,nicht die Aufgabe des Staates sein [könne], für die Wohnraumversorgung der Staatsbürger aufzukommen“ (Schneider, 1964a, 105).

Neben dem Institut für Wohnungs- und Siedlungsfragen an der Universität Münster berief sich auch ein zweites neues Institut mit Arnold Knoblauch auf ein Mitglied des wohnungswirtschaftlichen Beirates. Das Institut für Städtebau, Wohnungswirtschaft und Bausparwesen, auch Arnold-Knoblauch-Institut genannt, wurde jedoch nicht als Institut einer Universität, sondern als eingetragener Verein gegründet, der maßgeblich ,die Interessen der privaten Bausparkassen publizistisch ver- 
tritt" und mit mehreren Abgeordneten der CDU eng verbunden war (Chabrowski, 1963). Eine eigene wissenschaftliche Tätigkeit über das Kommentieren anderer Forschungsergebnisse hinaus wurde dem Institut von Anfang an abgesprochen (Chabrowski, 1963), sodass das Institut als ein Think Tank und nicht als ein wissenschaftliches Forschungsinstitut angesehen werden sollte. Dennoch gelang es dem Institut mit der Zeit an Renommee zu gewinnen und gemeinsam mit dem Bundesministerium für Wohnungswesen und Städtebau eine Broschüre über das Wohnungseigentum herauszugeben (Institut für Städtebau, Wohnungswirtschaft und Bausparwesen $u$. Bundesministerium für Wohnungwesen und Städtebau, 1966). Weitere Publikationen des Institutes erscheinen zudem in einer gesonderten Schriftenreihe.

Im Zentrum eines Gutachtens des Arnold-Knoblauch-Institutes stand unter dem seit einem Jahr amtierenden FDP-Minister Bucher die Frage nach den Förderungsmöglichkeiten für Eigentum an Geschosswohnungen in städtischen Zentren. Dem Minister erschien es notwendig durch das Gutachten „die Bevölkerung über die Eigentumswohnung besser [zu] informier[en] und [...] sich vor allem die unternehmerische Wohnungswirtschaft der Entwicklung guter Wohnungstypen noch stärker annimmt als bisher" (Institut für Städtebau, Wohnungswirtschaft und Bausparwesen u. Bundesministerium für Wohnungwesen und Städtebau, 1966, 3).

Diese sich herausbildende Praxis gutachterliche Tätigkeit, die sich in der zweiten Phase wissenschaftlicher Politikberatung deutlich intensivieren sollte (vgl. Abschnitt 6.3.2), kann jedoch nicht alleine auf die Beschaffung der gewünschten Information der Bürgerinnen und Bürger beschränkt gesehen werden. So wurden vor allem in den 60er Jahren 
vonseiten des federführenden Ministeriums Gutachten in Auftrag gegeben, in denen sich Ingenieure wie Architekten mit der möglichst wirtschaftlichen Planung auseinandersetzen (so Triebel u.a., 1965) oder auch Meinungsumfragen durchführten. Somit erweist sich das konsultierte Expertenspektrum als überaus breit gegenüber dem Beratungsangebot im Rahmen der allgemeinen Wirtschaftspolitik.

Das breite Spektrum der in den politischen Debatten zurate gezogenen Experten wird in der ersten Phase wissenschaftlicher Politikberatung besonders deutlich an der Einbeziehung von Theologen. Diese erfolgte maßgeblich durch Vertreter der christlichen Parteien und bildete die inhaltliche Grundlage für das breite Bündnis für eine stärkere Förderung des Familienheimes in der ersten Phase bundesdeutscher Wohnungspolitik. Es zeigt sich dabei, dass ein christliches Weltbild alleine noch keine Erklärung für die Befürwortung des Eigenheimgedankens in moderner Leseart bietet - geschweige denn bietet dieser die ausformulierten policy-Ideen als Grundlage für die Förderung von Wohneigentum. Diese musste zunächst aus den christlichen Lehren heraus interpretiert werden. Dies wurde maßgeblich durch Experten auf dem Gebiet der christlichen Theologie geleistet, die vonseiten der Politik zurate gezogen wurden. Dazu zählte der Dominikaner und Sozialethiker Eberhard Welty, der mit seiner Schrift über christlichen Sozialismus (Welty, 1946) als ein Experte für den christlichen Sozialismusbegriff galt, und im weiteren Verlauf eine wichtige Figur im Sozialausschuss stellen sollte (vgl. Uertz, 1981, 92ff.).

Ebenso ist der Theologe und Nationalökonom Oswald v. Nell-Breuning zu nennen, der sich als Berater von Papst Pius XI. und als Hauptautor der christlichen Sozialenzyklika quadragesimo anno (Nach vierzig 
Jahren) etabliert hatte, sich im Rahen der katholischen Arbeitnehmerbewegung mit gewerkschaftlichen Fragen befasste und zahlreiche persönliche Kontakte zum engeren Kreis der CDU pflegte (vgl. Fußnote 12 Uertz, 1981, 92). Er studierte nach seiner Weihung zum Priester Volkswirtschaftslehre und promovierte 1928 über die „Grundzüge der Börsenmoral" (Janssen, 2012, 595). Dabei bekannte er selber stets, dass er ,in Nationalökonomie [bloß] eine einzige Grundvorlesung“" gehört habe (Klein, 1989, 33). Als wirtschaftspolitisch einflussreich kann seine Arbeit innerhalb Deutschlands erst seit dem Ende des Zweiten Weltkrieges gelten, da ihm von 1936 bis zum Kriegsende sogar verboten wurde zu publizieren (Janssen, 2012, 595). Die eigentumsbezogenen Grundsätze der Enzyklika und die hierzu verfassten Kommentare von Oswald von Nell-Breuning von 1932 bildeten das Fundament des 1947 beschlossenen Programmes der nordrhein-westfälischen CDU (Uertz, 1981, 98).

Im zweiten Abschnitt der genannten Enzyklika „Machtvollkommenheit der Kirche über Gesellschaft und Wirtschaft" wird die Bedeutung des privaten Eigentums gesondert hervorgehoben. Als Grundlage dient dazu die Feststellung ,das Sondereigentumsrecht sei von der Natur, ja vom Schöpfer selbst dem Menschen verliehen, einmal, damit jeder für sich und die Seinen sorgen könne, zum andernmal, damit mittels dieser Institution die vom Schöpfer der ganzen Menschheitsfamilie gewidmeten Erdengüter diesen ihren Widmungszweck wirklich erfüllen“ (Pius XI., 1931, Ziff. 45). Diese Berufung auf das natürliche Recht des Menschen an Eigentum entspricht der traditionellen Auffassung der Kirche wie sie bereits von Thomas v. Aquin im Sinne eines ius gentium erwähnte (vgl. Uertz, 1981, 149ff.). In den Enzyklika aus den 30er Jahren des 
20. Jahrhunderts findet sich diesem Naturrecht gegenüber jedoch eine wesentliche Erweiterung, die eine Legitimation zur staatlichen Förderung des Privateigentums bildet. Sie dient der ,Überwindung der Proletarität durch Vermögensbildung":

,So wahr es ist, dass Pauperismus und Proletarität wohl zu unterscheidende Begriffe sind, so ist doch die überwältigende Massenerscheinung des Proletariats gegenüber einem kleinen Kreise von Überreichen ein unwidersprechlicher Beweis dafür, dass die Erdengüter, die in unserm Zeitalter des sogenannten Industrialismus in so reicher Fülle erzeugt werden, nicht richtig verteilt und den verschiedenen gesellschaftlichen Klassen nicht entsprechend zugute gekommen sind. [...] Darum ist mit aller Macht und Anstrengung dahin $\mathrm{zu}$ arbeiten, dass wenigstens in Zukunft die neu geschaffene Güterfülle nur in einem billigen Verhältnis bei den besitzenden Kreisen sich anhäufe, dagegen in breitem Strom der Lohnarbeiterschaft zufließe. Gewiß nicht, damit der Arbeiter von der Arbeit ablasse - ist doch der Mensch zur Arbeit geboren wie der Vogel zum Fluge - , sondern damit er durch Sparsamkeit seine Habe mehre, durch ihre sorgsame Verwaltung mit größerer Leichtigkeit und Sicherheit die Familienlasten bestreite und der Daseinsunsicherheit, die so recht eigentlich Proletarierschicksal geworden ist, überhoben, nicht bloß den Wechselfällen des Lebens gerüstet gegenüberstehe, sondern noch über dieses Leben hinaus die beruhigende Gewißheit habe, dass seine Hinterbliebenen nicht ganz unversorgt dastehen." (Pius XI., 1931, Ziff. 60f.) 
In diesem Abschnitt wird nicht nur deutlich, dass diese Übersetzung des Weltbildes der katholischen Soziallehre maßgebliche Präzisierungen von Ideen für die Eigentumsbildung beisteuerte. So finden sich darin Argumente aus politischen Debatten wieder wie die Entproletarisierung bei gleichzeitiger Betonung der zu belohnenden Sparsamkeit. Die Bedeutung von Nell-Breuning in der Ausgestaltung der Sozialpolitik in der Bundesrepublik darf über die Parteigrenzen hinaus nicht unterschätzt werden. Der politisch überaus engagierte Priester hatte eine langjährige Beratertätigkeit für deutsche Gewerkschaften und wirkte bei der Gründung des Bundes katholischer Unternehmer mit, beriet Parteien, wobei er nicht in einer von ihnen Mitglied wurde (Klein, 1989, 47, 49). So fanden seine Ideen aufgrund seiner engen Verbindung zur katholischen Arbeitnehmerbewegung auch Eingang in das Bad-GodesbergerProgramm der SPD von 1959, das bis 1989 wirksam war (siehe hierzu Abschnitt 6.3.1). Zudem war Nell-Breuning von 1948 bis 1965 Mitglied im Wissenschaftlichen Beirat beim Bundeswirtschaftsminister ,umgeben von Wirtschaftsexperten und Wirtschaftsprofessoren, von denen er [...] nach eigenen Angaben sehr viel in nationalökonomischen Fragen gelernt hat" (Klein, 1989, 49). 1950 wurde er zudem stellvertretender Vorsitzender des Wohnungswirtschaftlichen Beirats und ,von 1953 bis 1955 berät er den Zentralverband Deutscher Konsumgenossenschaften“ (Klein, 1989, 49).

In dieser Hinsicht erweist sich die Position der Wirtschaftswissenschaften gegenüber den Theologen als äußerst gering. Im Gegensatz zum zeitgleichen Aufschwung wirtschaftswissenschaftlicher Politikberatung in der allgemeinen Wirtschaftspolitik, kommen in der Wissensproduktion für die Wohnungspolitik vor allem die Bande zwischen den 
Koalitionen von Parteien, Verbänden und einzelnen Wissenschaftlern zum Tragen, die gemeinsame Positionierungen teilen. Dies spricht nicht nur für starke parteipolitische und Verbandsinteressen, sondern spiegelt auch die in Abschnitt 3.1.3 bereits beschriebenen Schwäche der Wirtschaftswissenschaften als eigenständige Disziplin in der jungen Bundesrepublik wieder. Zwar verfügen die Honoratioren über ein großes Renommee, werden in Beiräte berufen und erhalten erste Aufträge für Gutachten. Zugleich gibt es allerdings nur wenige Professoren, die sich mit dem Themenbereich der Wohnungspolitik von einem wirtschaftswissenschaftlichen Standpunkt aus befassen.

\subsubsection{Aufbau staatlicher Wissensproduktion}

Die große Bedeutung, welche der Wohnungspolitik nach dem Zweiten Weltkrieg zugemessen wurde, wird an der Einrichtung eines speziell für den Wohnungsbau ausgerichteten Ministeriums auf Bundesebene 1949 deutlich. Das eigenständige Ministerium muss als ein „Bruch mit der Verwaltungstradition" gesehen werden, da seit der Weimarer Republik ,stets von Ministerien betraut [wurde], deren Schwergewicht auf anderen Gebieten lag“" (Schulz, 1994, 330). Das separate Ministerium wurde aufgrund der als umfangreich und diffizil eingestuften Problemlage in der ersten Regierung Adenauer installiert, ,sollte allerdings nur für eine gewisse Zeit existieren“ (Kornemann, 1996, 124). Die Aufgaben des Ministeriums umfassten „die Förderung des Wohnungsbaus und Siedlungswesens auf Bundesebene einschließlich der damit zusammenhängenden Fragen der Wohnungswirtschaft, des Mietrechts, -preisrechts, der Kleinsiedlung, des Landarbeiterwohnungsbaus, Kleingartenwesens, der Wohnungsfürsorge für Bundesbedienstete und der Förderung der bautech- 
nischen Entwicklung“" (Schulz, 1994, 185). Als erster Wohnungsbauminister wurde Eberhard Wildermuth von der FDP ernannt, der das Bestehen seines Ministeriums als eine zeitbedingte Angelegenheit ansah: „Das Ressort werde klein gehalten und könne nach Erledigung seiner Aufgabe in ein anderes Ministerium eingegliedert werden" (Schulz, 1994, 185). Konflikte mit den dirigistischen Vorstellungen des sozialpolitischen Flügels der CDU/CSU waren unter diesen Voraussetzungen vorprogrammiert (Schulz 1994, 290ff.). In der vierten Legislaturperiode des Bundestages verschob sich der Schwerpunkt der Wohnungspolitik hin zu städtebaulichen und Raumordnungsfragen (vgl. Kornemann, 1996, 129ff.). Mit dem neuen Zuschnitt des Wohnungsbauministeriums als das Bundesministerium für Wohnungswesen, Städtebau und Raumordnung 1962 in dem fünften Kabinett Adenauer übernahm es die Federführung für die Raumordnung einschließlich der Fachaufsicht über das Institut für Raumforschung in der Bundesanstalt für Landeskunde und Raumforschung ${ }^{28}$ in Bad Godesberg vom Bundesinnenministerium, wodurch die ministeriumseigene Forschungsarbeit erstmals gestärkt wurde.

Der bereits in Abschnitt 5.3.1 beschriebenen streng katholischen Wohnungsbauminister Lücke sah das Ministerium entgegen seinem Vorgänger Wildermuth als ein Schlüsselministerium an, um über den Weg der staatlichen Wohnungspolitik auf gesellschaftspolitische Entwicklun-

28 Die Vorgängerinstitutionen (Reichsstelle für Raumforschung und Abteilung für Landeskunde) wurden in den Jahren des Nationalsozialismus als Instrumente der nationalsozialistischen "Volk-ohne-RaumIdeologie gegründet. Nach ihrer Zwangsauflösung nach dem Zweiten Weltkrieg wurden die beiden Institutionen wiedergegründet und ,dienten den Alliierten für landeskundliche Erhebungen und der jungen Bundesrepublik als Steuerungsinstrument für die Integration von Flüchtlingen“ (Bundesinstitut für Bau-, Stadt- und Raumforschung (BBSR) im Bundesamt für Bauwesen und Raumentwicklung, 2018a). 
gen Einfluss nehmen zu können (vgl. Schulz, 1994, 258f.). Lücke positionierte sich und sein Ministerium durch eigene Publikationen mehrfach zum Thema Eigenheimförderung und brachte sich so auch selber in wohnungspolitische Debatten mit ein (vgl. Lücke, 1959). Die wohnungspolitischen Zielsetzungen wurden im Rahmen von Schriften des Bundesministeriums herausgegeben und sollten nach dem Vorwort von Lücke „Unterlagen vor allem für Politiker, Soziologen, Wirtschaftler, Journalisten u.a., die sich mit Fragen der Wohnungspolitik auseinandersetzen wollen und müssen" bereitstellen (Bundesministerium für Wohnungsbau, 1961, 1).

Als wesentliches, durch das Ministerium berufenes Gremium zur Wissensproduktion in der Eigenheimförderung ist der in Abschnitt 5.3.2 bereits skizzierte wohnungswirtschaftliche Beirat zu nennen. Trotz den Unterschieden zwischen den Gremien kann davon ausgegangen werden, dass der Wohnungswirtschaftliche Beirat zumindest als eine Art Pendant zum Wissenschaftlichen Beirat des Bundeswirtschaftsministeriums gedacht war. So beschreibt Nell-Breuning (1959), dass der damalige Bundesbauminister Wildermuth im Mai 1950 zu einer Sitzung des Wissenschaftlichen Beirates geladen war, als dieser sich mit wohnungswirtschaftlichen Themen auseinandersetzte (vgl. Wissenschaftlicher Beirat beim Bundesministerium für Wirtschaft, 1950) ${ }^{29}$ und infolge dessen noch im selben Jahr den wohnungswirtschaftlichen Beirat ins Leben rief (Nell-Breuning, 1959, 35). Nell-Breuning zitiert aus der Einladung zur konstituierenden Sitzung:

29 Das Gutachten des wissenschaftlichen Beirates befasst sich maßgeblich mit der Frage inwiefern die staatliche Bewirtschaftung des Wohnungsmarktes mit dem Übergang zur Sozialen Marktwirtschaft vereinbar sein kann. 
„Bei verschiedenen Bundesministerien haben sich wissenschaftliche Beiräte, die die ministeriellen Arbeiten durch Erstattung wissenschaftlicher Gutachten unterstützen, besonders bewährt. Ich möchte daher auch bei meinem Ministerium einen solchen Beirat in Form eines Wohnungspolitischen Sachverständigenbeitats ins Leben rufen." (Nell-Breuning, $1959,35)$

Der Beirat erstellte von 1951 bis 1958 diverse Gutachten, wobei der Schwerpunkt der Tätigkeit ,mit sieben von zwölf Gutachten und 12 von 29 Sitzungen in den ersten beiden Jahren seiner Arbeit" lagen (Wilhelm, 1968, 43). Die Gutachten, die in verschiedenen Sammelbänden zusammengetragen wurden, können zumindest eingeschränkt als politisch unabhängig erstellte Expertisen eingestuft werden. Es lag zwar im Belieben des Ministeriums, „ob es die Gutachten berücksichtigte, andererseits wurden die Gutachten keineswegs als amtliche Äußerungen des Ministeriums verstanden" (Wilhelm, 1968, 44). Dabei war der Beirat nicht frei in der Befassung mit allen Fragen. Diese mussten entweder vom Ministerium gestellt oder aber durch den Bundesminister bestätigt werden" (Wilhelm, 1968, 46). Gegenüber dem nahezu mythischen Ruf des wissenschaftlichen Beirates beim Bundeswirtschaftsministeriums ist somit von einer insgesamt geringen Unabhängigkeit des Beirates auszugehen, dessen Tätigkeit alsbald zugunsten von Auftragsforschung eingestellt wurde.

Neben der neuen ministeriellen Struktur auf Bundesebene waren in der ersten Phase wohnungspolitischer Wissensproduktion auch staatliche Akteure auf Landes- und Kommunalebene an den Debatten beteiligt, was auch anhand von Eingaben in dem beim Zweiten Wohnungs- 
baugesetz beratenden Ausschuss für Bau- und Bodenrecht erkennbar wird. Genannt werden dabei ,insbesondere [die Eingaben] [...] vom Deutschen Städtetag, vom Deutschen Gemeindetag, vom Deutschen Landkreistag, [und] vom Ausschuß für Wohnungs- und Siedlungswesen in Essen“ (BT-Drs. 02/142, 7450B). Zu den weiteren staatlichen Akteuren in den Debatten um die Eigenheimförderung zählten somit auch Vertreter von Ländern und Kommunen, die sich über den Deutschen Städtetag, lokale Ausschüsse für Wohnungs- und Siedlungswesen sowie über die ARGEBAU-Fachkommission ${ }^{30}$ an den politischen Debatten über Eingaben beteiligten, oder aber von Politikern als Experten vor Ort zitiert wurden (vgl. BT-Drs. 02/142, 7380D; BT-Drs. 02/142, 7450B; Schulz 1994, 204ff.). Dabei hatten die Voten kommunaler Verbände ,prinzipiell nicht mehr Gewicht als die anderer Lobbygruppen“ (Schulz, 1994, 207f.). Gegenüber den Ländern, die innerhalb des Bundesrates noch eine wichtige Entscheidungsinstanz einnehmen, kommt den Kommunen somit zwar eine geringere Machtposition zu. Allerdings liefern kommunale Ämter insbesondere in den ersten wohnungspolitischen Debatten wesentliche Informationen zum Status Quo der Wohnraumversorgung, auf welche die Abgeordneten in den Bundestagsdebatten auch referierten. So verwies der SPD-Abgeordnete Stierle in der Debatte um das Zweite Wohnungsbaugesetz auf Daten des Frankfurter Wohnungsamtes, um auf den Wohnraummangel vor Ort zu

30 Die ARGEBAU wurde noch vor der Gründung der Bundesrepublik ,,von den acht für das Bauwesen zuständigen Ministerien der deutschen Länder in der amerikanischen und britischen Zone als Arbeitsgemeinschaft des Bau-, Wohnungs- und Siedlungswesens des Vereinigten Wirtschaftsgebetes gegründet, um die Interessen der Länderressorts gegenüber den bizonalen Verwaltungen zu vertreten“ (Egner, 2004b, 149f.). 
belegen (BT-Drs. 02/142, 7421A). Dieser Umstand verdeutlicht, dass es zum Zeitpunkt des Zweiten Wohnungsbaugesetzes noch keine verlässlichen Daten zum Wohnungsbestand in der Bundesrepublik gab. Tatsächlich verfügten einzelne Städte und Länder in ihren Karteien in der Nachkriegszeit über die einzigen halbwegs verlässlichen Daten zum Wohnungsbestand und Wohnungsbedarf (Leutner, 1990, 24):

„Zusammenfassende Daten zum seinerzeitigen Wohnungsbedarf und dessen Entwicklung in der Zukunft waren demgegenüber Überraschungsgrößen, bei deren Bestimmung man mangels exakter Unterlagen auf grobe Schätzungen angewiesen war." (Leutner, 1990, 24)

Daher ist der Aufbau einer amtlichen und umfassenden Statistik in der ersten Phase der bundesdeutschen Wohnungspolitik als der wohl wichtigste Schritt hinsichtlich der Wissensproduktion für politische Entscheidungen $\mathrm{zu}$ bewerten. Vor dem Zweiten Weltkrieg hatten lediglich zwei Wohnungszählungen in Deutschland stattgefunden (1918 und 1927), die sich auf Gemeinden mit mehr als 5.000 Einwohnern beschränkten (Statistisches Bundesamt, 1956, 5). Bei allen Zählungen waren bis dahin die Eigentümergruppen nicht mit berücksichtigt worden (Statistisches Bundesamt, 1956, 14). Nachdem bereits 1946 die Gebäude- und Wohnungsstatistik ,zur Feststellung der Kriegszerstörungen und Unterbringung der Flüchtlinge“ (Hölder u. Ehling, 1991, 26) ausgeweitet wurde, wurde basierend auf dem Volkszählungsgesetz von 1950 noch im selben Jahr die erste umfassende Wohnungszählung nach dem Zweiten Weltkrieg durchgeführt und 1956 veröffentlicht (Glatzer 1980, 15; Statistisches Bundesamt 1956). Der erste Wohnungsbauminister Wildermuth gab 1951 beim Statistischen Bundesamt und 
den Bundesländern eine weitere, umfassendere Bestandsaufnahme zur Wohnungsbau in der Bundesrepublik Deutschland in Auftrag, die im Hauptteil von dem volkswirtschaftlichen Referenten des Bundesbauministeriums Walter Fey bearbeitet wurde und auch maßgeblich auf dessen Initiative zurückzuführen ist (Fey, 1951). Die Auffassung Feys zur Notwendigkeit dieser Erfassung wird im Vorwort des Bandes deutlich:

„Die Kenntnis der Tatsachen in der klaren Form statistischer Angaben, richtig gedeutet und verstanden, ist eine unentbehrliche Grundlage auch für die wohnungspolitische Arbeit. [...] Eine notwendige Voraussetzung für den exakten Unterbau der Wohnungspolitik ist ein zuverlässiges statistisches Berichtswesen." (Fey, 1951, 2)

Vor seiner Karriere im Wohnungsbauministerium war Fey wissenschaftlicher Mitarbeiter im 1925 gegründeten Institut für Konjunkturforschung (IfK, heute DIW, vgl. Abschnitt 3.1.3) gewesen und hatte sich im Rahmen seiner Tätigkeit als Fachmann mit wohnungswirtschaftlichen Themen und dem dringenden Bedarf an einer besseren statistischen Erfassung der Wohnungsbestände auseinandergesetzt (Fey 1936; vgl. hierzu auch Führer 1997) und galt als anerkannter Fachmann in der jungen Bundesrepublik (vgl. Lütge, 1949). Als späterer Ministerialrat war Fey nicht nur der Anstoßgeber für die Einführung der Wohnungsstatistik, sondern auch bis März 1958 der Verbindungsreferent des Ministeriums für Angelegenheiten des wohnungswirtschaftlichen Beirates und nahm selber an allen Sitzungen teil um Anliegen des Ministeriums vorzubringen wie auch dem Ministerium Bericht zu erstatten (Wilhelm, 1968, 45). Auch auf Fachtagungen zur weiteren Entwicklung der Wohnungspolitik nahm Fey gemeinsam mit Wissenschaftlern 
und Praktikern (siehe Schneider, 1964a, 107) in seiner Position teil und sprach sich in einer Linie mit Lücke primär für eine verstärkte Eigenheimförderung aus (Fey, 1964, 84). Die Einstellung Feys kann als Indiz dafür gewertet werden, dass einerseits seine Sachkenntnis für die Wissensproduktion innerhalb der Ministerialbürokratie anerkannt und auch gewünscht wurde.

Im weiteren Verlauf sollte die amtliche Statistik zu Wohnungsmärkten weiter ausgebaut und deren Ergebnisse regelmäßiger veröffentlicht werden. Basierend auf dem bereits im Mai 1956 erlassenen Gesetz über eine Statistik der Wohn- und Mietverhältnisse und des Wohnungsbedarfs (Wohnungsstatistik 1956/57) wurden erstmals in größerem Umfang Art und Größe von Wohnungen mit der Anzahl der Haushaltsmitglieder und die Mietverhältnisse in der Bundesrepublik mithilfe einer 10-\%-Wohnungsstichprobe erfasst (vgl. BGBl 1956, Nr. 23, 427ff.; Glatzer 1980, 15; Wandersleb 1959, 11). Mit der Erhebung wurde das Statistische Bundesamt betraut, das seitdem die Ergebnisse in der Reihe „Wirtschaft und Statistik“ veröffentlicht (Statistisches Bundesamt, 1959). 1957 wurde eine weitere 1-\%-Zusatzerhebung und 1960 erneut eine 1-\% Wohnungsstichprobe durchgeführt. Erneuert wurde dieses Vorgehen durch das Gesetz über die Durchführung einer Repräsentativstatistik auf dem Gebiete des Wohnungswesens (Wohnungsstichprobengesetz) im August 1965, mit dem abermals und mit weitreichenden Details die Wohnungsversorgung in der Bundesrepublik erfasst werden sollte (vgl. BGBl. 1965, Nr. 41, 893). Die Ergebnisse dienten nicht nur zunehmend der politischen Debatte an sich, sondern sollten nach Auffassung des nunmaligen Wohnungsbauministers Lauritzen auch für ein breiteres Fachpublikum und interessierte Bürgerinnen und Bürger durch Veröf- 
fentlichungen zugänglich gemacht werden (so Lauritzen, 1972).

Statistische Darstellungen des Wohnungsmarktes waren - wie bereits in Abschnitt 5.2 beschrieben - überaus umstritten, was sich an den Gegendarstellungen von anderen Akteuren äußerte. Ebenso wenig existierten zu diesem Zeitpunkt verlässliche Angaben zu der Höhe der öffentlichen Förderungsmittel, die ,dem sozialen Eigenheim- bzw. Eigentumswohnungsbau zugute gekommen sind, da diese Summe nicht direkt statistisch ausgewiesen wird“ (Höhnen, 1968, 111).

\subsection{Phase 1: Vermarktlichung ohne Markt- wissen}

Trotz der anfänglichen Förderung des Wohneigentums nach Kriegsende kam es zunächst zu sinkenden Eigentumsquoten. Diese Entwicklung ist darauf zurückzuführen, dass der Mietwohnungsbau nach dem Motto „Zunächst jedem eine Wohnung, später jedem seine Wohnung“ (Bentzien, 2012, 19) forciert wurde - vor allem durch die Förderung der gemeinnützigen Wohnungswirtschaft. Die mangelnden Realisierbarkeit der Idealvorstellungen breit gestreuten Wohneigentums bringt Schulz (1994) wie folgt auf den Punkt:

„Es läßt sich wohl kaum eine größere Diskrepanz vorstellen als diejenige zwischen der Vision eines Volkes von selbstbewußten Eigenheimbesitzern und der herrschenden Realität eines Volkes im Wohnungselend.“(Schulz, 1994, 170)

Dennoch bildete der Bau von Eigenheimen aufgrund der neuen Fördermaßnahmen stets eine breite Grundlast der Neubautätigkeit (vgl. 
Schulz, 2000, 70). Mit ihnen wurden die Grundlagen für die Eigenheimförderung in Deutschland geschaffen: Neben dem Sozialen Wohnungsbau, in dessen Rahmen der Eigenheimförderung ein klarer Vorrang eingeräumt wurde, wurde die Bausparförderung sowie sie erste steuerliche Förderung installiert. So ergab sich bereits früh „ein umfangreiches und vielfältiges Instrumentarium zur Förderung der breit gestreuten Wohnungseigentumsbildung“" (Höhnen, 1968, 55).

Aus den Darstellungen der wohnungswirtschaftlichen Rahmenbedingungen heraus lässt sich die Eigenheimförderung in dieser Phase allerdings nicht erklären. Zwar herrschte zum Ende des Zweiten Weltkrieges eine massive Wohnungsnot, die jedoch vor dem Hintergrund knappen Kapitals durch den Bau von Mehrfamilienhäusern deutlich kostengünstiger gewesen wäre (Hilpert 2012; Kramper 2008). Aus materiellen Zwängen alleine lässt sich die frühe Wohneigentumsförderung der Bundesrepublik somit nicht erklären. Dementsprechend finden sich in den Begründungen für die Förderung von Wohneigentum auch keine harten wohnungspolitischen Notwendigkeiten, sondern vorrangig in normativen Begründungen aus dem sozial- und gesellschaftspolitischen Bereich (vgl. Leidner, 1981, 283f.). In den hier betrachteten Debatten lassen sich vor allem familienpolitische Zielsetzungen sowie die Förderung des Wohneigentums als "Bollwerk gegen den Kommunismus“ als Zielsetzungen der Eigenheimförderung nachweisen. Während beim Zweiten Wohnungsbaugesetz vor allem die Unterstützung von Bedürftigen im Vordergrund stand, sollte der förderberechtigte Personenkreis im Zeitverlauf auf breitere Schichten ausgeweitet werden.

An dieser Stelle wird deutlich, dass die Vermarktlichung auf der poli$c y$-Ebene der Eigenheimförderung in ihrer ideellen Dimension weniger 
in liberalen wirtschaftswissenschaftlichen Theorien verhaftet ist, sondern maßgeblich auf der katholischen Soziallehre beruht. Als prominentes empirisches Beispiel in der Wohlfahrtsstaatenforschung sei auf die Arbeit von Esping-Andersen verwiesen, der die Bedeutung der katholischen Soziallehre als abstrakten Wertekomplex für die Ausgestaltung des konservativen Wohlfahrtsstaates hervorhebt (Esping-Andersen, 1990), die vor allem von den christdemokratischen Parteien vorangetrieben wurde (siehe auch van Kersbergen 1995). Dabei geben abstrakte Weltbilder Akteuren eine wesentliche Grundorientierung, „die konstitutiv für ihre Situationsdefinitionen und Handlungsorientierungen" sein kann (Sachweh, 2011, 375). Wirtschaftsliberale Ideen sind demgegenüber in den Debatten zur Eigenheimförderung zwar vorhanden, beziehen sich allerdings meist auf eine Abschaffung des sozialen Wohnungsbaus und insbesondere auf eine Minderung der Macht der gemeinnützigen Wohnungswirtschaft. Der freie Markt wird befürwortet, allerdings nicht auf Kosten der Subventionierung des Eigenheimbaus.

Auch in ihrer kognitiven Dimension wurde die Eigenheimförderung von Ideen zur gesellschaftspolitischen Wirkung des Eigenheimes dominiert und nicht durch die parallel vorhandenen Paradigmen der Marktwissenschaftler. Analytische Tools der Ökonomie spielten in dieser Phase kaum eine Rolle. Von einer Konstruktion des Politikgegenstandes „Eigenheim" im Sinne der konstruktivistischen Perspektive wie etwa der Science and Technology Studies kann in dieser Phase nicht die Rede sein. Wie wenig selbstverständlich die Betrachtung der Wohnungspolitik durch die statistische Brille war, zeigt sich in den Debatten um die statistisch ausgeglichenen Wohnungsmärkte im Rahmen der Abbaugesetzgebung. Um es mit den Worten des Volkswirtes Seraphim auszu- 
drücken, wurde der Wert der Eigenheimförderung als ,umfassender und nachhaltiger [angesehen] als dies mit einer noch so exakten quantitativen Analyse auszudrücken ist" (Seraphim, 1955, 224). Somit lässt sich der institutionelle Wandel der Eigenheimförderung in der ersten Phase der Wissensproduktion als eine Vermarktlichung ohne Marktwissen beschreiben.

Allerdings ist an dieser Stelle noch nicht geklärt, wer die entsprechenden Ideen für den Politikbereich der Eigenheimförderung zu handlungsanleitenden Ideen bereitgestellt hat. Dominiert wurden die politischen Forderungen nach einer verstärkten Eigenheimförderung durch die christdemokratischen Parteien und ihre Verbündeten aus der Verbandslandschaft, wobei auch die anderen Parteien der Eigenheimidee vor allem in den frühen Jahren der Bundesrepublik überaus positiv gegenüberstanden. $\mathrm{Zu}$ den bedeutendsten an der Wissensproduktion beteiligten Verbänden gehören die katholischen Siedlungsdienste, Wirtschaftsverbände sowie der Mieterbund und der DGB. Von wissenschaftlicher Seite muss eine wesentliche Bedeutung einzelnen einflussreichen katholischen Theologen zugesprochen werden, die in ihren Beziehungen zu den christlichen Parteien die Ideenproduktion zur Eigenheimförderung voran trieben. Das prominenteste Beispiel ist Oswald von NellBreuning, der sowohl für die sozialpolitische Ausrichtung der Union als auch für die Neuorientierung der SPD einen wichtigen Stellenwert einnahm. Experten wie von Nell-Breuning trugen dazu bei, das abstraktere Vorstellungen der christlichen Soziallehre für den wohnungspolitischen Kontext zu konkretisieren und somit den Akteuren handlungsanleitendes Wissen zur Verfügung zu stellen. Die dominanten Ideen lassen sich somit auf das Akteursspektrum zurückführen, das sie im Rahmen der 
politischen Wissensproduktion verhandelt und diskutiert. Der in der ersten Hypothese herausgestellte Einfluss der Wissensmaschinerie auf die verfügbaren Ideen kann somit als bestätigt angesehen werden.

Mit Blick auf die Mechanismen im knowledge regime kann in der ersten Phase von einer erweiterten Dezision im Habermasschen Sinne ausgegangen werden: Der Kern der politischen Zielvorstellungen bleibt den gewählten Politikern und ihren normativen Wertvorstellungen vorbehalten. Die Ideen der machthabenden politischen Vertreter geben in dieser Phase den maßgeblichen Anstoß zu Reformen. Besonders hervorzuheben ist an dieser Stelle das Wirken des Wohnungsbauministers Lücke, der bereits im Rahmen des Ausschusses für Wiederaufbau große Anstrengungen unternahm, das „Familienheim“ stärker zu fördern. Dazu nahm er gezielt die Expertise von gleichgesinnten Verbänden und theologischen Experten in Anspruch, denen eine dienende Funktion zukam. Die Aufgabe der Experten aus Wissenschaft und Wirtschaft besteht in ihren Beiratstätigkeiten vor allem darin, Fragen des Wohnungsbauministers zu bearbeiten und nicht in einem hohen Maße eigenständig tätig zu werden. Von einer herausragenden Bedeutung der wissenschaftlichen Expertise im technokratischen Sinne kann daher in der ersten Phase nicht die Rede sein.

Eine Abhängigkeit der politischen Akteure von wissenschaftlichen Erkenntnissen ist bereits durch den Umstand auszuschließen, dass sich zunächst kaum Wissenschaftler mit der Eigenheimförderung befassten. Zwar sollte auch die Wohnungspolitik nach den Vorstellungen des ersten Wohnungsbauministers Preusker durch einen wissenschaftlichen Beirat nach dem Vorbild des wirtschaftswissenschaftlichen Beirates flankiert werden. Allerdings wurde diese Vorstellung schnell aufgegeben zuguns- 
ten eines wohnungswirtschaftlichen Beirats, der maßgeblich mit Verbandsvertretern besetzt wurde. Laut Wilhelm waren rund 80 Prozent der beratenden Praktiker stets Vertreter von Verbänden und der öffentlichen Verwaltung (Wilhelm, 1968, 47). Interessenverbände bilden in der ersten Phase wesentlicheren Produzenten von Ideen und Wissen für policy-Reformen. Sogar auf wissenschaftlichen Tagungen waren sie mit mehr Experten vertreten als die Wissenschaftler (vgl. Schneider, 1964a). Selbst die ausgewiesenen und anerkannten wissenschaftlichen Experten auf dem Gebiet waren nicht auf Wohnungspolitik spezialisiert, sondern meist Wirtschaftswissenschaftler mit einem ursprünglich anderen Betätigungsfeld. Innerhalb der wirtschaftswissenschaftlichen Expertise herrschte zudem Uneinigkeit, die sich auch in der unklaren Positionierung des wohnungswirtschaftlichen Beirates deutlich wurde (vgl. Seraphim, 1955).

Ein starker Einfluss der Ministerialbürokratie auf die verfügbaren Ideen gemäß der zweiten Hypothese kann für die erste Phase nicht bestätigt werden. Damit stimmt das Bild der Verwaltung in der ersten Phase der Eigenheimförderung stärker mit dem ersten Modell der Beziehung zwischen Staat und Verwaltung von Skocpol 1985 überein: staatliche Administrationen übernehmen hier eine dienende Funktion gegenüber anderen Akteuren und helfen primär dabei, Interessen gesellschaftlicher Gruppen zu verhandeln, um einen Ausgleich herbeizuführen. Allerdings werden in dieser Phase die Grundlagen für die spätere Entwicklung der Wissensproduktion durch die öffentliche Verwaltung geschaffen: Durch die Beauftragung von Beiräten durch die Ministerialbürokratie und den Aufbau der amtlichen Statistik installierten öffentliche Stellen in dieser ersten Phase nach dem zweiten Weltkrieg eine 
Reihe von Beratungsmechanismen und Kennzahlen, welche die künftige Entwicklung wissenschaftlicher Expertise im wohnungspolitischen Umfeld maßgeblich bestimmen sollten.

Die Vermarktlichung qua Eigenheimförderung in der policy-Dimension ging somit nicht mit einer Vermarktlichung auf der normativen Dimension einher. Allerdings werden innerhalb dieser ersten Phase durch die öffentliche Verwaltung die Grundlagen zur kognitiven Vermarktlichung geschaffen: Erst das Verfügbarmachen von Marktdaten ermöglichte die analytische Betrachtung von Wohnungsmärkten und die ideelle Weiterentwicklung durch wirtschaftswissenschaftliche Expertise, die in der zweiten Phase an Bedeutung gewinnen sollte. 


\section{Kapitel 6}

\section{Phase 2: Vom Sozialen}

\section{Wohnungsbau zur}

\section{individuellen steuerlichen}

\section{Förderung}

In der zweiten Phase lassen die zunehmend ausgeglichenen Wohnungsmärkte und der Wechsel zur sozialliberalen Regierungskoalition einen Wandel in der Wohnungspolitik vermuten. Hinsichtlich der Förderung von Wohneigentum ist dies jedoch nicht der Fall: Sie wurde unter der sozialliberalen Koalition weiter ausgebaut, sodass ein merklicher Anstieg der Wohneigentumsquoten zu verzeichnen ist (Abschnitt 6.1). Kennzeichnend für die Bundestagsdebatten ist in dieser Phase ein langsamer, aber dennoch merklicher ideeller Wandel: So wurde die Famili- 
enförderung zwar als zentrale Zielvorstellung der Eigenheimförderung beibehalten. Zeitgleich rückten jedoch zunehmend vermögenspolitische Zielvorstellungen in den Vordergrund (Abschnitt 6.2). Neu ist in diesem Zusammenhang auch die Orientierung der Politik an Wohneigentumsquoten als relevanter Zielgröße. Bereitgestellt wurden diese Ideen durch eine zunehmende wirtschaftswissenschaftliche Expertise, die sich vor allem in den vermehrt in Auftrag gegebenen Gutachten manifestiert (Abschnitt 6.3). Dabei muss allerdings angemerkt werden, dass diese Gutachten mehr für eine Verwissenschaftlichung des politischen Diskurses stehen und nicht für eine größere Macht der Wissenschaftler an sich. So wurde eine erkennbare Zahl relevanter Gutachten durch Interessenverbände in Auftrag gegeben, die damit ihre politischen Forderungen untermauern wollten. Sie orientierten sich dabei an einem allgemeinen administrativen Trend zu mehr wissenschaftlichen Gutachten und der Anerkennung externer Expertise für wohnungspolitische Entscheidungen. So war es insbesondere die Ministerialbürokratie, die den Wissenschaftlern über Auftragsforschung Ressourcen für ihre weitere Arbeit zur Verfügung stellte und Zugang zur politischen Wissensproduktion verschaffte. Die Analysen und Argumentationsmuster in den entsprechenden Debatten wurden dabei durch eine in größerem Umfang bereitgestellte amtliche Statistik überhaupt erst ermöglicht. Die zentralen Erkenntnisse im Hinblick auf den Zusammenhang zwischen den politischen Ideen und deren Produktion werden in Abschnitt 6.4 zusammengefasst. 


\subsection{Ende der Wohnungsnot und Ideenwan- del}

Im Jahr 1966 erlebte das Wirtschaftswunder der Nachkriegszeit rezessionsbedingt einen Dämpfer. Der erste große Einbruch in der gesamtwirtschaftlichen Konjunktur nach dem Zweiten Weltkrieg rückte „die wirtschaftliche Stabilität in Deutschland stärker ins öffentliche Bewußtsein" und hatte zur Folge, dass CDU und CSU ihr bis dato bestehendes „Image als Partei des wirtschaftlichen Erfolges“ einbüßten (Seeger 1995, 98; siehe auch Krummacher 1978, 226). Nachdem Ludwig Erhard vom Amt des Bundeskanzlers zurückgetreten war, kam es 1966 zur Bildung einer großen Koalition unter Bundeskanzler Kiesinger. Nun bestimmte nach Einschätzung von Seeger die SPD in Regierungsverantwortung die Wirtschafts- und Finanzpolitik maßgeblich mit:

„Bundeswirtschaftsminister Schiller (SPD) ging dazu über, eine Politik der Globalsteuerung, Stabilisierung und der antizyklischen Finanzierung zu betreiben sowie das kreditpolitische Instrumentarium der Deutschen Bundesbank stärker zum Zuge kommen zu lassen." (Seeger, 1995, 98)

Für die neue Regierung war es vor allem von großer Bedeutung, „die Wirtschaftskrise zu bewältigen und eine Reihe notwendiger Verfassungsreformen durchzuführen. Mit dem [keynesianisch orientierten] Stabilitätsgesetz von 1967 wurden die Instrumente geschaffen, die der Bundesregierung die Stabilisierung der Konjunktur erlauben sollten, und das Arbeitsförderungsgesetz von 1969 ergänzte die passive Arbeitsmarktpolitik um Instrumente der aktiven Arbeitsmarktpolitik“ (Ostheim u. Schmidt, 2007b, 165). Sowohl in der wirtschafts- als auch in 
der Sozialpolitik agierte die neue Bundesregierung unter der Annahme einer Plan- und Berechenbarkeit von Wirkungen politischer Maßnahmen. So ließ die Regierung „,keinen Zweifel daran, daß die Realisierung von Reformvorhaben nur bei kräftigem Wirtschaftswachstum und erfolgreicher Stabilitätspolitik möglich sei“ (Krummacher, 1978, 227).

Mit der Wahl der sozialliberalen Koalition und dem Machtwechsel 1969 wurden nicht nur die Globalsteuerung, sondern auch sozialstaatliche Maßnahmen weiter ausgebaut. Die Zielsetzungen „Demokratisierung, Chancengleichheit, sozialer Ausgleich und Bekämpfung öffentlicher Armut" ließen die neue Regierung im Charakter des gesellschaftlichen Umbruchs erscheinen (Ostheim u. Schmidt, 2007b, 166). Streeck beschreibt die Aufbruchstimmung wie folgt:

„Modernisierung war Ziel und Mittel zugleich, galt weithin als überfällig, und was sie bedeutete, erschien klar: keynesianische "Globalsteuerung" in der Wirtschaftspolitik und „Reformen“, etwa in Schulen und Universitäten, die zusammen aus Deutschland nach der Restauration unter Adenauer und der Stagnation unter Erhard eine „moderne Industriegesellschaft" machen sollten." (Streeck, 2015, 70)

Folgt man dieser Einschätzung, sollte unter der sozialliberalen Koalition eine Abkehr von der vorherigen, situationsorientierten Wohnungspolitik und ein stärker egalisierendes Fördermodell erwartet werden (Leutner, 1990, 131). Tatsächlich gab es in einigen Politikbereichen eine Abkehr von bestehenden Fördersystemen. Dazu zählen „die Streichung der steuerlichen Kinderfreibeträge, durch die nach Meinung der Koalition die Reichen begünstigt werden, und die gleichzeitige Einführung eines [...] für alle gleichen Kindergeldes“" (Feige, 1979, 50). Demge- 
genüber hatten sich für viele Bürgerinnen und Bürger sozialpolitische Schutzmaßnahmen während der ersten Regierungsjahren der sozialliberalen Koalition verbessert (Ostheim u. Schmidt, 2007b, 167). Ein wohnungspolitischer Wandel gegenüber den Vorgängerregierungen war im Bereich des Mietrechts zu verzeichnen. Unter der sozialliberalen Regierung sollte nicht nur der Mieterschutz eine deutliche Stärkung erfahren. Auch die Neubauförderung vonseiten des Staates wurde unter konjunkturpolitischen Gesichtspunkten deutlich ausgebaut (Mayer, 1998, 191) und damit die Wohnungspolitik konjunktur- und stabilitätspolitischen Zielsetzungen zeitweise untergeordnet (Harlander, 2008, 526). 1971 sollte der Mieterschutz mit dem „Gesetz zur Verbesserung des Mietrechts und zur Begrenzung des Mietanstiegs sowie zur Regelung von Ingenieur- und Architektenleistungen“ (MietVerbG) und mit dem „Gesetz über den Kündigungsschutz für Mietverhältnisse über Wohnraum“ (Erstes Wohnraumkündigungsschutzgesetz) eine erneute Stärkung erfahren. So wurde zum einen der Kündigungsschutz der Mieter durch Regelungen zur Nichtbeschaffbarkeit angemessenen Wohnraums verstärkt, was diesen eine größere Sicherheit bei allgemeinen Wohnungsknappheiten bieten sollte (Kofner, 2004, 169). Zum anderen bot die neue Gesetzgebung die Grundlage für eine Wiederaufnahme der Mietpreisbindungen für Altbauwohnungen sowie für freifinanzierte und steuerbegünstigte Wohnungen (Mayer, 1998, 191). Zusätzlich konnten bei bestehenden Mietverhältnissen Mieterhöhungen nur noch einmal pro Jahr und bis zur Höhe der sogenannten ortsüblichen Vergleichsmiete $^{1}$ erfolgen (Mayer, 1998, 192). Darüber hinaus sollt sich nach dem

1 Die örtlichen Vergleichsmieten sollen den durchschnittlichen Mieten für ,,vergleichbaren“ Wohnraum abbilden - also solchen von vergleichbarer Art, Größe, Ausstattung, Beschaffenheit und Lage (Kofner, 2004, 171). Das Instrument un- 
neuen Mietwucherparagraphen ein Vermieter strafbar machen, der „die Zwangslage, die Unerfahrenheit, oder den Leichtsinn [...] eines anderen dadurch ausbeutet, daß er sich oder einem Dritten für die Vermietung von Räumen zum Wohnen [...] Vermögensvorteile versprechen oder gewähren läßt, die in einem auffälligen Mißverhältnis ${ }^{2}$ zu seiner Leistung stehen"(Kofner, 2004, 170).

Als quantitative Engpässe der Wohnungsversorgung beseitigt zu sein schienen, wollte sich die Regierung nun auch zunehmend mit der besseren qualitativen Ausstattung des Wohnraumes befassen (Harlander, 2008, 826). Mit dem „Gesetz zur Förderung der Modernisierung von Wohnungen“ (Wohnungsmodernisierungsgesetz) von 1976 und dem „Wohnungsmodernisierungs- und Energieeinsparungsgesetz“ von 1978 sollte die Wohnqualität gefördert werden (Pauli, 2005, 73f.). Dem aufkommenden Problem der Modernisierungsverdrängung aufgrund durchgeführter Modernisierungsmaßnahmen sollte mit einer „Einschränkung der Duldungspflicht der Mieter bei Modernisierungsmaßnahmen“ entgegen gewirkt werden (Mayer, 1998, 200).

Demgegenüber wurde die Familienförderung in der Eigenheimpolitik nicht beschnitten, sondern sogar weiter ausgebaut. In seiner Regierungserklärung vom 28. Oktober 1969 plädierte Willy Brandt „für eine forcierte Vermögensbildung, unter anderem durch die Erweiterung des Bausparens [und] kündigte ein langfristiges Programm des sozialen

terscheidet sich insofern von einer festgelegten Referenzmiete als dass es auch qualitative Unterschiede auf Wohnungsmärkten mit berücksichtigt und diesen somit in Teilsegmente zu einer besseren Marktabbildung gliedert.

2 Ein solches Missverhältnis liegt nach heutiger Auslegung dann vor, wenn die verlangte Miete die ortsübliche Miete um mehr als 50 Prozent übersteigt (Kofner, 2004, 170). 
Wohnungsbaus, die Verabschiedung des Städtebauförderungsgesetzes sowie eine Verbesserung des Wohngeldgesetzes an" (Kornemann, 1996, 133). Darüber hinaus bekannte sich Brandt zur breiten Streuung des privaten Eigentums (Kornemann, 1996, 134). Somit kam es nicht zu einer Kehrtwende in der Wohneigentumsförderung unter der sozialliberalen Koalition - auch wenn diese zu erwarten gewesen wäre. Harlander (2008) merkt an, dass ein zentrales Problem für eine vollkommene Neuorientierung der sozialliberalen Koalition zwar darin bestanden hat, dass sie ab 1976 mit einer Bundesratsmehrheit von CDU/CSU-regierter Länder Kompromisse finden musste (Harlander, 2008, 826). Allerdings zeigen sich auch in den von SPD-Politikern formulierten wohnungspolitischen Zielsetzungen, dass es auch inhaltlich nur wenige Differenzen gegenüber den christdemokratischen Parteien in der Wohneigentumsförderung gab (vgl. Abschnitt 6.2).

Trotz aller Bestrebungen der vorherigen Gesetzgebungen konnte die Eigentumsquote seit dem Inkrafttreten des Zweiten Wohnungsbaugesetzes keine Steigerung verzeichnen. Sie war sogar von den 38 Prozent im Jahr 1956 auf 36 Prozent im Jahr 1972 gesunken - noch geringer fiel die Quote in den unteren Einkommensschichten aus (Müller-Heine, 1984, 39), wobei diese den politischen Debatten zufolge noch gesondert hätten gefördert werden sollen. Die massiv ansteigenden Inflationsraten der frühen 70er Jahre blieben allerdings auch für die Wohnungsmärkte nicht ohne Folgen. Steigenden Baupreisen zum trotz führte ein starker Anstieg der Neubautätigkeit als „Flucht in die Sachwerte“ (Dringenberg 1983, 153; Leutner 1990, 183) oder in „Betongold“ (Wissenschaftlicher Beirat beim Bundesministerium für Wirtschaft, 1982, 6) zu einer deutlichen Verbesserung der quantitativen Wohnungsver- 
sorgung (Müller-Heine, 1984, 38f.). In den Jahren 1971/72 lässt sich von einer regelrechten Boomphase des Hochbaus sprechen, die bis 1973 anhalten sollte (Krummacher, 1978, 276). Lediglich auf lokaler Ebene zeigten sich noch Versorgungsengpässe, von denen vor allem großstädtische Wohnungsmärkte betroffen waren (Mayer 1998, 194; Schulz 2000, 71). Für 1976 wiesen die amtlichen Statistiken sogar einen deutlichen Wohnungsüberschuss aus (Müller-Heine, 1984, 57). Dringenberg (1983) spricht vor dem Hintergrund der sich ändernden Wohnungsmarktlage auch von einer Umorientierung der Wohnungspolitik, während Krummacher (1978) lediglich von einer Akzentverschiebung ausgeht: „Die Neubaupolitik wurde schwerpunktmäßig von der Bestandspolitik abgelöst, Modernisierungsprogramme traten in den Vordergrund; an die Stelle der Wohnungspolitik im engeren Sinne rückte zunehmend eine übergreifende Städtebaupolitik [...]; im Subventionssystem fand eine Verlagerung von der Mietwohnungs- zur Eigentumsförderung statt sowie tendenziell von der Objektförderung zur Individualförderung durch Wohngeld“ (Dringenberg, 1983, 153).

Zahlreiche Reformen der sozialliberalen Koalition waren „unter reichlich optimistischen Annahmen über die zukünftige Wirtschaftsentwicklung getroffen" worden (Ostheim u. Schmidt, 2007b, 167f.). Mit dem ersten Ölpreisschock 1973/74 verschlechterte sich die gesamtwirtschaftliche Lage deutlich und die Grenzen des Wachstums rückten ins öffentliche Bewusstsein (Harlander, 2008, 825). Das Wirtschaftswachstum ging deutlich zurück, während Arbeitslosenraten stiegen. Basierend auf dem Stabilitätsgesetz von 1967 reagierte die sozialliberale Koalition auf den wirtschaftlichen Einbruch mit stabilisierenden Maßnahmen sowie mit Kürzungen im sozialpolitischen Bereich (Ostheim u. Schmidt, 2007b, 
168). Es ging nicht mehr um eine Ausweitung des Interventionsstaates, „sondern in erster Linie um Deregulierung“ (Harlander, 2008, 825). Hatte die Rezession Ende der 60er Jahre das Vertrauen der Bevölkerung in die Wirtschaftspolitik der Konservativen geschwächt, so lässt sich mit Lehnert seit der Jahreswende 1973/74 von einer „Identitäts\& Legitimationskrise der sozialliberalen Koalition" sprechen (Lehnert, 1984, 20):

,[I]m Zeichen des ökonomischen Kriseneinbruchs, der von einem Mentalitätswechsel (Sicherheitsdenken statt Reformerwartungen) begleitet war, [fand] die Rückkehr zu früheren, historisch und strukturell eingewurzelten, politischen Kräften, politischen Kräfteverhältnissen verstärkte Resonanz." (Lehnert, 1984, 20)

Nach einer stagnativen Phase der Bauwirtschaft in den Jahren 1969/70 schlug die die plötzliche Boomphase Anfang der 70er Jahre, in die bislang schwerste Krise des ohnehin krisenanfälligen Baugewerbes“ um (Krummacher, 1978, 277). Zunächst hatte die sozialliberale Koalition versucht den Bauboom im Rahmen der Stabilitätspolitik einzudämmen - so zum Beispiel durch die temporäre Aussetzung der steuerlichen Abschreibungsmöglichkeiten nach §7b EStG. Diese wirkten sich jedoch prozyklisch auf die Krise der Bauwirtschaft (Krummacher, 1978, 278). Es kam zu einer massiven Zunahme der Konkurse von Baubetrieben, die laut Krummacher 1976 etwa 3 mal höher lag als noch zu Beginn der 70er Jahre (Krummacher, 1978, 279).

Nach einer kurzweiligen wirtschaftlichen Erholungsphase kam es zu Beginn der 1980er Jahre zu einer erneuten Rezession, die zu steigenden Arbeitslosenzahlen und ,erneut verschärften Finanzierungsproblemen 
des Sozialstaats" führte (Ostheim u. Schmidt, 2007b, 169). Erneut zerbrach eine Regierungskoalition vor dem Hintergrund eines wirtschaftlichen Abschwungs. In der Folge führte eine Koalition aus Unionsparteien und FDP die begonnenen Sparmaßnahmen der sozialliberalen Koalition weiter - ,zunächst in höherem Tempo und mit größerem Nachdruck“ (Ostheim u. Schmidt, 2007b, 169). Lehnert beschreibt diesen politische Wandel als einen „Rückfall in die traditionelle konservativ-rechtsliberale Hegemonie nach einem leicht abgemilderten Muster der 50er und frühen 60er Jahre“ (Lehnert, 1984, 21). Deutliche Parallelen zu den konservativen Politiken der 50er und 60er Jahre sind dabei insbesondere darin auszumachen, dass der Konsolidierungskurs zu Beginn der 80er Jahre durch mehrere sozialpolitische Maßnahmen in der zweiten Hälfte der 80er Jahre deutlich abgemildert wurde. Insbesondere im Bereich der Familienpolitik wurde der Sozialstaat weiter ausgebaut. Ostheim und Schmidt nennen in diesem Zusammenhang die Einführung des Erziehungsgeldes und eines Erziehungsurlaubs für Väter und Mütter sowie die Anrechenbarkeit von Kindererziehungszeiten als Beitragszeiten in der Altersrente (Ostheim u. Schmidt, 2007b, 170). Diese Neuerungen wurden begleitet von abermals familienpolitisch begründeten Änderungen im Bereich der Wohneigentumsförderung (vgl. Abschnitt 6.2).

Dabei waren insbesondere die frühen 80er Jahre von erneuten Engpässen auf den Wohnungsmärkten geprägt. Trotz den inflationsgetriebenen Neubautätigkeiten der 70er Jahre ,traten in der Wohnungsversorgung Engpässe auf, die sich unter anderem durch spektakuläre Aktionen (z.B. Hausbesetzungen) mit Nachdruck in das Bewußtsein der Öffentlichkeit einprägten (Dringenberg 1983, 153; Harlander 2008, 842). Diese Hausbesetzungen sind als Teil einer der wichtigsten sozialen Be- 
wegungen der 1970er und 1980er Jahre zu sehen, die sich in vielen westeuropäischen Großstädten herausbildeten (vgl. Kuhn, 2012). In Deutschland äußerte sich diese Bewegung vor allem in Westberlin, wo massive Ausschreitungen im Rahmen einer Räumungsaktion in der Nacht vom 12. auf den 13. Dezember 1980 große mediale Öffentlichkeit erregten (Kuhn, 2012, 38f.). Anlass waren die großräumigen Sanierungsund Neubauarbeiten seit den 60er Jahren, in deren Rahmen große Teile der besonders kostengünstigen Altbaubestände modernisiert und damit verteuert wurden (Kuhn, 2012, 39). In der so genannten ,Schlacht am Fraenkelufer“ vom Dezember 1980 ,kulminierten die langjährigen stadtpolitischen Proteste, welche die Akzeptanz der Sanierungspolitik und der zentralistisch-autoritären Verwaltung der Stadt nachhaltig untergraben und dagegen ihre Vision des städtischen Lebens gesetzt hatten“ (Kuhn, 2012, 42).

Entgegen diesen Engpässen im kostengünstigen Mietwohnungsbau wurde der klassische Soziale Wohnungsbau unter der konservativ-liberalen Bundesregierung weiter geschwächt, wobei sich der Staat zunehmend als Investor auf dem Wohnungsmarkt zurückzog (Mayer, 1998, 199, 201f.). Mit dem „Gesetz zur Änderung des Wohnungsbindungsgesetzes und des Zweiten Wohnungsbaugesetzes“ (Wohnungsbauänderungsgesetz) von 1980 wurde eine bereits seit mehreren Jahren diskutierte Lockerung der Bindungen im Sozialen Wohnungsbau hinsichtlich der Miethöhe und Belegung umgesetzt (Peters, 1984, 218). Als zentrales Argument für die Preisgabe des Sozialen Wohnungsbaus galt das Problem der Fehlbelegung (Mayer, 1998, 201). „Fehlbelegungen“ kamen dadurch zustande, dass Bedürftige zwar eine Sozialwohnung erhalten konnten, diese aber nicht mit dem Ende ihrer Bedürftigkeit verlassen 
mussten (vgl. Egner, 2014). Mit dem 1981 erlassenen „Gesetz zum Abbau der Fehlsubventionierung und der Mietverzerrung im Wohnungswesen“ (AFWoG) sollten weitergehende Mängel der Objektförderung kompensiert werden. Wie bei den vorhergegangenen Deregulierungen wurde auch dieser Rückzug des Staates mit neuen Schutzbestimmungen für Mieter flankiert, um nach Möglichkeit Härten für Betroffene zu vermeiden (vgl. Peters, 1984, 218ff.). Einen wichtigen Meilenstein für diesen Abbau markierte ironischerweise das sogenannte, ,Sonderprogramm zur Belebung des Sozialen Wohnungsbaus und der Baunachfrage" für die Jahre 1983 und 1984. Im Rahmen dieses Programms sollten die Bundesmittel künftig nicht mehr in den klassischen Sozialmietwohnungsbau, sondern in den Zweiten Förderweg und die Förderung von Eigentümerwohnungen fließen. Seit 1986 wurden Bundesmittel im Rahmen des Sozialen Wohnungsbaus nur noch für Eigentümerwohnungen zur Verfügung gestellt, während Investitionen für Mietwohnungen im Rahmen des Sozialen Wohnungsbaus vollständig von Ländern und Kommunen aufgebracht werden mussten (Mayer, 1998, 206).

Mit der Abnahme der bereitgestellten Mittel für den Sozialen Wohnungsbau erlangte die steuerliche Förderung für das selbstgenutzte Wohneigentum immer größere Bedeutung. Dies war jedoch nicht nur im Bereich der Wohnungspolitik der Fall. So hatte sich bereits die sozialliberale Koalition mit ihrer ,als Jahrhundertwerk gepriesene[n] Steuerreform, die am 1. Januar 1975 in Kraft trat" (Feige, 1979, 49), darum bemüht, vor allem durch steuerliche Erleichterungen Konsumanreize zur Krisenbewältigung einzusetzen. Mit dem Steueränderungsgesetz von 1978 wurde zudem ,ein neuer, diesmal durchgehend progressiver Steuertarif eingeführt" (Feige, 1979, 50). Auch die Steuervergünstigun- 
gen im Rahmen der Eigenheimförderung waren unter der sozialliberalen Koalition bereits mehrfach in kleinerem Umfang angepasst worden (Leutner, 1990, 213ff.). So wurden 1977 steuerliche Abschreibungsmöglichkeiten erstmals auch für den Kauf bestehender Objekte geschaffen (Kofner 2004, 181; Leutner 1990, 380; Jokl u. Zehnder 1996, 407). Dementsprechend waren auch deutliche Anstiege in der Nutzung der steuerlichen Abschreibungsmöglichkeiten im Eigentümerwohnungsbau zu verzeichnen. Laut Leutner (1990) stiegen die Steuerverzichte von 1969 bis 1975 von 690 Millionen auf 1,55 Milliarden DM an und „bedingt durch die Ausweitung der Regelungen auf Gebrauchtwohnungen - zwischen 1967 und 1982 von 2,5 [sogar] auf 4,8 Milliarden DM“" (Leutner, 1990, 236). Auch unter der seit Ende 1982 regierenden CDU/CSUFDP-Koalition wurden vor allem steuerliche Erleichterungen zur Förderung der Eigenheimquote forciert. Dazu wurden weitere steuerliche Erleichterungen und direkte Förderungen zur Wohneigentumsbildung und Bausparförderung beibehalten und weiter ausgebaut (Mayer, 1998, 205). 1982 wurde zusätzlich mit dem Haushaltsstrukturgesetz das sogenannte „Baukindergeld“ erstmals eingeführt, das eine gesonderte Unterstützung für Bauherren mit Kindern beinhaltete und „die monetären Anreize zum Erwerb von Eigenheimen nochmals beträchtlich [erhöhte]“ (Egner 2014; siehe auch Kofner 2004, 187). ${ }^{3}$ Das Baukindergeld wurde in den Jahren 1990 und 1991 schrittweise angehoben. Im selben Jahr wurde das ,Sofortprogramm zur Wiederbelebung des Wohnungsbaus“

3 Das Baukindergeld ist keine direkte Förderung im Sinne einer Unterstützungszahlung, sondern eine steuerliche Sonderbehandlung von Bauherren mit Kindern. So konnte nach §34f. EStG „,vom zweiten Kind an jährlich 600 DM je Kind als Abzug von der Steuerschuld geltend gemacht werden“ Eigenheimerverband Deutschland e.V (2016) 
verabschiedet, mit dem die neue Regierung ein wirtschaftspolitisches Zeichen setzen wollte (Harlander, 2005, 688). Entgegen der Erwartungshaltung der neuen Bundesregierung trug das Programm jedoch nicht zu einer anhaltenden Verbesserung der Wohnraumversorgung bei.

Insgesamt wurden während der zweiten Phase der Wohneigentumsförderung die massiven Wohnungsnöte der Nachkriegszeit beseitigt. Allerdings kam es immer wieder zu lokalen Versorgungsengpässen, die sich in den wohnungspolitischen Diskussionen wiederfinden. Aus der allgemeinen Versorgungslage mit Wohnraum lässt sich der weitere Ausbau der Eigenheimförderung somit weiterhin nicht erklären. Eine alternative Erklärung für die unter der sozialliberalen Koalition ausgebaute Wohneigentumsförderung wäre eine konjunkturpolitische Maßnahme zur Stärkung der Bauwirtschaft Mitte der siebziger Jahre. Bereits in den 60er Jahren war die Bauförderung immer wieder als konjunkturpolitische Maßnahme genutzt worden (vgl. Jokl u. Zehnder 1996; Kofner 2004, 186). Wie nun insbesondere die Förderung von Wohneigentum zu einer Förderung der Bauwirtschaft beitragen kann, erläutert der CDUAbgeordnete Daniels wie folgt:

„Das führt dazu, daß sich Wohneigentümer z. B. sehr viel mehr um ihre Wohnung kümmern, als das Mieter tun. Sie geben im Durchschnitt einen sehr viel höheren Anteil ihres Einkommens für ihre Wohnung aus als Mieter. Sie stecken auch mehr eigene Leistung in die Wohnung hinein. Das bedeutet indirekt auch eine Förderung der Bauwirtschaft." (BT-Drs. 10/156, 11684B)

Mit dem Anspruch möglichst starke Wachstumsimpulse zu erzielen, setzte die sozialliberale Koalition stärker auf den Bau von Eigenheimen, 
deren Herstellungskosten erheblich über denen des Mietwohnungsbaus liegen (Krummacher, 1978, 285). Hinzu kommt laut Krummacher die durchschnittlich höhere Eigenkapitaleinbringung der Bauherren von Eigenheimen, was einen höheren Wachstumsimpuls bei gleichem Subventionsvolumen erlaubt (Krummacher, 1978, 285). Im Vordergrund der politischen Debatten rund um die Eigenheimförderung standen jedoch andere Zielsetzungen, die im folgenden Abschnitt eingehender beschrieben werden.

\subsection{Individuelle Vermögensbildung durch Wohneigentum}

Durch das Gesetz zur Förderung von Wohneigentum von 1976 (Wohnbesitzgesetz) sollte das Wohneigentum in Deutschland weiter über den Sozialen Wohnungsbau gefördert werden. Nach jahrelangen Debatten und dreimaligem Aufrufen des Vermittlungsausschusses konnte das Gesetz einstimmig verabschiedet werden (vgl. Krummacher, 1978, 340). Ausgangspunkt waren konkurrierende Gesetzesentwürfe der Regierungsparteien sowie von CDU und CSU, ,denen gemeinsam die jeweils ausdrückliche Erklärung war, mit neuen Fördermodellen den einkommensschwächeren Bevölkerungsschichten den Zugang zur Vermögensbildung in Form des Wohnungseigentums zu ermöglichen" (Krummacher, 1978, $341)$.

Als Kernstück des Gesetzes wird häufig die Einführung der sogenannten „Wohnbesitzwohnung“ beschrieben: Diese beinhaltete als eine Mischform aus Eigentums- und Mietwohnung ein Dauerrecht in einer Mietwohnung und einen vom dort Wohnenden gehaltener Anteil an 
einem geschlossenen Immobilienfonds (Simian 2000, 86; Müller-Heine 1984, 42). Laut Simian (2000) konnte sich das neue Instrument aufgrund der komplizierten rechtlichen Ausgestaltung sowie der in der Praxis nur geringen Vorteile für den Mieter nicht durchsetzen. ${ }^{4}$ Auch Krummacher bewertet das Instrument der Wohnbesitzwohnung überaus kritisch und bemerkt, dass sowohl Länge und Intensität der Debatten ,in einem krassen Mißverhältnis zur Bedeutung der eigentlichen Neuerung" gestanden hatte (Krummacher, 1978, 340). Als wesentlicheren Punkt in der rechtlichen Neuerung sieht er die im Rahmen dieses Gesetzes vorgenommene Anpassung des Zweiten Wohnungsbaugesetzes, „daß künftig die Förderung des Wohnungsbaus überwiegend der Bildung von Einzeleigentum (Familienheime und eigengenutzte Eigentumswohnungen) dienen muß, d.h. mit mehr als der Hälfte der öffentlichen Mittel zu fördern ist"(Krummacher, 1978, 288). Zuvor war der Vorrang der Eigenheimförderung zwar bereits im Gesetz enthalten, jedoch noch nicht verpflichtend gewesen (Krummacher, 1978, 352). Ein weiterer wichtiger Impuls des neuen Gesetzes war die Umwandlung von Sozialwohnungen in Eigentumswohnungen.

Die Abschreibungsmöglichkeiten für den Wohnungsbau wurden seit der Phase des Wiederaufbaus durch den $\S 7 \mathrm{~b}$ des Einkommenssteuergesetzbuches (EStG) geregelt. Die ursprünglich nur auf Bauherren von Mehrfamilienhäusern beschränkte Regelung wurde bereits 1953 im

4 Die oppositionellen Gegenstimmen der CDU befassen sich einerseits überaus intensiv mit der Kritik an dem neuen Konzept der Wohnbesitzwohnungen und plädierten energisch dafür „echtes privates Eigentum“ (BT-Drs. 07/036, 2019C) zu schaffen - unter anderem auch, weil durch einen größeren privaten Anteil an der gesamten Finanzierungsleistung auch die öffentlichen Kassen deutlich weniger belastet werden würden. 
Rahmen einer allgemeinen Wohnraumförderung auf Ersterwerber von Einfamilienhäuser und Eigentumswohnungen ausgeweitet. Allerdings sollte eine 1958 eingeführte Kostenobergrenze von 120.000 DM sicherstellen, dass Besserverdiener nur noch in geringerem Maße von den Abschreibungsmöglichkeiten profitieren (Kofner, 2004, 186). Bereits 1977 wurde von der sozialliberalen Koalition der $\S 7 \mathrm{~b}$ erstmals ausgeweitet auf Bestandswohnungen und Modernisierungsinvestitionen, um im Rahmen konjunkturfördernder Maßnahmen die Wohnqualität auch in den Städten zu erhöhen (vgl. Abschnitt 6.1). Die 80er Jahre sollten zahlreichere Änderungen für die steuerlichen Abschreibungsmöglichkeiten von Wohneigentum mit sich bringen. Die grundlegendste Reform bestand im Steueränderungsgesetz von 1987: Die bis dahin bestehende Nutzungswertbesteuerung für Wohneigentum nach §7b EStG wurde gestrichen und im $\S 10$ e den steuerabzugsberechtigten Sonderausgaben zugeordnet (Leutner, 1990, 382ff.). Mit den Anpassungen wurde nicht nur die steuerliche Belastung des Wohneigentums gestrichen, sondern auch die Bemessungsgrundlagen der steuerlichen Absetzbarkeit von 200.000 DM auf 250.000 DM für Einfamilienhäuser und Eigentumswohnungen deutlich ausgeweitet (Kofner 2004, 187f.; Jokl u. Zehnder 1996, 408). Entgegen der direkten Förderung ist diese steuerliche Förderung als regressiv zu bewerten. So weist Leutner (1990) darauf hin, dass Haushalte mit mittlerem und hohem Einkommen durch die steuerliche Abzugsfähigkeit überproportional begünstigt wurden (Leutner, 1990, 377).

Diese Zielsetzung der möglichst zielgenauen Förderung findet sich auch in den kritischen Stimmen auf Seiten der SPD und der Grünen als „fehlgeleitete Förderung“" So kritisieren die SPD-Abgeordneten gemeinsam mit den seit 1983 erstmals im Bundestag vertretenen Grünen, 
dass die progressiv steigenden steuerlichen Abschreibungsmöglichkeiten insbesondere den Steuerzahlern zugute kämen, die auch viele Steuern abzusetzen hätten:

„nach dem Motto also: Wer nur über ein geringes Einkommen verfügt und deshalb dringend auf staatliche Hilfe beim Erwerb von Wohneigentum angewiesen ist, bekommt wenig staatliche Förderung; wer viel verdient und entsprechend weniger oder überhaupt keine staatliche Förderung benötigt, bekommt viel.“ (BT-Drs. 10/156, 11688B)

Auch von der im Gesetz enthaltenden steigenden Kinderkomponente würden progressionsbedingt Familien mit einem höheren Einkommen deutlich stärker profitieren können als schlechter Verdienende. In diesem Zusammenhang erwähnen die oppositionellen Abgeordneten auch die hohen Kosten, die durch diese Subventionierung der Wohneigentumsförderung entstünden. War es in den vergangenen Debatten vor allem der Vermerk auf den zu fördernden Mietwohnungsbau - zur Not auch mit höherem Aufwand öffentlicher Gelder - kritisieren die Abgeordneten in dieser Debatte die hohen Gesamtkosten als die größte Einzelsubvention im Budget des Staatshaushalts (vgl. insbes. BT-Drs. 10/11680B) und bedienen damit staatliche Sparsamkeit als eine der im weiteren Verlauf bedeutender werdenden Zieldimension der Eigenheimförderung.

Zwar blieben bei den erneuten Fördermaßnahmen die bisherigen familienpolitischen Zielsetzungen erhalten. Allerdings kamen in dieser Phase auch neue Ideen hinzu, die sich als Zielsetzungen und Argumente in den wohnungspolitischen Diskussionen wiederfinden lassen. Zusätzlich wurden nun individualitäts- und freiheitsfördernde Eigen- 
schaften des Wohneigentums betont und die vermögenspolitische Bedeutung des eigenen Wohnraums hervorgehoben (vgl. auch Szypulski, 2008, 81). Hinzu kam als Novum in der bundesdeutschen Eigenheimförderung die Orientierung an statistischen Kennzahlen wie der Wohneigentumsquote. Diese wurde nicht nur als Kennzahl zur allgemeinen Zielerreichung verwendet, sondern oftmals bereits als Ziel an sich. So war es das erklärte Ziel der konservativ-liberalen Regierung die als zu niedrig empfundene Eigenheimquote auf 50 Prozent zu steigern (Mayer 1998, 205; Müller-Heine 1984; Mertens 2015, 311; vgl. auch BT-Drs. 10/156, 11667A). Gegenüber dem Quotenziel verloren andere politische Zielsetzungen zunehmend an Bedeutung:

„Große ideologische Debatten wie in den vorhergehenden Jahrzehnten gab es um ein ein solches Ziel in den achtziger Jahren nicht mehr." (Harlander, 2005, 690)

Als entscheidende Grundlage für diese neuen Zielvorstellungen ist die generelle Verfügbarkeit von wohnungsmarktspezifischen Daten, deren Entwicklung bereits in der ersten Phase durch die amtliche Statistik vorangetrieben worden war und die sich in der zweiten Phase zunehmend etablierten (vgl. Abschnitt 6.3). Gerahmt wurden diese Zielvorstellungen erstens von der Annahme, dass sich die Wohnungsmärkte in einem Gleichgewicht befinden und somit enge wohnungspolitische Zielsetzungen in den Debatten rund um die Eigenheimförderung keine Bedeutung mehr haben mussten. So erklärt der CDU-Abgeordnete Jahn: „Unser Ziel lautet nicht, jedem eine, sondern jedem seine Wohnung. [...] Das ist mehr als eine Politik der bloßen Sicherung des Wohnens, die wir bereits verwirklicht haben" (BT-Drs. 07/126, 11346D). Die zweite grundlegende Annahme hinter den wohnungspolitischen Debatten 
besteht in einer generellen Kontrollierbarkeit der wohnungswirtschaftlichen Entwicklung, wie sie sich auch in anderen Politikbereichen dieser Zeit finden lassen (vgl. Abschnitt 6.1).

\subsubsection{Zielgenauigkeit \& individualisierte Förderung}

Im Rahmen der wohnungspolitischen Debatten der 70er Jahre spielte bereits die Treffsicherheit eigentumsfördernder Maßnahmen eine bedeutende Rolle (vgl. Harlander, 2008, 831f.). Die Argumentation der FDP, welche sich bereits bei vorhergegangenen Gesetzesinitiativen für die Ausweitung der Förderung der Eigentumswohnung stark gemacht hatte, orientierte sich in diesem Punkt besonders an den Bedarfen der Ballungsgebiete und städtebaulichen wie auch raumordnerischen Gesichtspunkten. Dementsprechend forderten ihre Abgeordneten auch eine möglichst gezieltere Förderung in Bezug auf Lokalität als auch auf die jeweiligen Bedürfnisse der Geförderten:

„Es wird klargestellt, daß die Förderung in den Gebieten vordringlich ist, wo ein besonderer Wohnungsbedarf besteht, und daß zunächst einmal jener Personenkreis gefördert wird [...], der zu den wirtschaftlich Schwächeren gehört und sich nicht aus eigener Kraft in gleicher Weise wie andere helfen kann. Das reicht von den kinderreichen Familien über die jungen Ehepaare, die älteren Menschen bis hin zu den Schwerbehinderten.“ (BT-Drs. 07/036, 2021C)

Bereits in der Zielsetzung zum Gesetzentwurf der Bundesregierung steht zu Beginn, es sei die Aufgabe der öffentlichen Wohnungsbauförderung, ,sich um den Ausgleich des Spannungsverhältnisses auf den 
regionalen Wohnungsmärkten zu bemühen und das Wohnungsangebot nach Wohnungsart, Wohnungsform und Preis so aufzufächern, daß dabei den unterschiedlichen Bedürfnissen der Wohnungssuchenden möglichst weitgehend Rechnung getragen werden kann“ (BT-Drs. 07/577A). Wohneigentum - so der Gedanke - ,ist eine ganz wesentliche Möglichkeit, den einzelnen unabhängiger zu machen und seinen Freiheitsraum zu erweitern“ (BT-Drs. 07/036, 2021D). Auch die CDU-Abgeordneten verweisen in ihren Argumentationen mehrfach darauf, dass vor allem ein „breit gefächertes Angebot auf den Markt zu bringen“ sei, sodass „der Konsument [...] entscheiden [soll], welches Angebot er akzeptieren will“" (BT-Drs. 07/036, 2019C). Staatliche Unterstützung wird damit nicht mehr nur zur Befriedigung allgemeiner Wohnbedarfe verwendet, sondern um gezielt ihre individuellen Bedürfnisse als Konsumenten zu befriedigen. Dementsprechend bestand die Herausforderung darin, möglichst präzise der bestehenden individuellen Nachfrage gerecht werden können.

Auch vonseiten der SPD und FDP wurde in den 70er Jahren die Sicherstellung der bedarfsgerechten Wohnraumversorgung über das Mittel der Eigenheimförderung in den Vordergrund gestellt. So erklärt der SPD-Abgeordnete Krockert:

,[...] wir orientieren uns schon längst nicht mehr daran, ob irgendwann alle Leute ein Dach über dem Kopf haben. Wir orientieren uns vielmehr wohnungspolitisch an der sich weiter entwickelnden Bedürfnisvielfalt, der ein entsprechend differenziertes Angebot gegenüberstehen soll“ (BTDrs. 07/126, 11352A)

Der FDP-Abgeordnete Engelhard erklärt hierzu: 
„Ich glaube, es ist insbesondere gerade im Bereich des sozialen Wohnungsbaus ein sehr wichtiges Anliegen, daßs es uns künftig weniger um die bloße Unterbringung der Menschen gehen darf, sondern zunehmend um die Befriedigung ihrer eigentlichen Wohnbedürfnisse." (BT-Drs. 07/126, 11365D)

Die generellen Unterschiede in der allgemeinen Zielsetzung erscheinen mit etwas Distanz jedoch deutlich kleiner als es die zu diesem Zeitpunkt bereits lange währenden Debatten vermuten lassen. So stellt der CDU-Abgeordnete Orgaß in seinem Redebeitrag besonders prägnant heraus, dass ,die Auffassungen hinsichtlich der grundsätzlichen Zielsetzung der breiten Eigentums- und Vermögensstreuung näher beieinanderliegen, als es der Verlauf der Diskussion bisher vermuten läßt" (BTDrs. 07/126, 11371D). Gegenüber den vorherigen Debatten der 50er und 60er Jahre wird deutlich, dass es keine Grundsatzdebatte mehr um eine groß angelegte Sozialisierung von Wohneigentum vonseiten der SPD gibt. Vielmehr erscheint die SPD hinsichtlich der Wohneigentumsförderung nun deutlicher in Richtung der Positionen der Unionsparteien sowie der FDP gerückt zu sein.

Vorstellungen zur Präzisierbarkeit politischer Maßnahmen sind nicht nur im Bereich der Wohnungspolitik - eine wesentliche Änderungen gegenüber den Anfangsjahren der Bundesrepublik. Vor dem Hintergrund der in Abschnitt 6.1 geschilderten Vorstellungen zur Globalsteuerung und dem großen Vertrauen in die technischen Möglichkeiten der erfolgsichernden Steuerbarkeit politischer Maßnahmen erscheinen diese Vorstellungen im Bereich der Wohneigentumsförderung als Spiegelung eines politikfeldübergreifenden Trends.

Die angestrebte Zielgenauigkeit und Präzision der Förderung birgt 
neben der individuellen Zuschneidbarkeit noch ein weiteres Potenzial mit sich: Einsparungen durch effizienteren Mitteleinsatz. So werden Bestrebungen in der Zusicherung von mehr Unabhängigkeit und Individualität der Bürger in den Bundestagsdebatten der 1980er Jahre zunehmend mit der Vorstellung eines schlanken Staates verknüpft, der sich mit bürokratischen Umständlichkeiten aus dem Leben der Bürgerinnen und Bürger zurückziehen sollte. So erklärt der parlamentarische Staatssekretär Dr. Häferle in seinen Ausführungen: „Der Kernpunkt ist, daß künftig die Besteuerung des selbstgenutzten Wohneigentums wegfallen soll. Dies ist eine wichtige Weichenstellung in Richtung weniger Staat, weniger Bürokratie" (BT-Drs. 10/156, 11666D). Diese Argumentation entspricht weitestgehend den Forderungen der FDP-Abgeordneten, die einen weiteren Bürokratieabbau und zugleich eine Intensivierung der Eigenheimförderung auf Basis steuerlicher Abschreibungsmöglichkeiten fordern. Im Einklang mit diesem Ideal des freien Bürgers erscheint auf Seiten der Regierungsparteien wiederholt die bereits in den 70er Jahren aufkommende Argumentation, dass Eigentum insbesondere eine befreiende Wirkung für den Eigentümer habe. So erklärt der CDU-Abgeordnete Daniels: „Eigentum an der selbstgenutzten Wohnung stärkt die Unabhängigkeit. Es erhöht die Selbständigkeit, und es fördert auch die Selbstverantwortung“" (BT-Drs. 10/156, 11684B). Wie eng der Abgeordnete die Ziele des Bürokratieabbaus und der Selbstverwirklichung der Bürger miteinander verwoben sieht, wird vor allem in seinem Schlussplädoyer der ersten Lesung des Gesetzes erkennbar: „Abbau von Staat und Bürokratie auf der einen, Förderung von Eigentum, Selbständigkeit und Selbstverantwortung auf der anderen Seite, gezielte Hilfe, wo sie nötig ist, das sind die Grundprinzipien dieses Gesetzes" 
(BT-Drs. 10/156, 11685C).

Die Gegenthese zur individuellen Freiheit durch Eigentum wird hingegen von Abgeordneten der seit 1983 im Bundestag vertretenen Grünen vorgebracht: „Eigentum macht ja — das muß man sich auch einmal deutlich machen - nicht frei, sondern bringt den normalen Erwerber von Hauseigentum in eine 30-jährige Abhängigkeit von den Banken“ (BT-Drs. 10/156, 11674B), erklärt der Abgeordnete Vogel und bringt damit einen Kritikpunkt hervor, der in vorherigen Debatten kaum zur Geltung kam: die Abhängigkeit und eingeschränkte Handlungsfähigkeit durch die mit dem Wohneigentum verbundenen Kredite. Zuvor hatten lediglich am Rande Möglichkeiten des arbeitsbedingten Umzugs und der damit notwendigen Veräußerung des Eigenheims eine Rolle gespielt. Insgesamt plädieren die Grünen als einzige Partei gegen die Förderung des klassischen Wohneigentums mit öffentlichen Mitteln.

Die gesteigerte Abhängigkeit von Finanzinstituten anstelle von staatlicher Hilfe zeigt sich auch in der nachlassenden staatlichen Förderung der Eigenkapitalbildung. Zwar wurden neue Möglichkeiten zur Zinsverbilligung von Bausparkrediten eingeführt (vgl. Kofner, 2004, 187). Allerdings wurde zugleich das Bausparen immer weniger gefördert. Die ursprüngliche Bausparprämie sank von den ursprünglich 25 Prozent aus dem Jahr 1952 schrittweise auf 14 Prozent bis 1982 und auf 10 Prozent im Jahr 1989 (Jokl u. Zehnder, 1996, 408). Zusätzlich kam es zu immer mehr Zwangsversteigerungen, die auch von Abgeordneten der SPD als Argument dafür genutzt werden, dass durch die immer schlechter werdende Eigenkapitalausstattung insbesondere die einkommensschwachen Schichten durch die Wohneigentumsförderung keine maßgeblichen Vorteile erzielen konnten (vgl. BT-Drs. 10/156, 11682A). 


\subsubsection{Erlebbares und anlegbares Vermögen}

In den 70er Jahren treten erstmals vermögenspolitische Zielsetzungen der Wohneigentumsförderung in den Vordergrund. War in der ersten Phase der Eigenheimförderung die Vermögensbildung noch ein Mittel zur Entproletarisierung, wurde sie in der zweiten hier betarchteten Phase zu einem Ziel an sich. Dabei gab es allerdings verschiedene Begründungszusammenhänge. Die SPD-eigene Akzentuierung der neuen Eigentumsförderung besteht in der „Beteiligung der Arbeitnehmer am Produktivvermögen“ (BT-Drs. 07/036, 2015D), so der zuständige Bundesminister für Raumordnung, Städtebau und Bauwesen Vogel. Da das Produktivvermögen der gewerblichen Wirtschaft nur um rund 20 Prozent größer sei als das Wohnungsvermögen, müsse man „,neben dem Produktivvermögen auch das Grund- und Gebäudevermögen im Auge behalten, seiner übermäßigen Konzentration entgegenwirken und seine möglichst breite Streuung anstreben“ (BT-Drs. 07/036, 2016A, HvH. i. Orig.). Und weiter: ,Alle, die es mit der Vermögensbildung breiter Schichten unseres Volkes ernst meinen, können dies bei der Mitarbeit an diesem Gesetz unter Beweis stellen“ (BT-Drs. 07/036, 2017D). Ein wesentliches Argument vonseiten der FDP bestand weiterhin darin, dass durch die Schaffung von Einzeleigentum auch die ,wirtschaftliche Machtkonzentrationen auf dem Wohnungsmarkt" (BT-Drs. 07/036, 2022B) namentlich auf Seiten der gemeinschaftlichen Wohnungsunternehmen stärker entflechtet werden müssten.

Der Hintergrund zu dieser Forderung besteht in der Diagnose eines verteilungspolitischen Problems, dessen Ursprung den hohen Bau- und Bodenpreissteigerungen zugeschrieben wurde (vgl. Müller-Heine, 1984, 40): Aufgrund der größeren Nutzungsmöglichkeiten und Wertsicherheit 
der Immobilien gegenüber dem Produktivvermögen sahen Vermögenspolitiker in der Eigentumsförderung die Möglichkeit, zu einer dauerhaften Vermögensbildung in weiten Kreisen der Bevölkerung beizutragen. Für den vermögenspolitischen Aspekt des Wohneigentums ist es ebenso von Bedeutung, dass die Sparleistung der Bauherren in einem neuen Bedeutungszusammenhang steht. Auch die Möglichkeiten mithilfe des Wohneigentums die ,eigene Sparleistung mit möglichst großer Unabhängigkeit im Wohnen zu verbinden“ (BT-Drs. 07/036, 2020A) tritt erstmals explizit und wiederholt in den Debatten zur Förderung von Wohneigentum auf. Thematisiert wird dieser Punkt sowohl von Mitgliedern der SPD- auch von denen der CDU/CSU-Fraktion. In früheren Debatten war die eigene Sparleistung der Förderungsempfänger stets als zu belohnende Tugend stilisiert worden, welche in praktischer Hinsicht das fehlende Kapital des Staates zur ausreichenden Wohnraumversorgung substituieren, und entsprechend gefördert werden sollte. Nun floss die Spartätigkeit als individuelle Präferenz der Bürgerinnen und Bürger selbst in die Debatten mit ein. Auch in der Zielsetzung des Gesetzentwurfs der Bundesregierung heißt es, dass sich „bei einer ständig wachsenden Zahl von Arbeitnehmerhaushalten nicht unerhebliche Spar- und Vermögensbeträge" angesammelt hätten, die einer weiteren Anlageform bedürften (BT-Drs. 07/577A). Somit wird die vorherige Sparsamkeitserfordernis im Sinne der Frage Wie können Bürgerinnen und Bürger ihre Wohnraumversorgung am besten absichern? zu einer vermögenspolitischen Überlegung: Wie können Bürgerinnen und Bürger Ersparnisse generieren und ihr Vermögen vermehren? Diese Debatte um Wohnen als Vermögensanlage, als Wohnvermögen oder auch Betongold, trat derart explizit erstmals in diesen Debatten der Eigen- 
tumsförderung auf und verweist auf eine wesentliche Änderung der mit Wohneigentum verknüpften Ideen im Rahmen der Wohneigentumsförderung in Deutschland.

Vor dem Hintergrund dieser vermögenspolitischen Debatte erhielt auch die Bausparförderung einen wesentlichen Anteil in den Debatten. Kornemann hebt hervor, dass die Maßnahmen der Neuregelungen im Bereich der Bausparförderung jenen in der Wohneigentumsförderung entgegengelaufen wären. So seien durch das Einkommenssteuerreformgesetz (EStRG) vom 5. August 1974 über die Einführung von Einkommensgrenzen sowie die Kürzung der Höchstsparleistungen und Prämiensätze die staatlichen Fördermaßnahmen reduziert worden. Verstärkt worden wäre dies zusätzlich durch das von Sparmaßnahmen im Zuge der Wirtschaftskrise bestimmte Haushaltsstrukturgesetz vom 18. Dezember 1975, in dem die Prämiensätze erneut herabgesetzt wurden (vgl. Kornemann, 1996, 137). Dieser Einschätzung steht diejenige Krummachers entgegen, der anführt, dass selbst nach der Einführung der Einkommensobergrenzen nach den damaligen Einkommensstrukturen weiterhin 80 Prozent aller Haushalte von der Prämie profitieren konnten (Krummacher, 1978, 334). ,Alternativ [...] oder bei Überschreitung der dort geltenden Einkommensgrenzen bietet sich für Bausparer die Möglichkeit der steuerlichen Abzugsfähigkeit von Bausparbeiträgen im Rahmen des Sonderausgabenabzugs von Vorsorgeaufwendungen bei der Einkommenssteuer" (Krummacher, 1978, 335). Unter Berücksichtigung dieser Rahmenbedingungen erscheinen die Reformen der Bausparförderung unter der sozialliberalen Koalition deutlich weniger einschneidend als dies von Kornemann angenommen wird. Auch der zuständige Bundesminister Ravens betonte, dass ,die Bausparförderung trotz einer 
notwendigen Straffung der öffentlichen Fördermittel" erhalten werden müsste und setzte sich entsprechend dafür ein (Seeger, 1995, 123f.).

Gegenüber der allgemeinen Vermögensbildung tritt in den 1980er Jahren der Aspekt der Altersvorsorge in den Bundestagsdebatten als Argument ebenfalls deutlicher zum Vorschein als dies bis dahin der Fall gewesen ist. Gegenüber der Beteiligung möglichst breiter Schichten am Produktivvermögen, treten die privaten Investitionsleistungen zur individuellen Altersvorsorge in den Vordergrund. Mitunter wird das Ziel der privaten Altersvorsorge dem der Familienförderung gleichgestellt, wie die Aussage des zuständigen parlamentarischen Staatssekretärs beim Bundesministerium der Finanzen Häferle (CDU) zeigen:

„Die Eigentumsförderung steht hier im Vordergrund. Wir wollen als die vierte Säule der Lebens- und Altersvorsorge — neben der allgemeinen Alters- und Sozialrente, der Betriebsrente und dem Privatsparen und -versichern — das „erlebte Eigentum“ des eigenen „Häusles“ verstärken. Zugleich wollen wir die Familie stärken, indem die Förderungsmaßnahmen für die Familien besonders ausgeprägt werden." (BT-Drs. 10/156, 11666D)

In der zweiten Lesung des Gesetzes geht Häferle auf den Kontext der neuen altersvorsorgeorientierten Diskussion ein: „Denn wir alle wissen, daß die Altersversorgung im nächsten Jahrhundert vermehrt den eigenverantwortlichen Anteil braucht. Das ist ein wesentlicher Bestandteil dieses Gesetzes" (BT-Drs. 10/208, 15992A). Die Einordnung der Wohneigentumsförderung als familienfördernde Maßnahme mit gleichrangiger Bedeutung für die private Altersvorsorge entspricht auch derjenigen des CDU-Abgeordneten Schulhoff: 
„Hinzu kommt, daß die gleichrangige Förderung des Erwerbs von gebrauchtem Wohnraum vor allem jungen Familien helfen wird, die schon früh Eigentum erwerben wollen. Damit leistet die Regierung einen weiteren Beitrag zur Vermögensbildung. Gleichzeitig ist dies als ein weiterer Schritt zur privaten Altersvorsorge zu sehen; denn Bildung von Wohneigentum ist ein wichtiger Bestandteil der Alterssicherung, meine Damen und Herren." (BT-Drs. 10/156, 11671C)

Auch FDP-Politiker sehen die Vermögensbildung als einen wesentlichen Aspekt der Wohneigentumsbildung, wobei jedoch an den Äußerungen des Abgeordneten Gattermann deutlich wird, dass die Interpretation als Altersvorsorge noch keine überbordende Dominanz in der Debatte besitzt: „Man kann es gut und gerne als dritte Säule der Alterssicherung bezeichnen“ (BT-Drs. 10/156, 11676D).

Durch diese Zielsetzung wird auch die breite Streuung von Wohneigentum zugleich zu einem Teil der Zielsetzung die private (sic!) Alterssicherung für breitere Schichten zu sichern und nicht nur die eigene Wohnraumversorgung von einer Steigerung der Mieten unabhängiger zu machen. Diese Zuordnung zur Altersvorsorge ist dabei jedoch nicht bloß als ein diskursiver Wechsel zu verstehen, sondern entspricht auch der steuerrechtlichen Neuzuordnung zum Sonderausgabenbereich im $§ 10$ EStG, wie Staatssekretär Häfele erklärt:

„Damit wird deutlich, daß die Förderung des Wohneigentums nach unserem Steuerrecht in der gleichen Linie zu sehen ist wie die eigenverantwortliche Alters- und Lebensvorsorge, etwa beim Abzug der Sozialversicherungsbeiträge, beim Abzug der Lebensversicherungsbeiträge oder der 
Bausparprämien. Gerade beim Abzug von der Bemessungsgrundlage für alle vier Arten zeigt sich, daßs weniger eine Bauförderung hinter dieser Regelung steckt, sondern die Eigentumsidee im Sinne der Lebens- und Altersvorsorge." (BT-Drs. 10/208, 15922B-C)

Allerdings betonen selbst Abgeordnete der schwarz-gelben Regierungskoalition, dass mit dieser Form der Förderung „die bloße Kapitalanlage im Wohnungsbau nicht mehr gefördert werden“ sollte (BTDrs. 10/156, 11683C), um sich von Vorwürfen der Spekulationsförderung der Opposition zu lösen. Begründet wird diese Abwehr damit, dass mit der neuen Regelung Spitzenverdiener nicht mehr ihre Investitionen in mehrere Häuser steuerlich geltend machen könnten. Somit besteht zwar durchaus das klare Ziel die Wohneigentumsförderung zu einer Art staatlich geförderten privaten Altersvorsorge zu stilisieren, allerdings mit einer klaren Distanz zu Spekulationsförderung im Bereich des Wohneigentums. Vor dem Hintergrund der später noch deutlich werdenden Gleichstellung der Investitionen in Wohnungseigentum mit anderen Anlageformen zeigt sich hier noch ein relevanter Unterschied in der Behandlung von Wohneigentum und Kapitalanlagen anderer Art.

\subsubsection{Familienförderung als Rahmenerzählung}

Im Rahmen der Bundestagsdebatten um das Gesetz zur Förderung von Wohneigentum von 1976 zeigen sich weiterhin familienpolitische Idealvorstellungen, die mit der Eigenheimförderung erreicht werden sollten. Auch in Artikel 1 des Wohnbesitzgesetzes von 1976 heißt es: ,In ausreichendem Maße sind solche Wohnungen zu fördern, die die Entfaltung eines gesunden Familienlebens, namentlich für kinderreiche Familien, 
gewährleisten“ (BGBl 1976, Nr. 32, 737), was eine große Konstanz gegenüber der bisherigen Gesetzgebung aufweist. Allerdings kommt Familienförderung in den betrachteten Plenarreden der 70er Jahre deutlich weniger als eigentliche Zielsetzung der Abgeordneten zum Ausdruck. In den Bundestagsdebatten rund um das Steueränderungsgesetz der 80er Jahre zeigt sich demgegenüber eine regelrechte Renaissance familienpolitischer Zielvorstellungen. So erklärt der Staatssekretär beim federführenden Bundesfinanzministerium Häfele (CDU):

„Wir haben bewußt wie bisher die Form des Abzugs von der Bemessungsgrundlage gewählt, weil die Erfahrung lehrt, daß es keine größere Anreizwirkung gibt, als etwas progressionsmildernd zu fördern. Gerade aufstrebende Familien, gerade die, die eine Familie gründen und parallel dazu beruflich vorankommen wollen, werden von dieser Anreizwirkung besonders angesprochen." (BT-Drs. 10/156 11667A)

In der zweiten Lesung ergänzt Häfele, dass auch weitere Verbesserungen ,,vor allem für die Familie“ in Betracht kämen und dass ,gerade für Familien mit mehreren Kindern" die Förderung von Um- und Ausbauten besonders bedeutsam wären (BT-Drs. 10/208, 15992B). Auch die SPD bestärkte ihren Anspruch Eigenheime zu fördern, da dies auch dem Wunsch vieler Bürgerinnen und Bürger entsprechen würde. So erklärt der Abgeordnete Müntefering:

„Es besteht kein Zweifel: Der Wunsch nach Wohneigentum steht bei vielen Bundesbürgern, seien es nun 65 oder $80 \%$, in der Wunschskala ganz oben. Die 100 Milliarden DM an Bausparvertragssummen sind ein schlagender Beweis dafür. 
Auch die Tatsache, daß auch in schwierigen Zeiten der Neubau von Eigenheimen deutlich weniger zurückgeht als der von Mietwohnungen, ist ein Zeichen für diesen Wunsch vieler Mitbürger. Insgesamt aber dümpeln wir mit der Wohneigentumsquote so um die $40 \%$ herum, und es geht nicht so recht voran. (BT-Drs. 10/208, 15993A)

Eine Familienförderung, so Müntefering sei jedoch weniger über steuerliche Förderung, sondern über direkte Zuschüsse zu erreichen, da steuerliche Vergünstigungen ,besonders bei kinderreichen Familien“ nicht reichen würden (BT-Drs. 10/208, 15994B). Das Ideal des Einfamilienhauses als Wohnform für Familien erscheint dabei nicht nur in der Ausgestaltung des neuen Gesetzes, sondern auch im Familienbericht der Bundesregierung 5 aus dem Jahr 1986:

„Die Verfügbarkeit von Wohnung und Garten, auch wenn die Grundstücke und Häuser noch so klein sind, ist ein herausragendes Kriterium, denn nur wer über beides verfügen kann, ist zu einer selbstbestimmten Anpassung der baulichen Gestalt, der Ausstattung und der Nutzung, an die sich ständig im Lebenslauf und Familienzyklus wandelnden Anforderungen in der Lage. In Mietwohnungen ist dies nur in sehr unzureichender Weise möglich [...]. Die zentrale förderungswürdige und -bedürftige Gruppe sind auch unter diesem Gesichtspunkt junge Familien mit Kindern. Sie benötigen und wünschen Wohnungen in der Niedrigbauweise."

5 Die Familienberichte werden in regelmäßigen Abständen - mindestens in jeder zweiten Wahlperiode - von einer Sachverständigenkommission erstellt (siehe hierzu Tiemann u. Wagner, 2013, 4f.). 
(zitiert nach Jokl u. Zehnder, 1996, 392f.)

Allerdings finden sich im Familienbericht auch deutliche Bezüge zu vermögenspolitischen Aspekten der Wohneigentumsförderung, wie sie bereits in den Bundestagsdebatten um das Wohnbesitzgesetz aufkamen. Eine breit angelegte Streuung von Vermögen in Form von Wohneigentum sollte demnach einen, zukunftsorientierten Beitrag zur Daseinsvorsorge" (zitiert nach Jokl u. Zehnder, 1996, 393) leisten. Insgesamt erscheint die Familienförderung in den Bundestagsdebatten zwar häufiger als dies in den betrachteten Debatten der 70er Jahre der Fall war. Bei einer genaueren Betrachtung der Positionierung dieser Zielsetzung fällt jedoch auf, dass sie nie an erster Stelle genannt werden und zumeist als begleitende Zielsetzung zu vermögenspolitischen Zielvorstellungen verwendet werden.

Gegenüber der ersten Phase der Wohneigentumsförderung in der Bundesrepublik zeigt sich eine größere Vielfalt im Spektrum der Ideen in den politischen Debatten der zweiten Phase. Besonders deutlich wird in diesem Zusammenhang die Bedeutungsabnahme der gesellschaftspolitischen Zielvorstellungen durch die Wohneigentumsförderung gegenüber der Befriedigung individueller Wohnbedürfnisse. Dieser Wandel kann als Ausdruck der geringer gewordenen Wohnungsnot gegenüber der Zeit des Wiederaufbaus bewertet werden: Von einer umfassenden Wohnungsnot konnte nicht mehr die Rede sein, sodass es nun weniger um quantitative denn um qualitative Förderungen gehen konnte. Allerdings mussten diese realen Änderungen auch den politischen Akteuren vermittelt werden - ebenso wie die neuen Ideen zur qualitativen Verbesserung der Wohnraumversorgung. Daher soll im folgenden Abschnitt genauer betrachtet werden, wie sich die Wissens- und Ideenproduktion 
in diesem Zeitraum gegenüber der ersten Phase der wohnungspolitischen Wissensproduktion geändert hat.

\subsection{Nachfragegesteuerte Ideenproduktion}

Die grundlegende Annahme der Planbarkeit im Gesamtkonzept der Globalsteuerung spiegelte sich in der Wissensproduktion durch eine stetig wachsende Anzahl an Politikberatern aus der Wissenschaft. Laut Dreitzel wuchs deren Zahl soweit an, dass manche Ministerien nicht mehr genau wussten, ,,von wie vielen Gremien sie nun eigentlich beraten“" wurden (Dreitzel, 1966, 295). Die Entwicklungen zu einer verstärkten gutachterlichen Tätigkeit, die ihre Anfänge Mitte der 60er Jahren nahmen, intensivierten sich deutlich in den 70er Jahren, in denen deutlich mehr durch Bundesministerien in Auftrag gegebene Studien zu verzeichnen sind als in der vorherigen Phase. Eng verbunden ist damit eine regelrechte „Planungseuphorie [der sozialliberalen Koalition], in der interne und externe wissenschaftliche Politikberatung zunahm und das Beiratswesen ausdifferenziert wurde. Politikberatung in ihren unterschiedlichsten Formen galt als modern" (Siefken, 2007, 24). Dieser Trend zu mehr Planungsversuchen verlief ,quer durch alle Politikbereiche" (Lehnert, 1984, 24) und lässt sich somit als ein wesentlicher Trend im Bereich der allgemeinen staatlich-administrativen Techniken verorten. So wies Brandt in seiner Regierungserklärung 1969 darauf hin, dass „eine systematische Vorausschau und Planung immer wichtiger werde“ (Kornemann, 1996, 134). Dabei stand die sozialliberale Koalition in einem regelrechten ,Spannungsverhältnis von reformpolitischen Zielsetzungen und technokratischen Planungsmethoden" (Lehnert, 1984, 24). Auch in der Wohnungspolitik ,waren nun immer weniger die Traditio- 
nen der Wohnungsreform, immer mehr dagegen technokratische Politikansätze [...] maßgebend“ (Leutner, 1990, 175). Besonders prägnant kommt dieser Umstand im Vorwort einer Publikation des Bundesministeriums für Städtebau und Wohnungswesen zum Ausdruck, in dem der zu diesem Zeitpunkt amtierende Minister Lauritzen bekräftigt:

„Vielleicht wächst dann auch das Verständnis der Öffentlichkeit dafür, daß zu einer Bilanz auch die Inventur, daß zu wirtschaftlich und politisch sinnvollen Entscheidungen auch die Statistik gehört." (Lauritzen, 1972, 2)

In den folgenden Abschnitten soll entsprechend zur ersten betrachteten Phase darauf eingegangen werden, wo die immer relevanter werdende Expertise im Bereich der Eigenheimförderung zu verorten war. Auch in der zweiten Phase waren Parteien und die ihnen nahestehenden Verbände wesentliche Akteure in der Wissensproduktion (Abschnitt 6.3.1). Allerdings bedienten sich insbesondere die Verbände zunehmend der wissenschaftlichen Expertise in Form von Gutachten, die einem allgemeinen Trend zu einer stärkeren Verwissenschaftlichung der politischen Debatte entsprachen (Abschnitt 6.3.2). Den Anstoß für diesen Trend hatte vor allem die Ministerialverwaltung gegeben, die wie auch in anderen Ministerien eine verstärkte gutachterliche Tätigkeit für ihre eigenen Tätigkeiten nutzte (Abschnitt 6.3.3).

\subsubsection{Parteien \& Verbände in Wissenschaftseupho- rie}

Vor ihrem Eintritt in die Große Koalition in der zweiten Hälfte der 60er Jahre vollzog sich innerhalb der SPD ein programmatischer Wan- 
del, der für ihre spätere Weiterentwicklung entscheidend sein sollte. Mit dem Aufsetzen ihres Godesberger Programms von 1959 wandelte sie sich von einer „Klassen- und Facharbeiterpartei in der Tradition des marxistisch beeinflußten Heidelberger Programms von 1925“ zu einer „gemeinwohlorientierten sozialreformerischen Volkspartei“" (Frenzel, 2002, 98f.). Ursächlich für die neue inhaltliche Debatte war das als desaströs wahrgenommene Ergebnis der Bundestagswahlen von 1957, bei denen die CDU die absolute Mehrheit erringen konnte (Nonn, 2002, 72). Um sich einer weiteren Wählerschaft zu öffnen, sollte im folgenden Programm die „Unpopularität jeder Sozialisierung“ vermieden werden, um ,[b]ürgerliche Schichten und Strömungen im Wahlvolk [...] wie potentielle Koalitionspartner unter den bürgerlichen Parteien" nicht zu verschrecken (Nonn, 2002, 76).

Im Rahmen dieser inhaltlichen Öffnung der SPD gegenüber der Mitte lässt sich auch eine Öffnung gegenüber der in Phase 1 ausgiebig beschriebenen Inhalte der katholischen Soziallehre finden, wie Oswald von Nell-Breuning befand (Klein, 1989, 52). Allerdings bestritt dieser selber am Programm mitgearbeitet zu haben (Lafontaine, 1989). Obwohl im Vorfeld des Godesberger Programmparteitages Gespräche zwischen Vertretern der SPD und der katholischen Kirche auf Hinwirken des bereits erwähnten Eberhard Welty stattfanden (Brehm, 1989, 94), ist insgesamt festzustellen, dass den Vertretern der SPD die Kontakte zu Vertretern der katholischen Kirche als nicht besonders wichtig erschienen (Brehm, 1989, 96). In Bezug auf Wohnungspolitik heißt es im Programm:

„Jeder hat ein Recht auf eine menschenwürdige Wohnung.

Sie ist die Heimstätte der Familie. Sie muß deshalb auch 
weiterhin sozialen Schutz genießen und darf nicht nur privatem Gewinnstreben überlassen werden." (SPD, 1959)

Dieser starke familienpolitische Bezug in der Wohnungspolitik erscheint deutlich anschlussfähiger zur Position der konservativen Parteien als die zuvor vorherrschende Rhetorik. Zwar findet sich in diesem Programm kein klares Bekenntnis zur staatlichen Eigenheimförderung, jedoch ist auch keine strikte Ablehnung dieser Förderung zu finden. Mit Lauritz Lauritzen leitete unter der großen Koalition von Kiesinger erstmals ein Mitglied der SPD das für Wohnungswesen zuständige Bundesministerium. Dieser lehnte den zuvor verfolgten Deregulierungskurs seiner Vorgänger Preusker, Lücke und Buchner ab. Seinen Aussagen zufolge müsste ,[d]as Leitbild der sozialen Marktwirtschaft hinter den gesellschafts- und sozialpolitischen Aspekten der Wohnungspolitik zurücktreten“ (Seeger, 1995, 99). Kornemann stellt allerdings fest, dass zu diesem Zeitpunkt der wohnungs- und städtebauliche Bereich keine übergeordnete Priorität mehr besaß. So wurde das noch unter Adenauer 1962 zum Ministerium für Wohnungswesen, Städtebau und Raumordnung ausgeweitete Bundesministerium zum Bundesministerium für Wohnungswesen und Städtebau geschrumpft und der Bereich Raumordnung dem Innenministerium angegliedert (Kornemann, 1996, 132f.). Zudem war das Ministerium durch den Koalitionsvertrag eng an die Vorstellungen der Unionsparteien gebunden und Lauritzen sollte auf Ermahnung des Kanzlers Kiesinger „keine Politik auf eigene Faust“ betreiben dürfen (Führer 2015, 260; siehe auch Leutner 1990, 132). Größere Änderungen der Wohnungspolitik erfolgten unter Lauritzen in der Großen Koalition nicht und auch der Eigentumspolitik wurde weiterhin Vorrang eingeräumt (Leutner, 1990, 132f.). Insgesamt bezeich- 
net Kornemann die Wohnungspolitik unter der ersten großen Koalition auch als eine ,abstinente Phase“ (Kornemann, 1996, 132). Nichtsdestotrotz konnte Lauritzen sich in seiner Zeit als Bundesminister ,zu einer Schlüsselfigur in den Debatten um die [künftige] Ausgestaltung des Wohnungs- und Städtebaus" etablieren (Kramper, 2008, 356). So übernahm er 1968 die Leitung der „Bundesarbeitsgemeinschaft Sozialdemokratischer Wohnungswirtschaftler und Wohnungspolitiker, die ab 1969 als Bundesarbeitsgemeinschaft für Städtebau und Wohnungspolitik der SPD firmierte“ (Kramper, 2008, 356). Laut Kramper konnte diese Arbeitsgemeinschaft, die neben Lauritzen auch von dem Vorsitzenden der Neuen Heimat Albert Vietor geleitet wurde, die Wohnungsund Städtebaupolitik erneut zu einem wesentlichen Feld sozialdemokratischer Reformpolitik machen (vgl. Kramper, 2008, 356).

Mit ihrem neuen Programm „Orientierungsrahmen '85“ positionierte sich die SPD schließllich deutlich gemäßigter hinsichtlich der Wohnungsmarktregulierung und befürwortete verstärkt die Förderung von Wohneigentumsbildung (Seeger, 1995, 127). Dabei fanden auch innerhalb der SPD familien- und gesellschaftspolitische Ziele der Eigenheimförderung breit Akzeptanz (Harlander, 2008, 829). Auch der neue Wohnungsbauminister Karl Ravens forderte, daß der Wohnungsbau stärker vom wirtschaftlichen Wachstum abhängig gemacht würde und das selbstgenutzte Wohneigentum die dominierende Rolle im künftigen Wohnungsbau einnähme“ (Seeger, 1995, 123f.). Seeger führt den endgültigen Umschwung der SPD zu mehr Wohneigentumsförderung auch darauf zurück, dass die FDP ihren Einfluss stärker geltend machen konnte. Zudem seien die Auswirkungen den Konjunktureinbruches mit der erneuten Wahlphase zusammen gefallen, sodass alle Parteien ,konzeptionelle Zugeständnisse 
an den politischen Gegenspieler" machten (Seeger, 1995, 126). Die FDP hatte in ihren sogenannten Freiburger Thesen von 1971 erklärt, Wohneigentum sollte „die persönliche Unabhängigkeit und eine wirtschaftliche Sicherheit gewährleisten [...], so daß die Wohneigentumsbildung [zu einem] wesentlichen Bestandteil der Selbstbestimmung des wirtschaftlichen Individuums" wurde (Seeger, 1995, 100). Eng verbunden waren damit die weitere Überführung der Wohnungswirtschaft in eine marktwirtschaftliche Ordnung" (Seeger, 1995, 100). So sprach sich die FDP stärker als die SPD für neue wohneigentumsfördernde Maßnahmen im Rahmen des Sozialen Wohnungsbaus aus, ,damit auf der einen Seite die öffentlichen Haushalte entlastet würden und auf der anderen Seite den Bedürfnissen der Menschen stärker Rechnung getragen werden könnte“ (Seeger, 1995, 123).

Angesichts des engen Bündnisses mit der gemeinnützigen Wohnungswirtschaft und insbesondere der neuen Heimat erscheint es zunächst kontraintuitiv, dass sich die SPD nach und nach auch zugunsten der Eigenheimförderung positionierte. Allerdings hatten auch die gemeinnützigen Wohnungsgesellschaften ein großes Interesse an der Möglichkeit aus ihren Wohnungsbeständen einzelne Wohneinheiten an Selbstnutzer zu verkaufen. Zudem erstellten auch gemeinnützige Wohnungsunternehmen schon früh Einfamilienhäuser, die sie an ihre Bewohner verkauften (vgl. Ifo-Institut für Wirtschaftsforschung, 1960, 65). Unter Bundesminister Lauritzen wurde die Zusammenarbeit mit den Gemeinnützigen auch in der Wissensproduktion intensiviert. Sein Ministerium griff ,in hohem Maße auf das enge Geflecht von Experten zurück, das sich zwischen Gewerkschaften, SPD und Gemeinnützigen gebildet hatte und in dem Vertreter der Neuen Heimat eine überaus bedeutsame 
Rolle spielten“ (Kramper, 2008, 357). Zu Beginn der 70er Jahre war die Neue Heimat nach der Einschätzung von Kramper auf dem Höhepunkt ihrer Macht und ihres Einflusses, dessen Ende sich jedoch mit der Wirtschaftskrise von 1973/74 abzeichnete (Kramper, 2008, 30).

Auf Initiative der „Neuen Heimat“ wurde ab 1977 über steuerliche Vergünstigungen die Umwandlung von Sozialwohnungen in Eigentumswohnungen gefördert (Mayer 1998, 197; Hämmerlein 1996, 59). Besonders deutlich trat der politische Einfluss der Neuen Heimat in den Verhandlungen um das Gesetz zur Förderung von Wohneigentum (1976) zutage, sodass der Nachfolger Lauritzens Vogel später berichtete, „die $\mathrm{NH}$ habe das Ministerium zu diesem Zeitpunkt als eine Art nachgeordnete Dienststelle des Vorstandes behandelt" (Kramper, 2008, 358). So legten Vertreter der Neuen Heimat und des Deutschen Mieterbundes ein „Gutachten zum Wohnbesitzrecht vor, welches zusammen mit dem einstimmigen Votum der übrigen 11 Mitglieder des Sachverständigenausschusses der Öffentlichkeit ebenfalls zugänglich gemacht wurde“ (Peters 1984, 216; vgl. Nevermann u.a. 1970). Neben der Neuen Heimat waren auch weitere Partner aus dem korporatistischen Bereich an Expertisen zur Gesetzgebung beteiligt. 1974 hatten Sachverständige im Auftrag des Deutschen Verbandes für Wohnungswesen, Städtebau und Raumplanung,,verschiedener Sparten der Wohnungswirtschaft, Banken, Bausparkassen usw. [...] in einem Gutachten für den Deutschen Verband für Wohnungswesen, Städtebau und Raumplanung Stellung“" bezogen (Peters, 1984, 215) und darin angesichts mehrerer offener Fragen „Bedenken gegen die Anwendung des gebotenen Instrumentariums zur Finanzierung des Wohnungsbaues und damit zur ergänzenden Unternehmensfinanzierung“" (Peters, 1984, 215) geäußert. 
Der Anspruch, Wissen über weitere Akteure aus dem Bereich der Wohnungspolitik zugänglich zu machen, wird auch in dem Anhörungsverfahren des Ausschusses für Raumordnung, Bauwesen und Städtebau zu dem von der Bundesregierung eingebrachten Entwurf eines Gesetzes zur Förderung von Wohnungseigentum und Wohnbesitz im sozialen Wohnungsbau deutlich (BT-Drs. 07/3314). Dort kamen im September 1973 im Rahmen einer öffentlichen Sitzung als Experten Vertreter vom Deutschen Mieterbund, dem Deutschen Volksheimstättenwerk, dem Gesamtverband gemeinnütziger Wohnungsunternehmen, der Geschäftsstelle Öffentliche Bausparkassen, dem Verband der Privaten Bausparkassen, dem Verband Freier Wohnungsunternehmen, dem Verband privater Hypothekenbanken (VDH, inzwischen vdp), dem Zentralverband der Deutschen Haus-, Wohnungs- und Grundeigentümer sowie der Bundesvereinigung Deutscher Heimstätten zu Wort.

Mit dem Wechsel zum Kabinett Kohl blieb die vorherige Praxis der Expertisen zur Wohneigentumsförderung erhalten und wurde sogar noch weiter ausgebaut. Dabei ließ ,die marktwirtschaftliche Programmatik der neuen Koalitionspartner [...] erhebliche Politikveränderungen erwarten (Jaedicke u. Wollmann, 1991, 427). Dabei positionierten sich auch die christlichen Parteien zunehmend liberaler als dies in den vorherigen Legislaturperioden der Fall gewesen war (Hemmelmann, 2017, 155ff.). Dem Wohnungsbau sollten insgesamt ,neue Impulse gegeben werden. Neben der unverzüglichen Verbesserung der steuerlichen Förderung des Eigenheimbaus durch Zulassung eines begrenzten Schuldzinsabzugs [war auch] ein Programm zur Bausparzwischenfinanzierung“" vorgesehen (Kornemann, 1996, 139). Dabei sollten insgesamt die wohnungspolitischen Aufgaben des Bundes verringert werden 
(Leutner, 1990, 249). Wesentliche Beiträge zur Positionierung der CDU leistete in diesem Zusammenhang auch die Konrad-Adenauer-Stiftung, die zunehmend als Think Tank ihrer Partei fungierte (vgl. Abschnitt 3.1.2). Dabei berief sie sich in ihrem Gutachten zur Wohnungspolitik allerdings primär auf die historisch gewachsenen Eigenheimvorstellungen innerhalb der CDU, anstatt auf jene marktwirtschaftlichen Konzepte, die zunehmend auf Seiten der wissenschaftlichen Expertise anzutreffen waren (vgl. Groser, 1979). Von einer durch die schwarz-gelbe Regierungskoalition propagierten „Wende in der Wohnungspolitik“ kann jedoch nicht die Rede sein, wie Harlander (2008) feststellt:

„Die Konturen der durch die christlich-liberale Koalition ab 1982 durchgesetzten Deregulierungspolitik der 1980er Jahre [...] wurde in wesentlichen Bestandteilen auch bereits in der Endphase der sozialliberalen Koalition vorgezeichnet". (Harlander, 2008, 850)

Als auffällig gegenüber den vorherigen politischen Debatten zeigt sich in den Diskussionen um das Steueränderungsgesetz eine deutlich geringere Berufung von Experten aus Verbänden und Wirtschaft. Lediglich auf Stellungnahmen des Städtetages (BT-Drs. 10/208, 15986B), von Banken und Sparassen (BT-Drs. 10208, 15986D) und des Zentralverbands des Handwerks (BT-Drs. 10/208, 15986D) nahmen die Abgeordneten in den hier betrachteten Debatten Bezug. Dies kann als ein Symptom für den „Bedeutungsverlust industriell-korporatistischer Wohnungsmarktelemente" (Mertens, 2015, 313) in den 1980er Jahren gedeutet werden. So wurden häufig Werkswohnungsbestände veräußert oder von Wohnungsgesellschaften an deren Mieter veräußert (Mertens, 2015, 313). Eine gesonderte Erwähnung bedarf in diesem Zusammen- 
hang der Skandal um die Neue Heimat. 1982 veröffentlichte der Spiegel mit seiner Titelgeschichte „Gut getarnt im Dickicht der Firmen - Neue Heimat: Die dunklen Geschäfte von Vietor und Genossen“ Material, das den Niedergang des Unternehmens einleiten sollte. Mehrere Vorstandsmitglieder sollten sich - zum Teil auch direkt an den Mietern - bereichert haben. Im weiteren Verlauf zeigte sich, dass die mit diesen Bereicherungen einhergehenden finanziellen Schäden für die Gesellschaft in Millionenhöhe lagen, sodass der DGB die Gesellschaft veräußern musste. Dieser „groteske Untergang der Neuen Heimat“ (Bartholomäi, 2004, 26) blieb in der wohnungspolitischen Landschaft nicht ohne Folgen: „Der Bundestag und fast alle Landtage setzten erwartungsgemäß Untersuchungsausschüsse ein, mit dem Ziel, das Wohnungsgemeinnützigkeitsgesetz zu verschärfen“ (Bartholomäi 2004, 26; siehe auch Leutner 1990, 276ff.; Harlander 2005, 700ff.). Schließlich wurde den Gemeinnützigen Wohnungsunternehmen mit dem Steuerreformgesetz von 1990 die gesetzliche Grundlage entzogen. Eichner weist jedoch darauf hin, dass der Skandal um die Neue Heimat eher Anlass als Ursache für diese massiven Folgen war. Vielmehr seien „die Unternehmen zu groß geworden [...], daß sie ein gewaltiges Machtpotential darstellten. Die Neue Heimat ist ein erhellendes Beispiel dafür, daßs die Gemeinnützigen von der Wohnungspolitik der Bundesrepublik alles in allem erheblich profitierten - obwohl die von starke Kräften in der CDU/CSU bekämpft wurden“" (Eichener u. a., 2000, 75f-). Gegenüber diesem Sturz der Neuen Heimat in der öffentlichen Wahrnehmung - sowohl als Wohnungsanbieter als auch als Bereitsteller von Expertisen zu wohnungspolitischen Themen - gewannen Wissenschaftler, insbesondere aus dem Feld der Wirtschaftswissenschaften zunehmend an Renommee. Diese Entwick- 
lung soll in Abschnitt 6.3.2 dargestellt werden.

Wie in der ersten Phase zeigt sich auch in dem hier betrachteten Abschnitt, dass die jeweils regierenden Parteien und die mit ihnen in guter Verbindung stehenden Verbände wesentlich an der korporatistischen Wissensproduktion für die Eigenheimförderung beteiligt waren. Besonders an den fortgeführten Initiativen auch nach Regierungswechseln wird deutlich, dass sowohl die christdemokratischen Parteien als auch die SPD und die ihnen nahestehenden Verbände ein Interesse an der Eigenheimförderung hatten. Besonders deutlich wird dies im Verweis auf die entsprechenden Bemühungen der SPD in ihren Regierungsjahren durch den Abgeordneten Huonker: „Wir Sozialdemokraten sind stolz darauf, daß noch niemals so viel Wohneigentum wie in den 70er Jahren unter sozialdemokratisch geführten Bundesregierungen gebaut worden ist" (BT-Drs. 10/156, 11667D). So lässt sich anhand der Untersuchung der Bundestagsdebatten die Einschätzung Krummachers bestätigen, dass alle im Bundestag vertretenen Parteien sich nun bemühen „,sich selbst als die jeweils "besseren" Verfechter der Wohneigentumsförderung auszuweisen" (Krummacher, 1978, 352). Die Ideen hinter der Eigenheimförderung waren somit nicht abhängig von parteipolitischen Interessen, sondern wurden von allen Parteien vertreten. Dabei bedienten sich Politiker und insbesondere Verbände zunehmend der wissenschaftlichen Expertise, um ihren Stellungnahmen Gewicht zu verleihen. Diese zunehmende Verwissenschaftlichung der politischen Debatte soll im folgenden Abschnitt näher beleuchtet werden. 


\subsubsection{Wissenschaft auf Nachfrage}

Die zweite Phase wissenschaftlicher Politikberatung wird von Weingart oftmals charakterisiert als eine "Politisierung der Wissenschaft" (Weingart, 2006, 42), wobei sich die politische Interessenvertretung auf die wissenschaftliche Expertise selbst ausgedehnt hat (vgl. Abschnitt 3.1.3). Tatsächlich zeigt sich auch im Bereich der Wohnungspolitik eine zunehmende Präsenz insbesondere von wirtschaftswissenschaftlichen Expertisen. So treten in den analysierten Bundestagsdebatten Vertreter von Verbänden gegenüber wissenschaftlichen Experten zunehmend in den Hintergrund. In den Debatten der 80er Jahre beziehen sich selbst Abgeordnete der SPD in ihren Aussagen direkt auf Berechnungen des Deutschen Instituts für Wirtschaftsforschung, ebenso wie auf Aussagen des Wirtschaftswissenschaftlers Johann Eekhoff (vgl. BT-Drs. 10/156, 11680Bff.).

Besonders auffällig gegenüber der ersten Phase wissenschaftlicher Politikberatung erscheint die zunehmende gutachterliche Tätigkeit. Diese ist im wohnungspolitischen Bereich derart umfassend, dass sie in ganzen Reihen vonseiten des Ministeriums veröffentlicht werden. Zu nennen sind dabei zunächst die durch das Bundesministerium in Auftrag gegebenen Forschungsaufträge an das Deutsche Institut für Wirtschaftsforschung, die ,in Zusammenarbeit mit Wissenschaftlern der Universität Dortmund bereits 1976 eine methodische Studie über Probleme der Wohnungsnachfrage in der Bundesrepublik Deutschland und mögliche Prognoseansätze durchgeführt" hatte (Bartholmai u.a., 1980, 9). Das maßgebliche Ziel der Forschungen bestand darin, anhand von Prognosen wirtschaftlicher Rahmendaten auch Prognosen für eine sinnvolle Bautätigkeit abzuleiten, die der jeweiligen Nachfrageentwicklung ent- 
sprechen sollten, „damit Zukunftsaussagen abgeleitet werden können, die nicht nur den Charakter von Wünschen oder Richtlinien tragen, sondern Tendenzen der wahrscheinlichen Entwicklung in Abhängigkeit von bestimmten gesamtwirtschaftlichen Rahmenbedingungen aufzeigen" (Bartholmai u.a., 1980, 9). Ein wesentlicher Bestandteil der Untersuchungen war eine Wahrscheinlichkeitsbestimmung für Wohneigentum in Abhängigkeit von der Einkommenssituation festzustellen (Bartholmai u.a., 1980, 73ff.). Bartholomai et al. kommen dabei zu dem Ergebnis, dass ,sich die Eigentumsnachfrage auf einem relativ hohen Niveau halten wird“ (Bartholmai u.a., 1980, 119). Als Basis der Untersuchungen dienten die bis dahin erschienenen Erhebungen des Statistischen Bundesamtes sowie deren Fortschreibungen.

Weitere im Zusammenhang mit der Wohneigentumsförderung relevanten Gutachten befassen sich vor allem mit der Bestimmung der unterschiedlichen Wohneigentumsquoten in Belgien, Dänemark, Frankreich, Großbritannien, USA und der Bundesrepublik (Köster u. Mezler, 1979) und einer umfassenderen Bewertung wohnungspolitischer Strategien (Eekhoff u.a., 1979). Vor allem die Ergebnisse der vergleichenden Untersuchung zu Wohneigentumsquoten zeigen sich wiederholt in den politischen Debatten bis heute und sollen daher hier genauer betrachtet werden. Ausgangspunkt für den internationalen Vergleich bietet die bis heute gestellte Diagnose, dass die Wohneigentumsquoten in Deutschland gegenüber denen in anderen Ländern überaus gering sind. Wie bereits in früheren Debatten wurde auch zu Zeiten der sozialliberalen Koalition festgestellt, dass die bis dato erfolgten Fördermaßnahmen für Wohneigentum in der Bundesrepublik nicht ausreichend waren ${ }^{6}$, sodass

6 Ausgehend von in dem Gutachten zitierten Schätzungen hatte der Staat ,zwi- 
gezielt nach alternativen Handlungsstrategien gesucht wurde. Dieser Hinweis findet sich auch in dem ersten hierzu erschienenen Gutachten:

„Zumeist dient der Hinweis auf das höhere Niveau der Eigentumsquote in anderen europäischen Ländern dazu, Versäumnisse der Wohnungspolitik vergangener Jahre zu belegen, oder aber eine künftige Umorientierung der Wohnungspolitik zu fordern.“ (Köster u. Mezler, 1979, 10)

Wesentlich im Zusammenhang mit der Wohneigentumsquote als Kennzahl ist vor allem die Erkenntnis, dass sie ,in den letzten Jahren einen wichtigen Rang als (operationalisierbares) Zielkriterium der Wohnungspolitik erhalten hat, das in der fachlichen Diskussion häufig sogar als Erfolgskriterium oder vermuteter (Miß)-Erfolgsbeweis einzelner wohnungspolitischer Maßnahmen herhalten muß, obwohl derart komplexe Zusammenhänge sich anhand einzelner Daten kaum belegen lassen“ (Köster u. Mezler, 1979, 11). Das Gutachten kommt zu dem Schluss, dass nicht nur die im internationalen Vergleich höheren Baukosten für die vergleichsweise geringeren Wohneigentumsquoten verantwortlich sein konnten, sondern auch, dass der Erwerb ,,von Wohneigentum durch steuerliche Rahmenbedingungen und Förderungsprogramme [...] in der Bundesrepublik Deutschland weniger breit angelegt" ist, als dies in den Vergleichsländern der Fall ist (Köster u. Mezler, 1979, 6). So finden sich in diesem Gutachten erstmals konzentrierte Forderungen dafür, die Wohneigentumsförderung stärker über steuerliche Instrumentarien und damit primär über Abschreibungsmöglichkeiten zu fördern.

schen 1950 und 1974 insgesamt ca. 180 Mrd. DM zugunsten des Wohnungsbaus ausgegeben, davon entfielen ca. 80 Mrd. DM auf die Förderung von Wohneigentum“ (Köster u. Mezler, 1979, 9). 
In dem zweiten, breiter angelegten Gutachten wird allerdings kritisch hinterfragt, ,ob ein Aufholen in dieser Hinsicht für sich genommen ein sinnvolles wohnungspolitisches Ziel sein kann, vor allem wenn ein hoher Anteil von Eigentümern in Vergleichsländern zusammenfällt mit Beschränkungen auf dem Mietwohnungsmarkt" (Eekhoff u. a., 1979, 57). Vor dem Hintergrund erweiterter wohnungspolitischer Zielsetzungen wie der Förderung der unteren Einkommensklassen und der gesamten Wohnraumversorgung sehen die Ersteller des Gutachtens die Wohneigentumsförderung insgesamt kritisch.

Im Bereich der Auftragsforschung finden sich Studien, die eine möglichst präzise Eigenheimförderung fordern ,,sowie der Abbau von Mitnahmeeffekten " “ (Arras u.a., 1980, 5), die bereits in einem von der sozialliberalen Bundesregierung in Auftrag gegebenen Gutachten thematisiert und als „,relativ hoch eingeschätzt“" wurden (Eekhoff u. a. 1979, 5; siehe auch Leutner 1990, 378). Auch die Analyse von Ulbrich (1980) weist darauf hin, dass die bestehenden Formen der Eigenheimförderung tendenziell denjenigen nützen, die bereits über ein höheres Einkommen verfügen und geringere Einkommensklassen von diesen Subventionen nur in eingeschränktem Maße profitieren können. So finden sich in diesen Gutachten Kritikpunkte gegenüber der Eigenheimförderung, die im weiteren Verlauf vor allem von Abgeordneten der SPD in den Förderdebatten angeführt werden.

Neben der gutachterlichen Tätigkeit der Forschungsinstitute betätigten sich auch Verbände und Think Tanks zunehmend an der wis-

7 Unter Mitnahmeeffekten wird in der Volkswirtschaftslehre eine Inanspruchnahme von finanziellen Leistungen als Belohnung für ein Verhalten verstanden, das auch ohne den zusätzlichen finanziellen Anreiz stattgefunden hätte (Groot, 2008). 
senschaftlich gerahmten Wissensproduktion zur Eigenheimförderung. Auch das ehemalige Arnold-Knoblauch Institut (vgl. 5.3.2) ging mit einem weiteren Königsteiner Gespräch eine Woche vor der Bundestagswahl von 1980 in eine neue Runde und versammelte dabei Wohnungsund Vermögenspolitiker aller im Bundestag vertretenen Parteien (Institut für Städtebau, Wohnungswirtschaft und Bausparwesen, 1981, VII). Auch anhand der Teilnehmer dieser Gespräche lässt sich weiterhin eine starke korporatistische Prägung der Wissensproduktion nachzeichnen. So sind neben Verbandsvertreten der Bausparkassen auch der Generalsekretär des Deutschen Verbandes, ein Vorstandsmitglied der Gewos, der Hauptgeschäftsführer des Deutschen Volksheimstättenwerkes sowie MdBs und Vertreter der Ministerialbürokratie und Wissenschaftler anwesend (Institut für Städtebau, Wohnungswirtschaft und Bausparwesen, 1981, 112). Dabei lag der Fokus der Vorträge auf der Förderung von Wohneigentum. In seinem Vortrag thematisiert der Professor für Finanzwissenschaften Mackscheidt die verteilungspolitische Seite der Wohneigentumsförderung: „Meine These lautet: Obwohl die Wohnungspolitik von Anfang an verteilungspolitische Ziele oder Auflagen gekannt hat, ist der Frage nach der Effizienz der staatlichen Förderungsmaßnahmen hinsichtlich ihres verteilungspolitischen Gehaltes erst in den letzten Jahren nachgegangen worden. Solange das Ziel, einen der Nachfrage angemessenen Bestand an Wohnungen zu erreichen im Vordergrund stand, interessierten Fragen nach dem verteilungsmäßigen Niederschlag der staatlichen Förderungsmaßnahmen, von Einzelfällen abgesehen, kaum“ (Institut für Städtebau, Wohnungswirtschaft und Bausparwesen, 1981, $67)$.

Die enge Verbindung zwischen der wissenschaftlichen und der kor- 
poratistischen Wissensproduktion wird besonders deutlich anhand von Forschungsarbeiten, die gemeinsam durch das Ministerium und Verbänden in Auftrag gegeben wurden. Dies war sowohl unter der sozialliberalen Koalition als auch unter dem späteren Kabinett Kohl nicht ungewöhnlich. In Bezug auf die Eigenheimförderung ist dabei insbesondere ein Gutachten zu Formen der Wohnbau- und Bausparförderung von Interesse, das vom Bundesministerium für Raumordnung, Bauwesen und Städtebau ,unter Mitfinanzierung durch den Verband der Privaten Bausparkassen e.V. und die Geschäftsstelle Öffentliche Bausparkassen im Deutschen Sparkassen- und Giroverband e.V." in Auftrag gegeben wurde (Weissbarth, 1978). Diese sogenannte ,Sickerstudie“ (Harlander, 2008, 831) kam zu dem Ergebnis, das die Förderung von Eigenheimen Umzugsketten in Bewegung setzen würden, die dann auch zu einem verbesserten Angebot günstiger Mietwohnungen führen würden. Eigenheimförderung, so die Theorie der Sickereffekte, bewirkt so nicht nur eine verbesserte Wohnraumversorgung der mittleren Einkommensschichten, sondern auch für die unteren Einkommensklassen. Einer gesonderte Studie zur Eigentumsbildung im Wohnungsbau, die das Bundesministerium unter dem ersten Kabinett Kohl gemeinsam mit dem Verband der Privaten Bausparkassen und der Bundesgeschäftsstelle der Landesbausparlassen im Deutschen Sparkassen- und Giroverband e.V finanzierte, werden im Vorwort durch den amtierenden Minister Schneider die Sätze voraus gestellt: „Keine andere Vermögensart sichert und erweitert den Freiheitsraum des einzelnen (sic!) so sehr wie das Wohneigentum. Das direkte Erleben, einen von anderen nur wenig kontrollierbaren Verfügungsraum zu haben, zeichnet das Wohneigentum gegenüber anderen Vermögensformen [...] aus" (Weissbarth u. Hundt, 1983, 5). Um dies 
möglichst vielen zu ermöglichen, sollte nach Einschätzung der Gutachter insbesondere die Ansparung von Eigenkapital erleichtert und wenn möglich staatlich gefördert werden. $\mathrm{Zu}$ nennen ist ebenso das Gutachten von Köster u. Mezler (1979), das durch die GEWOS durchgeführt wurde, die maßgeblich aus den Mitteln der Neuen Heimat finanziert wurde.

$\mathrm{Zu}$ Beginn der 80er Jahre konstatieren Häußermann und Siebel, die Wohnungspolitik sei zu einer „Angelegenheit von Experten“ geworden (Häußermann u. Siebel, 1981, 316). „Komplizierte Finanzierungs-, Rechts- und Normierungsfragen bildeten eine Mauer, an der alle ,lediglich politisch" fundierten Initiativen wie laienhafte Marginalien abprallten“ (Häußermann u. Siebel, 1981, 317). Dieser Umstand der „unpolitischen Wohnungspolitik“ (Häußermann u. Siebel, 1981, 316) stand in einem starken Kontrast zu den stattfindenden Hausbesetzungsaktionen (vgl. Abschnitt 6.1). Während in politischen und Expertenkreisen vor allem darüber diskutiert wurde, „ob es schon an der Zeit sei, das Wohnungswesen ganz der marktwirtschaftlichen Regulierung zu überlassen“, spielten die Haltungen und Vorstellungen der Protestbewegungen in diesen „offiziell relevanten Diskussionen“ keine Rolle (Häußermann u. Siebel, 1981, 318). Der überwiegende Anteil der Referenzen in den politischen Debatten in der zweiten Hälfte der 80er Jahre geht in der Tat deutlich weniger an korporatistische Akteure oder bezieht sich gar auf Protestbewegungen. Stattdessen beziehen sich die politischen Entscheidungsträger maßgeblich auf Experten aus der Wissenschaft. Neben den Expertisen des Deutschen Instituts für Wirtschaftsforschung (DIW) wird vor allem auf die Expertise von Klaus Tipke, einem Steuerrechtswissenschaftler und dem Wirtschaftswissenschaftler und späte- 
rem Staatssekretär Johann Eekhoff Bezug genommen (vgl. Abschnitt 6.3.3).

Allerdings kann mit Bezug auf das betrachtete Politikfeld dabei kaum von einer tiefgreifenden Verwissenschaftlichung gesprochen werden. So waren auch in den Beiräten der vorherigen Phase die Verbandsvertreter sogar in der Überzahl. Hinsichtlich der Wissensproduktion für die Eigenheimförderung in Deutschland muss daher festgehalten werden, dass auch in der zweiten Phase Wissenschaftler gemeinsam mit korporatistischen Akteuren daran beteiligt gewesen sind, sich allerdings die Argumentationsmuster stark zugunsten de wissenschaftlichen Begründungsmuster verschoben haben. Letzteres ist vor allem daran erkennbar, dass sich nun auch zunehmend Verbandsvertreter auf wissenschaftliche Expertisen berufen und diese dazu auch in Auftrag geben. Ein wesentlicher Anstoß hierzu wurde durch die Ministerialbürokratie gegeben: Sie folgte dem allgemeine administrativen Trend zur Verwissenschaftlichung der externen Expertise und ermöglichte es somit vor allem Experten aus den Wirtschaftswissenschaften in der Wissensproduktion zur Eigenheimförderung Fuß zu fassen. Zusätzlich lieferten die ausgebauten amtlichen Statistiken mit ihren Daten den grundlegenden Baustein zur wirtschaftsiwssenschaftlichen Debatte. Diese Prozesse sollen im folgenden Abschnitt eingehender beschrieben werden.

\subsubsection{Etablierung von Ministerialbürokratie \& amt- licher Statistik}

Angesichts der ersten wohnungspolitischen Initiativen des Bundesministers Lauritzen in der ersten Großen Koalition überrascht es weniger, dass unter der sozialliberalen Koalition und dem weiterhin am- 
tierenden Bundesminister Lauritzen die wohnungspolitische Abstinenz überwunden werden sollte. Durch die Wiederaufnahme der Raumordnung wurde das zuständige Bundesministerium ab 1973 erneut gestärkt (Leutner, 1990, 135). Allerdings zeigt sich bereits an der Umbenennung des Ministeriums zum Bundesministerium für Raumordnung, Bauwesen und Städtebau, „daß der Wohnungsbau formal und sachlich hinter der Raumordnung und dem Städtebau zurücktreten sollte“ (Seeger, 1995, 110). Einem allgemeinen administeriellen Trend sollte sich ein deutlicher Ausbau der wissenschaftlich fundierten Analysen als Grundlage der Wohnungspolitik etablieren (vgl. hierzu Abschnitt 6.3.2; vgl. Kramper 2008, 357). Dies wurde bereits durch die in Abschnitt 6.3.2 beschriebenen Auftragsgutachten beschrieben. 1986, und damit ein Jahr vor der hier betrachteten Bundestagsdebatte, veröffentlichte das Bundesministerium eine zusätzliche Arbeit, die auch an das Risikopotenzial des Wohneigentums anknüpfte und in späteren Bundestagsdebatten thematisiert wurde: „Wenn die Voraussetzungen nicht stimmen“, so Bundesminister Schneider im Vorwort, ,kann der Erwerb von Wohneigentum jedoch auch zu einem schlechten Ende, zur Zwangsversteigerung führen“ (Drevermann u. Nordalm, 1986). Das Gutachten hatte zum Ziel, die zu diesem Zeitpunkt bestehende Diskussion ,auf eine sachliche Grundlage zu stellen“ (Drevermann u. Nordalm, 1986) und kam zu dem Ergebnis, dass die Zahl der Zwangsversteigerungen niedriger waren als angenommen und im darauffolgenden Jahr auch weiter abnehmen sollten. Zugunsten einer weiteren Eigenheimförderung wurde zudem vorgeschlagen das Ansparen von Eigenkapital weitreichender zu fördern.

Mit der Verschiebung der Instrumentarien der Wohneigentumsför- 
derung hin zur Steuerpolitik, übernahm von nun an das Bundesministerium der Finanzen die Federführung bei den entsprechenden Gesetzgebungsverfahren. Dabei waren die Mitarbeiter des Bundesfinanzministerium stets in engem Kontakt mit dem Mitarbeiterstab des für Wohnungsbau zuständigen Ministeriums. Dies beschreibt Kurt-Dieter Wagner, Ministerialdirektor beim Bundesministerium der Finanzen in einer Rede beim Königsteiner Gespräch von 1980 wie folgt:

„Wir haben nun versucht, uns parallel zu den Wohnungspolitikern die Köpfe zu zerbrechen. Ich muß sagen, das fällt natürlich Finanzpolitikern ausgesprochen schwer. Wir sind keine Fachleute auf diesem Gebiet. Von daher, würde ich sagen, sind alle Lösungen, die einseitig aus den Köpfen der Finanzpolitiker entspringen, sicherlich unschöner als solche, die in gemeinsamer Zusammenarbeit zwischen Wohnungspolitikern und Finanzpolitikern realisiert werden können." (zitiert nach Institut für Städtebau, Wohnungswirtschaft und Bausparwesen, 1981, 79)

Nach dem Regierungswechsel zur schwarz-gelben Koalition 1982 und unter dem neuen Bundesminister Oscar Schneider (CSU) wurde das Bundesministerium für Raumordnung, Bauwesen und Städtebau bis zum Ende der 1980er Jahre weitergeführt. Zwar wird der Bundesbauminister Schneider ,nicht zu den radikalen Verfechtern des marktwirtschaftlichen Kurses" gezählt, allerdings sehen Jaedicke u. Wollmann (1991) in der Berufung des Wirtschaftswissenschaftlers Johann Eekhoff, einem ,prononcierten und dezidierten ,Wohnungsmarktwirtschaftler”, zum Leiter der Abteilung „Wohnungspolitik“ im Bundesministerium für Raumordnung von 1983 bis 1991 und zum Staatssekretär im 
Bundesministerium für Wirtschaft von 1991 bis 1994 ein klares Anzeichen für eine wirtschaftsliberale Radikalisierung der Unionsparteien (Jaedicke u. Wollmann, 1991, 472). Tatsächlich positionierte sich Eekhoff stets deutlich für einen Rückzug staatlichen Engagements aus den Wohnungsmärkten und gegen die Vorstellung einer staatlich steuerbaren Wohnungswirtschaft (so Eekhoff, 1981). Eekhoff war Mitglied des 1982 gegründeten Kronberger Kreises, einem Verband von Wirtschaftsund Rechtswissenschaften, dessen Ziel nach eigenen Angaben darin besteht ,staatlicher Bevormundung zu verhindern und mit stimmigen ordnungspolitischen Reformkonzepten einen Beitrag zur Weiterentwicklung der freiheitlichen Ordnung in Deutschland und Europa zu leisten" (Stiftung Marktwirtschaft, 2018a). Als wissenschaftlicher Beirat der Stiftung Marktwirtschaft (vormals Frankfurter Institut), lässt sich der Kronberger Kreis als „marktwirtschaftliche[r] Think-Tank“ beschreiben (Stiftung Marktwirtschaft, 2018b). Eekhoff war selber nicht als ein Vertreter einer renommierten Universität oder eines Wirtschaftsforschungsinstituts zu einem Experten im Feld geworden, sondern hatte sich über seine Promotionsschrift zu einer „Nutzen-Kosten-Analyse der Stadtsanierung“ als einer der wenigen Wirtschaftswissenschaftler seiner Generation mit wohnungspolitischen Fragen befasst. Somit galt er bereits früh innerhalb der wirtschaftswissenschaftlichen Gemeinschaft insbesondere im Verein für Socialpolitik - als Experte für Wohnungspolitische Fragen (Eekhoff, 1981).

Bereits in den 70er Jahren hatte er gutachterliche Tätigkeiten im Auftrag des Wohnungsbauministeriums unter der sozial-liberalen Koalition geleistet (vgl. Eekhoff u.a. 1979; Eekhoff 1975) und war damit bereits vor seiner Berufung ins Ministerium im wohnungspolitischen 
Umfeld als Experte anerkannt. In seinem 1979 erschienenen Gutachten bewertete Eekhoff bestehende wohnungspolitische Förderinstrumente „,vor dem Hintergrund eines Marktes, der zum Gleichgewicht tendiert" (Eekhoff u.a., 1979, 5) und legte damit die Zielsetzung wohnungspolitischer Instrumentarien fest: Sie sollen maßgeblich dem Markt zum Gleichgewicht verhelfen, indem sie Marktunvollkommenheiten abmildern. Zudem findet sich hier die Feststellung, dass die Wohneigentumsförderung vonseiten der Bundesregierung maßgeblich unter der Zielsetzung der Vermögensbildung erfolgt, weil „Wohnungseigentum die Entfaltung eines gesunden Familienlebens gewährleiste, zum anderen, weil greifbares Vermögen [...] einen gesellschaftspolitischen Wert an sich darstellt (Eekhoff u.a., 1979, 57). In einem später erschienenen Tagungsband findet sich eine präzisere Stellungnahme zur Familienförderung durch Wohneigentum. Diese sei gerade deswegen gerechtfertigt, weil nur Familien mit Kindern ,[u]nter den bestehenden Bedingungen des Steuerrechts und des Rentenversicherungsrechts [...] in vollem Umfang den Dreigenerationenvertrag" erfüllen (Eekhoff u.a., 1985, 6). "Durch eine familienfreundliche Wohnungspolitik [sollte daher] teilweise ein Ausgleich geschaffen werden“ (Eekhoff u. a., 1985, 6).

Zwar wurde mit Eekhoff die wirtschaftswissenschaftliche Expertise innerhalb des Ministeriums mit einem bedeutenden Posten bedacht. Ein eigener wohnungspolitischer Beirat beim Ministerium wie in der ersten Phase wurde allerdings weder unter der sozialliberalen noch unter der schwarz-gelben Koalition installiert. Stattdessen befassten sich die Beiräte beim Bundeswirtschaftsministerium wie auch dem Familienministerium mit Fragen der Eigenheimförderung. So verfasste der Wissenschaftliche Beirat für Familienfragen beim Bundesministerium 
für Jugend, Familie und Gesundheit unter der sozialliberalen Koalition das Gutachten „Familie und Wohnen“ (Wissenschaftlicher Beirat für Familienfragen beim Bundesministerium für Jugend, familie und gesundheit, 1975), mit dessen Veröffentlichung das Ministerium die Ergebnisse ,einer breiteren interessierten Öffentlichkeit zugänglich machen" wollte (Wissenschaftlicher Beirat für Familienfragen beim Bundesministerium für Jugend, familie und gesundheit, 1975, 5). Wie der Titel bereits suggeriert, sollten dabei insbesondere „Analysen, Überlegungen und Empfehlungen zur Förderung familiengerechten Wohnens" zusammengefasst werden (Wissenschaftlicher Beirat für Familienfragen beim Bundesministerium für Jugend, familie und gesundheit, 1975, 5). Ein solches Gutachten in Zeiten der sozialliberalen Koalition mag zunächst verwundern, da eine Hervorhebung familienpolitischer Begründungsmuster in der Wohnungspolitik meist der Linie von CDU und CSU zuzuordnen ist. Allerdings wurde das Gutachten nicht im Auftrag des Ministeriums, sondern auf Betreiben des Beirates selber erstellt. So heißt es in der Einleitung:

„Das Problem familiengerechten Wohnens muß als integrierter Bestandteil einer wohnungspolitischen Gesamtkonzeption gesehen werden. Da die bisherigen wohnungspolitischen Maßnahmen keine klare Ausrichtung auf eine solche Konzeption erkennen lassen, sieht es der Beirat als notwendig an, sich im Rahmen seiner Untersuchungen mit der bisherigen Wohnungspolitik und ihren Mängeln auseinanderzusetzen, um familienpolitische Gesichtspunkte verstärkt in der Wohnungspolitik zur Geltung zu bringen." (Wissenschaftlicher Beirat für Familienfragen beim Bundesministerium für 
Jugend, familie und gesundheit, 1975, 11, eigene Hervorhebung)

Dabei wird deutlich, dass andere Beiräte gegenüber dem Wohnungswirtschaftlichen Beirat auch durchaus ohne Weisungen der Regierungen aktiv werden und somit auch die von ihnen selbst als wesentlich empfundenen Themen in die politische Agenda mit einbringen konnten. Der wissenschaftliche Beirat beim Bundesfamilienministerium lässt sich als ein überaus gemischt besetztes Gremium aus Pädagogen, Soziologen, weiteren Sozialwissenschaftlern und Volkswirten und einem Statistiker beschreiben. Besonders interessant ist im Zusammenhang mit dem vorliegenden Forschungsprojekt, dass der erste Vorsitzende zwar ein Soziologe war, der zweite war allerdings Willi Albers, ein Professor der Finanzwissenschaften, der laut Vorwort an dem erstellten Gutachten einen maßgeblichen Anteil hatte (Wissenschaftlicher Beirat für Familienfragen beim Bundesministerium für Jugend, familie und gesundheit, 1975, 5f.). Albers gilt als prominenter Sozialökonom (vgl. Hesse, 2010, 125) und prägte als Finanzwissenschaftler an der Universität Kiel die Disziplin maßgeblich (vgl. Kutz, 1997).

Die vermögenspolitische Dimension des Wohneigentums hatte bereits der wissenschaftliche Beirat beim Bundesfamilienministerium in seinem Gutachten von 1975 in den Vordergrund gestellt. In der Befassung mit der Wohneigentumsförderung stellt der Beirat zunächst fest, dass die bis dahin bestehenden Förderbedingungen bewirkt haben, „daß die zu Eigentum wohnenden Familien im Durchschnitt weit besser mit Wohnraum versorgt sind als die zur Miete wohnenden" (Wissenschaftlicher Beirat für Familienfragen beim Bundesministerium für Jugend, familie und gesundheit, 1975, 116) und spricht sich ,grundsätz- 
lich für die Beibehaltung einer besonderen Förderung der Eigentumsbildung im Wohnungsbereich aus" (Wissenschaftlicher Beirat für Familienfragen beim Bundesministerium für Jugend, familie und gesundheit, 1975, 116f.). Begründet wird dies damit, dass sich der Eigentumserwerb ,[g] [gade für diejenigen Sozialschichten, die sich bisher an den Maßnahmen zur Eigentumsbildung unterdurchschnittlich beteiligt haben [als wichtig erweist], da es sich um eine geldwertgesicherte Anlageform handelt, die mit einem geringeren Risiko behaftet ist als eine Beteiligung am Produktivvermögen. Darüber hinaus gibt es wenig andere Vermögensformen, die ihrem Eigentümer einen ähnlichen, von ihm auch real zu nutzenden Freiheitsspielraum verschaffen, wie das Grundeigentum (Wissenschaftlicher Beirat für Familienfragen beim Bundesministerium für Jugend, familie und gesundheit, 1975, 116). Diese Einschätzung ist deswegen bemerkenswert, weil der vermögenspolitische Aspekt in Vorgängergutachten noch nicht derart explizit zutage getreten ist. Insgesamt plädiert der Beirat für eine stärkere subjektgebundene Förderung über das Wohngeld und eine Abkehr der als nicht zielgenau genug empfundenen Objektförderung des Sozialen Wohnungsbaus.

Dabei war die Vermögensbildung bereits in der Regierungserklärung von Willy Brandt im Jahr 1969 zu einem wesentlichen Ziel der sozialliberalen Koalition erklärt worden (Kornemann, 1996, 133). Laut Ehrenberg sind die Ursachen dieses regelrechten Trends zu vermögenspolitisch motivierten Maßnahmen vor allem auf das Jahresgutachten des Sachverständigenrates zur Begutachtung der gesamtwirtschaftlichen Entwicklung von 1968/69 zurückzuführen. Durch dieses Gutachten sei die Vermögenspolitik, „wieder in den Mittelpunkt der wirtschaftspolitischen Diskussion“ gerückt worden (Ehrenberg, 1971, 49). 
Auch der wissenschaftliche Beirat Wissenschaftlichen Beirats beim Bundesministerium für Wirtschaft befasste sich im Rahmen eines Sondergutachtens mit den „Probleme[n] der Wohnungswirtschaft“. Als Anlass der gutachterlichen Tätigkeit des Beirates wird die „Neue Wohnungsnot" genannt, welche der Beirat selbst allerdings konsequent in Anführungszeichen setzt (Wissenschaftlicher Beirat beim Bundesministerium für Wirtschaft, 1982, 2). Damit knüpft er an eine entsprechende öffentliche Debatte an, die sich, ,angesichts der Besetzung leerstehender Häuser [...] verschärft" habe (Wissenschaftlicher Beirat beim Bundesministerium für Wirtschaft 1982, 3; vgl. Abschnitt 6.1). Die Anführungszeichen gehen einher mit der Beiratserklärung, dass „die Versorgung der Bevölkerung in der Bundesrepublik Deutschland mit Wohnraum insgesamt noch nie so gut war wie heute und [...] die Bundesrepublik zu den Ländern mit der besten Wohnraumversorgung in der Welt zählt" (Wissenschaftlicher Beirat beim Bundesministerium für Wirtschaft, 1982, 2). Auch wenn der Beirat die Bezeichnung des status quo als Wohnungsnot anerkennt, greift er die Debatten ,zum Anlaß einer Besinnung auf eine langfristige und grundsatzorientierte Wohnungspolitik“ auf, was ihm ,deshalb erforderlich [scheint], weil die im Gefolge staatlichen Engagements in der Wohnungswirtschaft aufgetretene Belastung öffentlicher Haushalte als nicht mehr tragbar erscheint und durch eine Neuorientierung der Wohnungspolitik eine erhebliche Entlastung der öffentlichen Haushalte erreichbar ist" (Wissenschaftlicher Beirat beim Bundesministerium für Wirtschaft, 1982, 3). Auch Häußermann und Siebel erklären, dass es schwer fällt angesichts der quantitativen wie auch qualitativen Verbesserungen der Wohnungsversorgung in der Bundesrepublik seit den Weltkriegen von einer tatsächlichen Wohnungsnot 
zu sprechen (Häußermann u. Siebel, 1981, 318).

Der Beirat plädiert in seinem Gutachten maßgeblich für eine weitere Deregulierung des Wohnungsmarktes und dafür ,schrittweise mehr marktwirtschaftliche Grundsätze zu verwirklichen [...] die inflationsbedingten volkswirtschaftlichen Störungen weitgehend auszuschalten [und] die der Wohnungspolitik gestellten sozialpolitischen Ziele mehr als bisher durch Subjektförderung zu verwirklichen" (Wissenschaftlicher Beirat beim Bundesministerium für Wirtschaft, 1982, 3). Kritisch äußert sich der Beirat in diesem Sondergutachten in Bezug auf die Eigenheimförderung insbesondere, dass diese vor allem die mittleren und oberen Schichten zugute kommen würde und damit „Probleme der unteren Schichten der Bevölkerung vernachlässigte" (Wissenschaftlicher Beirat beim Bundesministerium für Wirtschaft, 1982, 18). Diese ungerechtfertigten Begünstigungen bestünden laut Beirat sowohl bei steuerlichen Maßnahmen als auch im vermögenspolitischen Bereich. Insgesamt habe die bisherige Wohnungspolitik zwar einige sozialpolitische Probleme gelöst, aber dennoch auf diesem Gebiet ,wohl mehr Probleme geschaffen" (Wissenschaftlicher Beirat beim Bundesministerium für Wirtschaft, 1982, 20f.). Für die künftige Wohnungspolitik empfiehlt der Beirat demgegenüber: „Eine wesentliche Aufgabe der Wohnungspolitik sollte nach dem Erreichen eines globalen Ausgleichs von Angebot und Nachfrage auf dem Wohnungsmarkt darin bestehen, Bedingungen zu schaffen, die eine möglichst schnelle Anpassung des Angebots an Änderungen der Nachfrage sicherstellen" (Wissenschaftlicher Beirat beim Bundesministerium für Wirtschaft, 1982, 21). Hiermit gibt der Beirat über eine Problemdiagnose oder empfohlene Instrumente hinaus auch eine Zielsetzung für die Wohnungspolitik, die vor allem marktorientier- 
te Ideale zum Ausdruck bringt, was sich immer öfters in den künftigen Zielsetzungen wohnungspolitischer Debatten finden sollte. Diese gab es allerdings bereits in den 60er, aber auch schon in den 50er Jahren in den Debatten rund um die sogenannte „Wohnungszwangswirtschaft“. Nun allerdings scheinen sie auch in den allgemeinen wohnungspolitischen Diskussionen verbreiteter und allgemein akzeptierter als dies in der früheren Phase der Fall war.

In Bezug auf die Wohneigentumsförderung lassen sich in dem Gutachten des Wissenschaftlichen Beirates beim Bundeswirtschaftsministerium nur geringe Rechtfertigungen finden. Ganz im Gegenteil. Zwar sei eine staatliche Subventionsform sinnvoll, ,[s] ofern gewisse Formen des Wohnen oder das Eigentum an der genutzten Wohnung positive externe Effekte erzeugen" (Wissenschaftlicher Beirat beim Bundesministerium für Wirtschaft, 1982, 50f.). Allerdings sei dazu „diese Subventionspolitik gezielt auszurichten auf die Personen, deren Verhalten durch diese Maßnahme beeinflußt werden soll“, was am besten über Subjektförderung zu erreichen sei (Wissenschaftlicher Beirat beim Bundesministerium für Wirtschaft, 1982, 51).

„Hinsichtlich der steuerlichen Behandlung von Eigentumsmaßnahmen tritt der Beirat für eine Regelung ein, bei der die verschiedenen Gruppen von Bauherren gleich behandelt werden, und dafür, daß auch im Verhältnis zwischen Bauherren von Eigenheimen und Eigentumswohnungen einerseits und Bauherren im Mietwohnungsbau andererseits das Prinzip der Gleichbehandlung gilt.“ (Wissenschaftlicher Beirat beim Bundesministerium für Wirtschaft, 1982, 54)

Ebenso findet sich in dem Gutachten die Ansicht, Wohneigentum 
als Kapitalanlage zu betrachten. Gefordert wird dabei eine Gleichbehandlung der Immobilieninvestition zu anderen Kapitalanlagen, die im weiteren Verlauf noch an Bedeutung gewinnen wird:

„Der Beirat sieht im gegenwärtigen Steuersystem und Subventionssystem eine starke Begünstigung der Kapitalbildung im Wohnungssektor im Verhältnis zur Kapitalbildung, die direkt oder indirekt (über Kapitalsammelstellen) den Investoren im gewerblichen Sektor zugute kommt. [...] Durch Inflation wird diese unterschiedliche steuerliche Behandlung von Geldvermögen und Wohnungseigentum noch verschärft.“ (Wissenschaftlicher Beirat beim Bundesministerium für Wirtschaft, 1982, 51)

Eigenheimbesitzer, so die Analyse des Beirates, seien somit „,besser gestellt als derjenige, der sein Vermögen in Geldkapital anlegt" (Wissenschaftlicher Beirat beim Bundesministerium für Wirtschaft, 1982, 52). Auch die amtliche Statistik wurde mit weiteren Gebäudezählungen und Erfassungen zur Wohnsituation weiter ausgebaut (Glatzer, 1980). Basierend auf den Gesetzgebungen der 50er und 60er Jahre führte das Statistische Bundesamt 1972 und 1978 weitere Wohnungsstichproben durch. Anfang 1980 wurden die ersten Ergebnisse der Stichprobe von 1978 veröffentlicht und gaben ,den politischen Kräften bei divergierender Interpretation neues Material zur wohnungspolitischen Auseinandersetzung an die Hand" (Dringenberg, 1983, 153). Während gegen Ende 1979 basierend auf statistischen Fortschreibungen noch von einem ausgeglichenen Wohnungsmarkt ausgegangen wurde und ggf. sogar verstärkte Abrissmaßnahmen für die künftige Entwicklung vorgeschlagen wurden (so Bartholmai u.a., 1980), ,lagen [wenig später] die ersten 
Hochrechnungen aus der Wohnungsstichprobe von 1978 vor, derzufolge die Gesamtzahl der Wohnungen um 1,3 Mio. nach unten korrigiert wurde“ (Dringenberg, 1983, 154). Als Ursachen der ,neuen Wohnungsnot" nennt Harlander (2008) den Einbruch des Neubaus nach einer Steigerung von Baukosten und Grundstückspreisen in den Großstädten sowie die ,fortlaufende Verringerung der preisgünstigen Bestände durch Abriss, Zweckentfremdung, Luxusmodernisierung und Umwandlung von Miet- und Eigentumswohnungen“ (Harlander, 2008, 842). Im Rahmen der Debatten zur neuen Volkszählung sollte der Kenntnisstand durch eine erweiterte Zählung von Gebäude- und Wohnungsbeständen aktualisiert und präzisiert werden (vgl. BT-Drs. 09/451). Die Investitionsplanungen ,mit einem Zahlenwerk, dem beträchtliche Fehlerquoten unterstellt werden“ (Dringenberg, 1983, 154) erschien Akteuren aus Politik und auch aus der Verbändelandschaft nach Dringenberg unzumutbar (siehe hierzu auch Abschnitt 6.3.1). Somit wurde eine weitere Gebäude- und Wohnungszählung für 1981 angesetzt (Glatzer, 1980, 15). Waren bis 1977 nur in unregelmäßigen Abständen in der Veröffentlichungsserie „Wirtschaft und Statistik" des Statistischen Bundesamtes Beiträge zur Wohnungsstatistik erschienen (Glatzer, 1980, 229), wurde die Fachserie E zur Bauwirtschaft und Bautätigkeit ab 1977 zur Fachserie 5 ,Bautätigkeit und Wohnungen“ zusammengefasst (Glatzer, 1980, 228). Ab diesem Zeitpunkt erscheinen bis heute in regelmäßigeren Abständen die Ergebnisse neuer Wohnungszählungen sowie deren Fortschreibungen auf Grundlage der Bautätigkeit (Glatzer, 1980, 17). 


\subsection{Phase 2: Vermarktlichung mithilfe von Marktexperten}

Der allgemeine Wandel der Wohnungspolitik in der zweiten Phase wird oft als wohnungspolitische Wende von 1982 bezeichnet und maßgeblich dem Wechsel zur konservativ-liberalen Bundesregierung zugeschrieben. Allerdings konnte anhand der politischen Debatten sowie dem Instrumentenwandel gezeigt werden, dass die durch die schwarz-gelbe Koalition durchgeführten wohnungspolitischen Maßnahmen bereits in der sozial-liberalen Koalition vorbereitet und in Teilen bereits durchgeführt worden waren. Gegenüber dem sozialen Wohnungsbaus treten bereits in den 70er Jahren unter der sozialliberalen Koalition steuerliche Förderungsinstrumente in den Vordergrund. So bestand ,unter der Mehrheit der wohnungspolitischen Akteure ein Konsens darüber [...], dass der soziale Wohnungsbau in der Vergangenheit sinnvoll und notwendig war, aber seit den 1970er Jahren zunehmend seinen Sinn verloren und auch nicht intendierte Effekte gezeigt habe“ (Heinelt u. Egner 2006, 214; siehe auch Leutner 1990, 386).

Eine Ursache für den Wandel der Wohneigentumsförderung muss in einem Wandel der Positionierung der SPD gesehen werden. Während sie in der ersten Phase die Eigenheimförderung lediglich mitgetragen hatte, befürworten ihre Abgeordneten in der zweiten Phase stark die Förderung des Eigenheimes und brachten auch entsprechende Gesetze selber ein. Entgegen der Intentionen zur Förderung des gemeinnützigen Wohnungsbaus in der ersten Phase befürworteten nun auch die Gemeinnützigen, allen voran die Neue Heimat, die Förderung der Eigentumswohnungen - auch zugunsten von Verkäufen aus ihren eigenen 
Beständen. Sowohl CDU/CSU als auch die SPD waren darum bemüht, „sich selbst als die jeweils ,besseren“ Verfechter der Wohneigentumsförderung auszuweisen“ (Krummacher, 1978, 352). Der Wechsel zur sozialliberalen Koalition hatte somit keinen grundlegenden Wandel in der Eigenheimpolitik zufolge - ebensowenig der spätere erneute Wechsel zur konservativ-liberalen Bundesregierung zu Beginn der 80er Jahre. Die Folge war ein weiterer Ausbau der Eigenheimförderung im Sinne eines moving consensus (Jaedicke u. Wollmann 1991, 423f.; Harlander vgl. 2008, 850). Obwohl Wohnungspolitik häufig als ein politisch umkämpftes Feld angesehen wird, in dem die verschiedenen Parteien um seine Ausgestaltung kämpfen, kann für die zweite Phase gezeigt werden, dass selbst im Falle von Regierungswechseln die Wohnungspolitik in Deutschland durch einen überaus stetigen Wandel und nicht durch abrupte politische Richtungsänderungen geprägt ist. Regierungen übernahmen meist jene Maßnahmen und Weichenstellungen, die von ihren Vorgängern eingeführt worden waren, sodass von einem regelbezogenen institutionellem Wandel kaum die Rede sein kann. Die Förderung von Wohneigentum sollte erweitert werden, sodass nun nicht mehr nur dem klassischen Familienheim mit eigenem Garten entsprechen musste, sondern auch in Form einer Eigentumswohnung in städtischen Gebieten förderberechtigt war.

An dieser Stelle zeigt sich erneut die Bedeutung von mit politischen Maßnahmen fest verknüpften Ideen im Sinne von cognitve locking (Blyth, 2001): institutionalisierte Ideen können dazu beitragen, dass weitere policy-Änderungen in Bezug auf diese Ideen stattfinden. Familienpolitische Begründungen der Eigenheimförderung sind auch in der zweiten Phase weiterhin vorhanden - auch wenn diese zunehmend wie 
eine Rahmung vermögenspolitischer Argumentationen wirken. So weit mit einer Institution verknüpfte Ideen im Sinne des historischen Institutionalismus zu Pfadabhängigkeiten beitragen können, kann ideeller Wandel aus Perspektive des diskursiven Institutionalismus auch zu institutionellem Wandel beitragen. So findet sich anstelle der Belohnung sparsamen Verhaltens aus der ersten Phase nun die Ansicht, Wohneigentum solle ähnlich wie andere Kapitalanlagen zum Zweck der privaten Altersvorsorge verwendet werden. Gegenüber dem gesamtgesellschaftlichen Ideal der Eigentümer tritt das Ideal des sich selbst im eigenen Heim verwirklichenden Bürgers: Die Förderung von Individualität und Unabhängigkeit der Bürgerinnen und Bürger geht dabei einher mit der Forderung durch gezielte, bedarfsgerechte Förderung individuellen Bedürfnissen nach Möglichkeit gerecht zu werden.

Die Ursachen für ein Ausbleiben des Wandels nach dem Wechsel von Regierungen kann zwar den geringer werdenden wohnungspolitischen Unterschieden zwischen den Parteien zugeschrieben werden. Allerdings erklärt dies noch nicht den langsamen kognitiven wie auch normativen Wandel im Vergleich zur ersten hier betrachteten Phase. Dieser Wandel wird allerdings nachvollziehbarer, wenn Wandlungsprozesse im Regime der Wissensproduktion mit berücksichtigt werden. Auffällig ist dabei insbesondere ein regelrechter Boom gutachterlicher Tätigkeiten im Rahmen einer allgemeinen Planungseuphorie. Beauftragt wurden dabei zunächst verschiedene Experten aus dem sozialwissenschaftlichen Spektrum, wobei zunehmend Wirtschafts- und Sozialwissenschaftler mit quantitativen Ansätzen an Relevanz gewinnen konnten. Diese profitierten vor allem durch die nun zunehmend etablierten analytischen Tools der amtlichen Statistik, die einen entspre- 
chenden Zugang zum Politikgegenstand erst ermöglichten. Inhaltlich treten in dieser Phase vor allem die Marktwirtschaftler deutlicher hervor und übernehmen während und nach der Krise der Neuen Heimat eine wichtige, betont neutrale Position zu wohnungspolitischen Themen und bringen ihre Ideen und Analysen in die politischen Debatten mit ein. Ihr Bedeutungszuwachs im Bereich der Wohnungspolitik lässt sich besonders deutlich an der Karriere des Wirtschaftswissenschaftlers Johann Eekhoff erkennen, der zunächst als Wissenschaftler Auftragsgutachten für die sozialliberale Koalition etablieren konnte und unter der konservativ-liberalen Koalition schließlich zu einem einflussreichen Abteilungsleiter im Bundesministerium für Raumordnung, Bauwesen und Städtebau wird.

Wissenschaftliche Gutachten wurden nicht nur vonseiten Ministerialbürokratie in Auftrag gegeben, sondern auch von verschiedenen Verbänden der Wohnungswirtschaft, der Kreditwirtschaft sowie von Gewerkschaften und Mieterverbänden. Zusätzlich liefern den Verbänden nahestehende Think Tanks und Stiftungen Beiträge zur Wissensproduktion. In diesem Sinne kann im Bereich der Ideen- und Wissensproduktion in der Eigenheimförderung der Bundesrepublik zwar von einem relativen Bedeutungsverlust industriell-korporatistischer Wohnungsmarktelemente durch den Skandal der Neuen Heimat verzeichnet werden, jedoch bei weitem keine Auflösung. Als wesentlicher Mechanismus kann in der zweiten Phase korporatistische Politikberatung identifiziert werden, wobei Verbände und Parteien wie bereits in der ersten Phase eng miteinander verbunden sind. Allerdings ist die Kommunikation ihrer Position mithilfe wissenschaftlicher Publikationen eine andere geworden. Dabei spielen vor allem Kennzahlen der amtlichen Statistik 
wie die Wohneigentumsquote eine immer größere Rolle. Wurden statistische Beschreibungen wohnungspolitischer Zusammenhänge in der ersten Phase noch nicht anerkannt oder aber sogar bekämpft, haben sie sich diese in der zweiten Phase weitgehend etabliert, sodass sie sogar als Zielsetzungen für policy-Wandel fungieren können. Sie bilden nicht nur die Grundlage für Studien der Verbände: Sowohl die Unionsparteien als auch FDP und SPD verwenden diese Kennzahlen als Ausgangsbasis für Maßnahmen zur Erhöhung der Wohneigentumsquote ohne weitere Zieldimensionen dabei zu erwähnen. Der in der ersten Hypothese herausgestellte Einfluss der Wissensmaschinerie auf die verfügbaren Ideen in politischen Debatten kann auch in der zweiten Phase als bestätigt angesehen werden: der ideelle Wandel vollzieht sich nahezu spiegelbildlich zum sich wandelnden Akteursspektrum und den Beratungsmechanismen im knowledge regime.

Entscheidend für den Wandel in der Expertise erscheinen zwei Entwicklungen: Erstens führt die Idee der Planbarkeit und der Berechenbarkeit politischer Maßnahmen zu einer regelrechten Wissenschaftseuphorie politischer Akteure. Wissenschaftliche Begründungen für das eigene Handeln werden en vogue und erfordern die entsprechende gutachterliche Tätigkeit. Zwar steigt die absolute Anzahl der wirtschaftswissenschaftlichen Experten in der Wissensproduktion an. Diese sind zwar durch die Auftragsgutachten besser mit Ressourcen ausgestattet als dies noch in der ersten Phase der Fall war. Allerdings sind sie in diesen Tätigkeiten stark abhängig von der Ministerialbürokratie. Demgegenüber existiert kein Gremium mehr wie der wohnungswirtschaftliche Beirat, dem mehr Eigenständigkeit in der inhaltlichen Arbeit zugestanden worden war als den jeweils berufenen Gutachtern. Entgegen 
dieser institutionell schwächeren Position der wirtschaftswissenschaftlichen Expertise gewinnt das Expertenwissen jedoch zweitens an diskursiver Bedeutung: Durch zunehmend vorhandene Kennzahlen und Statistiken als analytische Tools erhielten die Wirtschaftswissenschaftler nicht nur die Grundlage für ihre Analysen, ihnen und ihrer Forschung wurden durch die Auftragsforschung auch neue Ressourcen zuteil. War das Eigenheim in der ersten Phase noch deutlich stärker mit gesellschaftspolitischen Idealvorstellungen verknüpft und dessen Diskussion anhand statistischer Kennzahlen tabu, wurde es im Verlauf der Jahre zu einer durch Kennzahlen bestimmbaren Größe. So konnte das „Familienheim" der ersten Phase erstmalig als eine Art Anlagegut betrachtet und politisch diskutiert werden. Von dieser Phase an ist somit zumindest eine teilweise anerkannten Konstruktion des Eigenheimes anhand von analytischen Tools im Sinne der Science and Technology Studies festzustellen: Politische Akteure wurden anhand von Kennzahlen und deren Interpretationen dazu befähigt die Lage auf dem Wohnungsmarkt aus einem anderen Blickwinkel zu erfassen (vgl. Lowe, 2011). Wohnungspolitik konnte so zunehmend zu einer „Angelegenheit von Experten“ (Häußermann u. Siebel, 1981, 316) werden.

Für beide relevanten Entwicklungen des knowledge regime der zweiten Phase sind Akteure der öffentlichen Verwaltung entscheidend. Anders als die nur kurzfristig zur Gutachtenerstellung einberufenen Experten, konnte die Ministerialbürokratie wie auch die amtliche Statistik durch ihre auf Dauer ausgerichteten Ressourcen profitieren und gegenüber der ersten Phase sichtlich an Einfluss gewinnen. Dies wird in einer zunehmenden Einbindung staatlich geförderter und privater Forschungseinrichtungen durch die Ministerialbürokratie deutlich. Wäh- 
rend die Ministerialbürokratie über den Zugang der Experten zu dem knowledge regime entscheidet und damit als gatekeeper fungiert, stellt die amtliche Statistik die zugrundeliegenden analytischen Tools bereit. Entsprechend der zweiten These von Skocpol 1985 wirken Handlungen der amtlichen Statistik und Weichenstellungen der Ministerialbürokratie in der Gutachtenvergabe nun sinngebend und sogar handlungsanleitend für andere Akteursgruppen aus Wirtschaft und Politik. Somit kann die zweite getroffene Hypothese zur Bedeutung staatlicher Stellen für die Wissensproduktion in der zweiten Phase als bestätigt angesehen werden.

Die zunehmende Vermarktlichung durch Eigenheimförderung in der policy-Dimension wurde somit in der zweiten Dimension verstärkt durch eine Vermarktlichung auf der kognitiven Dimension getragen. Auch normativ schlugen sich die marktorientierten Argumentationsmuster zunehmend nieder. Durch die ersten Betrachtungen des Wohneigentumsförderung nicht mehr nur als erlebbares, sondern auch anlegbares Vermögen legen zudem die Grundsteine für eine Finanzialisierung im Sinne von Epstein, da in dieser Hinsicht auch Finanzmotive in der Wohneigentumsförderung eine zunehmende Bedeutung erlangen (vgl. Epstein 2005b; Heeg 2013; Forrest u. Hirayama 2014). In der dritten Phase sollten vor allem die Finanzialisierungsaspekte des Wohneigentums markanter werden und die politischen Möglichkeiten der Förderung erweitern. 


\section{Kapitel 7}

\section{Phase 3: Verringerte}

\section{Wohneigentumsförderung}

als Teil der privaten

\section{Altersvorsorge}

Nachdem die Engpässe in der Wohnungsversorgung nach der Wiedervereinigung weitgehend behoben schienen, verlor die Wohnungspolitik auf Bundesebene im Laufe der dritten Phase ihren einstmaligen Stellenwert: Das Ministerium verlor seine Eigenständigkeit und auch Parteien engagierten sich deutlich weniger als zuvor im wohnungspolitischen Bereich (Abschnitt 7.1). Für die Wohneigentumsförderung kamen erschwerend die lauter werdenden Forderungen nach einem schlanken Staat hinzu, die das Ende der kostenintensivsten Einzelsubvention im 
Bundeshaushalt zur Folge haben sollten. Neben der Austeritätspolitik wurden die wohneigentumsbezogenen Debatten von dem bereits in der vorherigen Phase bedeutender gewordenen Zielvorstellung der individuellen Vermögensbildung beherrscht (Abschnitt 7.2). Familienpolitische Vorstellungen spielten zwar zu Beginn der 90er Jahre noch eine Rolle, verloren aber zunehmend an Bedeutung gegenüber der privaten Altersvorsorge und dem Austeritätsparadigma. Hatten sich bereits in der vorherigen Phase wohnungspolitische Debatten durch eine zunehmende Verwissenschaftlichung ausgezeichnet, intensivierte sich dieser Trend in der dritten Phase deutlich. Dabei wurde das Spektrum der Wissensproduktion sowohl um neue Akteure als auch um neue Formen der Wissensproduktion erweitert (Abschnitt 7.3). Für Ministerialbürokratie und amtliche Statistik bringen diese neuen Akteure und die von ihnen produzierten Daten neue Herausforderungen mit sich - ebenso wie Anforderungen internationaler Gremien an die Wissensproduktion zu Wohnungsmärkten durch die öffentlichen Stellen selbst. Wie bereits in den vorherigen Kapiteln sollen die zentralen Ergebnisse dieser Phase in Abschnitt 7.4 zusammengefasst werden.

\subsection{Bedeutungsverlust der Wohnungspolitik \& Haushaltsdisziplin}

Bedingt durch einen massiven Zustrom von Aussiedlern der ehemaligen Sowjetrepubliken kam es bereits Ende der 80er Jahre zu einem sprunghaften Anstieg der Wohnbevölkerung in der Bundesrepublik. Diese Umstände brachten den quantitativen Wohnraumbedarf gegenüber den qualitativen Anforderungen erneut auf die bundespolitische 
Tagesordnung (Leutner 1990, 255; Mayer 1998, 206f.). Die Wiedervereinigung stellte eine zusätzliche Herausforderung der Wohnungspolitik dar, da nun nicht nur zwei überaus verschiedene Wohnungsmärkte, sondern auch unterschiedliche Wohnungspolitiken zusammengeführt werden mussten. Mit der „Mietenreform Ost“ sollten im Wesentlichen die Grundsätze der westdeutschen Mietenpolitik mit Investitionsanreizen und Wohngeldunterstützung in vereinfachter Form auf die Wohnungsmärkte in den Neuen Ländern übertragen werden (Egner, 2014). Aufgrund zahlreicher ungeklärter Eigentumsverhältnisse im direkten Nachgang der Wende konnten in den Neuen Bundesländern zunächst lediglich Maßnahmen zur Bestandserhaltung und -verbesserung ergriffen werden (Mayer, 1998, 209). Der neuen Wohnungsknappheit in den Alten Bundesländern sollte zugleich mit einer Wiederbelebung des Sozialen Mietwohnungsbaus sowie durch die Förderung selbstgenutzten Wohneigentums entgegen gewirkt werden (Mayer, 1998, 212). Die weiterbestehende Regierung Kohl intensivierte ihre Förderung des selbstgenutzten Wohneigentums. Für die gesamte wiedervereinigte Bundesrepublik wurde zudem die steuerliche Förderung von Wohneigentum weiter ausgebaut. So wurde das in den 80er Jahren eingeführte Baukindergeld 1990 und 1991 schrittweise auf 1.000 DM pro Kind angehoben (Kofner, 2004, 188) und auch die steuerliche Berücksichtigung der Herstellungskosten stieg 1991 auf 330.000 DM (Kornemann, 2000, 95). Auch in der ehemaligen DDR sollte nach der Regierungserklärung von Kohl im Januar 1991 das Wohneigentum ,so schnell wie möglich attraktiv werden" (Bulletin der Bundesregierung, zitiert nach Kornemann 1996, 140). Die Bundesbauministerin Schwaetzer erklärte in ihrer Grundsatzrede vom 6. Februar 1991 ebenfalls: 
„Auch in den nächsten Jahren wird die Bildung von Wohneigentum ein wichtiger Schwerpunkt der Wohnungsbautätgkeit sein. Hier gab es in den letzten Monaten zusätzliche Belastungen durch die Baukosten- und Zinsentwicklung. Gerade jetzt dürfen wir nicht in der Wohneigentumsförderung nachlassen." (zitiert nach Gesamtverband der Wohnungswirtschaft, 1992, 18)

Erreicht werden sollte dies vor allem über einen zügig voranschreitenden Verkauf der Wohnungen an bisherige Mieter. Bis heute zeigen sich allerdings deutliche Unterschiede in den Wohneigentumsquoten zwischen Ost- und Westdeutschland (vgl. Abbildung 7.1). Angesichts der nicht maßgeblich angestiegenen Wohneigentumsquote und einem Höchststand von Subventionen für die Wohneigentumsförderung 1996, die Egner (2014) auf rund 12,8 Milliarden Euro an Mindereinnahmen von Steuergeldern schätzt, kam es zu einer wesentlichen Reform. Es schien klar geworden zu sein ,dass die Subventionen, die seit der Ära Adenauer geflossen waren, das eigentlich gesetzte Ziel, sehr viel mehr Deutsche zu Eigenheimbesitzern zu machen, nicht erreicht hatten“ (Egner, 2014). Mit dem Eigenheimzulagegesetz (EigZulG) wurde die alte Förderung, auf ein sogenanntes Konsumgutmodell umgestellt (vgl. Kofner, 2004, 190f.). Sie funktioniert entgegen dem vorher schon abgeschwächten Investitionsgutmodell nicht mehr über Steuerabzüge, sondern über reale Zulagen, die auf Antrag genehmigt werden konnten (Egner 2014; Georgakis 2004, 93).

Mit dem Amtsantritt der rot-grünen Bundesregierung 1998 erfolgte laut Heinelt u. Egner (2006) eine Wende in der Wohnungspolitik. Zentrales Merkmal der neuen Bundesregierung war politikfeldübergreifend 
Abbildung 7.1: Eigentümerquote nach Bundesländern im Zeitvergleich.

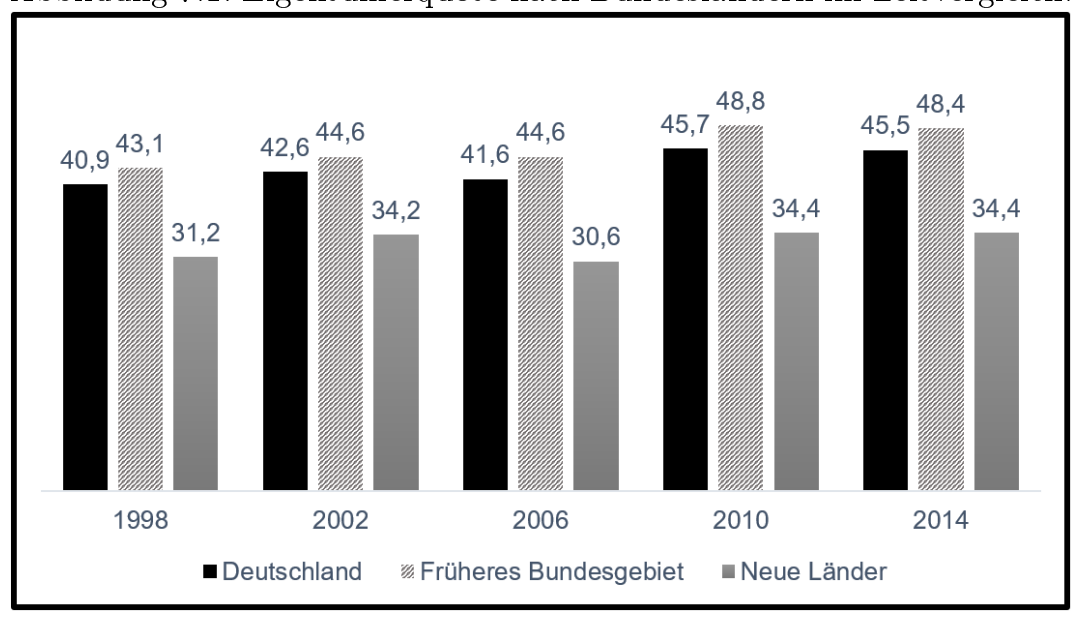

Quelle: Statistisches Bundesamt, eigene Darstellung

der von ihr verfolgte Sparkurs. Die Konsolidierung der Staatsfinanzen wurde ,zu einem wichtigen Ziel der sozialdemokratisch-grünen Regierungspolitik“, wobei allerdings „die gesamtwirtschaftliche Verantwortung staatlicher Finanzpolitik für die Stärkung des Wirtschaftswachstums im Vordergrund" bleiben sollte (Frenzel, 2002, 267). Mit dem Wechsel im Finanzministerium von Bundesminister Oscar Lafontaine zu Hans Eichel 1999 vollzog sich ein Wechsel hin zu einer deutlich regideren Austeritätspolitik (vgl. Frenzel, 2002, 265), die Seeleib-Kaiser (2003b) als eine programmatische Konvergenz in der Haushaltspolitik von SPD und Grünen mit dem liberal-konservativem Spektrum beschreibt (Seeleib-Kaiser, 2003b, 349). Die Sparpolitik der SPD ist vor dem Hintergrund der sich gegen Ende 2001 abschwächenden Konjunktur zu sehen: ,[D]ie Aktienkurse stürzten, die Arbeitslosenzahlen stiegen, die vorausgesagten Steuereinnahmen blieben aus“" (Seeleib-Kaiser, 
2003b, 348). So wurden im sozialpolitischen Bereich unter der rotgrünen Bundesregierung Einsparungen vorgenommen und Reformen vorangetrieben. Eine wesentliche Neuerung bestand in der Rentenreform 2001, benannt nach dem damaligen Bundesminister für Arbeit und Soziales, Walter Riester. Sie gilt als „Kernstück der sozialen Sicherungspolitik zwischen 1998 und 2003“ (Nullmeier, 2003, 167). Mit dem Altersvorsorgeverträge-Zertifizierungsgesetz (AltZertG) wurde eine die staatliche Förderung ergänzende, privat kapitalgedeckte Altersvorsorge beschlossen. Mit privaten Ersparnissen sollte die Rentenlücke der nach dem Umlageverfahren finanzierten Gesetzlichen Rentenversicherung kompensiert werden (Benölken u. Bröhl, 2018, 115ff.). Sie definiert somit förderungswürdige, der Altersvorsorge dienende Finanzprodukte, die hierfür zertifiziert werden müssen (Nullmeier, 2003, 173).

Im Feld der Wohnungspolitik musste zunächst das zuständige Bundesministerium seine Eigenständigkeit einbüßen, indem es mit dem Bundesverkehrsministerium zum Bundesministerium für Verkehr, Bauund Wohnungswesen zusammengelegt wurde (Bartholomäi, 2004, 31). Mit dem Gesetz zur Sozialen Wohnraumförderung (WoFG) von 2001 wurde zudem die Phase des Sozialen Wohnungsbaus ,faktisch beendet und durch das Konzept der Sozialen Wohnraumförderung ${ }^{1}$ ersetzt" (Egner, 2014, 17). Die Änderungen beziehen sich dabei vor allem auf die Verkleinerung der Zielgruppen der Wohnraumförderung, die Einkommensgrenzen und die individualisierten Ausgestaltungsmöglichkeiten der Fördermodelle auf Ebene der Länder (Szypulski, 2008, 102).

1 Die Änderungen zur Sozialen Wohnraumförderung beziehen sich vor allem auf eine Verkleinerung der förderungsberechtigten Zielgruppen, eine Verringerung der entsprechenden Einkommensgrenzen und die freieren Ausgestaltungsmöglichkeiten der Fördermodelle auf Ebene der Länder (Szypulski, 2008, 102). 
Zugleich wurde das Wohngeldgesetz erstmals nach einem Jahrzehnt an die höheren Verbraucherpreise angepasst.

Auch die erst 1996 eingeführte Eigenheimzulage sollte unter der neuen Bundesregierung reformiert werden. In der Kritik stand sie vor allem aufgrund von haushaltspolitischen Überlegungen. Im Gegensatz zu individuellen Steuerabzügen unter der alten steuerlichen Förderung waren die tatsächlichen Subventionskosten immer nur abschätzbar, aber nie konkret bestimmbar gewesen. Als direkt gezahlte Eigenheimzulage hingegen ,konnten sie leichter in das Blickfeld des Finanzministeriums und der Haushaltspolitiker geraten" (Georgakis, 2004, 103). Besonders deutlich wurde dies in den Subventionsberichten des Bundesfinanzministeriums: Die Eigenheimzulage war mit rund 9,4 Milliarden Euro im Rechnungsjahr 2002 (vgl. Bundesministerium der Finanzen, 2001) die größte Subventionszahlung des Bundes ,noch vor den Finanzhilfen des Bundes für die deutsche Steinkohle (3,1 Mrd. Euro)“ (Georgakis, 2004, 104).

Nachdem einige Anpassungen der Förderung bis 2004 vorgenommen wurden, die sich unter anderem auf den Zuschlag für Kinder des Bauherren bezogen, stand nach der Bundestagswahl von 2002 die komplette Streichung der Eigenheimzulage zur Diskussion (Georgakis, 2004, 102). Allerdings scheiterte die ersatzlose Streichung zunächst an der Unionsmehrheit im Bundesrat, sodass lediglich ein Kompromiss² in Form einer Umgestaltung der Eigenheimzulage 2003 beschlossen werden konnte.

Nach dem Regierungswechsel zur Großen Koalition 2005 schien die

2 Zu den Änderungen zählten eine Aufhebung der Förderungsunterschiede zwischen der Neu- und der Altbauförderung,die deutliche Absenkung der Einkommensgrenze und die Beschränkung der Förderung auf Haushalte mit Kindern (Georgakis, 2004, 102). 
Wohnungspolitik große Teile ihrer einstigen Bedeutung in sozialpolitischer Hinsicht verloren zu haben. So war die staatliche Wohnraumförderung auf ein neues Mindestmaß gekürzt worden und der Soziale Wohnungsbau aufgrund von Kosten und nicht intendierten Effekten wie Fehlbelegungen und der Entwicklung sozialer Brennpunkte derart diskreditiert, dass wohnungspolitische Akteure davon ausgingen, die dazugehörigen politischen Auseinandersetzungen seien abgeschlossen (Heinelt u. Egner, 2006, 214). Wohnungsnöte wurden als ein ,sozial selektives Risiko“ angesehen, sodass „ein politischer Handlungsbedarf im Hinblick auf die Wohnungsversorgung [...] zunehmend de-thematisiert werden konnte" (vgl. Heinelt u. Egner, 2006, 209).

Die allen vorgenommenen Anpassungen zum Trotz bestehenden Ausgaben in Zusammenhang mit der Eigenheimzulage wurden weiterhin als wesentliches Sparpotenzial angesehen. Angesichts „der guten Wohnraumversorgung und der zukünftigen Bedarfsentwicklung“" (Kirchner, 2006, 167) und den übermächtigen Konsolidierungsbestrebungen der ersten großen Koalition unter Angela Merkel wurde die Eigenheimzulage schließlich am 22. Dezember 2005 für Neufälle ab dem 1. Januar 2006 und mit Wirkung bis zum Jahr 2013 vollends abgeschafft, was erstmals einen deutlichen Bruch in der bundesdeutschen Wohneigentumsförderung markieren sollte. Zusammen genommen bezeichnet Egner (2014) die Reformen von der Abschaffung der Eigenheimzulage über das Ende des klassischen sozialen Wohnungsbaus und die Ausweitung des Wohnungsgeldes als weitere Schritte zur „Transformation der Wohnungspolitik zur Wohnungsmarktpolitik“ (Egner, 2014, 18; HvH.i.O.). Entscheidend war auch in dieser Phase der vorhandene Bestand von Wissen und Ideen, vor deren Hintergrund die politischen Akteure ih- 
re Entscheidungen treffen. In Bezug auf Austeritätspolitik weist Marschallek (2004) darauf hin, dass auch politische Handlungszwänge stets als ein Ergebnis politischer Deutungen zu verstehen sind (Marschallek, 2004, 289). Im folgenden Abschnitt werden die dominanten Ideen aus den Bundestagsdebatten zur Einführung der Eigenheimzulage, deren Abschaffung sowie der Überführung der Eigenheimförderung in das Konzept der Riesterrente dargestellt.

\subsection{Schlanker Staat \& Wohneigentum als Finanzanlage}

Angesichts der auch weiterhin nicht maßgeblich angestiegenen Wohneigentumsquoten bei einem Höchststand von Subventionen für die Wohneigentumsförderung 1996, die rund 12,8 Milliarden Euro an Mindereinnahmen von Steuergeldern betrugen (vgl. Bundesministerium der Finanzen, 2001), wurde eine Reform der steuerlichen Wohneigentumsförderung zunehmend als unausweichlich angesehen. In diesem Zusammenhang kam ebenfalls die lang angeführte Kritik der Opposition zum Tragen, dass ,Steuerpflichtige mit höheren Einkommen wegen ihrer progressionsbedingt höheren Steuersätze durch die steuerliche Absetzungsmöglichkeit intensiver gefördert wurden als solche mit niedrigeren Einkommen“ (Kofner 2004, 188; vgl. auch Harlander 2005, 692). Bei der Eigenheimförderung handelte sich demnach nicht nur um eine besonders kostenintensive, sondern auch um eine fehlgeleitete Subvention. Zwar wurde dieser Kritik bereits mit der Einführung von Einkommensgrenzen für die steuerliche Förderung begegnet (Kornemann, 2000, 95). Diese Anpassungen reichten jedoch nicht aus um darüber 
hinwegzutäuschen ,dass die Subventionen, die seit der Ära Adenauer geflossen waren, das eigentlich gesetzte Ziel, sehr viel mehr Deutsche zu Eigenheimbesitzern zu machen, nicht erreicht hatten“ (Egner, 2014).

Mit dem Eigenheimzulagegesetz (EigZulG) von 1996 wurde die vor allem in den 80er Jahren ausgebaute indirekte steuerliche Förderung zugunsten eines sogenannten Konsumgutmodells abgeschafft (vgl. Kofner, 2004, 190f.). Entgegen dem Investitionsgutmodell funktioniert diese nicht über Steuerabzüge, sondern über reale Zulagen, die auf Antrag genehmigt werden können (Egner 2014; Georgakis 2004, 93). Simian weist darauf hin, dass mit dem Eigenheimzuglagengesetz ,erstmals in Deutschland eine von der Steuerprogression unabhängige Förderung für die Finanzierung des sebstgenutzten Wohneigentums vergeben" wurde (Simian, 2000, 123). Die neu geschaffene Eigenheimzulage beinhaltete nochmals strengere Einkommensgrenzen als die steuerliche Förderung, die im Folgejahr sogar noch weiter eingegrenzt wurden (Kornemann, 2000, 95), war jedoch unabhängig von der Einkommenssteuerbelastung und damit auch - innerhalb der Einkommensgrenzen - vom Einkommen des Antragstellers (Georgakis, 2004, 96). Allerdings findet sich ein erheblicher Anteil der Rechtsgrundsätze des §10e EStG in den Regelungen zur Eigenheimzulage wieder (Kofner, 2004, 188). Der Förderbetrag lag zunächst bei 5.000 DM.

Dabei wurde die Eigenheimzulage durchaus noch als Familienförderung diskutiert. So ging Bundeskanzler Kohl in seiner Regierungserklärung vom 23. November 1994 vor allem auf die Wohnsituation von kinderreichen Familien und Alleinerziehenden ein: „Für Eltern und Alleinerziehende sind familiengerechte Wohnungen und ein kinderfreundliches Wohnumfeld von größter Bedeutung. Vor allem in den Ballungsgebieten 
besteht Mangel an bezahlbaren Wohnungen. Wir brauchen deshalb neben mehr Wohnraum auch eine Verstärkung der Wohneigentumsförderung, insbesondere für Familien mit Kindern"(zitiert nach Kornemann, 1996, 141). Auch die Beibehaltung des Baukindergeldes im Rahmen der neuen Eigenheimzulage deutet auf die weiterhin in den Debatten dominante familienpolitische Orientierung der Wohneigentumsförderung hin (Georgakis, 2004, 96). Mit ihr sollte laut Staatssekretär Falthauser (CSU) weiterhin ,ein richtiges familienpolitisches Signal“ gesendet werden (BT-Drs. 13055/4657B). Der CDU-Bundestagsabgeordnete Meister fügt dem hinzu: „Der Entwurf der Bundesregierung verstärkt die familienpolitische Komponente" (BT-Drs. 13055/4665C).

Trotz der vielfach genannten familienpolitischen Komponenten, erscheinen familienpolitische Zielsetzungen in den Debatten um die Eigenheimzulage zunehmend wie eine Zielsetzung unter anderen. Stattdessen erwecken einige Redebeiträge sowohl von den Regierungsparteien als auch vonseiten der SPD den Eindruck, das Wohneigentum müsse um des Wohneigentums willen selber gefördert werden. So werden die seit den 80er Jahren in den Debatten präsente Forderung der Quotenerhöhung in diesem Zusammenhang mit einem Aufholbedarf des deutschen Wohnungsmarktes gegenüber ausländischen Märkten als Argument in den Bundestagsdebatten mit angeführt. Exemplarisch seien hierfür die Ausführungen des Abgeordneten Braun (FDP) genannt:

„Ich weiß aber auch, daß andere Länder diesem Ziel viel näher sind als Deutschland. Ich frage mich, warum dies eigentlich so sein muß; denn der Wunsch nach den eigenen vier Wänden ist in Deutschland nicht weniger verbreitet als in Irland, in Spanien oder in Finnland. Die Deutschen sind 
auch nicht weniger wohlhabend als die Finnen, die Spanier und die Iren. Aber an irgend etwas muß es doch wohl liegen." (BT-Drs. 13055, 4662A)

Die in erster Linie an Austeritätspolitik orientierten Zielvorstellungen zu Zeiten der Umstellung auf die Eigenheimzulage deuteten bereits auf das wesentliche Kriterium ihrer späteren Abschaffung hin. Kleinteilige Reformen konnten nicht darüber hinwegtäuschen, dass es sich bei der Eigenheimzulage nicht nur um eines der umstrittensten Instrumente der Wohnungspolitik handelte (Georgakis, 2004, 94), sondern sogar um eine überaus kostenintensive Subvention. Die entsprechende Kritik war bereits seit den 80er Jahren wiederholt von Politikern der SPD, aber auch vonseiten der Grünen geäußert worden (vgl. Abschnitt 6.2). Mit diesem Argument war in den vorherigen Legislaturperioden jedoch meist versucht worden die Bevorzugung des Wohneigentums vor dem Mietwohnungsbau einzuschränken. Im Kontext der von der Regierung Schröder verfolgten Austeritätspolitik, in der das Sparen zu einer zentralen Grundlage der Sozialdemokratie wurde (vgl. Frenzel 2002, 272ff.; Abschnitt 7.3.1), weitete sich dieses Argument auf eine generelle Abschaffung der Eigenheimförderung über das Mittel der Eigenheimzulage aus.

Diese finale Abschaffung unter einer CDU-geführten Bundesregierung ist vor dem Hintergrund der langwierigen Debatten über die Kosten der Eigenheimförderung auf der einen und dem Ausbleiben der intendierten Wirkung auf der anderen Seite zu sehen: Auch unter der Eigenheimzulage waren die Wohneigentumsquoten wider Erwarten in Deutschland nicht beachtlich gestiegen. Zudem bot sich für die Streichung der Eigenheimzulage längst eine Umdeutung und damit eine Ver- 
schiebung in eine andere politische Systematik an. So heißt es im Koalitionsvertrag von CDU, CSU und SPD aus dem Jahr 2005 unter Punkt 2.4:

„Die Eigenheimzulage wird zum 1. Januar 2006 abgeschafft. Wohneigentum geniefst bei den Bürgern eine besonders hohe Akzeptanz im Rahmen der privaten Altersvorsorge. Daher werden wir das selbst genutzte Wohneigentum zum 1. Januar 2007 besser in die geförderte Altersvorsorge integrieren. Die Diskriminierung gegenüber anderen Formen der Altersvorsorge wird im Interesse einer echten Wahlfreiheit für die Bürger beseitigt" (CDU/CSU u. SPD 2005; e. HvH.).

2008 wurde mit dem sogenannten Eigenheimrentengesetz, auch WohnRiester genannt, selbstgenutztes Wohneigentum als gleichberechtigte Anlageform in die staatlich geförderte private Altersvorsorge aufgenommen. Grundsätzlich war die Anlage in Wohneigentum nach dem Altersvermögensgesetz von 2001 ausgeschlossen. Eine vollständige Übernahme der Wohneigentumsförderung in den Bereich der Riesterrente hätte eine Doppelförderung zur Folge gehabt (Lammerskitten, 2004, 24). Dieser Ausschluss wurde jedoch durch das sogenannte Entnahmemodell aufgelockert, wonach Entnahmemöglichkeiten ,für bereits angesparte Beiträge aus dem Vorsorgevertrag zwischen 10.000 und 50.000 Euro für den Bau oder Erwerb einer [selbstgenutzten] im Inland gelegenen Wohnung" bestanden, die jedoch bis zum 65. Lebensjahr wieder zurückgezahlt werden mussten (Runkel, 2004, 6f.). Dieses Modell galt laut Runkel jedoch als ,aufwändig, kompliziert und für den Betroffenen wegen der hohen Liquiditätsbelastung als ungünstig (Runkel, 2004, 7).

So wurde ein Gesetz verabschiedet, das die Wohneigentumsförde- 
rung nicht mehr bloß diskursiv mit der privaten Alterssicherung verknüpfte, sondern ,die Frage der Altersabsicherung der Haushalte explizit mit der Bildung von Wohneigentum verknüpfte und entsprechend förderte“ (Mertens, 2015, 278). Allerdings bestehen in Form der Riesterförderung keine Zwänge das angesparte Vermögen tatsächlich für eine Wohnimmobilie zu verwenden. Stattdessen können die Beträge vom sogenannten Wohnförderkonto auch als Alterssicherung ausgezahlt werden. Die Beanstandung der unklaren Zweckbestimmung der Mittel stellte sich als einer der zentralen Kritikpunkte in den Bundestagsdebatten heraus. Schließlich lässt sich der Mangel an Zweckbindung der Mittel auch als eine zentrale Begründung für die schrittweise Herabstufung der Bausparförderung in Deutschland sehen (vgl. Biedenkopf u. Miegel, 1979). Staatliche Wohneigentumsförderung ist nun fest im vermögens-, genauer, im rentenpolitischen Bereich verortet und somit weiter entfernt von ihrer ursprünglichen gesellschafts- und familienpolitischen Fundierung wie auch von der Wohnungspolitik. Im Koalitionsvertrag der schwarz-gelben Bundesregierung von 2009 wird dies nochmals deutlich: „Wohneigentum ist Altersvorsorge“ (CDU/CSU u. FDP, 2009, 42). Im Folgenden sollen mit der Austerität und der privaten Altersvorsorge die wesentlichen Zielvorstellungen hinter den Reformen der dritten Phase genauer betrachtet werden.

\subsubsection{Austerität}

Die große Bedeutung des Sparsamkeitsmotivs für den Staat, findet sich in den übergeordneten Zielsetzungen in den Aussagen der Bundestagsabgeordneten - unabhängig von der Parteizugehörigkeit. So fasst der Abgeordnete Meister die vier Zielsetzungen des neuen Gesetzes wie folgt 
zusammen: „die Aufkommensneutralität, das familienpolitische Signal, die Verwaltungsvereinfachung und die zielgenauere Förderung" (BTDrs. 13055/4666B). Für den Staatssekretär Falthauser sind die gleichberechtigten Ziele hingegen (1.) die Berechenbarkeit der „Förderung für die Bauwilligen“ und dass die Vorschriften (2.) insgesamt ,eine deutliche Vereinfachung" erfahren (parl. Staatssekretär beim BMF Faltlhauser in BT-Drs. 10/055, 4656B). Dementsprechend erscheinen familienpolitische Zielsetzungen nunmehr im Lichte einer Rahmung und nicht mehr wie zuvor als eigentlicher Anlass der Reformen. Kosten- und Effizienzargumente sind dabei nicht der zu diesem Zeitpunkt noch konservativliberalen Bundesregierung vorbehalten. So begrüßen SPD und Bündnis 90/Die Grünen nicht nur den Vorstoß der Bundesregierung, „den Mißbrauch der Wohnungspolitik zur Vermögensbildung der besser verdienenden Schichten wenigstens etwas einzudämmen" (BT-Drs. 13/ 055, 4660A). Stattdessen kritisiert die Opposition auch die Höhe der mit der Eigenheimzulage verbundenen Kosten und die ihrer Auffassung nach immer noch zu hoch angesetzte Einkommensbeschränkung für Förderberechtigte. Diese Kostenhöhe wird jedoch nicht allgemein diskutiert, sondern wie in den Debatten rund um die Förderung des Sozialen Wohnungsbaus mit den geringeren Ausgaben für Wohngeld und den sozialen Mietwohnungsbau verglichen (vgl. BT-Drs. 13/055, 4660A).

Insgesamt passierte die Abschaffung der Eigenheimzulage die sehr kurzen Lesungen des Bundestages am 1. und 15. Dezember 2005 nach dem Regierungswechsel zur Großen Koalition unter dem ersten Kabinett Merkel ohne nennenswerten Widerstand. Der SPD-Abgeordnete Hilsberg wird in den Debatten zur Abschaffung der Eigenheimzulage besonders deutlich: 
„Es gehört nun einmal zu einer verantwortlichen Politik, dass man Anachronismen, Dingen, die früher richtig waren und heute anachronistisch geworden sind, Rechnung trägt. Die Eigenheimzulage ist eine zunehmend sinnlose Subvention geworden." (BT-Drs. 16/5, 292D)

Lediglich ihre traditionellen Fürsprecher aus FDP und den Unionsparteien bekannten sich wiederholt zu dem Ziel der Eigenheimförderung auf anderem Wege: der privaten Altersvorsorge. Dabei positionierte sich die FDP klar gegen eine Abschaffung der Eigenheimförderung ohne einen zeitnahen Ausgleich (vgl. BT-Drs. 16/5, 285C-D). Unter anderem verweist der FDP-Abgeordnete Friedrich auf den Koalitionsvertrag der großen Koalition, in dem die Bundesregierung vorsieht die Wohneigentumsförderung an die Kreditanstalt für Wiederaufbau (KfW) zu delegieren. Sie wolle, so der Koalitionsvertrag, „mit der KfW-Förderbank Wege aufzeigen, wie die Beleihung im nachrangigen Bereich verbessert und verbilligt werden kann. Damit kann auch die Privatisierung von Wohnungen an Mieter unterstützt werden" (CDU/CSU u. SPD, 2005, Ziff. 2636ff.). Daran kritisiert der FDP-Abgeordnete, dass insbesondere junge Familien vor allem liquide Mittel benötigten und nicht neue Möglichkeiten der Kreditvergabe.

\subsubsection{Private Altersvorsorge}

Viel stärker als in den vorherigen Debatten tritt nun die Rolle der Eigenheimförderung als Förderung der privaten Altersvorsorge in Erscheinung. So erklärt der CDU-Abgeordnete Meister bereits in den Debatten zur Eigenheimzulage: „Die Bildung von Wohneigentum ist für viele Bürgerinnen und Bürger ein wichtiges Standbein der Vermögensbildung, 
aber auch der privaten Altersvorsorge“ (BT-Drs. 13055, 4665A). Dieser Aspekt wird insbesondere vom FDP-Abgeordneten Braun hervorgehoben:

„Wohneigentum ist eine besonders gute Form der Altersvorsorge. Wer die eigenen vier Wände rechtzeitig erworben hat, hat weniger Angst vor dem Leben im fortgeschrittenen Alter." (BT-Drs. 13065/5615A)

Die vermögenspolitische Verortung der Eigenheimförderung erscheint somit in den Debatten um die Eigenheimzulage bereits stärker als in den Vorhergegangenen. Dieser Umstand wird besonders daran deutlich, dass auch die Bausparförderung im Rahmen des Wohneigentumsförderungsgesetzes von 1996 erstmals seit langem wieder angehoben wurde

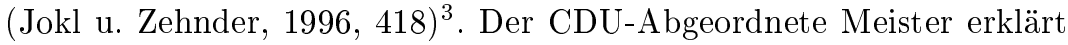
hierzu:

„Aus Sicht der Koalitionsfraktionen ist darüber hinaus positiv hervorzuheben, daß das Bausparen durch die Anhebung der Einkommensgrenzen auf 50000 DM für Ledige und 100 000 DM für Verheiratete eine deutliche Verbesserung erfährt. Auch dies entspricht unserer gesellschaftspolitischen Zielsetzung. Das Bausparen erleichtert die Schaffung von

3 ,,[D]ie begünstigten Höchstbeträge [wurden] von 800 DM/1.600 DM auf 1000 DM/ 2000 DM angehoben. Ferner wurden die 1975 eingeführten und seitdem nur ein Mal (1990) von 24.000 DM/18.000 DM (Ledige/Verheiratete) auf 27.000 DM/54.000 DM verbesserten Einkommensgrenzen für die Gewährung der Wohneigentumsprämie von 27.000 DM / 54.000 DM auf 50.000 DM/100.000 DM erhöht“ (Jokl u. Zehnder, 1996, 418) 
Wohneigentum durch die hierfür erforderliche Eigenkapitalbildung“". (BT-Drs. 13055/4666A)

Angesichts der finalen rechtlichen Eingrenzung auf den Bereich der privaten Altersvorsorge verwundert es wenig, dass auch die Debatten im Bundestag zum „Wohn-Riester“ ausschließlich von vermögens- und dabei insbesondere rentenspezifischen Zielvorstellungen geprägt sind. Die Neuverortung der Wohneigentumsförderung im Politikfeld der privaten Altersvorsorge bot eine Möglichkeit für Abgeordnete der CDU/CSU auch für eine Abschaffung der Eigenheimzulage zu plädieren. So erklärt der Abgeordnete Meister in der ersten Lesung des entsprechenden Gesetzesentwurfs der Regierung:

„Die Abschaffung der Eigenheimzulage haben wir in Verbindung mit der Frage der Integration der Immobilie in die private Altersvorsorge gesehen. Vor diesem Hintergrund sind wir bereit, zu sagen: Wenn die Immobilie in geeigneter Weise in die private Altersvorsorge integriert ist, kann auf das Förderinstrument der Eigenheimzulage verzichtet werden." (BT-Drs. 16/5, 230A)

In den Debatten des Bundestags wird diese Art der Förderung von ihren Befürwortern vor allem als eine von den Bürgerinnen und Bürgern selbst gewählte Zuordnung dargestellt, was stark an die bereits zitierte Formulierung aus dem Koalitionsvertrag erinnert. Als Belege für diese Präferenzen führen die Abgeordneten Umfrageergebnisse an, wonach stets ein Großteil der Deutschen eine eigene Immobilie als erstrebenswerte Altersvorsorge ansieht (vgl. BT-Drs. 16/170 18077B, 18078A, 18081A). Exemplarisch seien hier die Ausführungen des SPDAbgeordneten Krüger genannt: ,F F̈r viele Bürgerinnen und Bürger aber 
ist das mietfreie Wohnen im Alter die bevorzugte Art der individuellen Altersvorsorge“ (BT-Drs. 16/158, 16659C).

Die in den vorherigen Jahren stärksten Vertreter der Eigenheimsubventionierung von der FDP erklären stattdessen die Riesterförderung von Wohneigentum sei kein adäquater Ersatz für die weggefallenen Regelungen: „Sie ist unzureichend und ungeeignet, nennenswert mehr Wohneigentum zu schaffen. Die eingeplanten Mittel sind einfach zu gering" (Abg. Thiele, BT-Drs. 16/158, 16661A). Die Abgeordnete Scheel von Bündnis 90/ Die Grünen erklärt diesen Umstand in ihrer Ansprache wie folgt:

„Es ist wenig verwunderlich: Die SPD wollte eigentlich gar keine Änderungen, die Union wollte eigentlich einen Ersatz für die abgeschaffte Eigenheimzulage, und deshalb ist wohl auch der Name Eigenheimrente zustande gekommen." (BTDrs. 16/158, 16666B)

Verglichen mit den Debatten der vorherigen Jahrzehnte fällt besonders auf, dass die fast schon traditionelle Zielvorstellung der Familienförderung durch Eigenheimförderung nur noch in sehr wenigen Schlagworten präsent ist. Wird diese Vorstellung in den Auseinandersetzungen der 50er Jahre noch bis in die Gesetzestexte hinein weit vorangestellt, ist diese in den Wohn-Riester-Debatten nur noch am Rande präsent etwa in den Ausführungen des CDU-Abgeordneten Oswald:

„Familien und jungen Menschen, die sich ein Haus oder eine Wohnung zulegen wollen, geben wir mit diesem Gesetz eine neue Unterstützung an die Seite." (BT-Drs. 16/170, 18078A) 
Laut Egner u.a. (2004) war die relative Schwäche der Eigenheimförderung bereits zu Beginn der Nullerjahre und deren zunehmende Einschränkung auf die ,fragmentierten weitgehend politikfeld-extern begründeten Argumentationslinien“ zurückzuführen (Egner u.a., 2004, 255). Zwar wurde die Eigenheimförderung bereits in ihren Anfängen nicht primär wohnungspolitisch begründet, war jedoch eng mit familienund gesellschaftspolitischen Vorstellungen verknüpft gewesen. Letztendlich blieb ihren Verfechtern nur noch die vermögenspolitische Dimension zu ihrer Rechtfertigung, was vor dem Hintergrund der ,herausgestellten Sparzwänge“ nicht mehr ausreichte (Egner u.a., 2004, 255). Wie die entsprechenden Ideen Eingang in die politischen Debatten fanden, soll im folgenden Abschnitt dargestellt werden.

\subsection{Vielfalt datenbasierter Ideenproduktion}

Die Wissensproduktion der dritten hier betrachteten Phase wurde maßgeblich durch drei Entwicklungen gekennzeichnet: Erstens traten mehrere neue Akteure als Wissensproduzenten in Erscheinung, wobei sie allerdings das alte Akteursspektrum mehr ergänzten als ersetzten. Während in der korporatistischen Politikberatung neben dem Auftreten neuer Interessenvertreter eine geringere Kooperation der SPD mit ihren traditionellen Partnern in der Wohnungspolitik verzeichnet werden kann (Abschnitt 7.3.1), fällt in der wirtschaftswissenschaftlichen Expertise nicht nur das zunehmende Engagement halböffentlicher Forschungsinstitute auf, sondern auch das von privaten Beratungsunternehmen (Abschnitt 7.3.2). Diese neuen Akteure brachten, zweitens, selber neue und umfassendere Daten als Wissensbestände in die politischen Debatten mit ein, welche die amtliche Statistik bis dahin nicht 
bereitgestellt hatte. Hierbei leisten vor allem die nachgeordneten ministeriellen Forschungsabteilungen Beiträge zur Vernetzung der Experten und zur Vermittlung neuer Erkenntnisse für politische Akteure und Administration (Abschnitt 7.3.3). Als wesentliche Triebfeder zur Ausweitung der Wissensproduktion öffentlicher Stellen wie dem statistischen Bundesamt und der Bundesbank ist in dieser Phase erstmals nicht die nationale Ministerialbürokratie zu sehen, sondern transnationale Gremien, in denen die öffentlichen Stellen auch selber Daten zu den Entwicklungen innerhalb Deutschlands liefern sollen. Anhand neuer Datenbestände wird es den Wissensproduzenten jeder Gruppe zunehmend möglich auch ökonometrische Analysen des deutschen Wohnimmobilienmarktes zu erstellen. Die Ergebnisse ihrer Arbeit stellen die Wissensproduzenten, drittens, nicht mehr nur den politischen Akteuren, sondern auch zunehmend einer breiteren Öffentlichkeit zur Verfügung.

\subsubsection{Gelockerte Bande in Parteien \& Verbands- landschaft}

Hinsichtlich der im Bundestag vertretenen Parteien stellen Egner et al. eine markante Entwicklung in wohnungspolitischer Hinsicht fest: Für deren Programmatik spiele „offensichtlich die Wohnungspolitik keine zentrale Rolle mehr, denn in Partei- und Wahlkampfprogrammen sowie in sonstigen Veröffentlichungen der Parteien ist kaum noch etwas zum Politikfeld Wohnen zu finden - zumindest nicht in der Form, dass daraus in einer für die Analyse hinlänglichen Form auf konkrete Positionen in den untersuchten wohnungspolitischen Auseinandersetzungen hätte geschlossen werden können“ (Egner, 2004b, 138). So habe selbst die CDU/CSU-Bundestagsfraktion in der 15. Legislaturperiode keinen 
bau- und wohnungspolitischen Sprecher mehr benannt (Egner, 2004b, 138). Mit dem voranschreitenden Bedeutungsverlust der Wohnungspolitik wird es auch zunehmend schwerer, starke Koalitionen innerhalb der Wissensproduktion für die Politik zu identifizieren. Zahlreiche Verbände, die in der zweiten Phase noch in den Debatten oder zumindest in den entsprechenden Anhörungen aufgetaucht sind, treten nun nicht mehr in Erscheinung.

Hinsichtlich der Wohneigentumsförderung wurden erstmals wieder klare Unterschiede zwischen den großen Parteien erkennbar, was einem Wandel in der Positionierung der SPD zuzuschreiben ist: Während CDU und CSU unter der rot-grünen Bundesregierung weiter an der Eigenheimzulage festhalten wollten (Egner, 2004b, 142), sah die SPD „die Kürzung bzw. Neugestaltung der Eigenheimzulage“ als einen „Bestandteil der notwendigen Haushaltskonsolidierung" (Egner, 2004b, 147). Dieser programmatische Wandel hinsichtlich der Wohneigentumsförderung hatte sich Mitte der 90er Jahre vollzogen. Noch in ihrem Regierungsprogramm „Der Neue Weg“ zu Beginn der 90er Jahre wollte die SPD auf eine „Belebung der privaten Bautätigkeit“ hinwirken und „breite Schichten der Bevölkerung [sollten] in ihren eigenen vier Wänden leben" (zitiert nach Gesamtverband der Wohnungswirtschaft, 1992, 18). Der Kurswechsel der SPD zu einer vermehrten Austeritätspolitik wird besonders erkennbar im sogenannten Schröder-Blair-Papier, das 1999 im Vorfeld der Europawahl von Tony Blair und Gerhard Schröder als Modernisierungskonzept mit dem Titel „Der Weg nach vorne für Europas Sozialdemokraten" für die europäische Sozialdemokratie vorgelegt wurde (vgl. Frenzel, 2002, 155). Das Papier fordert „eine neue Kombination aus flexiblen Märkten und einer neu definierten Rolle eines 
Sozialinterventionsstaats“ (Frenzel, 2002, 155). Die „programmatische Fundamentalzäsur" dieses Papiers für die Sozialdemokratie bestand dabei laut Frenzel vor allem in dem übergeordneten „Paradigma, keine Politik gegen die Interessen der Wirtschaft zu treiben“ und stellt somit eine deutliche Verstärkung marktkonformer Elemente des 98er Wahlprogrammes dar (Frenzel, 2002, 154). Das Regierungsprogramm für die erste Amtsperiode von Schröder enthielt dementsprechend einerseits ,ein Bekenntnis zum bestehenden Sozialstaat“, forderte jedoch zugleich „die Neubestimmung des Verhältnisses von Solidarität (durch sozialstaatliche Rechte und Leistungen) und Individualität (durch zunehmende Eigenverantwortung und Eigeninitiative)" (Frenzel, 2002, 170f.). Somit war der Kurs der SPD nicht mehr nur in haushaltspolitischer Sicht verschärft, sondern enthielt auch klare wirtschaftsliberale Positionen.

Entgegen dieser Positionierung der SPD verläuft die Position der Friedrich-Ebert-Stiftung (vgl. Abschnitt 3.1.2), die die Eigenheimzulage als „wichtigste Komponente der Altersvorsorge befürwortet“, die „Konzentration der Zulage auf Familien mit Kindern [jedoch] mit dem Argument kritisiert, dass so ein wohnungspolitisches Instrument in ein familienpolitisches Instrument umgeformt wurde" (Egner, 2004b, 148). Der themenerarbeitende Arbeitskreis Wohnungspolitik der FES ist dabei nicht als ein Gremium aus SPD-Politikern zu verstehen, sondern besteht aus einem Netzwerk für verschiedene Experten der Wohnungspolitik. Unter seinen Mitgliedern finden sich neben Berufspolitikern Repräsentanten des GdW, des Bundesministeriums, des Städtetages, des Mieterbundes sowie des Forschungsinstitutes empirica [vgl. hierzu Abschnitt 7.3.2). Damit war in dem Arbeitskreis zwar ein breites Spektrum diverser Experten vertreten. Allerdings muss sein Einfluss auf die tat- 
sächliche Ausgestaltung wohnungspolitischer Ideen als gering angesehen werden (Egner, 2004b, 242). Auch Mieterbund und GdW ${ }^{4}$ warnten als langjähriger Partner der SPD in wohnungspolitischen Fragen vor einer Abschaffung der Eigenheimzulage (Egner 2004b, 161; Gesamtverband der Wohnungswirtschaft 1992, 7), tauchten jedoch mit ihren Einwänden im Rahmen der politischen Debatten nicht auf. Insbesondere der GdW hatte ein starkes Interesse an einer weiteren Förderung des privaten Wohneigentums, da es ihnen seit dem Wegfall des Wohnungsgemeinnützigkeitsgesetzes (vgl. Abschnitt 6.1) frei stand, ,sich im Bereich der Wohneigentumsbildung zu betätigen und sich auf diesem Gebiet neue Geschäftsfelder zu erschließen“, was viele gemeinnützige Wohnungsunternehmen auch taten (Gesamtverband der Wohnungswirtschaft, 1992, 7). Somit warb der GdW beständig dafür, dass breitere Schichten ,die realistische Chance haben [sollten], Wohneigentum zu erwerben" (Gesamtverband der Wohnungswirtschaft, 1992, 7) und wollte seine eigenen Mitgliedsunternehmen dazu ermutigen ,selbst im Bereich der Eigentumsmaßnahmen aktiv zu werden" (Gesamtverband der Wohnungswirtschaft, 1992, 8). Erst in den Debatten um die Integration der Wohneigentumsförderung in das System der Riesterrente waren Beteiligungen des GdW wieder nachweisbar (BT-Drs. 16/9688).

Auch der Eigentümerverband Haus- und Grund taucht erst 2008

4 Bereits in einem 1992 erschienenen Gutachten des GdW-Fachausschusses „Wohneigentum und Eigentumsverwaltung“ heißt es: „Die ganz überwiegende Mehrheit der Bevölkerung in Deutschland wünscht sich das Wohnen in der eigenen Wohnung oder im eigenen Haus. Die Politik reagiert darauf seit Jahrzehnten mit Förderprogrammen der verschiedensten Art. Trotzdem sind wir noch immer weit von dem Ziel entfernt, daß möglichst viele frei entscheiden und auch realisieren können, ob sie zur Miete oder im Eigentum wohnen wollen“ (Gesamtverband der Wohnungswirtschaft, 1992, 7). 
in den Debatten um die Einführung des sogenannten Wohnriester im Rahmen der öffentlichen Anhörung des Finanzausschusses erneut auf (BT-Drs. 16/9691). Die Förderung von Wohneigentum ist seit jeher eine zentrale Forderung des Verbandes und er plädierte zuvor heftig ,gegen die Einschnitte bei der Förderung des Wohneigentums" unter der rotgrünen Bundesregierung (Egner, 2004b, 163). Hinsichtlich der Eigenheimzulage ist aus der Verbandslandschaft insbesondere der Deutsche Verband für Wohnungswesen, Städtebau und Raumordnung (DV) als Aktivposten zu nennen. Er wurde bereits 1946 gegründet und „befasst sich mit Grundsatzfragen und Zukunftsperspektiven des Wohnungswesens, Städtebaus und der Raumordnung“", wobei er sich als unabhängig „,Von Standes- und Berufsinteressen“ beschreibt (Egner, 2004b, 176). Mit seiner maßgeblichen Tätigkeit in der Organisation von Symposien, Fachgesprächen und Kommissionen versteht sich der Verband dabei als „Plattform, auf der maßgebliche Persönlichkeiten aus Politik, Wirtschaft, Verwaltung, Rechtssprechung und Wissenschaft, die hier Verantwortung tragen, zu einem ständigen Erfahrungs- und Gedankenaustausch zusammengeführt werden sollen“ (Egner, 2004b, 176). Eine solche koordinierende Rolle nahm der Deutsche Verband in der Interessenkoordination in den Debatten zur Eigenheimzulage wahr und trieb diese Debatte auch maßgeblich mit voran (Egner, 2004b, 177). Auch zum Thema Altersvorsorge durch Wohnimmobilien leitete der DV eine Kommission ein, die sich mit der Fragestellung befassen sollte, „ob der Immobilie im Rahmen der Gesetzgebung zur Altersvorsorge eine Position eingeräumt worden ist, die ihrer Bedeutung als klassische Form der privaten Altersvorsorgung angemessen Rechnung trägt" (Lammerskitten, 2004, 16). Geleitet wurde die Kommission von dem Finanzexperten 
Peter Lammerskitten und dem bereits aus der vorherigen Phase wohnungspolitischer Expertise bekannten Johann Eekhoff (Lammerskitten, 2004, 16). Die Kommission begrüßte die Übernahme der Wohneigentumsförderung in den Bereich der privaten Altersvorsorge, ein Jahr bevor die Eigenheimzulage abgeschafft wurde.

Angesichts der hitzigen politischen Debatten um die Abschaffung der Eigenheimzulage (vgl. Abschnitt 7.2) verwundert es wenig, dass in der entsprechenden öffentlichen Anhörung des Finanzausschusses eine große Vielfalt an Experten zu Wort kommen (vgl. BT.-Drs. 16/250). Verglichen mit früheren Anhörungen finden sich wieder jene Verbände, die auch in den frühen Jahren der Bundesrepublik im Feld vertreten waren wie der Zentralverband des Deutschen Handwerks, der Zentralverband des Deutschen Baugewerbes, der dgb, ver.di, die Bundesvereinigung der Deutschen Arbeitgeberverbände (BDA) sowie die Bundesgeschäftsstelle der Landesbausparkassen, der Bundesverband Freier Immobilien- und Wohnungsunternehmen und die Bundesvereinigung der kommunalen Spitzenverbände. Hinzu kamen dabei das Präsidium des Bundes der Steuerzahler, die Deutsche Steuer-Gewerkschaft, der Deutsche Steuerberaterverband, der Bundesverband Investment und Asset Management (BVI) und der Verband Deutscher Medienfonds. Diese Vielfalt ist vergleichbar mit den Anhörungen des Finanzausschusses wenige Jahre später vor der Überführung der Wohneigentumsförderung in das Konzept der Riesterrente. Der einzige Unterschied besteht an dieser Stelle darin, dass mehr zusätzliche Vertreter der Kredit- und Versicherungswirtschaft mit anwesend sind (vgl. BT.-Drs. 16/9670). Als ein wesentlicher Wissensproduzent hinsichtlich der Eigenheimförderung ist auch der Verband der deutschen Pfandbriefbanken (zuvor 
Verband der deutschen Hypothekenbanken, VDH) zu nennen, der mit einem eigenen Index seit 2004 Daten zur durchschnittlichen Immobilienpreisentwicklung bereitstellt (Schürt, 2010, 30ff.). Vertreter des Verbandes waren schon zuvor an gutachterlichen Tätigkeiten mit anderen Akteuren aus dem wissenschaftlichen Spektrum beteiligt gewesen (vgl. Bundesamt für Bauwesen und Raumordnung, 2003). Dies zeigt abermals, dass auch in der dritten Phase nicht Wissenschaftler alleine die Wissensproduktion bestritten, sondern weiterhin die Verbände eine wesentliche Position einnahmen.

Somit ist für diese Phase der Wissensproduktion zunächst ein Wandel der beteiligten wissensbereitstellenden Akteure zu verzeichnen. Dies hängt einerseits damit zusammen, dass sich Abgeordnete der SPD nicht mehr in den Debatten auf ihre wohnungspolitischen Partner berufen und diese insbesondere in der Phase der Abschaffung der Eigenheimzulage nicht als Experten einbestellt wurden. Zu diesem Zeitpunkt vertraten sie mit einer eigentumsfreundlicheren Programmatik einen anderen Standpunkt als die SPD. Andererseits ist der Wandel des Akteursspektrums insbesondere in Richtung der Finanzindustrie maßgeblich durch den Wechsel des politischen Instrumentariums über die Riesterrente zu erklären. An Bedeutung gewannen gegenüber der Verbandslandschaft jedoch erneut Wirtschaftswissenschaftler. Diese Entwicklung soll im folgenden Abschnitt dargestellt werden.

\subsubsection{Aufstieg der Wirtschaftswissenschaften}

Auffällig gegenüber der vorherigen Phase der Wissensproduktion erscheint eine deutlich geringere Anzahl von Gutachten, die vonseiten des Ministeriums in Auftrag gegeben wurden. Zwar wurde in den aus- 
gehenden 80er Jahren noch ein Gutachten zur Geschichte der Wohnungspolitik in Auftrag gegeben, um der Frage nachzugehen, ,unter welchen Bedingungen die einzelnen Instrumente der Wohnungspolitik entwickelt und eingesetzt wurden, in welchem systematischen Zusammenhang mit anderen Instrumenten sie verwirklicht wurden und wie die Wirkungen der einzelnen Maßnahmen einzuschätzen waren“" (Leutner, 1990, I). Bis zur Einführung der Eigenheimzulage 1996 und in den ersten Folgejahren wurden aber zunächst keine weiteren Studien zur Wohneigentumsförderung durch das federführende Ministerium in Auftrag gegeben. Dies änderte sich jedoch im Rahmen der Debatten um die Neuregulierung der Eigenheimzulage zu Beginn der 2000er Jahre.

Die Mechanismen wissenschaftlicher Politikberatung seit Beginn der 90er Jahre sind laut Weingart (2006) durch eine, ,zumindest programmatische Demokratisierung" geprägt, wobei nicht mehr das Wissen an sich zur Legitimierung politischer Entscheidungen beiträgt, sondern vielmehr eine breit angelegte Partizipation in dessen Bereitstellung. Tatsächlich ist in der wissenschaftlichen Wissensproduktion für die Eigenheimförderung gegen Ende der 90er Jahre eine größere Vielzahl an Akteuren aus dem wissenschaftlichen Spektrum anzutreffen als in den vorherigen Episoden.

Dabei ist das 2001 erschienene Gutachten zu Hemmnissen der Wohneigentumsbildung zu nennen, das vom Bundesministerium für Verkehr, Bau und Wohnungswesen in Auftrag gegeben und durch das Unternehmen empirica bearbeitet wurde (Faller u.a., 2001). Ebenso wurde das Institut mit einem weiteren Gutachten zur Transaktionseffizienz und Transaktionsoptionen von Wohneigentum im internationalen Vergleich betraut (Faller u.a., 2006) und wurde auch zur öffentlichen Anhörung 
im Finanzausschuss zur Beratung des Eigenheimrentengesetzes geladen (BT-Drs. 16/9687). empirica ist verglichen mit anderen Forschungsinstituten im Bereich der Wohnungspolitik mit dem Gründungsjahr 1990 ein recht junges Institut und versteht sich als ,ein unabhängiges wirtschafts- und sozialwissenschaftliches Beratungsunternehmen" (Egner, 2004b, 196). Als Auftraggeber des Institutes nennt empirica selbst „Kommunen, Landes- und Bundesministerien [...], verschiedene Banken, Bausparkassen, Versicherungen sowie Bauträger und Immobilieninvestoren“ (Egner, 2004b). Hinsichtlich der Eigenheimzulage plädierte empirica für eine sofortige Abschaffung des Instrumentes, da diese „nur eine geringe Auswirkung auf die Zahl der neu geschaffenen Eigenheime, wohl aber eine preistreibende Wirkung auf deren Herstellungskosten“ habe (Egner, 2004b, 197).

Auch das genannte 2001 veröffentlichte empirica-Gutachten, in dem dargestellt werden sollte, warum die Wohneigentumsquote in der Bundesrepublik trotz der staatlichen Förderung nicht wie erhofft angestiegen war, kommt zu einem zweischneidigen, aber in der politischen Empfehlung eindeutigem Ergebnis: Einerseits sei der Wunsch nach Wohneigentum in der Bevölkerung weiter verbreitet als die Erfüllung der Wohnwünsche. Zudem sei ,auch vor dem Hintergrund der absehbaren Lücken in der Alterssicherung eine stärkere private Vorsorge erforderlich, und der Staat wird gleichzeitig dauerhaft unter dem Druck stehen, Subventionen für das Wohnungswesen abzubauen“ (Faller u.a., 2001, 1). Die Eigenheimzulage dient dem politischen Wunsch jedoch nicht, sondern kann sich sogar als kontraproduktiv erweisen. Damit stellt dieses Gutachten einen direkten Link zur Lösung von Problemen innerhalb der privaten Altersvorsorge und der Wohneigentumsförderung dar. Wie 
bereits vorherige Gutachten kommt die Studie zu dem Ergebnis, dass für eine steigende Wohneigentumsquote vor allem die preiswertere Bereitstellung von Neubauten vonnöten sei.

Empirica steht dabei sinnbildlich für eine neue Vielzahl an privaten wie auch öffentlichen und halböffentlichen Forschungseinrichtungen, die sich im Bereich der Wissensproduktion in der dritten Phase bewegen. Dazu zählen das Ifo-Institut für Wirtschaftsforschung (vgl. Abschnitt 3.1.3) sowie das Deutsche Institut für Urbanistik (Difu). Beide Institute wirkten an einem Sammelband des Bundesamtes zur Wohneigentumsbildung mit (vgl. Bundesamt für Bauwesen und Raumordnung, 2003). Als Experten aus der Wissenschaft kam im Rahmen der öffentlichen Anhörung zur Abschaffung der Eigenheimzulage neben einem Vertreter des Deutschen Instituts für Wirtschaftsforschung (DIW) mit Prof. Dr. Lorenz Jarass ein weiterer Wirtschaftswissenschaftler hinzu (vgl. Abschnitt 3.1.3). Jarass, Professor an der Hochschule Rhein/Main hatte sich in erster Linie als Experte für das deutsche Steuersystem ${ }^{5}$ profilieren können.

Die Vielfalt der Experten zeigt sich auch an einer deutlichen $\mathrm{Zu}-$ nahme der Datenanbieter zu wohnungspolitischen Fragestellungen (vgl. Schürt, 2010). Waren in den Jahren zuvor noch die Daten der amtlichen Statistik die wesentlichen Quellen für wissenschaftliche Analysen, wurde mit der bulwienGesa $\mathrm{AG}^{6}$ ein weiteres privatwirtschaftliches Be-

5 Er war von „2002 - 2003 Mitglied des wissenschaftlichen Beirats der Kommission zur Reform der Gemeindefinanzen [und von] 1998 - 2000 Mitglied der Kommission zur Reform der Unternehmensbesteuerung, die die Grundlagen für die deutsche Steuerreform im Zeitraum 2001 - 2005 legte“ (Jarass, 2018).

6 Die bulwienGesa AG entstand durch einen Zusammenschluss der Bulwien AG und der gesa Gesellschaft für Handels-, Standort- und Immobilienberatung 
ratungsunternehmen ein wichtiger Wissensanbieter für wohnungspolitische Entscheidungen. Gleiches gilt für die bereits genannte empirica $\mathrm{AG}$, das Beratungsunternehmen $\mathrm{F}+\mathrm{B}$ und das GEWOS Institut für Stadt-, Regional- und Wohnforschung. Allen gemeinsam ist das Angebot diverser Indices zur Immobilienpreisbeobachtung, die für Deutschland erst seit Mitte der 90er Jahre verfügbar ist (Schürt, 2010, 36). Zuvor wurden lediglich Mietpreise auf Bundesebene durch das Statistische Bundesamt zusammengetragen und von einzelnen Anbieter Indices auf lokaler Ebene für einzelne Städte erstellt.

Die Verfügbarkeit von Daten an sich zog eine Vielzahl an wirtschaftswissenschaftlichen Analysen nach sich, die ohne die neuen Datenquellen nicht möglich gewesen wären. Neben den Datenbeständen der neuen Anbieter bot sich auch zunehmend die Möglichkeit über Internetportale selber Daten über Immobilienmärkte zu sammeln. So berichtet einer der interviewten Experten:

"da: bin ich aufmerksam auf die äh auf die ähnlichen Daten bei Immoportalen aufmerksam geworden und da hab mir gesagt warum verwende ich diese Daten nicht also das ist eine sehr reiche Quelle an Daten die man verwenden kann und seitdem beschäftige ich mich ziemlich also hauptsächlich mit Immobilienmarktforschung also das kam von den Daten her dann kam also später kamen auch die Fragestellungen [...] ich war beeindruckt von der Fülle an Daten die man im Internet findet also ich machte damals das was man jetzt big data nennt ohne das zu wissen"

mbH 2004 und bietet Dienstleistungen in Form von Daten, Analyse, Bewertung und Consulting auf Immobilienmärkten (bulwien Gesa AG, 2018). 
Er macht in seiner Erzählung deutlich, dass für ihn die Verfügbarkeit von Angebotsdaten ${ }^{7}$ von Internetseiten den Ausschlag gegeben hat sich mit der Thematik eingehender zu befassen. Auch ein weiterer interviewter Experte eines Forschungsinstitutes, das über einen eigenen Datenbestand zu Immobilienpreisen verfügt, geht davon aus, dass ein Großteil der heutigen Studien auf den neuerdings in deutlich größerem Umfang verfügbaren Daten auf kleineren Ebenen beruht. Diese konnten die amtlichen Statistiken bis dahin nicht bieten.

,für ganz Deutschland es gibt keine Daten für einzelne Städte, gibt nur einen Index also man kann das nicht nach Bestand oder Neubau, kann auch nicht sagen ob das Mehrfamilienhäuser oder Reihenhäuser sind also damit kann man nichts machen (2) dann die Mieten also es gibt es als Teil vom Verbraucherpreisindex und manche Bundesländer wie Hamburg haben keinen Verbraucherpreisindex also wie kann ich dann die Mieten für Hamburg messen (2) es gibt keine Möglichkeit [...] ja und dann kann um über die Indices auf Kreis oder Gemeindeebene kann man vergessen also die gibt es gar nicht und wenn nicht alle Bundesländer solche Daten haben dann also Kreise und Gemeinden das ist unmöglich mit den amtlichen Daten“

Auch der Experte des BBSR verweist auf die zunehmende Bedeutung privater Anbieter von Wohnungsmarktstatistiken, die auch von staatlichen Stellen gekauft und für eigene Analysen verwendet werden.

7 Als Angebotsdaten werden solche Preisdaten verstanden, die nicht den tatsächlichen Preis bei der Transaktion, sondern den Preisvorschlag des Anbieters wiedergeben (Schürt, 2010). 
Eine weitere Entwicklung, die sich in der jüngsten Phase der wissenschaftlichen Politikberatung in der Wohnungspolitik abzeichnet und auf der neuen Datenverfügbarkeit beruht, ist eine zunehmende „Politikberatung der Öffentlichkeit" - vor allem vonseiten der Wirtschaftswissenschaften (Pitlik, 2001). Diese Entwicklung vollzieht sich vor allem in dem Bereich der Expertise für die mediale Öffentlichkeit und äußert sich in einem vermehrten Erscheinen frei verfügbarer, immobilienmarktspezifischer Publika. Wesentliche Ausgangspunkte für die Orientierung der Forschungsinstitute bilden laut Experteneinschätzung neben dem öffentlichen Interesse für die Ergebnisse der Analysen auch die politische Relevanz:

,wenn das Thema interessant ist und politisch relevant bei Immobilienforschung ist das fast immer so dann äh dann äh wird das als Wochenbericht ${ }^{8}$ auch veröffentlicht"

Somit scheint das Interesse der Veröffentlichungen nicht nur in einer großen und breiten Medienresonanz, sondern zumindest auch in der Beteiligung an tagespolitischen Diskursen zu liegen. Aufgrund der hohen medialen Aufmerksamkeit der Berichte zu Immobilienpreisentwicklungen empfahl schließlich auch der Abteilungsleiter dem Experten in jährlichen Abständen eine Art Aktualisierung der Berechnungen wie auch der Berichte vorzunehmen, sodass sich für den Experten ein Experiment zur neuen Routine entwickelte.

Trotz einer neuen Vielfalt an Experten im wohnungspolitischen Kon-

8 Der DIW Wochenbericht wird vonseiten des DIW selber als seine „FlaggschiffPublikation“ bezeichnet und soll ,,jede Woche komprimierte Informationen zu aktuellen wirtschaftspolitischen Fragen“ (Deutsches Institut für Wirtschaftsforschung, 2016) bieten. 
text, ist das Feld der wissenschaftlichen Expertise in der Wohnungspolitik weiterhin sehr überschaubar. So erklärt einer der interviewten Experten:

,also ich finds zum Beispiel bezeichnend dass allein die Forschungseinrichtungen die sich die sich näher damit beschäftigen doch sehr überschaubar sind in Deutschland es gibt in dem Bereich in dem ich mich zum Beispiel bewege [...] das kann ohne jetzt den Anspruch erheben zu wollen da wirklich alle Forscher zu kennen die sich in dem Bereich bewegen aber zumindest die die ich kenne das ist sehr überschaubar also die kann ich mehr oder weniger an 2 maximal eher an einer Hand abzählen“

Auch ein weiterer Experte äußert sich mit ironischem Unterton zu der geringen Anzahl an bestehenden Experten und wie einfach es dementsprechend sei als einer gewertet zu werden:

,ja $\mathrm{j}=\mathrm{ja}$ es reicht ein Papier zu schreiben ich hab vorher dieses Papier 2007 zur Veränderung der Preise geschrieben dann kommen die Leute aus China also ein Papier und schon ist man ein Experte ((lacht)) vielleicht wenn man sehr wenige hat dann sieht man die wenigen die es gibt die was zu dem Thema geschrieben haben als Experte ja"

Zusammenfassend lassen sich sowohl anhand der an der Wissensproduktion beteiligten Akteure als auch anhand des Wandels der Mechanismen der Wissensproduktion Änderungen in der dritten Phase verzeichnen. So zeichnet sich die dritte Phase sowohl im Bereich der korporatistischen als auch in der wissenschaftlichen Politikberatung 
durch eine neue Vielfalt der Akteure aus. Diese Entwicklung ist dabei nicht nur auf den Wandel der politischen Inhalte zurückzuführen, sondern wird vonseiten der Experten maßgeblich einer gestiegenen Verfügbarkeit von Daten zugeschrieben, welche den Ausgangspunkt ihrer Analysen darstellen. Zudem besteht eine Tendenz in der Expertise sich nicht mehr nur an andere Beteiligte im politischen Feld zu wenden, sondern auch vermehrt an die Öffentlichkeit. Ob eine solche „Politikberatung der Öffentlichkeit“ jedoch auch im politischen Kontext Bedeutung haben kann, is überaus zweifelhaft. So werden Beratungsangebote meist nicht als Beratung eingestuft, da ihnen ein im politischen Bereich handlungsfähiger Adressat fehlt (Buchholz, 2008, 79). Insgesamt wird die politische Bedeutung der hier genannten Forschungsinstitute von Egner (2004a) basierend auf einer Analyse des Politiknetzwerkes als gering eingestuft:

„Sie werden zwar unterschiedlich oft von anderen Akteuren als ,wichtig“ eingestuft, sind aber in den entsprechenden Cliquen kaum verankert, was für eine selektive Verbindung zwischen den anderen Akteuren und den einzelnen Instituten spricht." (Egner, 2004a, 242)

Allerdings führt Egner (2004a) an, dass die wissenschaftlichen Forschungsinstitute ,eine im Politikfeld anerkannte Funktion von „Ideengebern" haben“, wodurch „die von ihnen vertretenen Positionen in Zukunft wohnungspolitisch durchaus relevant werden" können (Egner u. a., 2004, 252). Diese Relevanz ist jedoch eng an jene Bedingungen geknüpft, die vonseiten der öffentlichen Verwaltung vorgegeben werden. Daher sollen im folgenden Abschnitt diese Bedingungen sowie die Wissensproduktion staatlicher Akteure selbst genau in Augenschein ge- 
nommen werden.

\subsubsection{Herausforderungen für Ministerialbürokratie \& amtliche Statistik}

Wie eingangs erwähnt, musste das Bundesministerium mit Amtsantritt der rot-grünen Bundesregierung 1998 seine Eigenständigkeit einbüßen, indem es mit dem Bundesverkehrsministerium zum Bundesministerium für Verkehr, Bau- und Wohnungswesen (BMVBW) zusammengelegt wurde (Bartholomäi 2004, 31; vgl. Abschnitt 7.1). Auch in der personellen Besetzung des Ministeriums wird laut Egner et al. die abnehmende Bedeutung der klassischen Wohnungspolitik mit dem Regierungswechsel zu rot-grün deutlich:

„Waren die Ministerinnen und Minister des für Wohnungspolitik zuständigen Ressorts in der Vergangenheit ausgewiesene wohnungspolitische Experten (beispielsweise Paul Lücke, Karl Ravens, Oscar Schneider oder Eduard Oswald), besteht im seit 1998 existierenden Ministerium kein wohnungspolitischer, sondern ein infrastrukturpolitischer Schwerpunkt. So sind von sieben Fachabteilungen nur noch zwei mit Wohnungspolitik beschäftigt [...].“ (Egner, 2004b, 134)

Das federführende Ministerium hinsichtlich der Reformen der Eigenheimzulage, ihrer Abschaffung sowie der Integration der Wohneigentumsförderung in das System der Riesterrente blieb das Bundesministerium der Finanzen. Für das Bundesfinanzministerium stellt der wohnungspolitische Bereich einen unter zahlreichen Aufgabenbereichen dar, da es ,nicht nur für die Haushalts- und Finanzpolitik, sondern 
auch für Zölle, Zollämter und Zollkriminalämter, die Verwaltung für die vom Bund ausgegebenen Wertpapiere [sowie] die Besoldung der Bundesbediensteten und die Bundesforstämter zuständig“ ist (Egner, 2004b, 136). Auch über den Bereich der Wohneigentumsförderung hinaus hat das Finanzministerium in dieser Phase eine bedeutende Rolle inne, da „die meisten wohnungspolitischen Gesetze mit direkten Kosten für den Bund im Rahmen von direkten Finanzhilfen oder steuerrechtlichen Fragen verbunden sind" (Egner, 2004b, 137). Trotz der zentralen Bedeutung des BMF schätzen Egner et al. in der gegebenen Phase den Einfluss des Bundesministerium für Verkehr, Bau- und Wohnungswesen dennoch als zentral ein, da es weiterhin als zentraler Akteur für den Informationsaustausch in wohnungspolitischen Fragestellungen gilt (Egner, 2004b, 234).

Wie bereits in Abschnitt 7.3.2 beschrieben, wurden insbesondere in den 90er Jahren deutlich weniger Expertengutachten in Auftrag gegeben als dies noch in der zweiten Phase wohnungspolitischer Wissensproduktion der Fall gewesen ist. Dies heißt jedoch nicht, dass in dieser Phase keine Experten zurate gezogen wurden. Stattdessen berief die Bundesregierung eine „Expertenkommission Wohnungspolitik“ ein, um ,auf Basis einer umfassenden Analyse Vorschläge für eine effiziente Nutzung wohnungspolitischer Instrumente auszuarbeiten“ (Sinn u. a., 1995, III). Ihre Zusammensetzung erinnert von der Beschreibung als Gremium aus unabhängigen „Experten aus Wissenschaft und Praxis“ zunächst stark an den wohnungswirtschaftlichen Beirat der 50er Jahre. Allerdings hatten in diesem Expertengremium die Wissenschaftler ein deutlich größeres Gewicht - sowohl ihrer Anzahl nach als auch in der Besetzung des Vorstandspostens durch Hans Werner Sinn, Professor für Nationalöko- 
nomie und Finanzwissenschaft. Die Berufung Sinns zum Vorsitzenden der Expertenkommission ist dabei nicht nur ein Zeichen für die hohe Wertschätzung der Wirtschaftswissenschaften im Allgemeinen, sondern beinhaltet auch eine politische Botschaft. Sinn, bis 2016 Präsident des ifo Instituts für Wirtschaftsforschung (vgl. Abschnitt 3.1.3), präsentierte sich stets als wirtschaftsliberal und gegen die staatliche Lenkung der Wirtschaft (Sinn, 1505). Neben Sinn waren mit Vertretern des Forschungsinstituts empirica und des Difu (Sinn u.a., 1995, 5) weitere relativ junge Wissensproduzenten im wohnungspolitischen Umfeld in der Expertenkommission vertreten (vgl. Abschnitt 6.3.2).

Die Ausrichtung des Gutachtens war insbesondere durch das Erreichen einer möglichst marktgerechten und effizienten Wohnungspolitik bestimmt, was bereits im Vorwort deutlich wird:

„Viele Eingriffe lähmen. Im ganzen hat das Ausmaß der Regulierung heute das Maß überschritten, das der Marktwirtschaft zuträglich ist. Die Kommission hat das Regulierungsgeflecht durchkämmt und versucht, die wichtigsten Mißstände sichtbar zu machen. [...] Die Grundlinie des Gutachtens wird von dem Bestreben bestimmt, Wege zur Verbesserung der wirtschaftlichen Effizienz im Wohnungswesen aufzuzeigen, ohne dabei die sozialen Aufgaben der Wohnungspolitik aus dem Auge zu verlieren." (Sinn u.a., 1995, III)

Der internationale Vergleich der Wohneigentumsquoten, dem im Rahmen der Debatten rund um die Eigenheimzulage ein großes Gewicht zukommt (vgl. Abschnitt 7.2), findet sich zuvor bereits im Gutachten der Expertenkommission Wohnungspolitik. Im Hinblick auf die Wohneigentumsförderung, der die Kommission ,ein besonderes Augen- 
merk" (Sinn u. a., 1995, IV) widmete, stellte sie fest, dass die Wohneigentumsquoten in Deutschland im internationalen Vergleich besonders niedrig ausfallen (Sinn u. a., 1995, 46ff.). Dies erscheint der Kommission als „bemerkenswert" vor dem Hintergrund, dass in anderen Ländern mit höheren Wohneigentumsquoten „das Einkommen [...] nicht höher ist als in Deutschland“ (Sinn u.a., 1995, 46). Allerdings erklärt die Kommission dass das Ziel einer Erhöhung der Eigentumsquoten nur unter der Maßgabe eines ,möglichst geringe[n] Subventionsaufwand[es] zu erreichen"sei (Sinn u.a., 1995, 256). Eine Eigenheimförderung müsste sich der Auffassung der Expertenkommission dementsprechend ,auf eine kleinere Zielgruppe, sogenannte Schwellenhaushalte" beschränken (Sinn u.a., 1995, 256). Auch die Regierungsparteien merken selber an, dass die mit der Eigenheimförderung verbundenen Kosten überaus hoch sind. Allerdings wird die Kostenhöhe nicht in Relation zu anderen wohnungspolitischen Ausgaben betrachtet, sondern in den Kontext der allgemeinen Staatsfinanzierung gestellt, was die immer bedeutender werdende Zielsetzung eines sparsamen Staates betont. Eine wie auch immer ausgestaltete Förderung des Wohneigentums hat somit bereits in den grundlegenden Debatten zur Eigenheimzulage ein bedeutendes Gewicht eingenommen.

Zudem sprach sich die Kommission für eine gezielte Sparförderung aus, die ,auf jedem Fall dem Vorsichtsmotiv beim Wohneigentumserwerb und der Notwendigkeit einer Mindestquote an eigenen Mitteln des Schuldners Rechnung tragen" sollte (Sinn u.a., 1995, 257). Neben dem traditionellen Bausparen kommen an dieser Stelle erstmals andere zweckgebundene Sparformen zum tragen, „beispielsweise höherverzinsliche Kapitalmarkttitel“ (Sinn u.a., 1995, 257). Staatliche Kre- 
ditbürgschaften sollten nach Auffassung der Kommission dafür sorgen, dass ,zusätzliche Finanzierungsrisiken für den Kreditgeber und damit letztendlich höhere Finanzierungskosten" vermieden werden (Sinn u.a., 1995, 258). Zwar sollte die Förderung entsprechender Kapitalmarkttitel im Rahmen der Eigenheimzulage nicht verwirklicht werden, allerdings durfte die Eigenheimzulage als fester Bestandteil des Eigentums anerkannt werden und hatte somit ,gegenüber Kreditinstituten einen eigenkapitalähnlichen Charakter“, sodass die Zulage nicht nur in der monetären Förderung ansetzt, sondern auch ,an der Kreditrationierung“ (Simian, 2000, 125).

Einen Ausbau erfuhr die ministerielle Forschungsarbeit mit der Zusammenlegung der Bundesbaudirektion (BBD) und der Bundesforschungsanstalt für Landeskunde und Raumordnung zum Bundesamt für Bauwesen und Raumordnung (BBR) 1998. Das BBR war damals eine von 22 nachgeordneten Behörden des BMVBW. 2004 wurde die Behörde um weitere Bundesämter erweitert und soll nun als Bundesanstalt für Bau-, Stadt- und Raumforschung (BBSR) die Bundesregierung mit fachlicher Expertise unterstützen. Einerseits erfolgt dies über selbst erstellte Studien und andererseits über die Betreuung der Vergabe und Auswertung externer Gutachten (Bundesinstitut für Bau-, Stadt- und Raumforschung (BBSR) im Bundesamt für Bauwesen und Raumentwicklung, 2018a). Die Praxis der Gutachtenvergabe beschreibt ein Experte wie folgt:

,wir betreuen ja verschiedenste Projekte des Ministeriums [...] wo ähm halt aktuelle Themen von Externen erforscht werden dann machen wir diese Projektsteuerung sagen wir mal vorher die Entwicklung der Leistungsbeschreibung dann 
in Zusammenarbeit mit dem Ministerium und ähm betreuen das Projekt dann nehmen es dann nachher noch ab“

Die finale Entscheidung ob ein Forschungsprojekt realisiert oder die Ergebnisse veröffentlicht werden, bleibt zwar dem Ministerium vorbehalten, das seinerseits allerdings in der inhaltlichen Ausgestaltung auf die Fachkompetenz innerhalb der nachgelagerten Behörde vertraut:

„,es gibt natürlich so auch gewisse Vorgaben über den Rahmen dass wir halt als nachgeordnete Behörde für das Bauministerium ja arbeiten und dann einiges an Vorgaben natürlich auch aus dem Bauministerium dann kommen das was beispielsweise so die Programmbetreuung angeht von Forschungsprojekten da ist sag ich mal machen wir auch Vorschläge das Ministerium sag ich mal hat natürlich die letztendliche Entscheidung ob das ob dieser Themenschwerpunkt jetzt bearbeitet wird oder dieser Themenschwerpunkt, bei den klassischen Marktanalysen was jetzt so die Wohnungsmarktbeobachtung angeht bei uns da sind wir eigentlich relativ frei gestellt was wir jetzt wie genau beleuchten und mit welchen Datenquellen da ähm stützt sich das Ministerium dann eigentlich auf unsere Fachkompetenz und ähm vertraut dem was wir jetzt als sinnvoll bewerten welche Quellen genutzt werden und wie wir diese Quellen dann auch auswerten und aufbereiten und ähm das =das ermöglicht dann auch diese Eigenverantwortung und eigene Kompetenz, die wir dann da mit reinbringen können"

Zu den selbst erstellten Gutachten zählt die Berichtsreihe „Bauland- 
und Immobilienmärkte“, in der das BBR im Auftrag des Bundesministeriums ,,[z]uverlässige Informationen über die Verhältnisse auf den Bauland- und Immobilienmärkten“ für die „Immobilien- und Wohnungswirtschaft, [...] Verbände, [...] Politik und vor allem auch [...] interessierten Bürger[n]" bieten soll (Bundesamt für Bauwesen und Raumordnung, 2001, 1). Die Ausweitung der Zielgruppe der Analysen kann sowohl als eine Fortschreibung des Öffentlichkeitsanspruches bisheriger Gutachten gewertet werden als auch als eine Möglichkeit, die maßgeblich aus den immer weiter anwachsenden Verbreitungsmöglichkeiten über das Internet ${ }^{9}$ resultiert.

Neben der eigenen wissenschaftlichen Tätigkeit ist die Bedeutung des BBSR in der Zusammenführung von Expertisen anderer Wissensproduzenten hervorzuheben. Dabei greift das BBSR nicht nur auf amtliche Datenbestände, sondern auch vermehrt auf die neu produzierten Daten anderer wissenschaftlicher Einrichtungen und von Verbänden zurück, wie ein Experte berichtet: ,in der Zwischenzeit gibts dann aber auch noch weitere Anbieter [...] dann greifen wir auch auf Daten von Verbänden zurück“".

Zusätzlich werden im Rahmen des BBSR auch wissenschaftliche Tagungen veranstaltet und daraus resultierende gemeinsame Gutachten herausgegeben (so Bundesamt für Bauwesen und Raumordnung 2003; Schürt 2010). Diese können auch in einem direkten politikberatenden Kontext stehen. So wurden im Zusammenhang mit den Debatten zur Eigenheimzulage Reformvorschläge „erstmals mittels empirischer Da-

$9 \quad$ Über die Seiten der wissenschaftlichen Abteilungen des Bundesamtes stehen die Berichte seit 2001 frei zum Download bereit (Bundesinstitut für Bau-, Stadtund Raumforschung (BBSR) im Bundesamt für Bauwesen und Raumentwicklung, 2018b). 
ten hinsichtlich ihrer sozialen und räumlichen Auswirkung analysiert" (Bundesamt für Bauwesen und Raumordnung, 2003) und durch das BBSR zusammengetragen. Die in diesem Sammelband zusammengelegten Beiträge von Mitarbeiterinnen und Mitarbeitern des BBR sowie des VDH, des Ifo Instituts für Wirtschaftsforschung und des Deutschen Instituts für Urbanistik (Difu), ,zeigen, dass das Wohneigentum an Bedeutung zugenommen hat" und somit auch vom BBR weiterhin beobachtet und analysiert werden sollte (Bundesamt für Bauwesen und Raumordnung, 2003). Die Wohneigentumsförderung in Form der Eigenheimzulage wird dabei abgesehen von den damit verbundenen Kosten als positiv bewertet.

Als wesentlicher Akteur in dieser Phase der Wohnungspolitik ist die ARGEBAU-Ministerkonferenz anzusehen, die letztmalig in den Debatten der 50er Jahre durch Eingebungen zum Zweiten Wohnungsbaugesetz im Bereich der Wohneigentumsförderung in Erscheinung getreten ist (vgl. Abschnitt 5.2). Als ,,beratendes Gremium im Vorfeld förmlicher Gesetze [...] bildet [sie] den Rahmen, in dem die Länder gemeinsame Rechtsvorschriften und Richtlinien für den Vollzug bundesstaatlicher Regelungen oder die einheitliche Regelung in allen Ländern erarbeiten“ (Egner, 2004b, 151). Die ARGEBAU tritt in dieser Phase nicht nur als Interessenvertretung der Länder auf, sondern auch als Auftraggeber eines Expertengutachtens, das 2002 durch das Bundesamt für Bauwesen und Raumordnung (BBR) mit der Arbeitsgruppe "Wirkungsanalyse Eigenheimzulage“ ihres Ausschusses für Wohnungswesen erstellt wurde (Müller u.a., 2002). Dabei sollte „die Wirkung der Eigenheimzulage im Hinblick auf das Verhältnis Neubau- und Bestandsförderung, die räumliche Zielgenauigkeit und die soziale Verteilungswirkung un- 
tersucht werden“ (Müller u.a., 2002, 1).

Ein wesentliches Gutachten mit Blick auf die Abschaffung der Eigenheimzulage bildet der 2002 veröffentlichte „Bericht zur Inanspruchnahme der Eigenheimzulage in den Jahren 1996-2000" im Auftrag der ARGEBAU-Ministerkonferenz. Hierzu wurden die beim Bundesamt für Finanzen verfügbaren Statistiken zur Eigenheimzulage herangezogen (Müller u. a., 2002). Die Gutachter kommen zu dem Ergebnis, dass der Eigenheimzulage zwar ein erheblicher Anteil an der Belebung der Eigentumsbildung auch bei Familien mit Kindern in der Bundesrepublik zu verdanken sei, allerdings sei bei einer Frage für oder gegen den Eigentumserwerb die Möglichkeit der öffentlichen Förderung nicht das wichtigste Kriterium. Nur dort, wo auch günstige Bodenpreise mit einem ausreichenden und nachfragegerechten Angebot vorliegen, ,leistet die Eigenheimzulage einen wirksamen Beitrag zur Eigentumsbildung" (Müller u. a., 2002, 31).

Mit den Expertisen der Deutschen Bundesbank ${ }^{10}$ entstand ein neuer Bereich der Wohnungsmarktanalyse abseits von den bis dato damit befassten Akteursgruppen aus dem wissenschaftlichen oder korporatistischen Bereich. Die Abhandlungen zu wohnungsmarktspezifischen Themen der Deutschen Bundesbank finden sich sowohl in den regelmäßig erscheinenden Monatsberichten ${ }^{11}$ als auch in den seit 2005 erscheinenden Finanzstabilitätsberichten. Mangels eigener Datenbestände und

10 Eine Übersicht der bis Ende 2015 von der Bundesbank herausgegebenen wohnungsmarktspezifischen Berichte findet sich Anhang B.2.

11 Die Monatsberichte der Deutschen Bundesbank erscheinen seit 1949 und behandeln diverse volkswirtschaftliche Themen, ,darunter insbesondere geld- und währungspolitische sowie finanz- und konjunkturpolitische Themen" (Deutsche Bundesbank, 2016a). 
ohne eine entsprechend verfügbare amtliche Statistik bezieht sich die Bundesbank dabei maßgeblich auf Daten der bereits genannten Bulwien AG (vgl.Deutsche Bundesbank 2003b; Deutsche Bundesbank 2003c), die seit 1975 Daten auf Städtebasis erhoben haben (Schürt, 2010, 39). Über einen langen Zeitraum hinweg kommt den Wohnungsmärkten in den Publikationen der Bundesbank jedoch nur eine untergeordnete Rolle zu. So werden Preisentwicklungen für Wohnraum in Deutschland lediglich im Zusammenhang mit der allgemeinen Verbraucherpreisentwicklung oder als Indikator für konjunkturelle Entwicklungen knapp thematisiert.

Als Startzeitpunkt einer gesonderten Berichterstattung zum deutschen Wohnimmobilienmarkt kann die Erstellung eines eigenen Preisindices für Wohnimmobilien durch die Bundesbank gewertet werden, auf den erstmals im Monatsbericht vom Mai des Jahres 2003 hingewiesen wird (Deutsche Bundesbank, 2003a, 39). ${ }^{12}$ Infolge erscheint der Index zur Wohnimmobilienmarktbetrachtung wiederholt in den Monatsberichten der Bundesbank ${ }^{13}$.

12 Im Monatsbericht aus dem September desselben Jahres wurden Ergebnisse dieser Analysen als ein eigenständiger Aufsatz mit dem Titel „Preisindikatoren für den Wohnungsmarkt" veröffentlicht (Deutsche Bundesbank, 2003c). Der Hinweis findet sich ebenso wie die folgenden wohnungsmarktspezifischen $\mathrm{Pu}-$ blikationen der Bundesbank in einem Unterabschnitt zur allgemeinen Preisentwicklung innerhalb eines Kapitels mit dem Titel „Konjunktur in Deutschland“. Bis 2008 berichtet die Bundesbank in ihren Monatsberichten meist nur knapp über die Entwicklungen des eigenen Häuserpreisindex im Kapitel „Konjunktur in Deutschland“. Diese Berichterstattung beläuft sich in der Regel auf ein bis zwei Absätze und einer dazugehörigen kleineren Grafik. Erst 2008 erhält der deutsche Wohnimmobilienmarkt als Untersuchungsgegenstand einen größeren, eigenen Abschnitt in den Monatsberichten von zwei bis drei Seiten, der bis ins aktuelle Jahr seriell in den Monatsberichten des Monats Februar erscheint. 
Als grundlegende Ursache für die Erstellung und Verbreitung eigener Immobilienpreisindices wird darauf verwiesen, dass ,,[z] u Beginn der neunziger Jahre [...] die Korrektur übersteigerter Immobilienpreise in einzelnen Fällen ganze Volkswirtschaften in die Krise gezogen und die Stabilität der betreffenden Finanzsysteme gefährdet" habe (Deutsche Bundesbank, 2003c, 45). Die zu diesem Zeitpunkt noch nicht vorhandene Datenbasis in Deutschland und das zugleich bestehende Interesse diesen Markt genauer beobachten zu können, wird in den späteren Berichten ergänzt um den Hinweis, dass in anderen Ländern wie Frankreich bereits entsprechende Datenbestände vorliegen würden:

\begin{abstract}
„Wünschenswert wäre es auch aus geldpolitischer Sicht, wenn zukünftig in Deutschland - wie in Frankreich - vierteljährliche, transaktionsbasierte Immobilienpreisindizes zeitnah bereitgestellt werden könnten." (Deutsche Bundesbank, 2008, 51)
\end{abstract}

Als entscheidender Faktor für diesen nun erscheinenden Bedarf an Immobilienpreisindices kann der Austausch und die Zusammenarbeit der Deutschen Bundesbank mit anderen Zentralbanken gesehen werden. Dies zeigt sich an einem Workshop von OECD und IMF zu Immobilienpreisindices im November 2006. In ihrem Paper stellen die Bundesbanker Hoffmann u. Lorenz (2006) dar, dass die Preisbeobachtung auf dem

Ein vergleichbarer Vorgang ist ebenso in den Finanzstabilitätsberichten der Deutschen Bundesbank ab November 2012 zu beobachten. Ab diesem Finanzstabilitätsbericht wird den Wohnungsmärkten in Deutschland mindestens ein Unterkapitel, wenn nicht sogar ein eigenes Kapitel gewidmet. Seit 2008 hat sich somit die wohnungsmarktspezifische Berichterstattung im Rahmen bereits bestehender Veröffentlichungen der Deutschen Bundesbank etablieren können. 
Markt für Wohnimmobilien in Deutschland bislang nur anhand einer kleinen Anzahl von ,indicators of sometimes dubious quality“ erfolgte (Hoffmann u. Lorenz, 2006).

Auch das Statistische Bundesamt arbeitete zu diesem Zeitpunkt an einem eigenen Immobilienpreisindex (vgl. Hoffmann u. Lorenz, 2006). Ein Experte beschreibt die Ursachen für diese Indexerstellung, die er und seine Kolleginnen und Kollegen wissenschaftlich begleitet haben, wie folgt:

„was in den letzten Jahren halt auch nochmal ne fundamentale Umsetzung ja auch erfolgte weil die aber wiederum auch die Vorgaben vom ähm europäischen statistischen Amt dann auch hatten in dem nämlich die Staaten in der ähm EU auch ähm ja im Prinzip verpflichtet wurden ähm Häuserpreisindices dann auch ähm zu nem bestimmten Turnus dann auch zu veröffentlichen nämlich auch quartalsweise zu veröffentlichen und so gab es halt von Eurostat schon so Vorgaben wie so etwas umgesetzt werden sollte“

Während die Motivationen für die privaten Anbieter einerseits in einem kommerziellen Vertrieb von Marktanalysen und die neuen regulatorischen Kapitalanforderungen für Banken zu sehen sind (Hoffmann u. Lorenz, 2006), besteht das Interesse des Statistischen Bundesamtes sowie der Bundesbank in der Erfüllung ihrer Berichtspflicht im Rahmen des Austauschs auf der internationalen Ebene, sei es die EU, die OECD oder der IMF. Hintergrund für das Interesse der Zentralbanken und Organisationen wie dem IMF oder der OECD waren steigende reale Preise für Wohneigentum in mehreren Ländern im Verlauf der 90er Jahre und die drohende Möglichkeit einer Blasenbildung in diesem Be- 
reich, der eine große Bedeutung hinsichtlich der Vermögensbildung in vielen Ländern einnimmt (Girouard u. a., 2006). Dabei gehen jedoch im internationalen Vergleich die Ansichten und Handlungsspielräume der Zentralbanken auseinander, wie mit diesen Fällen umzugehen ist:

,Some advocate central banks responding to house (or other asset) prices only to the extent that they contain information about future output growth and inflation, and, if desired, using alternative policy instruments (taxes and regulations) to stabilise housing cycles [...]. Others advocate that central banks should ,lean against the wind“ by having a tighter stance than would otherwise be warranted by overall demand conditions in the face of abnormally rapid increases in real house prices, particularly as there might be risks to financial stability [...]." (Girouard u. a., 2006, 32f.)

Somit werden die Preise von Wohneigentum in dieser maßgeblich makroökonomischen Perspektive nicht als eine Frage der Leistbarkeit von Wohnraum diskutiert, sondern als ein potenzielles Risiko für Finanzstabilität. Auch die seit 2005 erscheinenden Finanzstabilitätsberichte der Bundesbank ${ }^{14}$ behandeln zumindest am Rande die Preisentwicklungen am deutschen Immobilienmarkt. Eine größere Aufmerksamkeit widmet die Bundesbank in diesen ersten Finanzstabilitätsberichten noch dem US-amerikanischen Wohnimmobilienmarkt. So werden bereits im ersten Finanzstabilitätsbericht von 2005 potenzielle Auswir-

14 Die Finanzstabilitätsberichte enthalten ,Analysen und Einschätzungen der Deutschen Bundesbank zur Stabilität des deutschen Finanzsystems" (Deutsche Bundesbank, 2016b). Seit 2005 erscheinen diese als eigenständige Berichte und nicht mehr wie in den Jahren zuvor innerhalb der Monatsberichte. 
kungen von Krisen ausländischer Immobilienmärkte für das deutsche Finanzsystem thematisiert (Deutsche Bundesbank, 2005, 16). Demgegenüber rechtfertigen über einen längeren Zeitraum beobachtete stagnierende oder sogar fallende Preise an deutschen Immobilienmärkten die Kürze der Abhandlung in einem kurzen Absatz mit dazugehöriger Grafik (Deutsche Bundesbank, 2005, 21). Auch im darauf folgenden Finanzstabilitätsbericht wird der deutsche Wohnimmobilienmarkt lediglich in drei Absätzen innerhalb des Fließtextes kurz thematisiert (Deutsche Bundesbank, 2006, 42f.). Im Finanzstabilitätsbericht von 2007 steht angesichts der wirtschaftlichen Ereignisse in den USA weiterhin der US-Immobilienmarkt im Fokus. Der deutsche Wohnimmobilienmarkt wird zunächst lediglich dahingehend erwähnt, dass ,[d]as Risiko eines direkten Übergreifens der Subprime-Krise auf den deutschen Immobilien- und Hypothekenmarkt [...] als gering einzustufen“ sei (Deutsche Bundesbank, 2007, 41). Trotz ihrer neu ausgebauten Expertise, erscheinen die Daten der Deutschen Bundesbank nicht in den politischen Debatten.

Begrenzt wird der Ausbau der amtlichen Statistiken allerdings erneut durch Sparsamkeitsbemühungen auf Seiten der Politik, wie ein Experte zu berichten weiß:

„bei der klassischen amtlichen Statistik wie sie beim Statistischen Bundesamt läuft oder bei den Statistischen Landesämtern da hats halt nicht viel Bewegungen in den letzten Jahren gegeben weil das halt $\mathrm{n}$ sehr großer Apparat ist ähm wo auch halt eher immer wieder unter finanziellen Gesichtspunkten auch vom Finanzminister auf die Bremse gedrückt wird da jetzt nochmal wieder neue Statistiken zu etablieren 
sondern ähm ja dann wird da in der Regel auf den vorhandenen Bestand zurückgegriffen“"

\subsection{Phase 3: Finanzialisierung anhand wis- senschaftlicher Expertise}

Zusammenfassend lassen sich in dieser dritten Phase Veränderungen sowohl im Bereich der verwendeten politischen Instrumente und des Regelgehalts als auch in der wohneigentumsspezifischen Expertise erkennen. In Bezug auf den ideellen Gehalt der Eigenheimförderung muss angemerkt werden, dass sich hier die Änderungen innerhalb der zweiten Phase fortgeschrieben haben: Familienpolitische Argumente sind zwar weiterhin vorhanden, geraten jedoch weiter in den Hintergrund. Auf der ideellen Seite dominieren nun vermögenspolitische Vorstellungen die Zielvorstellungen der staatlichen Eigenheimförderung, deren Ansätze bereits in der zweiten Phase deutlich hervortraten und nun auf die private Altersvorsorge fokussiert sind. Mit der Austerität kommt eine politikfeldübergreifende Zielvorstellung hinzu, die der staatlichen Eigenheimförderung grundsätzlich entgegenläuft. Zunächst lässt sie sich in den politischen Debatten noch verknüpfen mit den in der zweiten Phase diskutierten Möglichkeiten zur Zielgenauigkeit der Förderinstrumente: je präziser die Förderung, desto weniger kann der Eindruck entstehen, dass Gelder verschwendet werden. Im Regelgehalt der policies spiegeln sich diese Prozesse zunächst in der Einführung der durch ihre Progressionsunabhängigkeit zielgenaueren Eigenheimzulage wieder. Schließlich tragen die Forderungen zur Sparsamkeit jedoch zur Abschaffung der Eigenheimzulage als der kostspieligsten Einzelsubvention im Bundes- 
haushalt bei. Somit zeigen sich weiterhin tradierte policy-spezifische Ideen in den politischen Debatten um die Eigenheimförderung. Impulsgeber für den institutionellen Wandel der Eigenheimförderung sind sind in der dritten Phase jedoch primär Ideen und Zielsetzungen aus anderen Politikfeldern wie die Förderung der privaten Altersvorsorge und Austeritätsbemühungen.

Hinsichtlich der Vermarktlichung zeigen sich in der dritten Phase somit neue Entwicklungen. Zunächst wird die zuvor immer weiter ausgebaute Wohneigentumsförderung erstmals merklich zurückgefahren und für einige Jahre sogar vollständig ausgesetzt bevor sie durch die Integration in die staatlich geförderte Riesterrente in kleinerem Umfang neu eingeführt wird. Allerdings finden sich in der kognitiven wie auch in der normativen Dimension Verfestigungen der bereits bestehenden Trends von Vermarktlichung und Finanzialisierung: Eigenheimförderung muss nicht nur marktkonform sein, sondern erfüllt nun maßgeblich das Ziel der privaten Vermögensbildung. Sie ist zwar weiterhin eine sozialpolitische Maßnahme, wird aber durch finanzpolitische Zielvorstellungen dominiert. Das entsprechende Wissen hierzu wird nahezu exklusiv in Form wirtschaftswissenschaftlicher Expertise von einem breiten Akteursspektrum bereitgestellt.

Die bereits in den vorherigen Phasen bestehenden Tendenzen der großen Parteien hinsichtlich der Eigenheimförderung bleiben zunächst bestehen. So zeigen sich CDU/CSU weiterhin als die stärksten Befürworter der Eigenheimförderung und auch die SPD spricht sich zu Beginn der 90er Jahre noch für eine Eigenheimförderung aus. Im Zusammenhang mit ihrer finanzpolitischen Umorientierung zeigt sich die SPD allerdings zuerst dazu bereit, die Eigenheimförderung zugunsten ei- 
nes schlankeren Staates zu opfern, während die konservativ-christlichen Parteien insbesondere über den Bundesrat eine Reform zunächst blockieren. Allgemein ist jedoch der Bedeutungsverlust der Wohnungspolitik auf Bundesebene in allen Parteien zu verzeichnen, was vor allem daran erkennbar wird, dass selbst die CDU in der 15. Legislaturperiode keinen eigenen wohnungspolitischen Sprecher mehr benannte (Egner, 2004b).

Sofern ein Thema keine große politische Relevanz mehr aufweist, müsste dies theoretisch dazu beitragen, dass Expertisen von Wissensproduzenten außerhalb der Parteienlandschaft ein größeres Gewicht erhalten (vgl. Hirschman u. Berman, 2014, 6). Tatsächlich lässt sich der Trend zu einer stärkeren Dominanz des Expertenwissens in der Wohnungspolitik aus der zweiten Phase auch in der dritten Phase beobachten. Dieser verläuft zunächst jedoch weniger über Expertengutachten, als durch die Einberufung einer Expertenkommission, die durch Finanzexperten wie Hans Werner Sinn dominiert wird. Zwar ist in dieser Kommission eine stärkere Präsenz wirtschaftswissenschaftlicher Experten $\mathrm{zu}$ verzeichnen als dies noch im wohnungswirtschaftlichen Beirat der ersten Phase der Fall war, allerdings sind in ihr auch weiterhin Vertreter wohnungspolitischer Verbände vertreten, von denen einige nun auch selber Daten für die wohnungspolitische Wissensproduktion bereitstellen. Von einem Verschwinden korporatistischer Expertise kann somit auch in der dritten Phase nicht die Rede sein. Insgesamt zeigt sich im Bereich der Expertise eine deutlich verstärkte Anwendung von quantitativen Modellen, die auf Wohnungsmärkte angewendet werden und sich auch in den politischen Debatten wieder finden. Gesellschaftspolitische Überlegungen finden dabei deutlich weniger Anwendung. Die 
Diagnose von Häußermann u. Siebel (1981) zu Beginn der 80er Jahre, Wohnungspolitik sei zu einer „Angelegenheit von Experten“ geworden, scheint sich nun vollends zu bestätigen.

Zugleich lässt sich ein deutlicher Ausbau der wissenschaftlichen Tätigkeiten im Rahmen ministerieller Forschungsstrukturen wie dem Bundesinstitut für Bau-Stadt- und Raumforschung nachweisen, das einerseits selber Expertisen erstellt, aber auch die Forschungstätigkeiten im Auftrag des Bundesministeriums koordiniert. Dabei greift das BBSR wie bereits das Ministerium in der vorherigen Phase auf die Arbeiten der halböffentlichen wie auch der privaten Forschungsinstitute zurück. Ebenso sind Vertreter von Verbänden und damit aus der „Praxis“ des alten korporatistischen Systems aktiv an der Ideenproduktion beteiligt. Die zunehmende Forschungstätigkeit zu wohnungspolitischen Themen an diesen Forschungsinstituten verläuft spiegelbildlich im Rahmen der Forschungstätigkeit der Deutschen Bundesbank wie auch am Statistischen Bundesamt. Dabei steht vor allem die Preisbeobachtung von Wohnimmobilien im Vordergrund. Der dominante Mechanismus korporatistischer Politikberatung aus der zweiten Phase ändert sich in der dritten Phase zu einer vornehmlich wissenschaftlichen Politikberatung unter Einbeziehung der Verbände. Mit einer weiteren Bedeutungsabnahme wohnungspolitischer Fragestellung auf Bundesebene können Akteure außerhalb der Parteienlandschaft an Einfluss gewinnen. Entscheidend sind dabei die bereits in den ersten Phasen installierten Mechanismen der Wissensproduktion, die maßgeblich durch die Ministerialbürokratie als gatekeeper gesteuert werden. Akteure der öffentlichen Verwaltung bestimmen weiterhin, wer als Experte anerkannt wird und wer Ressourcen für die eigene Forschung über Gutachtenerstellung oder 
Forschungsförderung erhält.

Die bestehenden koordinierenden Aufgaben der Ministerialbürokratie haben sich in Bezug auf die Wissensproduktion für die Eigenheimförderung weiter verfestigt. Zudem wurden die ministeriellen Ressourcen für eigene Forschungstätigkeiten durch das Bundesinstitut für Bau, Stadt- und Raumforschung gegen Ende der dritten Phase gestärkt und die amtliche Statistik weiter ausgebaut, sodass eine zusätzliche Stärkung des staatlichen „Fachmenschentums“ im Weberianischen Sinne erfolgt (vgl. Weber, 2013, 578). Mit der Deutschen Bundesbank ist ein weiterer staatlicher Akteur im Feld der Wissensproduktion aufgetreten, der maßgeblich in Kooperation mit dem Statistischen Bundesamt Kennzahlen zum Wohnimmobilienmarkt bereitstellt und damit die verfügbaren analytischen Tools für politische Akteure erweitert. Diese Befunde gehen einher mit Analysen zu Staatsstrukturen, die von einem Ausbau eigener staatlicher Expertise im Rahmen von Bürokratien ausgehen (Skocpol u. Rueschemeyer 1996, 6; Friedrich 1970, 17; Rueschemeyer u. Skocpol 1996, 297).

Die sich wandelnden ideellen Elemente der Wohneigentumsförderung mit mehr quantitativen Stellgrößen wäre ohne diesen Wandel in der Akteurszusammensetzung und deren analytischen Orientierung nur schwer vorstellbar. Die erste Hypothese zum Zusammenhang zwischen der Bedeutung der Akteursstruktur und Mechanismen der Wissensproduktion einerseits und dem ideellen Wandel wohlfahrtsstaatlicher Institutionen andererseits kann somit als bestätigt angesehen werden. Gleiches gilt für die in Hypothese zwei angenommene herausgehobene Bedeutung der Ministerialbürokratie im Feld der Wissensproduktion. Sie nimmt eine zentrale Position in der Vergabe der Gutachten und 
entsprechenden Ressourcen ein und koordiniert die Zusammenarbeit. Dabei ist die öffentliche Verwaltung allerdings auch stärker durch internationale Trends getrieben als dies in früheren Phasen der Fall war. Einen Ausblick auf die weitere Wissensproduktion bietet Abschnitt 8.3. 


\section{Teil IV}

Zusammenfassung und Ausblick 



\section{Kapitel 8}

\section{Vermarktlichung und ihre}

\section{Produzenten}

In international vergleichenden Untersuchungen wird die Förderung von Wohneigentum in Deutschland als Sonderfall beschrieben: Einerseits weist die staatliche Förderung von Wohneigentum eine vergleichsweise lange Geschichte auf, die im Gegensatz zu vielen anderen Ländern nicht erst in den 70er Jahren ihren Anfang nahm (Rolnik u. Rabinovich 2014; Ronald 2008; Bourdieu 2014), sondern bereits in den ersten Legislaturperioden nach dem Zweiten Weltkrieg. Andererseits erweisen sich die im internationalen Vergleich geringen Wohneigentumsquoten als auffällig: Deutschland ist bis heute das einzige Land in der EU, in dem mehr Menschen zur Miete wohnen als in ihren eigenen vier Wänden (Housing Europe 2017, 68). Im Rahmen der vorliegenden Untersuchungen konnten Besonderheiten der Vermarktlichung und Finanzialisierung innerhalb des policy-Subsystems der Eigenheimförderung 
in Deutschland offen gelegt und damit die Eigenheiten des kleinteiligen Institutionenwandels zwischen Pfadabhängigkeiten und Trends zur Vermarktlichung und Finanzialisierung beschrieben werden. Zwar kann Wohneigentumsförderung weiterhin als eine sozialpolitische Maßnahme bezeichnet werden. Sie wird jedoch nicht mehr von familien- und gesellschaftspolitischen Idealvorstellungen getragen, sondern als Bestandteil der staatlich geförderten privaten Altersvorsorge als ein Asset unter vielen betrachtet.

Zur Darstellung der zentralen Ergebnisse sollen hier zunächst die Dimensionen beschrieben werden, in denen Vermarktlichung und Finanzialisierung der bundesdeutschen Wohnungspolitik nachgewiesen werden konnten (Abschnitt 8.1). Die Ursachen für diese Entwicklungen sollen in Abschnitt 8.2 erläutert werden. Dabei zeigt sich, dass der institutionelle Wandel sowohl in ideeller Hinsicht als auch in Bezug auf den Regelgehalt eng mit dem sich ändernden Regime der Ideenproduktion zusammenhängt. Die Ergebnisse liefern Erkenntnisse für die Analyse institutionellen Wandels. So können Ideen vor allem in der langfristigen Perspektive eine entscheidende Rolle für kleinteiligen institutionellen Wandel spielen. Dementsprechend erweisen sich Akteure und Mechanismen der politikfeldspezifischen Ideenproduktion durchgehend als wesentliche Faktoren für den institutionellen Wandel. Im Zeitverlauf wird zudem die tragende Rolle der öffentlichen Verwaltung deutlich, deren Dominanz jedoch von unterschiedlichen Faktoren abhängt. In Abschnitt 8.3 schließe ich die Arbeit mit einem wohnungspolitischen Ausblick und Anregungen für künftige Forschungsprojekte. 


\subsection{Dimensionen der Vermarktlichung \& Wohneigentumsförderung}

In der hier betrachteten Zeitspanne wird deutlich, dass Prozesse der Vermarktlichung und Finanzialisierung wohlfahrtsstaatlicher Institutionen nicht immer in allen Dimensionen parallel verlaufen, sondern in unterschiedlichen Phasen den Regelgehalt oder aber die ideelle Dimension der policies stärker betreffen. Die wichtigsten Merkmale der betrachteten Phasen im Hinblick auf die zu untersuchenden Hypothesen sind in Tabelle 8.1 zusammengefasst (S. 330).

Der durchgängigste Trend der Vermarktlichung in der Eigenheimförderung betrifft den Regelgehalt der policies: Über den längsten Zeitraum hinweg wurde das Eigenheim staatlich gefördert, wobei seit den 70er Jahren auch eine Privatisierung der öffentlichen Wohnungsbestände zu verzeichnen ist. Diese Vermarktlichung in der Dimension des Regelgehalts ging jedoch nicht durchgehend mit einer Ökonomisierung in der ideellen policy-Dimension einher. So lässt sich die erste Phase der Wohneigentumsförderung in Deutschland als eine Vermarktlichung ohne Marktwissen beschreiben: Während durch mehrere policies die Vermarktlichung durch staatliche Eigenheimförderung im Bereich der Wohnungspolitik vorangetrieben wurde, waren wirtschaftstheoretische Ideen weder in kognitiver noch in normativer Hinsicht entscheidend. Stattdessen dominierten Vorstellungen der christlichen Soziallehre die Zielvorstellungen der frühen Eigenheimförderung. Wie umstritten demgegenüber in dieser ersten Phase noch statistische Kennzahlen bereits als Problemdefinitionen in politischen Debatten waren, zeigt sich in den 60er Jahren bei der Frage, ob Angebot und Nachfrage auf Wohnungs- 
Tabelle 8.1: Phasen der Ideenproduktion für die Wohneigentumsförderung

Phase 1: Fördervorrang des Familienheims zur Gesellschaftsstabilisierung Regelgehalt Eigenheimförderung wird im Sozialen Wohnungsbau verankert und erhält gesetzlichen Fördervorrang gegenüber dem Mietwohnungsbau

Ideen Dominanz der katholischen Soziallehre: Wohneigentum dient familien- \& gesellschaftspolitischen Zielen; ökonomische Betrachtungsweise wird abgelehnt

Ideen- Christliche Koalition aus Verbänden, theologischen Experproduktion ten \& christdemokratischen Parteien bestimmt Ideenproduktion; Ministerialbürokratie und Statistisches Bundesamt bauen eigene Expertise auf

Phase 2: Förderung von erlebbarem und anlegbarem Individualvermögen Regelgehalt Auch Wohnungen in Mehrfamilienhäusern werden als Wohneigentum gefördert; steuerliche Förderung setzt sich durch ggü. Sozialem Wohnungsbau

Ideen Familienförderung bleibt als Rahmung, aber Marktkonformität wird Grundlage der Förderung: Ausgleich von Angebot und Nachfrage soll erreicht werden; Kennzahl „Wohneigentumsquote" wird Orientierungspunkt

Ideen- $\quad$ Boom gutachterlicher Tätigkeiten im Auftrag der policyproduktion maker \& der policy-taker als administrativer Trend Phase 3: Förderung von Wohneigentum als Asset Regelgehalt Eigenheimzulage als Ersatz der steuerlichen Förderung; Eigenheimzulage wird abgeschafft zugunsten einer gleichberechtigten Anlageform in der Riesterrente

Ideen Wohneigentumsförderung soll unter Austeritätskriterien eine Förderung der privaten Altersvorsorge bewirken; Nutzen \& Kosten der Förderung sind präzise kalkulierbar Ideen- $\quad$ Berufung einer durch Finanzexperten dominierten Komproduktion mission; Ausbau der ministeriellen Forschungstätigkeit; Vielfalt privatwirtschaftlicher und halböffentlicher Forschungsinitiativen 
märkten als ausgeglichen gelten können (vgl. Abschnitt 5.2). Quantitative Analysen wurden selbst von wirtschaftswissenschaftlichen Experten als unzureichend für die Erfassung wohnungspolitischer Fragestellungen angesehen. Anhand dieser Phase wird deutlich, dass sich Vermarktlichung auch aus anderen Kulturbeständen speisen kann, die nicht notwendigerweise eine Nähe zum liberalen Credo haben (Münnich u. Sachweh, 2017, 13).

Allerdings nahm die Bedeutung wirtschaftstheoretischer Ideen in kognitiver Hinsicht bereits in der zweiten Phase der Ideenproduktion zu. Neben rahmenden familienpolitischen Zielsetzungen etablierte sich zunehmend Marktkonformität als Bedingung der Wohneigentumsförderung: Staatliche Eingriffe wurden nicht mehr gesellschaftspolitisch gerechtfertigt, sondern sollte dem Ausgleich von Angebot und Nachfrage befördern. Hinzu kam eine Orientierung von Politikern aller Parteien an statistischen Kennzahlen wie der Wohneigentumsquote, was in der vorherigen Phase noch undenkbar erschien. Nun wurde diese Kennziffer nicht nur als Bestandteil einer wohnungspolitischen Problemdefinition, sondern als eigenständige Zielgröße verwendet. So konnte auch unter der Rahmung als Familienförderung die Wohneigentumsförderung mithilfe statistischer Kennzahlen versachlicht und damit entpolitisiert werden im Sinne von de Goede (2004) und Forrest u. Hirayama (2014). Ob eine politische Maßnahme gut oder schlecht ist, entscheidet sich demnach weniger anhand normativer politischer Zielvorstellungen, sondern anhand von Expertisen und Kalkulationen. Die Logiken der Finanzialisierung (de Goede, 2004) und Vermarktlichung (Forrest u. Hirayama, 2014) verdrängten somit familien- und gesellschaftspolitische Elemente der Wohneigentumsförderung: ,political judgement [is replaced] with 
economic evaluation, including, but not exclusively, the evaluations offered by markets" (Davies, 2017, 6). Ökonomische Bewertungen verdrängen zunehmend andere Begründungsmuster und tragen somit über die Änderung ideeller Komponenten zu einer Vermarktlichung der policies bei.

Besonders deutlich kommt dieser Zusammenhang in der dritten Phase zum Vorschein, in der Kosten und Nutzen der Wohneigentumsförderung rechnerisch gegeneinander abgewogen und zugunsten einer allgemeinen Austeritätspolitik gemindert werden (vgl. Abschnitt 7.2). An dieser Stelle tragen die vermögenspolitischen Zielvorstellungen, die bereits während der zweiten Phase auftreten (vgl. Abschnitt 6.2), dazu bei, dass die Wohneigentumsförderung schließlich als Bestandteil der Riesterrente wieder eingeführt wird - allerdings in geringerem Umfang als die vorherige Eigenheimzulage. Damit wird die sich bereits im ideellen Wandel der zweiten Phase andeutende Finanzialisierung auch auf der Ebene der policy selbst übertragen: Wohneigentum ist nun ein Finanz- und Anlageprodukt. Gegenüber den Anfängen der Eigenheimförderung bieten wirtschaftswissenschaftliche Ideen und Konzepte ab der zweiten Phase nicht nur die Grundlage für Problemdefinitionen in der Eigenheimförderung, sie ermöglichen Wohneigentum als Asset zu betrachten und begünstigen damit den entsprechenden Wandel des Regelgehaltes der Wohneigentumsförderung. Zugleich erweist sich diese Entwicklung der Dimensionen der Vermarktlichung als paradox, da eine zunehmende Vermarktlichung der Ideen mit einer geminderten Vermarktlichung des Regelgehalts einhergeht. Vermarktlichung und Finanzialisierung begünstigen somit über die kognitive wie auch die normative Dimension der Eigenheimförderung eine Änderung des Regelgehaltes 
der wohlfahrtsstaatlichen Institution „Eigenheimförderung“ - allerdings ohne in diesem notwendigerweise eine stärkere Vermarktlichung hervorrufen zu müssen.

Insgesamt wird anhand der Betrachtung der unterschiedlichen Dimensionen der Eigenheimförderung in der Bundesrepublik deutlich, dass ideeller Wandel ein entscheidender Faktor für den Wandel des Regelgehalts der Eigenheimförderung in Deutschland war und seinerseits wiederum mit den vorherigen Wandlungsprozessen eng verbunden ist. Allerdings lässt sich durch eine bloße Diagnose der Wandlungsprozesse noch nicht beschreiben, wie dieser Wandel zustande kommen konnte. Die entsprechenden Faktoren sollen im folgenden Abschnitt erläutert werden.

\subsection{Wahlverwandtschaft: Verwaltung \& Ver- marktlichung}

Für den sich langsam vollziehenden institutionellen Wandel der Eigenheimförderung in Deutschland kommen, basierend auf den theoretischen Überlegungen in Kapitel 2, mehrere Erklärungen infrage. Klassische Institutionalismen betonen meist den Wandel der Rahmenbedingungen und die am Wandel beteiligten Akteure. Tatsächlich wurden wohnungspolitische Entscheidungen oftmals durch Engpässe in der Wohnungsversorgung beeinflusst (Jaedicke u. Wollmann, 1991, 433). Besonders deutlich zeigt sich dieser Zusammenhang zwischen Wohnungsmangel und institutionellem Wandel in der Wohnungspolitik in der Phase des Wiederaufbaus nach dem Zweiten Weltkrieg (vgl. Abschnitt 5.1). Allerdings ist in dieser Phase die Förderung von freiste- 
henden Familienheimen keineswegs eine alternativlose Reaktion der Politik: Die Wohnungsnot hätte auch nach Kenntnis der damaligen Akteure mit dem Bau von Mehrfamilienhäusern deutlich effektiver realisiert werden können (Hilpert 2012; Kramper 2008). Insbesondere vor dem Hintergrund der zunehmend als ausgeglichen wahrgenommenen Wohnungsmärkte der zweiten und dritten Phase erscheint die Wohneigentumsförderung als solches schwerlich aus wohnungspolitischen Notwendigkeiten heraus begründbar. Dies trifft auch auf die Zeiten steigender Inflationsraten in den 70er Jahren zu, in der eine regelrechte Flucht in die Sachwerte erfolgte und die Wohneigentumsquoten auch ohne zusätzliches staatliches Eingreifen gestiegen wären (vgl. Abschnitt 6.1). Angesichts der abnehmenden Engpässe auf Wohnungsmärkten hatte die allgemeine Wohnungspolitik in der dritten Phase sogar weitgehend ihren einstmaligen Stellenwert eingebüßt (vgl. Abschnitt 5.1). Aus einer Berücksichtigung der reinen wohnungspolitischen Rahmenbedingungen alleine lässt sich die über einen langen Zeitraum hinweg bestehende und weiter ausgebaute Wohneigentumsförderung in der Bundesrepublik nicht erklären.

Auch die These, wonach Regierungswechsel einen entscheidenden Einfluss auf wohnungspolitische Entscheidungen haben sollten, hält den Betrachtungen im Hinblick auf die Wohneigentumsförderung nicht stand. So lassen die Wechsel zwischen der sozialliberalen Koalition und christlich-konservativ dominierten Regierungen in der zweiten Phase zunächst wohnungspolitische Trendwenden erwarten (vgl. Abschnitt 6.1). Allerdings blieb die Eigenheimförderung unter der sozialliberalen Regierung nicht nur erhalten, sondern wurde noch weiter ausgebaut. Ebenso legte diese Regierung die Grundlagen für jene Reformen, 
welche die liberal-konservative Regierung später umsetzen sollte (vgl. Jaedicke u. Wollmann 1991; Harlander 2008). Nach Einschätzung von Schulz (1994) lassen sich diese geringen Abweichungen der verfolgten Eigenheimpolitik auch damit begründen, dass die oft vorgenommene Polarisierung zwischen den Parteien tatsächlich vorhandene Gegensätze überzeichnete (Schulz, 1994, 109). Waren die Unterschiede zwischen CDU und SPD in Bezug auf die Eigenheimförderung in der ersten Phase bereits geringer als gedacht, zeigte sich bereits in der zweiten Phase, dass beide Parteien darauf bedacht waren, sich als Verfechter der Wohneigentumsförderung auszuweisen (Krummacher, 1978, 352). Auch das Ausscheiden der Neuen Heimat, als dem bedeutendsten Vertreter der gemeinnützigen Wohnungswirtschaft aus dem Kreis der Interessenvertreter, lässt sich der Wandel der Eigenheimförderung nicht erklären. Die weitere Verbandslandschaft blieb, abgesehen von einzelnen Umstrukturierungen, im Zeitverlauf ebenfalls konstant. Lediglich bei der Abschaffung der Eigenheimzulage in der dritten Phase zeigen sich Konfliktlinien zwischen den großen Parteien: Im Zusammenhang mit ihrer finanzpolitischen Umorientierung ist die SPD dazu bereit, die Eigenheimförderung zugunsten eines schlankeren Staates zu opfern, während die konservativ-christlichen Parteien insbesondere über den Bundesrat eine Reform zunächst blockieren. Allgemein ist jedoch über die betrachteten drei Phasen hinweg ein allgemeiner Bedeutungsverlust der Wohnungspolitik auf Bundesebene in allen Parteien zu verzeichnen, was vor allem daran erkennbar wird, dass selbst die CDU in der 15. Legislaturperiode keinen eigenen wohnungspolitischen Sprecher mehr benannte (Egner, 2004b). 
Wie bereits in Abschnitt 8.1 dargestellt, sind die mit dem Politikfeld verknüpften Ideen wesentliche Faktoren für institutionellen Wandel in der langen Frist. Bereits frühere Untersuchungen zu den Ursachen der Eigenheimförderung legen nahe, dass derartige Maßnahmen nicht aus wohnungspolitischen Begründungsmustern oder Interessenkonstellationen alleine gerechtfertigt werden können, sondern stets einer erweiterten ideellen Rechtfertigung bedürfen (DeSalvo 1976; Leidner 1981; Jokl u. Zehnder 1996). Damit ist jedoch noch nicht geklärt, was zu diesem ideellen Wandel beigetragen hat.

Die vorliegende Untersuchung zeigt, dass Akteure eines politikfeldspezifischen knowledge regime Ideen für die unterschiedlichen Formen der Vermarktlichung bereitstellen: Die jeweiligen Akteure konkretisieren abstraktere Weltbilder zu politischen Zielsetzungen und Instrumenten und stellen Problemdefinitionen sowie analytische Tools zu deren Erfassung bereit. Bei den beobachtbaren Prozessen der Vermarktlichung handelt es sich im Fall der Eigenheimförderung weder um den bloßen Ausdruck eines internationalen ,neoliberalen Trends“ oder anonymer Globalisierungsprozesse (Lowe, 2011, 5). Stattdessen sind Ideen als eine Bedingung institutionellen Wandels von den an ihrer Produktion beteiligten Akteuren und den Kontextbedingungen der Wissensproduktion abhängig. Die Ideenproduktion ist somit ein entscheidender Faktor für den spezifischen Pfad der Vermarktlichung, der in der bundesdeutschen Eigenheimförderung eingeschlagen wurde.

Die erste hier aufgestellte Hypothese zum Einfluss von Akteursstruktur und Mechanismen der Ideenproduktion auf den ideellen Wandel wohlfahrtsstaatlicher Institutionen kann für alle drei betrachteten Phasen bestätigt werden. In der ersten Phase zeigt sich die Dominanz 
einer christlichen Koalition aus Verbänden, Parteien und theologischen Experten in der Formulierung und Konkretisierung politischer Zielvorstellungen und Maßnahmen, basierend auf der katholischen Soziallehre (vgl. Abschnitt 5.3). Parteien, Verbände und die ihnen nahestehenden theologischen Experten trugen zur Institutionalisierung der gesellschaftspolitischen Zielvorstellungen im Rahmen der Eigenheimförderung der Bundesrepublik Deutschland bei. Theologische Experten wie der Theologe Oswald von Nell-Breuning trugen dazu bei, das abstraktere Vorstellungen der christlichen Soziallehre für den wohnungspolitischen Kontext zu konkretisieren und dem an Reformen beteiligten Akteuren handlungsanleitendes Wissen zur Verfügung zu stellen. Die Aufgabe der Experten aus Theologie und Wirtschaft besteht in der ersten Phase maßgeblich darin, vorgegebene Fragestellungen des Wohnungsbauministers $\mathrm{zu}$ bearbeiten und nicht eigenständig tätig zu werden. Von einer herausragenden Bedeutung der wissenschaftlichen Expertise im technokratischen Sinne kann daher in der ersten Phase nicht die Rede sein. Stattdessen muss in der ersten Phase von einer erweiterten Dezision im Habermasschen Sinne als wesentlicher Mechanismus ausgegangen werden: Der Kern der politischen Zielvorstellungen bleibt den gewählten Politikern und ihren normativen Wertvorstellungen vorbehalten. In diesem Rahmen haben sowohl Wirtschaftswissenschaftler als auch die sich im Aufbau befindende öffentliche Verwaltung nur einen geringen Einfluss auf die in den politischen Debatten verfügbaren Ideen. Staatliche Administrationen übernehmen hier eine dienende Funktion gegenüber anderen Akteuren und helfen primär dabei, Interessen gesellschaftlicher Gruppen zu verhandeln, um einen Ausgleich herbeizuführen. Die zweite Hypothese zur herausragenden Bedeutung der öf- 
fentlichen Verwaltung muss somit für die erste Phase widerlegt werden. Allerdings wurden zugleich durch Mitarbeiter der Ministerialbürokraie wesentliche Mechanismen der zukünftigen Ideenproduktion initialisiert. Dazu gehören neben Beiratstätigkeiten und den ersten Vergaben von Auftragsgutachten auch der Aufbau der amtlichen Statistik.

Wie wesentlich diese Doppelrolle der öffentlichen Verwaltung in der Wissensproduktion als gatekeeper der Wissensproduktion einerseits und grundlegender Wissensproduzent andererseits war, wird in der zweiten Phase deutlich. Entsprechend der ersten Phase zeigt sich zunächst, dass der ideelle Wandel hinter der Wohneigentumsförderung mit einem Wandel in der Wissensmaschinerie verbunden ist. Gegenüber der ersten Phase spielen nun allerdings weniger Theologen eine wichtige Rolle, sondern eine Vielzahl an Sozial- und Wirtschaftswissenschaftlern aus verschiedenen Forschungsinstituten und Verbänden. Die Mechanismen der Wissensproduktion können in der zweiten Phase mit dem Begriff der korporatistischen Politikberatung beschrieben werden (vgl. Weingart, 2006): Repräsentanten von Interessengruppen oder von ihnen bestimmte Experten sind in Beratungsgremien und Gutachtenerstellung eingebunden. Zugang zum Regime der Wissensproduktion für den politischen Prozess erhalten diese Berater allerdings durch die Ministerialbürokratie. Wie viele andere Ministerien vergibt nun auch das zuständige Wohnungsbauministerium zahlreiche Forschungsaufträge zur verbesserten Planung wohnungspolitischer Maßnahmen. Ermöglicht wird die Gutachtenerstellung für die Wirtschaftswissenschaftler vor allem durch die zunehmend etablierten analytischen Tools der amtlichen Statistik. Anhand der Daten werden Wirtschaftswissenschaftler erstmals dazu in die Lage versetzt, quantitative Analysen zur Ideenproduktion beizu- 
steuern und Marktkonformität als berechenbares Ideal von Politiken in den Vordergrund zu stellen. Ohne den Ausbau der amtlichen Statistik war diese entscheidende Grundlage der wirtschaftswissenschaftlichen Analyse nicht gegeben.

Die Möglichkeiten der wirtschaftswissenschaftlichen Ideenproduktion lassen sich vor allem auf administrative Trends in der staatlichen Wissensverwaltung und -produktion zurückführen. Ohne die Bereitstellung von Kennziffern und Daten durch die amtliche Statistik und die Aufträge zur wirtschaftswissenschaftlichen Analyse in Form von Gutachtertätigkeiten, hätten diese entpolitisierenden Argumentationsmuster in der Wohnungspolitik keinen Eingang finden können. Die Grundlage der Vermarktlichung auf ideeller Ebene besteht somit in ,the explicitness and transparency of quantitative, economic indicators, of which the market price system is the model" (Davies, 2017, 6). Auf eigene Initiative erstellten die Experten in diesem Zeitraum noch keine Expertisen und auch die Anzahl jener wissenschaftlichen Experten, die sich mit wohnungspolitischen Fragestellungen auseinandersetzten, muss weiterhin als überaus gering eingestuft werden. Ihren Einfluss gewinnen die wenigen Experten allerdings durch Forschungsaufträge der Ministerialbürokratie, die ihnen neben Ressourcen zu weiterer Forschung auch das Renommee eines Experten und damit den Zugang zum Regime der Wissensproduktion liefern. Entsprechend der These von Skocpol 1985 wirken Handlungen der amtlichen Statistik und Weichenstellungen der Ministerialbürokratie in der Gutachtenvergabe sinngebend und sogar handlungsanleitend für andere Akteursgruppen aus Wirtschaft und Politik. Die zweite getroffene Hypothese zur Bedeutung staatlicher Stellen für die Wissensproduktion kann für die zweite Phase bestätigt 
werden.

Diese zentrale Rolle der öffentlichen Verwaltung im Regime der Ideenproduktion lässt sich auch in der dritten Phase nachweisen. Die bestehenden koordinierenden Aufgaben der Ministerialbürokratie haben sich in Bezug auf die Wissensproduktion für die Eigenheimförderung weiter verfestigt. Mit der Deutschen Bundesbank ist ein weiterer staatlicher Akteur im Feld der Wissensproduktion aufgetreten, der neben dem Statistischen Bundesamt Kennzahlen zum Wohnimmobilienmarkt bereitstellt und damit die verfügbaren analytischen Tools für politische Akteure erweitert. Diese Befunde gehen einher mit Analysen zu Staatsstrukturen, die von einem Ausbau eigener staatlicher Expertise im Rahmen von Bürokratien ausgehen (Skocpol u. Rueschemeyer 1996, 6; Friedrich 1970, 17; Rueschemeyer u. Skocpol 1996, 297). Zwar werden zugunsten einer Expertenkommission deutlich weniger Gutachten in Auftrag gegeben. Die Kommissionsberufung geht in dieser Phase allerdings von der Ministerialbürokratie aus, die auch ihre Arbeit eng begleitet. Insgesamt zeigt sich im Bereich der Expertise eine vermehrte Anwendung quantitativer Modelle, die auf Wohnungsmärkte angewendet werden und sich auch in den politischen Debatten wieder finden. Gesellschaftspolitische Überlegungen finden dabei deutlich weniger Anwendung. Die Diagnose von Häußermann u. Siebel (1981) zu Beginn der 80er Jahre, Wohnungspolitik sei zu einer „Angelegenheit von Experten“ geworden, scheint sich nun vollends zu bestätigen. Es zeigt sich, wie sehr sich die kognitive Infrastruktur der Wirtschaftswissenschaftler im Sinne von Hirschman u. Berman (2014) etablieren konnte (vgl. Abschnitt $3.1 .3)$.

Die neue Vielfalt der Produzenten eigenheimpolitischer Ideen zeich- 
net sich mehrheitlich durch wirtschaftswissenschaftliche Analysen aus. Bereits in der vorherigen Phase hatten auch Verbände die wirtschaftswissenschaftliche Analysemethoden zur Untermauerung ihrer Argumente genutzt und die entsprechenden Studien in Auftrag gegeben (vgl. Abschnitt 6.3.1). Andere Formen der Ideenproduktion sind kaum noch vorhanden (vgl. Abschnitt 7.3.1). Besonders deutlich wird dies durch einen Vergleich des wohnungswirtschaftlichen Beirates und der Expertenkommission Wohnungspolitik: In beiden Gremien sind Verbandsexperten und Wissenschaftler vertreten, aber die Rolle der Wirtschaftswissenschaftler hat sich geändert. Wird der wohnungswirtschaftliche Beirat in der ersten Phase noch durch einen Verbandsvertreter geleitet, entfiel diese Aufgabe in der Expertenkommission der dritten Phase auf den Finanzwissenschaftler Hans-Werner Sinn. Der dominante Mechanismus korporatistischer Politikberatung aus der zweiten Phase ändert sich in der dritten Phase zu einer vornehmlich wissenschaftlichen Politikberatung unter Einbeziehung der Verbände. Waren die Analysen des Beirates noch ordnungspolitischen Argumentationen verhaftet, dominieren im Bericht der Expertenkommission quantitative Analysen als Grundlage der gesammelten Argumentationen. Diese Form der Analyse erweist sich als zentral für die Betrachtung des Wohneigentums als Finanzanlage. Musste zunächst die Möglichkeit geschaffen werden, einen Wohnungsmarkt zu analysieren, so ergibt sich die Möglichkeit der Immobilienanlage als Finanzanlage erst durch die Möglichkeiten der Preis- und Renditebeobachtung durch analytische Tools (vgl. Abschnitt 2.2.1).

Folgt man den Schlussfolgerungen von Campbell u. Pedersen (2014), so wurde die wissenschaftliche Ideenproduktion um so bedeutender, je 
mehr sich Regierungen mit den komplexer werdenden Herausforderungen der Globalisierung befassen mussten (Campbell u. Pedersen, 2014, 5). Damit stimmen sie mit Erkenntnissen international vergleichenden Untersuchungen überein, wonach Unsicherheiten der politischen Akteure eine wesentliche Ursache für das Hinzuziehen externer Expertise darstellen (Hall 1989; Adler u. Haas 1992; Blyth 2002). Im Zusammenhang mit der Eigenheimförderung kann dies zwar im Zusammenhang mit der stärkeren Integration der Immobilienfinanzierung in das System der internationalen Finanzmärkte gesehen werden (vgl. Abschnitt $8.3)$.

Für die Bedeutungszunahme der wissenschaftlichen Expertise innerhalb der Wissensmaschinerie der Eigenheimförderung waren jedoch zwei andere Bedingungen entscheidend: Erstens wurde die Entpolitisierung der Debatten anhand wissenschaftlicher Analysen dadurch begünstigt, dass für die Parteien Wohnungspolitik zunehmend an Bedeutung verloren hat. Anders als in der ersten Phase, in der angesichts einer massiven Wohnungsnot Wohnungspolitik ein zentrales Wahlkampfthema darstellte (vgl. Abschnitt 5.1), verfügen in der dritten Phase Parteien nicht einmal mehr über einen wohnungspolitischen Sprecher (vgl. 7.1). Wie bereits Hirschman u. Berman (2014) feststellen, wird die Dominanz von Experten wahrscheinlicher, je weniger Bedeutung den politischen Fragestellungen vonseiten der Parteien zugesprochen wird.

Zweitens ist der gestiegene Einfluss der wirtschaftlichen Deutungen nicht ohne deren gezielte Einbeziehung durch die öffentliche Verwaltung denkbar. Thre Vertreter sorgen nicht nur dafür, dass die notwendigen Daten für die Analysen bereitgestellt werden, sondern ermöglichten den Experten auch über Auftragsgutachten und Beiratstätigkeiten den $\mathrm{Zu}$ - 
gang zur Wissensmaschinerie. An dieser Stelle ist darauf hinzuweisen, dass die langfristigen Folgen dieser Maßnahmen der Ministerialbürokratie wahrscheinlich nicht deren Intention entsprach. Gerade bei langfristigen Folgen ist stets von Paradoxien und nicht intendierten Konsequenzen von Akteurshandeln auszugehen, da sich die Akteure in der konkreten Handlungssituation auch Unsicherheiten gegenübersehen, die ihr eigenes Handeln und das der anderen betrifft (vgl. Nölke, 2014, 79f.). Stattdessen wird hier von einer Wahlverwandtschaft im Weberianischen Sinne zwischen den Tätigkeiten der öffentlichen Verwaltung einerseits und der De-Politisierung eines sozialpolitischen Instruments andererseits ausgegangen. Weber zufolge bestehen Wahlverwandtschaften ,Zwischen gewissen Formen des religiösen Glaubens und der Berufsethik" (Weber u.a., 2013, 256): Aspekte des calvinistischen Glaubens finden sich ebenso in dem spezifischen Ethos des modernen Kapitalismus wieder. Ist laut Weber ,[e]iner der konstitutiven Bestandteile des modernen kapitalistischen Geistes [...] geboren aus dem Geist der christlichen Askese“ (Weber u.a., 2013, 484f.), so ist die zunehmende ökonomische Betrachtung der Eigenheimförderung in der Bundesrepublik nicht denkbar ohne die durch die Ministerialverwaltung verfolgte Zielvorstellung, wohnungspolitische Arbeit an exaktes statistisches Berichtswesen zu knüpfen. Diese Bedeutungsüberschneidung zwischen der Vermarktlichung begünstigenden Ökonomisierung auf der einen und der präzisen statistischen Erfassung des Regulierungsgegenstandes auf der anderen Seite begünstigte schließlich die zunehmende De-Politisierung der Eigenheimförderung in Deutschland. 


\subsection{Forschungsperspektiven und Ausblick}

Die Ergebnisse der vorliegenden Untersuchung bieten neben Erkenntnissen zur langfristigen Entwicklung der bundesdeutschen Eigenheimförderung Impulse für künftige Forschungsarbeiten zu institutionellem Wandel. Zwar sind die hier getroffenen Schlussfolgerungen zum Zusammenhang zwischen Ideenproduktion und Vermarktlichung oder Finanzialisierung nicht ohne weiteres auf andere sozialpolitische Bereiche übertragbar, da sich einzelne Politikbereiche zu stark durch unterschiedliche Akteurskonstellationen, institutionelle Strukturen sowie inhaltliche Eigenheiten auszeichnen (vgl. Beyme u. Schmidt 2013; Lowe 2011, 10; vgl. Abschnitt 4.4). Allerdings liefert die Arbeit wichtige Implikationen für künftige Forschungsprojekte zur Weiterentwicklung von Theorien institutionellen Wandels durch Ideen (Béland u. Cox, 2011) sowie zu knowledge regimes (Campbell u. Pedersen, 2014).

Zunächst konnte durch das Einnehmen der langfristigen Perspektive gezeigt werden, dass insbesondere für kleinteiligen institutionellen Wandel Ideen einen entscheidenden Einfluss auf die langfristige Entwicklung einer Institution haben können. Als Bestandteile von Institutionen geben sie diesen einen Rahmen, in dem sie bestehen, aber sich auch weiterentwickeln können (vgl. Blyth, 2001). Die empirischen Ergebnisse der vorliegenden Arbeit legen nahe, dass sich Änderungen des institutionellen Gefüges zuerst auf der ideellen Ebene vollziehen bevor eine entsprechende Reform auf der Regelebene erfolgen kann. Institutionen sollten demnach nicht eng definiert werden als ,norms with teeth" (so Rueschemeyer, 2009, 210) und sich damit auf deren Regelgehalt konzentrieren. Vielmehr sollte ein breiter gefasster Institutionenbegriff diese ideelle Dimension mit berücksichtigen. Darüber hinaus 
spielen Ideen eine zentrale Rolle in der Sinnstiftung bei Handlungsentscheidungen von Akteuren, die zu institutionellem Wandel beitragen (vgl. Münnich, 2011). Ein ideeller Wandel erzwingt zwar keinen institutionellen Wandel, kann diesen aber über die Sinnstrukturierung der Akteure ermöglichen (vgl. Seeleib-Kaiser, 2003b). Das Set der zur Verfügung stehenden Ideen wirkt gemeinsam mit der materiellen Position des Akteurs im jeweiligen situativen Kontext als sinnstiftendes Element institutionellen Wandels. Wandeln sich die Ideen, ändert sich auch die Voraussetzungen für die Akteurshandlungen.

Sofern Ideen eine gesonderte Rolle für institutionellen Wandel zukommt, ist entscheidend herauszufinden, woher diese Ideen stammen. Mit ihrem Konzept der knowledge regime haben Campbell u. Pedersen (2014) auf die organisationale und institutionelle Maschinerie aufmerksam gemacht, die Daten, Forschungsergebnisse, Politikempfehlungen und andere Ideen für die politische Entscheidungsfindung produzieren. In ihrer international vergleichend ausgelegten Untersuchung zeigen sie, dass die Ausgestaltung dieser Maschinerie entscheidend dafür ist, wie Ideen der Vermarktlichung und Finanzialisierung auf nationaler Ebene formuliert und debattiert und schließlich für die Akteure bereitgestellt werden (Campbell u. Pedersen, 2014, 6). Hier entscheidet sich, welche Ideen den Entscheidungsträgern für institutionellen Wandel als Handlungsorientierung zur Verfügung stehen. Die vorliegende Untersuchung liefert darüber hinaus zunächst die Erkenntnis, dass es nicht ein einziges, politikfeldübergreifendes knowledge regime gibt, sondern dass diese je nach Politikbereich sehr unterschiedlich ausgestaltet sein können. Zusätzlich lassen sich innerhalb des Regimes im Zeitverlauf Veränderungen nachweisen, die sowohl die beteiligten Akteure als auch 
die Mechanismen der Ideenproduktion betreffen. Vor allem für die Untersuchung ideellen Wandels über einen längeren Zeitverlauf hinweg ergeben sich aus derartigen tiefergehenden Analysen der Wissensproduktion Erkenntnisse, die sich nicht alleine auf das jeweilige Produktionsund Politikregime zurückführen lassen.

Eine wesentliche Ergänzung des Modells von Campbell u. Pedersen (2014) ergibt sich aus der Berücksichtigung von Staatsstrukturen für die Wissensproduktion. Bereits die Arbeiten von Evans u.a. (1985a) legen nahe, dass Staatsstrukturen relevante Rahmenbedingungen darstellen, die von den am institutionellen Wandel beteiligten Akteuren berücksichtigt werden müssen. Empirisch konnte in dieser Arbeit gezeigt werden, dass staatliche Stellen wie die amtliche Statistik oder die Deutsche Bundesbank nicht nur selber als Akteure des knowledge regimes fungieren und Wissensbestände zur Verfügung stellen (vgl. Heclo 1975; Skocpol u. Rueschemeyer 1996; Rueschemeyer u. Skocpol 1996). Darüber hinaus kommt der Ministerialbürokratie eine bedeutende Funktion als gatekeeper der Ideenproduktion für den politischen Entscheidungsprozess zu. Empirisch konnte hier gezeigt werden, dass die Ministerialbürokratie für wesentliche Mechanismen der Gutachtenerstellung und Beiratstätigkeiten der Wissensproduzenten eine Schlüsselposition einnimmt: sie beauftragt und beruft Experten, stellt die konkreten inhaltlichen wie formellen Anforderungen an die Forschungsarbeiten und vergibt somit auch Ressourcen in einem Politikbereich, der sich über einen langen Zeitraum hinweg durch sehr kleine Expertenkreise ausgezeichnet hat. In diesem Zusammenhang bedarf es tiefgehender Fallstudien, die für andere Politikbereiche nicht nur die verschiedenen Dimensionen von Vermarktlichungs- und Finanzialisierungstendenzen 
aufschlüsseln, sondern auch Besonderheiten in der dahinterstehenden Ideenproduktion beleuchten. Als interessante Themenfelder bieten sich hierfür die Bedeutung der amtlichen Statistik für Vermarktlichungstendenzen sowie die sich ändernde Rolle der Ministerialbürokratie in den politikfeldspezifischen Maschinerien der Ideenproduktion an, da beide Bereiche bislang unzureichend durch aktuelle Forschung beschrieben wurden.

Die geschilderte Entwicklung der Ideenproduktion im Bereich der Eigenheimförderung ist mit deren Integration in der Riesterrente keineswegs zum Stillstand gekommen. So zeichnet sich seit einigen Jahren ein neues Spannungsfeld der wohnungspolitischen Ideenproduktion ab, das sich auf zwei gegenläufige Trends zurückführen lässt: die erneute Bedeutungszunahme der Wohnungspolitik innerhalb Deutschlands sowie die Tendenz zu einer an internationalen Expertenstandards orientierten Regulierung der Finanzmärkte.

Nach dem Regierungswechsel zur Großen Koalition 2005 schien Wohnungspolitik große Teile ihrer einstigen Bedeutung verloren zu haben. Wohnungsnöte wurden als ein ,sozial selektives Risiko“ angesehen, sodass ,ein politischer Handlungsbedarf im Hinblick auf die Wohnungsversorgung [...] zunehmend de-thematisiert werden konnte" (Heinelt u. Egner, 2006, 209). Die Ära der kleingedachten Wohnungspolitik endete jedoch spätestens mit dem Bundestagswahlkampf von 2013. Angesichts lokal steigender Mieten und Kaufpreise für Wohnraum rückte staatliche Wohnungspolitik erstmals seit langem wieder in das Zentrum politischer Debatten (Egner, 2014, 19). Der erneute Bedeutungszuwachs der bundesdeutschen Wohnungspolitik spiegelt sich auch im Koalitionsvertrag der Großen Koalition von 2013 wieder. Als Leitlinien 
stehen laut Koalitionsvertrag eine „Wiederbelebung des Sozialen Wohnungsbaus" sowie eine ,mietrechtliche und sozialpolitische Flankierung“ (CDU/CSU u. SPD, 2013, 114) erneut auf der wohnungspolitischen Agenda. Nach abgeschlossenen Koalitionsverhandlungen wechselten die Zuständigkeiten für die Bereiche Stadtentwicklung, Wohnen, ländliche Infrastruktur, öffentliches Baurecht sowie Bauwesen, Bauwirtschaft und Bundesbauten zum Bundesministerium für Umwelt, das zwischenzeitlich die Bezeichnung „Bundesministerium für Umwelt, Naturschutz, Bau und Reaktorsicherheit" (BMUB) trug. Nach der Bundestagswahl 2017 wurde der Bereich Wohnungsbau dem Bundesministerium des Innern zugeordnet, das nun die Bezeichnung „Bundesministerium des Innern, für Bau und Heimat" trägt. Zudem wurde auf Basis des neuen Koalitionsvertrages das sogenannte „Bündnis für Wohnen“ geschaffen, in dem neben Politikern auch korporatistische Akteure aus dem Feld der Wohnungswirtschaft vertreten sind.

Als prägnantes und zugleich stark umstrittenes Zeichen einer möglichen Renaissance wohnungspolitischer Maßnahmen kann die sogenannte Mietpreisbremse ${ }^{1}$ angesehen werden. In Bezug auf die Eigenheimförderung kann eine Re-Politisierung anhand des wieder eingeführten Baukindergeldes vermutet werden, mit dem seit September 2018 der erstmaligen Erwerb von selbstgenutztem Wohneigentum für Familien mit Kindern gefördert werden soll (Bundesministerium des Innern, für Bau und Heimat, 1309). Diese maßgeblich von den christdemokratischen Parteien vorangetriebene Maßnahme wurde aus dem Kreis der

1 Dabei handelt es sich um die erneute Einführung einer Mietpreisregulierung für neu vermieteten Wohnraum. Für ausgewiesene ,,angespannte Wohnungsmärkte" sollen die Neuvertragsmieten nun nicht mehr als zehn Prozent über der ortsüblichen Vergleichsmiete liegen. 
etablierten wohnungspolitischen Experten als kostspielige und wenig zielführende Fördermaßnahme stark kritisiert (vgl. Gavrillis u. Fröhndrich, 2018). An dieser Wiederbelebung der familienpolitischen Argumentationsmuster zeigt sich einerseits, dass diese normative Verknüpfung der Eigenheimförderung eine der weitreichendsten Konstanten in der bundesdeutschen Wohnungspolitik darstellt (vgl. Szypulski, 2008, 81). Andererseits zeigt sich dabei, dass von einer ausschließlichen Dominanz außerpartelicher Expertise weiterhin nicht die Rede sein kann.

Ein gegenläufiger Trend lässt sich in den medial weniger aufgegriffenen neuen Eingriffsrechten der Bundesanstalt für Finanzdienstleistungsaufsicht (BaFin) in die Wohnimmobilienkreditvergabe von Banken verzeichnen. Konkret sind damit Obergrenzen für die Baufinanzierung verbunden, welche sich am Maße der Gesamtverschuldung eines Verbrauchers sowie an dessen verfügbarem Einkommen orientieren sollen. Dieses Vorhaben geht auf das Expertengremium des Ausschusses für Finanzdienstleistungsaufsicht (AFS) ${ }^{2}$ zurück, der sich aus Vertretern der Deutschen Bundesbank, des Bundesministeriums der Finanzen, der BaFin sowie des Leitungsausschusses der Bundesanstalt für Finanzmarktstabilisierung (FMSA) zusammensetzt (Bundesanstalt für Finanzdienstleistungsaufsicht, 2016). Mit den Empfehlungen zur Neuregulierung im Bereich der Wohnungskreditvergabe greifen die Mitglieder des AFS explizit den internationalen Expertendiskurs zur Verhinderung möglicher Immobilienblasen auf (Ausschuss für Finanzstabilität,

2 Dieser Ausschuss hat die Aufgabe basierend auf Analysen der Deutschen Bundesbank ,für die Finanzstabilität maßgeblichen Sachverhalte regelmäßig zu erörtern und bei identifizierten Gefahren vor diesen zu warnen und Empfehlungen zu ihrer Abwehr abzugeben“ (Bundesanstalt für Finanzdienstleistungsaufsicht, 2016). 
2015a, 4). Die Maßnahmen sollen nach Angaben des AFS vor allem „einer nicht-tragfähigen Verschuldung entgegenwirken und somit das Risiko senken, dass ein Schuldner seinen finanziellen Verpflichtungen nicht mehr nachkommen kann" (Ausschuss für Finanzstabilität, 2015b, 34). Mit dieser Regulierung tragen die Expertenausschüsse Entwicklungen Rechnung, die sich durch eine zunehmende Internationalisierung der Hypothekenmärkte und den damit verbundenen Potentialen für die Entstehung erneuter Finanzkrisen Rechnung. Wohnungsmärkte werden damit regulatorisch nicht mehr als nationale Märkte angesehen, sondern sind eng mit den Entwicklungen auf den internationalen Finanzmärkten verknüpft (Lowe, 2011, 5). Aalbers bringt diesen Umstand wie folgt auf den Punkt:

„Due to the commodification and financialization of housing, housing risks are increasingly financial market risks these days." (Aalbers, 2012, 121)

Während sich durch den Bedeutungszuwachs wohnungspolitischer Themen auf nationaler Ebene eine Repolitisierung der Eigenheimförderung andeutet, weisen die in transnationalen Gremien vorbereiteten und von nationalen Experten ausgearbeiteten Gesetzesvorschläge auf eine weitere, durch Expertisen bestimmte Depolitisierung wohnungspolitischer Entscheidungen hin. Welcher Trend langfristig überwiegt, und ob auch neue politische Ideen vonseiten der Parteien oder anderen Ideenproduzenten bereitgestellt werden, wird sich in den kommenden Jahren zeigen.

Durch die Herausforderungen der zunehmenden internationalen $\mathrm{Zu}$ sammenarbeit in der Wissensproduktion entfallen auf die öffentliche Verwaltung vermehrt die Aufgaben zwischen den Ideenproduzenten 
durch deren Vernetzung zu vermitteln und deren unterschiedlichen Erkenntnisse für policy-maker aufzubereiten und ihnen zur Verfügung zu stellen. Gleiches gilt für den Umstand, dass Vertreter wirtschaftswissenschaftlicher Forschungsinstitute, von Think Tanks sowie von privaten Beratungsfirmen bereits seit der ausgehenden dritten Phase Analysen und Daten auf eigene Initiative und gezielt für eine Politikberatung der Öffentlichkeit erstellen. Ermöglicht wird ihnen dies durch neue Daten, die sie beispielsweise anhand von Online-Portalen auch unabhängig von der amtlichen Statistik beziehen können. Staatliche Stellen verfügen zwar weiterhin über die wohl zentralste Position in der Ideenproduktion für die Eigenheimförderung. Über ihre tatsächlichen Einflussmöglichkeiten wird allerdings in Zukunft entscheiden, wie viel Spielraum und Ressourcen ihr vonseiten der Politik zugestanden werden (vgl. Bourdieu, 2014, 118). 



\section{Literaturverzeichnis}

[Aalbers 2012] AAlbers, Manuel B.: European Mortgage Markets Before and After the Financial Crisis. In: Aalbers, Manuel B. (Hrsg.): Subprime cities. Malden, Mass : Wiley-Blackwell, 2012 (Studies in Urban and Social Change). - ISBN 9781444337761, S. 120-150

[Aalbers 2016] AALBers, Manuel B.: Routledge studies in the modern world economy. Bd. 158: The financialization of housing: A political economy approach. London and New York : Routledge, 2016. - ISBN 9781315668666

[Aalbers u. Christophers 2014] Aalbers, Manuel B. ; Christophers, Brett: Centring housing in political economy. In: Housing, Theory and Society 31 (2014), Nr. 4, S. 373-394. - ISSN 1403-6096

[Abbott 1988] Аввотт, Andrew: The system of professions: An essay on the division of expert labor. Chicago : University of Chicago Press, 1988. - ISBN 0-226-00069-9

[Adler u. Haas 1992] Adler, Emanuel ; HaAs, Peter M.: Conclusion: epistemic communities, world order, and the creation of a reflective research program. In: International Organization 46 (1992), Nr. 1, S. $367-390$

[von Alemann u. a. 2018] Alemann, Ulrich von ; Erbentraut, Philipp ; Walther, Jens: Das Parteiensystem der Bundesrepublik Deutschland: Eine Einführung. 5. Auflage 2018. Wiesbaden : Springer Fach- 
medien Wiesbaden and Springer-Verlag, 2018 (Grundwissen Politik). - ISBN 978-3-658-21158-5

[Arbaci 2007] ARBACI, Sonia: Ethnic segregation, housing systems and welfare regimes in Europe. In: European Journal of Housing Policy 7 (2007), Nr. 4, S. 401-433. - ISSN 1461-6718

[Arentz u. a. 2010] Arentz, Oliver ; Eekhoff, Johann ; Wolfgramm, Christine ; Institut FÜR Wirtschaftspolitik AN DER UnIVERSITÄT ZU KÖLN (Hrsg.): Zur Finanzmarktkrise: Die Rolle der Immobilienbewertung. Köln, 2010 (IWP Discussion Paper)

[Arras u.a. 1980] Arras, Hartmut E. ; HÜBschl, Jörg ; Afheldt, Heik ; Hogeforster, Jürgen ; SÄttler, Martin ; Bundesminister Für Raumordnung, Bauwesen und Städtebau (Hrsg.): Wohnungspolitik und Stadtentwicklung: Klischees, Probleme, Instrumente, Wirkungen, Rahmenbedingungen: Im Auftrag des Bundesministers für Raumordnung, Bauwesen und Städtebau, durchgeführt von der Prognos AG. 1980 (Schriftenreihe "Städtebauliche Forschung" des Bundesministers für Raumordnung, Bauwesen und Städtebau)

[Ausschuss für Finanzstabilität 2015a] Ausschuss FüR Finanzstabilität: Empfehlung vom 30. Juni 2015. https: //www . bundesfinanzministerium.de/Content/

DE/Pressemitteilungen/Finanzpolitik/2015/06/ 2015-06-30-PM22-empfehlung .pdf?_-blob=publicationFile\&v= 4. Version: 2015

[Ausschuss für Finanzstabilität 2015b] Ausschuss FÜr Finanzsta BILITÄT: Zweiter Bericht an den Deutschen Bundestag zur Finanzstabilität in Deutschland. Berlin, 2015

[Austen-Smith 1993] Austen-Smith, David: Information and Influence: Lobbying for Agendas and Votes. In: American Journal of Political Science 37 (1993), Nr. 3, S. 799. http://dx.doi.org/10. 2307/2111575. - DOI 10.2307/2111575. - ISSN 00925853 
[Ban 2016] BAN, Cornel: Ruling Ideas: How global neoliberalism goes local. New York, NY : Oxford University Press, 2016. - ISBN 9780190600396

[Bartholmai u. a. 1980] Bartholmai, Bernd ; BedaU, Klaus-Dieter ; Ulbrich, Rudi: Beiträge zur Strukturforschung. Bd. 58: Analyse und Prognose der Wohnungsnachfrage in der Bundesrepublik Deutschland. Berlin : Duncker \& Humblot, 1980. - ISBN 9783428446315

[Bartholomäi 2004] BartholomäI, Reinhart C.: Die Entwicklung des Politikfelds Wohnen. In: Egner, Björn (Hrsg.): Wohnungspolitik in Deutschland. Darmstadt : Schader-Stiftung, 2004. - ISBN 9783932736124, S. $15-34$

[Baur 2018] BAUR, Sophia C.: Die Legitimität von Stiftungen im Handlungsfeld Umwelt: Verständnis und Praktiken. Wiesbaden : SpringerVerlag, 2018 (Studien der NRW School of Governance). - ISBN 978-3-658-18719-4

[Béland u. Cox 2011] BÉland, Daniel ; Cox, Robert H.: Introduction. In: BÉland, Daniel (Hrsg.) ; Cox, Robert H. (Hrsg.): Ideas and politics in social science research. Oxford : Oxford University Press, 2011. - ISBN 978-0-19-973687-4, S. 3-46

[Belina 2017] BelinA, Bernd: Kapitalistischer Wohnungsbau: Ware, Spekulation, Finanzialisierung. In: Barbara Schönig, Justin Kadi und Sebastian Schipper (Hg.): Wohnraum für alle (2017), S. 31-46

[Benölken u. Bröhl 2018] Benölken, Heinz ; BRöHL, Nils: Altersvorsorge am Scheideweg: Erfolgreiche Strategien gegen Altersarmut. Wiesbaden : GABLER, 2018. - ISBN 978-3-658-21836-2

[Bentzien 2012] Bentzien, Verena: Schriftenreihe zur immobilienwirtschaftlichen Forschung. Bd. 7: Erschwinglichkeit von Wohneigentum in Deutschland: Entwicklung, Berechnung und Bedeutung von Erschwinglichkeitsindikatoren für die Wohneigentumsbildung in Deutschland unter Berücksichtigung objektspezifischer und lokaler 
wertbeeinflussender Faktoren. Köln : Immobilien Manager Verlag IMV, 2012. - ISBN 9783899842968

[Benzner 1989] Benzner, Bodo: Fundamenta Juridica. Bd. 8: Ministerialbürokratie und Interessengruppen: Eine empirische Analyse der personellen Verflechtung zwischen bundesstaatlicher Ministerialorganisation und gesellschaftlichen Gruppeninteressen in der Bundesrepublik Deutschland im Zeitraum 1949-1984. Baden-Baden : Nomos, 1989. - ISBN 3789017744

[Berman 1998] Berman, Sheri: The social democratic moment: Ideas and politics in the making of interwar Europe. Cambridge, Ma. : Harvard University Press, 1998. - ISBN 0674020847

[Bernhagen 2018] Bernhagen, Patrick: Lobbyismus in der Politikberatung. In: Falk, Svenja (Hrsg.) ; GlaAB, Manuela (Hrsg.) ; RömmeLe, Andrea (Hrsg.) ; Schober, Henrik (Hrsg.) ; Thunert, Martin (Hrsg.): Handbuch Politikberatung. Wiesbaden : Springer Fachmedien Wiesbaden, 2018. - ISBN 978-3-658-07461-6, S. 1-13

[Beyme 1986] Beyme, Klaus v.: Das Gespräch mit der Lobby: Parteien und Verbände. In: Krockow, Christian v. (Hrsg.): Parteien in der Krise Bd. 313. München : Beck, 1986. - ISBN 340631595X, S. 94-104

[Beyme u. Schmidt 2013] Beyme, Klaus v. ; Schmidt, Manfred G.: Politik in der Bundesrepublik Deutschland. Opladen : Westdeutscher Verlag, 2013. - ISBN 9783531119113

[Biedenkopf u. Miegel 1979] Biedenkopf, Kurt H. ; Miegel, Meinhard: IWG-Impulse. Bd. 2: Wohnungsbau am Wendepunkt: Wohnungspolitik in der sozialen Marktwirtschaft. 2. Aufl., (überarb. u. aktualis.), Sonderausg. Stuttgart : Verlag Bonn Aktuell, 1979. ISBN 3879590990

[Blätte 2018] BLÄTTE, Andreas: Politikberatung aus sozialwissenschaftlicher Perspektive. Version:2018. http://dx.doi.org/10.1007/ 978-3-658-07461-6\{_\}3-1. In: FAlK, Svenja (Hrsg.) ; GlaAB, Manuela (Hrsg.) ; Römmele, Andrea (Hrsg.) ; Schober, Henrik (Hrsg.) 
; Thunert, Martin (Hrsg.): Handbuch Politikberatung. Wiesbaden : Springer Fachmedien Wiesbaden, 2018. - DOI 10.1007/978-3-65807461-6_3-1. - ISBN 978-3-658-07461-6, S. 1-14

[Blattmann u. a. 2014] Blattmann, Heidi ; Jarren, otfried ; SchnaBel, Ulrich ; Weingart, Peter ; Wormer, Holger: Zum Verhältnis Medien: Wissenschaft in der Demokratie. In: Weingart, Peter (Hrsg.) ; Schulz, Patricia (Hrsg.): Wissen - Nachricht - Sensation. Weilerswist : Velbrück Wissenschaft, 2014. - ISBN 9783942393805, S. $391-412$

[Blyth 2001] Blyth, Mark: The Transformation of the Swedish Model: Economic Ideas, distributional conflict, and institutional change. In: World politics (2001), S. 1-26

[Blyth 2002] BLYTh, Mark: Great transformations: Economic ideas and institutional change in the twentieth century. Cambridge, UK and New York : Cambridge University Press, 2002. - ISBN 9780521010528

[BMWi 2017] BMWı: Beiräte beim Bundesministerium für Wirtschaft und Energie. http://www.bmwi.de/DE/Ministerium/beiraete, did=161984.html. Version: 2017

[Bogner u. Menz 2002] Bogner, Alexander ; Menz, Wolfgang: Wissenschaftliche Politikberatung? Der Dissens der Experten und die Autorität der Politik. In: Leviathan 30 (2002), Nr. 3, S. 384-399

[Bogner u. Menz 2009] Bogner, Alexander ; Menz, Wolfgang: Das theoriegenerierende Experteninterview: Erkenntnisinteresse, Wissensformen, Interaktion. In: Bogner, Alexander (Hrsg.): Experteninterviews. Wiesbaden : VS Verlag für Sozialwissenschaften, 2009. ISBN 9783531162591, S. 61-98

[Bogumil 2007] Bogumil, Jörg: Regierung und Verwaltung. In: Politische Bildung 4 (2007), S. 38-55 
[Bogumil 2016] BogumiL, Jörg: Die verschlungenen Wege der Politikberatung: Erfahrungen aus dem Bereich der Verwaltungspolitik der Bundesländer. In: Hoose, Fabian (Hrsg.) ; Beckmann, Fabian (Hrsg.) ; Schönauer, Anna-Lena (Hrsg.): Fortsetzung folgt. Springer Science and Business Media and Springer-Verlag, 2016. - ISBN 978-3-658-15449-3, S. 431-445

[Bogumil u. a. 2012] Bogumil, Jörg ; Ebinger, Falk ; Jochheim LinDA: Spitzenbeamte und ihr Verhalten bei politisch relevanten Entscheidungen. In: Derlien, Hans-Ulrich (Hrsg.) ; Schimanke, Dieter (Hrsg.) ; Veit, Sylvia (Hrsg.) ; Bull, Hans P. (Hrsg.): Bürokratie im Irrgarten der Politik Bd. Bd. 36. Baden-Baden : Nomos, 2012.ISBN 9783832971427, S. 151-174

[Bohne 2018] BoHne, Eberhard: Verwaltungswissenschaft: Eine interdisziplinäre Einführung in die Grundlagen. Wiesbaden : Springer Fachmedien Wiesbaden, 2018. - ISBN 978-3-531-17281-1

[Bonanno 2017] Bonanno, Alessandro: The Legitimation Crisis of Neoliberalism: The State, Will-Formation, and Resistance. New York : Palgrave Macmillan, 2017. - ISBN 9781137592453

[Bourdieu 1998a] Bourdieu, Pierre: Schriften zu Politik 83 Kultur. Bd. 3: Der Einzige und sein Eigenheim. Hamburg : VSA, 1998. ISBN 3879757178

[Bourdieu 1998b] Bourdieu, Pierre: Eine sichere Geldanlage für die Familie: Das Einfamilienhaus: Produktspezifik und Logik des Produktionsfeldes. In: STEINRÜCKE, Margareta (Hrsg.): Der Einzige und sein Eigenheim Bd. 3. Hamburg: VSA, 1998. - ISBN 3879757178, S. $26-83$

[Bourdieu 2014] Bourdieu, Pierre: The Social Structures of the Economy. 1. Aufl. Polity, 2014 http://gbv.eblib.com/patron/ FullRecord . aspx?p=1651140. - ISBN 0745625401

[Brehm 1989] Brenm, Thomas: Erlanger historische Studien. Bd. 14: 
SPD und Katholizismus - 1957 bis 1966. Frankfurt am Main : P. Lang, 1989. - ISBN 3631421389

[Breidenstein u.a. 2013] Breidenstein, Georg ; Hirschauer, Stefan ; Kalthoff, Herbert: UTB : Sozialwissenschaften, Kulturwissenschaften. Bd. 3979: Ethnografie: Die Praxis der Feldforschung. Konstanz : UTB, 2013. - ISBN 9783825239794

[Brie 2015] BRIE, Michael: Beiträge zur kritischen Transformationsforschung. Bd. 1: Polanyi neu entdecken: Das hellblaue Bändchen zu einem möglichen Dialog von Nancy Fraser und Karl Polanyi. Hamburg : VSA, 2015. - ISBN 978-3-89965-642-8

[Bröchler 2004] BRÖchler, Stephan: Kalliope im Wunderland. Version:2004. http://dx.doi.org/10.1007/ 978-3-322-80554-6\{_\}2. In: SchützeICHEL, Rainer (Hrsg.) ; Brüsemeister, Thomas (Hrsg.): Die beratene Gesellschaft: Zur gesellschaftichen Bedeutung von Beratung. Wiesbaden : VS Verlag für Sozialwissenschaften, 2004. - DOI 10.1007/978-3-322-805546_2. - ISBN 978-3-322-80554-6, S. 19-38

[Brown u. a. 2006] Brown, Mark B. ; Lentsch, Justus ; Weingart, Peter: Politikberatung und Parlament. Opladen : Budrich, 2006. ISBN 9783938094006

[Buchholz 2008] BuchHolz, Kai: Professionalisierung der wissenschaftlichen Politikberatung? Interaktions- und professionssoziologische Perspektiven. Bielefeld : Transcript, 2008 (Science Studies). http://dx.doi.org/10.14361/9783839409367. http://dx.doi. org/10.14361/9783839409367. - ISBN 9783899429367

[bulwienGesa AG 2018] BulwienGesa AG: Kompetenz in vier Dimensionen. http://www . bulwiengesa.de/de/leistungsprogramm. Version: 2018

[Bundesamt für Bauwesen und Raumordnung 2001] Bundesamt FÜR Baumesen und Raumordnung: Bauland- und Immobilienmärkte. Umsätze und Preise, Strukturen, Entwicklungen, Auswirkungen: Im 
Auftrag des Bundesministers für Verkehr, Bau und Wohnungswesen $(B M V B W)$. Bonn, 2001

[Bundesamt für Bauwesen und Raumordnung 2003] Bundesamt Für Bauwesen und Raumordnung ; Bundesamt Für Bauwesen und Raumordnung (Hrsg.): Wohneigentum. http://www.bbsr.bund.de/BBSR/DE/Veroeffentlichungen/ IzR/2003/Hef t06Wohneigentum.html?nn=422250. Version: 2003

[Bundesamt für Bauwesen und Raumordnung 2018a] BunDesamt FÜr Bauwesen und Raumordnung: Geschichte. http://www.bbsr.bund.de/BBSR/DE/Bundesinstitut/ Geschichte/geschichte_node.html. Version: 2018

[Bundesamt für Bauwesen und Raumordnung 2018b] Bundesamt Für Bauwesen und Raumordnung: Kernaufgaben des Bundesinstituts. http://www.bbsr.bund.de/BBSR/DE/Bundesinstitut/ Aufgaben/aufgaben_node.html. Version: 2018

[Bundesanstalt für Finanzdienstleistungsaufsicht 2016] BunDESANSTALT FÜR FinANZDIENSTLEISTUNGSAUfSICHT: Ausschuss für Finanzstabilität. http://www.bafin. $\mathrm{de} / \mathrm{DE} / \mathrm{DieBaFin} /$ GrundlagenOrganisation/Gremien/ AusschussFinanzstabilitaet/finanzstabilitaet_node.html. Version: 2016

[Bundesinstitut für Bau-, Stadt- und Raumforschung (BBSR) im Bundesamt für Bauwesen und Raumentwicklung 2018a] Bundesinstitut FÜr Bau-, Stadt- und Raumforschung (BBSR) im BunDesamt FÜr BauWesen und Raumentwicklung: Geschichte des Wissenschaftlichen Bereichs des BBR. http://www.bbsr.bund.de/ BBSR/DE/Bundesinstitut/Geschichte/geschichte_node.html. Version: 2018

[Bundesinstitut für Bau-, Stadt- und Raumforschung (BBSR) im Bundesamt für Bauwesen und Raumentwicklung 2018b] Bundesinstitut FÜr Bau-, Stadt- und Raumforschung 
(BBSR) Im Bundesamt FÜr Bauwesen und RaumentwickLUNG: Veröffentlichungen. https://www.bbsr.bund.de/BBSR/ DE/Veroeffentlichungen/veroeffentlichungen_node.html. Version: 2018

[Bundesministerium der Finanzen 2001] Bundesministerium Der F Inanzen ; Bundesministerium Der Finanzen (Hrsg.): Achtzehnter Subventionsbericht: Bericht der Bundesregierungüber die Entwicklung derFinanzhilfen des Bundes undder Steuervergünstigungenfür die Jahre 1999 - 2002. Berlin, 2001

[Bundesministerium des Innern, für Bau und Heimat 1309] BunDesministerium Des Innern, FÜr BAU und Heimat: Baukindergeld geht an den Start: Gefördert wird der erstmalige Neubau oder Erwerb von Wohneigentum zur Selbstnutzung. https://www . bmi . bund.de/SharedDocs/pressemitteilungen/ DE/2018/09/baukindergeld.html. Version: 13.09.2018

[Bundesministerium für Wirtschaft und Energie 2018] BunDESMINISTERIUM FÜR WIRTSChAFT UND ENERgIE: Veröffentlichungen des Wissenschaftlichen Beirats. http: //www.bmwi.de/Navigation/DE/Ministerium/Beiraete/ Veroeffentlichungen-Wissenschaftlicher-Beirat/ veroeffentlichungen-wissenschaftlicher-beirat.html. Version: 2018

[Bundesministerium für Wohnungsbau 1961] Bundesministerium FÜr Wohnungsbau: Grundsätze, Leistungen, Aufgaben der Wohnungsbaupolitik der Bundesregierung. Bonn, 1961

[Bundesministerium für Wohnungwesen und Städtebau 1967] BunDesministerium Für Wohnungwesen und StÄdtebau (Hrsg.): Schriftenreihe des Bundesministers für Wohnungswesen und Städtebau. Bd. 22: Beiträge zu den Grundlagen eines Wohnungswirtschaftsgesetzes: Referate im Sachverständigenbeirat des Bundesministeriums für Wohnungswesen, Städtebau und Raumordnung. Hamburg : Harmonia-Verlag GmbH, 1967 
[Burkhardt 2003] BuRkHARdT, Armin: Reihe Germanistische Linguistik. Bd. 241: Das Parlament und seine Sprache: Studien zu Theorie und Geschichte parlamentarischer Kommunikation. Tübingen : Niemeyer, 2003. - ISBN 3484312416

[Callon 1998] Callon, Michel: Introduction: The Embeddedness of Economic Markets in Economics. In: Callon, Michel (Hrsg.): The Laws of the Markets. Oxford : Blackwell Publishing Inc, 1998. ISBN 9780631206088, S. 1-57

[Campbell 1998] CampBell, John L.: Institutional analysis and the role of ideas in political economy. In: Theory and Society (1998), S. 377-409

[Campbell 2004] CampBell, John L.: Institutional change and globalization. Princeton, NJ : Princeton University Press, 2004. - ISBN 0-691-08921-3

[Campbell u. Pedersen 2001a] Campbell, John L. ; Pedersen, Ove K.: Conclusion: The Second Movement in Institutioal Analysis. In: Campbell, John L. (Hrsg.) ; Pedersen, Ove K. (Hrsg.): The rise of neoliberalism and institutional analysis. Princeton, N.J. : Princeton University Press, 2001. - ISBN 0691070865, S. 249-281

[Campbell u. Pedersen 2001b] Campbell, John L. ; Pedersen, Ove K.: Introduction: The Rise of Neoliberalism and Institutional Analysis. In: Campbell, John L. (Hrsg.) ; Pedersen, Ove K. (Hrsg.): The rise of neoliberalism and institutional analysis. Princeton, N.J. : Princeton University Press, 2001. - ISBN 0691070865, S. $1-23$

[Campbell u. Pedersen 2001c] Campbell, John L. (Hrsg.) ; PederSEN, Ove K. (Hrsg.): The rise of neoliberalism and institutional analysis. Princeton, N.J. : Princeton University Press, 2001. - ISBN 0691070865

[Campbell u. Pedersen 2014] Campbell, John L. ; Pedersen, Ove K.: The National Origins of Policy Ideas: Knowledge Regimes in the Uni- 
ted States, France, Germany, and Denmark. Princeton, New Jersey : Princeton University Press, 2014 http://laisumedu.org/DESIN Ibarra/desin/imagenescampbell/dos-d.pdf. - ISBN 1400850363

[Cassel u. Baumann 2018] Cassel, Susanne ; Baumann, Elke: Wissenschaftliche Beratung der Wirtschaftspolitik in Deutschland und Bedingungen für ihren Erfolg. Version: 2018. http://dx.doi.org/ 10.1007/978-3-658-07461-6\{_\}37-1. In: FALK, Svenja (Hrsg.) ; Glaab, Manuela (Hrsg.) ; Römmele, Andrea (Hrsg.) ; Schober, Henrik (Hrsg.) ; Thunert, Martin (Hrsg.): Handbuch Politikberatung. Wiesbaden : Springer Fachmedien Wiesbaden, 2018. - DOI 10.1007/978-3-658-07461-6_37-1. - ISBN 978-3-658-07461-6, S. $1-18$

[CDU/CSU 1949] CDU/CSU: Düsseldorfer Leitsätze. http://www. kas.de/wf/de/33.814/. Version: 1949

[CDU/CSU u. FDP 2009] CDU/CSU ; FDP: Wachstum. Bildung. Zusammenhalt. Der Koalitionsvertrag zwischen CDU, CSU und FDP. Berlin, 2009

[CDU/CSU u. SPD 2005] CDU/CSU ; SPD: Gemeinsam für Deutschland - mit Mut und Menschlichkeit: Koalitionsvertrag zwischen CDU, CSU und SPD. Berlin, 2005

[CDU/CSU u. SPD 2013] CDU/CSU ; SPD: Deutschlands Zukunft gestalten: Koalitionsvertrag zur 18. Legislaturperiode. Berlin, 2013

[Centeno u. Cohen 2012] Centeno, Miguel A. ; Cohen, Joseph N.: The arc of neoliberalism. In: Annual Review of Sociology (2012), S. 317-340. - ISSN 0360-0572

[Chabrowski 1963] Chabrowski, Sigmund: Verbände in unserer Zeit: Unter dem Mantel der Wissenschaft. In: Die Zeit (1963). http: //www.zeit.de/1963/36/unter-dem-mantel-der-wissenschaft

[Christliche Demokraten Kölns 1945] Christluche Demokraten KÖLNS: Kölner Leitsätze: Vorläufiger Entwurf zu einem Programm der Christlichen Demokratischen Union Deutschlands. 
http://www.kas.de/upload/ACDP/CDU/Programme_Beschluesse/ 1945_Koelner-Leitsaetze.pdf. Version: 1945

[Clemens 1994] Clemens, Gabriele (Hrsg.): Kulturpolitik im besetzten Deutschland 1945 - 1949. Stuttgart : Steiner, 1994 (Historische Mitteilungen). - ISBN 3-515-06324-2

[Crouch 2013] Crouch, Colin: Edition Suhrkamp. Bd. 2: Das befremdliche Überleben des Neoliberalismus. 4. Aufl. Berlin : Suhrkamp, 2013. - ISBN 9783518422748

[Czada 1994] Czada, Roland: Konjunkturen des Korporatismus: Zur Geschichte eines Paradigmenwechsels in der Verbändeforschung. In: Streeck, Wolfgang (Hrsg.) ; Czada, Roland (Hrsg.): Staat und Verbände Bd. 25. Opladen : Westdeutscher Verlag, 1994. - ISBN 353112661X, S. 37-64

[Czarniawska 2009] Czarniawska, Barbara: Narrative, Diskurse und Organisationsforschung. In: DiAz-Bone, Rainer (Hrsg.): Diskurs und Ökonomie. Wiesbaden : VS Verlag für Sozialwissenschaften, 2009 (Interdisziplinäre Diskursforschung). - ISBN 9783531156224, S. 6384

[Davies 2017] DaviEs, William: The Limits of Neoliberalism: Authority, Sovereignty and the Logic of Competition. Rev. ed. Los Angeles : SAGE, 2017 (Theory, Culture \& Society). - ISBN 9781526403520

[DeSalvo 1976] DeSalvo, Joseph S.: Housing subsidies: Do we know what we are doing? In: Policy analysis : publ. quarterly for the Graduate School of Public Policy, University of California 2 (1976), Nr. 1 , S. $39-60$

[Deutsche Bundesbank 1996] Deutsche Bundesbank: Zur Entwicklung der gegenwärtigen Lage der bauwirtschaft in Deutschland. In: Deutsche Bundesbank (Hrsg.): Monatsbericht April 1996. Frankfurt am Main, 1996, S. 19-33

[Deutsche Bundesbank 2003a] Deutsche Bundesbank: Monatsbericht Mai 2003. Frankfurt am Main, 2003 
[Deutsche Bundesbank 2003b] Deutsche Bundesbank: Neue Preisindices für Wohnimmobilien in Deutschland. In: Monatsbericht Mai 2003. Frankfurt am Main, 2003, S. 39

[Deutsche Bundesbank 2003c] Deutsche Bundesbank: Preisindikatoren für den Wohnungsmarkt. In: Deutsche Bundesbank (Hrsg.): Monatsbericht September. Frankfurt am Main, 2003, S. 45-59

[Deutsche Bundesbank 2005] Deutsche Bundesbank: Finanzstabilitätsbericht 2005. Frankfurt am Main, 2005

[Deutsche Bundesbank 2006] Deutsche Bundesbank: Finanzstabilitätsbericht 2006. Frankfurt am Main, 2006

[Deutsche Bundesbank 2007] Deutsche Bundesbank: Finanzstabilitätsbericht 200\%. Frankfurt am Main, 2007

[Deutsche Bundesbank 2008] Deutsche Bundesbank: Immobilienpreise im Jahr 2007. In: Deutsche Bundesbank (Hrsg.): Monatsbericht Februar 2008. Frankfurt am Main, 2008, S. 50-51

[Deutsche Bundesbank 2016a] Deutsche Bundesbank: Publikationen der Deutschen Bundesbank: Berichte. https: //www.bundesbank.de/Navigation/DE/Publikationen/ Berichte/Monatsberichte/monatsberichte.html. Version: 2016

[Deutsche Bundesbank 2016b] Deutsche Bundesbank: $P u$ blikationen der Deutschen Bundesbank: Berichte. https:// www . bundesbank. de/Navigation/DE/Publikationen/Berichte/ Finanzstabilitaetsberichte/f inanzstabilitaetsberichte. html. Version: 7, 2016

[Deutsche Bundesbank 2018] Deutsche Bundesbank: Das Forschungszentrum der Deutschen Bundesbank. https://www . bundesbank.de/de/bundesbank/forschung/forschungszentrum. Version: 2018

[Deutscher Bundestag 2018] Deutscher Bundestag: Aktuelle Fassung der öffentlichen Liste über die Registrierung von Verbänden 
und deren Vertretern. https://www.bundestag.de/blob/189476/ 7e407b7649ece88d629dffa5b239a7fe/lobbylisteaktuell-data. pdf. Version: 2018

[Deutsches Institut für Wirtschaftsforschung 2016] DeutSches Institut FÜR Wirtschaftsforschung: DIW Wochenbericht. http://www.diw.de/de/diw_01.c.100404. de/publikationen_veranstaltungen/publikationen/ wochenbericht/wochenbericht.html. Version: 2016

[Dobbin u.a. 2007] Dobbin, Frank ; Simmons, Beth ; Garrett, Geoffrey: The global diffusion of public policies: Social construction, coercion, competition, or learning? In: Annual Review of Sociology (2007), S. 449-472. - ISSN 0360-0572

[Döring u. Bortz 2016] Döring, Nicola ; BorTz, Jürgen: Forschungsmethoden und Evaluation in den Sozial- und Humanwissenschaften. 5. vollst. überarb., aktualisierte und erw. Aufl. Berlin : Springer Berlin Heidelberg, 2016 (Springer-Lehrbuch). - ISBN 9783642410888

[Dreitzel 1966] Dreitzel, Doris: Die Bundesregierung und ihre Wissenschaftler. In: atomzeitalter. Information und Meinung 10 (1966), S. $295-299$

[Drevermann u. Nordalm 1986] Drevermann, Marlis ; Nordalm, Volker ; Bundesminister für Raumordnung, Bauwesen und StÄDTEBAU (Hrsg.): Zahlungsschwierigkeiten von Wohneigentümern: Ergebnisbericht des Forschungsprojektes "Ansatzpunkte für die Behebung von zahlungsschwierigkeiten von Wohneigentümern". 1986 (Schriftenreihe "Wohnungsmarkt und Wohnungspolitik" des Bundesministers für Raumordnung, Bauwesen und Städtebau)

[Dringenberg 1983] Dringenberg, Rainer: Tendenzen der Wohnungspolitik in den 80er- Jahren. In: Sozialer Fortschritt (1983), Nr. 7, S. $152-158$

[Ebbinghaus 2009] EbBinghaus, Bernhard: Vergleichende Politische Soziologie: Quantitative Analyse- oder qualitative Fallstudiendesi- 
gns? In: Kaina, Viktoria (Hrsg.) ; Römmele, Andrea (Hrsg.): Politische Soziologie. Wiesbaden : VS Verlag für Sozialwissenschaften, 2009 (Lehrbuch). - ISBN 9783531150499, S. 481-501

[Ebner 2014] EBner, Alexander: Vermarktlichung, Finanzialisierung und das Austeritätsparadigma der europäischen Krisenbewältigung: Eine Polyanische Perspektive. In: Heires, Marcel (Hrsg.) ; Nölke, Andreas (Hrsg.): Politische Ökonomie der Finanzialisierung. Wiesbaden : Springer-Verlag, 2014 (Globale Politische Ökonomie). - ISBN 9783658037772, S. 49-61

[Eekhoff 1975] EeKHoff, Johann ; INSTITUT FÜr EMPIRISCHE WIRTSCHAFTSFORSCHUNG AN DER UnIVERSitÄT DES SAAVRlandeS (Hrsg.): Erfolgskontrolle in der Stadtentwicklung. Saarbrücken, 1975 (Arbeitspapiere)

[Eekhoff 1981] EekHoff, Johann: Wohnungspolitik in der Sozialen Marktwirtschaft. In: Issing, Otman (Hrsg.): Zukunftsprobleme der Sozialen Marktwirtschaft: Verhandlungen dauf der jahrestagung der Gesellschaft für Wirtschafts- und Sozialwissenschaften - Verein für Socialpolitik - in Nürnberg vom 15.-17. September 1980. Berlin : Duncker \& Humblot, 1981. - ISBN 3428048733, S. 455-479

[Eekhoff u.a. 1985] Eekhoff, Johann ; Paul, Theodor ; PreissLER, Egon K.-H. ; SAllandT, Peter: Materialien-Sammlung für Wohnungs- und Siedlingswesen und zur Raumplanung. Bd. 28: Rückzug des Staates aus der Wohnungspolitik? Münster : Selbstverlag des Instituts für Siedlungs-und Wohnungswesen und des Zentralinstituts für Raumplanung der Universität Münster, 1985. - ISBN 3884970534

[Eekhoff u.a. 1979] Eekhoff, Johann ; Sievert, Olaf ; Werth, G. ; Bundesminister für Raumordnung, Bauwesen und StÄdteBAU (Hrsg.): Bewertung wohnungspolitischer Strategien: Modernisierungsförderung versus Neubauförderung ; Forschungsprojekt BMBau RS II 4 - 704102 - 239 (1979). 1979 (BRD / Bundesminister für Raumordnung, Bauwesen und Städtebau : Schriftenreihe des Bundesministers für Raumordnung, Bauwesen und Städtebau) 
[Egner 2004a] EgneR, Björn: Akteursnetzwerk im Politikfeld Wohnen. In: Egner, Björn (Hrsg.): Wohnungspolitik in Deutschland. Darmstadt : Schader-Stiftung, 2004. - ISBN 9783932736124, S. 205-243

[Egner 2004b] EgneR, Björn (Hrsg.): Wohnungspolitik in Deutschland: Positionen, Akteure, Instrumente. Darmstadt : Schader-Stiftung, 2004. - ISBN 9783932736124

[Egner 2014] Egner, Björn: Wohnungspolitik seit 1945. In: Aus Politik und Zeitgeschichte 64 (2014), Nr. 20-21, 13-19. http://www . bpb.de/ apuz/183442/wohnungspolitik-seit-1945?p=all

[Egner u. Georgakis 2004] Egner, Björn ; Georgakis, Nikolaos: Die wichtigsten Akteure im Politikfeld Wohnen. In: Egner, Björn (Hrsg.): Wohnungspolitik in Deutschland. Darmstadt : SchaderStiftung, 2004. - ISBN 9783932736124, S. 133-203

[Egner u.a. 2004] Egner, Björn ; Georgakis, Nikolaos ; Heinelt, Hubert: Handlungsleitende Orientierungen wohnungspolitischer Akteure. In: Egner, Björn (Hrsg.): Wohnungspolitik in Deutschland. Darmstadt : Schader-Stiftung, 2004. - ISBN 9783932736124, S. 245259

[Ehrenberg 1971] Ehrenberg, Herbert: Vermögenspolitik für die siebziger Jahre. Stuttgart and Berlin and Köln : W. Kohlhammer, 1971

[Eichener u. a. 2000] Eichener, Volker (Hrsg.) ; VAn Emmerich, Horst (Hrsg.) ; Petzina, Dietmar (Hrsg.): Die unternehmerische Wohnungswirtschaft: Emanzipation einer Branche : der Strukturwandel der deutschen Wohnungswirtschaft seit dem ausgehenden 19. Jahrhundert. Frankfurt am Main : F. Knapp, 2000. - ISBN 3781906434

[Eigenheimerverband Deutschland e.V 2016] Eigenheimerverband Deutschland e.V ; Eigenheimerverband Deutschland e.V (Hrsg.): Geschichte der Wohneigentumsförderung. http://www . bdse-ev.de/historie/geschichte_foerderung. Version: 2016

[Epstein 2005a] Epstein, Gerald A. (Hrsg.): Financialization and the world economy. Edward Elgar Publishing, 2005. - ISBN 1781008264 
[Epstein 2005b] Epstein, Gerald A.: Introduction. In: Epstein, Gerald A. (Hrsg.): Financialization and the world economy. Edward Elgar Publishing, 2005. - ISBN 1781008264, S. 3-17

[Esping-Andersen 1990] Esping-Andersen, Gøsta: The three worlds of welfare capitalism. Cambridge : Polity Press, 1990. - ISBN 07456-0796-9

[Esping-Andersen 1998] Esping-Andersen, Gøsta: Die drei Welten des Wohlfahrtskapitalismus: Zur politischen Ökonomie des Wohlfahrtsstaates. In: Lessenich, Stephan (Hrsg.) ; Ostner, Ilona (Hrsg.): Welten des Wohlfahrtskapitalismus Bd. 40. Frankfurt/Main : Campus, 1998. - ISBN 3593359669, S. 19-58

[Evans u. a. 1985a] Evans, Peter B. (Hrsg.) ; Rueschemeyer, Dietrich (Hrsg.) ; Skocpol, Theda (Hrsg.): Bringing the State back in. Cambridge : Cambridge University Press, 1985. - ISBN 9780521313131

[Evans u. a. 1985b] Evans, Peter B. ; Rueschemeyer, Dietrich ; SkocPOL, Theda: On the Road toward a More Adequate Understanding of the State. In: Evans, Peter B. (Hrsg.) ; Rueschemeyer, Dietrich (Hrsg.) ; Skocpol, Theda (Hrsg.): Bringing the State back in. Cambridge : Cambridge University Press, 1985. - ISBN 9780521313131, S. $347-366$

[Falk u.a. 2006] Falk, Svenja ; Rehfeld, Dieter ; Römmele, Andrea ; Thunert, Martin: Einführung: Politikberatung - Themen, Fragestellungen, Begriffsdimensionen, Konzepte, Akteure, Institutionen und Politikfelder. In: FAlK, Svenja (Hrsg.) ; Rehfeld, Dieter (Hrsg.) ; Römmele, Andrea (Hrsg.) ; Thunert, Martin (Hrsg.): Handbuch Politikberatung. Wiesbaden : VS Verlag für Sozialwissenschaften, 2006. - ISBN 9783531142500, S. 11-22

[Faller u.a. 2001] Faller, Bernhard ; Braun, Reiner ; Heyn, Timo ; PfEIfFer, Ulrich: Hemmnisse der Wohneigentumsbildung. In: $B B R$ Reihe Forschungen 106 (2001) 
[Faller u.a. 2006] Faller, Bernhard ; Heising, Petra ; DüBel, Achim ; Bundesamt für Bauwesen und Raumordnung (Hrsg.): Internationaler Vergleich von Kosten und Dienstleistungseffizienz bei der Transaktion von Wohneigentum: Optionen für Deutschland: Ein Projekt des Forschungsprogramms "Allgemeine Ressortforschung" des Bundesministeriums für Verkehr, Bau und Stadtentwicklung (BMVBS) und des Bundesamtes für Bauwesen und Raumordnung (BBR). Bonn, 2006 (Forschungen)

[Feige 1979] FeIGE, Ulrich M.: Sozialpolitik: Netz mit Fehlern. In: Keller, Horst (Hrsg.): Das Jahrzehnt der Utopisten Bd. 70 : Bonner Texte. Bonn : Verlag Bonn Aktuell, 1979. - ISBN 3879591199, S. $47-54$

[Fey 1936] FEY, Walter ; FeY, Walter (Hrsg.): Leistungen und Aufgaben im Deutschen Wohnungs-und Siedlungsbau. 1936 (Sonderhefte des Instituts für Konjunkturforschung)

[Fey 1951] FEY, Walter: Der Wohnungsbau in der Bundersrepublik Deutschland: Zwischenbilanz und Vorschau: Im Auftrag des Bundesministers für Wohnungsbau. Domus-Verlag, 1951

[Fey 1964] Fey, Walter: Diskussionsbeitrag zum Referat "Die Auswirkungen der Wohnungszwangswirtschaft auf die künftigen Aufgaben der Wohnungsbaufinanzierung". In: SchneIdeR, Hans K. (Hrsg.): Der Abbau der Wohnungszwangswirtschaft. Köln : Verlagsgesellschaft Rudolf Müller, 1964 (Materialien-Sammlung für Wohnungsund Siedlingswesen), S. 84-86

[Fischer 1990] Fischer, Frank: Technocracy and the Politics of Expertise. SAGE, 1990. - ISBN 0803933797

[Forrest u. Hirayama 2014] Forrest, Ray ; Hirayama, Yosuke: The financialisation of the social project: Embedded liberalism, neoliberalism and home ownership. In: Urban Studies 52 (2014), Nr. 2, S. 233-244. http://dx.doi.org/10.1177/0042098014528394. - DOI 10.1177/0042098014528394. - ISSN 0042-0980 
[Forsthoff 1971] FonsthofF, Ernst: Der Staat der Industriegesellschaft: Dargestellt am Beispiel der Bundesrepublik Deutschland. München : Beck, 1971

[Fourcade 2006] Fourcade, Marion: The Construction of a Global Profession: The Transnationalization of Economics. In: American Journal of Sociology 112 (2006), Nr. 1, S. 145-194

[Fourcade 2009] Fourcade, Marion: Economists and Societies: Discipline and profession in the United States, Britain, and France, $1890 \mathrm{~s}$ to 1990s. Princeton : Princeton University Press, 2009 (Princeton studies in cultural sociology). - ISBN 9780691117607

[Frenzel 2002] Frenzel, Martin: Neue Wege der Sozialdemokratie: Dänemark und Deutschland im Vergleich (1982-2002). Wiesbaden : Deutscher Universitäts-Verlag and Imprint, 2002. - ISBN 3663108910

[Frey u. Kirchgässner 2002] Frey, Bruno S. ; Kirchgässner, Gebhard: Demokratische Wirtschaftspolitik: Theorie und Anwendung. 3. München : Vahlen, 2002 (Vahlens Handbücher der Wirtschafts- und Sozialwissenschaften). - ISBN 3-8006-2737-X

[Fricke u. Siewert 2015] Fricke, Carola ; Siewert, Markus B.: Bürokratie und öffentliche Verwaltung: Aufbau - Internationaler Vergleich - New Public Management. In: Wagschal, Uwe (Hrsg.) ; JÄckle, Sebastian (Hrsg.) ; Wenzelburger, Georg (Hrsg.): Einführung in die Vergleichende Politikwissenschaft. Stuttgart : Kohlhammer, 2015. - ISBN 9783170289642, S. 237-250

[Friedman 1953] Friedman, Milton: Essays in positive economics. Chicago : University of Chicago Press, 1953. - ISBN 978-0226264035

[Friedrich 1970] FrIEDRICH, Hannes: Staatliche Verwaltung und Wissenschaft. Frankfurt am Main : Europäische Verlagsanstalt, 1970

[Führer 1997] FÜHrER, Karl C.: Anspruch und realität. Das Scheitern der nationalsozialistischen Wohnungsbaupolitik 1933-1945. In: Vierteljahrshefte für Zeitgeschichte 45 (1997), Nr. 2, S. 225-256. - ISSN 0042-5702 
[Führer 2015] FüHRER, Karl C.: Studien zur Zeitgeschichte. Bd. Bd. 89: Die Stadt, das Geld und der Markt: Immobilienspekulation in der Bundesrepublik 1960-1985. Berlin : De Gruyter Oldenbourg, 2015. ISBN 3110415410

[Gavrillis u. Fröhndrich 2018] Gavrillis, Panajotis ; Fröhndrich, Sina: Diskussion um das Baukindergeld. https://ww. deutschlandfunk.de/ foerderung-von-wohneigentum-diskussion-um-das-baukindergeld . 724.de.html?dram:article_id=424721. Version: 2018

[Georgakis 2004] Georgakis, Nikolaos: Eigenheimzulage. In: EGNER, Björn (Hrsg.): Wohnungspolitik in Deutschland. Darmstadt : Schader-Stiftung, 2004. - ISBN 9783932736124, S. 93-105

[Gesamtverband der Wohnungswirtschaft 1992] Gesamtverband DeR Wohnungswirtschaft (Hrsg.): Wohneigentum in Deutschland: Geschichte, Bedeutung, Perspektiven. 1. Wissen : Reiner Winters, 1992

[Gesellschaft für Immobilienwirtschaftliche Forschung e.V. 2012] Gesellschaft FÜr Immobilienwirtschaftliche Forschung E.V.: Geschichte. https://www.gif-ev.de/cms.172/show/ verein\#cms-104. Version: 2012

[Girouard u.a. 2006] Girouard, Nathalie ; van Den Noord, Paul ; Kennedy, Mike ; André, Christophe ; Organisation for Economic Co-operation and Development (Hrsg.): Recent House Price Developments: The Role of Fundamentals. http://dx.doi. org/10.1787/864035447847. Version: 2006 (OECD Economics Department Working Papers)

[Glatter 2007] Glatter, Jan: Die Privatisierung der kommunalen Wohnungsbestände in Dresden. In: Raumplanung 131 (2007), S. 7984

[Glatzer 1980] Glatzer, Wolfgang: Schriftenreihe. Bd. 1: Wohnungsversorgung im Wohlfahrtsstaat: Objektive und subjektive Indikato- 
ren zur Wohlfahrtsentwicklung in der Bundesrepublik Deutschland. Frankfurt/Main : Campus, 1980. - ISBN 3593326213

[de Goede 2004] Goede, Marieke de: Repoliticizing financial risk. In: Economy and Society 33 (2004), Nr. 2, S. 197-217. - ISSN 0308-5147 [Goldstein u. Keohane 1993] Goldstein, Judith S. (Hrsg.) ; Keohane, Robert O. (Hrsg.): Ideas and foreign policy: Beliefs, institutions, and political change. 1. print. Ithaca : Cornell University Press, 1993 (Cornell studies in political economy). - ISBN 0801429315

[Gormley 2007] Gormley, William T.: Public policy analysis: Ideas and impacts. In: Annual Review of Political Science (2007), S. 297313

[Groot 2008] Groot, Loek: Redesigning the Welfare State: Germany's Current Agenda for an Activating Social Assistance. In: Journal of Income Distribution 17 (2008), Nr. 3-4, S. 115-118

[Groser 1979] Groser, Manfred: Forschungsbericht / KonradAdenauer-Stiftung. Bd. 2: Die neue soziale Frage: Theoretische, programmatische und ordnungspolitische Grundlagen : empirische Befunde. Melle : Knoth, 1979. - ISBN 3-88368-014-1

[Grundmann u. Stehr 2011] Grundmann, Reiner ; Stehr, Nico: Suhrkamp Taschenbuch Wissenschaft. Bd. 1990: Die Macht der Erkenntnis. 1., Auflage. Berlin : Suhrkamp, 2011. - ISBN 9783518295908

[Haas 1992] HaAs, Peter M.: Introduction Epistemic communities and international policy coordination. In: International Organization (1992), S. 1-36

[Haas 1993] HaAs, Peter M.: Stratospheric Ozone: Regime Formation in Stages. In: Young, Oran R. (Hrsg.) ; Osherenko, Gail (Hrsg.): Polar politics. Ithaca : Cornell University Press, 1993 (Cornell studies in political economy). - ISBN 0801480698, S. 152-185

[Habermas 1968] Habermas, Jürgen: Verwissenschaftlichte Politik und öffentliche Meinung. In: Habermas, Jürgen (Hrsg.): Technik und 
Wissenschaft als "Ideologie" Bd. 287. Frankfurt am Main : Suhrkamp, 1968, S. 120-145

[Habermas 1990] Habermas, Jürgen: Strukturwandel der Öffentlichkeit: Untersuchungen $z u$ einer Kategorie der bürgerlichen Gesellschaft. 1. Frankfurt am Main : Suhrkamp, 1990 https: //soth-1alexanderstreet-1com-1cvym6akn0bb3. han.sub.uni-goettingen.de/cgi-bin/asp/philo/soth/ documentidx.pl?sourceid=S10023116

[Hall 1989] Hall, Peter A. (Hrsg.): The political power of economic ideas: Keynesianism across nations. Princeton : Princeton University Press, 1989. - ISBN 0-691-07799-1

[Hall 1993] Hall, Peter A.: Policy paradigms, social learning, and the state: The case of economic policymaking in Britain. In: Comparative Politics (1993), S. 275-296

[Hall 2006] Hall, Peter A.: Stabilität und Wandel in den Spielarten des Kapitalismus. In: Beckert, Jens (Hrsg.) ; EbBinghaus, Bernhard (Hrsg.) ; Hassel, Anke (Hrsg.) ; Manow, Philip (Hrsg.): Transformationen des Kapitalismus Bd. Band 57. Frankfurt : Campus, 2006. - ISBN 9783593382814, S. 181-204

[Hall u. Taylor 1996] Hall, Peter A. ; Taylor, Rosemary C. R.: Political science and the three new institutionalisms. Köln : MPIfG, 1996 (MPIfG Discussion Paper)

[Hall u. Thelen 2009] Hall, Peter A. ; Thelen, Kathleen A.: Institutional change in varieties of capitalism. In: Socio-Economic Review 7 (2009), Nr. 7, S. $7-34$

[Hamann 2012] Hamann, Julian: Der geisteswissenschaftliche Bildungsdiskurs der preußischen Universitätsreform. Versuch einer wissenssoziologischen Feld- und Diskursanalyse. Version: 2012. http:// dx.doi .org/10.1007/978-3-531-94259-9\{_\}13. In: BERNHARD, 
Stefan (Hrsg.) ; SchmidT-Wellenburg, Christian (Hrsg.): Feldanalyse als Forschungsprogramm 1. Wiesbaden : VS Verlag für Sozialwissenschaften, 2012. - DOI 10.1007/978-3-531-94259-9_13. - ISBN 978-3-531-17871-4, S. 345-379

[Hämmerlein 1996] HäMmeRLEIN, Hans: Einführung in die Wohnungswirtchaft: Ein Leitfaden in Thesen und Übersichten. 1. Aufl. BadenBaden : Nomos, 1996. - ISBN 3789043532

[Hanke 1994] Hanke, Hans H.: Eigenheime - bewohnte Bollwerke der Demokratie: Schweizer Pestalozzi-Dörfer für Berglehrlinge und amerikanische MSA-Bergabeitersiedlungen als Beispiele für die Neuordnung der westdeutschen Wohnkultur. In: Clemens, Gabriele (Hrsg.): Kulturpolitik im besetzten Deutschland 1945 - 1949. Stuttgart : Steiner, 1994 (Historische Mitteilungen). - ISBN 3-515-06324-2, S. 9-54

[Harlander 2005] Harlander, Tilman: Wohnungspolitik. In: Schmidt, Manfred G. (Hrsg.): Geschichte der Sozialpolitik in Deutschland seit 1945. Baden-Baden : Nomos, 2005. - ISBN 3789073253, S. 685-712

[Harlander 2008] Harlander, Tilman: Wohnungspolitik. In: Geyer, Martin H. (Hrsg.) ; Bundesministerium FÜr Arbeit und SoZIAles (Hrsg.) ; Bundesarchiv (Hrsg.): 1974-1982 Bundesrepublik Deutschland Bd. 6. [S.1.] : Nomos, 2008. - ISBN 9783789073236, S. $825-850$

[Harvey 2007a] HaRvey, David: Kleine Geschichte des Neoliberalismus. Zürich : Rotpunktverl., 2007. - ISBN 9783858693433

[Harvey 2007b] HaRVEY, David: Räume der Neoliberalisierung: Zur Theorie der ungleichen Entwicklung. Hamburg : VSA, 2007. - ISBN 978-3-89965-230-7

[Hassel u. Trampusch 2006] Hassel, Anke ; Trampusch, Christine: Verbände und Parteien: Die Dynamik von Parteikonflikten und die Erosion des Korporatismus. In: Beckert, Jens (Hrsg.) ; EBbingHaus, Bernhard (Hrsg.) ; Hassel, Anke (Hrsg.) ; Manow, Philip 
(Hrsg.): Transformationen des Kapitalismus Bd. Band 57. Frankfurt : Campus, 2006. - ISBN 9783593382814, S. 111-132

[Häußermann u. Siebel 1981] Häussermann, Hartmut ; Siebel, Walter: Unpolitische Wohnungspolitik? In: Leviathan 9 (1981), Nr. 3/4, S. $317-331$

[Heclo 1975] Heclo, Hugh: Modern social politics in Britain and Sweden: From relief to income maintenance. Cop. 1974, 2nd print. New Haven, Conn. <etc.> : Yale University Press, 1975. - ISBN 0300014805

[Heeg 2013] Heeg, Susanne: Wohnungen als Finanzanlage: Auswirkungen von Responsibilisierung und Finanzialisierung im Bereich des Wohnens. In: sub\urban. zeitschrift für kritische stadtforschung 1 (2013), Nr. 1, 75-99. http://nbn-resolving.de/urn:nbn:de:101: 1-201308249524. - ISSN 2197-2567

[Heinelt 2004] Heinelt, Hubert: Rahmenbedingungen, Politikinhalte und Politikprozesse. In: EGneR, Björn (Hrsg.): Wohnungspolitik in Deutschland. Darmstadt : Schader-Stiftung, 2004. - ISBN 9783932736124, S. $35-48$

[Heinelt u. Egner 2006] Heinelt, Hubert ; Egner, Björn: Wohnungspolitik - von der Wohnraumzwangsbewirtschaftung zur Wohnungsmarktpolitik. In: Schmidt, Manfred G. (Hrsg.) ; Zohlnhöfer, Reimut (Hrsg.): Regieren in der Bundesrepublik Deutschland. Wiesbaden : VS Verlag für Sozialwissenschaften, 2006. - ISBN 3531143441, S. 203-220

[Heires u. Nölke 2014] Heires, Marcel (Hrsg.) ; Nölke, Andreas (Hrsg.): Politische Ökonomie der Finanzialisierung. Wiesbaden : Springer-Verlag, 2014 (Globale Politische Ökonomie). - ISBN 9783658037772

[Heisterkamp 2017] Heisterkamp, Ulich: Think Tanks der Parteien? Eine vergleichende Analyse der deutschen politischen Stiftungen. 2., 
aktualisierte Auflage. Wiesbaden and Erscheinungsort nicht ermittelbar : Springer Fachmedien Wiesbaden, 2017 (Research). - ISBN 978-3-658-18521-3

[Hemmelmann 2017] Hemmelmann, Petra: Der Kompass der CDU: Analyse der Grundsatz- und Wahlprogramme von Adenauer bis Merkel. Wiesbaden : Springer-Verlag, 2017 (Research). - ISBN 978-3658-18114-7

[Hesse 2010] Hesse, Jan-Otmar: Campus Forschung. Bd. 947: Wirtschaft als Wissenschaft: Die Volkswirtschaftslehre in der frühen Bundesrepublik. Frankfurt am Main : Campus, 2010. - ISBN 9783593393155

[Hesse u. Preckwinkel 2009] Hesse, Markus ; Preckwinkel, Wiebke: Globalisierung der Wohnungswirtschaft. In: Zeitschrift für Wirtschaftsgeographie 53 (2009), Nr. 1-2, S. 156-171. - ISSN 2365-7693

[Hilpert 2012] HilperT, Dagmar: Wohlfahrtsstaat der Mittelschichten? Sozialpolitik und gesellschaftlicher Wandel in der Bundesrepublik Deutschland (1949-1975). Göttingen : Vandenhoeck \& Ruprecht, 2012. - ISBN 9783525370278

[Hirschman u. Berman 2014] Hirschman, Daniel ; Berman, Elizabeth P.: Do economists make policies? On the political effects of economics. In: Socio-Economic Review 12 (2014), Nr. 4, S. 779-811

[Hoesli u. MacGregor 1997] Hoesli, Martin ; MacGregor, Bryan: European Real Estate Research and Education: Development, Globalization, and Maturity. In: The Journal of Real Estate Finance and Economics 15 (1997), Nr. 1, 5-9. http://dx.doi.org/10.1023/A\% 3A1007722020421. - ISSN 0895-5638

[Hoffmann u. Lorenz 2006] Hoffmann, Johannes ; Lorenz, Andreas: Real Estate Price Indices for Germany: Past, Present and Future. Paper for the OECD-IMF Workshop 7. 2006 
[Höhnen 1968] HöHnEn, Wilfried: Die vermögensploitischen Gesetze und Massnahmen in der Bundesrepublick Deutschland; Entwicklung, Ergebnisse und kritische Beurteilung. Köln : Bund-Verlag, 1968

[Hölder u. Ehling 1991] Hölder, Egon ; Ehling, Manfred: Zur Entwicklung der amtlichen Statistik in Deutschland. In: Fischer, Wolfram (Hrsg.) ; KunZ, Andreas (Hrsg.): Grundlagen der Historischen Statistik von Deutschland. VS Verlag für Sozialwissenschaften, 1991. - ISBN 3531122460, S. 15-31

[Holm 2010] Holm, Andrej: Privare heisst rauben-Zur Ökonomie von Wohnungsprivatisierungen. In: Z-Zeitschrift marxistische Erneurung 21 (2010), Nr. 83, S. 46-59

[Holm 2011] Holm, Andrej: Wohnung als Ware: zur Ökonomie und Politik der Wohnungsversorgung. In: Widersprüche: Zeitschrift für sozialistische Politik im Bildungs-, Gesundheits-und Sozialbereich 31 (2011), Nr. 121, S. 9-20. - ISSN 0721-8834

[Hopf 2013] Hopf, Christel: Qualitative Interviews: Ein Überblick. In: Flick, Uwe (Hrsg.): Qualitative Forschung Bd. 55628. Reinbek bei Hamburg : Rowohlt, 2013. - ISBN 9783499556289, S. 349-360

[Housing Europe 2017] Housing Europe: The State of Housing in the EU 2017. Brüssel, 2017

[Hustedt u. Houlberg Salomonsen 2014] Hustedt, Thuris ; Houlberg Salomonsen, Heidi: Politikberatung durch die Ministerialverwaltung: Funktionale Differenzierung oder Integration unterschiedlicher Wissensformen? In: Kropp, Sabine (Hrsg.) ; Kuhlmann, Sabine (Hrsg.): Wissen und Expertise in Politik und Verwaltung Bd. 1. Opladen and Toronto : Verlag Barbara Budrich, 2014. - ISBN 9783847401179, S. 203-221

[Hüther u. a. 2015] Hüther, Michael ; Voigtländer, Michael ; HaAs, Heide ; Deschermeier, Philipp: IW-Analysen. Bd. Nr. 102: The importance of long-term financing by banks: Advantages and future 
challenges. Köln : IW, Institut der Deutschen Wirtschaft Köln Medien GmbH, 2015. - ISBN 9783602149483

[Ifo-Institut für Wirtschaftsforschung 1960] IFO-INSTITUT FÜR WIRTSCHAFTSFORSCHUNG: Wohnungswirtschaft und Marktwirtschaft: Aufgaben der Wohnungsunternehmen: Gutachten des IfO-Institutes für Wirtschaftsforschung, e.V., München, erstattet im Auftrage des Gesamtverbandes Gemeinnütziger Wohnungsunternehmen, e.V. Köln. Hamburg : Harmonia-Verlag GmbH, 1960

[Institut für Städtebau, Wohnungswirtschaft und Bausparwesen 1981] Institut FÜr StÄDTEBAU, Wohnungswirtschaft Und BaUSPARWESEN (Hrsg.): Wohnversorgung und Wohneigentum in den achtziger Jahren: XVI. Königsteiner Gespräch : Referate und Diskussion. Bd. 36. Bonn : Domus-Verlag, 1981 (Schriftenreihe des Instituts für Städtebau, Wohnungswirtschaft und Bausparwesen e.V). - ISBN 3871692786

[Institut für Städtebau, Wohnungswirtschaft und Bausparwesen u. Bundesministerium für Wohnungwesen und Städtebau 1966] INSTITUT FÜr StÄDTEBAu, Wohnungswirtschaft und BausparWesen (Hrsg.) ; Bundesministerium FÜr WohnungWesen und StÄDtebau (Hrsg.): Das Eigenheim in der Etage: Eine Broschüre übe das Wohnungseigentum. Bonn : Domus-Verlag, 1966

[Jaedicke u. Wollmann 1991] Jaedicke, Wolfgang ; Wollmann, Hellmut: Wohnungspolitik und Regierungswechsel. In: Blanke, Bernhard (Hrsg.) ; Wollmann, Hellmut (Hrsg.): Die Alte Bundesrepublik Bd. 12. Opladen : Westdeutscher Verlag, 1991. - ISBN 3531121979, S. $420-436$

[Jäger u. Voigtländer 2006] JÄGER, Manfred ; VoigtlÄnder, Michael: IW-Analysen. Bd. Nr. 22: Immobilienfinanzierung: Hypothekenmärkte und ihre gesamtwirtschaftliche Bedeutung. Köln : Deutscher Instituts Verlag, 2006. - ISBN 3602147444

[Janssen 2012] Janssen, Hauke: Beiträge zur Geschichte der deutschsprachigen Ökonomie. Bd. Band 10: Nationalökonomie und Natio- 
nalsozialismus: Die deutsche Volkswirtschaftslehre in den dreißiger Jahren des 20. Jahrhunderts. 4. Marburg : Metropolis-Verlag, 2012. - ISBN 9783895188756

[Jarass 2018] JARASS, Lorenz: Lebenslauf. http://www.jarass.com/ home/de/lebenslauf. Version: 2018

[Jenkis 1996] Jenkis, Helmut W.: Einführung in die Wohnungswirtschaftspolitik. In: Jenkis, Helmut W. (Hrsg.) ; Dieterich, Hartmut (Hrsg.): Kompendium der Wohnungswirtschaft. München and Wien : Oldenbourg, 1996. - ISBN 3486233009, S. 65-122

[Jokl u. Zehnder 1996] JokL, Stefan ; Zennder, Andreas: Wohneigentumsbildung: Wünsche, Forderungen, Grenzen. In: Jenkis, Helmut W. (Hrsg.) ; Dieterich, Hartmut (Hrsg.): Kompendium der Wohnungswirtschaft. München and Wien : Oldenbourg, 1996. - ISBN 3486233009, S. 392-418

[Jun 2018] Jun, Uwe: Spitzenakteure der Parteien. In: Falk, Svenja (Hrsg.) ; GlaAb, Manuela (Hrsg.) ; Römmele, Andrea (Hrsg.) ; Schober, Henrik (Hrsg.) ; Thunert, Martin (Hrsg.): Handbuch Politikberatung. Wiesbaden : Springer Fachmedien Wiesbaden, 2018. - ISBN 978-3-658-07461-6, S. 1-12

[Kalthoff 2007] Kalthoff, Herbert: Rechnende Organisation: Zur Anthropologie des Risikomanagements. In: BECKERT, Jens (Hrsg.): Märkte als soziale Strukturen. Frankfurt am Main : Campus, 2007. - ISBN 9783593384719, S. 151-165

[Kalthoff u. Köllner 2015] Kalthoff, Herbert ; Köllner, Sonja: Finanzkommunikation als Praxis ökonomischen Darstellens. In: Heintz, Bettina (Hrsg.) ; Tyrell, Hartmann (Hrsg.): Interaktion - Organisation - Gesellschaft revisited Bd. 43, Sonderheft. Stuttgart : Lucius \& Lucius, 2015. - ISBN 9783828206052, S. 390-407

[Kaufmann 1988] Kaufmann, Franz-Xaver: Christentum und Wohlfahrtsstaat. In: Zeitschrift für Sozialreform 34 (1988), Nr. 2 
[Kelstrup 2016] KelstRup, Jesper D.: Routledge research in comparative politics. Bd. 67: The politics of think tanks in Europe. Abingdon, Oxon and New York, NY : Routledge, 2016. - ISBN 1138918326

[Keynes 2008] Keynes, John M.: The general theory of employment, interest, and money. Princeton, N.J. : Recording for the Blind \& Dyslexic, 2008 (Great minds series)

[King 2005] KInG, Michael: Epistemic Communities and the Diffusion of Ideas: Central Bank Reform in the United Kingdom. In: West European Politics 28 (2005), Nr. 1, S. 94-123. http://dx.doi.org/10. 1080/0140238042000297107. - DOI 10.1080/0140238042000297107. - ISSN 0140-2382

[Kirchheimer 1965] Kirchheimer, Otto: Der Wandel des westeuropäischen Parteisystems. In: Politische Vierteljahresschrift 6 (1965), Nr. 1, S. $20-41$

[Kirchner 2006] KIRCHNER, Joachim: Wohnungsversorgung für unterstützungsbedürftige Haushalte: Deutsche Wohnungspolitik im europäischen Vergleich. Wiesbaden : Deutscher Universitäts-Verlag / GWV Fachverlage GmbH, Wiesbaden, 2006 (SpringerLink). - ISBN 3835096532

[Klein 1989] KLEIN, Heribert (Hrsg.): Oswald von Nell-Breuning: Unbeugsam für den Menschen : Lebensbild, Begegnungen, ausgewählte Texte. Freiburg i. Br. and Basel : Herder, 1989. - ISBN 3451214830

[Kleinman 1996] Kleinman, Mark: Housing, welfare, and the state in Europe: a comparative analysis of Britain, France, and Germany. Edward Elgar Publishing, 1996

[Kofner 2004] Kofner, Stefan: Wohnungsmarkt und Wohnungswirtschaft. München : R. Oldenbourg, 2004. - ISBN 3486576054

[Kohl 2015] KoHL, Sebastian: The Power of Institutional Legacies: How Nineteenth Century Housing Associations Shaped Twentieth 
Century Housing Regime Differences between Germany and the United States. In: European Journal of Sociology 56 (2015), Nr. 02, S. 271-306. http://dx.doi.org/10.1017/S0003975615000132. DOI 10.1017/S0003975615000132. - ISSN 0003-9756

[Kohl 2016] KoHL, Sebastian: Urban History Matters: Explaining the German-American Homeownership Gap. In: Housing Studies 31 (2016), Nr. 6, S. 694-713. http://dx.doi.org/10.1080/02673037. 2015.1121213. - DOI 10.1080/02673037.2015.1121213. - ISSN 02673037

[Kohl 2017] KoHL, Sebastian: Homeownership, renting and society: Historical and comparative perspective. 2017 (Routledge advances in sociology). - ISBN 978-1-138-64494-6

[Kornemann 1996] Kornemann, Rolf: Die wohnungspolitischen Zielsetzungen in den Regierungserklärungen. In: JENKIS, Helmut W. (Hrsg.) ; Dieterich, Hartmut (Hrsg.): Kompendium der Wohnungswirtschaft. München and Wien : Oldenbourg, 1996. - ISBN 3486233009, S. 123-143

[Kornemann 2000] Kornemann, Rolf: Paradigmenwechsel: Von der Steueroptimierung zur Objektorientierung. In: Wohnungsnot und Leerstände - die Wohnungsmärkte im neuen Jahrtausend $\mathrm{Bd}$. Bd. 62. Berlin : Domus-Verlag, 2000. - ISBN 3871694789, S. 92-103

[Korpi u. Palme 1998] KorPI, Walter ; PAlme, Joakim: LIS working paper series. $\mathrm{Bd}$. 174: The paradox of redistribution and strategies of equality: Welfare state institutions, inequality and poverty in the Western countries. Walferdange : Luxembourg Income Study, 1998

[Köster u. Mezler 1979] Köster, Jörn ; Mezler, Johannes: Wohneigentumsquote: Bestimmungsgründe der Wohneigentumsquote in den Ländern Belgien, Dänemarkt, Frankreich, Großbritannien, USA, Bundesrepublik Deutschland: Im Auftrag des Bundesministers für Raumordnung, Bauwesen und Städtebau, Bonn, voregegt von der Gesellschaft für Wohnungs- und Siedlungswesen mbH (GEWOS), Hamburg. Bonn, 1979 
[Kramper 2008] Kramper, Peter: VSWG. Vierteljahrschrift für Sozialund Wirtschaftsgeschichte, Beihefte. Bd. 200: Neue Heimat: Unternehmenspolitik und Unternehmensentwicklung im gewerkschaftlichen Wohnungs- und Städtebau 1950-1982. Stuttgart : F. Steiner, 2008. ISBN 9783515092456

[Krasner 1993] Krasner, Stephen D.: Westphalia and All That. In: Goldstein, Judith S. (Hrsg.) ; Keohane, Robert O. (Hrsg.): Ideas and foreign policy. Ithaca : Cornell University Press, 1993 (Cornell studies in political economy). - ISBN 0801429315, S. 235-264

[Krevert 1993] Krevert, Peter: Studien zur Politikwissenschaft Abt. B, Forschungsberichte und Dissertationen. Bd. 79: Funktionswandel der wissenschaftlichen Politikberatung in der Bundesrepublik Deutschland: Entwicklungslinien, Probleme und Perspektiven im Kooperationsfeld von Politik, Wissenschaft und Öffentlichkeit: Univ., Diss.Münster (Westfalen), 1992. Münster : Lit, 1993. - ISBN 3894735813

[Kropp u. Kuhlmann 2014] Kropp, Sabine ; Kuhlmann, Sabine: Wissen und Expertise in Politik und Verwaltung: Eine einleitende Zusammenfassung. In: Kropp, Sabine (Hrsg.) ; Kuhlmann, Sabine (Hrsg.): Wissen und Expertise in Politik und Verwaltung Bd. 1. Opladen and Toronto : Verlag Barbara Budrich, 2014. - ISBN 9783847401179, S. $7-19$

[Krummacher 1978] KRummacher, Michael: Wohnungspolitik und Sozialstaatspostulat in der Bundesrepublik Deutschland: Eine politikwissenschaftliche Analyse des Anspruchs, der Maßnahmen und Wirkungen der staatlichen Wohnungspolitik der BRD. Hannover, Technische Universität, Diss., 1978

[Kuhn 2012] KuHN, Armin: Zwischen gesellschaftlicher Intervention und radikaler Nischenpolitik. Häuserkämpfe in Berlin und Barcelona am Übergang zur neoliberalen Stadt. In: Hanno Balz/JanHenrik Friedrichs (Hg.), "All we ever wanted..." Eine Kulturgeschichte europä ischer Protestbewegungen der 1980er Jahre. Berlin: Karl Dietz Verlag 37 (2012), S. 52 
xxxii

[Kuhnle 1996] Kuhnle, Stein: International Modeling, States and Statistics: Scandinavian Sociel Security Solutions in the 1890s. In: RuESchemeyer, Dietrich (Hrsg.) ; Skocpol, Theda (Hrsg.): States, social knowledge, and the origins of modern social policies. Princeton N.J and New York : Princeton University Press and Russell Sage Foundation, 1996 (Princeton paperbacks). - ISBN 9780691001128, S. $233-263$

[Kunz 2003] KunZ, Andreas (Hrsg.): Die Akte Neue Heimat: Krise und Abwicklung des größten Wohnungsbaukonzerns Europas 1982-1998. Bd. 1. Frankfurt [u.a.] : Campus, 2003. - ISBN 3593371642

[Kusche 2008] Kusche, Isabel: Politikberatung und die Herstellung von Entscheidungssicherheit im politischen System. 1. Aufl. Wiesbaden : VS Verlag für Sozialwissenschaften, 2008 (SpringerLink). - ISBN 9783531160481

[Kutz 1997] Kutz, Karl-Heinz: Ehrung für Professor Dr. Willi Albers. https://idw-online.de/de/news2961. Version: 1997

[Lafontaine 1989] Lafontaine, Oskar: Mitgestalter des Sozialstaates. In: KLeIN, Heribert (Hrsg.): Oswald von Nell-Breuning. Freiburg i. Br. and Basel : Herder, 1989. - ISBN 3451214830, S. 80-83

[Lammerskitten 2004] Lammerskitten, Peter: Immobilien in der Riester-Rente. In: Michels, Winfried (Hrsg.): Wohneigentum und Altersvorsorge Bd. 14. Münster : Institut für Siedlungs- und Wohnungswesen, 2004. - ISBN 3884971972, S. 16-27

[Lampert 1994] LamperT, Heinz: Wohnungspolitik. Version: 1994. http://dx.doi.org/10.1007/978-3-662-08340-6\{_\}12. In: LAMPERT, Heinz (Hrsg.): Lehrbuch der Sozialpolitik. Berlin, Heidelberg : Springer Berlin Heidelberg, 1994. - DOI 10.1007/9783-662-08340-6_12. - ISBN 978-3-662-08340-6, S. 306-319

[Langenohl 2009] Langenohl, Andreas: Finanzmarktöffentlichkeiten: Die funktionale Beziehung zwischen Finanzmarkt und öffentlichem Diskurs. In: Diaz-Bone, Rainer (Hrsg.): Diskurs und Ökonomie. 
Wiesbaden : VS Verlag für Sozialwissenschaften, 2009 (Interdisziplinäre Diskursforschung). - ISBN 9783531156224, S. 245-266

[Lasswell u. Kaplan 1968] Lasswell, Harold D. ; Kaplan, Abraham: Power and society: A framework for political inquiry. 7. New Haven : Yale University Press, 1968

[Lasswell 1956] Lasswell, Harold H.: The political science of science: an inquiry into the possible reconciliation of mastery and freedom. In: American Political Science Review 50 (1956), Nr. 4, S. 961-979

[Lauritzen 1972] Lauritzen, Lauritz ; Bundesministerium FÜR Städtebau und Wohnungswesen (Hrsg.): Das Wohnen in der Bundesrepublik. Bonn, 1972

[Lee u. Strang 2006] LeE, Chang K. ; StRAng, David: The international diffusion of public-sector downsizing: Network emulation and theorydriven learning. In: International Organization (2006), S. 883-909

[Lehmbruch 1977] LeHmBRuch, Gerhard: Liberal corporatism and party government. In: Comparative Political Studies 10 (1977), Nr. 1, S. 91-126. - ISSN 0010-4140

[Lehmbruch 1979] Lenmbruch, Gerhard: Parteiensystem und Interessenverbände in der Politikentwicklung. In: Matthes, Joachim (Hrsg.): Sozialer Wandel in Westeuropa. Frankfurt : Campus, 1979. - ISBN 3593326205, S. 591-610

[Lehmbruch 1986] Lehmbruch, Gerhard: Grenzen des Parteienwettbewerbs: Parteien im Netzwerk der Politikverflechtung. In: Krockow, Christian v. (Hrsg.): Parteien in der Krise Bd. 313. München : Beck, 1986. - ISBN 340631595X, S. 105-114

[Lehnert 1984] LehnerT, Detlef: Die sozial-liberale Koalition: Vom "historischen Bündnis" zum wahltaktischen Bruch? In: Glaessner, Gert-Joachim (Hrsg.) ; Holz, Jürgen (Hrsg.) ; Schlüter, Thomas (Hrsg.): Die Bundesrepublik in den siebziger Jahren. VS Verlag für Sozialwissenschaften, 1984. - ISBN 3810004588, S. 15-31 
[Leidner 1981] LEIDNER, Rüdiger M.: Schriften des Instituts für Wohnungsrecht und Wohnungswirtschaft an der Universität Köln. Bd. 49: Wohnungspolitik und Wohnungsmarktwirtschaft: Gegensatz oder Ergänzung? Frankfurt am Main : Fritz Knapp Verlag, 1981

[Lengwiler 2016] LengwiLeR, Martin: Kontinuitäten und Umbruche in der deutschen Wissenschaftspolitik (1900-1990). In: Simon, Dagmar (Hrsg.) ; Knie, Andreas (Hrsg.) ; Hornbostel, Stefan (Hrsg.) ; Zimmermann, Karin (Hrsg.): Handbuch Wissenschaftspolitik. Wiesbaden : Springer-Verlag, 2016 (Springer Reference Sozialwissenschaften). - ISBN 9783658054540, S. 3-19

[Lepsius 1966] Lepsius, Mario R.: Extremer Nationalismus: Strukturbedingungen vor der nationalsozialistischen Machtergreifung. Bd. 15. Kohlhammer, 1966

[Lessenich 2003] Lessenich, Stephan: Dynamischer Immobilismus: Kontinuität und Wandel im deutschen Sozialmodell. Frankfurt/Main and New York : Campus, 2003. - ISBN 9783593373768

[Lessenich 2013] Lessenich, Stephan: Die Neuerfindung des Sozialen: Der Sozialstaat im flexiblen Kapitalismus. 3., unveränderte Auflage 2013. Bielefeld : Transcript, 2013 (X-Texte zu Kultur und Gesellschaft). http://www . content-select . com/index .php?id=bib_ view\&ean=9783839407462. - ISBN 9783899427462

[Leutner 1990] Leutner, Bernd ; Bundesminister Für RaumordNUng, BAUWESEn Und StÄDTEBAU (Hrsg.): Wohnungspolitik nach dem 2. Weltkrieg: Im Auftrag des Bundesministers für Raumordnung, Bauwesen und Städtebau. Bonn, 1990 (Schriftenreihe "Forschung" des Bundesministers für Raumordnung, Bauwesen und Städtebau)

[Lill 2005] LiLL, Rudolf: Über die Anfänge der CDU in Köln, 19451948. In: Historisch-Politische Mitteilungen 12 (2005), S. 157-172

[Lipset u. Rokkan 1967] Lipset, Seymour M. (Hrsg.) ; Rokkan, Stein (Hrsg.): Party systems and voter aligments: Cross-national perspec- 
tives. 2. pr. New York : Free Press, 1967 (International yearbook of political behavior research)

[Lowe 2011] Lowe, Stuart: The Housing Debate. Bristol : Policy Press, 2011 (Policy and politics in the twenty-first century). - ISBN 9781847422736

[Lücke 1959] Lücke, Paul: Zum Geleit. In: Fischer-DieskaU, Joachim (Hrsg.): Beiträge zur Theorie und Praxis des Wohnungsbaues: Arnold Knoblauch als Festschrift zum 80. Geburtstag gewidmet von seinen Freunden. Domus-Verlag, 1959, S. 5

[Luhmann 1966] Luhmann, Niklas: Theorie der Verwaltungswissenschaft: Bestandsaufnahme und Entwurf. Köln : Grote, 1966

[Luhmann 1992] Luhmann, Niklas: Die Wissenschaft der Gesellschaft. 1. Frankfurt am Main : Suhrkamp, 1992. - ISBN 3-518-58065-5

[Luhmann 2000] Luhmann, Niklas: Die Politik der Gesellschaft. 1. Aufl. Frankfurt am Main : Suhrkamp, 2000. - ISBN 3-518-58290-9

[Lütge 1949] LÜTGE, Friedrich: Wohnungswirtschaft. Piscator-Verlag, 1949

[MacKenzie 2008] MacKenzIE, Donald A.: An Engine, not a Camera: How Financial Models Shape Markets. 1. Cambridge, MA and London : MIT Press, 2008 (Inside technology). - ISBN 9780262633673

[MacKenzie 2010] MacKenzie, Donald A.: Opening the Black Boxes of Global Finance. In: KyrTses, Alexandros-Andreas (Hrsg.): Financial markets and organizational technologies. Houndmills, Basingstoke, Hampshire and New York : Palgrave Macmillan, 2010 (Palgrave Macmillan studies in banking and financial institutions). - ISBN 9780230234055, S. 92-116

[MacKenzie u. Millo 2003] MacKenzie, Donald A. ; Millo, Yuval: Constructing a Market, Performing Theory: The Historical Sociology of a Financial Derivatives Exchange. In: American Journal of Sociology (2003), S. 107-145 
[Maeße 2013a] Maesse, Jens: Das Feld und der Diskurs der Ökonomie. In: Maesse, Jens (Hrsg.): Ökonomie, Diskurs, Regierung. Wiesbaden : Springer Berlin Heidelberg, 2013 (Globale Politische Ökonomie). ISBN 9783658012946, S. 241-275

[Maeße 2013b] Maesse, Jens (Hrsg.): Ökonomie, Diskurs, Regierung: Interdisziplinäre Perspektiven. Wiesbaden : Springer Berlin Heidelberg, 2013 (Globale Politische Ökonomie). - ISBN 9783658012946

[Mahoney u. Rueschemeyer 2003] Mahoney, James ; RueschemeyER, Dietrich: Comparative historical analysis in the social sciences. Cambridge University Press, 2003. - ISBN 0521016452

[Mahoney u. Thelen 2010] Mahoney, James ; Thelen, Kathleen A.: A theory of gradual institutional change. In: MAHONEY, James (Hrsg.) ; Thelen, Kathleen A. (Hrsg.): Explaining institutional change : ambiguity, agency, and power. Cambridge [u.a.] : Cambridge University Press, 2010. - ISBN 0-521-11883-2, S. 1-37

[Marschallek 2004] Marschallek, Christian: Die "schlichte Notwendigkeit" privater Altersvorsorge: Zur Wissenssoziologie der deutschen Rentenpolitik. In: Zeitschrift für Soziologie (2004), S. 285-302

[Maurach 1963] Maurach, Reinhart: In Memoriam Hans-Jürgen Seraphim. In: Jahrbücher für Geschichte Osteuropas 11 (1963), Nr. 1, 158-160. http://daten.digitale-sammlungen.de/ db/ bsb00003811/images/index . html?id=00003811\&groesser $=\& f \mathrm{ip}=$ qrsyzt seayasdaseayawfsdreayaenyzts\&no=7\&seite=178. - ISSN 00214019

[Mayer 1998] MaYer, Annette: Volkswirtschaftliche Schriften. Bd. Heft 484: Theorie und Politik des Wohnungsmarktes: Eine Analyse der Wohnungspolitik in Deutschland unter besonderer Berücksichtigung der ökonomischen Theorie der Politik. Berlin : Duncker \& Humblot, 1998. - ISBN 9783428094875

[Mayntz 2006] Mayntz, Renate: Die Organisation wissenschaftlicher Politikberatung in Deutschland. In: Politikberatung in Deutschland. 
Wiesbaden : VS Verlag für Sozialwissenschaften, 2006. - ISBN 9783-531-14375-0, S. 115-122

[Mayntz 2009] Mayntz, Renate: Speaking Truth to Power: Leitlinien für die Regelung wissenschaftlicher Politikberatung. In: der moderne staat 2 (2009), Nr. 1, S. 5-16. - ISSN 1865-7192

[Mayntz u. Derlien 1989] Mayntz, Renate ; Derlien, Hans-Ulrich: Party Patronage and Politicization of the West German Administrative Elite 1970-1987 - Toward Hybridization? In: Governance 2 (1989), Nr. 4, S. 384-404. http://dx.doi.org/ 10.1111/j.1468-0491.1989.tb00099.x. - DOI 10.1111/j.14680491.1989.tb00099.x. - ISSN 0952-1895

[McCloskey 2009] McCloskey, Deidre N.: Ökonomen leben in Metaphern. In: Diaz-Bone, Rainer (Hrsg.): Diskurs und Ökonomie. Wiesbaden : VS Verlag für Sozialwissenschaften, 2009 (Interdisziplinäre Diskursforschung). - ISBN 9783531156224, S. 109-124

[McNamara 1998] McNamara, Kathleen R.: The currency of ideas: Monetary politics in the European Union. Ithaca, NY : Cornell University Press, 1998 (Cornell studies in political economy). - ISBN 0801434327

[Mertens 2015] Mertens, Daniel: Schriften aus dem Max-PlanckInstitut für Gesellschaftsforschung Köln. Bd. Band 82: Erst sparen, dann kaufen? Privatverschuldung in Deutschland. Frankfurt : Campus, 2015. - ISBN 9783593505008

[Metha 2011] Metha, Jal: The Varied Roles of Ideas in Politics: From Wheter to How. In: BÉland, Daniel (Hrsg.) ; Cox, Robert H. (Hrsg.): Ideas and politics in social science research. Oxford : Oxford University Press, 2011. - ISBN 978-0-19-973687-4, S. 23-47

[Meuser u. Nagel 1991] Meuser, Michael ; Nagel, Ulrike: ExpertInneninterviews: Vielfach erprobt, wenig bedacht. Ein Beitrag zur qualitativen Methodendiskussion. In: Qualitativ-empirische Sozialforschung (1991), S. 441-471 
xxxviii

[Michels 2004] Michels, Winfried (Hrsg.): Münsteraner Wohnungswirtschaftliche Gespräche. Bd. 14: Wohneigentum und Altersvorsorge. Münster : Institut für Siedlungs- und Wohnungswesen, 2004. ISBN 3884971972

[Mirowski u. Plehwe 2015] Mirowski, Philip (Hrsg.) ; Plehwe, Dieter (Hrsg.): The road from Mont Pèlerin: The making of the neoliberal thought collective. Cambridge, Mass. and London : Harvard University Press, 2015. - ISBN 9780674033184

[Morgan 2001] Morgan, Glenn: Transnational communities and business systems. In: Global Networks 1 (2001), Nr. 2, S. 113-130.- ISSN $1471-0374$

[Müller u.a. 2002] Müller, Rolf ; Rohrbach, Iris ; Sigismund, Markus ; Schlömer, Claus ; Bundesamt Für BAuwesen UND RAUMORDNUNG (Hrsg.): Bericht zur Inanspruchnahme der Eigenheimzulage in den Jahren 1996-2000: Im Auftrag der ARGEBAU-Ministerkonferenz. http://www.bbsr.bund.de/ BBSR/DE/WohnenImmobilien/Immobilienmarktbeobachtung/ ProjekteFachbeitraege/Wohneigentum/DL_Bericht_

Eigenheimzulagenbericht.pdf?__blob=publicationFile\&v=3. Version: 2002

[Müller-Heine 1984] MülleR-HEINE, Karin: Studien zur Sozialpolitik. Bd. Bd. 1: Entwicklungsphasen der Wohnungspolitik in der Bundesrepublik Deutschland. Krefeld : Marchal und Matzenbacher, 1984. ISBN 3883580430

[Münnich 2010] Münnich, Sascha: Schriften aus dem Max-PlanckInstitut für Gesellschaftsforschung Köln. Bd. 70: Interessen und Ideen: Die Entstehung der Arbeitslosenversicherung in Deutschland und den USA. Frankfurt am Main [u.a.] : Campus, 2010. - ISBN 9783593393001

[Münnich 2011] MüNnICH, Sascha: Interessen und Ideen: Soziologische Kritik einer problematischen Unterscheidung/Interests and Ideas: A 
Sociological Critique of a Problematic Distinction. In: Zeitschrift für Soziologie 40 (2011), Nr. 5, S. 371-387

[Münnich u. Sachweh 2017] Münnich, Sascha ; Sachwen, Patrick: Einleitung: Varianten des kapitalistischen Geistes im Wandel. In: Sachweh, Patrick (Hrsg.) ; MÜnNICH, Sascha (Hrsg.): Kapitalismus als Lebensform? Wiesbaden : Springer-Verlag, 2017 (Wirtschaft und Gesellschaft). - ISBN 978-3-658-12916-3, S. 3-26

[Musgrave 2005] Musgrave, Richard A.: Merit goods. In: Readings in public finance (2005), S. 126-131

[Mützel 2009] Mützel, Sophie: Geschichten als Signale: Zur diskursiven Konstruktion von Märkten. In: Diaz-Bone, Rainer (Hrsg.): Diskurs und Ökonomie. Wiesbaden : VS Verlag für Sozialwissenschaften, 2009 (Interdisziplinäre Diskursforschung). - ISBN 9783531156224, S. $225-244$

[Nell-Breuning 1959] Nell-Breuning, Oswald: Der Wohnungswirtschaftliche Beirat beim Bundesministerium für Wohnungsbau. In: Fischer-Dieskau, Joachim (Hrsg.): Beiträge zur Theorie und Praxis des Wohnungsbaues: Arnold Knoblauch als Festschrift zum 80. Geburtstag gewidmet von seinen Freunden. Domus-Verlag, 1959, S. $35-44$

[Nevermann u.a. 1970] Nevermann, Paul ; Sasse, Rudolf ; Thiel, Eberhard: Wohnungspolitik im Kreuzfeuer. In: Wirtschaftsdienst 50 (1970), Nr. 9, S. 515-526. - ISSN 0043-6275

[Niederhafner u. Speth 2004] Niederhafner, Stefan ; Speth, Rudolf: Die Ministerialbürokratie in Deutschland: Vom Kellner zum Koch? In: Forschungsjournal Neue Soziale Bewegungen 17 (2004), Nr. 3, S. $23-35$

[Nölke 2009] NöLKE, Andreas: Finanzkrise, Finanzialisierung und vergleichende Kapitalismusforschung. In: Zeitschrift für internationale Beziehungen (2009), S. 123-139 
[Nölke 2014] NöLke, Andreas: Politik der Finanzialisierung: Zum Wohlfahrtsbeitrag des Finanzsektors in Rechnungslegungsstandards und volkswirtschaftlicher Gesamtrechnung. In: HeIREs, Marcel (Hrsg.) ; NöLKE, Andreas (Hrsg.): Politische Ökonomie der Finanzialisierung. Wiesbaden : Springer-Verlag, 2014 (Globale Politische Ökonomie). - ISBN 9783658037772, S. 79-94

[Nonn 2002] Nonn, Christoph: Das Godesberger Programm und die Krise des Ruhrbergbaus. Zum Wandel der deutschen Sozialdemokratie von Ollenhauer zu Brandt. In: Vierteljahrshefte für Zeitgeschichte (2002), S. 71-97. - ISSN 0042-5702

[North 1981] North, Douglass C.: Structure and Change in Economic History. New York, London : W. W. Norton Company, 1981.- ISBN 0393014789

[Nullmeier 2003] Nullmeier, Frank: Alterssicherungspolitik im Zeichen der "Riester-Rente". In: GoHR, Antonia (Hrsg.): Sozial- und Wirtschaftspolitik unter Rot-Grün. Wiesbaden : Westdeutscher Verlag, 2003. - ISBN 3531140647, S. 167-187

[Nullmeier u. Rüb 1993] Nullmeier, Frank; RüB, Friedbert W.: Theorie und Gesellschaft. Bd. 28: Die Transformation der Sozialpolitik: Vom Sozialstaat zum Sicherungsstaat. Frankfurt am Main and New York : Campus, 1993. - ISBN 9783593348575

[Nützenadel 2005] NÜtzenadel, Alexander: Kritische Studien zur Geschichtswissenschaft. Bd. Bd. 166: Stunde der Ökonomen: Wissenschaft, Politik und Expertenkultur in der Bundesrepublik 1949-1974. Göttingen : Vandenhoeck \& Ruprecht, 2005. - ISBN 3525351496

[Ostheim u. Schmidt 2007a] Ostheim, Tobias ; Schmidt, Manfred G.: Gründungskrise und Sozialpolitik: Die 50er Jahre der Bundesrepublik Deutschland. In: Schmidt, Manfred G. (Hrsg.): Der Wohlfahrtsstaat. Wiesbaden : VS Verlag für Sozialwissenschaften, 2007 (Lehrbuch). ISBN 9783531151984, S. 153-164 
[Ostheim u. Schmidt 2007b] Ostheim, Tobias ; Schmidt, Manfred G.: Vom Ausbau zur Konsolidierung: Sozialpolitik von der sozialliberalen Koalition bis zur Wiedervereinigung. In: SchMidT, Manfred G. (Hrsg.): Der Wohlfahrtsstaat. Wiesbaden : VS Verlag für Sozialwissenschaften, 2007 (Lehrbuch). - ISBN 9783531151984, S. $165-172$

[Pätsch 2018] Pätsch, Anke: Politikberatung durch Stiftungen. In: Falk, Svenja (Hrsg.) ; GlaAb, Manuela (Hrsg.) ; Römmele, Andrea (Hrsg.) ; Schober, Henrik (Hrsg.) ; Thunert, Martin (Hrsg.): Handbuch Politikberatung. Wiesbaden : Springer Fachmedien Wiesbaden, 2018. - ISBN 978-3-658-07461-6, S. 1-19

[Pauli 2005] PAUli, Martin: Hannoversche geographische Arbeiten. Bd. Bd. 58: Öffentliche und private Steuerung von Stadtentwicklung in unterschiedlichen Gesellschaftssystemen: Ein Vergleich ost- und westdeutscher Städte. Münster : Lit, 2005. - ISBN 9783825883775

[Peck 2010] PECK, Jamie: Constructions of Neoliberal Reason. First edition. Oxford, United Kingdom : Oxford University Press, 2010.ISBN 9780199662081

[Peters 1984] PeTERs, Karl-Heinz: Volkswirtschaftliche Schriften. Bd. Heft 343: Wohnungspolitik am Scheideweg: Wohnungswesen, Wohnungswirtschaft, Wohnungspolitik. Berlin : Duncker \& Humblot, 1984. - ISBN 3428055861

[Pielke 2007] PiElke, Roger A.: The honest broker: Making sense of science in policy and politics. Cambridge : Cambridge University Press, 2007. - ISBN 9780521694810

[Pierson 2000] Pierson, Paul: Increasing returns, path dependence, and the study of politics. In: American Political Science Review (2000), S. 251-268

[Pitlik 2001] PITLIK, Hans: Politikberatung der Öffentlichkeit? In: Perspektiven der Wirtschaftspolitik : eine Zeitschrift des Vereins für Socialpolitik ; PWP 2 (2001), Nr. 1, S. 61-73 
[Pius XI. 1931] PIUS XI.: Enzyklika Quadragesimo anno unseres Heiligen Vaters Pius XI: über die Gesellschaftliche Ordnung, ihre Wiederherstellung und ihre Vollendung nach dem Heilsplan der Frohbotschaft, zum 40. Jahrestag des Rundschreibens Leo's XIII. Rerum novarum: an die Ehrwürdigen Brüder, die Patriarchen, Primaten, Erzbischöfe und Bischöfe und die anderen Oberhirten,. http://www.kathpedia.com/index.php?title= Quadragesimo_anno_(Wortlaut). Version: 1931

[Plehwe 2015] Plehwe, Dieter: Introduction. In: Mirowski, Philip (Hrsg.) ; Plenwe, Dieter (Hrsg.): The road from Mont Pèlerin. Cambridge, Mass. and London : Harvard University Press, 2015. ISBN 9780674033184, S. 1-42

[Polanyi 1978] Polanyi, Karl: Suhrkamp Taschenbuch Wissenschaft. Bd. 260: The great transformation: Politische und ökonomische Ursprünge von Gesellschaften und Wirtschaftssystemen. Frankfurt am Main : Suhrkamp, 1978. - ISBN 3518278606

[Rickert 1983] RickerT, Gottfried: Technokratie und Demokratie. In: Zum Technokratieproblem in der (1983)

[Rolnik u. Rabinovich 2014] Rolnik, Raquel ; Rabinovich, Lidia: Late-neoliberalism: The financialisation of homeownership and the housing rights of the poor. In: Economic and social rights after the global financial crises. Cambridge : Cambridge University Press, 2014. - ISBN 978-1-107-04325-1, S. 57-89

[Ronald 2008] Ronald, Richard: The ideology of home ownership: Homeowner societies and the role of housing. Basingstoke and New York : Palgrave Macmillan, 2008. http://dx.doi.org/10.1057/ 9780230582286. http://dx.doi.org/10.1057/9780230582286. ISBN 9781403989451

[Rosenthal 2008] Rosenthal, Gabriele: Interpretative Sozialforschung: Eine Einführung. 2. Weinheim : Juventa, 2008 (Grundlagentexte Soziologie). http://www.socialnet.de/rezensionen/isbn.php? isbn $=978-3-7799-1482-2$. - ISBN 9783779914822 
[Rosenthal 2015] Rosenthal, Gabriele: Interpretative Sozialforschung: Eine Einführung. 5., überarbeitete Aufl. Weinheim, Bergstr : Beltz Juventa, 2015 (Grundlagentexte Soziologie). - ISBN 9783779926146

[Rottke 2009] RotTke, Nico B.: Immobilienwirtschaftslehre als wissenschaftliche Disziplin. In: Rottke, Nico B. (Hrsg.) ; Thomas, Matthias (Hrsg.): Immobilienwirtschaftslehre. Köln : Immobilien Manager Verlag IMV, 2009. - ISBN 9783899842081, S. 27-71

[Rueschemeyer 2009] Rueschemeyer, Dietrich: Usable theory: Analytic tools for social and political research. Princeton, N.J : Princeton University Press, 2009 http://search.ebscohost.com/login. aspx?direct=true\&scope $=$ site\&db=nlebk\&db=nlabk\&AN=335593. - ISBN 9781282754614

[Rueschemeyer u. Skocpol 1996] Rueschemeyer, Dietrich ; Skocpol, Theda: Conclusion. In: Rueschemeyer, Dietrich (Hrsg.) ; SkocPOL, Theda (Hrsg.): States, social knowledge, and the origins of modern social policies. Princeton N.J and New York : Princeton University Press and Russell Sage Foundation, 1996 (Princeton paperbacks). - ISBN 9780691001128, S. 296-312

[Runkel 2004] RunkeL, peter: Wohneigentumsbildung und private Altersvorsorge. In: Michels, Winfried (Hrsg.): Wohneigentum und Altersvorsorge $\mathrm{Bd}$. 14. Münster : Institut für Siedlungs- und Wohnungswesen, 2004. - ISBN 3884971972, S. 1-16

[Sabatier u. Jenkins-Smith 1999a] SAbatier, Paul A. (Hrsg.) ; Jenkins-Smith, Hank C. (Hrsg.): Policy change and learning: An advocacy coalition approach. Boulder, Colo. : Westview Press, 1999 (Theoretical lenses on public policy). http://www.loc.gov/catdir/ enhancements/fy0830/93002987-b.html. - ISBN 9780813316499

[Sabatier u. Jenkins-Smith 1999b] Sabatier, Paul A. ; JenkinsSmith, Hank C.: The Advocacy Coalition Framework: An Assessment. In: Sabatier, Paul A. (Hrsg.) ; Jenkins-Smith, Hank C. (Hrsg.): Policy change and learning. Boulder, Colo. : Westview Press, 
1999 (Theoretical lenses on public policy). - ISBN 9780813316499, S. $117-169$

[Sachweh 2011] SAchweH, Patrick: Ideen, Werte und Kultur als Erklärungsfaktoren in der Wohlfahrtsstaatsforschung. In: Zeitschrift für Sozialreform 57 (2011), Nr. 4, S. 371-382

[Scharpf 2000] ScHARPF, Fritz W.: Interaktionsformen: akteurzentrierter Institutionalismus in der Politikforschung. Leske + Budrich, 2000. - ISBN 381002709X

[Scharpf u. Schmidt 2004] Scharpf, Fritz W. (Hrsg.) ; Schmidt, Vivien A. (Hrsg.): Oxford Scholarship Online. Bd. 1: From vulnerability to competitiveness. Oxford : Oxford University Press, 2004. - ISBN 9780199240883

[Schelsky 1961] ScHELSKY, Helmut: Arbeitsgemeinschaft für Forschung des Landes Nordrhein-Westfalen, Geisteswissenschaften. Bd. 96: Der Mensch in der wissenschaftlichen Zivilisation. Wiesbaden : VS Verlag für Sozialwissenschaften, 1961. http://dx.doi. org/10.1007/978-3-663-02159-9. http://dx.doi.org/10.1007/ 978-3-663-02159-9. - ISBN 9783663002468

[Schimank u. Volkmann 2017] Schimank, Uwe ; Volkmann, Ute: Ökonomisierung der Gesellschaft. In: MAurer, Andrea (Hrsg.): Handbuch der Wirtschaftssoziologie. Wiesbaden : VS Verlag für Sozialwissenschaften, 2017 (Wirtschaft und Gesellschaft). - ISBN 978-3-53115259-2, S. 382-393

[Schipper 2017] SchIPPER, Sebastian: Wohnraum dem Markt entziehen? Wohnungspolitik und städtische soziale Bewegungen in Frankfurt und Tel Aviv. Wiesbaden, Germany : Springer-Verlag, 2017 (Stadt, Raum und Gesellschaft). http://www.springer.com/. ISBN 9783658179939

[Schloder 1959] Schloder, Georg: Eingliederung der privaten Bausparkassen in den Realkredit. In: Fischer-DiEskau, Joachim (Hrsg.): 
Beiträge zur Theorie und Praxis des Wohnungsbaues: Arnold Knoblauch als Festschrift zum 80. Geburtstag gewidmet von seinen Freunden. Domus-Verlag, 1959, S. 83-115

[Schmidt 2001] SchmidT, Manfred G.: ZeS-Arbeitspapier. Bd. 2/2001: Parteien und Staatstätigkeit. Bremen : Zentrum für Sozialpolitik Universität Bremen, 2001

[Schmidt 2010a] SchmidT, Manfred G.: Wörterbuch zur Politik. 3., überarb. und aktualisierte Aufl. Stuttgart : Kröner, 2010. - ISBN 9783520404039

[Schmidt 2008] Schmidt, Vivien A.: Discursive institutionalism: The explanatory power of ideas and discourse. In: Annual Review of Political Science (2008), Nr. 11, S. 303-326

[Schmidt 2010b] SchmidT, Vivien A.: Taking ideas and discourse seriously: Explaining change through discursive institutionalism as the fourth "new institutionalism". In: European Political Science Review (2010), S. $1-25$

[Schnapp 2004] Schnapp, Kai-Uwe: Ministerialbürokratien in westlichen Demokratien: Eine vergleichende Analyse. Opladen : Leske + Budrich, 2004. - ISBN 3810038008

[Schneider 1964a] SchneIder, Hans K. (Hrsg.): Der Abbau der Wohnungszwangswirtschaft. Köln : Verlagsgesellschaft Rudolf Müller, 1964 (Materialien-Sammlung für Wohnungs- und Siedlingswesen)

[Schneider 1964b] SchneIDer, Hans K. (Hrsg.): Grundlagen, Zielsetzungen und Methoden der Wohnungspolitik in der Sozialen Marktwirtschaft: Sonderveröffentlichung des Instituts für Siedlungs- und Wohnungswesen der Westfälischen Wilhelms-Universität Münster. Köln : Verlagsgesellschaft Rudolf Müller, 1964

[Schneider u. Janning 2006] SchneIDer, Volker ; JAnning, Frank: Lehrbuch. Bd. Bd. 43: Politikfeldanalyse: Akteure, Diskurse und Netzwerke in der öffentlichen Politik. 1. Aufl. Wiesbaden : VS Verlag für Sozialwissenschaften, 2006. - ISBN 9783531145495 
[Schubert 1991] Schubert, Klaus: Grundwissen Politik. Bd. Bd. 6: Politikfeldanalyse: Eine Einführung. Opladen : Leske + Budrich, 1991. - ISBN 3810008826

[Schulte 2013] Schulte, Martin: Politik mitgestalten: Möglichkeiten wissenschaftlicher politikberatung durch private think tanks. Marburg, Germany : Tectum Verlag, 2013. - ISBN 9783828858374

[Schulz 1994] Schulz, Günther: Wiederaufbau in Deutschland: Die Wohnungsbaupolitik in den Westzonen und der Bundesrepublik von 1945 bis 1957. Düsseldorf : Droste, 1994. - ISBN 3-7700-0975-4

[Schulz 2000] Schulz, Günther: Wohnungspolitik und Wohnungswirtschaft seit 1945. In: Eichener, Volker (Hrsg.) ; van EMmerich, Horst (Hrsg.) ; Petzina, Dietmar (Hrsg.): Die unternehmerische Wohnungswirtschaft. Frankfurt am Main : F. Knapp, 2000. - ISBN 3781906434 , S. $63-77$

[Schürt 2010] Schürt, Alexander ; Bundesinstitut fÜr BaU-, Stadt- Und Raumforschung (BBSR) im Bundesamt Für Baumesen und Raumentwicklung (Hrsg.): Synopse Immobilienpreisbeobachtung: Anforderungen - Datengrundlagen Verfahren - Produkte. https://www.bbsr.bund.de/BBSR/DE/ Veroeffentlichungen/BBSROnline/2010/DL_ON012010.pdf? _-_blob=publicationFile\&v=2. Version: 2010 (BBSR-OnlinePublikation)

[Schwartz 2012] Schwartz, Herman M.: Finance and the State in the Housing Bubble. In: Aalbers, Manuel B. (Hrsg.): Subprime cities. Malden, Mass : Wiley-Blackwell, 2012 (Studies in Urban and Social Change). - ISBN 9781444337761, S. 53-73

[Schwartz u. Seabrooke 2008] Schwartz, Herman M. ; Seabrooke, Leonard: Varieties of residential capitalism in the international political economy: Old welfare states and the new politics of housing. In: Comparative European politics (2008), S. 237-261 
[Seabrooke u. Schwartz 2009] Seabrooke, Leonard ; Schwartz, Herman M.: The Politics of Housing Booms and Busts. Basingstoke : Palgrave, 2009. - ISBN 0230280447

[Sebaldt 2001] SebaldT, Martin: Parlamentarische Demokratie und gesellschaftliche Modernisierung: Der Deutsche Bundestag. In: OBERReuter, Heinrich (Hrsg.) ; Kranenpohl, Uwe (Hrsg.) ; Sebaldt, Martin (Hrsg.): Der Deutsche Bundestag im Wandel. Wiesbaden : Westdeutscher Verlag, 2001. - ISBN 3-531-13684-4, S. 123-125

[Seeger 1995] SEEger, Hagen R. T.: Wohnungswirtschaft im Wahlzyklus der Politik. Sinzheim : Pro-Universitate-Verl., 1995 (Wissenschaftliche Schriften : Wirtschaft). - ISBN 3930747243

[Seeleib-Kaiser 2003a] Seeleib-Kaiser, Martin: Politikwechsel nach Machtwechsel? In: GoHR, Antonia (Hrsg.): Sozial- und Wirtschaftspolitik unter Rot-Grün. Wiesbaden : Westdeutscher Verlag, 2003. ISBN 3531140647, S. 11-27

[Seeleib-Kaiser 2003b] Seeleib-Kaiser, Martin: Rot-Grün am Ende? In: GoHr, Antonia (Hrsg.): Sozial- und Wirtschaftspolitik unter RotGrün. Wiesbaden : Westdeutscher Verlag, 2003. - ISBN 3531140647, S. $347-361$

[Seraphim 1955] Seraphim, Hans-Jürgen (Hrsg.): Beiträge und Untersuchungen / Institut für Siedlungs- und Wohnungswesen der Westfälischen Wilhelms-Universität Münster. Bd. 48: Das Eigenheim: Eine soziologische und volkswirtschaftliche Analyse. Köln-Braunsfeld : Rudolf Müller, 1955

[Siefken 2007] Siefken, Sven T.: Expertenkommissionen im politischen Prozess: Eine Bilanz zur rot-grünen Bundesregierung 1998 - 2005. 1. Aufl. Wiesbaden : VS Verlag für Sozialwissenschaften, 2007. - ISBN 9783531156910

[Simian 2000] Simian, Jose M.: Kooperations- und genossenschaftswissenchaftliche Beiträge. Bd. Bd. 46: Eigentumsorientierte Wohnungspolitik in Deutschland und Chile: Eine Analyse unter Berücksichti- 
xlviii

gung der Wohnungsgenossenschaften. Münster : Regensberg, 2000. - ISBN 3792307448

[Sinn 1505] Sinn, Hans-Werner: Der wahre Neoliberalismus braucht klare Regeln. In: Die Welt (15.05.2010). https://www.welt.de/debatte/kommentare/article7641497/ Der-wahre-Neoliberalismus-braucht-klare-Regeln .html

[Sinn u.a. 1995] Sinn, Hans-Werner ; Fenbert, Hermann ; JAKob, Wolfgang ; NachtKamp, Hans H. ; Pfeiffer, Ulrich ; Schunk, Edmund ; Siebertz, Paul ; Sievert, Olaf ; Sonnenschein, Jürgen ; Staender, Ludwig ; Steffens, Dietrich ; Tomann, Horst ; Wullkopf, Uwe: Wohnungspolitik auf dem Prüfstand. Tübingen : Mohr, 1995. - ISBN 316146396x

[Skocpol 1985] Skocpol, Theda: Bringing the State Back In: Strategies of Analysis in Current Research. In: Evans, Peter B. (Hrsg.) ; RuEschemeyer, Dietrich (Hrsg.) ; Skocpol, Theda (Hrsg.): Bringing the State back in. Cambridge : Cambridge University Press, 1985. ISBN 9780521313131, S. 3-37

[Skocpol u. Rueschemeyer 1996] Skocpol, Theda ; Rueschemeyer, Dietrich: Introduction. In: Rueschemeyer, Dietrich (Hrsg.) ; SkocPOL, Theda (Hrsg.): States, social knowledge, and the origins of modern social policies. Princeton N.J and New York : Princeton University Press and Russell Sage Foundation, 1996 (Princeton paperbacks). - ISBN 9780691001128, S. 3-14

[SPD 1959] SPD: Godesberger Programm: Grundsatzprogramm der Sozialdemokratischen Partei Deutschlands. Beschlossen vom Außerordentlichen Parteitag der Sozialdemokratischen Partei Deutschlands in Bad Godesberg vom 13. bis 15. November 1959. http://www . spd. de/show/1682029/spd_godesbergerprogramm.pdf. Version: 1959

[Spruijt u.a. 2014] SpruiJt, Pita ; Knol, Anne B. ; Vasileiadou, Eleftheria ; Devilee, Jeroen ; Lebret, Erik ; Petersen, Arthur C.: 
Roles of scientists as policy advisers on complex issues: A literature review. In: Environmental Science \& Policy 40 (2014), S. 1625. http://dx.doi.org/10.1016/j.envsci.2014.03.002. - DOI 10.1016/j.envsci.2014.03.002. - ISSN 14629011

[Starke 2015] Starke, Peter: Prozessanalyse. In: Wenzelburger, Georg (Hrsg.) ; Zohlnhöfer, Reimut (Hrsg.): Handbuch PolicyForschung. Wiesbaden : Springer-Verlag, 2015 (Springer VS Handbuch). - ISBN 9783658019679, S. 453-482

[Statistisches Bundesamt 1956] Statistisches Bundesamt: Einführung in die Methoden und die Organisation der Zählung. In: Gebäude- und Wohnungszählung in der Bundesrepublik Deutschland 1 (1956), Nr. 1, S. 2-19

[Statistisches Bundesamt 1959] Statistisches Bundesamt: Der Wohnungsbedarf aus der Sicht der Haushalte: Erste vorläufige Ergebnisse der $1 \mathrm{vH-Zusatzerhebung} \mathrm{zur} \mathrm{Wohnungsstatistik} \mathrm{1957.} \mathrm{In:} \mathrm{Wirtschaft}$ und Statistik 11 (1959), S. 66-71

[Statistisches Bundesamt 2014] Statistisches Bundesamt: Bauen und Wohnen: Mikrozensus - Zusatzerhebung 2014. In: Fachserie 5 (2014), Nr. 1, S. 4-450

[Steinmo u. a. 1992] Steinmo, Sven (Hrsg.) ; Thelen, Kathleen A. (Hrsg.) ; Longstreth, Frank (Hrsg.): Structuring politics: Historical institutionalism in comparative analysis. Cambridge : Cambridge University Press, 1992 (Cambridge studies in comparative politics). - ISBN 0-521-41780-5

[Stiftung Marktwirtschaft 2018a] STIFTUng MARKTwiRTSCHAFT: Kronberger Kreis. https://www.stiftung-marktwirtschaft.de/ inhalte/kronberger-kreis/. Version: 2018

[Stiftung Marktwirtschaft 2018b] Stiftung MarkTwiRTschaft: Über Uns. https://www.stiftung-marktwirtschaft.de/ inhalte/die-stiftung/ueber-uns/. Version: 2018 
[Streeck 1994] Streeck, Wolfgang: Staat und Verbände: Neue Fragen.

Neue Antworten? In: Streeck, Wolfgang (Hrsg.) ; Czada, Roland (Hrsg.): Staat und Verbände Bd. 25. Opladen : Westdeutscher Verlag, 1994. - ISBN 353112661X, S. 7-34

[Streeck 2015] STREECK, Wolfgang: Von der Gesellschaftssteuerung zur sozialen Kontrolle: Rückblick auf ein halbes Jahrhundert Soziologie in Theorie und Praxis. In: Blätter für deutsche und internationale Politik 60 (2015), Nr. 1, S. 63-80. - ISSN 0006-4416

[Streeck 2016] Streeck, Wolfgang: Wissenschaftliche Politikberatung: Was wird verlangt, was kann sie bieten? In: Hoose, Fabian (Hrsg.) ; Beckmann, Fabian (Hrsg.) ; Schönauer, Anna-Lena (Hrsg.): Fortsetzung folgt. Springer Science and Business Media and SpringerVerlag, 2016. - ISBN 978-3-658-15449-3, S. 489-506

[Streeck u. Thelen 2005a] Streeck, Wolfgang (Hrsg.) ; Thelen, Kathleen A. (Hrsg.): Beyond continuity: Institutional change in advanced political economies. 1. publ. Oxford : Oxford University Press, 2005. - ISBN 0-19-928046-0

[Streeck u. Thelen 2005b] Streeck, Wolfgang ; Thelen, Kathleen A.: Introduction: Institutional Change in Advanced Political Economies. In: Streeck, Wolfgang (Hrsg.) ; Thelen, Kathleen A. (Hrsg.): Beyond continuity. Oxford : Oxford University Press, 2005. - ISBN 0-19-928046-0, S. 3-39

[Szypulski 2008] Szypulski, Anja: Gemeinsam bauen - gemeinsam wohnen: Wohneigentumsbildung durch Selbsthilfe. Wiesbaden : VS Verlag für Sozialwissenschaften, 2008 (SpringerLink). - ISBN $383505578 \mathrm{X}$

[Thelen 2004] Thelen, Kathleen A.: How institutions evolve: The political economy of skills in Germany, Britain, the United States, and Japan. Cambridge : Cambridge University Press, 2004 (Cambridge studies in comparative politics). http://www.loc.gov/catdir/ description/cam041/2004040785.html. - ISBN 9780511302893 
[Thelen 2012] Thelen, Kathleen A.: Varieties of Capitalism: Trajectories of Liberalization and the New Politics of Social Solidarity. In: $A n-$ nual Review of Political Science 15 (2012), Nr. 1, S. 137-159. http: //dx.doi.org/10.1146/annurev-polisci-070110-122959. - DOI 10.1146/annurev-polisci-070110-122959

[Thunert 1999] ThunerT, Martin: Think Tanks als Ressourcen der Politikberatung. In: Forschungsjournal Neue Soziale Bewegungen 12 (1999), Nr. 3, S. 10-19

[Thunert 2001] Thunert, Martin: Politikberatung in der Bundesrepublik Deutschland seit 1949. In: Willems, Ulrich (Hrsg.): Demokratie und Politik in der Bundesrepublik 1949-1999. VS Verlag für Sozialwissenschaften, 2001. - ISBN 3810031208, S. 223-242

[Tiemann u. Wagner 2013] Tiemann, Heinrich ; Wagner, Gert G.: Working Paper Series des Rates für Sozial- und Wirtschaftsdaten. Bd. 220: Die wissenschaftliche Politikberatung der Bundesregierung neu organisieren. Berlin : Rat für Sozial- und Wirtschaftsdaten (RatSWD), 2013 http://www.ratswd.de/dl/RatSWD_WP_220.pdf

[Tooze 2001] Tooze, J. A.: Cambridge studies in modern economic history. Bd. 9: Statistics and the German State, 1900 - 1945: The making of modern economic knowledge. Cambridge : Cambridge University Press, 2001 http: //www . loc.gov/catdir/samples/cam031/ 00067609.html. - ISBN 9780521803182

[Triebel u.a. 1965] Triebel, Wolfgang ; KrÄntzer, Karl R. ; JANIK, Engelbert: Wirtschaftliche Planung und rationelle Ausführung von Einfamilienhäusern: Querschnittliche Untersuchungen und Erfahrungen bei Demonstrativbauten und Versuchs- und Vergleichsbauten des Bundesministeriums für Wohnungswesen, Städtebau und Raumordnung: Im Auftrage des Bundesministeriumns für Wohnungswesen, Städtebau und Raumordnung. Bad Godesberg, 1965 (Informationen)

[Uertz 1981] UERTZ, Rudolf: Schriftenreihe der Vierteljahreshefte für Zeitgeschichte. Bd. 43: Christentum und Sozialismus in der frü- 
hen CDU. Stuttgart : Deutsche Verlags-Anstalt, 1981. - ISBN 3421060665

[Ulbrich 1980] Ulbrich, Rudi ; Bundesminister fÜr RaumordNung, BAUwesen und StÄDTEBAU (Hrsg.): Verteilungswirkungen des Förderungssystems für den Wohnungabsu: Im Auftrag des Bundesministers für Raumordnung, Bauwesen und Städtebau, durchgeführt vom Deutschen Institut für Wirtschaftsforschung. 1980 (Schriftenreihe "Wohnungsmarkt und Wohnungspolitik" des Bundesministers für Raumordnung, Bauwesen und Städtebau)

[Ullrich 2003] Ullrich, Carsten G.: Wohlfahrtsstaat und Wohlfahrtskultur: Zu den Perspektiven kultur- und wissenssoziologischer Sozialpolitikforschung. Mannheim : Mannheimer Zentrum für Europäische Sozialforschung, 2003 (Arbeitspapiere / Mannheimer Zentrum für Europäische Sozialforschung)

[van Horn u. Mirowski 2015] van Horn, Rob ; Mirowski, Philip: The Rise of the Chicago School of Economics and the Birth of Neoliberalism. In: Mirowski, Philip (Hrsg.) ; Plehwe, Dieter (Hrsg.): The road from Mont Pèlerin. Cambridge, Mass. and London : Harvard University Press, 2015. - ISBN 9780674033184, S. 139-178

[van Kersbergen 1995] van Kersbergen, Kees: Social capitalism: A study of Christian democracy and the welfare state. London : Routledge, 1995. - ISBN 0415116708

[Verband deutscher Pfandbriefbanken 2016] Verband Deutscher PfandbriefBanken: Aufgaben. http://pf andbrief.de/cms/ _internet.nsf/tindex/de_91.htm. Version: 2016

[Wagschal u. Grasl 2015] Wagschal, Uwe ; Grasl, Maximilian: Verbände und Korporatismus: Funktionen - Theorien - PolicyWirkungen. In: WaGschal, Uwe (Hrsg.) ; JäCKLE, Sebastian (Hrsg.) ; Wenzelburger, Georg (Hrsg.): Einführung in die Vergleichende Politikwissenschaft. Stuttgart : Kohlhammer, 2015. - ISBN 9783170289642, S. 203-220 
[Walter 1994] WALTER, Wolfgang: Strategien der Politikberatung: Die Interpretation der Sachverständigen-Rolle im Lichte von Experteninterviews. In: HitzleR, Ronald (Hrsg.): Expertenwissen. Opladen : Westdeutscher Verlag, 1994. - ISBN 3531125818, S. 268-284

[Wandersleb 1959] Wandersleb, Hermann: Entwicklungstendenzen im Wohnungsbau in der Bundesrepublik Deutschland. In: FischerDieskau, Joachim (Hrsg.): Beiträge zur Theorie und Praxis des Wohnungsbaues: Arnold Knoblauch als Festschrift zum 80. Geburtstag gewidmet von seinen Freunden. Domus-Verlag, 1959, S. 7-19

[Wansleben 2011] Wansleben, Leon: Wie wird bewertbar, ob ein Staat zu viele Schulden hat? Finanzexperten und ihr Bewertungswissen in der griechischen Schuldenkrise. In: Berliner Journal für Soziologie (2011), S. 495-519. - ISSN 1862-2593

[Weber 1977] WeBER, Jürgen: Die Interessengruppen im politischen System der Bundesrepublik Deutschland. Stuttgart : Kohlhammer, 1977 (Ein systematisches Programm Politikwissenschaft). - ISBN 3170042130

[Weber 1988] WeBER, Max: Einleitung in die Wirtschaftsethik der Weltreligionen. In: Weber, Max (Hrsg.) ; Weber, Marianne (Hrsg.): Gesammelte Aufsätze Bd. 1490. Tübingen : Mohr, 1988. - ISBN 3168453684, S. 237-275

[Weber 2013] WeBer, Max: Wirtschaft und Gesellschaft: Grundriss der verstehenden Soziologie. 5. Tübingen : Mohr, 2013. - ISBN 3161477499

[Weber u.a. 2013] Weber, Max ; Aldenhoff-Hübinger, Rita ; BaIer, Horst ; Borchardt, Knut ; Dahlmann, Dittmar ; Deininger, Jürgen ; Dilcher, Gerhard ; Fehlemann, Silke ; Frommer, Sabine ; Golzıo, Karl-Heinz ; Hanke, Edith ; Hübinger, Gangolf ; Kroll, Thomas ; Kurth, Peter ; Kolonko, Petra ; Krumeich, Gerd ; Lepsius, M. R. ; Lepsius, Susanne ; Meyer-Stoll, Cornelia ; Mommsen, Wolfgang J. ; Morgenbrod, Birgitt ; Nippel, 
Wilfried ; Offermann, Julia ; Otto, Eckart ; Riesebrodt, Martin ; Schluchter, Wolfgang ; Schmidt-Glintzer, Helwig ; SchröDer, Joachim ; Schwentker, Wolfgang ; Terwey, Andreas: Gesamtausgabe. Bd. 1/18: Die protestantische Ethik und der Geist des Kapitalismus. Tübingen : Mohr Siebeck, 2013. - ISBN 3161532716

[Wehlau 2009] Wehlau, Diana: Lobbyismus und Rentenreform: Der Einfluss der Finanzdienstleistungsbranche auf die Teil-Privatisierung der Alterssicherung. 1. Aufl. Wiesbaden : VS Verlag für Sozialwissenschaften, 2009. - ISBN 3531165305

[Weible u. a. 2009] Weible, Christopher M. ; SAbatier, Paul A. ; MCQueen, Kelly: Themes and variations: Taking stock of the advocacy coalition framework. In: Policy Studies Journal 37 (2009), Nr. 1, S. 121-140. - ISSN 1541-0072

[Weingart 2006] WeingarT, Peter: Erst denken, dann handeln? Wissenschaftliche Politikberatung aus der Perspektive der Wissens(chafts)soziologie. In: FAlk, Svenja (Hrsg.) ; Rehfeld, Dieter (Hrsg.) ; Römmele, Andrea (Hrsg.) ; Thunert, Martin (Hrsg.): Handbuch Politikberatung. Wiesbaden : VS Verlag für Sozialwissenschaften, 2006. - ISBN 9783531142500, S. 35-44

[Weissbarth 1978] Weissbarth, Reinhold ; Bundesminister FÜr Raumordnung, Bauwesen und Städtebau (Hrsg.): Sickereffekte verschiedener Formen der Wohnbau-und Bausparförderung: Im Auftrag des Bundesministeriums für Raumordnung, Bauwesen und Städtebau unter Mitfinanzierung durch den Verband der Privaten Bausparkassen e.V. und die Geschäftsstelle Öffentliche Bausparkassen im Deutschen Sparkassen- und Giroverband e.V., durchgeführt von Infratest Wirtschaftsforschung GmbH. 1978 (Schriftenreihe "Wohnungsmarkt und Wohnungspolitik" des Bundesministers für Raumordnung, Bauwesen und Städtebau)

[Weissbarth u. Hundt 1983] Weissbarth, Reinhold ; Hundt, Berthold: Schriftenreihe des Bundesministers für Raumordnung, Bauwe- 
sen und Städtebau. Bd. 07.014: Die Eigentumsbildung im Wohnungsbau. Braunschweig : Waisenhaus-Buchdruckerei und Verlag, 1983

[Weitnauer 1951] Weitnauer, Hermann: Das Wohnungseigentumsgesetz. In: Juristenzeitung 6 (1951), Nr. 6, S. 161-166

[Welty 1946] Welty, Eberhard: Christlicher Sozialismus. In: Neue Ordnung 1 (1946), S. 39-70

[Wiese-von Ofen 2016] WIESE-von Ofen, Irene: 70 Jahre DV: Rückblick auf unsere bewegte Verbandsgeschichte. Berlin, 2016

[Wijburg u. Aalbers 2017] Wijburg, Gertjan ; Aalbers, Manuel B.: The alternative financialization of the German housing market. In: Housing Studies 32 (2017), Nr. 7, S. 968-989. http://dx.doi.org/10.1080/02673037.2017.1291917. - DOI 10.1080/02673037.2017.1291917. - ISSN 0267-3037

[Wilensky 1975] WiLENSKy, Harold L.: The welfare state and equality: Structural and ideological roots of public expenditures. Berkeley : University of California Press, 1975. - ISBN 0520028007

[Wilhelm 1968] Wilhelm, Wendelin: Wissenschaftliche Beratung der Politik in der Bundesrepublik Deutschland: Probleme wiss. Beiräte bei Bundesmin. unter besonderer Berücksichtigung des Wohnungswirtschaftlichen Beirates beim Bundeswohnungsbaumin: Univ., Wirtschafts- u. Sozialwiss. Fak., Diss. 1966 u. d. T.: Wilhelm, Wendelin: Probleme wissenschaftlicher Beiräte bei Bundesministerien unter besonderer Berücksichtigung des Wohnungswirtschaftlichen Beirates beim Bundeswohnungsbauministerium.-Zugleich: Köln, 1966. Berlin, Köln, Frankfurt (Main) : Beuth, 1968

[Williamson 1985] Williamson, Oliver E.: The economic institutions of capitalism. New York, 1985

[Wissenschaftlicher Beirat beim Bundesministerium für Wirtschaft 1950] Wissenschaftlicher Beirat Beim Bundesministerium FÜR WirTschafT: Stellung des Wohnungswesens in der sozialen Marktwirtschaft: Gutachten vom 7.5.1950. Königstein, 1950 
[Wissenschaftlicher Beirat beim Bundesministerium für Wirtschaft 1982] Wissenschaftlicher Beirat Beim Bundesministerium FÜR WIRTSChaft: Probleme der Wohnungswirtschaft. Bonn, 1982

[Wissenschaftlicher Beirat für Familienfragen beim Bundesministerium für Jugend, familie und gesundheit 1975] Wissenschaftlicher Beirat FÜr FAmilienfragen Beim Bundesministerium für JuGEND, FAMILIE UND GESUNDHEIT: Schriftenreihe des Bundesministers für Jugend, Familie und Gesundheit. Bd. 20: Familie und Wohnen: Analysen und Empfehlungen zur Wohnungsversorgung der Familien und zur Förderung familiengerechten Wohnens in der Bundesrepublik Deutschland; Gutachten des Wissenschaftlichen Beirats für Familienfragen beim Bundesministerium für Jugend, Familie und Gesundheit. 2. Aufl. Stuttgart : Kohlhammer, 1975. - ISBN 3170024361

[Wohnungswirtschaftlicher Beirat 1954] WohnungswiRTschaftliCHER BEIRAT: Zur Frage der familiengerechten Wohnungen. In: Bundesministerium Für Wohnungsbau (Hrsg.): Gutachten des Wohnungswirtschaftlichen Beirats 1951-1953. Bonn, 1954 (Schriftenreihe des Bundesministers für Wohnungsbau), S. 72-90

[Wolff 2013] Wolff, Stephan: Wege ins Feld und ihre Varianten. In: Flick, Uwe (Hrsg.): Qualitative Forschung Bd. 55628. Reinbek bei Hamburg : Rowohlt, 2013. - ISBN 9783499556289, S. 334-349

[Wollmann 2002] Wollmann, Hellmut: Politikberatung. In: Nohlen, Dieter (Hrsg.): Kleines Lexikon der Politik. München : C.H. Beck, 2002. - ISBN 3-406-45958-7, S. 376-380

[Wullkopf u. Zinn 1981] Wullkopf, Uwe ; Zinn, Hermann: Zur Geschichte des Instituts Wohnen und Umwelt. In: InstiTuT FÜR WoHNEN UND UMWelt (Hrsg.): Wohnungspolitik am Ende? Opladen : Westdeutscher Verlag, 1981, S. 291-297

[Zentraler Immobilien Ausschuss e.V. 2018] Zentraler Immobilien Ausschuss E.V.: Geschichte. https://www.zia-deutschland.de/ ueber-den-zia/geschichte/. Version: 2018 
Anhang 



\section{Anhang A}

\section{Rahmenbedingungen}

\section{A.1 Wechselnde Zuschnitte des Wohnungs- bauministeriums}

\begin{tabular}{llll} 
Jahr & Bundesregierung & Ministerium & Minister \\
\hline \multirow{2}{*}{1949} & Kabinett Adenauer I: & BundesministeriunEberhard Wildermuth \\
& CDU/CSU, FDP, DP & für Wohnungs- & (FDP) \\
& & bau
\end{tabular}

1953 Kabinett Adenauer II: CDU/CSU, FDP, DP, GB/BHE (55-56 ohne GB/BHE; 56-57 mit FVP und DP) 


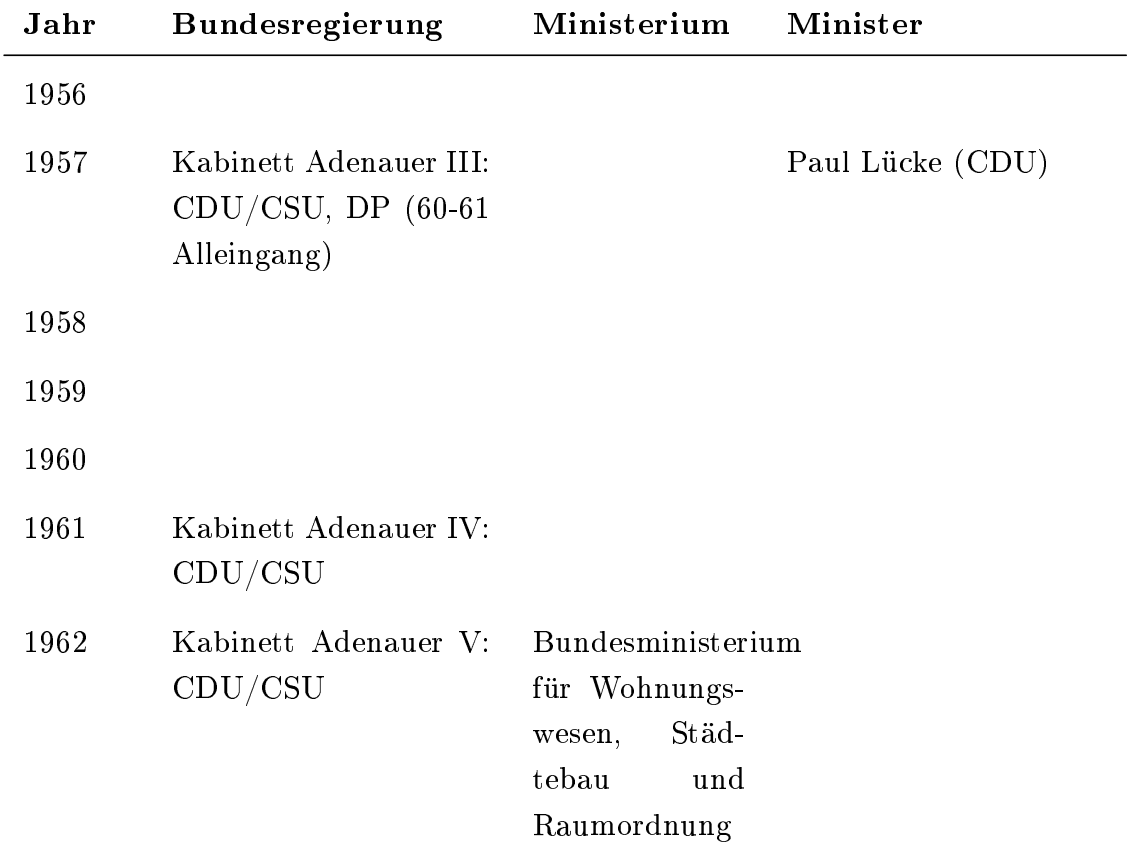

1963 Kabinett Erhard I:

CDU/CSU, FDP

1964

1965 Kabinett Erhard II

Dr. Ewald Bucher (FDP)

1966 Kabinett Kiesinger: BundesministeriunDr. Bruno Heck (CDU) $\mathrm{CDU} / \mathrm{CSU}, \mathrm{SPD}$ für Wohnungswesen und Städtebau 1967 1968 Dr. Lauritz Lauritzen (SPD)

1969 Kabinett Brandt I: SPD, FDP 


\begin{tabular}{|c|c|c|}
\hline Jahr & Bundesregierung & Ministerium \\
\hline \multirow[t]{4}{*}{1970} & & Bundesministerium \\
\hline & & für Städtebau \\
\hline & & und Woh- \\
\hline & & nungswesen \\
\hline
\end{tabular}

1972 Kabinett Brandt II: Dr. Hand-Jochen VoSPD, FDP gel (SPD)

Bundesministerium

für Raumordnung, Bauwesen und

Städtebau

1974 Kabinett Schmidt I: SPD, FDP

1976 Kabinett Schmidt II: SPD, FDP (SPD)

1980 Kabinett Schmidt III: SPD, FDP

1982 Kabinett Kohl I: BundesministeriunDr. Oscar Schneider CDU/CSU, FDP für Raumord- (CSU) nung, Bauwesen und Städtebau 


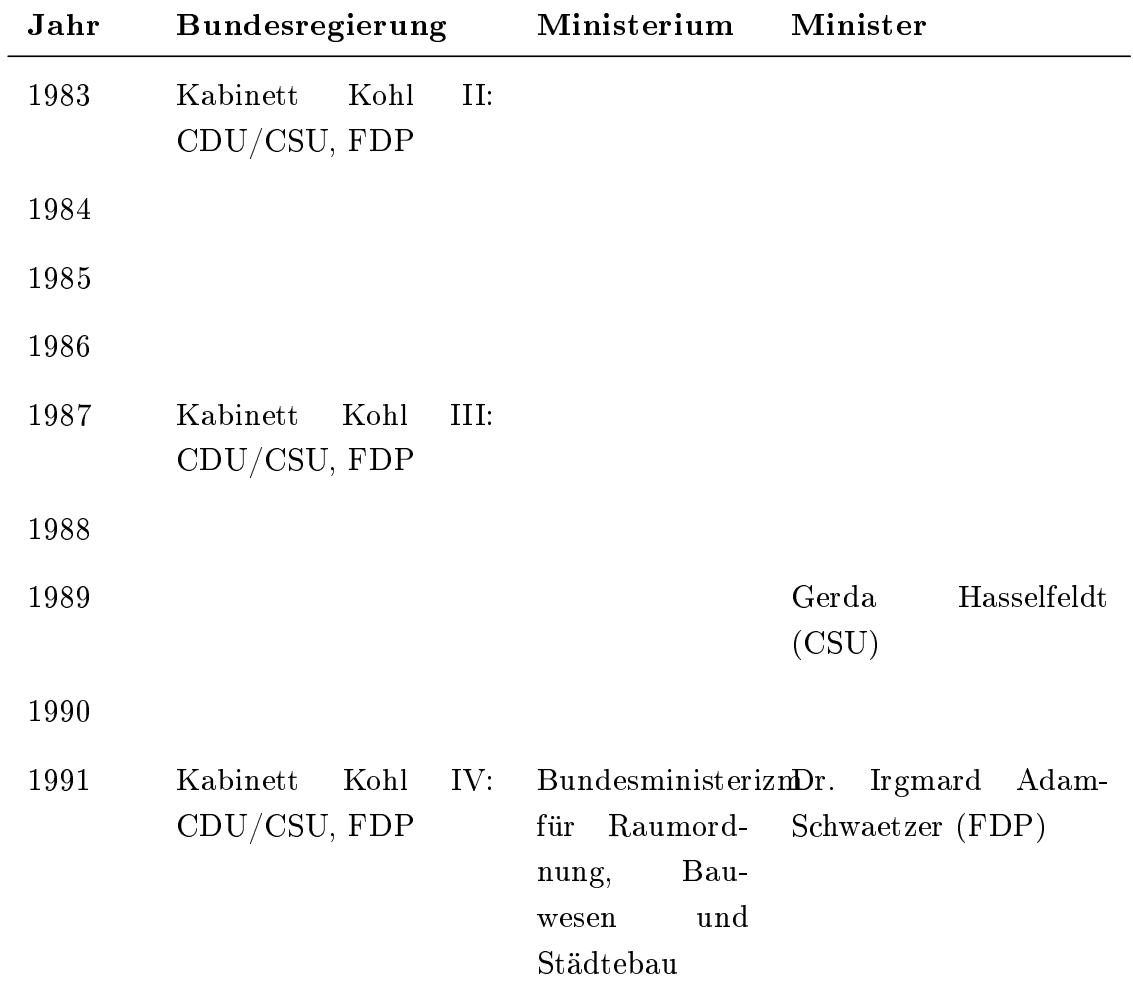

1994 Kabinett Kohl V: BundesministeriunProf. Dr. Klaus Töpfer CDU/CSU, FDP 


\begin{tabular}{|c|c|c|c|c|}
\hline Jahr & Bundesregierung & Ministerium & Ministe & \\
\hline 1998 & $\begin{array}{l}\text { Kabinett Schröder I: } \\
\text { SPD, Bündnis } 90 / \text { Grü- } \\
\text { ne }\end{array}$ & $\begin{array}{l}\text { Bundesministeriv } \\
\text { für Verkehr, } \\
\text { Bau- und Woh- } \\
\text { nungswesen }\end{array}$ & $\begin{array}{l}\text { niranz } \\
\text { (SPD) }\end{array}$ & Müntefehring \\
\hline 1999 & & & $\begin{array}{l}\text { Reihard } \\
\text { (SPD) }\end{array}$ & Klimmt \\
\hline $\begin{array}{l}2000 \\
2001\end{array}$ & & & \multicolumn{2}{|c|}{ Kurt Bodewig (SPD) } \\
\hline 2002 & $\begin{array}{l}\text { Kabinett Schröder I: } \\
\text { SPD, Bündnis } 90 / \text { Grü- } \\
\text { ne }\end{array}$ & & \multicolumn{2}{|c|}{ Manfred Stolpe (SPD) } \\
\hline
\end{tabular}

2005 Kabinett Merkel I: BundesministeriunWolfgang Tiefensee CDU/CSU, SPD für Verkehr, (SPD) Bau und Stadtentwicklung 
lxiv

\begin{tabular}{llll} 
Jahr & Bundesregierung & Ministerium & Minister \\
\hline 2013 & Kabinett Merkel III: & BundesministeriunBarbara & Hendricks \\
& CDU/CSU, SPD & für Umwelt, (SPD) & \\
& & Naturschutz, & \\
& & Bau und Reak- \\
& & torsicherheit
\end{tabular}

2014

2015

Quelle: Kornemann 1996, 142; eigene Ergänzungen 


\section{Anhang B}

\section{Methodik}

\section{B.1 Schriftenreihen des Bundesministeriums für Wohnungsbau}

- Schriftenreihe des Bundesministers für Wohnungsbau (1952-1962)

- Schriftenreihe des Bundesministers für Wohnungswesen, Städtebau und Raumordnung (1963-1964)

- Schriftenreihe des Bundesministers für Wohnungswesen und Städtebau (1967-1969)

- Schriftenreihe des Bundesministers für Städtebau und Wohnungswesen (1971)

- Schriftenreihe des Bundesministers für Raumordnung, Bauwesen und Städtebau (1988-1987)

- (01) Modellvorhaben, Versuchs- und Vergleichsbauvorhaben

- (02) Stadtentwicklung

- (03) Städtebauliche Forschung

- (04) Bau- und Wohnforschung

- (05) Bundeswettbewerbe

- (06) Raumordnung 
- (07) Wohnungsmarkt und Wohnungspolitik

- Schriftenreihe Materialien zur Raumentwicklung der Bundesforschungsanstalt für Landeskunde und Raumordnung (1991-1997)

- Schriftenreihe Forschung des Bundesministers für Raumordnung, Bauwesen und Städtebau (1989-1998)

- Schriftenreihe Forschungen des Bundesministeriums für Verkehr, Bau und Stadtentwicklung (1998-2013)

- Sonderveröffentlichungen BMVBS (1994-2013)

- Schriftenreihe Berichte (1998-2009)

- Informationen aus der Forschung des BBSR (2000-derz.)

- BBSR-Online-Publikation (2002-derz.)

- BBSR-Berichte KOMPAKT (2009-derz.)

- BMVBS-Online-Publikation (2010-derz.)

- Schriftenreihe des BBSR: Analysen Bau.Stadt.Raum (2010-derz.)

- BBSR-Analysen KOMPAKT (2011-derz.) 


\section{B.2 Wohnimmobilienmarktspezifischen Ver- öffentlichungen der Deutschen Bundes- bank}

Veröffentlichung Titel

Mai $03 M B$

Neue Preisindizes für Wohnimmo- $1 / 2$

bilien in Deutschland

Sep $03 M B$

Preisindikatoren für den Woh- 15 nungsmarkt

Feb $08 M B$

Immobilienpreise im Jahr 2007

2

Feb $09 M B$

Immobilienpreise in Deutschland 2 im Jahr 2008

Feb $10 M B$

Preise für Wohnimmobilien in 2

Deutschland im Jahr 2009

Feb $11 M B$

Die Preise für Wohnimmobilien in 2

Deutschland im Jahr 2010

Feb $12 M B$

Die Preise für Wohnimmobilien in 2

Deutschland 2011

Nov 12 FStB

Deutscher Wohnimmobilienmarkt 11 in Bewegung

Feb $13 M B$

Die Preise für Wohnimmobilien in 3

Deutschland 2012

Okt $13 M B$

Die Preissteigerungen bei Wohnim18 mobilien seit dem Jahr 2010: Einflussfaktoren und regionale Abhängigkeiten 
lxviii

Veröffentlichung Titel

Seitenzahl

Nov 13 FStB Wohnimmobilienmarkt weiter dy- 6 namisch

Feb $14 M B \quad$ Die Preise für Wohnimmobilien in 6

Deutschland im Jahr 2013

Nov 14 FStB Immobilienkredite unter Beobach- 12

tung

Feb $15 M B$

Die Preise für Wohnimmobilien in 3

Deutschland im Jahr 2014

Nov 15 FStB

Neue Instrumente für die Wohnim- 6

mobilienfinanzierung in Vorberei-

tung

Quelle: eigene Darstellung; $M B=$ Monatsbericht; FStB $=$ Finanzstabilitätsbericht 


\section{B.3 Transkriptionsregeln}

\begin{tabular}{ll}
\hline Zeichen & Bedeutung \\
\hline, & kurzes Absetzen \\
$(4)$ & Dauer der Pause in Sekunden \\
ja: & Dehnung \\
$(($ lachen $))$ & Kommentar des Transkribierenden (z. B. räuspern, lachen) \\
nein & betont \\
viel- & Abbruch \\
$>$ nein $<$ & leise \\
$\ldots$ & Auslassungen im Transkript \\
$($ ) & Inhalt der Äußerung ist unvollständig \\
\hline
\end{tabular}




\section{Heide Haas}

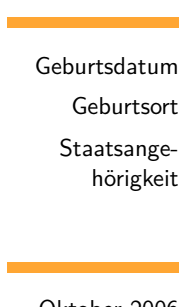

Oktober 2006 bis Juli 2012

\section{Persönliche Daten}

24. März 1987

Bergisch Gladbach

deutsch

\section{Studium}

Diplom, Universität zu Köln, Volkswirtschaftslehre sozialwissenschaftlicher Richtung. Nebenfach: Soziologie.

Wahlpflichtfächer: Empirische Wirtschafts- und Sozialforschung sowie Finanzwissenschaften. Diplomarbeitsthema: "Das internationale Netzwerk der Zentralbanker im Wandel" (Note: 1,3). Gesamtergebnis der Diplomprüfung: 1,5 (sehr gut).

\section{Ausgewählte Publikationen}

\section{Monographien}

2016 Konsequenzen veränderter Finanzierungsbedingungen für die Bauwirtschaft, gemeinsam mit Michael Voigtländer, Ralph Henger, Daniel Bendel und Michael Schier, Bundesinstitut für Bau-, Stadt- und Raumforschung, Online Publikation 01/2016, Bonn.

2015 Die Bedeutung der Langfristfinanzierung durch Banken. Vorteile und zukünftige Herausforderungen, gemeinsam mit Michael Hüther, Michael Voigtländer und Philipp Deschermeier, IW-Analysen, Nr. 101, Köln.

2013 Wirtschaftsfaktor Immobilien 2013. Gesamtwirtschaftliche Bedeutung der Immobilienwirtschaft, gemeinsam mit Michael Voigtländer et al., Berlin.

\section{Zeitschriftenbeiträge}

2016 A first analysis of the new German rent regulation, gemeinsam mit Philipp Deschermeier, Marcel Hude und Michael Voigtländer, International Journal of Housing Policy 16 (3), S. 293-315.

2015 Die Bedeutung makroprudenzieller Regulierung für den deutschen Immobilienmarkt, gemeinsam mit Markus Demary, IW-Trends, 42. Jg., Nr. 4, S. 55-71.

2013 Die Folgen von Solvency II für die Immobilienwirtschaft, gemeinsam mit Kirill Pomogajko und Michael Voigtländer, IW-Trends, 40. Jg., Nr. 4, S. 33-49. 


\section{Policy Papers}

2015 The Impact of Bank Capital Regulation on Financing the Economy. Comments on the Public Consultation of the European Commission on the Possible Impact of the CRR and CRD IV, gemeinsam mit Markus Demary, IW policy paper, Nr. 27, Köln.

2015 Building a Capital Markets Union. Comments on the Public consultation, gemeinsam mit Markus Demary, Vera Demary, Matthias Diermeier, Tobias Henze, Klaus-Heiner Röhl und Jochen Pimpertz, IW Report, 18. Mai, Köln.

2015 Mietpreisdynamik in Köln und Berlin, gemeinsam mit Philipp Deschermeier, IW policy paper, Nr. 21, Köln.

2015 Bankenregulierung und die Folgen für die Immobilienwirtschaft, IW policy paper, Nr. 6/2015, Köln.

2014 Die Folgen der Mietpreisbremse - Eine Analyse am Beispiel der Wohnungsmärkte in Köln und Berlin, gemeinsam mit Philipp Deschermeier, Marcel Hude und Michael Voigtländer, IW policy paper, Nr. 17, Köln.

2014 Transparenz statt Deckelung. Reformpotenziale des Vorfälligkeitsentgelts, gemeinsam mit Michael Voigtländer, IW policy paper, Nr. 13, Köln.

2014 Immobilienkreditfonds. Systemisches Risiko oder Ergänzung zur Bankfinanzierung?, gemeinsam mit Daniel Bendel und Markus Demary, IW policy paper, Nr. 10, Köln.

2013 Reale Nachfrage oder bloße Spekulation. Ist der deutsche Wohnimmobilienmarkt überhitzt?, gemeinsam mit Ralph Henger und Michael Voigtländer, IW policy paper, Nr. 8, Köln.

\section{Ausgewählte Konferenzbeiträge}

Januar 2018 Paradigms of Home Ownership Promotion. The changing Role of Economists in the Knowledge Production for Housing Politics in Germany, Workshop "Housing, planning and urban renewal: enduring challenges in German Politics", Funded by the International Association for the Study of German Politics, Berlin.

Juli 2017 Knowledge Production and Home-Ownership Promotion. The Field of Real Estate Market Analysis in Germany, Tirana ENHR 2017, European Network for Housing Research, Lissabon.

Juni 2015 A first analysis of the new German rent regulation, ENHR Lisboa 2015, European Network for Housing Research, Lissabon. 